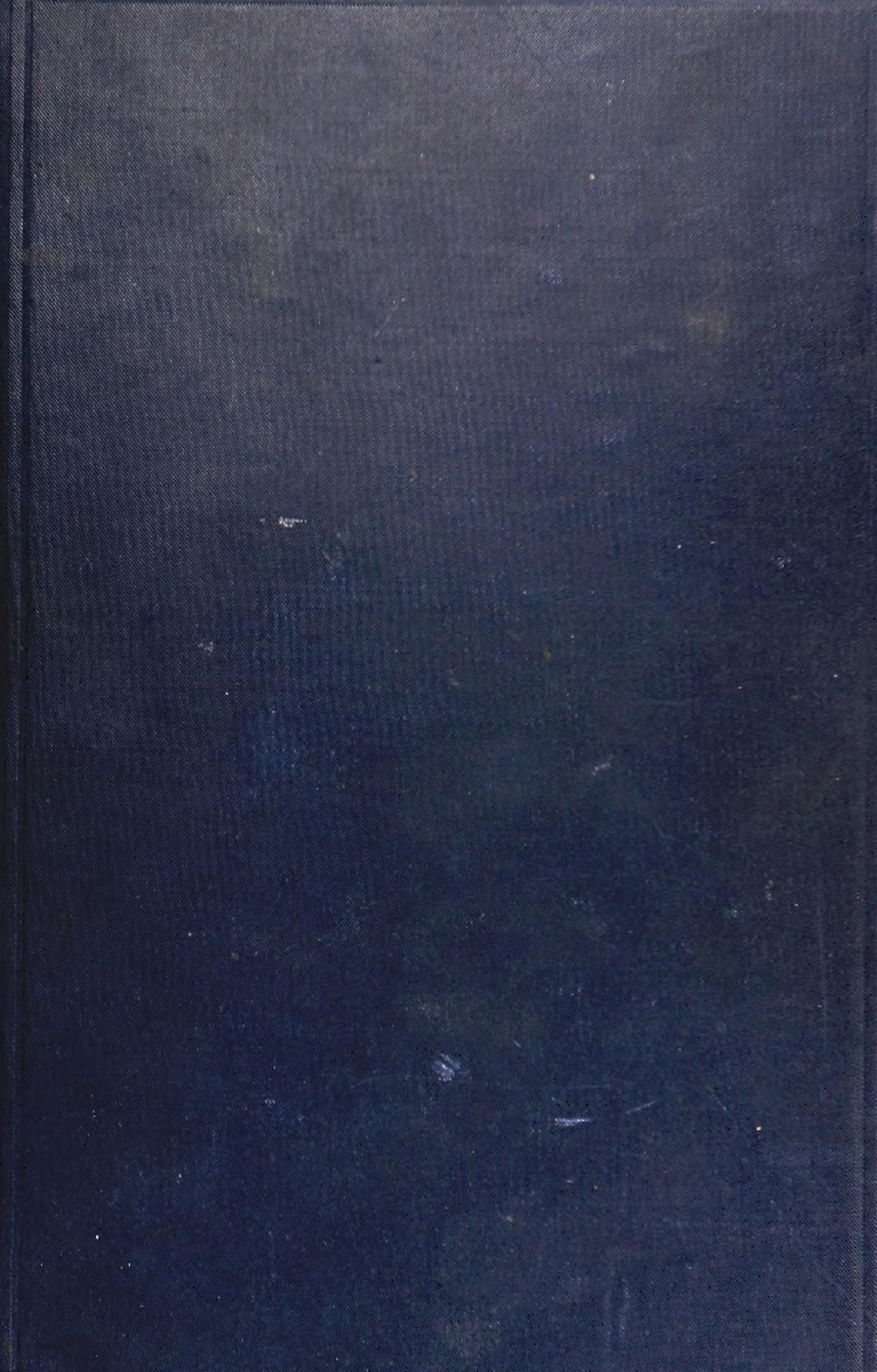




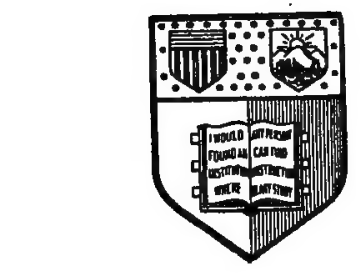

\section{象ew 国ork}

State College of Agriculture

At Cornell antibersity

Jtbaca, ․․ 8 .

\section{Ylibrary}




\section{Cornell University Library}

The original of this book is in the Cornell University Library.

There are no known copyright restrictions in the United States on the use of the text.

http://www.archive.org/details/cu31924052310335 




\section{e QK 901 C62}

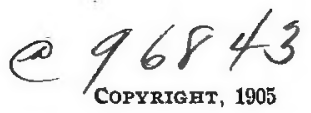

BY FREDERIC E. CLEMENTS AND IRVING S. CUTTER All rights reserved 


\section{PREFACE}

The present volume is intended as a handbook for investigators and for advanced students of ecology, and not as a text-book of the subject. An elementary text-book covering the same field, but adapted to the needs of undergraduate students, is in preparation. The handbook is essentially an account of the methods used by the author in his studies of the last eight years, during which a serious attempt has been made to discover and to correlate the fundamental points of view in the vast field of vegetation. No endeavor is made to treat any portion of the subject exhaustively, since a discussion of general methods and general principles is of much greater value in the present condition of ecology. The somewhat unequal treatment given the different subjects is, due to the fact that it has been found possible to develop some of these more rapidly than others. Finally, it must be constantly kept in mind that ecology is still in a very plastic condition, and in consequence, niethods, fundlamental principles, and matters of nomenclature and terminology must be approached without prejudice in order that the best possible development of this field may be attained.

Grateful acknowledgment for criticisms and suggestions is made to Professor Doctor Charles E. Bessey and Professor Doctor Roscoe Pound, who have read the text. The author is under especial obligations to Doctor Edith $\mathrm{S}$. Clements for the drawings of leaf types, as well as for reading and criticising the manuscript. Professor Goodwin D. Swezey, Professor of Astronomy in the University of Nebraska, has kindly furnished much material for the determination of the sun's altitude, and consequent light intensities, and has read the section devoted to light. Mr. George A. Loveland, Director of the Nebraska Section of the U. S. Weather Bureau, has contributed mary helpful suggestions to the discussion of meteorological instruments. To Nella Schlesinger, Alice Venters, and George L. Fawcett, advanced students in experimental ecology, the author is indebted for many experiments which have been used in the discussion of adjustment and adaptation.

Acknowledgment is also made to the following for various cuts: Henry J. Green, Brooklyn, New York; Julien P. Friez, Baltimore, Maryland; C. H. Stoelting Co., Chicago, Illinois; Draper Manufacturing Co., New York city; Gundlach-Manhattan Optical Co., Rochester, New York; Rochester Optical Co., Rochester, New York; Bausch and Lomb Optical Co., Rochester, New York.

FREDERIC EDWARD CLEMENTS.

The University of Nebraska,

May, 1905. 



\section{CONTENTS}

Chapter I. The Foundation of Ecology

THE NEED OF A SYSTEM

PAGE

I. The scope of ecology . . . . . . . . I I

2. Ecology and physiology . . . . . . . . I I

Historical Development

3. Geographical distribution

4. The plant formation

5. Plant sticcession

6. Ecological phytogeography

7. Experimental ecology

8. Ecology of the habitat

9. The evidence from historical development

Present Status of Ecology

Io. The lack of special training . . . . . . 6

I I. Descriptive ecology . . . . . . . . . 7

r2. The value of floristic . . . . . . . 8

I3. Reconnaissance and investigation . . . . . 8

I4. Resident investigation . . . . . . . . 9

I5. The dangers of a restricted field . . . . . . . 9

Applications of Ecology

I6. The subjects touched by ecology . . . . . . Io

I7. Physiology and pathology . . . . . . . II

I8. Experimental evolution . . . . . . . . II

I9. Taxonomy $. \quad . \quad . \quad . \quad . \quad . \quad . \quad$. $\quad$ I2

20. Forestry . . . . . . . . . . . 14

2I. Physiography . . . . . . . . . . I5

22. Soil physics . . . . . . . . . . 15

23. Zoogeography . . . . . . . . . . $\quad$ I5

24. Sociology . . . . . . . . . . . . 16

THF ESSENTIALS OF A SYSTEM

25. Cause and effect: habitat and plant . . . . . I6

26. The place of function . . . . . . . I7 


\section{Chapter II. The Habitat}

CONCEPT AND ANÄLYSIS

PAGE

27. Definition of the habitat . . . . . . . . I8

28. Factors . . . . . . . . . . I8

Classification of Factors

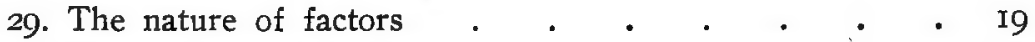

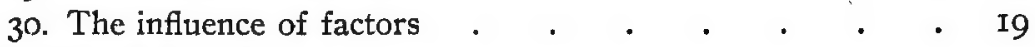

Determination of Factors

3I. The need of exact measurement . . . . . 20

32. The value of meteorological methods . . . . . 20

33. Habitat determination . . . . . . . 2 I

Instrumentation

34. Determinable and efficient differences . . . . 2 I

35. Methods . . . . . . . . . . 22

36. Method of simple instruments . . . . . 22

37. Method of automatic instruments . . . . $\quad 23$

38. Combined methods $\cdot$.

CONSTRUCTION AND USE OF INSTRUMENTS

39. The selection of instruments . . . . . . 24 Water-content

40. Value of different instruments . . . . . 25

Geotome methods

4r. The geotome

42. Soil borers

43. Taking samples of soil

44. Weighing

45. Computation

46. Time and location of readings . . . . $\quad$. 28

47. Location of readings , . . . . . . . . . . . 29

48. Depth of samples . . . . . . . . 30

49. Check and control instruments . . . . . 30

Physical and Physiological, Water

50. The availability of soil water . . . . . . 30

5I. Terms . . . . . . . . . . $3 \mathrm{I}$

52. Chresard determination under control . . . . 32

53. Chresard readings in the field . . . . . . 33

54. Chresard values of different soils . . . . $\quad$. 34 
Records and Results

55. The field record . . . . . . . . 35

56. The pernanent record . . . . . . . . 36

57. Sums and means . . . . . . . . . 36

58. Curves . . . . . . . . . . . . 37

Humidity

59. Instruments $\quad . \quad$. $\quad . \quad$. $\quad . \quad$. $\quad 37$

Psychrometers

6o. Kinds . . . . . . . . . . . . 37

6r. The sling psychrometer . . . . . . . . $\quad . \quad 38$

62. Readings . . . . . . . . . . . . 39

63. Cog psychrometer . . . . . . . . . . 39

64. Construction and use . . . . . . . 40

65. Hygrometers . . . . . . . . . 40

Psychrographs

66. The Draper psychrograph . . . . . . $4 \mathrm{I}$

67. Placing the instrument . . . . . . . 42

68. Regulating and operating the instrument . . . 43

69. The weekly visit . . . . . . . . 44

Humidity Readings and Records

70. The time of readings . . . . . . . 44

71. Place and height . . . . . . . . . 45

72. Check instruments . . . . . . . . 45

73. Humidity tables . . . . . . . . . 46

74. Sums, means, and curves . . . . . . 47

Conversion scale for temperatures

Light

75. Records . . . . . . . . . . 48

76. Methods . . . . . . . . . . 48 .

The Photometer

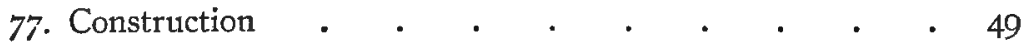

78. Filling the photometer . . . . . . 50

79. Making readings . . . . . . . . 50

80. The Dawson-Lander sun recorder . . . . . $5 \mathrm{I}$

8I: The selagraph . . . . . . . . . 52

Standards

8z. Use . . . . . . . . . . . . 53

83. Making a standard . . . . . . . . 53

Readings

84. Kinds of standards . . . . . . . . $\quad$. 54

85. Time . . . . . . . . $\quad$. $\quad$. 55

Chart for determining sun's altitude $. \quad . \quad . \quad$. 57 
PAGE

86. Taßle for determining apparent noon . . . $\quad 5^{8}$

87. Place . . . . . . . . . . . 59

Table of intensity at various angles . . . . 60

Reflected and Absorbed Light

88. The fate of incident light . . . . . . 60

89. Methods of determination . . . . . . 6I

90. Leaf and epidermis prints . . . . . . . 62

Expression of Results

9r. Light records . $\quad . \quad$. $\quad . \quad$. . . . 63

92. Light sums, means and curves . . . . . 63

\section{Temperature}

93.

Thermometers

94. Air thermometers

95. Soil thermometers

96. Maximum-minimum thermometers

97. Radiation thermometers

Readings

98. Thermographs . . . . . . . . . 67

99. 'Time

I0O. Place and height.

Expression of Results

IOI. Temperature records

102. Temperature sums and means . . . . . . 70

Io3. Temperature curves . . . . . . . . . . 7 I

I04. Plant temperatures . . . . . . . . 7 I

Precipitation

I05. General relations

I06. The rain gauge

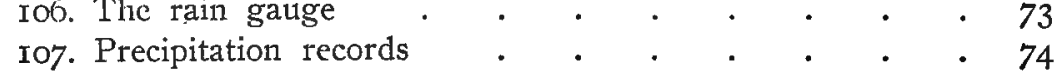

$\begin{array}{llllllll} & \cdot & \cdot & \cdot & \cdot & \cdot & \cdot & \\ 72\end{array}$

Wind

I08. Value of readings

I09. The anemometer

IIO. Records

Soil

III. Soil as a factor

Ir2. The value of soil surveys

II3. The origin of soils

II4. The structure of soils

II5. Mechanical analysis 
I 16. Kinds of soils

II7. The chemical nature of soils . . . . . . . 80 Physiography

I 18 . Factors

Altitude

II9. Analysis into factors

I20. The barometer . . . . . 82

Slope

I2r. Concept

122. The clinometer

I23. The trechometer

Exposure

124. Exposure

125. Surface

126. Record of physiographic factors

127. Topography

Biotic Factors

128. Influence and importance

129. Animals

I30. Plants

METHODS OF HABITAT INVESTIGATION

I3I.

Method of Simple Instruments

r32. Choice of stations

133. Time of readings

I34. Details of the method.

I35. Records

Method of Ecograph! Batteries

I36.

Expression of Physical Factor Results

I37. The form of results

Factor Records

I38.

Factor Curves

I39. Plotting

I4O. Kinds of curves .

I4I. Combinations of curves

142. The amplitude of curves

Factor Means and Sums 


\section{Chapter III. The Plant}

\section{STIMULUS AND RESFONSE}

General Relations

PAGE

I44. The nature of stimuli

145. The kinds of stimtili

I46. The nature of response

I47. Adjustment and adaptation

I48. The measurement of response

I49. Plasticity and fixity

I50. The law of extremes

I5 I. The method of working hypotheses

Hydroharmose

\section{Adjustment}

I52. Water as a stimulus . . . . . . . . I07

I53. The influence of other factors upon water. . . . 107

I54. Response . . . . . . . . . . . 108

155. The measurement of absorption . . . . . . . . I09

I56. The quantitative relation of absorption and transpiration . I I I

I57. Measurement of transpiration . . . . . . II3

I58. Field methods . . . . . . . . . . II4

I59. Expression of results . . . . . . . . . II6

I60. Coefficient of transpiration . . . . . . II7

Adaptation

I6r. Modifications due to water stimuli . . . . . II8

I62. Modifications due to a small water supply . . . . I I8

I63. The decrease of water loss . . . . . . . . . II8

I64. The increase of water supply. . . . . . . I2I

165. Modifications due to an excessive water supply . . . I2I

I66. Plant types . . . . . . . . . . I22

I67. Xerophytic types . . . . . . . . . $\quad$. 122

I68. Types of leaf xerophytes . . . . . . . . I23

I69. Types of stem xerophytes . . . . . . . . 125

I70. Bog plants . . . . . . . . . . . 126

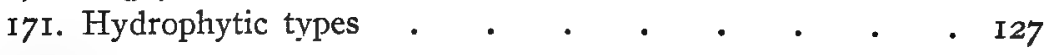

Photoliarmose

Adjustment

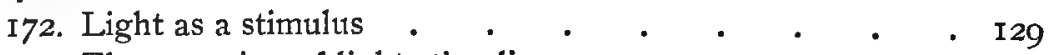

I73. The reception of light stimuli . . . . . . I3I

I74. The response of the chloroplast. . . . . . ${ }^{132}$

I75. Aeration and translocation . . . . . . . . I34 
I76. The measurement of responses to light Adaptation

177. Influence of chloroplasts upon form and structure . . I38

I78. Form of leaves and stems . . . . . . . $\quad$ I39

I79. Modification of the epidermis . . . . . . I40

180. The differentiation of the chlorenchym . . . . I42

I8I. Types of leaves . . . . . . . . . I44

I82. Heliophytes and sciophytes . . . . . . I44

EXPERIMENTAL EVOLIJION

183. Scope . . . . . . . . . . . I45

184. Fundamental lines of inquiry . . . . . . I46

I85. Ancestral form and structure . . . . . . I46

186. Variation and mutation . . . . . . . . . I47

I87. Methods . . . . . . . . . . I49

Method of Natural Experiment

I88. Selection of species . . . . . . . . . . I49

I89. Determination of factors . . . . . . . I5I

Igo. Method of record . . . . . . . . . . I52

Method of Habitat Cultures

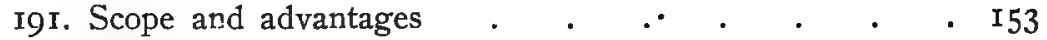

I92. Methods . . . . . . . . . . . I53

I93. Transfer . . . . . . . . . . . . . I54

I94. Modification of the habitat . . . . . . . I56

Method of Control Cultures

I95. Scope and procedure . . . . . . . . I57

I96. Water-content series . . . . . . . . I58

I97. Light series . . . . . . . . . I60

Chapter IV. The Formation

METHODS OF INVESTIGATION AND RECORD

I98. The need of exact methods . . . . . . . I6I

Quadrats

I99. Uses . . . . . . . . . . . I6I

200. Possible objections . . . . . . . . . . $\quad$ I63

Kinds of Quadrats and Their Use

201. Size and kinds . . . . . . . . . . . I64

202. Tapes and stakes $. \quad . \quad . \quad . \quad . \quad . \quad . \quad . \quad$ I64

203. Locating quadrats . . . . . . . . . . $\quad$. 165 
The List Quadrat

204. Description . . . . . 165

205. Manner of use . . . . . . . . . 166

206. Table of abundance. . . . . . . . . . 166

The Chart Quadrat

207. Description and use . . . . . . . I67

208. The chart . . . . . . . . . . I68

209. Mapping . . . . . . . . . . . . . . . . . . .

2IO. Factors and photographs . . . . . . . I70

The Permanent Quadrat

2 II. Description and uses. . . . . . . . I70

2I2. Manner of use . . . . . . . . . 172

The Denuded Quadrat

213. Description . . . . . . . • . 173

2I4. Methods of denuding and recording . . . . I74

2I 5. Physical factors . . . . . . . . . I75

Aquatic Quadrats

216. Scope .

175

Transects

2I7. The transect

The Line Transect

218. Description and method . . . . . . . I76

219. The location and size. . . . . . . . . . 177

The Belt Transect

220. Details

The Permanent 'Transect

221. Advantages

222. Details

The Denuded Transect

223.

The Layer Transect

224.

Ecotone Charts

225.

The Migration Circle

226. Purpose

227. Location and method.

228. The denuded circle

229. Photographs

Cartography

230. Value of cartographic methods . 
23r. Standard scale

\section{DEVELOPMENT AND STRUCTURE}

247. Vegetation an organism

248. Vegetation essentially dynamic

249. Functions and structurés

250. Concept .

251. Causes

252. Aggregation

Kinds of Association

253. Categories .

254. Stratum association

. . . . . . . 204

255. Ground association . . . . . . . . . 205

256. Species guild association . . . . . . . 206

257. Light association . . . . . . . . 206

258. Water-content association . • . . • . . 208

Invasion

THE DEVELOPMENT OF THE FORMATION

259.

260.

Migration $26 \pi$. 
262. Mobility . . . . . . . . . . 2 II

263. Organs for dissemination . . . . . . 2 I I

264. Contrivances for dissemination . . . . . . 212

265. Position of disseminules . . . . . . . . 214

266. Seed production. . . . . . . . . . 2 I5

267. Agents of migration . . . . . . . . . . . 216

268. The direction of migration $\quad . \quad . \quad . \quad . \quad . \quad$. 2 I9

Ecesis

269. Concept . . . . . . . . . . . . 220

270. Germination of the seed . . . . . . . . 22 I

27I. Adjustment to the habitat . . . . . . . 223

Barriers

272. Concept . . . . . . . . . . . 224

273. Physical barriers . . . . . . . . 225

274. Biological barriers . . . . . . . . . 225

275. Influence of barriers . . . . . . . . 226

Endemism

276. Concept . . . . . . . . . . 227

277. Causes . . . . . . . . . . . 228

278. Significance . . . . . . . . . . 228

Polyphylesis and Polygenesis

279. Concept

280. Proofs of polygenesis

281. Origin by polyphylesis

Kinds of Invasion

282. Continuous and intermittent invasion

283. Complete and partial invasion

284. Permanent and temporary invasion.

Manner of Invasion

285. Entrance into the habitat . . . . $\therefore .236$

286. Influence of levels . . . . . . . . 238

Investigation of Invasion

287.

Succession

288. Concept

289. Kinds of succession .

- 239

Primary Successions

290. . . . . . . . . . . . . 24I

29I. Succession through elevation . . . . . . $24 \mathrm{I}$

292. Succession through volcanic action . . . . . 242

293. Weathering . . . . . . . . . . 243 
294. Succession in residuary soils 


\section{The relict method
THE STRUCTURE OF THE FORMATION}

Causes of Zonation

333. Growth

334. Reactions

335. Physical factors .

336. Physiographic symmetry

Kinds of Zonation

337.

338. Radial zonation .

339. Bilateral zonation

340. Vertical zonation

Alternation

34I. Vegetation zones

342. Concept

343. Causes

344. Competition

345 . Kinds of alternation

The Formation in Detail

346. The rank of the formation. . . . . . . 292

347. The parts of a formation . . . . . . . . 295

348. Nomenclature of the divisions . . . . . . . . 299

349. The investigation of a particular formation . . . 299 Classification and Relationship

350. Basis . . . . . . . . . • . 300

35I. Habitat classification. . . . . . . . . . 301

352. Nomenciature . . . . . . . . . 302

353. Developmental classification . . . . . . 304

354. Regional classification . . . . . . . 304

355. Mixed formations . . . . . . . . 304

\section{EXPERIMENTAL VEGETATION}

356. Scope and methods

Method of Natural Habitats

357. Natural experiments .

Method of Artificial Habitats

3.58. Modification of habitat 
362. Details of culture methods . . . . . . 3II

GLOSSARY

BIBLIOGRAPHY 



\title{
RESEARCH METHODS IN ECOLOGY
}

\author{
CHAPTER I. THE FOUNDATION OF ECOLOGY \\ The Need of a System
}

1. The scope of ecology. The clue to the field of ecology is found in the Greek word, oikos, home. The point of view in the following treatise is constantly that which is inherent in the term itself. Ecology is therefore considered the dominant theme in the study of plants, indeed, as the central and vital part of botany. This statement may at first appear startling, if not unfounded, but mature reflection will show that all the questions of botanical science lead sooner or later to the two ultimate facts: plant and habitat. The essential truth of this has been much obscured by detached methods of study in physiology, morphology, and histology, which are too often treated as independent fields. These have suffered incomplete and unsymmetric development in consequence of extreme specialistic tendencies. Analytic methods have dominated research to the exclusion of synthetic ones, which, in a greatly diversified field, must be final, if botanical knowledge is something to be systematized and not merely catalogued. Physiology in particular has suffered at the hands of detached specialists. Originally conceived as an inquiry into the origin and nature of plants, it has been developed strictly as a study of plant activities. It all but ignores the physical factors that control function, and the organs and tissues that refiect it.

2. Ecology and physiology. There can be little question in regard to the essential identity of physiology and ecology. This is evident when it is clearly seen that the present difference between the two fields is superficial. Ecology has been largely the descriptive study of vegetation; physiology has concerned itself with function; but, when carefully analyzed, both are seen to rest upon the same foundation. In each, the development is incomplete: ecology has so far been merely superficial, physiology too highly specialized. The one is chaotic and unsystematized, the other too often a minute study of function under abnormal circumstances. The greatest need of the former is the introduction of method and system, of the latter, a broadening of scope and new objectives. The growing recognition of the identity of the two makes it desirable to anticipate their final merging, and 
to formulate a system that will combine the good in each, and at the same time eliminate superficial and extreme tendencies. In this connection, it becomes necessary to point out to ecologist and physiologist alike that, while they have been working on the confines of the same great field, each must familiarize himself with the work and methods of the other, before his preparation is complete. Both must broaden their horizons, and rearrange their views. The ecologist is sadly in need of the more intimate and exact methods of the physiologist: the latter must take his experiments into the field, and must recognize more fully that function is but the middleman between habitat and plant. It seems probable that the final name for the whole field will be physiology, although the term ecology has distinct advantages of brevity and of meaning. In this event, however, it should be clearly recognized that, although the name remains the same, the field has become greatly broadened by new viewpoints and new methods.

\section{HISTORICAL DEVELOPMENT}

3. Gengraphical distribution. The systematic analysis of the great field of ecology is essential to its proper development in the future. A glance at its history shows that, while a number of essential points of attack have been discovered, only one or two of these have been organized, and that there is still an almost entire lack of correlation and coordination between these. The earliest and simplest development of the subject was concerned with the distribution of plants. This was at first merely an off-shoot of taxonomy, and, in spite of the work of Humboldt and Schouw, has persisted in much of its primitive form to the present time, where it is represented by innumerable lists and catalogues. Geographical distribution was grounded upon the species, a fact which early caused it to become stereotyped as a statistical study of little value. This tendency was emphasized by the general practice of determining distribution for more or less artificial areas, and of instituting comparisons between tegions or continents too little known or too widely remote. The fixed character of the subject is conclusively shown by the fact that it still persists in almost the original form more than a half century after Grisebach pointed out that the formation was the real unit of vegetation, and hence of distribution.

4. The plant formation. The corner-stone of ecology was laid by Grisebach in 1838 by his recognition of the plant formation as the fundamental feature of vegetation. Earlier writers, notably Linné (1737, I75I), Biberg ( 1749 ), and Hedenberg ( 1754 ), had perceived this relation more or less clearly, but failed to reduce it to a definite guiding principle. This was a natural result of the dominance of descriptive botany in the I8th century, 
by virtue of which all other lines of botanical inquiry languished. This tendency had spent itself to a certain degree by the opening of the Igth century, and both plant distribution and plant physiology began to take form. The stimulus given the former by Humboldt ( 1807 ) turned the attention of botanists more critically to the study of vegetation as a field in itself, and the growing feeling for structure in the latter led to Grisebach's concept of the formation, which he defined as follows: "I would term a group of plants which bears a definite physiognomic character, such as a meadow, a forest, etc., a phytogeographic formation. The latter may be characterized by a single social species, by a complex of dominant species belonging to one family, or, finally, it may show an aggregate of species, which, though of various taxonomic character, have a common peculiarity; thus, the alpine meadows consist almost exclusively of perennial herbs." The acceptance of the formation as the unit of vegetation took place slowly, but as a result of the work of Kerner (1863), Grisebach (1872), Engler (I879), Hult (I88I, I885), Goeze (I882), Beck (I884), Drude (I889), and Warming ( 1889 ), this point of view came tc be more and more prevalent. It was not, however, until the appearance of three works of great importance, Warming ( 1895 ), Drude ( 1896 ), and Schimper (I898), that the concept of the formation became generally predominant. With the growing recognition of the fornution during the last decade has appeared the inevitable tendency to stereotype the subject of ecology in this stage. The present need, in consequence, is to show very clearly that the idea of the formation is a fundamental, and not an ultimate one, and that the proper superstructure of ecology is yet to be reared upon this as the foundation.

5. Plant succession. The fact that formations arise and disappear was perceived by Biberg as early as I749, but it received slight attention until Steenstrup's study of the succession in the forests of Zealand (I844 prox.). In the development of formations, as well as in their recognition, nearly all workers have confined themselves to the investigation of particular changes. Berg (I844), Vaupell (I85I), Hoffmann (I856), Middendorff (I864), Hult (I88I), Senft ( I888), Warming (I890), and others have added nutuch to our detailed knowledge of formational development. Notwithstanding the lapse of more than a half century, the study of plant successions is by no means a general practice among ecologists. This is a ready explanation of the fact that the vast field has so far yielded but few generalizations. Warming (1895) was the first to compile the few general principles of development clearly indicated up to this time. The first critical attempt to systematize the investigation of succession was made by Clements (1904), though this can be considered as little more than a beginning on account of 
the small number of successions so far studied. Future progress in this field will be conditioned not only by the more frequent study of developmental problems by working ecologists, but also, and most especially, by the application of known principles of succession, and by the working out of new ones.

6. Ecological phytogeography. Until recent years, the almost universal tendency was to give attention to formations from the standpoint of vegetation alone. While the habitat was touched here and there by isolated workers, and plant functions were being studied intensively by physiologists, both were practically ignored by ecologists as a class. The appearance of Warming's Lehrbuch der oecologischen PAlanzengeographie (1896) and of Schimper's Pflanzengeographie auf physiologischer Grundlage (1898) remedied this condition in a measure by a general discussion of the habitat, and by emphasizing the importance of the ecological or physiological point of view. Despite their frank recognition of the unique value of the habitat, the major part of both books was necessarily given to what may be termed the general description of formations. For this reason, and for others arising out of an almost complete dearth of methods of investigation, ecology is still almost entirely a floristic study in practice, although there is a universal recognition of the much greater value of the viewpoint which rests upon the relation between the formation and its habitat.

7. Experimental ecology. Properly speaking, the experimental study of ecology dates from Bonnier ${ }^{1}$ ( 1890,1895 ), though it is well understood that experimental adjustment of plants to certain physical factors had been the subject of investigation before this time. The chief merit of Bonnier's work, however, lies in the fact that it was done out of doors, under natural conditions, and for these reasons it should be regarded as the real beginning of this subject. Bonnier's experiments were made for the purpose of determining the effect of altitude. Culture plots of certain species were located in the Alps and the Pyrenees, and the results were compared with control cultures made in the lowlands about Paris. In I894 he also made

'BONNIER, G.

Les Plantes Arctiques Comparées aux Mêmes Espèces des Alps et des Pyrénkes. Rev. Gen. Bot. 6:505. 1894.

Cultures Expérimentales dans les Alps et lẹs Pyrénées. Rev. Gen. Bot. 2:514. 1890. Recherches Expérimentales sur l'Adaptation des Plantes au Climat Alpin. Ann. Nat. Sci. $7: 20: 218$. 1895 .

Bonnier, G., et Ch. Flahault

Modifications des végétaux sur l'influence des conditions physiques du milieu. Ann. Nat. Sci. 6:7:93. 1878, 
a comparative study of certain polydemic species common to the arctic islands, Jan Meyen and Spitzenberg, and to the Alps. Both of these methods are fundamental to field experiment, but the results are inconclusive, inasmuch as altitude is a complex of factors. As no careful study was made of the latter, it was manifestly impossible to refer changes and differences of structure to the definite cause. In a paper that has just appeared, E. S. Clements (Ig05) has applied the method of polydemic comparison to nearly a hundred species of the Rocky mountains. In this work, the all-important advance has been made of determining accurately the decisive differences between the two or more habitats of the same species in terms of direct factors, water-content, humidity, and light. In his own investigations of Colorado mountain vegetation, the author has applied the method of field cultures by planting seeds of somewhat plastic species in habitats of measured value, and has thought to initiate a new line of research by applying experimental methods to the study of vegetation as an organism. In connection with this, there has also been developed a method of control experiment in the plant house under definitely measured differences of water and light.

8. Ecology of the habitat. Since the time of Humboldt, there have been desultory attempts to determine the physical factors of habitats with some degree of accuracy. The first real achievement in this line was in the measurement of light values by Wiesner in 1896 . In 1898 the writer first began to study the structure of habitats by the determination of watercontent, light, humidity, temperature, wind, etc., by means of instruments. These methods were used by one of his pupils, Thornber (IgOI), in the study of a particular formation, and by another, Hedgcock (1902), in a critical investigation of water-content. Two years later, similar methods of measuring physical factors were put into operation in connection with experimental evolution under control in the plant house. E. S. Clements (1905), as already indicated, has made the use of factor instruments the foundation of a detailed study of polydemic species, i. e., those which grow in two or more habitats, and which are, indeed, the most perfect of all experiments in the production of new forms. In a volume in preparation upon the mountain vegetation of Colorado, the writer has brought the use of physical factor instruments to a logical conclusion, and has made the study of the habitat the basis of the whole work. Out of this investigation has come a new concept of vegetation (Clements I904), namely, that it is to be regarded as a complex organism with structures and with functions susceptible of exact methods of study. 
9. The evidence from historical development. This extremely brief resume of what has been accomplished in the several lines of ecological research makes evident the almost complete absence of correlation and of system. The whole field not merely lacks system, but it also demands a much keener perception of the relative value of the different tendencies already developed. It is inevitable from the great number of tyros, and of dilettante students of ecology in comparison with the few specialists, that the surface of the field should have received all of the attention. It is, however, both unfortunate and unscientific that great lines of development should be entirely unknown to all but a few. There is no other department of botany in which the superficial study of more than half a century ago still prevails to the exclusion of better methods, many of which have been known for a decade or more. It is clear, then, that the imperative need of ecology is the proper coordination of its various points of view, and the working out of a definite system which will make possible a ready recognition of that which is fundamental and of that which is merely collateral. The historical development, as is well understood, can furnish but a slight clite to this. It is a fact of common knowledge that the first development of any subject is general, and usually superficial also. True values come out clearly only after the whole field has been surveyed. For these reasons, as will be pointed out in detail later, the newer viewpoints are regarded as either the most important or the most fundamental. Experimental ecology will throw a flood of light upon plant structure and function, while exact methods of studying the habitat are practically certain of universal application in the future.

\section{PRESENT STATUS OF ECOLOGY}

10. The lack of special training. The bane of the recent development popularly known as ecology has been a widespread feeling that anyone can do ecological work, regardless of preparation. There is nothing in modern botany more erroneous than this feeling. The whole task of ecology is to find out what the living plant and the living formation are doing and have done in response to definite complexes of factors, i. e., habitats. In this sense, ecology is practically coextensive with botany, and the student of a local flora who knows a few hundred species is no more competent to do ecological work than he is to reconstruct the phylogeny of the vegetable kingdom, or to explain the transmission of ancestral qualities. The comprehensive and fundamental character of the subject makes a broad special training even more requisite than in more restricted lines of botanical inquiry. The ecologist must first of all be a botanist, not a mere cataloguer of plants, and he must also possess a particular training in the special meth- 
ods of ecological research. He must be familiar with the various points of attack in this field, and he must know the history of his subject thoroughly. Ecology affords the most striking example of the prevalent evil of American botanical study, i. e., an indifference to, or an ignorance of the literature of the subject. The trouble is much aggravated here, however, by the breadth of the field, and the common assumption that a special training is unnecessary, if not, indeed, superfluous. Ignorance of the important ecological literature has been a most fertile source of crude and superficial studies, a condition that will become more apparent as these fields are worked again by carefully trained investigators.

11. Descriptive ecology. The stage of development of the subject at the present time may be designated as descriptive ecology, for purposes of discussion merely. This is concerned with the superficial description of vegetation in general terms, and results from the fact that the development has begun on the surface, and has scarcely penetrated beneath it. The organic connection between ecology and floristic has produced an erroneous impression as to the relative value of the two. Floristic has required little knowledge, and less preparation: it lends itself with insidious ease to chance journeys or to vacation trips, the fruits of which are found in vague descriptive articles, and in the multiplication of fictitious formations. While there is good reason that a record should be left of any serious reconnaissance, even though it be of a few weeks' duration, the resulting lists and descriptive articles can have only the most rudimentary value, and it is absurd to regard them as ecological contributions at all. No statement admits of stronger emphasis, and there is none that should be taken more closely to heart by botanists who have supposed that they were doing ecological work. An almost equally fertile source of valueless work is the independent treatment of a restricted local area. The great readiness with which floristic lists and descriptions can be made has led many a botanist, working in a small area, or passing hurriedly through an extended region, to try his hand at formation making. From this practice have resulted scores of so-called formations, which are mere patches, consocies, or stages in development, or which have, incleed, no existence other than in the minds of their discoverers. The misleading definiteness which a photograph seems to give a bit of vegetation has been responsible for a surplus of photographic formations, which have no counterparts in nature. Indispensable as the photograph is to any systematic record of vegetation, its use up to the present time has but too often served to bring it into disrepute. There has been a marked tendency to apply the current methods of descriptive botany to vegetation, and to fegard every slightly different piece of the floral covering 
as a formation. No method can yield results further from the truth. It is evident that the recognition and limitation of formations should be left absolutely to the broadly trained specialist, who has a thorough preparation by virtue of having acquainted himself carefully with the development and structure of typical formations over large areas.

12. The value of floristic. In what has been said above, there is no intent to decry the value of floristic. The skilled workman can spare the material. which he is fashioning as readily as the ecologist can work without an accurate knowledge of the genera and species which make up a particular vegetation. Some botanists whose knoivledge of ecology is that of the study or the laboratory have maintained that it is possible to investigate vegetation without knowing the plants which compose it. Ecology is to be wrought out in the field, however, and the field ecologist-none other, indeed, should bear the name-understands that floristic alone can furnish the crude material which takes form under his hands. It is the absolute need of a thorough acquaintance with the flora of a region which makes it impossible for a traveler to obtain anything of real ecological value in his first journey through a country. As the very first step, he must gain at least a fair knowledge of the floristic, which will alone take the major part of one or more growing seasons. This information the student of a local flora already has at the tip of his tongue; in itself it can not constitute a contribution to ecology, but merely the basis for one. In this connection, moreover, it can not be used independently, but becomes of value only after an acquaintance with a wide field. Floristic study and floristic lists, then, are indispensable, but to be of real value their proper function must be clearly recognized. They do not constitute ecology.

13. Reconnaissance and investigation. In striving to indicate the true value and worth of ecological study, it becomes necessary to draw a definite line between what we may term reconnaissance and investigation. By the former is understood the preliminary survey of a region, extending over one or two years. The objects of such a survey are to obtain a comprehensive general knowledge of the topography and vegetation of the region, and of its relation to the other regions about it. The chief purpose, however, is to gain a good working acquaintance with the flora: a reconnaissance to be of value must do this at all events. Certain general facts will inevitably appear during this process, but they will invariably need the confirmation of future study. It would be an advantage to real ecology if reconnaissance were to confine itself entirely to the matter of making a careful floristic survey. Investigation begins when the inquiry is directed 
to the habitat, or to the development and structure of the formation which it bears, i. e., when it takes up the manifold problems of the oikos. Such a study must be based upon floristic, but the latter becomes a part of inrestigation only in so far as it leads to it. Standing by itself, it is not ecological research: it is the preparation for it. This distinction deserves careful thought. The numerous recruits to ecology have turned their attention to what lay nearest to hand, with little question as to its value, or to where it might lead. The result has been to make reconnaissance far outweigh investigation in amount, and to give it a value which properly belongs to the latter. Furthermore, this mistaken conception has in many cases, without doubt, prevented its leading to valuable research work.

14. Resident investigation. Obviously, if reconnaissance is a superficial survey, and investigation thorough extensive study, an important distinction between then is in the time required. While one nuay well be the result of a journey of some duration, the other is essentially dependent upon residence. In the past the great disparity between the size of the field and the number of workers has made resident study too often an ideal, but in the future it will be increasingly the case that a particular region will be worked by a trained ecologist resident in it. This mav never be altogether true of inaccessible and' sterile portions of the globe. It may be pointed out, however, that, between the tropics and the poles, residence during the summer or growing period is in essence continuous residence. In the ultimate analysis, winter conditions have of course some influence upon the development of vegetation during the summer, but the important problems which a vegetation presents must be worked out during the period of development. For temperate, arctic, and alpine regions, then, repeated study during the growing period for a term of years has practically all the advantages of continuous residence. For all practical purposes, it is resident study.

15. The dangers of a restricted field.. In the resident study of a particular region, the temptation to make an intensive investigation of a circumscribed area is very strong. The limits imposed by distance are alone a sufficient explanation of this, but it is greatly increased by the inclination toward detailed study for which a small field offers opportunity. This temptation can be overcome only by a general preliminary study of the larger region in which the particular field is located. The broader outlook gained in this way will throw needed light upon many obscure facts of the latter, and at the same time it will act as a necessary corrective of the tendency to consider the problems of the local field in a detached manner, and to magnify the value of the distinctions made and the results obtained. 
Such a general survey has the purpose and value of a reconnaissance, and is always the first step in the accurate and detailed investigation of the local area or formation. Each corrects the extreme tendency of the other, and thorough comprehensive work can be done only by combining the two methods. When the field of inquiry is a large area or covers a whole region, the procedure should be essentially the same. A third stage must be added, however, in which a more careful survey is made of the entire field in the light of the thorough study of the local area. The writer's methods in the investigation of the Colorado vegetation illustrate this procedure. The summers of $1896,1897,1898$ were devoted to reconnaissance; those of I899-I904 were given to detailed and comprehensive study by instrument and quadrat of a highly diversified, representative area less than 20 miles square, while the work of the final summer will be the application of the results obtained in this localized area to the region traversed from I896-98. This is practically the application of methods of precision to an area of more than 100,000 square miles. It also serves to call attention to another point not properly appreciated as yet by those who would do ecological work. This is the need of taking up field problems as a result of serious forethought, and not as a matter of accident or mere propinquity. A carefully matured plan of attack which contemplates an expenditure of time and energy for a number of years will yield results of value, no matter how much attention an area may have received. On the other hand, an aimless or hurried excursion into the least known or richest of regions will lead to nothing but a waste of time, especially upon the part of the ecologist, who mitst read the articles which result, if only for the purpose of making sure that there is nothing in them.

\section{APPLICATIONS OF ECOLOGY}

16. The subjects touched by ecology. The applications of ecological methods and results to other departments of botany, and to other fields of research are numerous. Many of these are both intimate and fundamental, and give promise of affording new and extremely fruitful points of view. It has already been indicated that ecology bears the closest of relations to morphology and histology on the one side, and to physiology on the otherthat it is, indeed, nothing but a rational field physiology, which regards form and function as inseparable phenomena. The closeness with which it touches plant pathology follows directly from this, as pathology is nothing more than abnormal form and functioning. Experimental work in ecology is purely a study of evolution, and the facts of the latter are the materials with which taxonomy deals. Forestry has already been termed "applied 
ecology" and in its scientific aspects, which are its foundation, it is precisely the ecology of woody plants, and of the vegetation which they constitute. Apart from botany, the physical side of ecology is largely a question of soil physics, and of physiography. On the other hand, vegetation is coming more and more to be regarded as a fundamental factor in zoogeography and in sociology. Furthermore, with respect to the latter, it will be pointed out below that the principles of association which have been determined for plants, viz., invasion, succession, zonation, and alternation, apply with almost equal force to man.

17. Physiology and pathology. The effect of ecology in emphasizing the intrinsically close connection between physiology and morphology has already been mentioned. Its influence in normalizing the former by forcing it into the field as the place for experiment, and by directing the chief attention to the plant as an organism rather than a complex of organs, is also rapidly coming to be felt. Ecology will doubtless exert a corrective influence upon pathology in the near future. This is inevitable as the latter ceases to be the merely formal study of specific pathogenic organisms, and turns its attention to the cause of all abnormality, which is to be found in the habitat, whether this be physical, as when the water-content is low, or biotic, when a parasitic fungus is present. The relative ease with which specific diseases can be studied has helped to obscure the essential fact that the approach to pathology must be through physiology. Much indeed of the observational physiology of the laboratories has been pathology, and it will be impossible to draw a clear line between them until precise experiment in the habitat has come into vogue.

18. Experimental evolution. As a result of the extremely fragmentary character of the geological record, nothing is more absolute than that there can be no positive knowledge of the exact origin of a form or species, except in those rare cases of the present day, where the whole process has taken place under the eye of a trained observer. The origin of the plant forms known at present must forever lie without the domain of direct knowledge. If it were possible by a marvel of ingenuity and patience to develop experimentally Myosurus from Selaginella, this would not be absolutely conclusive proof that Myosurus was first derived in this way. When all is said, however, this would be the very best of presumptive evidence. It must also be recognized that this is the nearest to complete proof that we shall ever attain, and with this in mind it becomes apparent at once that evidence from experiment is of paramount importance in the study of evolution (the origin of species). 
The phase of experimental ecology which has to do with the plant has well been called experimental evolution. While this is a field almost wholly without development at present, there can be little question that it is to be one of the most fertile and important in the future. Attention will be directer first to those forms which are undergoing modification at the present time. The cause and direction of change will be ascertained, and its amount and rapidity measured by biometrical methods. The next step will be to actually change the habitat of representative types, and to determine for each the general trend of adaptation, as well as the exact details. By means of the methods used and the results obtained in these investigations, it will he possible to attack the much more difficult problem of retracing the development of species already definitely constituted. This will be accomplished by the study of the derived and the supposed ancestral form, but owing to the great preponderance of evolution over reversion, the study of the ancestral form will yield much more valuable results.

The general application of the methods of experimental ecology will mark a new era in the study of evolution. There has been a surplus of literary investigation, but altogether too little actual experiment. The great value of De Vries ${ }^{3}$ work lies not in the importance of the results obtained, but in calling attention to the unique importance of experimental methods in contributing to a knowledge of evolution. The development of the latter has been greatly hindered by the dearth of actual facts, and by a marked tendency to compensate for this by verbiage and dogmatism. This is well illustrated by the present position of the "mutation theory," which, so far as the evidence available is concerned, is merely a working hypothesis. An incredible amount of bias and looseness of thought have characterized the discussion of evolution. It is earnestly to be hoped that the future will bring more work and less argument, and that the literary evolutionists will become less and less reluctant to leave the relative merits of variation and mutation to experiment.

19. Taxonomy. Taxonomy is classified evolution. It is distinct from descriptive botany, which is merely a cataloguing of all known forms, with little regard to development and relationship. The consideration of the latter is peculiarly the problem of taxonomy, but the solution must be sought through experimental evolution. The first task of the latter is to determine the course of modification in related forms, and the relationships existing between them. With this information, taxonomy can group forms according to their rank, i. e., their descent. The same method is applicable to the species of a genus, and, in a less degree, perhaps, to the genera which constitute a family. The use to which it may be put in indicating family re- 
lationships will depend largely upon the gap existing between the families concerned. While interpretation will always play a part in taxonomy, the general use of experiment will leave much less opportunity for the personal equation than is at present the case. Taxonomy, like descriptive botany, is based tpon the species, but, while there may exist a passable kind of descriptive botany, there can be no real taxonomy as long as the sole criterion of a species is the difference which any observer thinks he sees between one plant and another. The so-called species of to-day range in value from mere variations to true species which are groups of great constancy and definiteness. The reasons for this are obvious when one recalls that "species" are still the product of the herbarium, not of the field, and that the more intensive the study, the greater the output in "species." It would seen that careful field study of a form for several seasons would be the first requisite for the making of a species, but it is a precaution which is entirely ignored in the vast majority of cases. The thought of subjecting forms presumed to be species to conclusive test by experiment has apparently not even occurred to descriptive botanists as yet. Notwithstanding. there can be no serious doubt that the existing practice of re-splitting hairs must come to an end sooner or later. The remedy will come from without through the application of experimental methods in the hands of the ecologist, and the cataloguing of slight and unrelated differences will yield to an ordered taxonomy.

Experimental evolution will solve a taxonomic problem as yet untouched, namely, the effect of recent environment upon the production of species. It is well understood that some species grow in nature in various habitats without suffering material change, while others are modified to constitute a new form in each habitat. It is at once clear that these forms (or ecads) are of more recent descent than the species, i. e., of lower rank. It must a!so be recognized that a constant group and a highly plastic one are essentially different. If constancy is made a necessary quality of a species, one is a species, the other is not. If both are species, then two different kinds must be distinguished. Among the species of our manuals are found many ecads, alongside of constant and inconstant species. These can be distinguished only by field experiment, and their proper coordination is possible cnly after this has been done. Indeed, the whole question of the ability or the inability of environmental variation to produce constant species is one that must be referred to repeated and long-continued experiment in the field.

A minor service of considerable value can be rendered taxonomy by working over the diagnosis from the ecological standpoint. Many ecological facts are of real diagnostic value, while others are at least of much interest, and serve to direct attention to the plant as a living thing. The loose use of 
terms denoting abundance, which prevails in lists and manuals, should be replaced by the exact usage which the quadrat method has made possible for vegetation. The designation of habitats could be made much more exact, and the formation, as well as the habitat-form or ecad, and the vegetation-form or phyad, should be indicated in addition. The general terms drawn from pollination, seed-production, and dissemination might also be included to advantage.

20. Forestry, if the purely conmercial aspects be disregarded, is the ecology of a particular kind of vegetation, the forest. Therefore, in pointing out the connection between them, it is only necessary to say that whatever contributes to the ecology of the forest is a contribution to forestry. There are, however, certain lines of inquiry which are of fundamental importance. First among these, and of primary interest from the practical point of view, are the questions pertaining to the distribution of forests and their structure. Of even greater significance are the problems of forest development, movement, and of reforestation, which are comprised in succession. The gradual invasion of the plains and prairies by the forest belt of the east and north is in full conformity with the laws of invasion, and the ecological methods to be employed here serve not merely to determine the actual conditions at present, but also to forecast them with a great deal of accuracy. The slow but certain development of forests on new soils, and their more rapid re-establishment where the woody vegetation has been destroyed by burning or lumbering, are ordinary phenomena of succession, for which the ecologist has already worked out the laws, and determined the methods of investigation. Having once ascertained the original and adjacent vegetation and the character of the habitat, the ecologist can indicate with accuracy not only the character of the new forest that will appear, but also the nature of the antecedent formations. A full knowledge of the character and laws of succession will prove of the greatest value to the forester in all studies of forestation and reforestation. Forests which now seem entirely unrelated will be seen to possess the most intimate developmental connection, and the fuller insight into the life-history gained in this way will have a direct bearing upon methods of conservation, etc. It will further show that the forester must know other vegetations as well, since grassland and thicket formations have an intimate influence upon the course of the succession, as well as upon the advance of a forest frontier.

One of the greatest aids which modern ecology can furnish forestry, however, is the method of determining the physical nature of the habitat. So far, foresters have been obliged to content themselves with a more or less superficial study of the structure of forest formations, without being 
able to do more than guess at the physical causes which control both structure and development. This handicap is especially noticeable in the case of forest plantings in non-forested regions, where it has been impossible to estimate the chances of success, or to determine the most favorable areas except by actual plantations. Equipped with the proper instruments for measuring water-content, humidity, light and temperature, the ecologist is able to determine the precise conditions under which reproduction is occurring, and to ascertain what non-forested areas offer the most nearly similar conditions. A knowledge of habitats and the means of measuring them enables the forester to discover the causes which control the vegetation with which he is already familiar, and to forecast results otherwise hidden. Furthermore, it makes it possible for him to enter a new region and to determine its nature and capabilities at a minimum of time and energy.

21. Physiography. Physiographic features play an important part in determining the quantity of certain direct factors of the habitat. Perhaps a more important connection between physiography and ecology is to be found in succession. The beginning of all primary, and of many secondary successions is to be sought in the physiographic processes which produce new habitats, or modify old ones. On the other hand, most of the reactions which continue successions exert a direct influence upon the form of the land. The most pronounced influence of terrestrial successions is found in the stabilization which their ultimate stages exert upon land forms, even where these are highly immature. The chief effect of aquatic successions is to be found in the "silting up" and the formation of new land, which result from the action of vegetation upon silt-bearing waters. The closeness of the relation between succession and the forms of the land has led to the application of the term "physiographic ecology" to that part of the subject which deals with the development of vegetation, i. e., succession.

22. Soil physics. This subject is as much a part of ecology as is forestry. It is intrinsically that subdivision of ecology which deals with the edaphic factors of the habitat, and their relation to the plant. Since the basis is physics, there has been a general tendency to overvalue the determination of soil properties, and to ignore the fact that these are decisive only when considered with reference to the living plant. As the soil contains the water which is the factor of greatest importance to plants, soil physics is an especially important part of ecology. Its methods are discussed under the habitat.

23. Zoogeography. Since animals are free for the most part, and hence not confined so strictly to one spot as plants, their dependence upon the 
habitat is not so evident. The relation is further obscured by the fact that no physical factor has the direct effect upon them which water or light exerts upon the plant. Vegetation, indeed, as the source of food and protection, plays a more obvious, if not a more important part. This is especially true of anthophilous insects, but it also holds for all herbivorous animals, and, through them, for carnivorous ones. The animal ecology of a particular region can only be properly investigated after the habitats and plant formations have been carefully studied. Here, as in floristics, a great deal can be done in the way of listing the fauna, or studying the life habits of its species, without any knowledge of plant ecology; but an adequate study must be based upon a knowledge of the vegetation. Although animal formations are often poorly defined, there can be no doubt of their existence. Frequently they coincide with plant formations, and then have very definite limits. They exhibit both development and structure, and are subject to the laws of invasion, succession, zonation, and alternation, though these are not altogether similar to those known for plants, a fact readily explained by the motility of animals. Considered from the above point of view, zoogeography is a virgin field, and it promises great things to the student who approaches it with the proper training.

24. Sociology. In its fundamental aspects, sociology is the ecology of a particular species of animal, and has in consequence, a similar close connection with plant ecology. The widespread migration of man and his social nature have resulted in the production of groups or communities which have much more in common with plant formations than do formations of other animals. The laws of association apply with especial force to the family, tribe, community, etc., while the laws of succession are essentially the same for both plants and man. At first thought it might seem that man's ability to change his dwelling-place and to modify his environment exempts him in large measure from the influence of the habitat. The exemption, however, is only apparent, as the control exerted by climate, soil, and physiography is all but absolute, particularly when man's dependence upon vegetation, both natural and cultural, is called to mind.

\section{The Essentials of a System}

25. Cause and effect: habitat and plant. In seeking to lay the foundation for a broad and thorough system of ecological research, it is necessary to scan the whole field, and to discriminate carefully between what is fundamental and what is merely collateral. The chief task is to discover, if possible, such a guiding principle as will furnish a basis for a permanent and logical superstructure. In ecology, the one relation which is precedent 
to all others is the one that exists between the habitat and the plant. This relation has long been known, but its full value has yet to be appreciated. It is precisely the relation that exists between cause and effect, and its fundamental importance lies in the fact that all questions concerning the plant lead back to it ultimately. Other relations are important, but no other is paramount, or able to serve as the basis of ecology. Ecology sums up this relation of cause and effect in a single word, and it may be- that this advantage will finally cause its general acceptance as the proper name for this great field.

In the further analysis of the connection between the habitat and the plant, it is evident that the causes or factors of the habitat act directly upon the plant as an individual, and at the same time upon plants as groups of individuals. The latter in no wise decreases the importance of the plant as the primary effect of the habitat, but it gives form to research by making it possible to consider two great natural groups of phenomena, each characterized by very different categories of effects. Ecology thus falls naturally into three great fundamental fields of inquiry: habitat, plant, and formation (or vegetation). To be sure, the last can be approached only through the plant, but as the latter is not an individual, but the unit of a complex from the formational standpoint, the formation itself may be regarded as a sort of multiple organism, which is in many ways at least a direct effect of the habitat. In emphasizing this fundamental relation of habitat and vegetation, it is imperative not to ignore the fact that neither plant nor formation is altogether the effect of its present habitat. A third element must always be considered, namely, the historical fact, by which is meant the ancestral structure. Upon analysis, however, this is in its turn found to be the product of antecedent habitats, and in consequence the essential connection between the habitat and the plant is seen to be absolute.

26. The place of function. In the foregoing it is understood that the immediate effect of the physical factors of the habitat is to be found in the functions of the plant, and that these determine the plant structure. Function has so long been the especial theme of plant physiology that methods of investigation are numerous and well known, and it is unnecessary here to consider it further than to indicate its general bearing. The essential sequence in .ecological, research, then, is the one already indicated, viz., habitat, plant, and formation, and this will constitute the order of treatment in the following pages. That portion of floristic which is not mere descriptive botany belongs to the consideration of the formation, and in consequence there will be no special treatment of floristic as a subdivision of ecology. 


\section{CHAPTER II. THE HABITAT}

\section{Concept and Analysis}

27. Definition of the habitat. The habitat is the sum of all the forces or factors present in a given area. It is the exact equivalent of the term environment, though the latter is commonly used in a more general sense. As an ecological concept, the habitat refers to an area much more definite in character, and more sharply limited in extent than the habitat of species as indicated in the manuals. Since the careful study of habitats has scarcely begun, it is impossible to recognize and delimit them in an absolute sense. Visible topographic boundaries often exist, but in many cases, the limit, though actual, is not readily perceived. Contiguous habitats may be sharply limited, or they may pass into each other so gradually that no real line of demarcation can be drawn. Whatever variations they may show, however, all' habitats agree in the possession of certain essential factors, which are universally present. On the other hand, a few factors are merely incidental and may be present or absent. The relative value and amount of these is probably similar for no two habitats, though the latter readily fall into groups with reference to the amount of some particular factor.

28. Factors. The factors of a habitat are water-content, humidity, light, temperature, soil, wind, precipitation, pressure, altitude, exposure, slope, surface (cover), and animals. To these should be added gravity and polarity, which are practically uniform for all habitats, and may, in consequence, be ignored in this treatise. Length of season, while it plays an important part in vegetation, is clearly a complex and is to be treated under its constituents. Of the factors given, all are regularly found in each habitat, though some are not constantly present. The first five, water-content, humidity, light, temperature, and soil are the most important, and any one may well serve as a basis for grouping habitats into particular classes with reference to quantity. As will be pointed out later, however, water-content and light furnish the most striking differences between habitats, and offer the best means of classification. As habitats are inseparable from the formations which they bear, the discussion of the kinds of habitats is reserved for chapter IV. 


\section{Classification of Factors}

29. The nature of factors. The factors of a habitat are arranged in two groups according to their nature: (I) physical, (2) biotic. In the strict sense, the physical factors constitute the habitat proper, and are the real causative forces. No habitat escapes the influence of biotic factors, however, as the formation always reacts upon it, and the influence of animals is usually felt in some measure. Physical factors are further grouped into (I) climatic and (2) edaphic, with respect to source, or, better, the medium in which they are found. Climatic, or atmospheric factors are humidity, light, temperature, wind, pressure, and precipitation. Axiomatically., the stimuli which they produce are especially related to the leaf. Edaphic or soil factors are confined to the soil, as the term denotes; and are immediately concerned with the functions of the root. Water-content is by far the most important of these; the others are soil composition (nutrientcontent), soil temperature, altitude, slope, exposure, and surface. The last four are of a more general character than the others, and are usually referred to as physiographic factors. Cover, when dead, might well be placed among these also, but as it is little different from the living cover in effect, it seems most logical to refer it to biotic factors.

30. The influence of factors. While the above classification is both obvious and convenient, a more logical and intimate grouping may be made upon the influence which the factor exerts. On this basis, factors are divided into (I) direct, (2) indirect, and (3) remote. Direct factors are those which act directly upon an important function of the plant and produce a formative effect: for example, an increase in humidity produces an immediate decrease in transpiration. They are water-content, humidity, and light. Other factors have a direct action: thus temperature has an immediate influence upon respiration and probably assimilation also, but it is not structurally formative. Wind has a direct mechanical effect upon woody plants, but it does not fall within our definition. Indirect factors are those that affect a formative function of the plant through another factor; thus a change in temperature causes a change in humidity and this in turn calls forth a change in transpiration; or, a change in soil texture increases the water-content, and this affects the imbibition of the root-hairs. Indirect factors, then, are temperature, wind, pressure, precipitation, and soil composition. Remote factors are, for the most part, physiographic and biotic: they require at least two other factors to act as middlemen. Altitude affects plants through pressure, which modifies humidity, and hence transpiration. Slope determines in large degree the run-off during a rain-storm, thus 
affecting water-content and the amount of water absorbed. Earthworms and plant parts change the texture of the soil, and thereby the water-content. Indirect factors often exert a remote influence also, as may be seen in the effect which temperature and wind have in increasing evaporation from the soil, and thus reducing the water-content. This distinction between factors may. seem insufficiently grounded. In this event, it should be noted that it centers the effects of all factors upon the three direct ones, water-content, humidity, and light. If it further be recalled that these are the only factors which produce qualitative structural changes, and that the classification of ecads and formations is based upon them, the validity of the distinction is clear.

\section{The Determination of Factors}

31. The need of exact measurement. Any serious endeavor to find in the habitat those-causes which are producing modification in the plant and in vegetation can not stop with the factors merely. The next step is to determine the quantity of each. It is not sufficient to hazard a guess at this, or to make a rough estimate of it. Habitats differ in all degrees, and it is impossible to institute comparisons between them without an exact measure of each factor. Similarly, one can not trace the adaptations of species to their proper causes unless the quantity of each factor is known. It is of little value to know the general effect of a factor, unless it is known to what degree this effect is exerted. For this purpose it becomes necessary to appeal to instruments, in order to determine the exact amount of each factor that is present in a particular habitat, and hence to determine the ratio between the stimulus and the amount of structural adjustment which results. The employment of instruments of precision is clearly indispensable for the task which we have set for ecology, and every student that intends to strike at the root of the subject, and to make lasting contributions to it, must familiarize himself with instrumental methods. One great benefit will accrue to ecology as soon as this fact is generally recognized. The use of instruments and the application of results obtained from them demand much patience and seriousness of purpose upon the part of the student. As a consequence, there will be a general exodus from ecology of those that have been attracted to it as the latest botanical fad, and have done so much to bring it into disrepute.

32. The value of meteorological methods. At the outset there must be a very clear understanding that weather records and readings have only a very general value. This is in spite of the fact that the instruments employed are of standard precision. An important reason for this lack of 
value is that readings are not made in a particular habitat; as a rule, indeed, they are made in towns and cities, and hence are far removed from masses of vegetation. They are usually taken at considerable heights, and give but a general indication of the conditions at the level of vegetation. The chief difficulty, however, is that the factors observed at weather stations--temperature, pressure, wind, and precipitation-are those which have the least value for the ecologist. It is true that a knowledge of the temperature and rainfall of a great region will afford some idea of the general character of its vegetation. A proper understanding of such a vegetation is, however, to be gained only through the exact study of its component formations. Ecology has already incurred sufficient censure as a subject composed of very general ideas, and the use of meteorological data, which can never be connected definitely with anything in the plant or the formation, should be discontinued. This must not be understood to mean that meteorological instruments can not be used in the proper place and manner, i. e., in the habitat.

33. Habitat determination. It is self-evident that determinations of factors by instruments can only be of value in the habitat where they are made. In other words, a habitat is a unit for purposes of measuring its factors, and measures of one habitat have no exact value in another. This fact can not be overstated. Thus, 'while it is perfectly legitimate, and indeed highly desirable, to locate thermographs in different mountain zones for ascertaining the rate at which temperature decreases with altitude, the data obtained in this way are not directly applicable in explanation of plant or formation changes, except when the same species occurs at different altitudes. Special methods are valuable and often absolutely necessary, but in view of the fact that the plant as well as the formation is the definite product of a definite habitat, the fundamental rule in instrumentation is that complete readings must be made within a habitat for that habitat alone. This necessarily presupposes a certain preliminary acquaintance with the habitat to be investigated, as it is imperative that the station for making readings be located well within the formation, in order to avoid transition conditions. In vegetation, there are as many habitats as formations, and in addition a large number of new and denuded habitats upon which successions have not yet started; a knowledge of each formation or succession must rest ultimately upon the factors of its particular habitat.

34. Determinable and efficient differences. The instruments employed in studying habitats can not be too exact, as there is no adequate knowledge as yet concerning the real differences which exist between related or contiguous formations. This is particularly true of differences which are 
efficient in producing a recognizable structural change in plant or formation. Investigations made by the writer have shown that standard instruments will measure differences of quantity quite too small to produce a visible reaction. Efficient differences are not the same for different factors, and perhaps also for the same factor when found in various combinations. They vary widely for different species, being in direct relation to the plasticity of the latter. The point necessary to bear in mind in formulating methods for habitat investigation and in making use of instruments is that standard instruments should be used for the very reason that we do not yet know the relation between determinable and efficient differences. On the other hand, it is unnecessary to insist upon.absolute exactness as soon as it is found that the determinable difference lies well within the efficient one. This by no means indicates that instruments are not to be carefully standardized and frequently checked, or that accurate readings should not be made. It means that a slight margin of error may be permitted in a machine which registers well within the efficient difference for that factor, and that instruments that read to the last degree of nicety are not absolutely necessary. In the fundamental work of determining efficient differences, however, instruments can not have too great precision. Moreover, these differences must be based upon the most plastic species of a formation, and the readings must be made under normal conditions.

\section{INSTRUMENTATION}

35. Methods. In the field use of instruments two methods have been developed. The first in point of time was the method of simple instruments, devised especially for class work, and capable of being used only where a number of trained students are available. The method of automatic instruments was an immediate outgrowth of this, due to the necessity which confronts the solitary investigator of being in different habitats at the same time. In the gradual evolution of this subject, it has become possible to combine the two methods in such a way as to retain all the advantages of the automatic method, and most of those of the method of simple instruments.

36. Method of simple instruments. By simple instruments are denoted those that do not record, but must be read by the observer at the time. They are standard instruments of precision, but possess the disadvantage of requiring an observer for each one. They are well illustrated by the thermometers and psychrometers used by the Weather Bureau. In the hands of trained observers the results obtained are unimpeachable; in fact, standard simple instruments must be constantly employed to check automatic 
ones. As physical factors vary greatly through the day and through the year, it is all-important that the readings in habitats which are being compared should be made at the same instant. This requires a number of observers; as many as twelve stations have been read at one time, and there is of course no limit to the number. It is very important, also, that observers be carefully trained in the handling of instruments, and in reading them accurately and intelligently at the proper moment. In practice it has been found impossible to do such work in elementary classes, and, even in using small advanced classes, prolonged drill has been necessary before trustworthy results could be obtained. When a class has once been thoroughly trained in making accurate simultaneous readings, there is practically no limit, other than that set by time, to the valuable work that can be done, both in instruction and investigation.

37. Method of automatic instruments. The solitary investigator must replace trained helpers by automatic instruments or ecographs. These have the very great advantages of giving continuous simultaneous records for long periods, and of laving no personal equation. They must be regulated and checked, to be sure, but as this is all done by the same person, the error is negligible. There is nothing more satisfactory in resident investigation than a series of accurate recording instruments in various habitats. Ecographs have two disadvantages. The chief perhaps is cost. The expense of a single "battery" which will record light, water-content, humidity, and temperature is about $\$ 250$. Another difficulty is that they can be used only within a few miles of the base, since they require attention every week for regulation, change of record, etc. While this means that ecographs in their present form are not adapted to reconnaissance, this is not a real disadvantage, as the scattered observations posisible on such a journey can best be made by simple instruments.

38. Combined methods. The best results by far are to be obtained by the combined use of simple and automatic instruments. This is particularly true in research, but it applies also to class instruction. The ecographs afford a continuous, accurate basal record, to which a single reading made at any time or place can be readily referred for comparison. On the other hand, it is an easy matter to carry a full complement of simple instruments on the daily field trips, and to make accurate readings in a score or more of formations in a single day. An isolated reading, especially of a climatic factor, has little or no value in itself, but when it can be compared with a reading made at the same time in the base station by an ecograph, it is the equivalent of an automatic reading. This method renders a set of simple instruments 
more desirable for a long trip or reconnaissance than a battery of automatic ones. It is practically impossible to carry the latter into the field, and in any event a continuous record is out of the question. As there are other tasks at such times also, it becomes evident that the taking of single readings which can be compared with a continuous record offers the most satisfactory solution.

\section{Construiction and Use of Instruments}

39. The selection of instruments. In selecting and devising instruments for the investigation of physical factors, emphasis has first been laid upon accuracy. This is the result of a feeling that it is better to have instruments that read too minutely than those which do not make distinctions that are sufficiently close, particularly until more has been learned about efficient differences. On the other hand, no hesitation has been felt in employing instruments which are not absolutely accurate, when it was clear that the error was less than the efficient difference. Similarly, the margin of error practically eliminates itself in the case of simultaneous comparative readings, when the instruments have been checked to the same standard. Simplicity of construction and operation are of great importance, especially in saving time where a large number of instruments are in operation. Expense is likewise to be carefully considered. It is impossible to have too many instruments, but cost practically determines the number that can be obtained. It is further necessary to secure or invent both simple and automatic instruments for all factors, except such invariable ones as altitude; slope, etc. Simple instruments must be of a kind that can be easily carried, and so constructed that they can be used at a minimum of risk. The sling psychrometer, for example, is very readily broken in field use, and it has been replaced by a protected modification, the rotating form.

In describing the construction and operation of the many factor instruments, there has been no attempt to make the treatment exhaustive. Those instruments which the author has found of greatest value in his own work are given precedence, and the manner of using them is described in detail. Other instruments of value are also considered, though with greater brevity. Some of the most complex and expensive ones have been ignored, as it is altogether improbable that they can come into general use in their present form. While the conviction is felt that the methods described below will enable the most advanced investigators to carry on thorough work, it is hoped that they will be seen to be so fundamental, and so attractive, that they will appeal to all who are planning serious ecological study. 


\section{WATER-CONTENT}

40. Value of different instruments. The paramount importance of watercontent as a direct factor in the modification of plant form and distribution gives a fundamental value to the methods used for its determination. Automatic instruments for ascertaining the water in the soil are costly, in addition to being complicated, and often inaccurate. For these reasons, much attention has been given to developing the simpler but more reliable methods in which a soil-borer or geotome is used. The latter is simple, inexpensive, and accurate. It can be carried easily upon daily trips or upon longer reconnaissances, and is always ready for instant use. In the determination of physiological water-content, it is practically indispensable. . Indeed, the readiness with which geotome determinations of water-content can be made should hasten the universal recognition of the fact that it is the available, and not the total amount of water in the soil, which determines the effect upon the plant.

\section{Geotome Methods}

41. The geotome. In its simplest form, the geotome is merely a stout iron tube with a sharp cutting edge at one end and a firmly attached handle at the

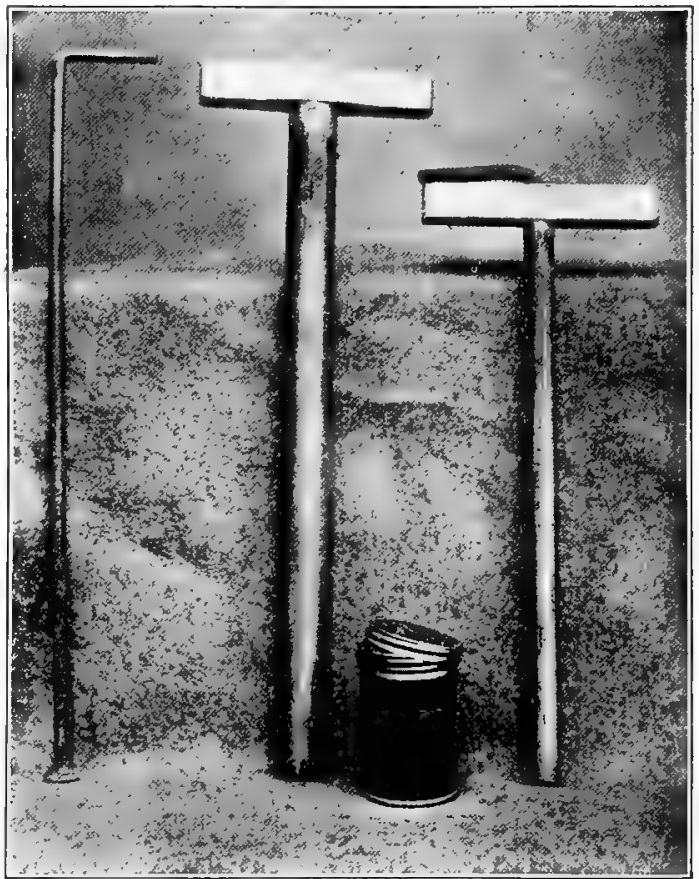

Fig. 1. Geotomes and soil can. other. The length is variable and is primarily determined by the location of the active root surface of the plant. In xerophytic habitats, generally a longer tube is necessary than in mesophytic ones. The bore is largely determined by the character of the soil ; for example, a larger one is necessary for gravel than for loam. Tubes of small bore also tend to pack the soil below them, and to give a correspondingly incomplete core. The best results have been obtained with geotomes of $1 / 2-I$ inch tube. Each geotome 
has a removable rod, flattened into a disk at one end, and bent at the other, for forcing out the core after it has been cut from the soil. Sets of geotomes have been made in lengths of $5,10,12,15,20$, and 25 inches. The 12- and I5-inch forms have been commonly used for herbaceous formations and layers. They are marked in inches so that a sample of any lesser depth may be readily taken. Such a device is very necessary for gravel soils and in mountain regions, where the subsoil of rock lies close to the surface.

42. Soil borers. There is a large variety of soil borers to choose from, but none have been found as simple and satisfactory for relatively shallow readings as the geotome just described. For deep-rooted plants, many xerophytes, shrubs, and trees, borers of the auger type are necessary. These are large and heavy, and of necessity slow in operation. They can not well be carried in an ordinary outfit of instruments, and the size of the soil sample itself precludes the use of such instruments far from the base station, except on trips made expressly for obtaining samples from deep-seated layers. For, depths from two to eight feet, the Fraenkel borer is perhaps the most satisfactory, except for the coarser gravels; it costs $\$ 14$. or $\$ 20$ according to the length. For greater depths, or when a larger core is desirable, the Bausch \& Lomb borer, number 16536 , which costs $\$ 5.25$, should be made use of. This is a ponderous affair and can be employed only on special occasions. On account of the size of samples obtained by these borers, it is usually most satisfactory to take a small sample from the core at different depths. Frequently, indeed,

Fig. 2. Fraenkel a hand trowel may be readily used to obtain a good sample at a particular depth.

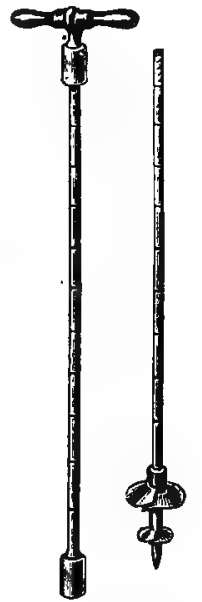

Fig. 3. American soil borer.

43. Taking samples of soil. In obtaining soil samples, the usual practice is to remove the air-dried surface, noting its depth, and to sink the geotome with a slow, gentle, boring movement, in order to avoid packing the soil. This difficulty is further obviated by deep notches with sharp, beveled edges which are cut at the lower end. In obtaining a fifteen-inch core, there is also less compression if it be cut five inches at a time. Repeated tests have shown, however, that the single compressed sample is practically as trustworthy as the one made in sections. The water-content of the former constantly fell within .5 per cent of that of the latter, and both varied less 
than I per cent from the dug sample used as a check. As soon as dug, the core is pressed out of the geotome by the plunger directly into an air-tight soil can. Bottles may be used as containers, but tin cans are lighter and more durable. Aluminum cans have been devised for this purpose, but on account of the expense, "Antikamnia" cans have been used instead. These are tested, and those that are not water-tight are rejected, although it has been found that, even in these, ordinary soils do not lose an appreciable amount of water in twenty-four hours. The lid should be screwed on as quickly as possible, and, as an added precaution, the cans are kept in a close case until they have been weighed. The cans are numbered consecutively on both lid and side in such a way that the number may be read at a glance. The numbers are painted, as a label wears off too rapidly, and scratched numbers are not quickly discerned.

44. Weighing. Although soil samples have been kept in tight cans outside of cases for several days without losing a milligram of moisture, the safest plan is to make it a rule to weigh cans as quickly as possible after bringing them in from the field. Morcover, when delicate balances are available, it is a good practice to weigh to the milligram. At remote bases, however, ancl particularly in the field,

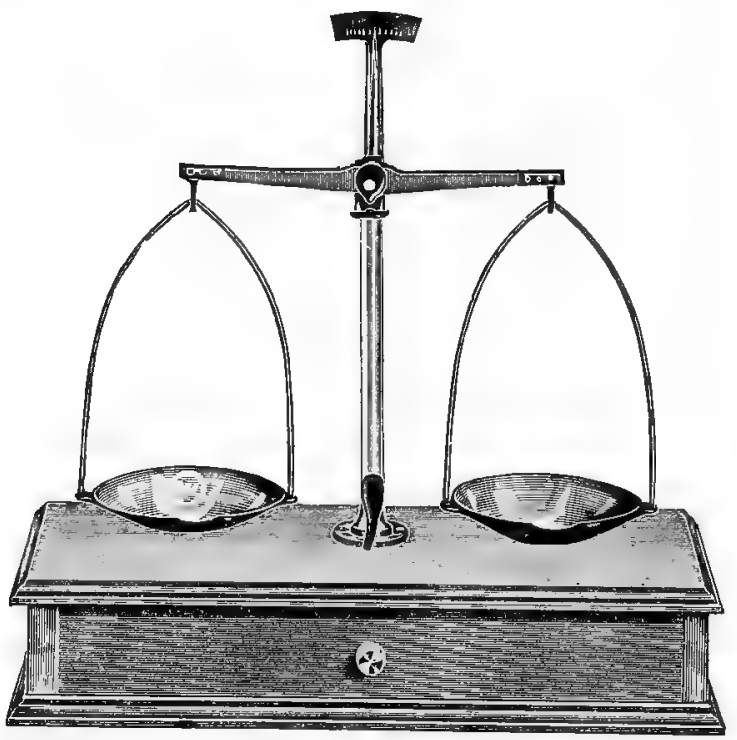

Fig. 4. Field balance. and on reconnaissance, where delicate, expensive instruments are out of place, coarser balances, which weigh accurately to one centigram, give satisfactory results. The study of efficient water-content values has already gone far enough to indicate that differences less than I per cent are negligible. Indeed, the soil variation in a single square meter is often as great as this. The greatest difference possible in the third place, i. e., that of 9 milligrams, does not produce a difference of .I of $I$ per cent in the water-content value. 'In consequence, such strong portable balances as Bausch \& Lomb I2308 (\$2), which can be carried anywhere, give entirely 
reliable results. The best procedure is to weigh the soil with the can. Turning the soil out upon the pan or upon paper obviates one weighing, but there is always some slight loss, and the chances of serious mishap are many. After weighing, the sample is dricd as rapidly as possible in a water bath or oven. At a temperature of $100^{\circ} \mathrm{C}$. this is accomplished ordinarily in twenty-four hours; the most tenacious clays require a longer time, or a higher temperature. High temperatures should be avoided, however, for soils that contain much leaf mould or other organic matter, in order that this may not be destroyed. When it is necessary on trips, soil samples can be dried in the sun or even in the air. This usually takes several days, however, and a test weighing is generally required before one can be certain that the moisture is entirely gone. The weighing of the . dried soil is made as before, and the can is carefully brushed out and weighed. The weight of aluminum cans may be determined once for all, but with painted cans it has been the practice to weigh them each time.

45. Computation. The most direct method of expressing the watercontent is by per cents figured upon the moist soil as a basis. The ideal way would be to determine the actual amount of water per unit volume, but as this would necessitate weighing one unit volume at least in every habitat studied, as a preliminary step, it is not practicable. The actual process of computation is extremely simple. The weight of the dried sample, $z w^{1}$, is subtracted from the weight of the original sample, $w$, and the weight of the can, $\tau^{2}$, is likewise subtracted from $w$. The first result is then divided by the second, giving the per cent of water, or the physical water-content. The iormula is : $\frac{w-w^{1}}{w-w^{2}}=W$. The result is expressed preferably in grams per hundred grams of moist soil; thus $20 / 100$, from which the per cent of water-content may readily be figured on the basis of dry or moist soil.

46. Time and location of readings. Owing to the daily change in the amount of soil water due to evaporation, gravity, and rainfall, an isolated determination of water-content has very little value. It is a primary requisite that a basis for comparison be established by making (I) a series of readings in the same place, (2) a series at practically the same time in a number of different places or habitats, or (3) by combining the two methods, and following the daily changes of a series of stations throughout an entire season, or at least for a period sufficient to determine the approximate maximum and minimum. The last procedure can hardly be carried out except at a base station, but here it is practically indispensable. It has been followed both at Lincoln and at Minnehaha, resulting in a basal series for each place that is of the greatest importance. When such a 
base already exists, or, better, while it is being established, scattered readings may be used somewhat profitably. As a practical working rule, however, it is most convenient and satisfactory to make all determinations consecutively, i. e., in a series of stations or of successive days. Under ordinary conditions, the time of day at which a particular sample is taken is of little importance, as the variation during a day is usually slight. This does not hold for exposed wet soils, and especially for soils which have just been wetted by rains. In all comparative series, however, the samples should be taken at the same hour whenever possible. This is particularly necessary when it is desired to ascertain the daily decrease of water-content in the same spot. In the case of a series of stations, these should be read always in the same order, at the same time of day, and as rapidly as possible. When a daily station series is being run, i. e., a series by days and stations both, the daily reading for each place should fall at the same time. While there are certain advantages in making readings either early or late in the day, they may be made at any time if the above precautions are followed.

47. Location of readings. Samples should invariably be taken in spots which are both typical and normal, especially when they are to be used as representative of a particular area or habitat. A slight change in slope, soilcomposition, in the amount of dead or living cover, etc., will produce considerable change in the amount of water present. Where habitat and formation are uniform, fewer precautions are necessary. This is a rare circumstance, and as a rule determinations must be made wherever appreciable differences are in evidence. The problem is simpler when readings are taken with reference to the structure or modifications of a particular species, but even here, check-readings in several places are of great value. The variation of water in a spot apparently uniform has been found to be slight in the prairies and the mountains. In taking three samples in spots a few inches to several feet apart, the rlifference in the amount of water has rarely exceeded I per cent, which is practically negligible. Gardner ${ }^{1}$ found that i6 samples taken to a depth of 3 inches, in as many different portions of a carefully prepared, denuded soil plot, showed a variation of $7 \mathrm{I} / 2$ per cent. This is partially explained by the shallowness of the samples, but even then the results of the two investigations are in serious conflict and indicate that the question needs especial study. It should be further pointed out that all readings should be made well within a partictular area, and not near its edge, and that. in the case of large diversified habitats, it is the consocies and the society which indicate the obvious variations in the structure of the habitat.

${ }^{2}$ Gardner, F. D. The Electrical Methods of Moisture Determination in Soils. Bull. Div. Soils, 12:12. 1898. 
48. Depth of samples. The general rule is that the depth of soil samples is determined by the layer to which the roots penetrate, The practice is to remove the air-dried surface in which no roots are found, and to take a sample to the proper depth. This method is open to some objection, as the actively absorbing root surfaces are often localized. There is no practical way of taking account of this as yet, except in the case of deeprooted xerophytes and woody plants. It is practicable to determine the lucation of the active root area of a particular plant and hence the watercontent of the soil layer, but in most formations, roots penetrate to such different depths that a sample which includes the greater part of the distance concerned is satisfactory. Some knowledge of the soil of a formation is also recessary, since shallow soils do not require as deep samples as others. The same is true of shaded soils without reference to their depth, and, in large measure, of soils supplied with telluric water. In all cases, it is highly desirable to have numerous control-samples at different depths. The normal cores are 12 or I 5 inches; control-samples are taken every 5 inches to the depth desired, and in some cases 3 -inch sections are made. It has been found a great saving of time to combine these methods. A 5-inch sample is taken and placed in one can, then a second one, and a third in like manner. In this way the water-content of each 5 -inch layer is determined, and from the combined weight the total content is readily ascertained.

49. Check and control instruments. A number of instruments throw much light upon the general relations of soil water. The rain-gauge, or ombrometer, measures the periodical replenishment of the water supply, and has a direct bearing upon seasonal variation. The atmometer affords a clue to the daily decrease of water by evaporation, and thus supplements the rain-gauge. The run-off gauge enables one to establish a direct connection between water-content and the slope and character of the surface. The amount and rapidity of absorption are determined by means of a simple instrument termed a rloptometer. The gravitation water of a soil is ascertained by a hizometer, and some clue to the hygroscopic and capillary water may be obtained by an artificial osmotic cell. All of these are of importance because they serve to explain the water-content of a particular soil with especial reference to the other factors of the habitat. It is evident that none of them can actually be used in exact determinations of the amount of water, and they will be considered under the factors with which they are more immediately concerned.

\section{Physical and Physiological Water}

50. The availability of soil water. The amount of water present in a soil is no real index to the influence of water-content as a factor of the 
habitat. All soils contain more water than can be absorbed by the plants which grow in them. This residual water, which is not available for use, varies for different soils. It is greatest in the compact soils, such as clay and loam, and least in the loose ones, as sand and gravel. It differs, but to a much less degree, from one species to another. A plant of xerophytic tendency is naturally able to remove more water from the same soil than one of mesophytic or hydrophytic character. As the species of a particular formation owe their association chiefly to their common relation to the watercontent of the habitat, this difference is of little importance in the field. In comparing the structure of formations, and especially that of the plants which are found in them, the need to distinguish the available water from the total amount is imperative. Thus, water-contents of 15 per cent in gravel and in clay are in no wise con!parable. A coarse gravel containing I5 per cent of water is practically saturated. The plants which grow upon it, are mesophytes of a strong hydrophytic tendency, and they are able to use $14 \frac{1}{2}$ or all but .5 out of the 15 . per cent of water. In a compact clay, only $3 \mathrm{I} / 2$ of the I.5 per cent are available, and the plants growing in it are marked xerophytes. It is evident that a knowledge merely of the physical watercontent is actually misleading in such cases, and this holds true of comparisons of any soils which differ considerably in texture. After one has determined the physiological water for the great groups of soils, it is more or less possible to estimate the amounts in the various types of each. As an analysis is necessary to show how close soils are in texture, this is jittle better than a guess, and for accurate work it is indispensable that the available water be determined for each habitat. Within the same formation, however, after this has once been carefully ascertained, it is perfectly satisfactory to convert physical water-content into available by subtracting the non-available water, which under normal conditions in the field remains practically the same.

The importance of knowing the available water is even greater in those habitats in which salts, acids, cold, or other factors than the molecular attraction of soil-particles increase the amount of water which the plant can not absorb. Few careful investigations of such soils have yet been made, and the relation of available to non-available water in them is almost entirely unknown. It is probable that the phenomena in some of these will be found to be produced by other factors.

51. Terms. The terms, physiological water-content, and physical watercontent, are awkward and not altogether clear in their application. It is here proposed to replace them by short words which will refer directly to the availability of the soil water for absorption by the plant. Accordingly, 
the total amount of water in the soil is divided into the available and the non-available water-content. The terms suggested for these are respect-

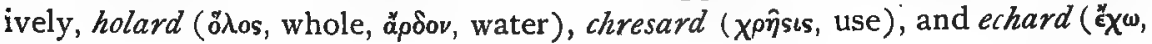
to withlold)..

52. Chresard determinations under control. The determination of the shresard in the field is attended with peculiar difficulties. In consequence, the method of obtaining it under control will first be described. The inquiry may be made with reference to soils in general or to the soil of a particular formation. In the last case, if the plants used are from the same formation, the results will have almost the value of a field determination. When no definite habitat is the subject of investigation, an actual soil, and not an artificial mixture, should be used, and the plants employed should be mesophytes. The individual plants are grown from seeds in the proper soils, and are repotted sufficiently often to keep the roots away from the surface. The last transfer is made to a pot large enough to permit the plant to become full-grown without crowding the roots. The pot should be glazed inside and out in order to prevent the escape of moisture. This interferes siightly with the aeration of the soil, but it will not cause any real difficulty. The plant is watered in such a way as to make the growth as normal as possible. After it has become well established, three soil samples are taken in such a manner that they will give the variation in different parts of the pot. One is taken near the plant, the second midway between the plant and the edge of the pot, and the third near the edge. The depth is determined by the size of the pot and the position of the roots. The holard is determined for these in the usual way, but the result is expressed with reference to roo grams of dry soil; the average is taken as representative. The soil is then allowed to dry out slowly, as sudden drouth will sometimes impair the power of absorption and a plant will wilt although considerable available water remains. Plants often wilt in the field daily for several successive hot dry clays, and become completely turgid again during the night. If the drying out takes place slowly, the plant will not recover after it has once begun to wilt. The proper time to make the second reading is indicated by the pronounced wilting of the leaves and shoots. Complete wilting occurs, as a rule, only after the younger parts have drawn for some time upon the watery tissues of the stem and root; by which time evaporation has considerably deceased the water in the soil. It is a well-known fact that young keaves do not wilt easily, especially in watery or succulent plants. Three samples are again taken and the average water-content determined as above: This is the non-available water or the echard. The latter is then computed on the basis of Ioo grams of dry soil, and this result is subtracted 
from the holard to give the chresard in grams for each Ioo grams of dry weight. The chresard may also be expressed with respect to Ioo grams of moist soil. As a final precaution in basal work, it is advisable to determine the chresard for six individuals of the same species under as-nearly the same conditions as possible. When it is desired, however, to find the average chresard for a particular soil, it is necessary to employ various species representing diverse phyads and ecads. Such an investigation is necessarily very complicated, and must be made the subject of special inquiry.

53. Chresard readings in the field. The especial difficulties which must be overcome in the field are the exclusion of rain and dew and the cutting off of the capillary water. It is evident, of course, that experiments of this sort must also be entirely free from outside disturbance. The choice of an area depends upon the scope of the study. If the chresard is sought for a particular consocies, the block of soil to be studied should show several species which are fairly representative. In case the chresard of a certain species is to be obtained, this species alone need be present, but it should be represented by several individuals. Check plots are desirable in either event, and at least two or three which are as nearly uniform as possible should be chosen. The size and depth of the soil block depends upon the plants concerned. It must be large enough that the roots of the particular individuals under investigation are not disturbed. There is a limit to the size of the mass that can be handled readily, and in consequence the test plants must not be too large or ton deeply rooted. The task of cutting out the soil block requires a spade with a long sharp blade. After ascertaining the spread and depth of the roots, the block is cut so that a margin of several inches free from the roots concerned is left on the sides and bottom. If the block is to be lifted out of place, so that the sides are exposed to evaporation, this allowance should he greater. In some cases, it may be found more convenient to dig the plant up, place it in a large pot, and put the latter back in the hole. As a general practice, however, this is much less satisfactory.

After the block has been cut, it may be moved if the soil is sufficiently compact, and then allowed to dry out in its own formation or elsewhere. The results are most valuable in the first case, though it is often an advantage to remove blocks cut from shade or wet formations to dry, sunny stations where they will dry more rapidly. The most satisfactory and natural method, however, is to leave the block in place, and to prevent the reestab: lishment of capillary action by enclosing it within plates. This is accomplished by slipping thin sheet-iron plates into position along the cut surfaces. 
The plate for the bottom should be somewhat wider than the block, and is slipped into place by raising the block if the soil is not too loose; in the latter event, it is carefully driven in. The side plates are then pushed down to meet the former. The size of the plates depends upon the block; in general, plates of $\mathrm{I}, 2$, and 3 feet square, with the bottom plates a trifle larger, are the most serviceable. Access of rain and dew is prevented by an awning of heavy canvas which projects far enough heyond each side of the block to prevent wetting. The height will depend of course upon the size of the plants. The awning must be used only when rain or heavy dew is threatened, as the shade which it produces changes the power of the plant to draw water from the soil.

The time necessary to cause wilting varies with the habitat and the weather. When the block is large and in position, two or three weeks are required. This period of drying incidentally furnishes an excellent opportunity for determining the rate at which the particular soil loses water. The holard sample is taken daily for several days before the block is cut out, in order to obtain an average, care being taken of course to avoid a period of extreme weather. The echard samples are taken as soon as the wilting is sufficient to indicate that the limit of available water is reached. The air-dry soil above the roots is first removed. The treatment of the samples and the computation of the chresard are as previously indicated.

54. Chresard values of different soils. The following table gives the water-content values of six representative soils. The per cents of holard (at saturation) and of echard are those determined by Hedgcock ${ }^{1}$ with six mesophytes as test plants for each soil. The chresard has been computed directly from these.

\begin{tabular}{|c|c|c|c|c|c|c|}
\hline \multirow{7}{*}{ 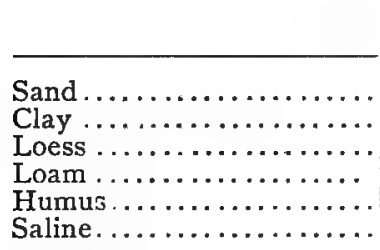 } & \multicolumn{2}{|c|}{ HOLARD } & \multicolumn{2}{|c|}{ ECHARD } & \multicolumn{2}{|c|}{ CHRESARD } \\
\hline & 14.3 & 12.6 & .3 & .25 & 14 & 12.3 \\
\hline & 47.4 & 32.5 & 9.3 & 6.3 & 38.1 & 26.2 \\
\hline & 59.3 & 37.1 & 10.1 & 6.4 & 49.2 & 30.7 \\
\hline & 64.1 & 39.1 & 10.9 & 6.6 & 53.2 & 32.5 \\
\hline & 65.3 & 39.6 & 11.9 & 7.2 & 53.4 & 32.4 \\
\hline & 68.5 & 40.8 & 16.2 & 9.6 & 52.3 & $\mathbf{3 1 . 2}$ \\
\hline
\end{tabular}

The first column indicates the per cent based upon the dry weight, the second upon the weight of the moist soil.

While these can not be considered absolute for a particular soil other than the ones investigated, they are found to correspond somewhat closely to the results obtained for other soils of the respective groups. For accurate

${ }^{1}$ HeDGCOCK, G. G. The Relation of the Water-Content of the Soil to Certain Plants, Principally Mesophytes. Rep. Bot. Surv. Nebr., 6:48: 1902. 
research, the chresard must of course be ascertained for each formation with respect to its peculiar plants and soil. The infuence of the ecad in more or less determining the echard is also shown by Hedgcock, who found that floating plants wilt at 25 per cent, amphibious ones at $\mathrm{I}^{-20}$ per cent, mesophytes at 6-12 per cent, and mesophytic xerophytes at $3-6$ per cent. The echard is also somewhat higher for shade plants than for heliophytes.

\section{Records and Results}

55. The field record. It is superfluous to point out that a definite form for field records saves much time and prevents many mistakes. The exact form may be left to personal taste, but there are certain features which are essential. Many of these are evident, while others may seem unnecessary; all, however, have been proved by experience to have some value in saving time or in preventing confusion. The two fundamental maxims of field work are that nothing is too trivial to be of importance, and that no detail should be entrusted to the memory. The field record should contain in unmistakable terms all that the field has yielded. These statements apply with especial force to water-content, in many senses the most important of physical factors. The precise character of the record depends upon the way in which the readings are made, whether scattered or in series. As the day-station series is of the greatest importance, the record is adapted for it especially, but it will also serve for all readings. The record is chronological, since this is the only convenient method for the field. A proper form for a field record of water-content is the following:

\begin{tabular}{|c|c|c|c|c|c|c|c|c|c|c|c|c|c|c|c|c|}
\hline \multirow{3}{*}{$\begin{array}{l}0 \\
0 \\
0 \\
0\end{array}$} & \multirow{3}{*}{ 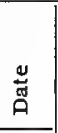 } & \multirow{3}{*}{ 尊 } & \multirow{3}{*}{ 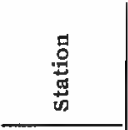 } & \multirow{3}{*}{ 总 } & \multirow{3}{*}{ 茄 } & \multirow{3}{*}{ 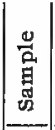 } & \multirow{2}{*}{\multicolumn{4}{|c|}{$\begin{array}{r}\text { HOLARD } \\
\text { Weighings }\end{array}$}} & \multirow{2}{*}{\multicolumn{3}{|c|}{$\begin{array}{r}\text { ECHARD } \\
\text { Weighings } \\
\end{array}$}} & \multirow{3}{*}{ 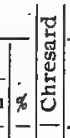 } & \multicolumn{2}{|c|}{ NOTES } \\
\hline & & & & & & & & & & & & & & & & \\
\hline & & & & & & & 1st & 2d & Can & $q$ & 1st & $2 \mathrm{~d}$ & $\operatorname{can}$ & & $\frac{8}{2}$ & का \\
\hline 10 & $2 / 8 / 04$ & Spruce forest & Jack Brook & Mertensiare & Loam & 10 & 58.7 & 50.1 & 25.59 & 2 & 58.7 & 53.41 & 25.52 & 1016 & 6 Cloudy & 0 \\
\hline 17 & $"$ & Spruce forest & Milky way & Gentianare & $\begin{array}{l}\text { 1/ mold } \\
\text { * gravel }\end{array}$ & $10: 2$ & 64.25 & 57.5 & 21.35 & & 64.25 & 53.6 & 21.35 & 511 & 1 Cloudy & 0 \\
\hline 40 & $"$ & Gravel slide. & Hiawatha & Asterare & Gravel & $2: 10$ & 78.55 & 74.3 & 2285 & 8 & 78.55 & 74.85 & 22.85 & 17 & 7 Cloudy| & 0 \\
\hline
\end{tabular}

A general designation of the soil-composition is a material aid, especially where there is a difference in the core. For example, in a mountain forest or meadow, the upper layer will usually be mold, the lower sand or gravel. A careful estimate of the relation between the two throws much light upon the chresard. Under "sample" the number taken to reach the desired depth, if more than one, is indicated by placing the number before the depth, thus 2 :Io. When two or more full cores are included in the same sample for a 
check, the order is I0:2. It has already been shown, however, that these frecautions are not necessary for ordinary purposes. In computing the holard and echard, there is no need to show the figuring, if the process is checked and then proved. Notes upon sky conditions aid in explaining the daily decrease in water-content. The amount of rain and the period during which it falls are of great importance in understanding the fluctuations of the holard. Under community it is highly desirable to have a list of all the species, but it is impossible to include this in the table, and a glance at the formation list will show them. The form indicated above serves for a daystation series, a daily series in one station for any number of check series in one spot, and for scattered readings. In many cases the echard will not be determined, but on account of its primary importance, there should be a space for it, especially since it may be desirable to determine it at some later time.

56. The permanent record. This should be kept by formation, or if the latter exhibits well-defined associations, the formational record may be divided accordingly. This may seem an unnecessary expenditure of time, but a slight experience in finding the water-content values of a particular habitat, when scattered through a chronological field record, will be convincing. The form of permanent record is the same as for the field, except that the column for the formation and that for the society are often unnecessary.

57. Sums and means. From the great difficulty of determining the absolute water-content, and of obtaining a standard of comparison between soils on account of the varying ratio between bulk and weight, water-content sums are impracticable. For the same reasons, means of actual water-content are practically impossible, and the mean water-content must be expressed in per cents. Daily readings are not essential to a satisfactory mean. In fact, a single reading at each extreme enables one to approximate the real mean very closely; thus, the average of 26 readings in the prairie formation is I 8 per cent. The extremes are 5 per cent and 28 per cent, and their average 16.5 per cent. A few readings properly scattered through moist and dry periods will give a reliable mean, as will also a series of daily readings from one heavy rain through a long dry period. The one difficulty with the last method is that such periods can not well be determined beforehand. Means permit ready comparison between habitats, but in connecting the modifications of a species with water-content as a cause, the extremes are significant as indicating the range of conditions. Furthermore, the ex: tremes, i. e., 5 per cent and 28 per cent, make it possible to approximate 
the mean, I8 per cent, while the latter gives little or no.clue to the extremes. It is hardly necessary to state that means and extremes should be determined for a certain habitat, or particular area of it, and that the results may be expressed with reference to holard and chresard.

58. Curves. The value of graphic methods and the details of plotting curves are reserved for a particular section. It will suffice in this place to indicate the water-content curves that are of especial value. Simple curves are made with regard to time, place, or depth. The day curve shows the fluctuations of the water-content of one station from day to day or from time to time. The station curve indicates the variation in water from station to station, while the depth curve represents the different values at various depths in the same station. These may be combined on the same sheet in such a way that the station curves of each day may be compared directly. Similar combinations may be used for comparing the day curves, or the depth curves of different stations, but these are of less importance. A combination of curves which is of the greatest value is one which admits of direct comparison between the station curves of saturation, holard, chresard, and echard.

\section{HUMIDITY}

59. Instruments. As a direct factor, humidity is intimately connected with water-content in determining the structure and distribution of plants. The one is in control of water loss; the other regulates water supply. Humidity as a climatic factor undergoes greater fluctuation in the same habitat, and the efficient difference is correspondingly greater. Accordingly, simple instruments are less valuable than automatic ones, since a continuous record is essential to a proper understanding of the real influence of humidity. As is the rule, however, the use of simple instruments, when they can be referred to an ecographic basis, greatly extends the field which can be studied. In investigation, both psychrometer and psychrograph have their proper place. In the consideration of simple instruments for obtaining humidity values, an arbitrary distinction is made between psychrometers and hygrometers. The former consist of a wet and a dry bulb thermometer, while the latter make use of a hygroscopic awn, hair, or other object.

\section{Psychrometers}

60. Kinds. There are three kinds of psychrometer; the sling, the cog, and the stationary. All consist of a wet bulb and a dry bulb thermometer set in a case; the first two are designed to be moved or whirled in the air. The same principle is applied in each, viz., that evaporation produces a 


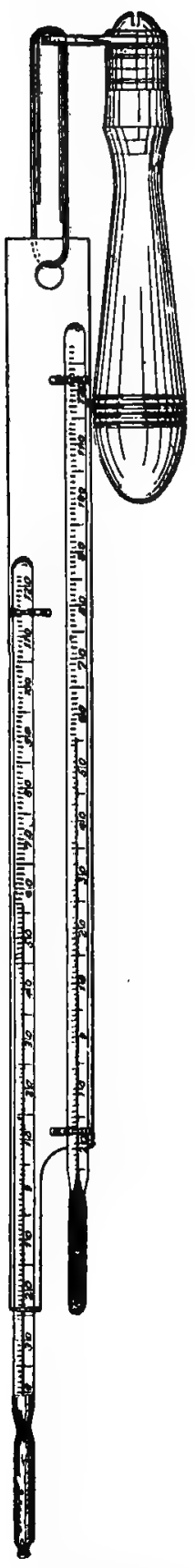

Fig. 5. Sling psychrometer. decrease in temperature proportional to the amount of moisture in the air. The dry bulb thermometer is an ordinary thermometer, while the wet bulb is covered with a cloth that can be moistened. The former indicates the normal temperature of the air, the latter gives the reduced temperature due to evaporation. The relative humidity of the air is ascertained by means of the proper tables, from two terms, i. e., the air temperature and the amount of reduction shown by the wet bulb. The sling and the cog psychrometers alone are in general use. The stationary form has been found to be unreliable, because the moisture, as it evaporates from the wet bulb, is not removed, and, in consequence, hinders evaporation to the proper degree.

61. The sling psychrometer. The standard form of this is shown in the illustration, and is the one used by the Weather Bureau. This instrument can be obtained from H. J. Green, IIgI Bedford Ave., Brooklyn, or Julien P. Friez, Io7 E. German St., Baltimore, at a cost of $\$ 5$. It consists of a metal frame to which are firmly attached two accurately standardized thermometers, reading usually from $-30^{\circ}$ to $130^{\circ}$. The frame is attached at the uppermost end to a handle in such fashion that it swings freely. The wet bulb thermometer is placed lower, chiefly to aid in wetting the cloth more readily. Tlie cloth for the wet bulb should be always of the same texture and quality; the standard used by the Weather Bureau can be obtained from the instrument makers. A slight difference in texture makes no appreciable error, but the results obtained with different instruments and by different observers will be more trustworthy and comparable if the same cloth be used in all cases. The jacket for the wet bulb may be sewed in the form of a close-fitting bag, which soon shrinks and clings tightly. It may be made in the field by wrapping the cloth so that the edges just overlap, and tying it tightly above and below the bulb. In either case, a single layer of cloth alone must be used. The cloth becomes soiled or thin after a few months' constant use and should be replaced. It is a wise precaution to carry a small piece of psychrometer cloth in the field outfit. 
62. Readings. All observations shonld be made facing the wind, and the observer should move one or two steps during the reading to prevent the possibility of error. The cloth of the wet bulb is moistened with water by means of a brush, or, much better, it is dippeel directly into a bottle of water. Distilled water is preferable, as it contains no dissolved material to accumulate in the cloth. Tap-water and the water of streams may be used without appreciable error, if the cloth is changed somewhat more frequently. The temperature of the water is practically negligible under ordinary conditions. Readings can be made more quickly, however, when the temperature is not too far from that of the air. The psychrometer is held firmly and swung rapidly through the air when the space is not too confined. Where there is danger of breakage, it is swung back and forth through a short arc, pendulum-fashion. As the reading must be made when the mercury of the wet bulb reaches the lowest point, the instrument is stopped from time to time and the position of the column noted. The lowest point is often indicated by the tendency of the mercury to remain stationary;

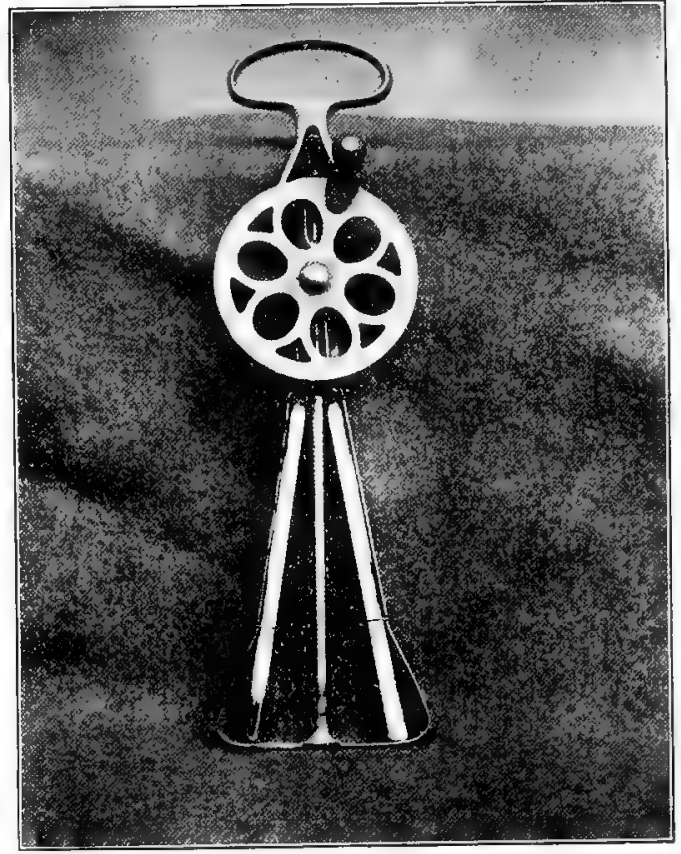

Fig. 6. Cog psychrometer.

as a rule it can be noted with certainty when the next glance shows a rise in the column. In following the movement, and especially in noting the final reading, great care must be taken to make the latter before the mercury begins to rise. For this reason it is desirable to shade the psychrometer with the body when looking at it, and to take pains not to breathe upon the bulbs nor to bring them too near the body. At the moment when the wet bulb registers the lowest point, the dry bulb should be read and the results recorded.

63. Cog psychrometer. This instrument, commonly called the "eggbeater" psychrometer, has been devised to obviate certain disadvantages of 
the sling psychrometer in field work, and has entirely supplanted the latter in the writer's own studies. It is smaller, more compact, and the danger of breaking in carriage or in use is almost nil. It has the great advantage of making it possible to take readings in a layer of air less than two inches in thickness, and in any position. Fairly accurate results can even be obtained from transpiring leaves. The instrument can readily be made by a good mechanic, at a cost for materials of $\$ I .75$, which is less than half the price for the sling form. A single drawback exists in the use of short, Centigrade thermometers, inasmuch as tables of relative humidity are issually expressed in Fahrenheit. It is a simple matter, however, to convert Centigrade degrees into Fahrenheit, mentally, or the difficulty may be avoided by the conversion table shown on page 47 , or by constructing a Centigrade series of humidity tables. The fact that the wet and dry bulbs revolve in the same path has raised a doubt concerning the accuracy of the results obtained with this instrument. Repeated comparisons with the sling psychrometer have not only removed this doubt completely, but have also proved that the standardization of the thermometers has been efficient.

64. Construction and use. A convenient form of egg-beater is the Lyon (Albany, New York), in which the revolving plates can be readily removed, leaving the axis and the frame. The thermometers used are of the short Centigrade type. They are $4 / 2$ inches long and read from $-5^{\circ}$ to $50^{\circ}$. Eimer and Amend, 205 Third Ave., New York city, furnish them at 75 cents each. The thermometers are carefully standardized and compared, and then gromped in pairs that read together. Each pair is used to construct a particular psychrometer. Each thermometer is strongly wired to one side of the frame, pieces of felt being used to protect the tube and increase the contact. The frame is also bent at the base angles to permit free circulation of air about the thermometer bulbs. The bulb of one thermometer is covered with the proper cloth, and the psychrometer is finished. Since the frame revolves with the thermometers, it is necessary to pour the water on the wet bulb, or to employ a pipette or brush. The thermometer bulbs are placed in the layer to be studied, and the frame rotated at an even rate and with moderate rapidity. The observation is further made as in the case of the sling psychrometer. As the circle of rotation is less than three inches in diameter, and the layer less than an inch, in place of nearly three feet for the sling form, the instrument should not be moved at all for extremely localized readings, but it must be moved considerably, a foot or more, if it is desirable to obtain a more general reading.

65. Hygrometers. While there are instruments designed to indicate the humidity by means of a hygroscopic substance, not one of them seems to 
be of sufficient accuracy for use in ecological study. The difficulty is that the hygroscopic reaction is inconstant, rather than that the instruments are not sufficiently sensitive. A number of hygrometers have been tested, and in all the error has been found to be great, varying usually from IO-20 per cent. In the middle of the scale they sometimes read more accurately, but toward either extreme they are very inexact. It seems probable that an accurate hygrometer can be constructed only after the model of the Draper psychrograph. Its weight and bulk would make it an impossible instrument for field trips, and the expense of one would provicle a dozen psychrometers. In consequence, it does not seem too sweeping to say that no hygrometer can furnish trustworthy results. Of simple. instruments for humidity, the psychrometer alone can be trusted to give reliable readings. Crova's hygrometer, used by Hesselmann, is not a hygrometer in the sense indicated. As it is much less convenient to handle and to operate than the $\operatorname{cog}$ psychrometer, it is not necessary to describe it.

\section{Psychrographs}

\section{The Draper psy-} chrograph. A year's trial of the Draper psychrograph in field and planthouse has left little ques-

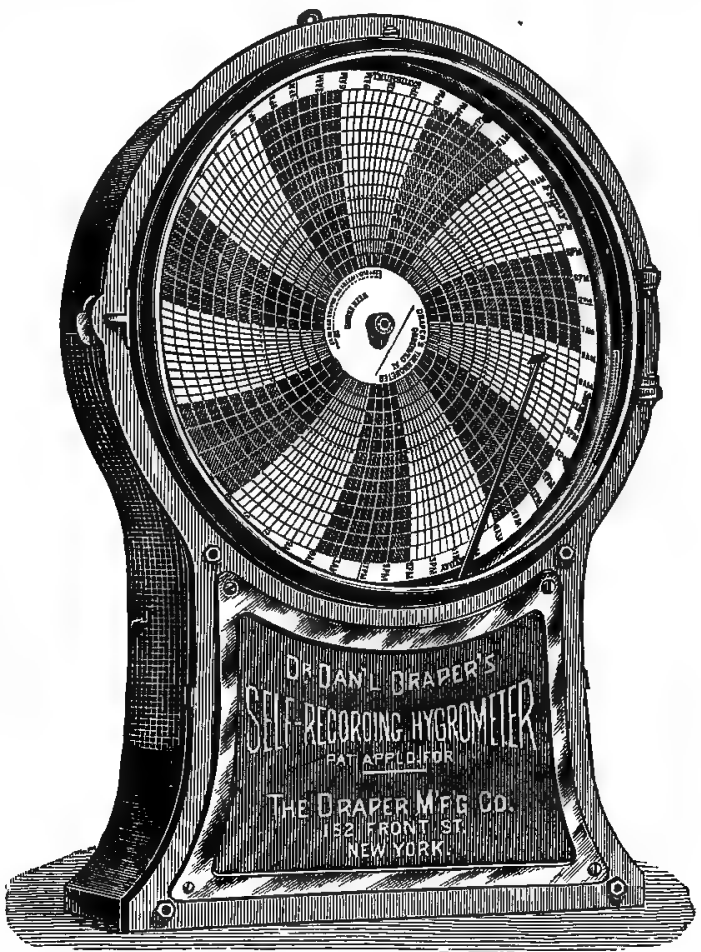

Fig. 7. Draper psychrograph. tion of its accuracy and its great usefulness. Essentially, it consists of a band of fine catgut strings, which are sensitive to changes in the moisturecontent of the air. The variations in the length of the band are communicated to a long pointer carrying an inking pen. The latter traces the record in per cent of relative humidity on a graduated paper disk, which is practically the face of an eight-day clock. The whole is enclosed in a metal case with a glass front. A glance at the illustration will show the general 
structure of the instrument. Continued psychrometric tests demonstrate that the margin of error is well within the efficient difference for humidity, which is taken to be 5 per cent. In the field tests of the past summer, two psychrographs placed side by side in the same habitat did not vary $i$ per cent from each other. The same instruments when in different habitats did not deviate more than I per cent from the psychrometric values, except when the air approached saturation. For humidities above go per cent, the deviation is considerable, but as these are temporary and incident upon rainfall, the error is not serious. For humidities varying from 1o- 85 per cent, the psychrograph is practically as accurate as the psychrometer. Per cents below Io are rare, and no tests have been made for them.

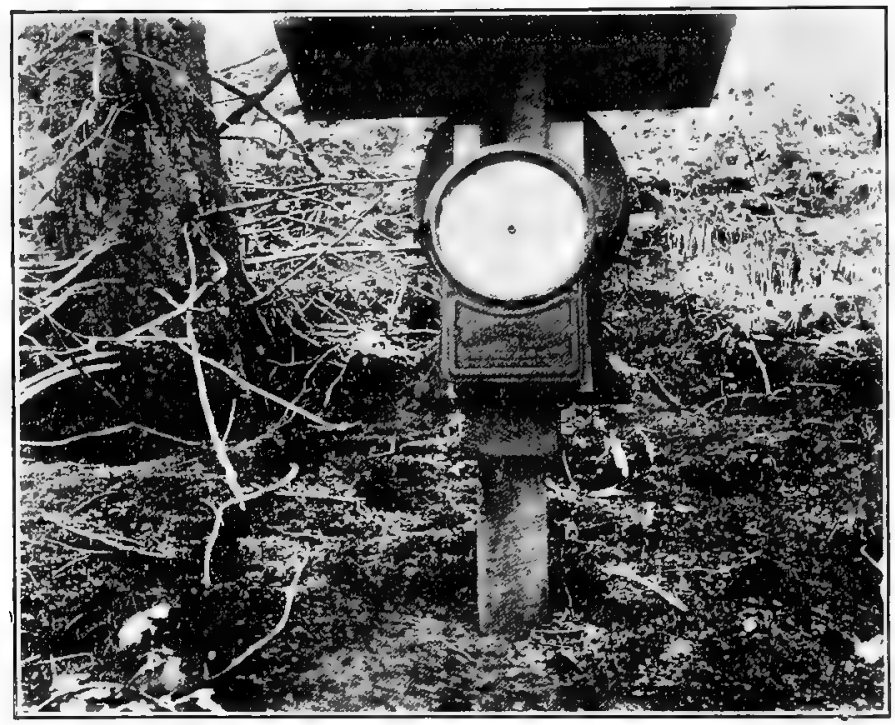

Fig. 8. Instrument shelter, showing thermograph and psychrograph in position.

67. Placing the instrument. The psychrograph should be located in a place where the circulation of the air is typical of the station observed. A satisfactory shelter will screen the instrument from sun and rain, and at the same time permit the air to pass freely through the perforations of the metal case. The form shown in figure 8 meets both of these conditions. A desirable modification is effected by fastening a strip about the cover of such depth as to prevent the sun's rays from striking the case except when the sun is near the horizon. A cross block is fastened on the 
post of the shelter after being exactly leveled. The psychrograph rests upon this block, which is three feet above the ground in order to avoid the influence of radiation. The instrument is held in position by slipping the eye over a small-headed nail driven obliquely. It does not hang from the latter, but must rest firmly upon the cross block. The post is set to a depth that prevents oscillation in the wind, which is liable to obscure the record. In shallow mountain soils stability is attained by fastening a broad board at the base of the post before setting it. When two or more psychrographs are established in different habitats, great pains are taken to set them up in exactly the same way. The shelters are alike, the height above the soil the same, and the instruments all face the south.

68. Regulating and operating the instrument. When two or more psychrographs are to be used in series, they must be compared with each other in the same spot for several days until they run exactly together with respect to per cent of humidity and to time. During this comparison they are checked by the psychrometer and so regulated that they register the proper humidity. When a single instrument is used alone as the basis to which simple readings may be referred, all rcgulating may well be done after the instrument is in position. This is a simple process; it is accomplished by obtaining the relative humidity beneath the shelter and at the proper height by a psychrometer. The pen hand is then moved to the proper line on the disk by means of the screws at its base. These are reached by removing the lettered glass face. The thumbscrew on the side opposite the direction in which the pen is to move is released, and the opposite screw simultaneously tightened, until the pen remains upon the proper line. Experience has proved that the record sheet should be correctly labeled and dated before being placed on the disk. In the press of field duties, records labeled after removal are liable to be confused. It is likewise a great saving of time to write the date of the month in the margin of each segment. Care is taken to place the sheet on the disk in the same position each time; this can easily be done by seeing that the sharp point on the disk penetrates the same spot on the paper. A single drop of ink in the pen will usually give the most satisfactory line. A thin line is read most accurately. If the pen point is too fine, however, the ink does not flow readily, and the point should be slightly blunted by means of a file. More often the line is too broad and the pen must be carefully pointed. Occasionally the pen does not touch the sheet, and it becomes necessary to bend the hand slightly. This is a frequent difficulty if the records are folded or wrinkled, and consequently the sheets should always be kept flat. 
69. The weekly visit. Psychrographs must be visited, checked, rewound, and inked every week. Whenever possible this should be done regularly at a specified day and hour. This is especially desirable if the same record sheet is used for more than one week. Time and energy are saved by a fixed order for the various tasks to be done at each visit. After opening the instrument the disk is removed, and the clock wound, and, if need be, regulated. The record sheet is replaced, the disk again put on the clock arbor, and the pen replenished with a drop of ink. A psychrometer reading is made, and the restlts in terms of relative humidity noted at the proper place on the disk sheet. If the psychrograph vary more than I per cent, it is adjusted to read accurately. In practice it has been found a great convenience to keep each record sheet in position for three weeks, and the time may easily be extended to four. In this event, the pen is carefully cleaned with blotting paper at each visit, and is then refilled with an ink of different color. To prevent confusion, the three different colored inks are always used in the same order, red for the first week, blue for the second, and green for the third. The advantages of this plan are obvious: fewer records are used and less time is spent in changing them. The records of several weeks are side by side instead of on separate sheets, and in working over the season's results, it is necessary to handle but a third as many sheets.

The Draper psychrograph is made by the Draper Manufacturing Company, i52 Front St., New York city. The price is \$30. A few record sheets and a bottle of red ink are furnished with it. Additional records can be obtained at 3 cents each. The inks are $25-50$ cents per bottle, depending ipon the color.

\section{Humidity Readings and Records}

70. The time of readings. If simple instruments alone are used for determining humidity, readings are practically without value unless made simultaneously through several stations, or successively at one. When it is possible to combine these, and to make psychrometer readings at different habitats for each hour of the day, or at the same hour for several days, the series is of very great value. Single readings are unreliable on account of the hourly and daily variations of humidity, but when these changes are recorded by a psychrograph, such readings at once become of use, whether made in the same habitat with the recording instrument or elsewhere. In the latter case, one reading will tell little about the normal humidity of the habitat, but several make a close estimate possible. When a series of psychrographs is in use, accurate observations can be made to advantage anywhere at any time. As a rule, however, it has been found most con- 
venient to make simple readings at 6:00 A.M., I :00 P.M., and 6:00 P.M., as these hours afford much evidence in regard to the daily range. A good time also is that at which the temperature maximum occurs each day, but this is movable and in the press of field work can rarely be taken advantage of. A very fair idea of the daily mean humidity is obtainable by averaging the readings made at the hours already indicated. The comparison of single readings with the psychrograph record should not be made at a time when a rapid change is occurring, as the automatic instrument does not respond inmediately. Such a condition is usually represented by a sudden rain, and is naturally not a satisfactory time for single readings in any event.

71. Place and height. As stated above, the psychrograph is placed three feet above the surface of the ground in making readings for the comparison of stations. In low, herbaceous formations, the instrument is usually placed within a few inches of the soil in order to record the humidity of the air in which the plants are growing. In forest formations, the moisture often varies considerably in the different layers. This variation is easily determined by simultaneous psychrometer readings in the several layers, or, if occasion warrants, a series of psychrographs may be used. In field work the rule has been to make observations with the psychrometer at 6 feet, 3 feet, and the surface of the soil, but the reading at the height of 3 feet is ordinarily sufficient. Humidity varies so easily that several readings in different parts of one formation are often desirable. In comparing different formations, the readings should be made in corresponding situations, for example, in the densest portion of each.

72. Check instruments. Humidity is so readily affected by temperature, wind, and pressure, that a knowledge of these factors is essential to an un-

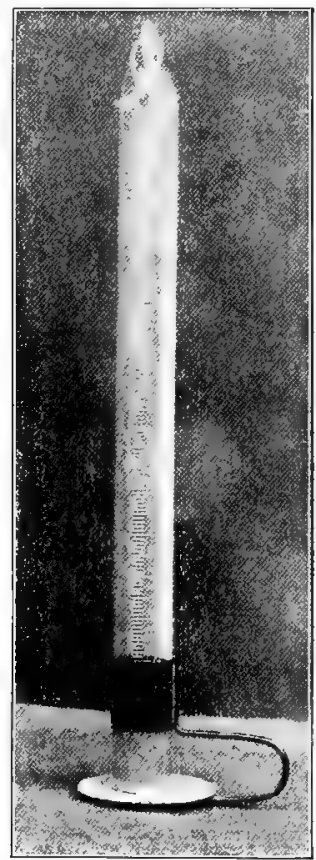

Fig. 9. Atmometer. derstanding of its fluctuations. Pressure, disregarding daily variation, is taken account of in the tables for ascertaining relative humidity, and is determined once for all when the altitude of a station has been carefully established. The temperature is obtained directly from the dry bulb reading. Its value is fundamental, as the amount of moisture in a-given space is directly affected by it; like pressure, it also is taken account of in the formula. The movement of the air has an immediate influence 
upon moisture by mixing the air of different habitats and layers. So far as the plant is concerned, it has practically the effect of increasing or decreasing the humidity by the removal of the air above it. Thus, while the anemometer can furnish no direct evidence as to the amount of variation, it is of aid in explaining the reason for it. Likewise, the rate of evaporation as indicated by a series of atmometers, affords a ready method of estimating the comparative effect of humidity in different habitats. Potometers and other instruments for measuring transpiration throw much light upon humidity values. Since they are concerned with the response of the plant to humidity, they are considered in the following chapter.

73. Humidity tables. To ascertain the relative humidity, the difference between the wet and dry bulb readings is obtained. This, with the dry bulb temperature, is referred to the tables, where the corresponding humidity is found. A variation in temperature has less effect than a variation in the difference; in consequence, the dry bulb reading is expressed in the nearest init, and the difference reckoned to the nearest .5 . The humidity varies with the air pressure. Hence, the altitude must be determined for the base station, and for all others that show much change in elevation. Within the ordinary range of growing-period temperatures, the effect of pressure is not great. For all ordinary cases, it suffices to compute tables for pressures of 30,29 , 27,25 , and 23 inches. The following table indicates the decrease in pressure which is due to altitude.

ALTITUDE

\begin{tabular}{rrcc}
\multicolumn{2}{c}{ ALTITUDE } & \multicolumn{2}{c}{ PRESSURE } \\
Feet & Meters & Inches & Centimeters \\
0 & 0 & 30 & 76 \\
910 & 277 & 29 & 73.5 \\
1850 & 574 & 28 & 71 \\
2820 & 860 & 27 & 68.5 \\
3820 & 1165 & 26 & 66 \\
4850 & 1477 & 25 & 63.5 \\
5910 & 1792 & 24 & 61 \\
7010 & 2138 & 23 & 58.5 \\
8150 & 2485 & 22 & 56 \\
9330 & 2845 & 21 & 53.5 \\
10550 & 3217 & 20 & 51 \\
13170 & 4016 & 18 & 46 \\
16000 & 4880 & 16 & 41
\end{tabular}

The fluctuations of pressure due to weather are usually so slight that their influence may be disregarded. An excellent series of tables of relative humidity is found in Marvin's Psychrometric Tables; published by the U. S. Weather Bureau, and to be obtained from the Division of Publications, Washington, D: C., for Io cents. A convenient field form is mađe by removing the portion containing the tables of relative humidity, and binding it in stiff oilcloth. 
74. Sums, means, and curves. An approximate humidity sum can be obtained by adding the absolute humidities for each of the twenty-four hours, and expressing the results in grains per cubic foot. It is possible to establish a general ratio between this sum and the transpiration sum of the plant, but its value is not great at present. Means of absolute and of relative humidity are readily determinable from the psychrograph records; the latter are the most useful. The mean of relative humidity for the twenty-four hours of a day is the average of the twentyfour hour humidities. From these means the seasonal mean is computed in the same manner. A close approximation, usually within I degree, may be obtained in either case by averaging the maximum and minimum for the period concerned. Various kinds of curves are of value in representing variation in humidity. $\mathrm{Ob}$ viously, these must be derived from the psychrograph, or from the psychrometer when the series is sufficiently complete. The level curve indicates the variation in different stations at the same time.

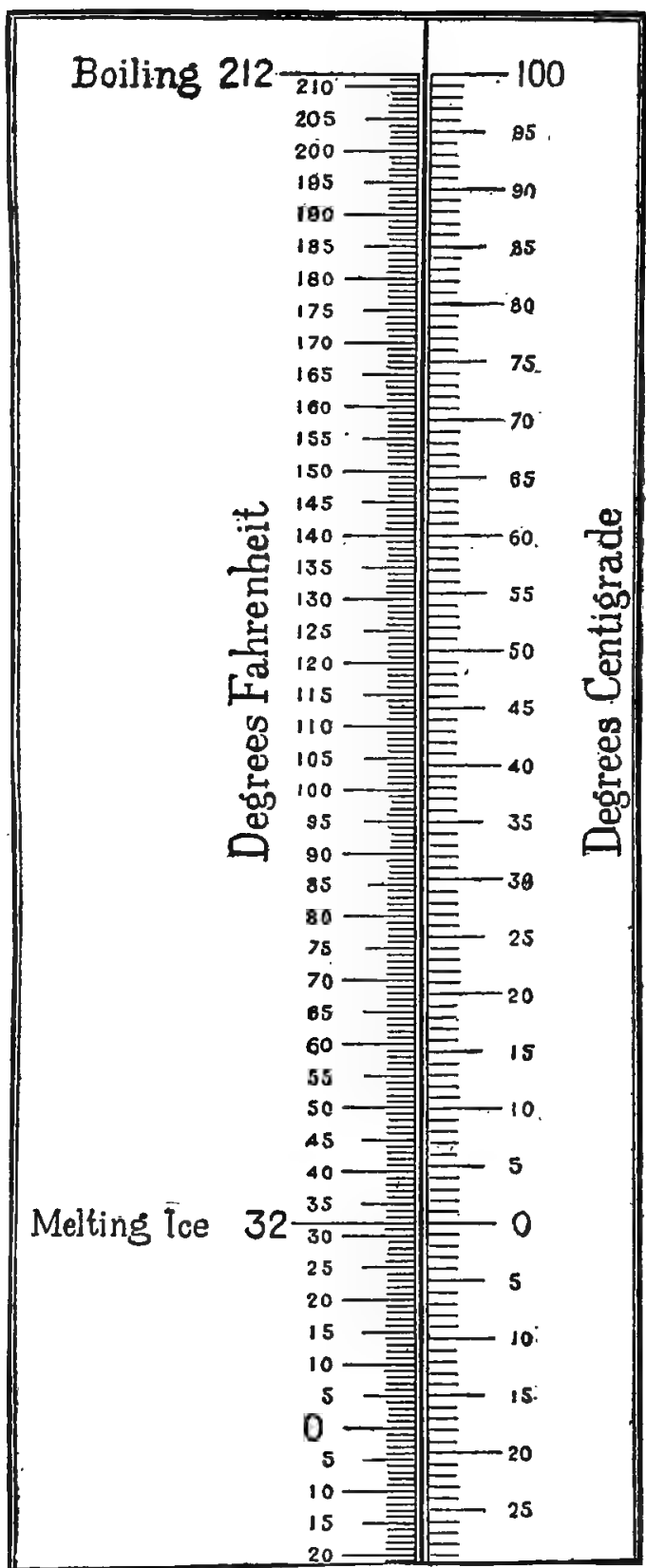

Fig. 10. Conversion scale for temperatures. 
These may be combined in a series for the comparison of readings made at various heights in the stations. The day or point curve shows the fluctuations during the day of one point, and the station curve the variation at different heights in the same station. The curves of successive days or of different stations may of course be combined on the same sheet for comparison. Level and station curves based upon mean relative humidities are especially valuable.

75. Records. A field form is obviously unnecessary for the psychrograph. The record sheets constitute both a field and permanent record. The altitude and other constant features of the station and the list of species, etc., are entered on the back of the first record sheet, or, better, they are noted in the permanent formation record. For psychrometer readings, whether single or in series, the following record form is employed:

\begin{tabular}{|c|c|c|c|c|c|c|c|c|c|c|c|c|c|c|c|}
\hline \multirow[b]{2}{*}{$\stackrel{\text { II }}{\text { Aू }}$} & \multirow[b]{2}{*}{ 总 } & \multirow[b]{2}{*}{ 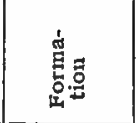 } & \multirow[b]{2}{*}{ 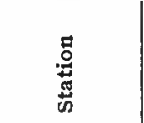 } & \multirow{2}{*}{ 葵 } & \multirow{2}{*}{ 兽 } & \multirow{2}{*}{ 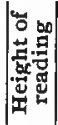 } & \multirow{2}{*}{$\mid \begin{array}{l}0 \\
\bar{\Xi} \\
\hat{\theta}\end{array}$} & \multirow{2}{*}{ 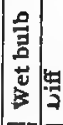 } & \multirow{2}{*}{\multicolumn{2}{|c|}{ 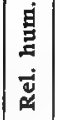 }} & \multirow{2}{*}{ 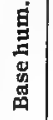 } & \multirow{2}{*}{ 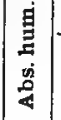 } & \multicolumn{3}{|c|}{ NOTES } \\
\hline & & & & & & & & & & & & & 焉 & 品 & 疍 \\
\hline $15 / 8 /{ }^{\prime} 04$ & 6:20 A.M. & Spruce .... & Brook bank & $2500 \mathrm{~m}$ & Mertensiare & $1 \mathrm{ft}$ & $51^{\circ}$ & $46^{\circ}$ & 57 & 726 & $63 \%$ & 2.9 & Clear & 0 & 0 \\
\hline 4 & 4 & Half gravel & Hiawatha & “ & Asterare ... & $"$ & $56^{\circ}$ & $49^{\circ}$ & 76 & $61 \%$ & $63 \%$ & 3.0 & "4 & 0 & 0 \\
\hline$"$ & 6:45 P M. & Spruce .... & Brook bank & $"$ & Mertensiare & $*$ & $54^{\circ}$ & $52^{\circ}$ & 2 & $89 \%$ & $69 \%$ & 4.2 & “. & $2 \mathrm{cc}$. & 0 \\
\hline “" & “" & Half gravel & Hiawatha .. & “ & Asterare .... & “ & $56^{\circ}$ & ธี20 & 47 & $79 \%$ & 696 & 4.0 & “" & $2 \mathrm{cc}$. & $\mathbf{0}$ \\
\hline
\end{tabular}

On page 47 is given a table for the conversion of Centigrade into Fahrenheit temperatures. This may be done mentally by means of the formula $F=\frac{C}{5} \times 9+32^{\circ}$.

\section{LIGIHT}

76. Methods. All methors for measuring light intensity, which have been at all satisfactory, arc based upon the fact that silver salts blacken in the light. The first photographic method was proposed by Bunsen and Roscoe in 1862 ; this has been taken up by Wiesner and variously modified. After considerable experiment by the writer, however, it seemed desirable to abandon all methods which require the use of "normal paper" and "normal black" and to develop a simpler one. As space is lacking for a satisfactory discussion of the Bunsen-Roscoe-Wiesner methods, the reader is referred to the works cited below. ${ }^{1}$ Simple photometers for making light readings simultaneously

'Bursen, R., And Roscoe, H. Photometrische Untersuchungen. Poggendorff's Annalen., 117 :599. 1862.

WIESNER, J.

Photometrische Untersuchungen auf pflanzenphysiologischen Gebiete. Sitzb. Akad. Wiss. Wien., I, 1893. II, 1895. 
or in series were constructed in I900, and have been in constant use since that time. An automatic instrument capable of making accurate continuous records proved to be a more difficult problem. A sunshine recorder was ultimately found which yields valuable results, and very recently a recording photometer which promises to be perfectly satisfactory has been devised. Since the hourly and daily variations of sunlight in the same habitat are relatively small, automatic photometers are perhaps a convenience rather than" a necessity.

\section{The Photometer}

77. Construction. The simple form of photometer shown in the illustration is a light-tight metal box with a central wheel upon which a strip of

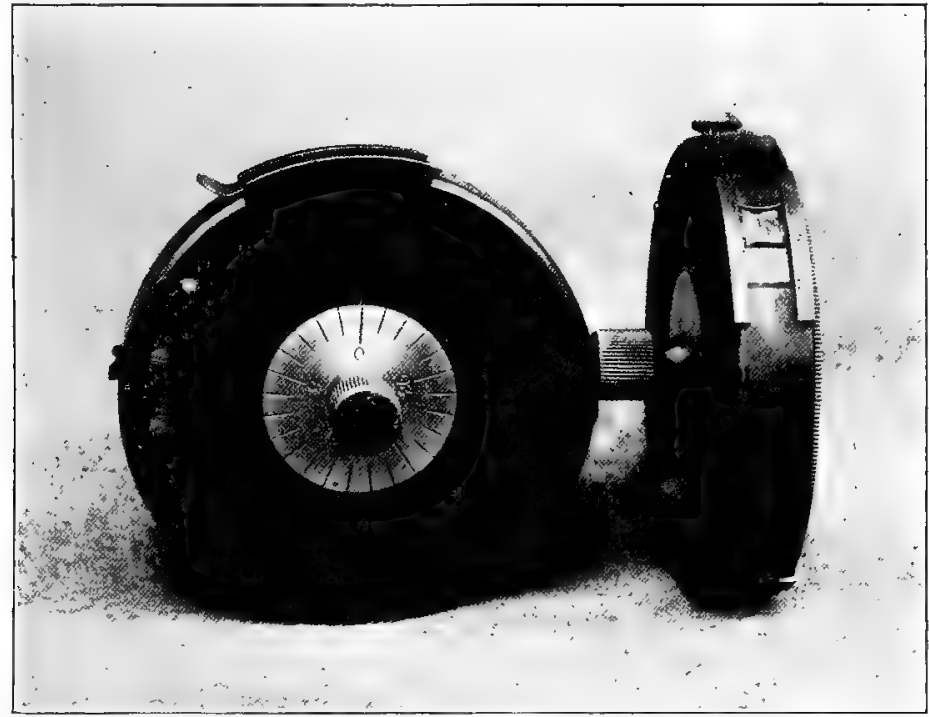

Fig. 11. Photometer, showing front and side view.

photographic paper is fastened. This wheel is revolved by the thumbscrew past an opening $6 \mathrm{~mm}$. square which is closed by means of a slide working closely between two flanges. At the edge of the opening, and beneath the slide is a hollow for the reception of a permanent light standard. The disk of the thumbscrew is graduated into twenty-five parts, and these are numbered. A line just beneath the opening coincides with

Untersuchungen über das photochemische Klima von Wien, Cairo, und Buitenzorg (Java) Denksch. Kais. Akad. Wien., 64. 1896.

Untersuchungen über den Lichtgenuss der Pflanzen im arktischen Gebiete. Sitzb. Kais. Akad. Wien., 109. 1900. 
the successive lines on the disk, and indicates the number of the exposure. The wheel contains twenty-five hollows in which the click works, thus moving each exposure just beyond the opening. The metal case is made in two parts, so that the bottom may be readily removed, and the photographic strip placed in position. The water-photometer is similar except that the opening is always covered with a transparent strip and the whole instrument is water-tight. These instruments have been made especially for measuring light by the C. H. Stoelting Co., 3I W. Randolph street, Chicago, Ill. The price is $\$ 5$.

78. Filling the photometer. The photographic paper called "solio" which is made by the Eastman Kodak Company, Rochester, N. Y., has proved to be much the best for photometric readings. The most convenient size is that of the $8 \times$ Io inch sheet, which can be obtained at any supply house in packages of a dozen sheets for 60 cents. New "emulsions," i. e., new lots of paper, are received by the dealers every week, but each emulsion can be preserved for three to six months without harm if kept in a cool, lighttight place. Furthermore, all emulsions are made in exactly the same way, and it has been impossible to detect any difference in them. To fill the photometer, a strip exactly $6 \mathrm{~mm}$. wide is cut lengthwise from the $8 \times$ Io sheet. This must be done in the dark room, or at night in very weak light. The strip is placed on the wheel, extreme care being taken not to touch the coated surface, and fixed in position by forcing the free ends into the slit of the wheel by a piece of cork 8-9 $\mathrm{mm}$. long. The wheel is replaced in the case, turned until the zero is opposite the index line, and the instrument is ready for use.

79. Making readings. An exposure is made by moving the slide quickly in such a way as to uncover the entire opening, and the standard if the exposure is to be very short. Care must be taken not to pull the slide entirely out of the groove, as it will be impossible to replace it with sufficient quickness. The time of exposure can be determined by any watch after a little practice. It is somewhat awkward for one person to manage the slide properly when his attention is fixed upon a second hand. This is obviated by having one observer handle the watch and another the photometer, but here the reaction time is a source of considerable error. The most satisfactory method is to use a stop-watch. This can be held in the left hand and started and stopped by the index finger. The photometer is held against it in the right hand in such a way that the two movements of stopping the watch and closing the slide may be made at the same instant. The length of exposure is that necessary to bring the tint of the paper to that of the 
standard beside it. A second method which is equally advantageous and sometimes preferable does away with the permanent standard in the field and the need for a stop-watch. In this event, the strip is exposed until a medium color is obtained, since very light or very deep prints are harder to match. This is later compared with the multiple standard. In both cases, the date, time of day, station, number of instrument and of exposure, and the length of the latter in seconds are carefully noted. The instrument is held with the edge toward the south at the level to be read, and the opening uppermost in the usual position of the leaf. When special readings are desired, as for isophotic leaves, reflected light, etc., the position is naturally changed to correspond. In practice, it is made an invariable rule to move the strip for the next exposure as soon as the slide is closed. Otherwise

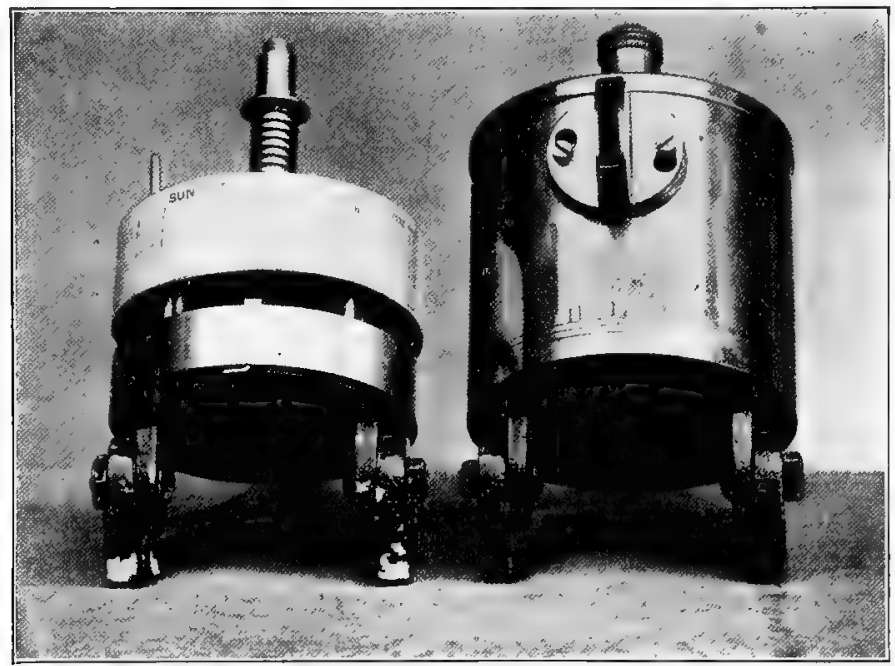

Fig. 12. Dawson-Lander sun recorder.

double exposures are liable to occur. When a strip is completely exposed it is removed in the dark, and a new one put in place. The former is carefully labeled and dated on the back, and put away in a light-tight box in a cool place.

80. The Dawson-Lander sun recorder. "The instrument consists of a small outer cylinder of copper which revolves with the sun, and through the side of which is cut a narrow slit to allow the sunshine to impinge on a strip of sensitive paper, wound round a drum which fits closely inside the outer cylinder, but is held by a pin so that it can not rotate. By means of 
a serew fixed to the lid of the outer cylinder, the drum holding the sensitive paper is made to travel endwise down the outer tube, one-eighth of an inch daily, so that a fresh portion of the sensitive surface is brought into position to receive the record." The instrument is driven by an eight-day clock placed in the base below the drum. The slit is covered by means of a flattened funnel-shaped hood, and the photographic strip is protected from rain by a perfectly transparent sheet of celluloid. The detailed structure of the instrument is shown in figure $\mathbf{I} 2$. This instrument may be obtained from Lander and Smith, Canterbury, England, for \$35.

In setting up the sunshine recorder, the axis should be placed in such a position that the angle which it makes with the base is the same as the altitude of the place where the observations are made. This is readily done by loosening the bolts at either side. The drum is removed, the celluloid sheet unwound by means of the key which holds it in place, the sensitive strip put in position, and the sheet again wound up. Strips of a special sensitive paper upon which the hours are indicated are furnished by the makers of the instrument, but it has been found preferable to use solio strips in order to facilitate comparison with the standards. The drum is placed on the axis, and is screwed up until it just escapes the collar at the top of the spiral. The clock is wound and started, and the outer cylinder put on so that the proper hour mark coincides with the index on the front of the base.

As a sunshine recorder, the instrument gives a perfect record, in which the varying intensities are readily recognizable. Since the cylinder moves one-half inch in an hour, and the slit is or of an inch, the time of each exposure is 72 seconds. This gives a very deep color on the solio paper, which results in a serious error in nuaking comparisons with the standard. On account of the hood, diffuse light is not recorded when it is too weak to cast a distinct shadow. It seems probable that this difficulty will be overcome by the use of a flat disk containing the proper slit, and in this event the instrument will become of especial value for measuring the diffuse light of layered formations. The celluloid sheet constitutes a source of error in sunlight on account of the reflection which it causes. This can be prevented by using the instrument only on sunny days, when the protection of the slicet can be dispensed with.

81. The selagraph. This instrument is at present under construction, and can only be described in a general way. In principle it is a simple photometer operating automatically. It consists of a light-tight box preferably of metal, which contains an eight-day lever clock. Attached to the arbor of the latter is a disk 7 inches in diameter bearing on its circumference a solio strip I $\mathrm{cm}$. wide and $59 \mathrm{~cm}$. long. The opening in the box 
fo: exposure is $6 \mathrm{~mm}$. square and is controlled by a photographic shutter. The latter is constructed so that it may be set for 5 , Io, or 20 seconds, since a single period of exposure can not serve for both sun and shade. The shutter is tripped once every two hours, by means of a special wheel revolving once a day. Each exposure is $6 \mathrm{~mm}$. square, and is separated by a small space from the next one. Twelve exposures are made every 24 hours, and 84 during the week, though, naturally, the daytime exposures alone are recorded. Comparisons with the multiple standard are made exactly as in the case of the simple photometer. The selagraph is made by the $\mathrm{C}$. $\mathrm{H}$. Stoelting Co., Chicago, Illinois.

\section{Standards}

82. Use. The light value of each exposure is determined by reference to a standard. Wher the photometer carries a permanent standard, each exposure is brought to the tint of the latter, and its value is indicated by the time ratio between them. Thus, if the standard is the result of a 5-second exposure to full sunlight at meridian, and a reading which corresponds in color requires Ioo seconds in the habitat concerned, the light of the latter is twenty times weaker or more diffuse. Usually, the standard is regarded as unity, and light values figured with reference to it, as .05. With the selagraph such a use of the standard is impossible, and often, also, with the photometer it is unnecessary or not desirable. The value of each exposure in such case is obtained by matching it with a multiple standard, after the entire strip has been exposed. The further steps are those already indicated. After the exact tint in the standard has been found, the length of the reading in seconds is divided by the time of the proper standard, and the result expressed as above.

83. Making a standard. Standards are obtained by exposing the photometer at meridian on a typically clear day, and in the field where there is the least dust and smoke. Exception to the latter may be made, of course, it1 obtaining standards for plant houses located in cities, though it is far better to have the same one for both field and control experiment. Usable standards can be obtained on any bright day at the base station. Indeed, valuable results are often secured by immediate successive sun and shade readings in adjacent habitats, where the sun reading series is the sole standard. Preferably, standards should be made at the solstices or equinoxes, and at a representative station. The June solstice is much to be preferred, as it represents the maximum light values of the year. Lincoln has been taken as the base station for the plains and mountains. It is desirable, however, 
that a national or international station be ultimately selected for this purpose, in order that light values taken in clifferent parts of the world may be readily compared.

84. Kinds of standards. The base standard is the one taken at Lincoln (latitude $4 \mathrm{I}^{\circ} \mathrm{N}$.) at meridian June $20-22$. This is properly the unit to which all exposures are referred, but it has been found convenient to employ the Minnehaha standard as the base for the Colorado mountains, in order to avoid reducing each time. Relative standards are frequently used for temporary purposes. Thus, in comparing the light intensities of a series of formations, one to five standards are exposed on the solio strip before beginning the series of readings. Proof standards are the exposed solio strips, which fade in the light, and can, in consequence, be kept only a few weeks without possibility of error. The fading can be prevented by "toning" the strip, but in this event the exposures must be fixed in like manner before they can be compared. This process is inconvenient and time-consuming. It is also open to considerable error, as the time of treatment, strength of solution, etc., must be exactly equivalent in all instances. Permanent standards are accurate water-color copies of the originals obtained by the photometer. These have the apparent disadvantage of requiring a double comparison or matching, but after a little practice it is possible to reproduce the solio tints so that the copy is practically indistinguishable from the original. The most satisfactory method is to make a long stroke of color on a pure white paper, since a broad wash is not quite homogeneous, and then to reject such parts of the stroke as do not match exactly. Permanent standards fade after a few month's use, and must be replaced by parts of the original stroke. Singlc standards are made by one exposure, while multiple ones have a series of exposures filling a whole light strip. These are regularly obtained by making the exposures from I-IO seconds respectively, and then increasing the length of each successive exposure by 2 seconds. Single exposures of $\mathrm{x}-5$ seconds as desired usually serve as the basis for permanent standards, but a multiple standard may also be copied in permanent form. Exposures for securing standards must be made only under the most favorable conditions, and the length in seconds must be exact. The use of the stopwatch is imperative, except where access may be had to an astronomical clock with a large second hand, which is even more satisfactory. The length of time necessary for the series desired is reckoned beforehand, and the exposures begun so that the meridian falls in the middle of the process.

Single standards are exceedingly convenient in photometer readings, but they are open to one objection. In the sunshine it is necessary to make instant decision upon the accuracy of the match, or the exposure becomes too 
deep. In the shade where the action is slower, this difficulty is not felt. For this reason it is usually desirable to check the results by a multiple standard, and in the case of selagraph records, where the various exposures show a wide range of tint, light values are obtainable only by direct comparison with the multiple standard. The exact matching of exposure and standard requires great accuracy, but with a little practice this may be done with slight chance of error by merely moving the exposure along the various tints of the standard until the proper shade is found. The requisite skill is soon acquired by running over a strip of exposures several times until the comparisons always yield the same results for each. The margin of error is practically negligible when the same person makes all the comparisons, and in the case of two or three working on the same reading the results diverge little or not at all. The efficient difference for light is much more of a variable than is the case with water-content. It has been determined so far only for a few species, all of which seem to indicate that appreciable modification in the form or structure of a leaf does not occur until the reduction in intensity reaches. I of the meridian sunlight at the June solstice. The error of comparison is far less than this, and consequently may be ignored, even in the most painstaking inquiry.

\section{Readings}

85. Time. The intensity of the light incident upon a habitat varies periodically with the hour and the day, and changes in accord with the changing conditions of the sky. The light variations on cloudy days can only be determined by the photometer. While these can not be ignored, proper comparisons can be instituted only between the readings taken on normal days of sunshine. The sunlight varies with the altitude of the sun, i. e., the angle which its rays make with the surface at a given latitude. This angle reaches a daily maximum at meridian. The yearly maximum falls on June 22, and the angle decreases in both directions through the year to a minimum on December 22. At equal distances from either solstice, the angle is the same, e. g., on March 2I and September 23. At Lincoln ( $4 \mathrm{I}^{\circ} \mathrm{N}$. latitude) the extremes at meridian are $73^{\circ}$ and $26^{\circ}$; at Minnehaha $\left(39^{\circ}\right)$ they are $75^{\circ}$ and $28^{\circ}$. The extremes for any latitude may be found by subtracting its distance in degrees north of the two tropics from 90. Thus, the 5oth parallel is $26.5^{\circ}$ north of the tropic of Cancer, and the maximum altitude of the sun at a place upon it is $63.5^{\circ}$. It is $73.5^{\circ}$ north of the tropic of Capricorn, and the minimum meridional altitude is $16.5^{\circ}$.

The changes in the amount of light due to the altitude of the sun are produced by the earth's atmosphere. The absorption of light rays is greatest near the horizon, where their pathway through the atmosphere is longest, 
and it is least at the zenith. The absorption, and, consequently, the relative intensity of sunlight, can be determined at a given place for each hour of any sunshiny day by the use of chart I3. This chart has been constructed for Lincoln, and will serve for all places within a few degrees of the 4oth parallel. The curves which show the altitude of the sun at the various times of the day and the year have been constructed by measurements upon the celestial globe. Each interval between the horizontal lines represents 2 degrees of the sun's altitude. The vertical lines indicate time before or after the apparent noon, the intervals corresponding to Io minutes. If the relative intensity at Lincoln on March I2 at $3: 00$ P.M. is desired, the apparent noon for this day must first be determined. A glance at the table shows that the sun crosses the meridian on this day at 9 minutes 53 seconds past noon at the goth meridian. The apparent noon at Lincoln is found by adding 26 minutes 19 seconds, the difference in time between Lincoln and a point on the goth meridian. When the sun is fast, the proper number of minutes is taken from 26 minutes 49 seconds. The apparent noon on March $\mathrm{I} 2$ is thus found to fall at $12: 37$ P.M., and $3: 00$ I.M. is 2 hours and 23 minutes later. The sun's altitude is accordingly $36^{\circ}$. If the intensity of the light which reaches the earth's surface when the sun is at zenith is taken as $I$, the table of the sun's altitudes gives the intensity at $3: 00 \mathrm{P} . \mathrm{Mr}$. on March 12 as .85 .

For places with a latitude differing by several degrees from that of Lincoln, it is necessary to construct a new table of altitude curves from the celestial globe. It is quite possible to make a close approximation of this from the table given, since the maximum and minimum meridional altitude, and hence the corresponding light intensity, can be obtained as indicated above. For Minnehaha, which is on the rosth meridian, and for other places on standard meridians, i. e., $60^{\circ}, 75^{\circ}, 90^{\circ}$, and $120^{\circ} \mathrm{W}$., the table of apparent noon indicates the number of minutes to be addled to $t 2$ noon, standard time, when the sun is slow, and to be subtracted when the sun is fast. The time at a place east or west of a standard meridian is respectively faster or slower than the latter. The exact difference in minutes is obtained from the difference in longitude by the equation, $15^{\mathrm{C}}=\mathrm{I}$ hour. Thus, Lincoln, $96^{\circ} 42^{\prime}$ $W$. is $6^{\circ} 42^{\prime}$ west of the standard meridian of $90^{\circ}$; it is consequently 26 minutes 49 seconds slower, and this time must always be added to the apparent noon as determined from the chart. At a place east of a standard meridian, the time difference is, of course, stubtracted.

The actual differences in the light intensity from hour to hour and day to day, which are caused by variations in the sun's altitude, are not as great as might be expected. For example, the maximum intensity at Lincoln, June 22, is .98; the minimum meridional intensity December 22 is .73. The extremes on June 22 are .98 and .33 (the latter at $6: 00$ A.M. and $6: 00$ P.M. 
approximately); between $8: 00$ A.M. and $4: 00$ P.M. the range in intensity is from .90 to .98 merely. On December 22, the greatest intensity is .52 , the least .20 (the latter at 8:00 A.M. and 4:00 P.M. approximately). If the growing season be taken as beginning with the rst of March and closing the Ist of October, the greatest variation in light intensity at Lincoln within a period of Io hours with the meridian at its center (cloudy days excepted) is from .33 to .98. In a period of 8 hours, the extremes are .65 to .98 , i. e., the greatest variation, .3 , is far within the efficient difference, which has been put at .9.

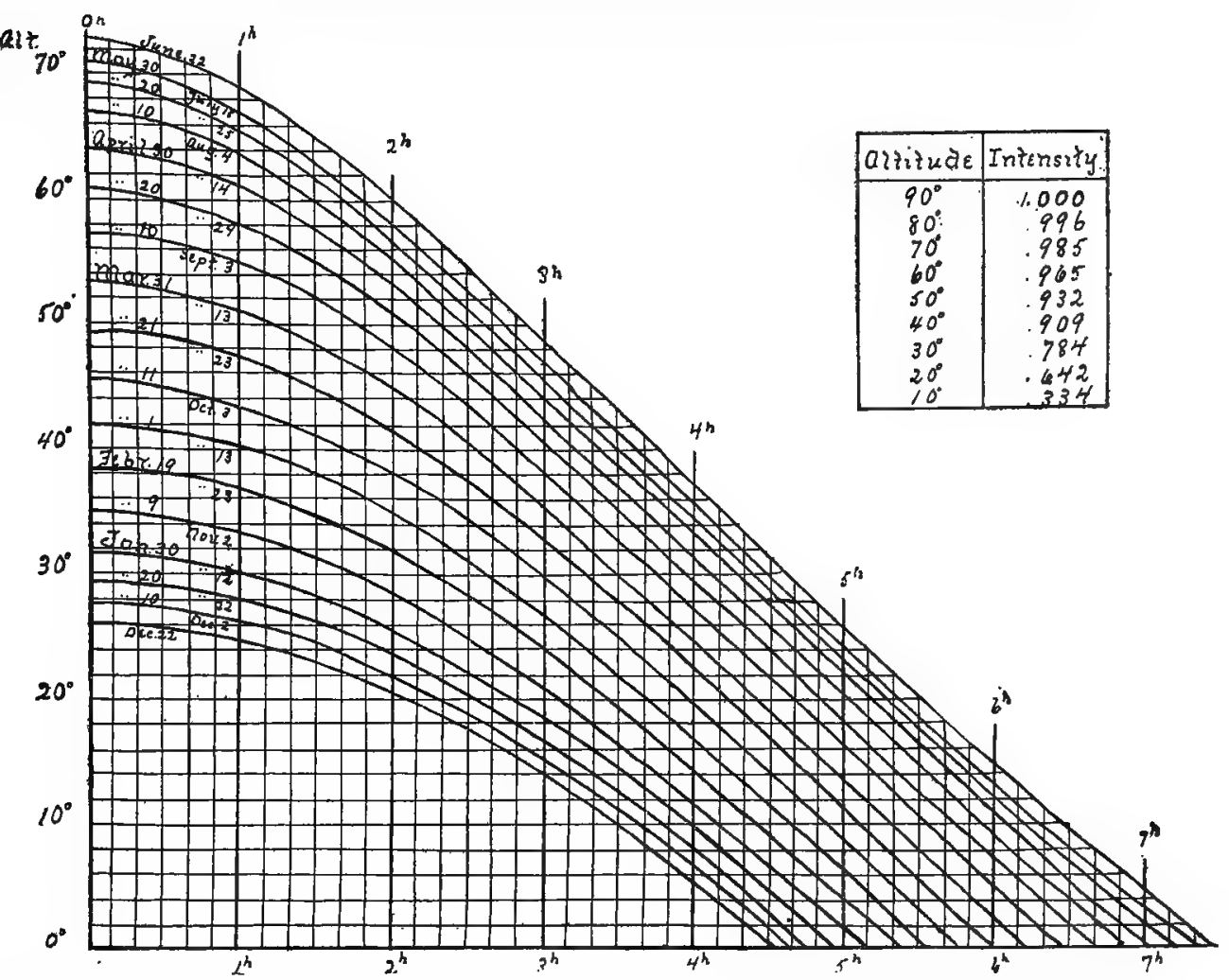

Fig. 13. Chart for the determination of the sun's altitude, and the corresponding light intensity.

For the growing period, then, readings made between $8: 00$ A.M. and $4: 00$ P.M. on normal sunshiny days may be compared directly, without taking into account the compensation for the sun's altitude. Until the efficient difference has been determined for a large number of species, however, it seems wise to err on the safe side and to compensate for great differences in time of day or year. In all doubtful cases, the intensity obtained by the astronomical 
method should also be checked by photometric readings. A slight error probably enters in, due to reflection from the surface of the paper, and to temperature, but this is negligible.

86. Table for determining apparent noon

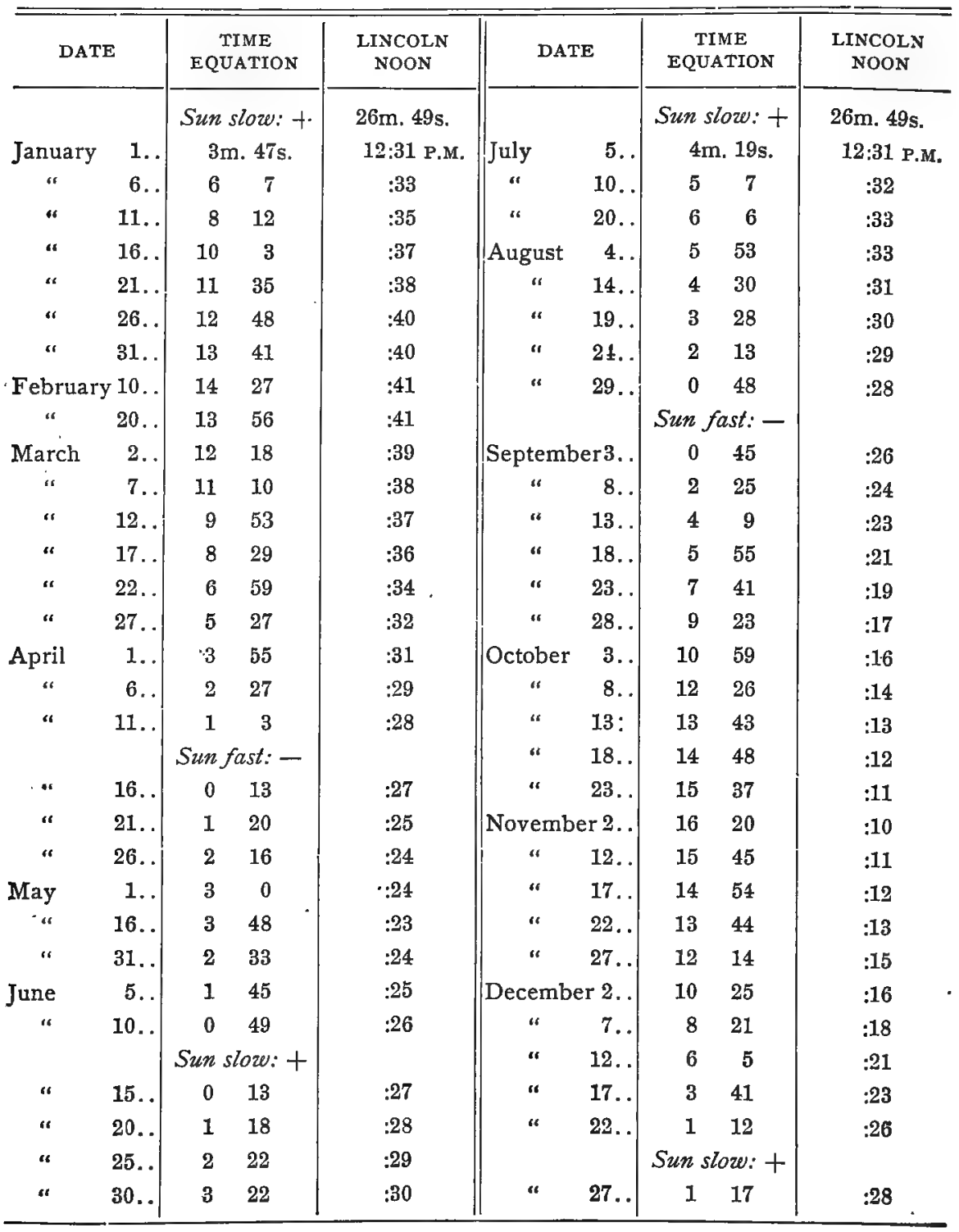


87. Place. The effect of latitude upon the sun's altitude, and the consequent light intensity have been discussed in the pages which precede. Latitude has also a profound influence upon the duration of daylight, but the importance of the latter apart from intensity is not altogether clear. The variation of intensity due to altitude has been greatly overestimated; it is practically certain, for example, that the dwarf habit of alpine plants is not to be ascribed to intense illumination, since the latter ircreases but slightly with the altitude. It has been demonstrated astronomically that about 20 per cent of a vertical ray of sunlight is absorbed by the atmosphere by the time it reaches sea level. At the summit of Pike's Peak, which is I 4,000 feet $(4,267$ meters $)$ high, the barometric pressure is I7 inches, and the absorption is approximately II per cent. In other words, the light at sea level is 80 per cent of that which enters the earth's atmosphere; on the summit of Pike's Peak it is 89 per cent. As the effect of the sun's altitude is the same in both places, the table of curves on page 57 will apply to both. Taking into account the difference in absorption, the maximum intensity at sea level and at I4,000 feet on the fortieth parallel is .98 and I.09 respectively. The minimum intensities between 8:00 A.M. and 4:00 P.M. of the growing period are .64 and .7I respectively. The correctness of these figures has been demonstrated by photometer readings, which have given almost exactly the same results. Stuch slight variations are quite insufficient to produce an appreciable adjustment, particularly in structure. They are far within the efficient difference, and Reinke ${ }^{1}$ has found, moreover, that photosynthetic activity in Eloded is not increased beyond the normal in sunlight sixty times concentrated. In consequence, it is entirely unnecessary to take account of different altitudes in obtaining light values.

The slope of a habitat exerts a considerable effect upon the intensity of the incident light. If the angle between the slope and the sun's ray be $90^{\circ}$, a square meter of surface will receive the maximum intensity, I. At an angle of $10^{\circ}$, the same area receives but.I 7 of the light. This relation between angle and intensity is shown in the table which follows. The influence of the light, however, is felt by the leaf, not by the slope. Since there is no connection between the position of the leaf and the slope of the habitat, the latter may be ignored. In consequence, it is unnecessary to make allowances for the direction of a slope, viz., whether north, east, south, or west, in so far as light values are concerned. The angle which a leaf makes with its stem determines the angle of incidence, and hence the amount of light received by the leaf surface. This is relatively unimportant for two reasons. This angle changes hourly and daily with the altitude of the

${ }^{1}$ ReINKE, J. Bot. Zeit., 41:713. 1883. 
sun, and the intensity constantly swings from one extreme to the other. Moreover, the extremes I.OO and 0.17 , even if constant, are hardly sufficient to produce a measurable result. When the angle of the leaf approaches $90^{\circ}$, there is the well-known differentiation of leaf surfaces and of chlorenchym, but this has no relation to the angle of incidence.

$\begin{array}{cc}\text { Table of Intensity } & \text { at } \\ & \text { Various } \\ \text { ANGLE } & \text { INTENSITY } \\ 90 & 1.00 \\ 80 & .98 \\ 70 & .94 \\ 60 & .87 \\ 50 & .77 \\ 40 & .64 \\ 30 & .50 \\ \mathbf{0} & .34 \\ 10 & .17\end{array}$

In the sunlight, it makes no difference at what height a light reading is taken. In forest and thicket as well as in some herbaceous formations, the intensity of the light, if there is any difference, is greatest just beneath the foliage of the facies. In forests especially, the light is increasingly diffuse toward the ground, particularly where layers intervene. In woodland formations, moreover, the exact spot in which a reading is made must be carefully chosen, unless the foliage is so dense that the shade is uniform. A very satisfactory plan is to take readings in two or more spots where the shade appears to be typical, and to make a check reading in a "sunfleck," a spot where sunlight shows through. In forests and thickets, the sunflecks are fleeting, and the light value is practically that of the shade. In passing into open woodland and thicket, the sunflecks increase in size and permanence, until finally they exceed the shade areas in amount and become typical of the formation.

\section{Reflected and Absorbed Light}

88. The fate of incident light. The light present in a habitat and incident upon a leaf is not all available for photosynthesis. Part is reflected or screened out by the epidermis, and a certain amount passes through the chlorenchym, except in very thick leaves. The light absorbed is by far the greatest in the majority of species. Many plants with dense coatings of hairs reflect or withhold more light than they absorb, and the amount of light reflected by a thick cuticule is likewise great. As light is imponderable, the actual amoint absorbed or reflected by the leaf can not be determined. It is possible, however, to express this in terms of the total amount 
received, by means of readings with solio paper, and the knowledge thus obtained is of great importance in interpreting the modifications of certain types of leaves. For example, a leaf with a densely hairy epidermis may receive light of the full intensity, $I$; the amount reflected or screened out by the hairs may be 95 per cent of this, the amount absorbed 5 per cent, and that transmitted, nil. In the majority of cases, however, the absorbed light is considerably more than the amount reflected or transmitted.

\section{Methods of determina-} tion. If results are to be of value, reflected and transmitted light must be determined in the habitat of the plant simultaneously with the total light which a leaf receives. An approximation of the light reflected from a leaf surface is secured by placing the photometer so that the light reflected is thrown upon the solio strip. A much more satisfactory method, however, is to determine it in connection with the amount of light transmitted through the epidermis. This is done by stripping a piece of epidermis from the upper surface of the leaf and placing it over the slit in the photometer for an exposure. An exposure in

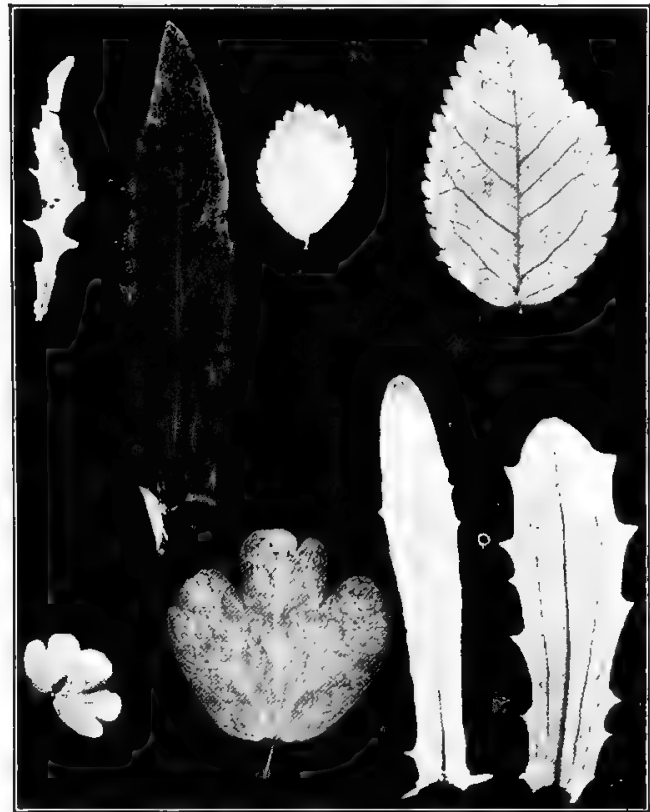

Fig. 14. Leaf print: exposed $10 \mathrm{~m}$., 11 A.M. August 20. The leaves are from sun and shade forms of Bursa bursa-pastoris, Rosa sayii, Thalictrum sparsiflorum, and Machaeranthera aspera. In each the shade leaf prints more deeply.

the full light of the habitat is made simultaneously with another photometer, or immediately afterward upon the same strip. When the epidermis is not too dense, both exposures are permitted to reach the same tint, and the relation between them is precisely that of their lengths of exposure. Ordinarily the two exposures are made absolutely simultaneous by placing the epidermis over half of the opening, leaving the other half to record the full light value, and the results, or epidermis prints, are referred to a multi- 
ple standard. The difference between the two values thus obtained represents the amount of reflected light together with that screened by the epidermis. 'The amount of light transmitted through the leaf may be measured in the same way by using the leaf itself in place of the epidermis alone. The time of exposure is necessarily long, however, and it has been found practicable to obtain leaf prints by exposing the leaf in a printing frame, upon solio paper, at the same time that the epidermis, print is made. In a few species both the upper and lower epidermis can be removed and the amount of light absorbed determined directly by exposing the strip covered

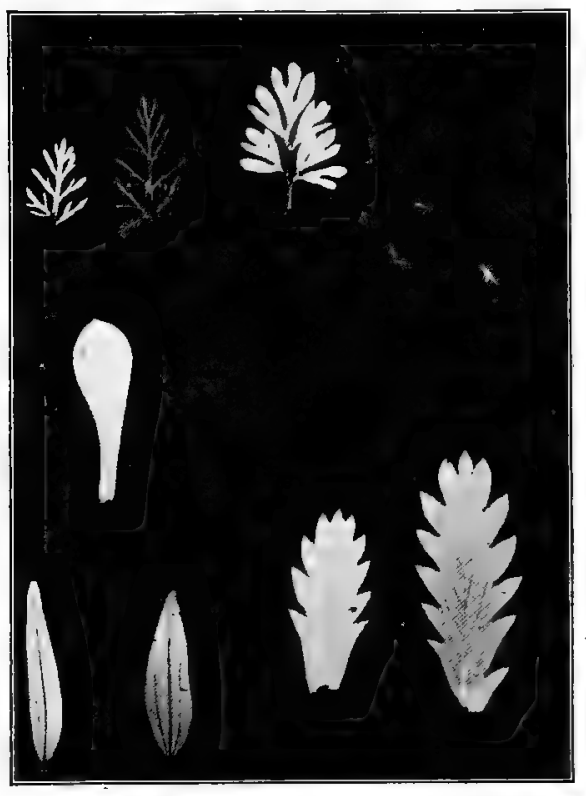

Fig. 15. Leaf print: exposure as before. Sun and shade leaves of Achillea lanulosa, Capnoides aureum, Antennaria umbrinella, Galium boreale, and Potentilla propinqua. with the chlorenchym. Generally, however, this must be computed by subtracting the sum of the per cents of reflected and transmitted light from too per cent, which represents the total light.

90. Leaf and epidermis prints. In diphotic leaves the screening effect of the lower epidermis may be ignored. Isophotic sun leaves, i. e., those nearly upright in position or found above light-colored, reflecting soils, are usually strongly illuminated on both sides, and the $a b-$ sorbed light can be obtained only by measuring the screening effect of both epiderms. Shade leaves and submerged leaves often contain chloroplasts in the epidermis, and the above method can not be applied to them. In fact, in habitats where the light is quite diffuse, practically all incident light is absorbed. The rare exceptions are those shade leaves with a distinct bloom. In addition to their use in obtaining the amount of light absorbed, both leaf and epidermis prints are extremely interesting for the direct comparison of light relations in the leaves of species belonging to different habitats. The relative screening value of the upper and lower epidermis, or of the corresponding epiderms of two ecads or two species, is readily ascertained by exposing the two side by side in sunshine, over the slit in the photometer. For leaf prints fresh leaves are desirable, though nearly the same results can be obtained from 
leaves dried under pressure. The leaves are grouped as desired on the glass of a printing frame, and covered with a sheet of solio. They are then exposed to full sunlight, preferably at meridian, and the prints evaluated by means of the multiple standard. This method is especially useful in the comparison of ecads of one species. These differences due to transmitted light are very graphic, and can easily be preserved by "toning" the print in the usual way.

\section{Expression of Results}

91. Light records. The actual photographic records obtained by photometer and selagraph can at most be kept but a few months, unless they are "toned" or fixed. "Toning" modifies the color of the exposure materially, and changes its intensity so that it can not be compared with readings not fixed. It would involve a great deal of inconvenience to make all comparisons by means of toned strips and standard, even if it were not for the fact that it is practically impossible to obtain exactly the same shade in lots toned at different times. The field record, if carefully and neatly made, may well take the place of a permanent one. The form is the following:

\begin{tabular}{|c|c|c|c|c|c|c|c|c|c|c|c|c|c|c|c|}
\hline 宛 & 点 & 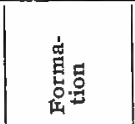 & 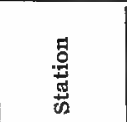 & 莺 & $\begin{array}{l}\text { 岁 } \\
\text { : } \\
\text { 总 } \\
\text { 空 }\end{array}$ & 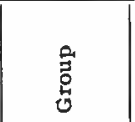 & 苟 & 完 & 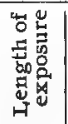 & 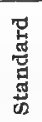 & $\underset{3}{4}$ & 岁 & 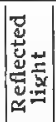 & 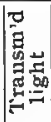 & 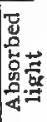 \\
\hline 14/9/04 & 12:C0 м. & Spruce & Milky Way & $2600 \mathrm{~m}$ & N.E. $: 0^{\circ}$ & Opulaster & 1 foot & $2: 10$ & $160 \mathrm{~s}$ & $3 \mathrm{~s}$ & .019 & & $\ldots$ & & \\
\hline “ & 12:05 P.M. & Spruce & Moss Glen & $2500 \mathrm{~m}$. & Level & Streptopus & “ & $2: 12$ & $240 \mathrm{~s}$ & $3 \mathrm{~s}$. & .012 & $\ldots$ & $\ldots$ & $\ldots$ & \\
\hline$"$ & 12:15 P.M. & Brook b'nk & Grotto & $2500 \mathrm{~m}$. & E. $3^{\circ}$ & Filix & Surface & $2: 13$ & $360 \mathrm{~s}$. & $3 \mathrm{~s}$. & .008 & & & $\therefore \ldots$ & \\
\hline
\end{tabular}

92. Light sums, means, and curves. Owing to the fact that the selagraph has not yet been used in the field, no endeavor has been made to de- termine the light value for every hour of the day in different habitats. Consequently there has been no attempt to compute light sums and means. Photometer readings have sufficed to interpret the effect of light in the structure of the formation, and of the individual, but they have not been sufficiently frequent for use in ascertaining sums and means. The latter are much less valuable than the extremes, especially when the relative duration of these is indicated. Means, however, are readily obtained from the continuous records. Light sums are probably impracticable, as the factor is not one that can be expressed in absolute terms. The various kinds and combinations of light curves are essentially the same as for humidity. The level curve through a series of habitats is the most illuminating, but the day curve of hour variations is of considerable value. The curve of 
daily duration, based upon full sunlight, is also of especial importance for plants, and stations which receive both sun and shade during the day.

\section{TEMPERATURE}

93. In consequence of its indirect action, temperature does not have a striking effect upon the form and structure of the plant, as is the case with water and light. Notwithstanding, it is a factor of fundamental importance. This is especially evident in the character and distribution of vegetation. It is also seen in the germination and growth of plants, in the length of season, and in the important influence of temperature upon humidity, and hence upon water-content. Because of its intimate relation with the comfort of mankind, the determination of temperature values has received more attention than that of any other factor, and excellent simple and recording instruments are numerous. For plants, it is also necessary to employ instruments for measuring soil temperatures. The latter unquestionably have much less meaning for the plant than the temperatures of the air; but they have a direct influence upon the imbibition of water, and upon germination.

\section{Thermometers}

94. Air thermometers. The accurate measurement of temperature requires standard thermometers. Reasonably accurate instruments may be standardized by determining their error, but they are extremely unsatisfactory in practice, since they result in a serious waste of time. Accurate thermometers 'which read to the degree are entirely serviceable as a rule, but instruments which read to a fraction of a degree are often very much to be desired. The writer has found the "cylindrical bulb thermometer, Centigrade scale" of H. J. Green, to be an exceedingly satisfactory instrument. The best numbers for general use are 247 and $25 \mathrm{I}$, which read from $-\mathrm{I} 5^{\circ}$ to $50^{\circ} \mathrm{C}$. and are graduated in $.2^{\circ}$. They are respectively 9 and $\mathrm{I2}$ inches long, and cost $\$ 2.75$ and $\$ 3.5 \circ$. These instruments are delicate and require careful handling, but even in class work this has proved to be an advantage rather than otherwise. In making readings of air temperatures with such thermometers, constant precautions must be taken to expose the bulb directly to the wind and to keep it away from the hand and person.

95. Soil thermometers. The thermometer described above has been used extensively for soil temperatures. The determination of the latter is conveniently combined with the taking of soil samples, by using the hole for a temperature reading. When carefully covered, these holes can be used from day to day throughout the season without appreciable error, even in 
gravel soils. Repeated tests of this have been made by simultaneous readings in permanent and newly made holes, and the results have always been the same. It has even been found that the error is usually less than I degree when the hole is left uncovered, if it is more than 9 inches deep. A slight source of error lies in the fact that the thermometer must be raised to make the reading. With a little practice, however, the top of the column of mercury may be raised to the surface and read before the change of temperature can react upon it. This is especially important in very moist or wet soils where the bulb becomes coated with a film of moisture. This evaporates when the bulb is brought into the air, and after a moment or two the mercury slowly falls.

Regular soil thermometers are indispensable when readings are desiréd at depths greater than I2-I8 inches. They possess several disadvantages which restrict their use almost wholly to permanent stations. It is scarcely possible to carry them on field trips, and the time required to place them in the soil renders them practically useless for single readings. Moreover, the instruments are expensive, ranging in price from $\$ 7$ for the two-foot thermometer, to \$19 for the eightfoot one. When it is recognized that deep-seated temperatures are extremely constant and that the slight fluctuations affect, as a rule, only the relatively stable shrubs and trees, it is evident that such temperatures are of restricted importance. Still, in any habitat, they must be ascertained before they can well be ignored, though it is unwise to spend much time and energy in their determination. Soil thermometers of the form illustrated may be obtained from H. J. Green, Brooklyn.

96. Maximum-minimum thermometers. These are used for determining the range of temperature within a given period, usually a day. Since they are much cheaper than thermographs, they can replace these in part, although they merely indicate the maxinum and minimum temperatures for

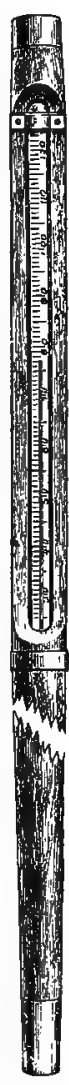

Fig. 16. Soil thermometer the day, and do not register the time when each occurs. The maximum is a mercurial thermometer with a constriction in the tube just above the bulb; this allows the mercury to pass out as it expands, but prevents it from running back, thus registering the maximum temperature. 'The minimum thermometer contains alcohol. The column carries a tiny dumbbell-shaped marker which moves down with it, but will not rise as 
the liquid expands. This is due to the fact that the fluid expands too slowly to carry the marker upward, while the surface tension causes it to be drawn downward as the fluid contracts. The minimum temperature is indicated by the upper end of the marker. In setting up the thermometers, they are attached by special thumbscrews to a support which holds them in an oblique position. The minimum is placed in a special holder

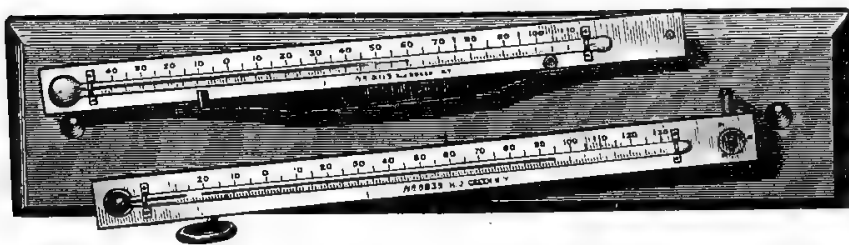

Fig. 17. Maximum-minimum thermometer. above the maximum which rests on a pin that is u sed also for screwing the pivot-screw into position. The support is screwed tightly to the cross-piece of a post, or in forest formations it is fastened directly to a board nailed upon a tree trunk. A shelter has not been used in ecological work, although it is the rule in meteorological observations. The minimum thermometer is set for registering by raising the free end, so that the marker runs to the end of the column. The mercury of the maximum is driven back into the bulb by whirling it rapidly on the pivot-screw after the pin has been taken out. 'This must be done with care in order that the bulb may not be . broken. As soon as the instrument comes to rest, it is raised and the pin replaced, great care being taken to lift it no higher than is necessary. When the night maximum is sought, the thermometer should be whirled several times in order to drive the column sufficiently low.

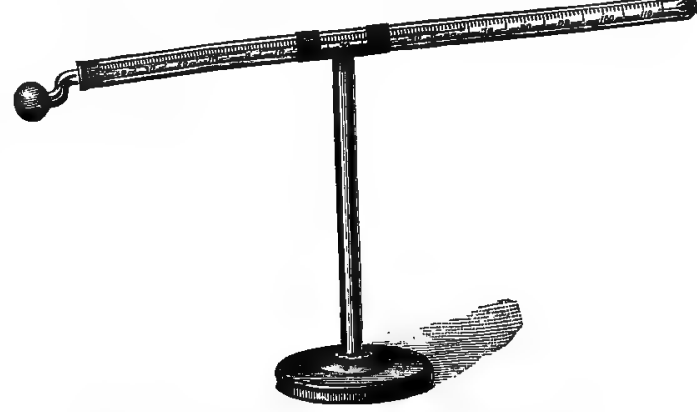

Fig. 18. Terrestrial radiation thermometer. Usually, in such cases, a record is made of this point to make sure that the maximum read is the actual one. If the pivot-screw is kept well oiled, less force will be required to drive the mercury back. In practice, the thermometers have been observed at $6: 00$ A.M. and 6:00 P.M. each day, thus permitting the reading of the maximum-minimum for both day and night. Pairs of maximum-minimum thermometers are to be obtained from H. J. Green, I I9I Bedford Ave., Brooklyn, or Julien P. Friez, Baltimore, Maryland, at a cost of $\$ 8.25$. 
97. Radiation thermometers. These are used to determine the radiation in the air, and from the soil, i. e., for solar and terrestrial radiation. The latter alone has been employed in the study of habitats, chiefly for the purpose of ascertaining the difference in the cooling of different soils at night. The terrestrial radiation thermometer is merely a special form of minimum thermometer, so arranged in a support that the bulb can be placed directly above the soil or plant to be studied. It is otherwise operated exactly like the minimum thermometer, and the reading gives the minimum temperature which the air above the plant or soil reaches, not the amount of radiation. As a consequence, these instruments are valuable only where read in connection with a pair of maximum-minimum thermometers in the air, or when read in a series of instruments placed above different soils or plants.

98. Thermographs. Two types of standard instruments are in general use for obtaining continuous records of air temperatures.

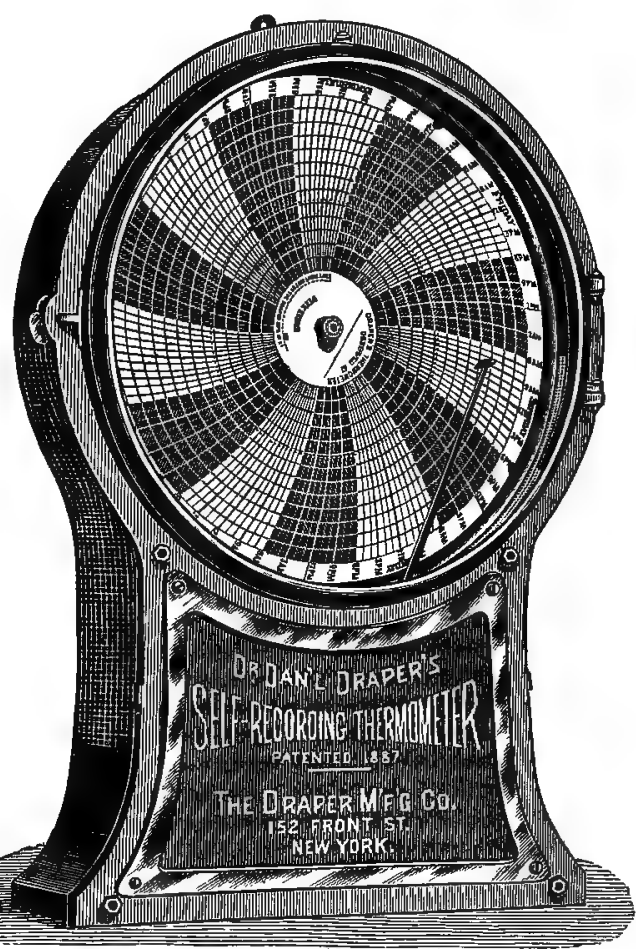

Fig. 19. Draper thermograph. These are the Draper thermograph, made by the Draper Manufacturing Company, I52 Front St., New York city ( $\$ 25$ and $\$ 30$ ), and the Richard thermograph sold by Julien P. Friez, Baltimore $(\$ 50)$. After careful trial had demonstrated that they were equally accurate, the matter of cost was considered decisive, and the Draper thermograph has been used exclusively in the writer's own work. This instrument closely resembles the psychrograph mantufactured by the same company. It is made in two sizes, of which the larger one is the more satisfactory on account of the greater distance between the lines of the recording disk. The thermometric part consists of two bimetallic strips, the contraction and expansion of which 
are communicated to a hand carrying a pen. The latter traces a line on the record.sheet which is attached to a metal disk made to revolve by an eightday clock. In practice the thermograph is set up in the shelter which contains the psychrograph, and in exactly the same manner. The clock is wound, the record put in place, and the pen inked in the same way-also. The proper position of the pen is determined by making a careful ther-

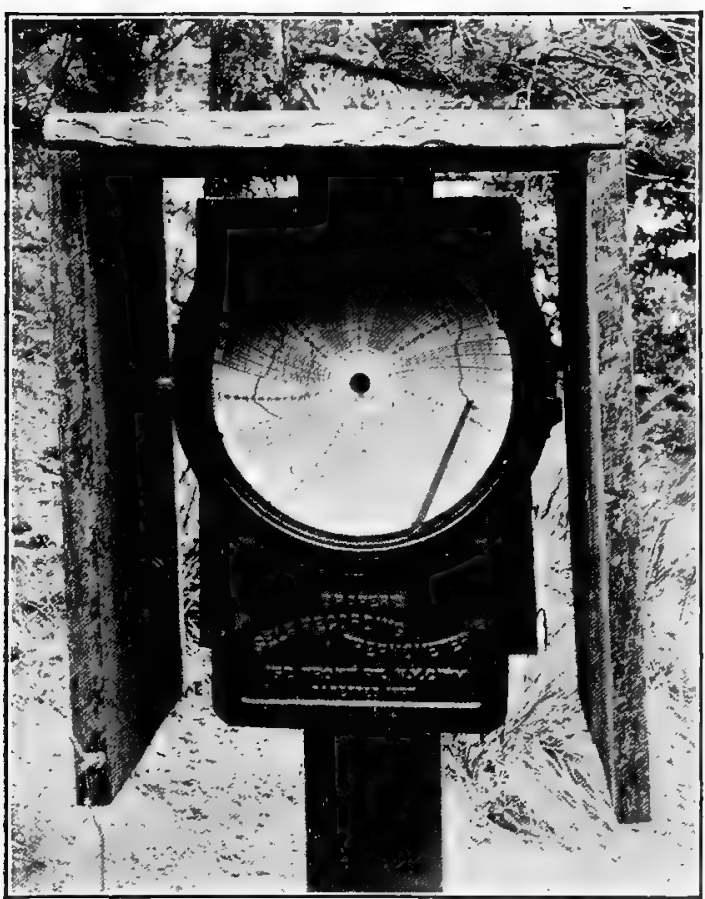

Fig. 20. Shelter for thermograph. mometer reading under the shelter, and then regulating the pen-hand by means of the screws at the base of it. A similar test reading is also made each week, when the clock is rewound. A record sheet may be left in position for three weeks, the pen being filled each week with a different ink. The fixed order of using the inks is red, blue, and green as already indicated.

Owing to the fact that they are practically stationary, soil thermographs are of slight value, except at base stations. Flere, the facts that they are expensive, that the soil-temperatures are of relatively little importance, and that they can be determined as easily, or nearly so, by simple thermometers, make the use of such instruments altogether unnecessary, if not, indeed, undesirable. In a perfectly equipped research station, they undoubtedly have their use, but at ordinary stations, and in the case of private investigators, their value is in no wise commensurate with their cost. 


\section{Readings}

99. Time. The hourly and daily fluctations of the temperature of the air render frequent readings desirable. It is this variation, indeed, which makes single readings, or even series of them, inconclusive, and renders the use of a recording instrument almost imperative in the base station at least. Undoubtedly, a set of simultaneous readings at different heights in one station, or at the same height in different stations, especially if made at the maximum, have much value for comparison, but their full significance is seen only when they are referred to a continuous base record. Such series, moreover, furnish good results for purposes of instruction. In re-

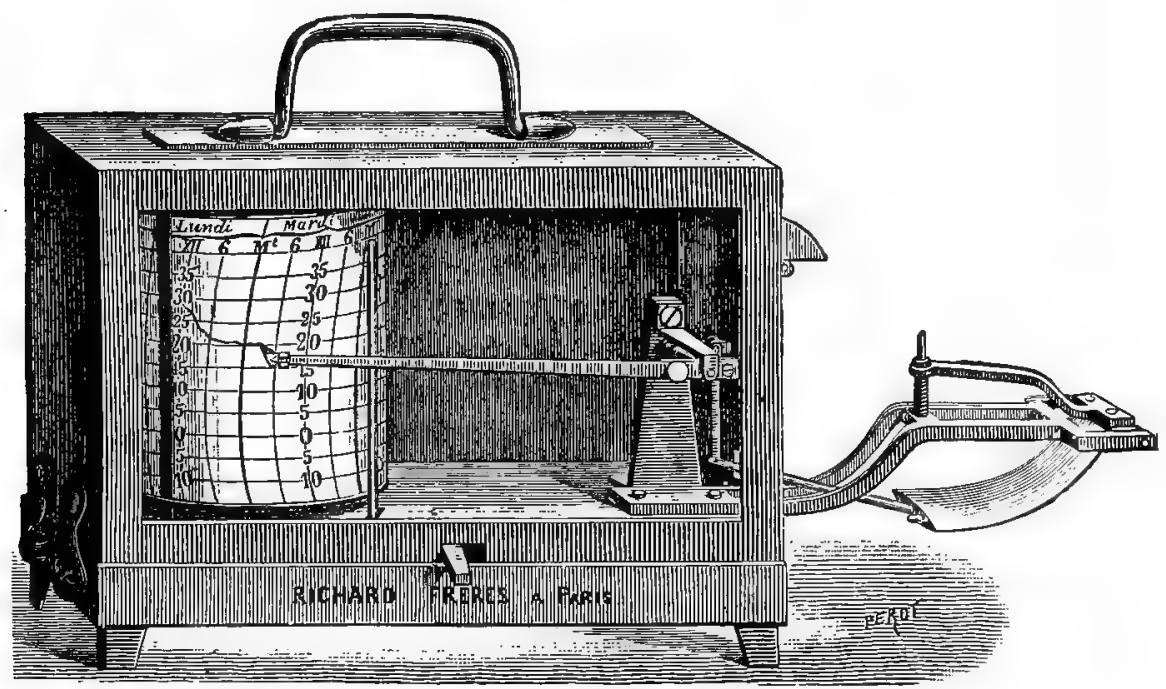

Fig. 21. Richard thermograph.

search work, however, it has been found imperative to have thermographs in habitats of widely different character. With these as bases, it is possible to eke them out with considerable satisfaction by means of maximumminimum thermometers in less different habitats, or in different parts of the same habitat. Naturally these are less satisfactory, and are used only when expense sets a limit to the number of thermographs. In a careful analysis of a single habitat, more can be gained by one base thermograph supplemented by three pairs of maximum-minimum thermometers in dissimilar areas of the habitat than by two thermographs, and the cost is the same. 
100. Place and height. For general air temperatures, thermograph and thermometer readings are made at a height of 3 feet (I meter). Soil temperatures are regularly taken at the surface and at a depth of $I$ foot. When a complete series of simultaneous readings is made in one station, the levels are 6 feet and 3 feet in the air, the surface of the soil, and 5, Io, and 15 inches in the soil. When sun and shade occur side by side in the same formation, as is true of many thickets and forests, surface readings are regularly made in both. Similarly, valuable results are obtained by making simultaneous readings on the bare soil, on dead cover, and upon a leaf, while the influence of cover is readily ascertained by readings upon it and beneath $i t$. A full series of station readings made at the same time upon north, east, south, and west slopes is of great importance in studying the effects of exposure.

\section{Expression of Results}

101. Temperature records. Neither field nor permanent form is required for thermographic records, other than the record sheet itself, which contains all the necessary information in a fairly convenient form. Although the temperature of a particular hour and day can not be read at a mere glance, it can be obtained so easily that it is a waste of time to make a tabular copy of each record sheet. For thermometer readings, either single or in series, the following form is used:

\begin{tabular}{|c|c|c|c|c|c|c|c|c|c|c|c|c|}
\hline \multirow{2}{*}{ 品 } & \multirow{2}{*}{ 暿 } & \multirow{2}{*}{ 密 } & \multirow{2}{*}{ 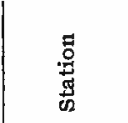 } & \multirow{2}{*}{ 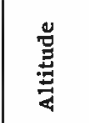 } & \multirow{2}{*}{ 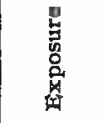 } & \multirow{2}{*}{ 兽 } & \multicolumn{3}{|c|}{$\begin{array}{l}\text { POSITION OF } \\
\text { READING }\end{array}$} & \multirow{2}{*}{ 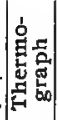 } & \multirow[b]{2}{*}{$\frac{y}{3}$} & \multirow[b]{2}{*}{3} \\
\hline & & & & & & & 3 feet & surf. & 12 in. & & & \\
\hline $17 / 8 / 04$ & 6:30 A.M & Spruce & Jack Brook & $2550 \mathrm{~m}$ & N.E. $5^{\circ}$ & Mertensiare & $9^{\circ}$ & $9^{\circ}$ & $9.8^{\circ}$ & $10^{\circ}$ & Clear & 0 \\
\hline " & “ & Half gravel & Hiawatha & $2550 \mathrm{~m}$ & N.E. $7^{\circ}$ & Asterare & $11.2^{\circ}$ & $11.2^{\circ}$ & $14.8^{\circ}$ & $10^{\circ}$ & Clear & 0 \\
\hline " & 6:00 P M & Spruce & Jack Brook & $2550 \mathrm{~m}$ & N.E. $5^{\circ}$ & Mertensiare & $11.4^{\circ}$ & $11.4^{\circ}$ & $98^{\circ}$ & $11^{\circ}$ & Cloudy & 0 \\
\hline ". & " & Half gravel & Hiawatha & $2550 \mathrm{~m}$. & N.E. $7^{\circ}$ & Asterare & $12^{\circ}$ & $13.8^{\circ}$ & $16.4^{\circ}$ & $11^{\circ}$ & cloudy & 0 \\
\hline
\end{tabular}

102. Temperature sums and means. The amount of heat, i. e., the number of calories received within a given time by a definite area of plant surface, can be determined by means of a calorimeter. From this the temperature sum of a particular period may be obtained by simple addition. In the present condition of our knowledge, it is impossible to establish any exact connection between such results and the functional or growth effect that can be traced directly to heat. As a consequence, temperature sums do not at present contribute anything of value to an understanding of the relation between cause and effect. The mean daily temperature is readily 
obtained by averaging twenty-four hour-temperatures recorded by the thermograph. The method employed by $\mathrm{Meyen}^{1}$, of deriving the mean directly from the maximum and minimum for the day, is not accurate; from a large number of computations, the error is always more than two degrees. On the other hand, the mean obtained by averaging the maximum and minimum for the day and night has been found to deviate less than I degree from the mean proper. This fact greatly increases the value of maximum-minimum instruments if they are read daily at 6:00 A.M. and 6:00 P.M.

103. Temperature curves. The kinds and combinations of temperature curves are almost without number. The simple curves of most interest are those for a series of stations or habitats, based upon the level of three feet, or the surface, or the daily mean. The curves for each station representing the different heights and depths and the season curve of the daily means for a habitat are also of much importance. One of the most illuminating combinations is that. which groups together the various level curves for a series of habitats. Other valuable combinations are obtained by grouping the curves of daily means of different habitats for the season, or the various station curves.

104. Plant temperatures. The direct effects of temperature as seen in nutrition and growth can be ascertained only by determining the temperature of plant tissues. The temperatures of the air and of the soil surface have an important effect upon humidity, and water-content, and through them upon the plant, but heat can influence assimilation, for example, only in so far as it is absorbed by the assimilating tissue. The temperatures of the leaf, as the most active nutritive organ of the plant, are especially important. While it is a well-known fact that internal temperatures follow those of the air and soil closely, though with varying rapidity of response, this holds less for leaves than for stems and roots. Owing to the very obvious difficulties, practically nothing has yet been done in this important field. A few preliminary results have been obtained at Minnehaha, which serve to show the need for such readings. Gravel slide rosettes in an air temperature of $24^{\circ} \mathrm{C}$. and a surface temperature of $40^{\circ} \mathrm{C}$. gave the following surface readings: Parnelia, $40^{\circ}$, Eriogonum, $38.6^{\circ}$, Arctostaphylus, $35^{\circ}$, Thlaspi, $31.8^{\circ}$, and Senecio, $31^{\circ}$. The leaf of Eriogonum flarum, which is smooth above and densely hairy below, indicated a temperature of $3^{\mathrm{I} .8^{\circ}}$ when rolled closely about the thermometer bulb with the smooth surface out; and $28^{\circ}$. when the hairy surface was outside. The surface read-

IMeyen, F. J. F. Grundriss der Pflanzengeographie, 12. 1836. 
ings of the same leaf were $.5^{\circ}-\mathrm{I}^{\circ}$ higher when made upon the upper smooth surface. This immediately suggests that the lower surface may be modified to protect the leaf from the great heat of the gravel, which often reaches $50^{\circ} \mathrm{C}$. $\left(122^{\circ} \mathrm{F}\right.$. .

\section{PRECIPITATION}

105. General relations. As the factor which exerts the most important control upon water-content and humidity, rainfall must be carefully considered by the ecologist. It is such an obvious factor, and is usually spoken of in such general terms that the need of following it accurately is not evident at once. When it is recognized that the fluctuations of water-content are directly traceable to it, it hecomes clear that its determination is as important as that of any indirect factor. This does not mean, however, that the amount of yearly rainfall is to be taken from the records of the nearest weather station, and the factor dismissed. Like other instruments, the rain gauge must be kept at the base station of the area under study; and when this is extensive or diverse, additional instruments should be put into commission. While the different parts of the same general climatic region may receive practically the same amount of precipitation during the year, it is not. necessarily true that the rainfall of any particular storm is equally distributed, especially in the mountains. Nothing less than an exact knowledge of the amount of rain that falls in the different areas will make it possible to tell how much of the water-content found at any particular time in these represents merely the chance differences of precipitation.

The forms of precipitation are rain, dew, hail, snow, and frost. Of these, hail is too infrequent to be taken into account, while frost.usually occurs only at the extremes of the growing season, and in its effect is rather to be reckoned with temperature. Snow rarely falls except during the period of rest, and, while it plays an important part as cover, it is merely one of several factors that determine the water-content of the soil at the beginning of spring. The influence of dew is not clearly understood. It is almost always too slight in amount and too fleeting to affect the watercontent of the soil. It seems probable that it may serve by its own evaporation to decrease in some degree the water loss from the soil, and from bedewed plants. If, however, the dew is largely formed by the water of the soil and of the plant, as is thought by some, then it is negligible as a reinforcement of water-content. From the above, it is evident that rainfall alone exerts a profound effect upon the habitat, and it is with its measurement that the ecologist is chiefly concerned. 
106. The rain gauge, as the illustration shows, is a cylindrical vessel with a funnel-sliaped receiver at the top, which is 8 inches in diameter. The receiver fits closely upon a narrower brass vessel or measuring tube in which the rain collects. The ratio of surface between receiver and tube is to to $\mathrm{I}$ : For readings covering a general area, the rain gauge is placed in the open, away from buildings or other obstructions, and is sunken in the ground sufficiently to keep it upright. In localities where winds are strong, it is usually braced at the sides also or stupported by a wooden frame. In measuring the amount of rain in the measuring tube, the depth is divided by ten in
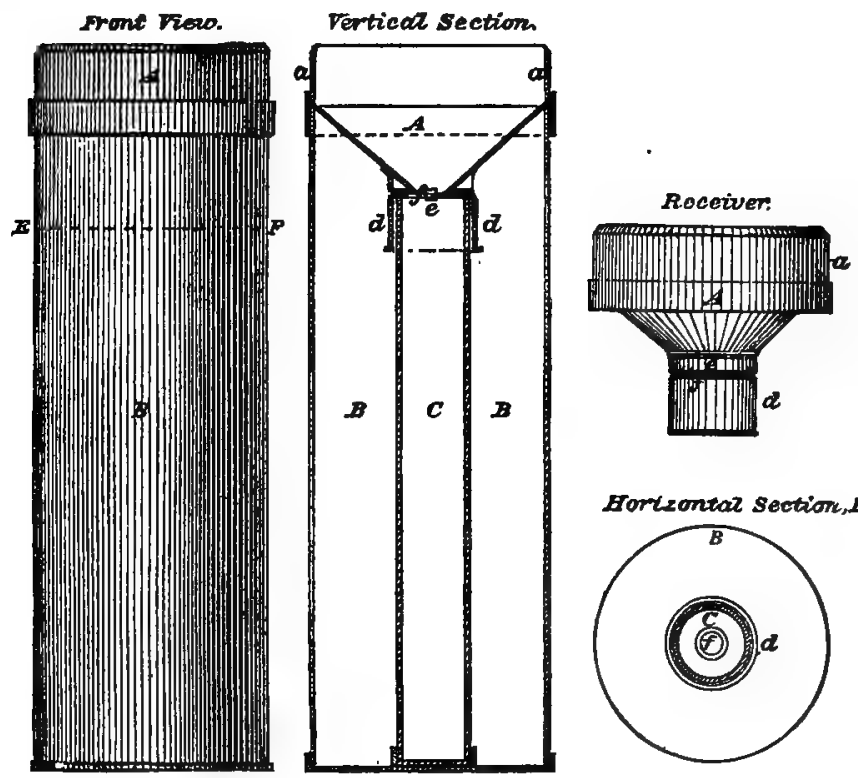

Fig. 22. Rain gauge showing construction.

order to ascertain the actual rainfall. The depth is measured by inserting the measuring-rod through the hole in the funnel until it touches the bottom. It is left for a second or so, quickly withdrawn, and the limit of the wetted portion noted. In the case of standard rods, the actual rainfall is read directly in hundredths, so that the division by ten is unnecessary. After each reading, the measuring-tube is carefully drained, replaced, and the receiver put in position. No regular time for making readings is necessary. During a rainy period, it is customary to make a measurement each day, but it has been found more satisfactory for ecological purposes to measure each shower, and to record its duration. These two facts furnish 
a ready clue to the relative amount of run-off in each fall of rain. The measurement of snowfall is often made merely by determining its depth. For comparison with rainfall, the rain gauge with receiver and tube withdrawn is used. The snow which falls is melted, poured into the measuring tube, and measured in the ordinary way. The U. S. Weather Bureau standard rain gauge, with measuring stick, may be obtained of $\mathrm{H}$. J. Green, or of J. P. Friez for $\$ 5.25$.

107. Precipitation records. From the periodic character of precipitation, rainfall sums, means, and curves have little importance in the careful study of the habitat. The rainfall curve for the growing season is an aid in explaining the curve of water-content, and the mean rainfall of a region gives some idea of its vegetation, though even here the matter of its distribution is of primary importance. The rain and snow charts published by the U. S. Weather Bureau furnish data of some importance for the general study of vegetation, but it is evident that they can play little part in a system which is founded upon the habitat. Precipitation records, for reasons of brevity and convenience, are united with wind records, and the form will be found under the discussion of this factor.

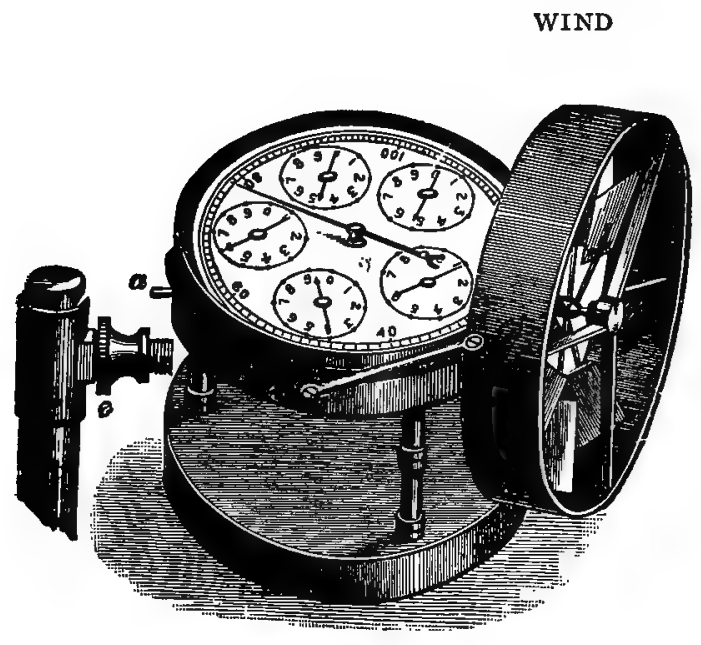

Fig. 23. Simple anemometer.

108. Value of readings. On account of its direct effect upon humidity, and its consequent influence upon water-content, the part which wind plays in a habitat can not be ignored in a thorough investigation. It is an important element in exposure, and accordingly has a marked mechanical effect upon the 'vegetation of exposed habitats, alpine slopes, seacoasts, plains, etc.

Owing to its inconstancy and its extreme variation in velocity, single wind readings are absolutely without value. When read in series, anemometers give some information upon the comparative air movement in different hab- 
itats, but the chance of error is great, except when the breeze is steady. Anemographs alone give real satisfaction. Accurate results, however, are not obtainable without a series of two or more in different habitats, and it is still an open question whether the results obtained justify the expense. For a completely equipped base station, anemometer, anemograph, and wind vane are desirable instruments, but the study of the habitat has by no means reached the stage of precision in which their general use is necessary.

109. The anemometer in its simplest form is adapted only to readings made under direct observation, as a stidden change in the direction of the wind reverses the movement of the indicator needle. This simple wind gauge, shown in figure 23 , has been used for instructional purposes, and to a slight extent, also, in ascertaining the effect of cover. In constant winds, successive single readings are found to have value, but, ordinarily, the observations must be simultaneous. Careful tests of this simple instrument show that it is essentially accurate. It may be obtained from the C. H. Stoelting Company, 3I W. Randolph St., Chicago, for $\$ 25$. The s tand a rd anemometer (Fig. 24) is practically a

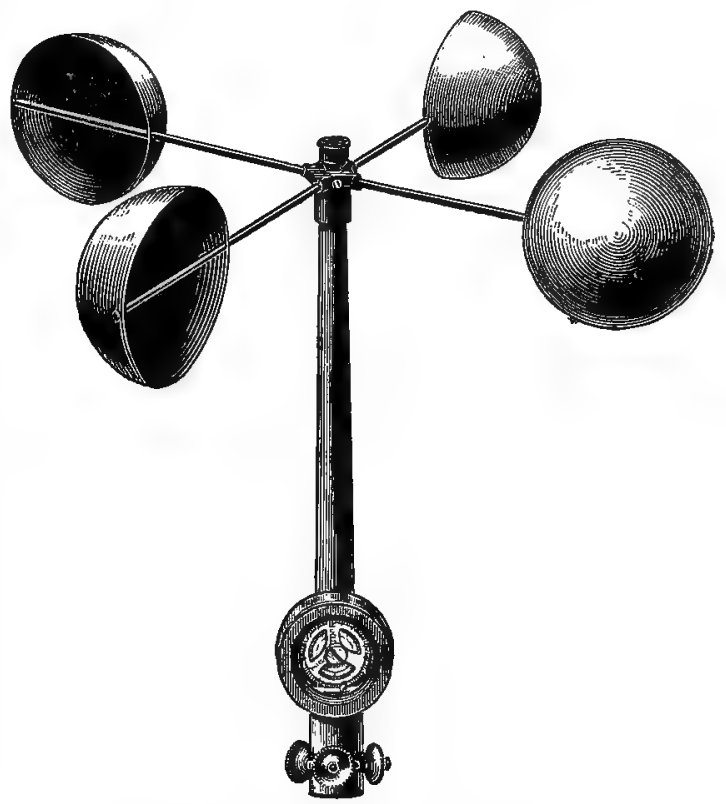

Fig. 24. Standard anemometer. recording instrument up to $\mathrm{I}, 000$ miles, but as the dials run on without any indication of the total number of revolutions, it must be visited and read each day. This renders its use difficult for habitats which are some distance apart. When exact determinations of wind values become necessary, the most successful method is to establish a series of three standard anemometers. One of these should be placed upon the most exposed part of a typically open habitat, the second in the most protected part of the same habitat, while the third is located in the midst of a representative forest formation. If the two habitats are close together, the daily visits 
can be made without serious inconvenience. The reading of the register: ing dials requires detailed explanation, and for this the reader is referred to the printed directions which accompany the instrument. In setting up the anemometer it must be borne in mind that the ecologist desires the wind velocity for a particular habitat. In consequence, the precautions which the meteorologist takes to place the instrument at a certain height and well away from surrounding obstructions do not hold here. Standard anemometers are furnished by H. J. Green, and J. P. Friez for $\$ 25$ each.

The anemograph is an anemometer electrically connected with an automatic register. It is the only instrument adapted to continuous weekly records in different habitats, but the price, $\$ 75$ ( $\$ 25$ for the anemometer and $\$ 50$ for the register) is practically prohibitive, at least until a complete series of ecographs for other factors has been obtained.

110. Records. The following form is used as a combined record for precipitation and wind:

\begin{tabular}{|c|c|c|c|c|c|c|c|c|c|c|c|c|c|}
\hline \multirow{2}{*}{ 品 } & \multirow[b]{2}{*}{ 息 } & \multirow[b]{2}{*}{ 递 } & \multirow[b]{2}{*}{$\begin{array}{l}\text { 号 } \\
\text { 苟 } \\
\text { in }\end{array}$} & \multirow[b]{2}{*}{ 营 } & \multirow{2}{*}{ 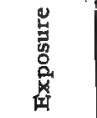 } & \multirow[b]{2}{*}{ 总 } & \multicolumn{2}{|c|}{ RAINFAIL } & \multicolumn{5}{|c|}{ WIND } \\
\hline & & & & & & & 莺 & 产号 & 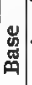 & 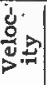 & 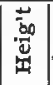 & 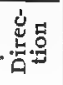 & 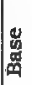 \\
\hline 29/8/04 & 6:30 P.M. & Half gravel & Hiawatha & $2550 \mathrm{~m}$. & N.E. $17^{\circ}$ & Asterare & 1 & 8 hours & .. & 5 & $3 \mathrm{ft}$. & N. w: & \\
\hline $31 / 8 / 04$ & 5:45 P.M & " & “" & “" & “ & “ & Trace & $10 \mathrm{~min}$. & 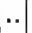 & 12 & “ & " & \\
\hline $2 / 9 / 04$ & 4:00 P.M. & "6 & “ & a & " & $" 4$ & .2 & 2 hours & ". & 7 & " & w. & \\
\hline $3 / 9 / 04$ & 10:00 P.M & " & “ & " & " & " & Trace & & & 18 & “ & : & \\
\hline
\end{tabular}

SOIL

111. Soil as a factor. In determining the value of the soil as a factor in a particular habitat, it must be clearly recognized that its importance lies solely in the control which it exerts apon water-content and nutrient-content. The former is directly connected with the texture or fineness of the soil, the latter with its chemical nature. Accordingly, the structure of the soil and its chemical composition are the fundamental points of attack. These are not at all of equal value, however. Water is both a food, and a solvent for the nutrient salts of the soil. Furthermore, the per cent of soluble salts, as determined in mechanical analyses, is practically the same for all ordinary soils. Indeed, the variations for the same soil types are as great as for entirely different types. For these reasons, solttble salt-content may be ignored except where it is readily seen to be excessive, as in alkaline soils; and determinations of chemical composition are necessary only in those soils which contain salts or acids to an injurious degree, e. g., 
alkaline soils, peat bogs, humus swamps, etc. The structure of the soil, on the other hand, in the usual absence of excessive amounts of solutes, absolutely controls the fate of the water that enters the ground, in addition to its influence upon the run-off. It determines the amount of gravitation water lost by percolation, as well as the water that can be raised by capillarity. The resultant of these, the total soil water or holard, is hence an effect of structure, while the size and compactness of the particles are conclusive factors in controlling the chresard. It must be recognized, however, that these are all factors which enable us to interpret the amount of holard or chresard found in a particular soil. They have no direct important effect upon the plant, but influence it only in so far as they affect the water present.

112. The value of soil surveys. The full appreciation of the preeminent value of water-content, particularly of the chresard, greatly simplifies the ecological study of soils. The ecologist is primarily concerned with soil water only in its relation to the plant, and while a fair knowledge of soil structure is essential to a proper understanding of this, he has little concern with the detailed study of the problems of soil physics. For the sake of a proper balance of values, he must avoid the tendency noted elsewhere of ignoring the claims of the plant, and of studying the soil simply as the seat of certain physical phenomena. Accordingly, it is felt that mechanical and chemical analyses, determinations of soluble salt-content, etc., have much less value than has been commonly supposed. The usual methods of soil survey, which pay little or no attention to water-content, and none at all to available water, are practically valueless for ecological research. This statement does not indicate a failure to appreciate the importance of the usual soil methods for many agricultural problems, such as the use of fertilizers, conservation of moisture, etc., though even here to focus the work upon water-content would give much more fundamental and serviceable results. For these reasons, slight attention will be paid to methods of mechanical and chemical analysis. In their stead is given a brief statement of the origin, structure, and character of soils with especial reference to water-content.

113. The origin of soils. Rocks form soils in consequence of weathering, under the influence of physical and biotic factors. Weathering consists of two processes, disintegration, by which the rock is broken into component particles of various sizes, and decomposition, in which the rock or its fragments are resolved into minute particles in consequence of the chemical disaggregation of its minerals, or of some other chemical change. These processes are usually concomitant, although, as a rule, one is more evident than the other. The relation between them is dependent upon the character 
of the rock and the forces which act upon it. Hard rocks, i. e., igneous and metamorphic ones, as a rule disintegrate more rapidly than they decompose; sedimentary rocks, on the other hand, tend to decompose more rapidly than they disintegrate. In many cases the two processes go hand in hand. This difference is the basis for the distinction, first proposed by Thurmann, between those rocks which weather with difficulty and those which weather readily. The former were called dysgeogenous, the latter eugeogenous. Thurmann restricted the application of the first term to those rocks which produce little 'soil, but it seems more logical to apply dysgeogenous to those in which disintegration is markedly in excess of decomposition, and eugeogenous to those rocks that break down rather readily into fine soils. With respect to the general character of the soil formed, rocks are pelogenous, clay-producing, psammogenous, sand-forming, or pelopsammogenous, producing mixed clay and sand. The first two are divided into perpelic, hemipelic, oligopelic, perpsammic, etc., with reference to the readiness with which they are weathered, but this distinction is not a very practicable one. The grouping of soils into silicious, calcareous, argillaceous, etc., with reference to the chemical nature of the original rock, is of no value to the ecologist, apart from the general clue to the physical properties which it furnishes.

114. The structure of soils. The water capacity of a soil is a direct result of the fineness of the particles. Since the water is held as a thin surface film by each particle or group of them, it follows that the amount of water increases with the water-holding surface. The latter increases as the particles become finer and more numerous, and thus produce a greater aggregate surface. 'The upward and downward movements of water in the soil are likewise in immediate connection with the size of particles. The upward or capillary movement increases as the particles become finer, thus making the irregular capillary spaces between them smaller, and magnifying the pull exerted. On the contrary, the downward movement of gravitation water, i. e., percolation, is retarded by a decrease in the size of the soil grains and hastened by an increase. Hence, the two properties, capillarity and porosity, are direct expressions of the structure of the soil, i. e., of its texture or fineness. Capillarity, however, increases the water-content of the upper layers permeated by the roots of the plant, while porosity decreases it. On the basis of these properties alone, soils would fall into two groups, capillary soils and porous soils, the former fine-grained and of high watercontent, the latter coarse-grained and with relatively little water. A third factor, however, of great importance must be taken into account. This is the pull exerted upon each water film by the soil particle itself. This pull ap- 
parently increases in strength as the film grows thinner, and explains why it finally becomes impossible for the root-hairs to draw moisture from the soil. This property, like capillarity, is most pronounced in fine-grained soils, such as clays, and is least evident in the coarser sands and gravels. It seems to furnish the direct explanation of non-available water, and, in consequence, to indicate that the chresard is an immediate result of soil texture.

115. Mechanical analysis. From the above it is evident that, with the same rainfall, coarse soils will be relatively dry, and fine soils correspondingly moist. However, this difference in holard is somewhat counterbalanced by the fact that the chresard is much greater in the former than in the latter. The basis of these relations can be obtained only from a study of the texture of the soil. The usual method of doing this is by mechanical analysis. This is far from satisfactory, since the use of the sieves often brings about the disaggregation of groups of particles which act as units in the soil. Furthermore, the analysis affords no exact evidence of the compactness of the soil in nature, and tests of capillarity and porosity made with soil samples out of position are open to serious error. Nevertheless, mechanical analyses furnish results of some value by making it possible to compare soils upon the basis of textlire. For ecological purposes, minute analyses are undesirable; their value in any work is doubtful. A separation of soil into gravel, sand, and silt-clay is sufficient, since the relative proportion of these will explain the holard and chresard of the soil concerned. The latter are also affected in rich soils, especially of forests, by the organic matter present. If this is in a finely divided condition, the amount is determined by calcining. When a definite layer of leafmold is present, as in forests and thickets, its water-value

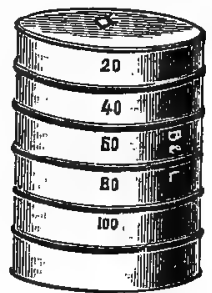

Fig. 25. Sieves for soil analysis. is found separately, since its power of retaining water is altogether out of proportion to its weight.

116. Kinds of soils. It is very doubtful whether it is worth while to attempt to distinguish soils upon the basis of mechanical analysis. Unquestionably, the most satisfactory method is to distinguish them with respect to holard and chresard, and to regard texture as of secondary importance. A series of soil classes which comprise various soil types has been proposed by the U. S. Bureau of Soils as follows: (I) stony loam, (2) gravel, (3) gravelly loam, (4) dunesand, (5) sand, (6) fine sand, (7) sandy loam, (8) fine sandy loam, (9) loam, (Io) shale loam, (II) silt loam, (I2) clay loam, (I3) clay, (I4) adobe. These are based

${ }^{1}$ Instructions to Field Parties and Descriptions of Soil Types, 35. 1903. 
entirely upon, mechanical analyses, and in some cases are too closely related to be useful. The line between them can nowhere be sharply drawn. Indeed, the variation within one class is so great that soils have frequently been referred to the wrong group. Thus, Cassadaga sand (gravel 22 per cent, sand 43 per cent, silt $2 \mathrm{I}$ per cent, clay Io per cent) is more closely related to Oxnard sandy loam $(26-37-18-12)$ and to Afton fine sandy loam $(28-43-18-8)$ than to Coral sand $(6 \mathrm{I}-29-3-4)$, Galveston sand (6-9II-I), or Salt Lake sand $(84-\mathrm{I} 5-\mathrm{I}-\mathrm{O})$. Elsinore sandy loam $(8-38-$ $35-10)$ is much nearer to Hanford fine sandy loam $\left(9-36-33^{-14}\right)$ than to Billings sandy loam $(\mathrm{I}-60-22-\mathrm{II})$ or to Utuado sandy loam (48-2319-8). The soil types are much more confused, and for ecological purposes at least are entirely valueless. Lake Charles fine sandy loam has the composition, I-34-52-9; Vernon fine sandy loam, I-37-54-7, while many other so-called types show nearly the same degree of identity.

117. The chemical nature of soils. The effect of alkaline and acid substances in the soil upon water-content and the activities of the plant is far from being well understood. It is generally recognized that salts and acids tend to inhibit the absorptive power of the root-hairs. In the case of saline soils, this inhibitive effect seems to be established, but the action of acids in bogs and swamps is still an open question. It is probable that the influence of organic acid has been overestimated, and that the curious anomaly of a structural xerophyte in a swamp is to be explained by the stability of the ancestral type and by the law of extremes. Apart from the effect which excessive amounts of acids and salts may have in. reducing the chresard, (the chemical character of the soil is powerless to produce structural modification in the plant.) Since Thurmann's researches there has been no real support of the contention that the chemical properties of the soil, not its physical nature, are the decisive factors in the distribution and adaptation of plants. It is not sufficient that the vegetation of a silicious soil rliffers from that of a calcareous one. A soil can modify the plants upon it only though its water-content, or the solutes it contains. Hence, the chemical composition of the original rock is immaterial, except in so far as it modifies these two factors. Humus, moreover, while an important factor in growth, has no formative influence beyond that which it exerts through water-content.

\section{PHYSIOGRAPHY}

118. Factors. The physiographic factors of a definite habitat are altitude, exposure, slope, and surface. In addition, topography is a general though less tangible factor of regions, while the dynamic forces of weathering, 
erosion, and sedimentation play a fundamental role in the change of habitats. It is evident, however, that these, except where they affect the destruction of. vegetation directly, can operate upon the plant only through more direct factors, such as water, light, and temperature. While they are themselves not susceptible of measurement, they can often be expressed in terms of determinable factors, i. e., slope, exposure, and surface. Fundamentally, they constitute the forces which change one habitat into another, and, in consequence, are really to be considered as the factors which produce succession. The static features of physiography, altitude, etc., lend themselves readily to determination by means of precise instruments. Thesefactors, though by no means negligible, are remote, and consequently their mere measurement is insufficient to indicate the nature or extent of their influence upon the plant. It is necessary to determine also the manner and degree in which they affect other factors, a task yet to be done. Readings of altitude, slope, and exposure are so easily' made that the student must carefully avoid the tendency to let them stand at' their own value, which is slight. Instead, they should be made the starting point for ascertaining the differences which they produce in water-content, humidity, wind, and temperature.

\section{Altitude}

119. Analysis into factors. Of all physiographic features, altitude is the most difficult to resolve into simple factors. Because of general geographic relations, it has a certain connection with rainfall, but this is vague and inconstant. Obviously, in its influence upon the plant, altitude is really pressure, and in consequence its effect is exerted upon the climatic and not the edaphic factors of the habitat. Theoretically, the decrease of airpressure in the increased altitude directly affects humidity, light, and temperatture. Actually, while there is unquestionably a decrease in the absorption of the light and heat rays owing to the fact that they traverse less atmosphere, which is at the same time less dense; this seems to be negligible. Photometric readings at elevations of 6,000 and $\mathrm{r}_{4}, 000$ feet have so far failed to show more than slight differences, which are altogether too small to be efficient. The effect upon humidity is greater, but the degree is uncertain. Continuous psychrographic records at different elevations for a full season, at least, will be necessary to determine this, since the psychrometric readings so far made, while referred to a base psychrograph, are too scattered to be conclusive. Finally, the length of the season, itself a composite, is directly dependent upon the altitude. This relation, though obscure, rests chiefly upon the rarefaction of the air which prevents the accumulation of heat in both the soil and the air. 
120. The barometer. To secure convenience and accuracy in the determination of altitude, it is necessary to use both a mercurial and an aneroid barometer. The latter is by far the most serviceable for field work, but it requires frequent standardizing by means of the former. The mercurial form is much more accurate and.should be read daily in the base station. It is practically impossible to carry it in the field, except in the so-called mountain form, which is of great service in establishing the altitudes of a series of stations. In use the aneroid barometer may be checked daily by the mercurial standard, or it may be set at the altitude of the base station. thus giving a direct reading. After the normal pressure at the base has once been ascertained, however, the most satisfactory method is to set the aneroid each day by the standard, at the same time noting the

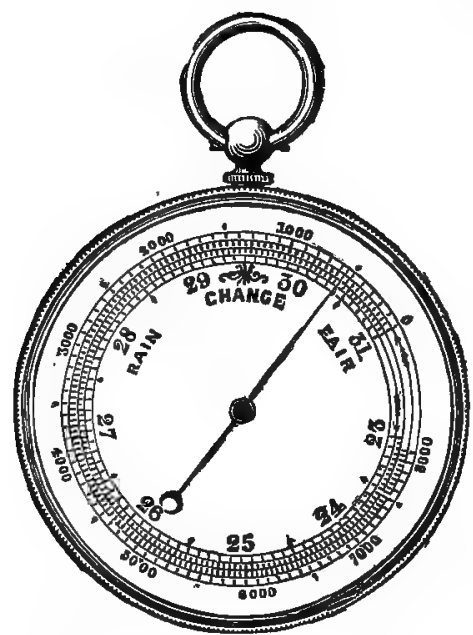

Fig. 26. Aneroid barometer. pressure deviation in feet of elevation (see p. 46). The absolute elevation of the various stations of a series may be determined either by adding or subtracting this deviation from the actual reading at the station, or by noting the change from the base station, and then adding or subtracting this from the normal of the latter. When it is impossible to check the aneroid by means of a mercurial barometer, the average of a series of readings made at different days at one station, especially if taken during settled weather, will practically eliminate the daily fluctuations, and yield a result essentially accurate. Even in this event, the accuracy of the aneroid should be checked as often as possible, since the mechanism may go wrong at any time. The barograph, while a valuable instrument for base stations, is not at all necessary. These instruments can be obtained from all makers of meteorological apparatus, such as H. J. Green, and J. P. Friez. Aneroid barometers reading to 16,000 feet cost about $\$ 20$; the price of the Richards aneroid barograph is $\$ 45$. Ordinary observatory barometers cost $\$ 30-\$ 40$; the standard instrument sells at $\$ 75-\$ 100$. The mountain barometer, which is altogether the most serviceable for the ecologist, ranges from $\$ 30-\$ 55$, depending upon accessories, etc. 
121. Concept. This term is used in the ordinary sense to indicate the relation of the surface of a habitat to the horizon. Although it is a complex of factors, or rather influences several factors, these are readily determinable. The primary effect of slope is seen in the control of runoff and drainage, and consequently of water-content, although these are likewise affected by soil texture and by surface. Slope, moreover, as a concomitant of exposure, has an important bearing upon light and heat by virtue of determining the angle of incidence, and also upon wind, and, through it, upon the distribution of snow. At present, while it can be expressed definitely in degrees, it has not yet been connected quantitatively with more direct factors. This is, how ever, not a difficult 'task, and it is probable that we shall soon come to express slope principally in amount of run-off, and of incident heat.

122. The clinometer. In the simplest form, this instrument is merely a semicircle of paper, with each half graduated from $1-90^{\circ}$. It is mounted on a board and placed base upward, upon a wooden strip, 2 feet long and 2 inches wide, which has a true edge. At the center of the circle is attached a line and plummet for reading the perpendicular. A more convenient form is shown in figure 28 , which is both clinometer and compass. This also necessitates the use of a

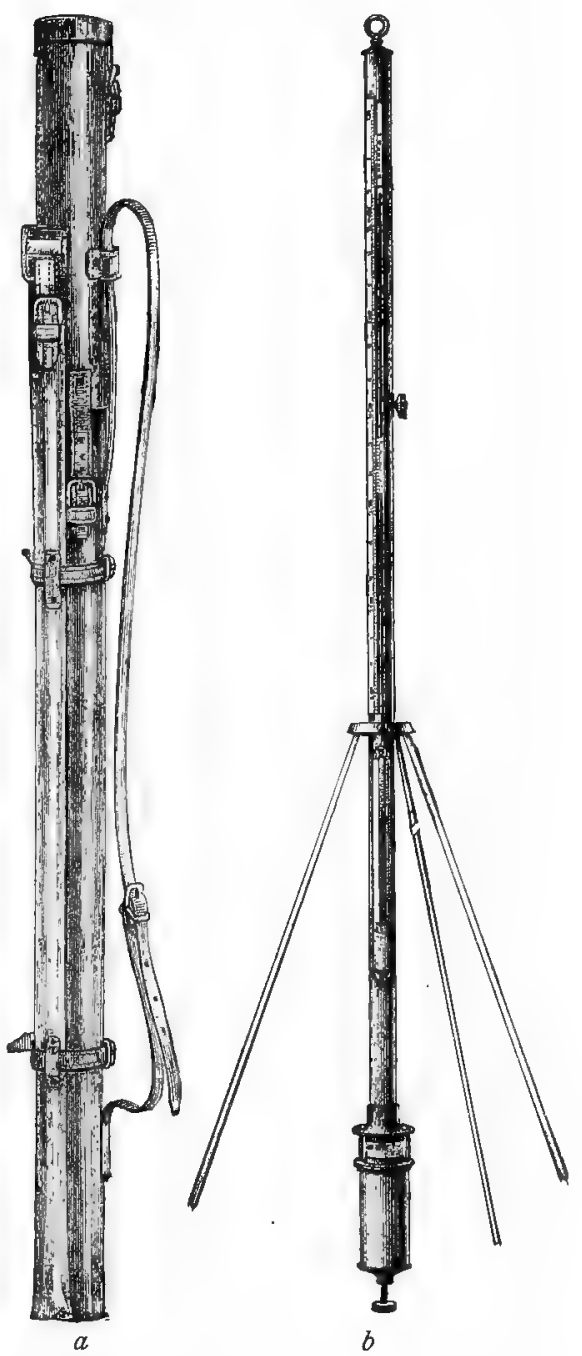

Fig. 27. Mountain barometer: (a) in carrying case; $(b)$ set up for use. 
basing strip to eliminate the inequalities of the surface. The dial face is graduated to show inches of rise per yard, as 'well as the number of degrees, but the latter, as the simpler term, is preferable for ecological work. In making a reading, the basing strip is placed upon a representative area of the slope, and pressed down firmly to equalize slight irregularities. The clinometer is moved slightly along the upper edge, causing the marker to swing freely; After the latter comes to rest, the instrument is carefully turned upon 'its back, when the angle of the slope in degrees may be read directly. Two or three such readings in different areas will suffice for the entire habitat, unless it be extremely irregular. The clinometer with compass may be obtained from the Keuffel and Esser Company, II I Madison St., Chicago, Illinois, for $\$ 5$.
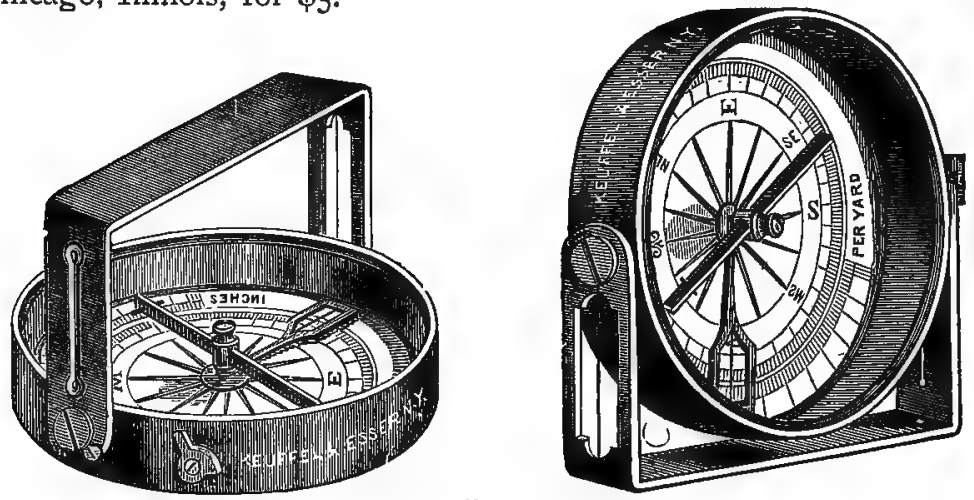

Fig. 28. Combined clinometer and compass.

123. The trechometer. For measuring the effect of slope upon run-off, a simple instrument called the trechometer ( $\tau \rho \rho^{\prime} \chi \omega$, to run off) has been devised. This consists merely of a metal tank, $3 \times 4 \times$ I2 inches, holding 144 square inches of water, with an opening $1 / 4 \times 12$ inches at the base in front, closed by a tight-fitting slide. Three metal strips, $2 \times 12$ inches, are fastened to the front of the tank in such a way as to enclose a square foot of soil into which the strips penetrate an inch. In the front strip is an opening, I inch square, provided with a drip from which the run-off is collected in a measuring vessel. In use, the instrument is put in position with the metal rim forced down I inch into the soil; the tank is filled, the graduate put in place, and the slide raised. The run-off for a square foot is the amount of water caught by the graduate, and is represented in cubic inches per square foot. For obtaining results which express slope alone, comparisons must be made upon the same soil, from which all cover, dead and living, has been removed. They must be as closely together in time as possible, at least during the same day, as rain or evaporation will 
cause considerable error. It is obvious that with the same slope or on a level the trechometer may also be used to advantage to determine the absorptive power of soils of different texture. It serves well a similar purpose when used in different habitats to measure the composite action of slope, soil, and cover in dividing the rainfall into run-off and absorbed water.

\section{Exposure}

124. Exposure refers primarily to the direction toward which a slope faces, i. e., its exposition or insolation with respect to sun and wind. It is not altogether separable from slope, however, inasmuch as the angle of the slope has some effect upon the degree of exposure. The chief influence of exposure is exerted through temperature, since slopes longest exposed to the sun's rays receive the most heat. This is supplemented in an important degree by the fact that a group of rays I foot square will occupy this area only on slopes upon which they fall at right angles. In all other cases the rays are spread over a longer area, with a consequent reduction in the amount of heat received. This effect is feit principally in evaporation from the soil, and in soil temperatures. For the leaf, it is largely if not entirely negligible, since the angle of incidence is determined by the position of the leaf, which is the same for each species whether on the level or upon a slope. On this account, exposure has little or no bearing upon light, except that the total amount of light received by the aggregate vegetation of a slope will be greater than for a level area of the same size. The effect of wind varies with the exposure. It is naturally most pronounced in those directions from which the prevailing dry or cold winds blow, and it is greatly emphasized by the fact that the opposite exposure is correspondingly protected. The infuence of wind, especially in producing evaporation from the plant and the soil, increases with the slope, since the mutual protection of the plants, or that afforded the soil by the cover, is much reduced. Finally, the distribution of the snow by the wind, a matter of considerable importance for early spring vegetation, is largely determined by exposure.

Exposure is expressed directly in terms of direction, to which is added the angle of the slope. A good field compass, reading to twelve points, is sufficient. It should be checked, of course, by the declination of the needle at the place under observation. A convenient instrument is the one already mentioned, in which compass and clinometer are combined, since these are regularly used at the same time.

125. Surface. The most important consideration with respect to surface is the presence or absence of cover, and the character of the latter. With 
the exception of snow, cover is, however, a question of vegetation, living and dead, and consequently is to be referred to the discussion of biotic factors. The surface of the soil itself often shows irregularities which must be taken into account. Such are the rocks of boulder and rock fields, the hummocks of meadows and bogs, the mounds of prairie dog towns, the innumerable minute gullies and ridges of bad lands, the raised tufts of sand-hills, etc. The influence of these is not profound, but they do have an appreciable effect upon the run-off, temperature, and wind. In many cases, this is distinctly measurable, but as a rule little more can be done than to indicate that the surface is even or uneven, and to describe the degree and kind of unevenness.

126. Record of physiographic factors. Altitude, slope, exposure, and surface are essentially constant factors, and are determined once for all, after a few check readings have been made, except in those relatively rare habitats in which dynamic forces are very active. The form of record used is the following:

\begin{tabular}{|c|c|c|c|c|c|c|c|}
\hline DATE & FORMATION & STATION & GROUP & ALTITUDE & SLOPE & EXPOSURE & SURFACE \\
\hline $10 / 7 / 02$ & Gravel slide.. & Golf Links... & Eriogonare... & $2700 \mathrm{~m}$. & $23^{\circ}$ & N.N.W. & Even \\
\hline “ & Brook bank.. & Jack Brook... & violare....... & $25550 \mathrm{~m}$. & 50 & E.N.E. & " \\
\hline “ & Half gravel.. & Hiawatha .... & Achilieare.... & $2600 \mathrm{mu}$. & $14^{3}$ & E. & Uneven \\
\hline “ & Spruce. & Milky Way... & Opulasterare. & $2625 \mathrm{~m}$. & $12^{\circ}$ & N. & Eiven \\
\hline
\end{tabular}

127. Topography. As heretofore indicated, questions pertaining ito the form and development of the land concern groups of habitats within which each habitat is the unit of investigation after the manner already laid down. A knowledge of topography is essential to the accurate mapping of a region, for which the simple methods of plane table and contour work are employed, while the geology of the surface is of primary importance in the study of successions.

\section{BIOTIC FACTORS}

128. Influence and importance. Biotic factors are animals and plants. With respect to influence they are usually remote, rarely direct. Nevertheless, they often play a decisive part in the vegetation. Their effect is, as a rule, felt directly by the formation rather than the habitat, but in either case the one reacts upon the other. Such factors are not themselves susceptible of exact measurement, but their influence upon the habitat is usually measurable in terns of the physical factors affected. In the case 
of biotic factors, it must be distinctly understood that these are not properly factors of the habitat as a physical complex, but that they are rather to be considered as reactions exerted by the effect, or formation, upon the cause or habitat. This is most especially true of plants.

129. Animals. The activities of man fall into two classes: (I) those that destroy vegetation, and (2) those that modify it. There are rare instances also where the work of man has changed a new or already denuded habitat. In the cases where the vegetaiion is destroyed, the habitat itself is sufficiently changed to permit the effect to be measured by physical factor instruments. Otherwise, the influence is felt only by the formation, as when man makes possible the migration of weeds, and it can be measured in terms of invasion by the quadrat alone. It becomes especially evident. then, in the case of man's activities, that where they produce a denuded habitat they are to be regarded as factors in the habitat; when they merely affect the formation, this is not strictly true. The changes wrought by other animals are essentially the same as those produced by man. They are not so marked nor so important, but their relation to habitat and formation is the same. As a rule, however, they affect the habitat much less than they do the formation.

130. Plants. As a dead cover, vegetation is a factor of the habitat proper, but it has relatively little importance, since it occurs regularly during the resting period. Its chief effects are in modifying soil temperature, and in holding snow and rain, and thereby increasing the water-content. By its gradual decay, moreover, it not only adds humus to the soil, but it thereby increases the water-retaining capacity of the latter also. The cover of living vegetation reacts upon the habitat in a much more vital fashion, exerting a powerful effect upon every physical factor of the habitat. The factors thus affected are distinctly measurable though it is often impossible to determine just how much of the factor is directly traceable to the vegetation. This is a simple problem in the case of most aerial factors, especially light, but it is extremely difficult for soil factors, such as water-content and soil texture. In the case of all habitats covered with formations, by far the great majority, it is impossible as well as unnecessary to separate the physical factors of the habitat proper from the reaction upon them which the plant covering exerts. Indeed, the great differentiation of habitats is largely due to the universal principle that in vegetation the effect or formation always reacts upon the cause or habitat in such a way as to modify it. As fundamental causes of succession, the discussion of the various reactions of vegetation is reserved for another place. 


\section{Methods of Habitat Investigation}

131. The use of the various instruments previously described depends largely upon the preponderance of simple instruments or recording ones. The former necessitate a number of well-trained assistants; the latter require only a part of the time of one investigator. For the most satisfactory results, however, an assistant is all but indispensable. Since simple instruments are most easily obtained because of their cheapness, and are especially adapted to purposes of instruction, the method of using them will be described first, and then that of ecograph batteries.

\section{THE METFLD OF SIMPLE INSTRUMENTS}

132. Choice of stations. This method is based upon simultaneous readings by means of simple instruments in a series of habitats, or of stations

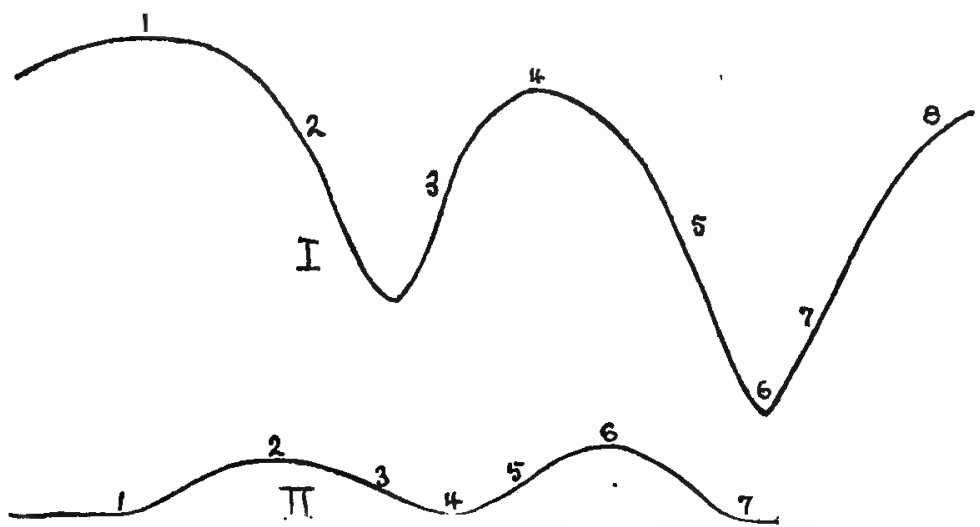

Fig. 29. Series of stations: I, at Minnehaha; II, at Lincoln in the prairie formation.

in a single habitat. Such readings are necessary for the variable atmospheric factors, humidity, light, temperature, and wind. . Frequent readings suffice for water-content and precipitation, while only two or three determinations, enough to check out the error, are necessary for the constant factors, altitude, slope, exposure, and surface. An account of the exact procedure employed in class study at Lincoln and Minnehaha will best serve to illustrate the use of this method. The series of stations chosen at Lincoln' were primarily within a single formation, for the purpose of determining the physical factor variation in different areas. One series was located in the prairie-grass formation (Koelera-Andropogon-psilium), and consisted of the following stations: (I) low prairie, (2) crest of ridge I, 
(3) northeast slope of ridge I, (4) grassy ravine, (5) southwest slope of ridge II, (6) bare irest of ridge II, (7) thicket ravine. The other series was established in the bur-oak-hickory forest (Quercus-Hicoria-hylium) at the following stations: (I) thicket, (2) woodland, (3) knoll in forest, (4) depression in forest, (5) level forest floor, (6) nettle thicket, (7) brook bank. At Minnehaha the series was primarily one of different formations: (1) the pine formation (Pinus'xerohylium), (2) the gravel-slide formation (Pseudocymopterus-Mentzelia-chalicium), (3) east slope of spruce forest (Picea-Pseudotsuga-hylium), (4) - ridge in the spruce forest, (5) north slope of spruce forest, (6) brook bank in forest, (7) the thicket formation (Quercus-Cercocarpus-lochmodium), (8) the aspen formation (Populus hylium). When permanent or temporary quadrats are established, they are ordinarily used as regular stations, - since this enables one to refer the physical factor readings to a few definite individual plants, as well as to the entire formation. The transects in figure 29 illustrate two of the above series of stations.

133. Time of readings. The frequency of simple readings and the times at which they are made must be regulated largely by opportunity and convenience. In addition to making readings once or twice a week throughout the season, the series should be read at least once every day for a representative week or two. It is also very desirable to have a series for each hour of a typical day, or of two days, one of which is clear, the other cloudy. When a single daily reading is made, it should be taken at or as near meridian as possible. The usual series is the one obtained by simultaneous observations at the same level in different stations. An important series is also secured by simultaneous readings at the various levels of the same station, though it is not necessary to take this series frequently.

134. Details of the method. After the stations have been selected by a careful preliminary survey of the habitat or series of habitats, their location is indicated by a small flag bearing a number, in case there is no danger of these being disturbed. Otherwise, less conspicuous stakes are used. The ordinary practice is to visit each station of the series, and to take readings of water-content, altitude, slope, and exposure. On the first trip these are all made by the instructor, but after a short time the determination of each factor may be assigned in rotation to each of the students. After these constant factors have been read and recorded, one student is equipped with photometer, thermometer, and psychrometer, and, if desirable, anemometer, and left at the first station. At each succeeding station the same plan is followed, so that at the end of the series the constant factors have all been 
read, and there is an observer at each station prepared to make readings of the variable ones. The task of acquainting the students with the operation of photometer, psychrometer, etc., can best be done in class or at a previous field period, as it is evident that they must be familiar with the instruments before they can use them accurately in the field series. The details of operation have already been given and need not be repeated here. The task of obtaining readings at the same moment may be met by supplying each observer with a watch, which runs exactly with all the others, or by making observations upon signal. The second means has been found most successfill in practice, sincc the signal fixes the attention at the exact moment.

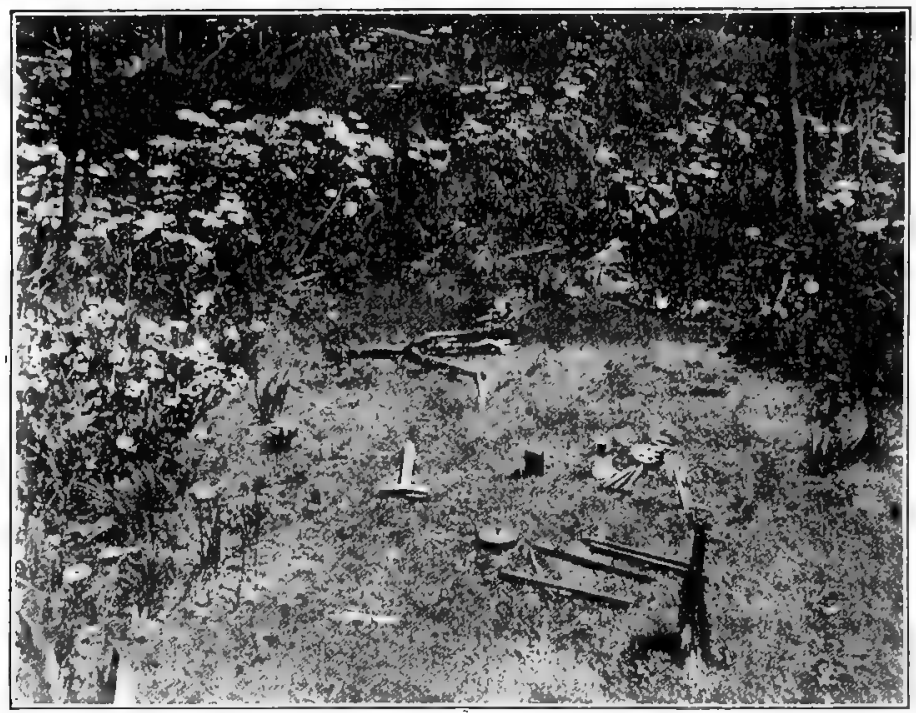

Fig. 30. A denuded station in the aspen formation.

The best plan is for the instructor to occupy a commanding position somewhere near the middlie of the series, and to give the signals by shout or whistle at the proper interval. Considerable care and experience are necessary to do the last satisfactorily. Sufficient time must be given for the operation of the instrument and the making of the record. In addition, a period must be permitted to elapse which is long enough for every instruinent to reach the proper reading. For example, in a series which contains a gravel slide and a forest, the thermometer which has just been used for an air reading will require four or five times as long an interval to respond to the temperature of the gravel as to that of the cool forest floor. In such series, the instructor should regularly take his place in the station where 
the response is slowest or greatest. He must record the exact time of each signal, and note any general changes of sky or wind that produce temporary fluctuations at the time of reading. When the readings extend over a whole day, the usual plan is to begin at the last station and take a second series of water-content samples, noting the exact time in order that the rate of water loss may be determined. A check series of physiographic factors may be made at this time also, or this may be left for future visits. While it is unnecessary to take soil samples oftener than once a day, it is important to make at least one series at each visit. Sometimes it becomes desirable to know the rate of water loss in different stations during the day, and in this event, samples are taken at one or two hour intervals for the entire day.

In making simultaneous readings at the different levels of one station, the observers are grouped in one spot in such a way that they do not interfere with the correct reading of each instrument. Readings of this sort are most valuable in the case of temperature, which shows greater differences at the various levels. Important differences of humidity and wind also are readily obtained, and, in layered formations, marked variations in the amount of light. In the open, the ordinary levels for temperature are 6 feet, 3 feet, surface, 5, Io, and 15 inches in the ground, and for wind and humidity, 6 feet, 3 feet, and surface. In forests the same levels are used for comparison with formations in the open, but a more desirable series for light especially is secured by making readings at the height of, or better, just below the various layers. Series of this sort are likewise made on signal. The best time of day is that of a period in which the middle station is read near meridian, since the variation due to time is sufficiently small to permit fairly accurate comparisons between the readings for the different stations.

135. Records. The form used for recording the observations made by means of simple instruments is shown below. It is hardly necessary to state that it may be readily modified to suit the needs of different investigators. Ordinarily, each sheet is 11sed for the records of one habitat or series alone, but for convenience sake, the records of two different series are here combined. The figures given are taken from records for the prairie and forest formations at Lincoln. 


\section{Koelera-Andropogon-psilium}

April 25, 1901. Clear. South wind.

\begin{tabular}{|c|c|c|c|c|c|c|c|c|c|c|c|c|c|c|}
\hline \multirow{3}{*}{$\frac{\text { TIME P.M. }}{\text { STATION }}$} & \multicolumn{5}{|c|}{ TEMPERATURE } & \multicolumn{2}{|c|}{ LIGHT } & \multicolumn{2}{|c|}{ HUMIDITY } & \multirow{2}{*}{\multicolumn{3}{|c|}{$\begin{array}{c}\text { WATER- } \\
\text { CONTENT } \\
\%\end{array}$}} & \multicolumn{2}{|c|}{ WIND } \\
\hline & $3: 20$ & $3: 24$ & $3: 30$ & $3: 35$ & $3: 40$ & & & $3: 45$ & $3: 55$ & & & & $2: 40$ & $3: 05$ \\
\hline & $11 / 2 \mathrm{~m}$. & Surf & 5 & 10 & 15 & $11 / 2 \mathrm{~m}$ & Surf. & $11 / 2 \mathrm{~m}$. & Surf & 5 & 10 & 15 & $11 / 2 \mathrm{~m}$. & Surf \\
\hline $\begin{array}{l}\cdots \\
\cdots \\
\cdots \\
. .\end{array}$ & $\begin{array}{l}27.8 \\
26.5 \\
26.9 \\
26.2 \\
28 \\
28 \\
26.4\end{array}$ & \begin{tabular}{|l}
29.6 \\
31.3 \\
28.5 \\
30 \\
32.4 \\
40.8 \\
30.8
\end{tabular} & $\begin{array}{l}17.8 \\
18.3 \\
18.2 \\
16.8 \\
18.6 \\
23.8 \\
16\end{array}$ & $\begin{array}{l}15.8 \\
16.6 \\
14.2 \\
13.2 \\
14.6 \\
16 \\
13\end{array}$ & $\begin{array}{l}10.9 \\
12.8 \\
13.5 \\
11.6 \\
14.2 \\
15 \\
11\end{array}$ & & $\therefore \cdots$ & $\begin{array}{l}58 \\
63 \\
59 \\
51 \\
68\end{array}$ & $\begin{array}{l}\mathbf{5 9} \\
\mathbf{5 9} \\
\mathbf{5 9} \\
66 \\
60 \\
\mathbf{5 1} \\
\mathbf{7 0}\end{array}$ & $\begin{array}{l}17.7 \\
17.9 \\
16.5 \\
24.4 \\
10.7 \\
5 \\
27\end{array}$ & $\begin{array}{c}14.6 \\
12.2 \\
16.9 \\
21.3 \\
17 \\
8.3 \\
24.3\end{array}$ & $\begin{array}{l}17.4 \\
14.3 \\
19 \\
24.8 \\
17.2 \\
10.3 \\
21.4\end{array}$ & $\begin{array}{r}740 \\
1100 \\
980 \\
920 \\
1080 \\
1010 \\
680\end{array}$ & $\begin{array}{r}280 \\
510 \\
520 \\
460 \\
490 \\
410 \\
52\end{array}$ \\
\hline
\end{tabular}

\section{Quercus-Hicoria-hylinm}

April 20, 1901. Clear. Southeast wind.

\begin{tabular}{|c|c|c|c|c|c|c|c|c|c|c|c|c|c|c|}
\hline \multirow{3}{*}{$\begin{array}{c}\text { TIME } \\
\text { A.M. } \\
\text { STA- } \\
\text { TION }\end{array}$} & \multicolumn{5}{|c|}{ TEMPERATURE } & \multicolumn{2}{|c|}{ IIGHT } & \multicolumn{2}{|c|}{ HUMIDITY } & \multirow{2}{*}{\multicolumn{3}{|c|}{$\begin{array}{c}\text { WATER- } \\
\text { CONTENT } \\
\%\end{array}$}} & \multicolumn{2}{|c|}{ - WIND } \\
\hline & \multirow{2}{*}{$\frac{10: 40}{11 / 2 \mathrm{~m} .}$} & \multirow{2}{*}{$\frac{10: 46}{\text { Surf }}$} & \multirow{2}{*}{$\frac{10: 50}{5}$} & \multirow{2}{*}{$\frac{10: 55}{10}$} & \multirow{2}{*}{$\frac{11: 00}{15}$} & \multirow{2}{*}{$\frac{12: 00}{11 / 2 \mathrm{~m}}$} & \multirow{2}{*}{$\frac{12: 05}{\text { Surf. }}$} & \multirow{2}{*}{$\frac{11: 10}{11 / 2 \mathrm{~m} .}$} & \multirow{2}{*}{$\frac{11: 20}{\text { Surf. }}$} & & & & \multirow{2}{*}{$\frac{11: 30}{11 / 2 \mathrm{~m} .}$} & \multirow{2}{*}{$\mid \frac{11: 45}{\text { Surf. }}$} \\
\hline & & & & & & & & & & 5 & 10 & 15 & & \\
\hline & 1 & 25 & 9. & 8.4 & 7.8 & .08 & .06 & 73 & 81 & 24.2 & 19.2 & 19.5 & ; 298 & 0 \\
\hline & 16.2 & 30.5 & 8 & 8.4 & 7.8 & .11 & .09 & 73 & 86 & 22 & 22.5 & 19.4 & 375 & 2 \\
\hline & 16.2 & 17.8 & 7. & 7.8 & & .08 & .06 & 73 & 95 & 22.1 & 20.4 & 21.6 & 640 & 6 \\
\hline & 15.6 & 26.2 & 10. & 8.4 & 8 & .06 & .0 & 81 & 95 & 25.4 & 23.1 & 22.4 & 275 & 12 \\
\hline & 1.7. & 25.4 & 7. & 7.4 & 7. & .03 & .02 & 90 & 95 & 27.2 & 19.8 & 18. & 178 & 2 \\
\hline , & 16. & 20.2 & 8. & 7 & 6. & 0 & .0 & 32 & 90 & 27.6 & 20.8 & 18.8 & 3 115 & 4 \\
\hline & 15 & 17 & 6 & 6.4 & 6.1 & .05 & .04 & 82 & 90 & 23.8 & 19 & 19.3 & 60 & 0 \\
\hline
\end{tabular}

\section{METHOD OF ECOGRAPH BATTERIES}

136. A battery of recording instruments. consists of a selagraph, a psychrograpl, and a thermograph, to which an anemograph is added when possible. As stated before, the determination of water-content by the geotome method is more satisfactory than by any automatic instrument yet devised. When the base station is located where the sunlight is unobstructed, which should be the case whenever possible, it is unnecessary to include a selagraph in those batteries placed in similarly exposed stations, since the light values will be the same. As a rule, batteries are established within different zones or different habitats, except where a highly diversified habitat is made the subject of special inquiry. Such a restriction arises from the fact that expense, care of operation, etc., place a limit upon the number of batteries, 
and, in such case, the task of primary importance is to establish the physical character of representative habitats. For these reasons, the first series of thermographs established in 1903 was located with respect to altitude, the instruments being placed at Manitou 2,000 m., Minnehaha, 2,600 m., and Mount Garfield 3,800 m. In I904, the stations established for the record of temperature and humidity were situated with respect to habitats representing the four formations: gravel slide, half gravel slide, spruce forest, and brook bank.

The batteries are located and set up according to the directions already given. A 2-meter quadrat with the battery as the middle is staked and mapped. Within this, all readings of water-content, soil temperature, and physiographic factors are made. Altitude, slope, exposure, and cover are recorded when each battery is located, and a soil sample is taken for mechanical analysis: When the position of the batteries permits it, water-content readings should be made frequently, once or twice a week at least. In addition, a complete series of samples should be taken daily for a period sufficient to indicate the ordinary extremes of water-content.

The ecograph battery of each habitat constitutes a standard to which the results obtained by simple instruments may be referred with accuracy. It not only does this, but it also serves as a basis for interpreting the readings of simple instruments in distant habitats of the same character. In this way a few batteries judiciously placed make possible the exact physical investigation of a large number of habitats, covering a considerable area. The only limit, indeed, upon this method is that placed by time. The proportionate use of batteries and of simple instruments must be largely determined by the conditions which confront the investigator. It is obvious that, where expense is not a decisive factor, the gain in time and in completeness of results is enormously in favor of the battery. There is an additional value in the automatic and continuous record which can not be overlooked. When the use of instruments in the study of habitat and formation becomes universal, the importance of the ecograph will be immeasurably enhanced. It will be possible to secure duplicate records of batteries located in the most remote and diverse regions, from the equator to the poles, and comparative phytogeography upon a scientific hasis will for the first time be possible. This opens an alluring vista of the future when ecologists the world over will cooperate in such a way that the results obtained by ecograph batteries anywhere on the globe will permit of exact comparison. 
THE EXPRESSION OF PHYSICAL FACTOR RESULTS

137. The form of results. It is almost inevitable that the general adoption of precise methods of measuring the habitat will result in a common form for expressing the physical character of the latter. An actual diagnosis of each habitat is not a difficult matter, after the factors are carefully measured, and will unquestionably lead to very desirable definiteness and precision. The accurate investigation of the physical factors of a number of habitats for one growing season furnishes the necessary material for a diagnosis based upon the mean for the growing season. Similar results for two or three seasons will yield a diagnosis as accurate and as final as that of a formation, or, indeed, as that of many species. The author's investigations have not yet gone far enough to warrant proposing a final form for this, but the following diagnosis is offered as a suggestion:

- Elymus-Muhlenbergia-chalicium. Habitat: holard 9 per cent, chresard 8 per cent, relative humidity 40 per cent, light 0.6 , soil colluvial gravel (gravel 70 per cent, sand 27 per cent, silt 3 per cent), air temperature $65^{\circ}$, surface $82^{\circ}$, soil $59^{\circ}$, wind 10 miles, rainfall 8 inches, altitude $2,800 \mathrm{~m}$., slope $23^{\circ}$, exposure south, surface even, cover open, no active biotic agencies.

The detailed comparison of habitats is made most readily by the graphic method of curves, which constitute the most desirable form of expression in connection with the original record upon which they are based. Factor means are particularly desirable for diagnostic purposes, and they furnish valuable curves also. Factor sums are impracticable at present, and it seems doubtful that they will ever be of much value. It is by no means impossible, however, that a more detailed and exact knowledge of the physiology of adaptation, coupled with methods of precision in the habitat, will render them necessary.

\section{Factor Records}

138. Experience has shown that the practice of making hasty and often formless records in the field is unwise and is apt to be inaccurate as well. The time saved in the field is more than counterbalanced by that consumed in copying the results into the permanent form. The danger of error in field notes rapidly taken is very grave, and the chance of confusion and the waste of time in deciphering them are great. Moreover, the task of checking a copy with the original, which is absolutely necessary for accuracy, involves a further expenditure of time and energy. For these reasons the field record should be made in permanent form. Definite record sheets are used, and the invariable rule is made that all readings are to be noted in ink at the time and spot where they are taken. On a long journey, or in the face of 
many observations, the tendency to take notes or to record observations rapidly is very great, but this will correct itself after a few attempts to use such notes. The record forms for various factors have been indicated in the proper place, as well as the one for simultaneous readings. Ecograph sheets are carefully filed, and constitute permanent records. With a little practice they may be read almost as easily as tables, and any attempt to put them into tabular form is a mere waste of time. For purposes of study and of publication, it often becomes necessary to bring together all the results obtained for a particular habitat, both by simple instruments and by ecographs. The form of record used for this is essentially that already indicated for simultaneous readings on page 92 , since general features and constant factors can not well be included in the table. Record sheets of this type have been printed at a cost of $\$ 5$ per thousand, and the various factor records can be obtained at about the same rate. The size of sheet used is $91 \% 2 \times 73 / 4$ inches. The record book is the usual note-book cover, which has been found neither too large nor too small. It is protected from dirt and rain by a covering of oilcloth which overlaps the edges. Record books should be carefully labeled, and each one should contain a single year's records.

\section{Factor Curves}

139. Plotting. The paper employed is divided into centimeter squares which are subdivided into 2-millimeter units. For ordinary curves the size of sheet is $9 \mathrm{r} / 2 \times 73 / 4$ inches, which makes it possible for curve sheets to be filed in the record book. Tablets containing 60 of these sheets can be obtained for 20 cents each from the Central School Supply House, Chicago. For curves longer than 9 inches special sizes of sheets must be used. On account of their inconvenience large sheets are avoided whenever possible. This can usually be accomplished by increasing the numerical value of the intervals. The inks employed in plotting are the waterproof inks of Chas. Higgins \& Co., Brooklyn, New York. These are made in ten or more colors, black, violet, indigo, blue, green, yellow, orange, brown, brick red, carmine, and scarlet, and cost 25 cents per bottle. In addition to being waterproof, they make it possible to combine curves in all conceivable ways without destroying their identity. Furthermore, it is a great advantage to use the same color invariably for the same kind of curve: thus, it has been the practice to indicate the 3 -foot, surface, 5 , IO, and 15 -inch temperature curves by violet, green, yellow, blue, and carmine respectively. A fine-pointed pen, such as the Spencerian No. I, is most satisfactory for inking; drawing pens, such as Gillott's Crowquill, are too finely pointed for ordinary use. 
In plotting a curve, it is first necessary to determine the value of the interval, and the extreme range of the curve or combination. For example, in the case of temperature, it is most convenient to assign a value of $I^{\circ}$ Centigrade to each centimeter, since the thermometers used read to onefifth of a degree, which corresponds exactly to the 2-millimeter units of each square. The length of the sheet permits a range of 22 degrees Centigrade, and the actual limits must be determined for the particular results to be employed. For the same region, it is very desirable that the unit interval and the range be the same, in order that all curve sheets may admit of direct comparison. Indeed, it is greatly to be hoped that in the future ecologists will agree to a uniform system of curve-plotting, cartograpliy, etc., as the geographers are beginning to do in the construction of maps. The major intervals are written, or, better, typewritten, at both sides of the sheet, and the time or space intervals are indicated at the top. Each curve sheet is properly labeled, and essential data indicated. The readings are taken from the field record, and their proper positions indicated by a dot. These are connected first by a pencil line, the curves being made abrupt rather than flowing; and the line, after having been carefully checked, is traced in ink.

140. Kinds of curves. Curves are named both with reference to the factor concerned and the position or sequence of the readings. The factors which lend themselves most readily to this method of represeritation are the variable ones, water-content, humidity, light, temperature, and wind, and corresponding curves are distinguished. Altitude and slope may likewise be shown by means of curves, but the use of cross section or contour lines serves the same purpose and is more natural. With regard to time and position, curves are distinguished as level, station, and point curves. A level curve is one based upon readings made at the same level through a series of stations or of habitats, e. g., the level curve of surface temperature. The station curve represents the various levels or points at which readings are made in a single station. The point curve has for a basis the hourly or daily variation of a factor at a particular point or level in a station. All of these may be simple curves, when established upon a single reading for a series, or mean curves when they are based upon the mean of a number of readings. Curves which show the extremes of a factor, i. e., the maximum and minimum, are also extremely. valuable, though a combination of the two for comparison is preferable.

141. Combinations of curves are invaluable for bringing similar curves together, and permitting ready comparison of them. For this, and also because they save space, they are regularly employed to the almost complete 
exclusion of single curves. Combinations are made simply by tracing the curves to be compared upon the same sheet, it being understood that dissimilar curves, e. g., level and station, can not be combined. Colored inks are an absolute necessity in combining; the primary principle underlying their use is that curves that approach closely or cross each other must be traced in inks that contrast sharply. As elsewhere stated, it has been made the invariable rule to use the same color for the same level or point. This applies especially to temperature, but holds also for humidity, light, wind, and water-content, so that the color always indicates the level. For the same reason, it is applied to a combination of point curves for one station, though it is inapplicable to a series of point curves when these lie in the same level. Light readings above 6 feet and water-content readings below $\mathrm{r}_{5}$ inches necessitate the use of additional colors.

Combinations may be made of the curves of a single factor for purposes of comparison, or they may consist of curves of different factors in order to aid in interpreting or indicating their relation to each other. Curves of the same factor may be combined to form various series. The level series consists of all the level curves for the stations under observation, e. g., the six levels for temperature, three levels for wind, etc. Similarly, the station series is a combination of all the station curves, and a corresponding arrangement may be made for point curves with reference either to station or to level. An extremely valuable combination of curves is that of the holard and chresard for a series of stations. The most important combinations of the curves of different factors are naturally those based upon factors intimately related to each other or to the plant. The grouping of water-content and humidity curves is of great value, especially when the transpiration curve is added. Light and temperature curves make an interesting combination, while a humidity, temperature, and wind series is of much aid in tracing the connection between these factors. Finally, it is altogether feasible to arrange the curves of water-content, humidity, light, temperature, and wind upon the same sheet in such fashion as to give a graphic representation of the whole physical nature of a single habitat or a series. In all combinations of curves representing different factors, it must be borne in mind that the position of a curve does not represent a definite value with reference to the others, since some are based upon per cents, others upon degrees, etc. The comparison must be based upon the character of the curves, but even then it is an important aid. An instructive grouping has been employed where series of readings on the same day, or on two successive days in forest and in prairie have yielded the usual level series of curves. The series for the two habitats are arranged on the same page, one at the right and the other at the left, and permit direct 
comparison of corresponding level or factor curves, both with respect to position and character.

142. The amplitude of all the curves described above is determined by the unit values of the factors concerned, while the length is dependent upon the number of stations, points, or times. The value assigned the latter upon the plotting paper is purely arbitrary, but it is most convenient to fix this at the centimeter square. The unit value for temperature is I" Centigrade per square, each subdivision of the latter representing 0.2, and the range being 22 degrees. For water-content curves, each square represents a value of 2 per cent, the smaller square being 0.4 per cent, and the range 2-48 per cent. The unit value for humidity is taken as 5 per cent, making each small square I per cent, and giving room on the sheet for the entire range from I-IOo per cent. Owing to the anemometer used, curves of wind velocity have been based upon the number of feet per minute. One hundred feet is taken as the unit value, and the range is from $0-2200$ feet. The unit value for the curve of light intensity is .005. Each small square is .oor, which permits a range from .oor to .or on one sheet. Consequently, when it is desired to plot the curve of a series of habitats with a range in intensity greater than this it is necessary to use a double sheet. This is the usual device when the range of curves is too great, except where the excess is slight. In this case the curve is left open at the top, and the value which the crest attains is indicated. All curves in combination are labeled at the beginning or left to indicate the level, station, or point, and at the end or right to show the time, or day, if this is not the basis of the curve or series.

The discussion that precedes deals exclusively with curves representing factors determined in the field. It applies with equal force to results obtained by instruments in control houses. In these, however, all factors except those directly experimented with, usually water-content and light, are practically equalized, and the curves based upon them are used chiefly to show how nearly equal they have become. The important curves are those of the water-content series, both holard and chresard, and of the shade tents. Where several houses are differentiated with respect to temperature or humidity, curve series of both these factors are necessary.

\section{Factor Means and Sums}

143. It has been shown elsewhere that the daily mean of temperature can be closely approximated from the maximum and minimum of both day and night. Maximum-minimum instruments for the other factors are lacking, however, and for light, humidity, and wind these values can only be ob- 
tained from the ecograph which makes it possible to get the exact mean from the sum of all the hour readings. When it comes to the seasonal mean, the ecograph is even more necessary, exception being made for water-content, in which case a number of readings on various days through the season will suffice. The value of factor means for diagnosis and for curves has already been sufficiently commented upon, and the feasibility of factor sums already indicated. 


\title{
CHAPTER III. THE PLANT
}

\author{
Stimulus And Response
}

\section{GENERAL REL.ATIONS}

144. The nature of stimuli. Whatever produces a change in the furictions of a plant is a stimulus. The latter may be a force or a material; it may be imponderable or ponderable; effect, not character, determines a stimulus. Consequently, reaction or response decides what constitutes a stimulus. The presence of the latter can be recognized only through an appreciable or visible response, since it is impossible to discriminate between an impact which produces no reaction and one which produces a merely latent one. From this it is evident that quantity is decisive in determining whether the impact becomes a stimulus. Plants grow constantly under the influence of many stimuli, all varying from time to time in amount. Small changes in these are so frequent that, in many cases at least, the plant no longer appreciably reacts to them. Such -changes, though usually measurable, are not stimuli. Futhermore, it must be clearly recognized that plants which are in constant response to stimuli are stimulated anew by an efficient increase or decrease in the amount of any one of these. As is well known, however, such increase or decrease is a stimulus only within certain limits, and the degree of change necessary to produce a response depends upon the amount of the factor normally present. The entire absence of a force usually present, moreover, often constitutes a stimulus, as is evident in the case of light. The nature of the plant itself has a profound bearing upon the factors that act as stimuli. Many species are extremely labile, and react strongly to relatively slight stimuli; others are correspondingly stable, and respond only to stimuli of much greater force. Some light is thrown upon the nature of this difference by the behavior of ecads. A form which has grown under comparatively uniform conditions for a long time seems to respond less readily, and is therefore less labile than one which is subject to constant fluctuation. In many cases this is not true, however, and the degree of stability, i. e., of response, can only be connected in a general way with taxonomic position.

145. The kinds of stimuli. The factors of a habitat are external to the plant, and consequently are termed external stimuli. Properly speaking, all stimuli are external, but since the response is often delayed or can 
not be clearly traced, it may be permissible to speak of internal stimuli, i. e., those which appear to originate within the plant. These, however, are extremely obscure, and it is hardly possible to deal with them until much more is known of the action of external stimuli. Of the latter, certain forces, gravity and polarity, act in a way not at all understood, and as they are essentially alike for all plants and all habitats, they can here be ignored. Stimuli are imponderable when, like light and heat, they are measured with reference to intensity, and ponderable, when, as in the case of water-content, humidity, and salt-content, they can be expressed in mass or weight. It is undesirable to insist upon this distinction, however, since the real character of a stimulus is determined by its effect, and the latter is not necessarily dependent upon whether the stimulus is one of force or one of material. There is, however, a fundamental difference between factors with respect to their relation to the plant. Direct factors alone are stimuli, since indirect factors must always act through them. For example, the wind, its mechanicai infuence excepted, can affect the plant only in so far as it is converted into the stinululus of increased or decreased humidity. Consequently, the normal stimuli of the plants of a formation are: (I) watercontent, (2) solutes, (3) himidity, (4) light, (5) temperature, (6) wind. Soil, pressure, physiography, and biotic factors influence plants only through these, and are not stimuli, though exceptions must be made of biotic factors in the case of sensitive, insectivorous, and gall-producing plants.

146. The nature of response. Since plants have no special organs for the perception of stimuli, nor sensory tracts for their transmission, an external stimulus acting upon a plant organ is ordinarily converted into a response at once. The latter as a rule becomes eviclent immediately; in some cases it is latent or imperceptible, or some time elapses before the chain of responses finds visible expression. A marked decrease in humidity calls forth an immediate increase of transpiration, but the ultimate response is seen in the closing of the stomata. A response to decreased light intensity, on the other hand, is much less rapid and obvious. This difference is largely due to the fact that the functional response is more marked, or at least more perceptible in one case than in the other.

Response is the reaction of the plant to a stimulus; it begins with the impact of an efficient factor, and ends only with the consequent final readjustment. The immediate reaction is always functional. The nature and intensity of the stimulus determine whether this functional response is followed by a corresponding change in structure. The consideration of this theme consequently gains in clearness if a functional and a structural 
response be distinguished. The chief value of this distinction lies in the fact that many reactions are functional alone; it serves also to emphasize the absolute interdependence of structure and function, and the imperative need of considering both in connection with the common stimulus. For these reasons, the logical treatment is to connect each stimulus with its proper functional change, and, through this, with the corresponding modification of structure. For the sake of convenience, the term adjustment is used to denote response in function, and adaptation, to indicate the response in structure.

147. Adjustment and adaptation. The adjustment of a plant to the stimuli of its habitat is a constant process. It is the daily task, seen in nutrition and growtl. So long as these take place under stimulation by factors which fall within the normal variation of the habitat, the problems belong to what has long been called physiology. When the stimuli become unusual in degree or in kind, by a change of habitat or a modification in it, adjustment is of much greater moment and is recorded in the plant's structure. These structural records are the foundation of proper ecological study. Since they are the clirect result of adjustment, however, this affords further evidence that a division of the field into ecology and physiology is illogical and superficial. Slight or periodical adjustment may concern function alone; it may be expressed in the movement of parts or organs, such as the closing of stomata or changes in the position of leaves, in growth, or in modifications of structure. This expression is fundamentally affected by the nature of the factor and is in direct relation to the intensity of the latter. Adaptation comprises all structural changes resulting from adjustment. It includes both growth and modification. The latter is merely growth in response to unusual stimuli, but this fact is the real clue to all evolution. Growth is periodic, and in a sense quantitative; it results from the normal continuous adjustment of the plant to the stimuli of its proper habitat. In contrast, modification is relatively permanent and qualitative; it is the response to stimuli unusual in kind or intensity. A definite knowledge of the processes of growth is indispensable to an understanding of modification. In the fundamental task of connecting plant and habitat, it is the modification of the plant, and not its growth, which records the significant responses to stimuli. For this reason the discussion of adaptation in the pages that follow is practically confined to modification of structure. This is particularly desirable, since growth has long been the theme of physiological study, while modification has too often been considered from the structural standpoint alone. The comparatively few studies that have taken function into account have been largely empirical; 
in them neither stimulus nor adaptation has received anything approaching adequate treatment.

148. The measurement of response. The amount of response to a stimulus is proportional to the intensity of the factor concerned. This does not mean that the same stimulus produces the same response in two distinct species, or necessarily in two plants of one species. In these cases the rule holds only when the plants or species are equally plastic. For each individual, however, this quantitative correspondence of stimulus and response is fundamental. It is uncertain whether an exact or constant ratio can be established between factor and function; the answer to this must await the general use of quantitative methods. There can be no doubt, however, that within certain limits the adjustment is proportional to the amount of stimulus, whereas reaction is well known to be abnormal or inhibited beyond certain extremes. It is quite erroneous to think that reaction is independent of quantity of stimulus, or to liken the stimulating factor to "the smallest spark (which) by igniting a mass of powder, produces an enormous mechanical effect." 1 Such a statement is only apparently true of the action of mechanical stimuli upon the few plants that may properly be said to possess irritability, such as sensitive plants and certain insectivorous ones. Of the normal relation of response to direct factors, water, light, etc., it is entirely untrue. Axiomatically, there is ordinarily an essential correspondence, also, between the amount of adjustment and of adaptation. This correspondence is profoundly affected, however, by the structural stability of the plant.

From the preceding it follows that the measurement of response and the relating it to definite amounts of direct factors as stimuli are two of the most fundamental tasks of ecology. The exact determination of physical factors has no value apart from its use for this purpose. It is perfectiy clear that precise methods of measuring stimuli call for. similar methods in determining the amount of adjustment and of adaptation. The problem is a difficult one, and it is possible at present only to indicate the direction which its development should take, and to describe a few methods which will at least serve as a beginning. To cover the ground adequately it is necessary to measure response by adjustment and by adaptation separately, and in the latter to find a measure for the individual and one for the species. The one is furnished by the methods of morphology and the other by biometry.

1Pfeffer-Ewart. Plyysiology of Plants, 1:13. $10 \mathrm{Co.}$ 
A primary requisite for any method for measuring adjustment is that it be applicable to field conditions. Many instruments for measuring transpiration, for example, are valueless, not because they are inaccurate, but because the plant studied is under abnormal conditions. To avoid the latter is absolutely necessary, a fact which makes it peculiarly difficult to devise a satisfactory field method. After the latter has been found and applied, it becomes possible to check other methods by it, and to give them real value. The final test of a field method is three-fold: (I) the plant must be studied while functioning normally in its own habitat; (2) the method must give accurate results; and (3) it must permit of extensive and fairly convenient application in the field. Until methods of this character, some of which are described later, have been employed for some time, it is impossible to connect definite intensities of factor stimuli with measured amounts of adjustment. Ultimately, it seems certain that researches will regularly take this form.

Adaptation is primarily indicated by changes in the arrangement and character of the cells of the plant. Since these determine the form of each organ, morphology also furnishes important evidence in regard to the course of adaptation, but form can be connected certainly with adjustment only through the study of cellular adaptation. In tracing the modifications of cell and of tissue, the usual methods of histology, viz., sectioning and drawing, suffice for the individual. It is merely necessary to select plants and organs which are as nearly typical as can be determined. The question of quantity becomes paramount, however, since it often gives the clue to qualitative changes, and hence it is imperative that complete and accurate measurements of cells, tissues, and organs be made. These measurements, when extended to a sufficiently large number of plants, serve to indicate the direction of adaptation in the species. They constitute the materials for determining biometrically the mean of adaptation for the species and the probable evolution of the latter. In its present development, biometry contains too much mathematics, and too little biology. This has perhaps been unavoidable, but it is to be hoped that the future will bring about a wise sifting of methods, which will make biometry the ready and invaluable servant of all serious students of experimental evolution. This condition does not obtain at present, and in consequence it seems unwise to consider the subject of biometry in this treatise.

149. Plasticity and fixity. As the product of accumulated responses, each species is characterized by a certain ability or inability to react to stimuli. Many facts seem to indicate that the degree of stability is connected with the length of time during which the species is acted upon by 
the same stimuli. It seems probable that plants which have reacted to sunlight for hundreds of years will respond less readily to shade than those which have grown in the sun for a much shorter period. This hypothesis is not susceptible of proof in nature because it is ordinarily impossible to distinguish species upon the basis of the time during which they have occupied one habitat. Evidence and ultimate proof, perhaps, can be obtained only by field and control experiments, in which the time of occupation of any habitat is definitely known. Even in this case, however, it is clear that antecedent haljitats will have left effects which can neither be traced nor ignored. Additional support is given this view by the fact that extreme) types, both ecological and taxonomic, are the most stable. Intense xerophytes and hydrophytes are much more fixed than mesophytes, though the intensity of the stimulus has dotrbtless as great an influence as its duration. Composites, labiates, grasses, orchids, etc., are less plastic than ranals, rosals, etc., but there are many exceptions to the apparent rule that fixity increases with taxonomic complexity. At present it seems quite impossible to suggest an explanation of the rule. Recent experiments indicate that there may be ancestral fixity of function, as well as of structure. It has been found, for example, that the flowers of certain species always react normally to the stimuli which procluce opening and closing, while others make extremely erratic response. If further work confirms this result and extends it to other functions, the necessity of arriving at a better understanding of fixity will be greatly emphasized.

It is impossible to make progress in the study of adaptation without recognizing the fundamental importance of ancestral fixity as a factor. E. S. Clements ${ }^{1}$ has shown that a number of species undergo pronounced changes in habitat without showing appreciable modification. Consequently, it is incorrect to assume that each habitat puts a structural impress upon every plant that enters it. For this reason, the writer feels that the current explanation of xerophytic bog plants, etc., is probably wrong, and that the discrepancy between the nature of the habitat and the structure of the plant is to be explained by the persistence of a fixed ancestral type. The anomaly is scarcely greater than in cases that have proved capable of being explained.

150. The law of extremes. When a stimulus approaches either the maximum or minimum of the factor for the species concerned, response becomes abnormal. The resulting modifications approach each other and in some respects at least become similar. Such effects are found chiefly in

1 The Relation of Leaf Structure to Physical Factors. 1905. 
growth, but they occur to some degree in structure also. It is imperative that they be recognized in nature as well as in field and control experiment, since they directly affect the ratio between response and stimulus. The data which bear upon the similarity of response to extremes of different factors are too meager to permit the formulation of a rule. It is permissible, however, to suggest the general principle that extreme stimuli produce similar growth responses, and to emphasize the need of testing its application to adaptation proper.

151. The method of working hypotheses. In the study of stimulus and response, where the unimpeachable facts are relatively few, and their present correlation slight, the working hypothesis is an indispensable aid. "The true course of inductive procedure . . . consists in anticipating nature, in the sense of forming hypotheses as to the laws which are probably in operation, and then observing whether the combinations of phenomena are such as would follow from the laws supposed. The investigator begins with facts and ends with them. He uses such facts as are in the first place known to him in suggesting probable hypotheses; deducing other facts which would happen if a particular hypothesis is true, he proceeds to test the truth of his notion by fresh observations or experiments. If any result prove different from what he expects, it leads him either to abandon or to modify his hypothesis; but every new fact may give some new suggestion as to the laws in action. Even if the result in any case agrees with his anticipations, he does not regard it as finally confirmatory of his theory, but proceeds to test the truth of the theory by new deductions and new trials." In the treatment of adjustment and adaptation which follows, the method of multiple working hypotheses is uniformly employed. No apology is felt to be necessary for this, since the whole endeavor is to indicate the proper points of attack, and not to distinguish between that which is conjectural and that which is known. If an hypothesis occasionally seem to be stated too strongly, it is merely that it appears, after a survey of the problem from all sides, to explain the facts most satisfactorily. The final proof of any hypothesis, however, rests not only upon its ability to explain all the facts, but also upon the inability of other hypotheses to meet the same test. The discovery and examination of all possible hypotheses, and the elimination of those that prove inadequate are the essential steps in the method of working hypotheses.

${ }^{1}$ Jevons, W. A. The Principles of Science, 2:137. 1874. 
HYDROHARMOSE

\section{ADJUSTMENT}

152. Water as a stimulus. Plants are continually subjected to the action of the water of the soil and of the air; exception must naturally be made of submerged plants. The stimulus of soil water acts upon the absorbing organ, the root, while that of humidity affects the part most exposed to the air, viz., the assimilative organ, which is normally the leaf. But since both are simultaneous water stimuli, a clearer conception is gained of this operation if they are viewed as two phases of the same stimulus. This point of view receives further warrant from the essential and intimate relation of humidity and water-content as determined by the plant. They are in fact largely compensatory, as is shown at some length later. In determining the intensity of the two, a significant difference between them must be recognized. The total humidity of the air at any one time constitutes a stimulus to the leaf which it touches. This is not true of the total soil water. Part of the latter is not available under any circumstances, and can not affect the plant, at least directly. The chresard alone can act as a stimulus, but even this is potential in the great majority of cases, since the actual stimulus is not the water available but the water absorbed. The latter, moreover, contains many nutrient salts which are in themselves stimuli, but as they normally have little bearing upon the action of water as a stimulus they are to be considered only when present in excessive) amounts.

153. The influence of other factors upon water. The amount of humidity is modified directly by temperature, wind, precipitation, and pressure,. and, through these, it is affected by altitude, slope, exposure, and cover. Naturally, also, the evaporation of soil water has a marked influence. In determining water-content, atmospheric factors, with the exception of precipitation, are usually subordinate to edaphic ones. Soil texture, slope, and precipitation act directly in determining soil water, while temperature, wind, and pressure can operate only through humidity. This is likewise true of altitude, exposure, and cover, though the latter has in addition a profound effect upon run-off. Biotic factors can affect humidity or water-content only through the medium of another factor. Light in itself has no action upon either, but through its conversion into heat within the chloroplast, it has a profound effect upon transpiration. The following table indicates the general relation between water and the other physical factors of the habitat. 
The order of the signs, \pm , denotes that the water increases and decreases with an increase and decrease of the factor, or the reverse, $\mp$.

Humidity \pm
Temperature $\mp$
Wind $\mp$
Precipitation \pm
Pressure \pm
Soil texture 0
Altitude $\mp$
Slope $\mp$
Exposure $\mp$
Cover \pm

Water-content \pm

Temperature $\mp$

Wind $\mp$

Precipitation \pm

Pressure $\mp$

Soil texture

Porosity $\mp$

Capillarity \pm

Slope $\mp$

Exposure $\mp$

Cover \pm

154. Response. The normal functional responses to water stimuli are absorption, diffusion, transport, and transpiration. Of these, absorption and transpiration alone are the immediate response to soil water and humidity, respectively. Consequently they are the critical points of attack in studying the fundamental relation of the plant to the water of its habitat. In determining the pathway of the response, it is necessary to trace the steps in diffusion and transport, but, as these are essentially alike for all vascular plants, this task lies outside the scope of the work in hand. As previously stiggested, the relation between absorption and transpiration is strictly compensatory, though, for obvious reasons, the amount of water transpired is usually somewhat less than the amount absorbed. Absorption falls below transpiration when extreme conditions cause temporary or permanent wilting; the two activities are essentially equal after a growing plant reaches maturity. In all cases, however, the rule is that an increase or decrease in water loss produces a corresponding change in the amount of water absorbed, and, conversely, variation in absorption produces a consequent change in transpiration. This is strictly true only when the stimuli are normal. For example, a decrease in humidity causes increased water loss, which, through diffusion and transport, is compensated by increased activity of the root surface. Frequently the water supply is insufficient to compensate for a greater stimulus, and the proper balance can be attained only by the closing of the stomata. In the case of excessive stimuli, neither compensation suffices, and the plant dies. Many mesophytes and all xerophytes lave probably resulted from stimuli which regularly approached the limit of compensation for each, and often overstepped, but never permanently exceeded it. For hydrophytes, the danger arises from excessive water supply, not water loss. There is a limit to the compensation afforded by transpiration, which is naturally dependent upon the amount of plant sur- 
face exposed to the air. No compensation occurs in the case of submerged plants; floating hydrophytes possess a single transpiring leaf surface, while the leaves of amphibious plants behave as do those of mesophytes. The whole question of response to water stimuli thus turns upon the compensation for water loss afforded by 'water supply where the latter is moderate or precarious, and upon the compensation for water supply furnished by water loss where the supply is excessive, submerged plants excepted.

155. The measurement of absorption. As responses to measured stimuli of water-content and humidity, it is imperative that the amount of absorption and of transpiration be determined quantitatively. It is also extremely desirable that this be done in the normal habitat of the plant. A careful examination of the problems to be met quickly discloses the great difficulty of obtaining a direct and accurate measure of absorption under normal conditions, especially in the field. For this purpose, the ordinary potometric experiments by means of cut stems are valueless. The use of the entire plant in a potometer yields much more trustworthy results, though the fact that the root is under abnormal conditions can not be overlooked, especially in the case of mesophytes and xerophytes. While potometric conditions are less abnormal for amphibious plants, the error is not wholly eliminated, since the roots normally grow in the soil. The potometer can be made of value for quantitative work only by checking the results it gives by means of an instrument or a method in which the plant functions normaily. In consequence, the potometer can not at present be used to measure absorption directly, othough, as is further indicated in the discussion of transpiration, it is a valuable supplementary instrument, after the check mentioned has been applied to its use with a particular species.

An estimate of the amount of absorption may be obtained either in the field or in the control house by taking samples from the protected soil at different times. Since it is impossible to determine the weight of the area in which the roots lie, and since the soil water is often unequally distributed, this method can not yield exact results. An accurate method of neasuring absorption under essentially normal conditions has been devised and tested in the control house. The essential feature of the process is the placing a plant in a soil containing a known quantity of water, and removing it after it has absorbed water from the soil for a certain period. In carrying out the experiment, a soil consisting of two parts of sod and one of sand was used, since the aeration is more perfect and the particles are more easily removed from the roots. The soil was completely dried out in a water bath and then placed in a five-inch battery jar. The latter, together with the rubber cloth used later to prevent evaporation, was weighed to the decigram. A weighed quantity of water was added, and the whole again weighed as a 
check. Two plants of Helianthus annuus were taken from the pots in which they had grown, and the soil was carefully washed from the roots. Each plant was weighed with its roots in a dish of water to prevent wilting, and then carefully potted, one in each battery jar. A thistle tube was placed in the soil of each jar to facilitate aeration, as well as the addition of weighed amounts of water, when necessary, and the rubber cloth attached in the usual manner to prevent evaporation. The entire outfit was weighed again, and the weighing repeated at 8:00 A.M. and 5:00 P.M. for five days, in order to determine the amount of transpiration and its relation to the water absorbed. The plants were kept in diffuse light to prevent excessive water loss while the roots were becoming established. At the close of the experiment, the jar and its contents were weighed finally. The plants were removed and weighed, the soil particles being shaken from the roots into the jar, which was also weighed. The results obtained were as follows:

\begin{tabular}{|c|c|c|c|c|c|c|c|}
\hline & $\begin{array}{l}\text { Wt. of pot } \\
\text { and dry soil }\end{array}$ & Wt. of pot & d wet soil & Total $\mathrm{H}_{2} \mathrm{O}$ & $\mathrm{H}_{2} \mathrm{O}$ left & $\begin{array}{c}\mathrm{H}_{2} \mathrm{O} \text { ab- } \\
\text { sorbed }\end{array}$ & $\begin{array}{c}\mathrm{H}_{2} \mathrm{O} \text { tran } \\
\text { spired }\end{array}$ \\
\hline II & $\begin{array}{l}1846.0 \mathrm{~g} . \\
1886.7 \mathrm{~g} .\end{array}$ & $\begin{array}{c}\mathrm{I} \\
2218.0 \mathrm{~g} . \\
2253.2 \mathrm{~g}\end{array}$ & $\begin{array}{c}\text { II } \\
2174.3 \mathrm{~g} . \\
2221.6 \mathrm{~g} .\end{array}$ & $\begin{array}{l}372.0 \mathrm{~g} . \\
366.5 \mathrm{~g} .\end{array}$ & $\begin{array}{l}328.3 \mathrm{~g} . \\
334.9 \mathrm{~g} .\end{array}$ & $\begin{array}{l}43.7 \mathrm{~g} . \\
31.6 \mathrm{~g} .\end{array}$ & $\begin{array}{l}43.7 \mathrm{~g} . \\
31.6 \mathrm{~g} .\end{array}$ \\
\hline
\end{tabular}

The amount of water absorbed may be obtained directly by subtracting the final weight of the jar and moist soil from their first weight, but a desirable check is obtained by taking the dry weight of jar and soil from the first, and the final weight of these, and subtracting the one from the other as indicated in the table. A second check is afforded by daily weighings, from which the amount of water transpired is determined. Since the two sunflower plants made practically no growth during the period of experiment, the exact correspondence between water absorbed and water lost is not startling, though it can not be expected that the results will always coincide.

This method has certain slight sources of error, all of which, it is thought, have been corrected in a new and more complete series of experiments now being carried on. The aeration of the soil is not entirely normal, as is also true of the capillary movements of the water, on account of the nonporous glass jar and the rubber cloth. Since the latter are necessary conditions of all accurate methods for measuring absorption and transpiration, the resulting error must be ignored. It can be reduced, however, by forcing air through the thistle lube from time to time. Sturdy plants, such as the sunflower, are the most satisfactory, since they recover more quickly from the shock of transplanting. Almost any plant can be used, however, if 
repotted in a loose sandy soil often enough. This permits the root system to develop normally, and also makes it possible to wash the soil away without injury to the root. The method is so recent that there has been no opportunity to test it in the field. It would seem that it can be applied without essential change to plants in their normal habitats. Very large herbs or plants with extensive root systems could not be used to advantage, and to be practicable the experiments would need to be carried on near the base station. The great value of the method, however, lies in its use as a check in determining the accuracy of other methods, and in practice it will

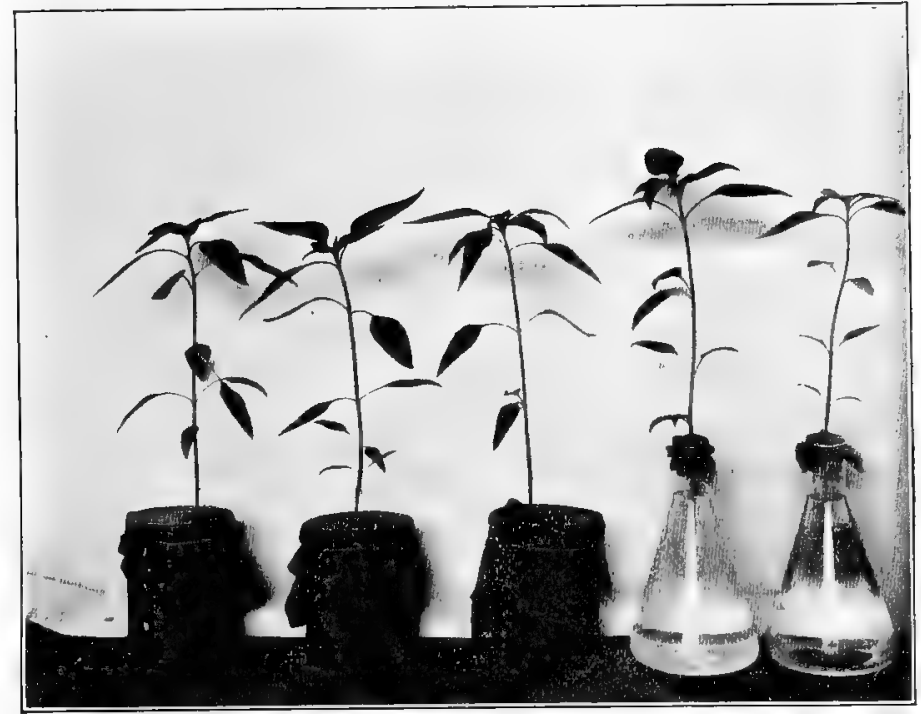

Fig. 31. Absorption and transpiration of Helianthus annuzs. I and II, plants repotted in soil of known weight and water-content; III, plant undistured in the original soil; IV, potometer containing plant with cut stem; V, potometer with entire plant.

often be found convenient and time-saving to use the latter, after they have once been carefully checked for different groups of species. This matter is further considered under measures of transpiration.

156. The quantitative relation of absorption and transpiration. Burgerstein $^{1}$ has summarized the results of various investigators in the statement "that between the quantitative absorption of water on the one hand and emission on the other there exists no constant parallelism or proportion,"

${ }^{1}$ Die Transpiration der Pflanzen, 14. 1904. 
and he has cited the work of Kröber, and of Eberdt in proof. This statement holds, however, only for short periods of a few hours, or more rarely, a day, and even here its truth still remains to be conclusively demonstrated. The discrepancy between absorption and transpiration for a short period is often greater than for a longer time, but it is evident that a transient change in behavior or a small ertor in the method would inevitably produce this result. Eberdt found the discrepancy for a few hours to be $1-2 \mathrm{ccm}$. in an entire plant of Helianthus annuns, while for a whole day the water absorbed was $33.57 \mathrm{ccm}$. and the water lost $33.98 \mathrm{ccm}$. Kröber's experiments with cut branches of Asclepias incarnata showed a maximum difference for I2 hours of $2.5 \mathrm{ccm}$., but the discrepancy for the first 24 hours was I ccm. and for the second $1.9 \mathrm{ccm}$. In both cases, the potometer was employed. Consequently, as will be shown later, Eberdt's results are not entirely trustworthy, while those of Kröber, made with cut stems, are altogether unreliable. Hence, it is clear that the discrepancy is slight for a period of several days or weeks, and that it may be ignored without serious error, except in a few plants that retain considerable water as cell sap, in consequence of extremely rapid growth. Accordingly, the amount of transpiration, which may be readily and accurately determined, can be employed as a measure of absorption that is sufficiently accurate for nearly all purposes. The truth of this statement may be easily confirmed. It is evident that the amount of water absorbed equals the amount transpired plus that retained by the plant as cell-sap, or used in the manufacture of organic compounds. In plants not actively growing, the amount lost equals that absorbed, as already shown in the experiment with Helianthus. According to Gain ${ }^{1}$, Dehérain has found that a plant rooted in ordinary soil transpired $680 \mathrm{~kg}$. of water for each kilogram of dry substance elaborated. In Helianthus annuus, the dry matter is Io per cent of the weight of the green plant. A well-grown plant weighing $\mathrm{r}, 000$ grams, therefore, consists of IoO grams of dry matter and goo of water. The length of the growing period for such a plant is approximately roo days, during which it transpires 68 kilograms of water. Assuming the rate of transpiration and of growth to be constant, the plant transpires 680 grams daily, adds 9 grams to its cell-sap, and I gram to its dry weight. The amount of water in a gram of cellulose and its isomers is about $3 / 5$. Consequently, the total water absorbed daily by the plant is 689.6 grams. The 680 grams transpired are 98.6 per cent of the amount absorbed; in other words, only 1.4 per cent of the water absorbed is retained by the plant. From this it is evident that the simplest

${ }^{1}$ Recherches sur le Rôle Physiologique de l'Eau dans la Végétation. Ann. Nat. Sci., 7:20:65. 1895. 
and most convenient measure of absorption under normal conditions can be obtained through transpiration, since the discrepancy between absorption and transpiration is scarcely larger than the error of any method applicable to the field. Conversely, the measure of absorption obtained by the process described in the preceding section serves also as a measure of transpiration. The determination of the latter in the field is so much simpler, however, that it is rarely desirable to apply the absorption method.

157. Measurement of transpiration. The water loss of a plant may be determined absolutely or relatively. Absolute or quantitative determinations are by (I) weighing, (2) collecting, or (3) measuring the water absorbed; relative values are indicated by hygroscopic substances. A number of methods have been employed more or less generally for measuring transpiration. The great majority of these can be used to advantage only in the laboratory, and practically all fail to meet the fundamental requirement for successful field work, namely, that the plant be studied under normal conditions in its own habitat. The following is a summary of the various methods, the details of which may be found in Burgerstein.

I. Weighing. This is the most satisfactory of all methods for determining water loss. It is more accurate than any other, and is unique in that it does not place the plant under abnormal conditions. On the score of convenience, moreover, it excels every other method capable of yielding quantitative results. Various modifications of weighing are employed, but none of these have all the advantages of a direct, simple weighing of the plant in its own soil.

2. Collecting the zrater transpired. This may be done by collecting and weighing the water vapor exhaled by a plant placed within a bell jar, or by weighing a deliquescent salt, such as calcium chloride, which is used to absorb the water of transpiration. The decisive disadvantage of these inethods is that transpiration is carried on in an atmosphere far more humid than normal. If an excessive amount of salt is used, the air is abnormally dry. In both cases, the water loss decreases until it reaches a point much below the usual amount. Finally, all methods of this kind are open to considerable error, and are inconvenient, especially in field work. They are of relatively slight value in comparison with weighing.

3. Potometers. It has already been shown that the amount of water absorbed is a close measure of the amount transpired. In consequence, the potometer can be used to determine the amount of transpiration provided the absorption is not abnormal. It is rarely and only with much difficulty that this condition can be met. The use of cut stems and branches does not meet it, and even in the case of plants with roots, the results must be 
compared with those obtained from absorption experiments made with plants rooted in soil before they can be relied upon. This necessity practically puts the potometer out of commission for accurate work, unless future study may show a somewhat constant ratio between the absorption of a plant in its own soil and that of a plant placed in a potometer.

4. Measuring absolute humidity. The cog psychrometer makes it possible to determine the increased relative humidity produced within a glass cylinder or special tin chamber by a transpiring plant. From this result the absolute humidity is readily obtained, and by means of the latter the actual amount of water given off. The evident drawback to this method is that the increasing humiclity within the chamber gives results entirely abnormal for the plant concerned.

5. Self-registering instruments. There are various methods for registering the amount of transpiration, based upon weighing, or upon the potometer. The Richard recording evaporimeter has all the advantages of weighing, inasmuch as the water loss is measured in this way, and in addition the amount is recorded upon a revolving drum, obviating the necessity of repeated attention in case it is desirable to know the exact course of transpiration. On the other hand, methods which depend upon the potometer, while graphic, are not sufficiently accurate to be of value.

6. The use of hygroscopic materials. Hygroscopic substances change their form or color in response to moisture. As they indicate comparative water loss alone, they are of value chiefly in the study of the stomatic surfaces of leaves. F. Darwin ${ }^{1}$ has used strips of horn, awns of Stipa, and epidermis of $Y u c c a$ to construct small hygroscopes for this purpose. In these instruments the error is large, but as no endeavor is made to obtain exact results, it is negligible. Filter paper impregnated with a 3-5 per cent aqueous solution of cobalt chloride is deep blue when dry. If a strip of cobalt paper is placed upon a leaf and covered with a glass slip it turns bright rose color, the rapidity of the change affording a clue to the amount of transpiration.

158. Field methods. The conditions which a satisfactory field method of measuring transpiration must fulfill have already been discussed; they are accuracy, simplicity, and normality. These conditions are met only by weighing the plant in its own soil and habitat. This has been accomplished by means of the sheet-iron soil box, already described under the determination of the chresard. The method is merely the familiar one of pot and balance, slightly modified for field use. The soil block, which contains the

${ }^{1}$ Observations on Stomata by a New Method. Proc. Camb. Phil. Soc., 9:303. 1897. 
plant to be studied, is cut out, and the metal plates put in position as indicated in section 53. Indeed, it is a great saving of time and effort to determine transpiration and chresard in the same experiment; this is particularly desirable in view of the close connection between them. In this event, the soil block must be small enough not to exceed the load of a field balance. After the block is cut and encased, all the plants are removed, except the one to be studied. If several individuals of the same species are present, it is an advantage to leave all of them, since the error arising from individuai variations of water loss may, in this way, be almost completely eliminated. A sheet of rubber or rubber cloth is carefully tied over the box to prevent evaporation from the soil. A broad band is passed under the box to aid in lifting it upon the scales. The latter must be of the platform type, and should have a capacity as great as consistent with the need for moving it about in the field. Weighings are made in the usual way, care being taken to free the surface of the box from soil. The aeration of the soil block is kept normal by removing the rubber for a few minutes from time to time, or by forcing air through a thistle tube. Water is also added through the latter, when it is desired to continue the experiment for a considerable period. After the study of transpiration is concluded, the rubber cloth is removed, soil samples taken, and the soil allowed to dry out until the plant becomes thoroughly wilted. If the box is weighed again, the difference represents the amount of available water. The per cent of chresard is also obtained in the usual way by taking samples for ascertaining the echard, and subtracting this from the holard. Field determinations of water loss yield the most valuable results when different habitat forms, or ecads, of the same species are used. There is little profit in comparing the transpiration of a typical sun plant, such as Touterea multiflora, with that of a shade plant, such as Washingtonia obtusa. But the simultaneous study of plants like Chamaenerium angustifolium, Gentiana acuta, Scutellaria brittonii etc., which grow in several different habitats, furnishes direct and fundamental evidence of the course of adjustment and adaptation.

Hesselmann ${ }^{1}$, in his study of open woodlands in Sweden, has employed a method essentially similar to the preceding. Young plants of various species were transferred to pots in the field, where they were allowed to grow for several months before a series of weighings was made to determine the amount of transpiration. Since weighing is the measure used in each, both methods are equally accurate. The one has a certain advantage in that the pots are, perhaps, more easily handled, while the other has the advantage

I Zur Kenntnis des Pflanzenlebens schwedischer Laubwiesen. Beih. Bot. Cent., 18:311. 1901. 
of maintaining the normal relation of soil and roots, a condition more or less impossible in a pot. In both instances the weighing should be done in the habitat, which was not the case in Hesselmann's researches.

The slight value of the potometer, which has had a vogue far beyond its merits, is indicated by the following table. These results were obtained from three plants of Helianthus annuns; III was left undisturbed in the pot where it had been growing, IV was placed in a potometer, after the root had been cut off, and $\mathrm{V}$ was an entire plant placed in a potometer. The amount of transpiration is indicated in grams per square decimeter of leaf surface. The plants were kept in diffuse light, except for a period of two hours (8:00 to 10:00 A.M.) on the last day, when they were in full sunshine at a temperature of $75^{\circ} \mathrm{F}$. Plant IV wilted so promptly in the sunshine that it was found necessary to conclude the experiment in diffuse light.

\begin{tabular}{c|c|c|c|c|c|c|c|c|c|c|c}
\hline \hline & 8 A.M. & 5 P.M. & 8 A.M. & 5 F.M. & 8 A.M. & 5 P.M. & 8 A.M. & 10 A.M. & 5 P.M. & 8 A.M. & Total \\
\cline { 3 - 9 } & & & & & & & & & & \\
III & 2.9 & 7.3 & 2.4 & 6.0 & 1.7 & 1.6 & 2.0 & 3.4 & 2.0 & 1.8 & 31.1 \\
IV & 4.7 & 7.2 & 2.9 & 2.3 & 1.0 & 0.6 & 0.9 & 0.5 & 0.5 & 0.4 & 21.0 \\
V & 3.7 & 5.3 & 3.2 & 4.8 & 2.5 & 1.6 & 3.0 & 2.6 & 1.6 & 2.6 & 30.9 \\
\hline
\end{tabular}

The cut plant, IV, lost more water the first day than either of the others, but the water loss soon decreased, and at the end of the period was almost nil. The total transpiration for III and $\mathrm{V}$ is much the same, but the range of variation for periods of $\mathrm{I} 2$ hours is from +2 to $-\mathrm{I}$ gram. This experiment is taken as a fair warrant that the use of cut stems in potometers can not give accurate results. It is inconclusive, however, as to the merits of potometric values obtained by means of the entire plant, and further studies are now being made with reference to this point.

159. Expression of results. From the previous discussion of the relation between them, it follows that an expression of the amount of transpiration likewise constitutes an expression of absorption. It is very desirable also that the latter be based upon root surface and chresard, but the difficulty of determining the former accurately and readily is at present too great to make such a basis practicable. In expressing transpiration in exact terms, the fact that plants of the same species or form are somewhat individual in their behavior must be constantly reckoned with. In consequence, experiments should be made upon two or three individuals whenever possible, in order to avoid the error arising from this source. 
Water loss may be expressed either in terms of transpiring surface or of dry weight. Since there is no constant relation between surface and weight, the terms are not interchangeable or comparable, and in practice it is necessary to use one to the exclusion of the other. Obviously, surface furnishes by far the best basis, on account of its intimate connection with stomata and air-spaces, a conclusion which Burgerstein (l. c., p. 6) has shown by experiment to be true. For the best results, the whole transpiring surface should be determined. This is especially necessary in making comparisons of different species. In those studies which are of the greatest valtie, viz., ecads of the same species, it is scarcely desirable to measure stem and petiole surfaces, unless these organs show unusual modification. The actual transpiring surface is constituted by the walls of the cells bordering the intercellular spaces, but, since it is impossible to determine the aggregate area of these, or the humidity of the air-spaces themselves, the leaf surface must be taken as a basis. Since the transpiration through the stomata is much greater than that through the epidermal walls, the number of stomata must be taken into account. Since they are usually less abundant on the upper surface, their number should be determined for both sides of the leaf. The errors arising from more or less irregular distribution are eliminated by making counts near the tip, base, and middle of two or three mature leaves. The most convenient unit of leaf surface is the square decimeter. The simplest way to determine the total leaf area of a plant is to outline the leaves upon a homogeneous paper, or to print them upon a photographic paper. The outlines are then cut out and weighed, and the leaf area obtained in square decimeters by dividing the total weight by the weight of a square decimeter of the paper used. The area may also be readily determined by means of a planimeter.

160. Coefficient of transpiration. At present it does not seem feasible to express the transpiration of a plant in the form of a definite coefficient, but it is probable that the application of exact methods to each part of the problem will finally bring about this result. Meanwhile the following formula is suggested as a step toward this goal: $t=g \frac{u}{l} L H T$, in which $t$, the transpiration relation of a plant, is expressed by the number of grams of water lost per hour, on a day of sunshine, by one square decimeter of leaf, considered with reference to the stomata of the two surfaces, and the amount of the controlling physical factors, light, humidity, and temperature, at the time of determination. For Helianthus annuns, this formula would appear as follows: $t=2 \frac{200}{250}: 1: 50: 75^{\circ}$. To avoid the large figures arising 
from the extent of surface considered, the number of stomata per square decimeter is divided by 10,000. This amounts to the number per square millimeter, and time may consequently be saved by using this figure directly. While this formula obviously leaves much to be desired, it has the great advantage of making it possible to compare ecads of one species, or species of the same habitat or of different habitats, upon an exact basis of factor, function, and structure.

\section{ADAPTATION}

161. Modifications due to water stimuli. In adaptation, the great desideratum is to connect each modification quantitatively with the corresponding adjustment. This is even more difficult than to ascertain the quantitative relation between stimulus and functional response, a task still beset with serious obstacles. At the present time, little more can be done than to indicate the relation of marked adaptations of organs and tissues to the direct factors operating upon them, and to attempt to point out among the functions possibly concerned the one which seems to be the most probable connection between the probable stimulus and the structure under investigation. In the pages that follow, no more than this is attempted. The general changes of organs and tissues produced by water are first discussed, and after this is given a summary of the structural features of the plant types based upon water-content.

162. Modifications due to a small water supply. A water supply which may become deficient at any time is compensated either by changes which decrease transpiration, or by those that increase the amount of water absorbed or stored. 'These operate upon the form and size of the organs concerned, as well as upon their structure. Modifications of the form of leaf and stem are alike in that they lessen transpiration by a reduction of the amount of surface exposed to the air. Structural adaptations, on the other hand, bring about the protection of epidermal cells and stomata, and often internal cells also, from the factors which cause transpiration, or they anticipate periods of excessive transpiration by the storage of water in specialized cells or tissues. In certain extreme types the epidermis is itself modified for the absorption of water vapor from the air.

163. The decrease of water loss. The following is a summary of the contrivances for reducing transpiration.

I. Position of the leaf. Since the energy of a ray of sunlight is greatest at the sun's highest altitudes, those leaves transpire least which are in such a 
position cluring midciay that the ray's strike them as obliquely as possible. A leaf at right angles to the noonday sun receives ten times as much light and heat upon a square decimeter of surface as does one placed at an angle of Io degrees. This device for reducing the intensity of insolation is best developed in the erect or hanging leaves of many tropical trees. In temperate zones, it is found in such plants as Silphium laciniatum and Lactuca scariola, and in species with equitant leaves. In such plants as Helianthus anmuus, the effect is just the opposite, since the turning of the crown keeps the leaves for a long time at a high angle to the incident rays. In the case of mats, it is the aggregation of plants which brings about the mutual protection of the leaves from insolation and wind.

2. Rolling of the lcaf. Many grasses and ericaceous plants possess leaves capable of rolling or folding themselves together when drouth threatens. In other cases, the leaves are permanently rolled or folded. The advantage of this device arises not only from the reduction of surface, but also from the fact that the stomata come to lie in a chamber more or less completely closed. In the case of those mosses whose leaves roll or twist, a reduction of surface alone is effected.

3. Reduction of leaf. The transpiring surface of a plant is reduced by decreasing the number of leaves, by reducing the size of each leaf, or by a change in its form. In so far as the stem is a leaf, a decrease in size or a change in shape brings about the same result. The final outcome of reduction in size or number is the complete loss of leaves, and more rarely, of the stem. Such marked decrease of leaf area is found only in interise xerophytes, though it occurs in all deciduous trees as a temporary adaptation. Changes in leaf form are nearly always accompanied by a decrease in size. Of the forms which restlt, the scale, the linear or cylindrical leaf, and the succulent leaf are the most common. Leaves which show a tendency to divide often increase the number of lobes or make them smaller.

4. Epidermal modifications. Excretions of wax and lime by the epidermis have a pronounced effect by increasing the impermeability of the cuticle, and, hence, decreasing epidermal transpiration. It seems improbable that a coating of wax on the lower surface of a diphotic leaf can have this purpose. The thickening of the outer wall of epidermal cells to form a cuticle is the most perfect of all contrivances for decreasing permeability and reducing transpiration. In many desert plants, the greatly thickened cuticle effectually prevents epidermal transpiration. In these also the cuticle is regularly developed in such a way as to protect the guard cells, and even to close the opening partially. An epidermis consisting of two or more layers of cells is an effective, though less frequent device against water loss. When combined with a cuticle, as is usually the case, the impermeability is almost complete. Hairs decrease transpiration by screening the 
epidermis so that the amount of light and heat is diminished, and the access and movement of dry air impeded. While hairs assume the most various forms, all hairy coverings serve the same purpose, even when, as in the case of Mesembryanthemum, they are primarily for water-storage. Hairs protect stomata as well as epidermal cells: the greater number of the former on the lower surface readily explains the occurrence of a hairy covering on this surface, even though absent on the more exposed upper side. In some cases, hairs are developed only where they serve to screen the stomata.

The modifications of the stomata with respect to transpiration are numerous, yet all may be classed with reference to changes of number or level. With the exception of aquatic and some shade plants, the number of stomata is normally greater on the less exposed, i. e., lower surface. The number on both surfaces decreases regularly as the danger of excessive water loss increases, but the decrease is usually more rapid on the upper surface, which finally loses its stomata entirely. It has been shown by many observers that species growing in dry places have fewer stomata to the same area than do those found in moist habitats. This result has been verified experimentally by the writer in the case of Ranunculus sceleratus, in which, however, the upper surface possesses the larger number of stomata. Plants of this species, which normally grow on wet banks, were grown in water so that the leaves floated, and in soils containing approximately Io, I5, 30, and 40 per cent of water. 'The averages for the respective forms were: upper 20, lower o; upper I8, lower I0; upper I8, lower II; upper II, lower 8; upper Io, lower 6 . Reduction of number is effective, however, only under moderate conditions of dryness. As the latter becomes intense, the guard cells are sunken below the epidermis, either singly or in groups. In both cases, the protection is the same, the guard cells and the opening between them being withdrawn from the intense insolation and the dry air. The sun rays penetrate the chimney-shaped chambers of sunken stomata only for a few minutes each day, and they are practically excluded from the stomatal hollows which are filled with hairs. The influence of dry winds is very greatly diminished, as is also true, though to a less degree, for leaves in which the stomata are arranged in furrows. Sunken stomata often have valve-like projections of cuticle which reduce the opening also. Finally, in a few plants, water loss in times of drouth is almost completely prevented by closing the opening with a wax excretion.

5. Modifications in the chlorenchym. A decrease in the size and number of the air passages in the leaf renders the movement of water-laden air to the stomata more difficult, and effects a corresponding decrease in transpiration. The increase of palisade tissue, though primarily dependent upon light, reduces the air-spaces, and consequently the amount of water lost: 
The development of sclereids below the epidermis likewise hinders the escape of water. Finally, the character of the cell sap often plays an important part, since cells with high salt-content or those containing mucilaginous substances give up their water with reluctance.

164. The increase of water supply. Plants of dry habitats can increase their absorption only by modifying the root system so that the absorbing surfaces are carried into the deep-seated layers of soil, and the surfaces in contact with the dry soil are protected by means of a cortex. Exception must be made for epiphytes and a few other plants that absorb rain water and dew through their leaves, and for those desert plants that seem to condense the moisture of the air by means of hygroscopic salts, and absorb it through the epidermis of the leaf. The storage of water in the leaf is a very important device; it increases the water supply by storing the surplus of absorbed water against the time of need. Modifications for water storage are occasionally found in roots and stems, but their chief development takes place in the leaf. The epidermis frequently serves as a reservoir for water, either by the use of the epidermal cells themselves, by the formation of hypodermal water layers, or by means of superficial bulliform cells. The water cells of the chlorenchym regularly appear in the form of large clear cells, scattered singly or arranged in groups. In this event, they occur either as transverse bands, or as horizontal layers, lying between the palisade and sponge areas, and connecting the bundles. A few plants possess tracheid-like cells which also serve to store water. In the case of succulent leaves, practically the whole chlorenchym is used for storing water, though they owe their ability to withstand transpiration to a combination of factors.

165. Modifications due to an excessive water supply. Water plants with aerial leaf surfaces are modified in such manner as to increase water loss and to decrease water supply, but the resulting modifications are rarely striking. There is a marked tendency to increase the exposed surface'. This is indicated by the fact that, while the leaves of mud and floating forms become larger, they change little or not at all in thickness. The lobing of leaves is also greatly reduced, or the lobes come to overlap. Leaves of water plants are practically destitute of all modifications of epidermis and stomata, which could serve to hinder transpiration. The stomata are usually more numerous on the upper surface, and in the same species their number is greater in the forms grown in wet places. These facts explain in part the extreme development of air-passages in water plants, though this is, in large measure, a response to the increasing diffictlty of aeration. The 
increase of air-spaces is correlated with reduction of the palisade, and a decided increase in the sponge. An increase in water supply is indicated by the absence of storage tissues, and the reduction of the vascular system, which, however, is more closely connected with a diminished need for mechanical support.

166. Plant types. The necessity for decreasing or increasing water loss in compensation of the water supply has made it possible to distinguish two fundamental groups of plants upon the twofold basis of habitat and structure. These familiar groups, xerophytes and hydrophytes, represent two extremes of habitat and structure, between which lies a more or less vague, intermediate condition represented by mesophytes. These show no characteristic modifications, and it is consequently impossible to arrange them in subgroups. Xerophytes and hydrophytes, on the other hand, exhibit marked diversity among themselves, a fact that makes it desirable to

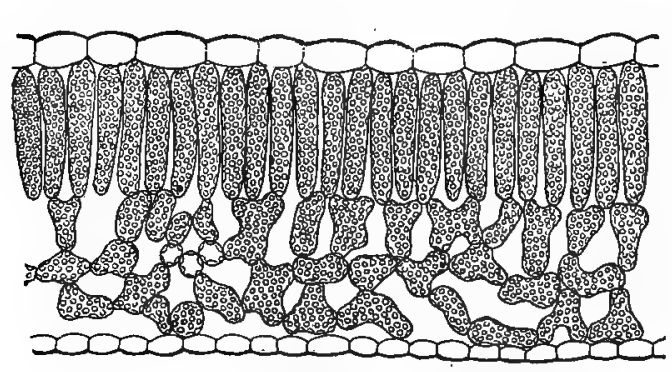

Fig. 32. Mesophyll of Pedicularis procera (chresard, 15\%, light, 1). $\times 130$. recognize subgroups, which correspond to fundamental differences of habitat or adaptation. It is hardly necessary to point out that these types are not sharply defined, or that a single plastic species may be so modified as to exhibit several of them. The extremes are always clearly defined, however, and they indicate the specific tendency of the adaptation shown by other members of the same group.

167. Xerophytic types. With the exception of dissophytes, all xerophytes agree in the possession of a deep-seated root system, adapted to withdraw water from the lower moist layers, and to conserve from loss from the upper dry layers. Reservoirs are developed in the root, however, in relatively few cases. The stem follows the leaf more or less closely in its modification, except when the leaf is greatly reduced or disappears, in which event the stem exhibits peculiar adaptations. While the leaf is by far the most strikingly modified, it is a difficult task to employ it satisfactorily as the basis. for distinguishing types. Several adaptations are often combined in the same leaf, and it is only where one of these is preeminently developed, as in the case of succulence, that the plant can be referred to a definite type. The latter does not happen in many species of the less 
intensely xerophytic habitats, and, consequently, it is difficult, if not undesirable, to place such xerophytes under a particular group. The best that can be done is to recognize the types arising from extreme or characteristic modification, and to connect the less marked forms as closely as possible with these. Halophytes differ from xerophytes only in the fact that the chresard is determined by the salt-content of the habitat, and not by the texture of the soil. In consequence, they should not be treated as a distinct group.

168. Types of leaf xerophytes. In these, adaptation has acted primarily upon the leaf, while the stem has remained normal for the most part. Even when the leaves have become scale-like, they persist throughout the growing season, and continue to play the primary part in photosynthesis. The following types may be distinguished:

I. The normal form. The leaf is of the usual dorsiventral character. In place of a reduction in size, structural modifications are $\mathrm{used}$ to decrease transpiration. With respect to the protective feature that is predominant, three subtypes may be recognized. The cutinized leaf compensates for a low water-content by means of a thick cuticle, often reinforced by a high development of palisade tissue. Such leaves are more or less

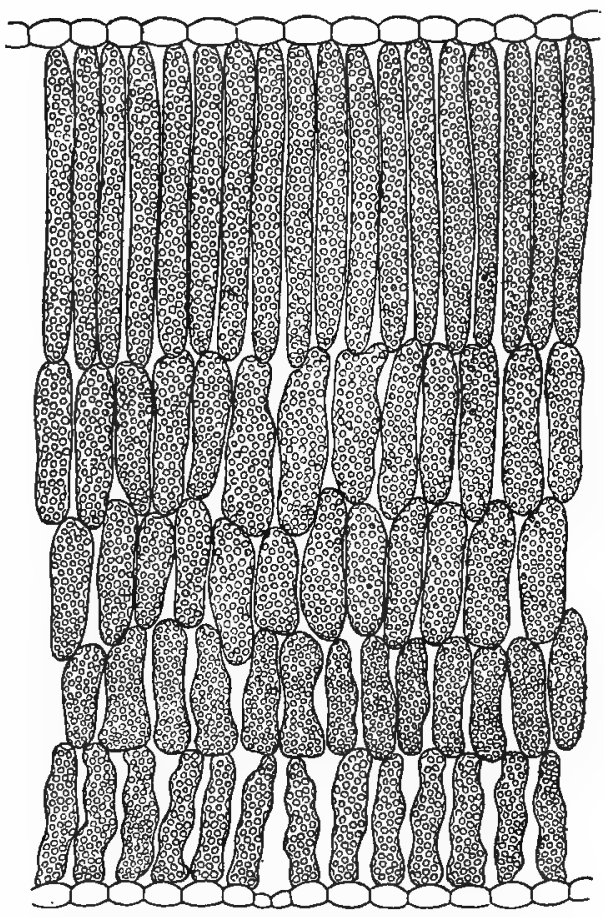

Fig. 33. Staurophyll of Bahia dissecta, showing extreme development of palisade (chresard, 3-9\%; light, 1). $\times 130$.

leathery, and they are often evergreen also. Arctostaphylus and many species of Pentstemon are good examples. Lanate leaves, i. e., those with dense hairy coverings on one or both surfaces, as Artemisia, Antennaria, etc., regularly lack both cuticle and palisade tissue. The protection against water loss, however, is so perfect that the chlorenchym often assumes the loose structure of a shade leaf. Storage leaves ustrally have a welldeveloped cuticle and several rows of palisade cells, but their characteristic feature is the water-storage tissue, which maintains a reserve supply of 
water for the time of extreme drouth. Xerophytic species of Helianthus furnish examples of transverse bundles of storage cells, while those of Mertensia illustrate the more frequent arrangement in which the water tissue forms horizontal layers.

2. The succulent form. Many succulent leaves are normal in shape and size, though always thicker than ordinary leaves. Ustally, however, they are reduced in size and are more or less cylindrical in form. The necessary decrease in transpiration is effected by the reduction in surface, the general storage of water, a waxy coating, and, often also, by a very thick cuticle. Agave, Mesembryanthemum, Sedum, and Senecio furnish excellent examples of this type.

3. The dissected form. The reduction in surface is brought about by the division of the leaf blade into narrow linear or thread-like lobes which

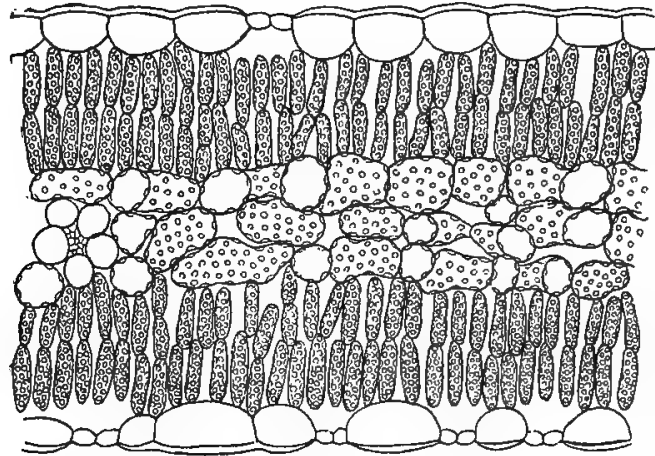

Fig. 34. Diplophyll of Mertensia linearis, showing water cells (chresard, 3-9\%, light, 1). $\times 130$. are widely separated. The latter are themselves protected by. a hairy covering or a thick cuticle, which is often supplemented by many rows of palisade, or by storage tissue. Artemisia, Senecio, and Gilia contain species which serve as good examples of this type.

4. The grass form. Xerophytic grasses and sedges have narrow filamentous 1 e a ves with longitudinal furrows which serve to protect the stomata. The furrows are sometimes filled with hairs which are an additional protection, and the leaves often protect themselves further by rolling up into a thread-like shape. The elongated subulate leaves of Juncus and certain Cyperaceae are essentially of this type, although they are usually not furrowed.

5. The needle form. This is the typical leaf of conifers, in which a sweeping reduction of the leaf surface is an absolute necessity. The relatively small water loss of the needle leaf is still further decreased by a thick cuticle, and usualiy also by hypodermal layers of sclerenchyma.

6. The roll form. Roll leaves are frequently small and linear. Their characteristic feature is produced by the rolling in of the margin on the under side, by which an almost completely closed chamber is formed for the protection of the stomata which are regularly confined to the lower surface of the leaf. The upper epidermis is heavily cutinized and the lower 
one often protected by hairs. This type is found especially among the genera of the Ericales, but it also occurs in a large number of related families.

7. The scale form. Reduction of leaf surface for preventing excessive water loss reaches its logical culmination in the scale leaf characteristic of many trees and shrubs, e. g., Cupressus, Tamarix, etc. Scale leaves are leathery in texture, short and broad, and closely appressed to the stem, as will as often overlapping.

169. Types of stem xerophytes. In these types the leaves are deciduous early in the growing period, reduced to functionless scales, or entirely absent. The functions of the leaf have been assumed by the stem, which exhibits many of the structural adaptations of the former. Warming ${ }^{1}$ has distinguished the following gromps:

I. The phyllode form. The petiole is broadened and takes the place of the leaf blade which is lacking. In other cases, the stem is flattened or winged, and it replaces the entire leaf. This type occurs in Acacia, Baccharis, Genista, etc.

2. The virgate form. The leaves either fall off early or they are reduced to functionless scales. The stems are thin, erect, and rod-like, and are often greatly branched. They are heavily cutinized and palisaded, and the stomata are frequently in longitudinal furrows. This type is characteristic of the Genisteae; it is also found in Ephedra, many species of Polygonum, Lygodesmia, etc.

3. The rush form. In Heleocharis, many species of Juncus, Scirpus, and other Cyperaceae, the stem, which is nearly or completely leafless, is cylindrical and unbranched. It usually possesses also a thick cuticle, and several rows of dense palisade tissue.

4. The cladophyll form. In Asparagus the leaves are reduced to mere functionless scales, and their function is assumed by the small needle-shaped branches.

5. The flattened form. As in the preceding type, the place of the scalelike leaves is taken by cladophylls, which are more or less flattened and leaflike. Ruscus is a familiar illustration of this form.

6. The thorn form. This is typical of many spiny desert shrubs, in which the leaves are lost very early, or, when present, are mere functionless scales. The stems have an extremely thick cuticle, and the stomata are deeply sunken, as a rule. Colletia and Holacantha are good examples of the type.

${ }^{1}$ Lehrbuch der Oekologischen Pflanzengeographie. 2d ed., 196. 1902. 
7. The succulent form. Plants with succulent stems such as the Cactaceae, Stapelia, and Euphorbia have not only decreased water loss by extreme reduction or loss of the leaves, and the reduction of stem surface, but they also offset transpiration by means of storage tissues containing a mucilaginous sap. The cuticle is usually highly developed and the stomata sunken. Thorns and spines are also more or less characteristic features.

170 Bog plants. Many of the xerophytic types just described are found in ponds, bogs, and swamps, where the water supply is excessive, and hydro-

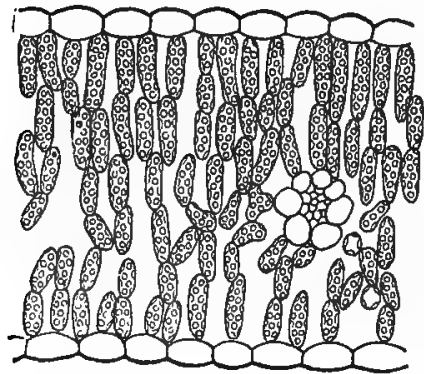

1

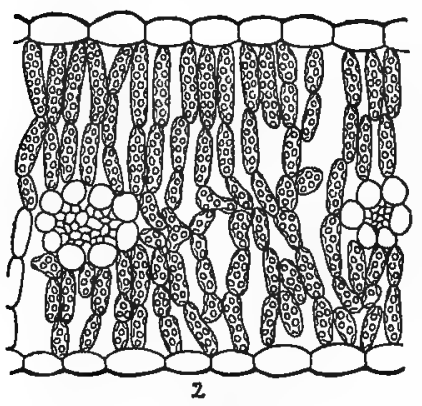

Fig. 35. Polygonum bistor toides, a stable type: 1 , mesophyll (chresard, 25\%);2, xerophyll (chresard, $3-5 \%$ ). $\times 130$. phytes would be expected. The explanation that "swamp xerophytes" are due to the presence of humic acids which inhibit absorption and aeration in the roots has been generally accepted. As Schimper has expresscd it, bogs and swamps are "physiologically dry", $i$. e., the available water is small in amount, in spite of the great total watercontent. Burgerstein (l. c., I42) has shown, however, that maize plants transpire, i. e., absorb, three times as much water in a solution of 0.5 per cent of oxalic acid as they do in distilled water, and that branches of Taxus in a solution containing I per cent of tartaric acid absorb more than twice as much as in distilled water. Consequently, it seems improbable that small quantities of humic acids should decrease absorption to the extent necessary for the production of xerophytes in ponds and bogs. Incleed, in many ponds and streams, where Heleocharis, Scirpus, Juncus, etc., grow, not a trace of acid is discoverable. Furthermore, plants with a characteristic hydrophytic structure throughout, such as Ramunculus, Caltha, Ludwigia, Sagittaria; etc., are regularly found growing alongside of apparent xerophytes. Many of the latter, furthermore, show a striking contrast in size and vigor of growth in places where they grow both upon dry gravel banks and in the water, indicating that the available water-content is much greater in the latter. Finally, many so-called "swamp xerophytes" possess typically hydrophytic structures, such as air-passages, diaphragms, etc. In spite of a growing feeling that the xerophytic features of certain amphibious plants can not be ascribed to a low chresard in ponds and swamps, a satisfactory explana- 
tion of them has been found but recently. This explanation has come from the work of E. S. Clements already cited, in which it was found that certain sun plants underwent no material structural change when grown in the shade, and that the same was true also of a few species which grew in two or more habitats of very different water-content. In accordance with this, it is felt that the xerophytic features found in amphibious plants are due to the persistence of stable structures, which were developed when these species were growing in xerophytic situations. When it is called to mind that monocotyledons, and especially the grasses, sedges, and rushes, are peculiarly stable, it may be readily understood how certain ancestral characters have persisted in spite of a striking change of habitat. Such a hypothesis can only be confirmed by the methods of experimental evolution, and a critical study of this sort is now under way.

171. Hydrophytic types. Hydrophytes permit a fairly sharp division into three groups, based primarily upon the relation of the leaf surface to the two media, air and water. In submerged plants, the leaves are con-
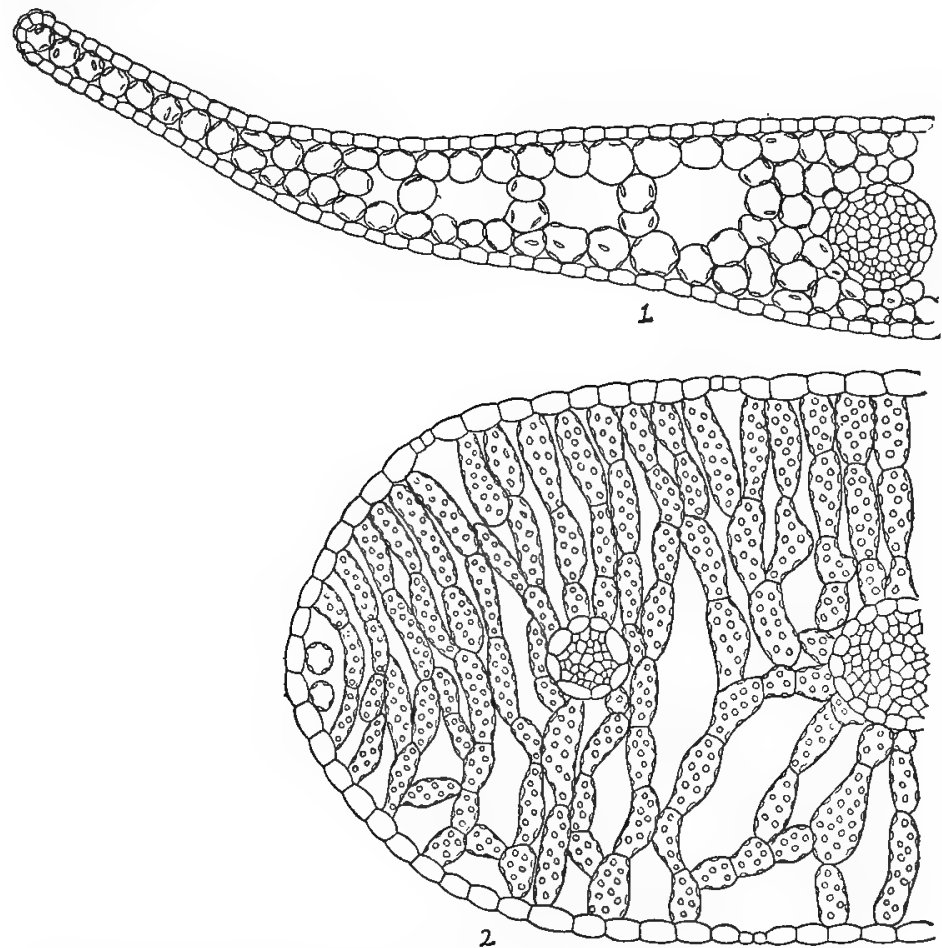

Fig. 36. Hippuris vulgaris: 1 , submerged leaf; 2 , aerial leaf. $\times 130$. 
stantly below the water; in amphibious ones, they grow normally in the air. Floating plants have leaves in which the upper surface is in contact with the air, and the lower in contact with the water. Transpiration is at a maximum in the amphibious plant; it is reduced by half in the floating type, and is altogether absent in submerged plants. Aeration reaches a high development in amphibious and floating forms, but air-passages are normally absent from submerged forms except as vestiges. Photosynthesis is marked in the former, but considerably weakened in the latter. The vascular system, which attains a moderate development in the amphibious type, is considerably reduced in floating forms, and it is little more than vestigiate in submerged ones.

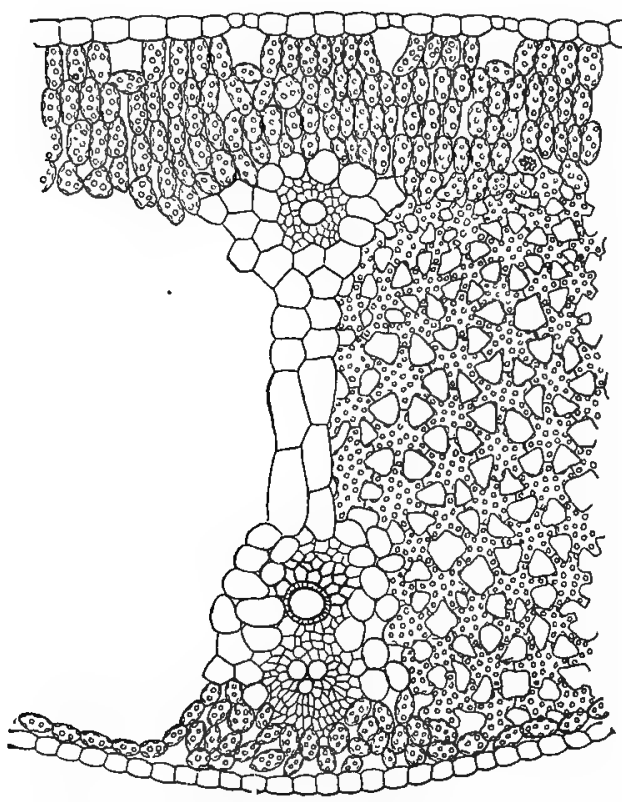

Fig. 37. Floating leaf of Sparganium angustifolium. $\times 130$.

on the upper than on the lower surface.

\section{The amphibious type.} Plants of this type grow in wet soil or in shallow water. The leaves are usually large and entire, the stem well developed, and the roots numerous and spreading. In the majority of cases the leaves are constantly above the water, but in some species the lower leaves are often covered, normally, or by a rise in level, and they take the form or structure of submerged leaves. This is illustrated by Callitriche autumnalis, Hippuris vulgaris, Ranunculus delphinifolius, Proserpinaca palustris, Roripa americana, etc. The epidermis has a thin cuticle, or none at all, and is destitute of hairs. The stomata are numerous and usually more abundant The palisade tissue is represented by one or more well-developed rows, but this portion of the leaf is regularly thinner than that of the sponge part. The latter contains, large air-passages, or, in the majority of cases, numerous air-chambers, usually provided with diaphragms. The stems are often palisaded, and are characterized by longitudinal air-chambers crossed by frequent diaphragms, which extend downward through the roots.

2. The floating typc. With respect to form and the structure of the upper part of the leaf, floating leaves are essentially similar to those of amphibious plants. They are usually lacquered or coated with wax to prevent the 
stoppage of the stomata by water. Stomata, except as vestiges, are found only on the upper surface, and the palisade tissue is much less developed than the sponge, which is uniformly characterized by large air-chambers. The stems are elongated, the aerating system is enormously developed, and the supportive tissues are reduced. In the Lemnaceae, the leaf and the stem are represented by a mere frond or thallus, and the roots are in the process of disappearance, e. g., Spirodela has several, Lemna one, and Woiffia none.

3. The submerged type. Both stem and root have been greatly reduced in submerged plants, owing to the generalization of absorption and the density of the water. The leaves are greatly reduced in size and thickness, chiefly, it would seem, for the purpose of insuring readier aeration and great illumination. The leaf may be ribbon-like, linear, cylindrical, or finely dissected. Stomata are sometimes present, but they are functionless and vestigial. A distinction into palisade and sponge tissues, when present, must also be regarded as a vestige; the chlorenchym is essentially that of a shade leaf. The air chambcrs are much reduced, and sometimes lacking; they function doubtless as reservoirs for air obtained from the water.

\section{PHOTOHARMOSE}

\section{AOJUSTMENT}

172. Light as a stimulus. In nature, light stimuli are determined by intensity and not by quality. A single exception is afforded by those aquatic habitats where the depth of water is great, and in consequence of which certain rays disappear by absorption more quickly than others. In forests and thickets, where the leaves transmit only the green and yellow rays, it would appear that the light which reaches the herbaceous layers is deficient in red and violet rays. The amount of light transmitted by an ordinary sun leat is so small, however, that it has no appreciable effect upon the quality of the light beneath the facies, which is diffuse white light that has passed between the leaves. Indeed, it is only in the densest forests that distinct sunflecks do not appear. Coniferous forests, with a light value less than .005, which suffices only for mosses, lichens, and a few flowering plants, show frequent sunflecks. This is convincing evidence that the light of such habitats is normal in quality. It warrants the conclusion that in all habitats with an intensity capable of supporting vascular plants the light, no matter how diffuse, is white light. The direction of the light ray is of slight importance in the field, apart from the difference in intensity which may result from it. In habitats with diffuse light, the latter comes normally and constantly from above. Likewise, in sunny situations, direction can 
have little influence, since both the direction and the angle of the incident rays change continually throughout the day, and the position of the leaf itself is more or less constantly changed by the wind. The influence of duration upon the character of light stimuli is difficult to determine. There can be no question that the time during which a stimulus acts has a profound bearing upon the response that is made to it. In nature the problem is complicated by the fact that light stimuli are both continuous and periodic. The duration of sunlight is determined by the periodic return of night as well as by the irregular occurrence of clouds. Since one is a regular, and the other at least a normal happening, it is necessary to consider duration only with respect to the time of actual sunlight on sunny days, except in the case of formations belonging to regions widely different in the amount of normal sunshine, i. e., the number of cloudy days. In consequence, duration is really a question of the intensities which succeed each other during the day. The differences between these have already been shown to fall within the efficient difference for light, and for this reason the ratio between the light intensity of a meadow and of a forest is essentially the ratio between the sums of light intensity for the two habitats, i. e., the duration. The latter is of importance only where there is a daily alternation between sunshire and shadow, as at the edge of forest and thicket, in open woodland, etc. In such places duration determines the actual stimulus by virtue of the sum of preponderant intensities. The periodicity of daylight is a stimulus to the guard cells of stomata, but its relation to intensity in this connection is not clear.

The amount of change in light intensity necessary to constitute an efficient stimulus seems to depend upon the existing intensity as well as upon the plant concerned. Apparently, a certain relative dectease is more efficient for sun plants than for shade plants. At least, many species sooner or later reach a point where a difference larger than that which has been efficient no longer produces a structural response. This has been observed by E. S. Clements $(l$. c.) in a number of shade ecads. For example, a form of Galinm boreale, which grew with difficulty in a light value of .002, showed essentially the leaf structure of the form growing in light of .03, while the form in full sunlight showed a striking difference in the leaf structure. In considering the light stimuli of habitats, it is unnecessary to discuss the stimulus of total darkness upon chlorophyllous plants, although this is of great importance in experimental evolution and in control experiment. The normal extremes of light intensity, i. e., those within which chlorenchym can function, are full sunshine represented by $\mathrm{I}$, and a diffuseness of .002 , though small flowering plants have once or twice been found in an intensity of .oDI. The maximum light value, even on high mountains, never exceeds I by more than an inconsiderable amount, except for the 
temporary concentration due to drops of dew, rain, etc. It seems improbable that the concentrating effect of epidermal papillae can do much more than compensate for the reflection and absorption of the epidermis. Experimental study has shown that the maximum intensity in nature may be increased several, if not many times, without injurious results and without an appreciable increase in the photosynthetic response, thus indicating that the efficient difference increases toward the maximum as well as toward the minimum.

173. The reception of light stimuli. Rays of light are received by the epidermis, by which they are more or less modified. Part of the light is reflected by the outer wall or by the cuticle, particularly when these present a shining surface. Hairs diffract the light rays, and hairy coverings consequently have a profound influence in determining stimuli. The walls and contents of epidermal cells furthermore absorb some of the light, especially when the cell sap is colored. In consequence of these effects, the amount of light that reaches the chlorenchym is always less than that incident upon the leaf, and in many plants, the difference is very great. According to Haberlandt ${ }^{1}$, the epidermal cells of some shade plants show modifications designed to concentrate the light rays. Of such devices, he cistinguishes two types: one in which the outer epidermal wall is arched, another in which the inner wall is deeply concave. Although there can be no question of the effect of lens-shaped epidermal cells, their occurrence does not altogether support Haberlandt's view. Arched and papillate epidermal cells are found in sun plants where they are unnecessary for increasing illumination, to say the least. A large number of shade plants show cells of this character, but in many the outer wall is practically a plane. Shade forms of a species ustually have the outer wall more arched or papillate, but this is not always true, and, in a few cases, it is the lower epidermis alone that shows this feature. Finally, a localization of this function in certain two-celled papillae, such as Haberlandt indicates for Fittonia werschaffelti, does not appear to be plausible.

The epidermis merely receives the light; the perception of the stimulus normally occurs in those cells that contain chloroplasts. The cytoplasm of the epidermal cells, as well as that of the chlorenchym cells, is sensitive to light, but the response produced by the latter is hardly discernible in the absence of plastids, except in those plants which possess streaming protoplasm. The daily opening and closing of the stomata, which is due to light, is evidently connected with the presence of chloroplasts in the guard cells. Naturally, the perception of light and the corresponding response occur in the epidermis of many shade and submerged plants which have

${ }^{1}$ Physiologische Pflanzenanatomie. 3d ed., 537. 1904. 
chloroplasts in the epidermal cells. Such cases merely serve to confirm the view that the perception of light stimuli is localized in the chloroplast. In conformity with this view, the initial response to such stimuli must be sought in the chloroplast, and the explanation of all adaptations due to light must be found in the adjustment shown by the chloroplasts.

174. Response of the chloroplast. The fundamental response of a plastid to light is the manufacture of chlcrophyll. In the presence of carbon lioxide and water, leucoplasts invariably make chlorophyll, and chloroplasts replace that lost by decomposition, in response to the stimulus exerted by light. The latter is normally the efficient factor, since water is always present in the living plant, and carbon dioxide absent only locally at most. Sun plants which possess a distinct cuticle, however, produce leucoplasts, not chloroplasts, in the epidermal cells, although these are as strongly iiluminated as the guard cells, which contain numerous chloroplasts. This is evidently explained by the lack of carbon dioxide in the epidermis. This gas is practically unable to penetrate the compact cuticle, at least in the small quantity present in the air. The supply obtained through the stomata is first levied upon by the guard cells and then by the cells of the chlorenchym, with the result that the carbon dioxide is all used before it can reach the epidermal cells. This view is also supported by the presence of chloroplasts along the sides and lower wall of palisade cells, where there is normally a narrow air-passage, and their absence along the upper wall when this is closely pressed against the epidermis, as is usually the case. Furthermore, the leaves of some mesophytes when grown in the sun develop a cuticle and contain leucoplasts. Under glass and in the humid air of the greenhouse, the same plants develop epidermal chloroplasts but no cuticle. This is in entire harmony with the well-known fact that shade plants and submerged plants often possess chloroplasts in the epidermis. Although growing. in different media, their leaves agree in the absence of a cuticle, and consequent absorption of gases through the epidermis. The size, shape, number, and position of the chloroplasts are largely determined by light, though a number of factors enter in. No accurate studies of changes in size and shape have yet been made, though casual measurements have indicated that the chloroplasts in the shade form of certain species are nearly hemispherical, while those of the sun form are plane. In the same plants, the number of chloroplasts is strikingly smaller in the shade form, but exact comparisons are yet to be made. The position and movement of chloroplasts have been the subject of repeated study, but the factors which control them are still to be conclusively indicated. Light is clearly the principal cause, although there are many cases where a marked change in the light intensity fails to call forth any readjustment of the 
plastids: The position of air-spaces as reservoirs of carbon dioxide and the movement of crude and elaborated materials from cell to cell frequently have much to do with this problem. Finally, it must be constantly kept in mind that the chloroplasts lie in the cytoplasm, which is in constant contact with a cell wall. Hence, any force that affects the shape of the cell will have a corresponding influence upon the position of the chloroplasts. When it is considered that in many leaves these four factors play some part in determining the arrangement of the plastids, it is not difficult to understand that anomalies frequently appear.

It may be laid down as a general principle that chloroplasts tend to place themselves at right angles to rays of diffuse light and parallel to rays of sunlight. This statement is borne out by an examination of the leaves of typical sun and shade species, or of sun and shade forms of the same species. Cells which receive diffuse light, i. e., sponge cells, normally have their rows of plastids parallel with the leaf surface, while those in full sunlight place the rows at right angles to the surface. This disposition at once suggests the generally accepted view that chloroplasts in diffuse light are placed in such a way as to receive all the light possible, while those in sunlight are so arranged as to be protected from the intense illumination. Many facts support this statement with respect to shade leaves, but the need of protection in the sun leaf is not clearly indicated. The regular occurrence of normal chloroplasts in the guard cells seems conclusive proof that full sunlight is not injurious to them. Although the upper wall of the outer row of palisade cells is usually free from chloroplasts, yet it is not at all uncommon to find it covered by them. These two conditions are often found in cells side by side, indicating that the difference is due to the presence of carbon dioxide and not to light. In certain species of monocotyledons; the arrangement of the chloroplasts is the same in both halves of the leaf, and there is no difference between the sun and shade leaves of the same species. The experimental results obtained with concentrated sunlight, though otherwise conflicting, seem to show conclusively that full sunlight does not injure the chloroplasts of sun plants, and that the position of plastids in palisade cells is not for the purpose of protection. This arrangement, which is known as apostrophe, is furthermore often found in shade forms of heliophytes. In typical shade species, and in submerged plants, the disposition of plastids on the wall parallel with the leaf surface, viz., epistrophe, is more regular, but even here there are numerous exceptions to the rule.

The absorption of the light stimulus by the green plastid results, under normal conditions, in the immediate production of carbohydrates, which in the vast majority of cases soon become visible as grains of starch. The appearance of starch in the chloroplasts of flowering "plants is such a 
regular response to the action of light that it is regarded as the normal indication of photosynthetic activity. The mere presence of chlorophyll is not an indication of the latter, since chlorophyll sometimes persists in light too diffuse for photosynthesis. The amount of starch formed is directly connected with the light intensity, and in consequence it affords a basis for the quantitative estimation of the response to light. Two responses to

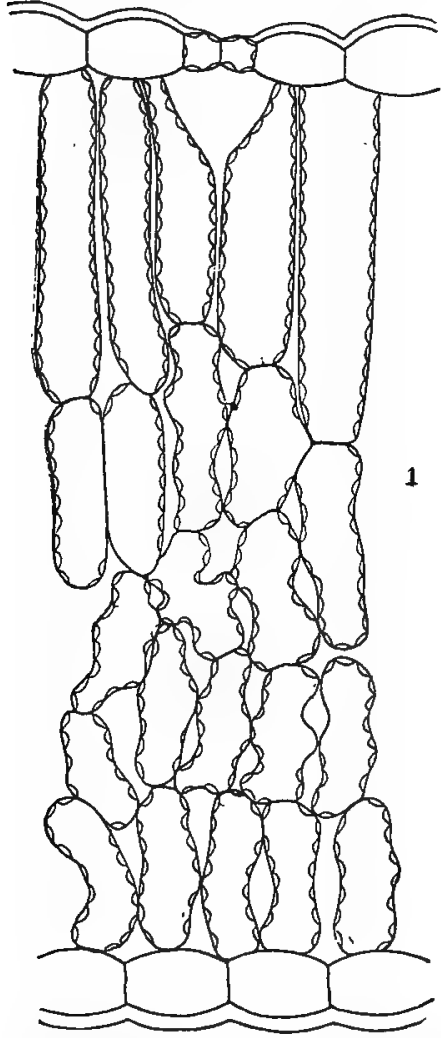

Fig. 38. Ecads of Allionia linearis, showing position of chloroplasts. The palisade shows apostrophe, the sponge epistrophe: 1 , sun leaf (chresard, $2-5 \%$, light, 1 ); 2, shade leaf (chresard, 11\%; light, .012); 3, shade leaf (chresard, 11\%; light, .003). $\times 250$. light stimuli have a direct effect upon the amount of transpiration. Of the light energy absorbed by the chloroplast, only 2.5 per cent is used in photosynthesis, while 95-98 per cent is converted into heat, and brings about marked increase in transpiration. Furthermore, in normal turgid plants, the direct action of light, as is well known, opens the stomata in the morning and closes them at night.

175. Aeration and translocation. The movements of gases and of solutions through the tissues of the leaf are intimately con n e c t ed with photosynthesis, and hence with responses to light stimuli. Aeration depends primarily upon the periodic opening of the stomata, for, while the carbon dioxide and oxygen of the air are able to pass through epidermal walls not highly cutinized, the amount obtainable in this manner is altogether inadequate, if not negligible. 'The development of sponge tissue or aerenchym is intimately connected with the stomata. The position and amount of aerenchym and the relative extent of sponge cells and air-spaces are in part determined by the number and position of the 
breathing pores. The disposition of air spaces has much to do with the arrangement of chloroplasts in both palisade and sponge tissues. Starch formation is also dependent upon the presence of air spaces, but, contrary to what would be expected, it seems to be independent of their size, since sun leaves, which assimilate much more actively than shade leaves, have the smallest air spaces. From this fact, it appears that the rapidity of aeration depends very largely upon the rapidity with which the gases are used. Translocation likewise affects the arrangement of the chloroplasts and the formation of starch. According to Haberlandt, it also plays the principal part in determining. the form and arrangement of the palisade cells. Chloroplasts are regularly absent at those points of contact where the transfer of materials is made from cell to cell, though this is not invariably true. Since air passages are necessarily absent where cell walls touch, it is possible that this disposition of the plastids is likewise due to the lack of aeration. Translocation is directly connected with the appearance of starch. As long as all the sugar made by the chloroplasts is transferred, no starch appears, but when assimilation begins to exceed translocation, the increasing concentration of the sugar solution results in the production of starch grains. The latter is normally the case in all flowering plants, with the exception of those that form sugar or oil, but no starch. The constant action of translocation is practically indispensable to starch formation, since an over-accumulation of carbohydrates decreases assimilation, and finally inhibits it altogether. In consequence,

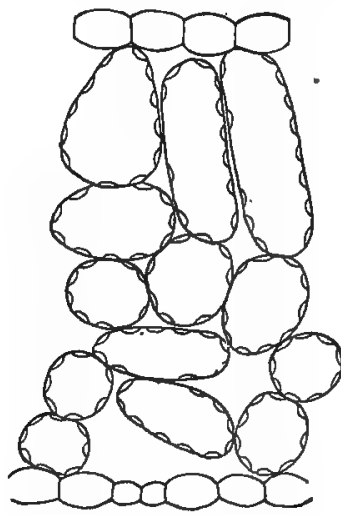

1

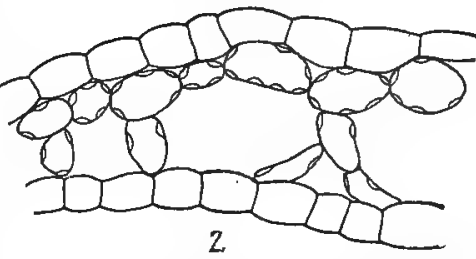

Fig. 39. Position of chloroplasts in aerial leaf (1) and submerged leaf (2) of Callitriche bifida. $\times 250$. translocation occurs throughout the day and night, and by this means the accumulated carbohydrates of one day are largely or entirely removed before the next.

176. The measurement of responses to light. Responses, such as the periodic opening and closing of stomata, which are practically the same for all leaves, are naturally not susceptible of measurement. This is also true of the transpiration produced by light, but the difficulty in this case is due 
to the impossibility of distinguishing between the water loss due to light and that caused by humirlity and other factors. If it were possible to determine the amount of chlorophyll or glucose produced, these could be used as satisfactory measures of response. As it is, they can only be determined approximately by counting the chloroplasts or starch grains. The arrangement of the chloroplasts can not furnish the measure sought, since it does not lend itself to quantitative methods, and since the relation to light intensity is too inconstant. Hesselmann (l. c., 400) has determined the amount of carbon dioxide respired, by means of a eudiometer, and has based comparisons of sun and shade plants upon the results. As he points out, however, light has no direct connection with respiration. Although the latter increases necessarily with increased nutrition, the relation between them is so obscure, and so far from exact, that the amount of respiration can in no wise serve as a measure of the response to light. As a result of the foregoing, it is clear that no functional response is able to furnish a satisfactory measure of adjustment to light, though one or two have perhaps sufficient value to warrant their tise. Indeed, structural adaptations offer a much better basis for the quantitative determination of the effects of light stimuli, as will be shown later.

In attempting to use the number of chloroplasts or starch grains as a measure of response, the study should be confined to the sun and shade forms of the same species, or, in some cases, to the forms of closely related. species. The margin of error is so great and the connection with light sufficiently remote that comparisons between unrelated forms or species. are almost wholly without value. It has already been stated that starch is. merely the surplus carbohydrate not removed by translocation; the amount of starch, even if accurately determined, can furnish no real clue to the amount of glucose manufactured. In like manner, the number of chloroplasts can furnish little more than an approximation of the amount of chlorophyll, unless size and color are taken into account. In sun and shade ecads of the same species, the general functional relations are essentially. the same, and whatever differences appear may properly be ascribed to different light intensities for the two habitats. The actual counting of chloroplasts and starch grains is a simple task. Pieces of the leaves of the two or more forms to be compared are killed and imbedded in paraffin in the usual way. To save time, the staining is done in toto. Methyl green is used for the chloroplasts and a strong solution of iodine for the starch grains. When counts are to be made of both, the leaves are first treated with iodin and then stained with the methyl green. The thickness of the microtome sections should be less than that of the palisade cells in order that the chloroplasts may appear in profile; thus facilitating the counting. The count is made for a segment roo $\mu$ in width across the entire leaf. 
Two segments in different parts of the section are counted, and the result multiplied by five to give the number for a segment I millimeter in width. Although sun and shade leaves regularly differ in size and thickness, no correction is necessary for these. Size and thickness stand in reciprocal relation to each other in ecads, and thickness is largely an expression of the absorption of light, and hence of its intensity. In the gravel, forest, and thicket ecads of Galium boreale, cotnts of the chloroplasts gave the following results. The gravel form (light I) showed 3,500 plastids in the I-mm. segment, the forest form (light .03) possessed I,350, and the thicket form (light .002), I,000. In these no attention was paid to the size and form of the plastids in the different leaves, since the differences were inappreciable. When this is not the case, both factors should be taken into account. Starch grains are counted in exactly the same way. Indeed, if care is taken to collect leaves of forms to be compared, at approximately the same time on sunshiny days, a count of the chloroplasts is equivalent to a count of the starch grains in the vast majority of cases. Measurements of the size of starch grains can be made with accuracy only when the leaves are killed in the field at the same time, preferably in the afternoon. Counts of chloroplasts alone can be used as measures of response in plants that produce sugar or oil, while either chloroplasts or starch grains or both may be made the basis in starch-forming leaves.

Hesselmann (l. c., 379) has employed Sachs's iodine test as a measure of photosynthesis. This has the advantage of permitting macroscopic examination, but the comparison of the stained leaves can give only a very general idea of the relative photosynthetic activity of two or more ecads. The iodine test is made as follows: ${ }^{-}$fresh leaves are placed for a few minutes in boiling water, and then in 95 per cent alcohol for $2-5$ minutes, in order to remove the chlorophyll and other soluble substances. The leaves are placed in the iodine solution for $1 / 2-3$ hours, or until no further change in color takes place. The strength of the solution is not clearly indicated by Sachs, who says: "I used an alcoholic solution of iodin which is best made by dissolving a large quantity of iodin in strong alcohol and adding to this sufficient distilled water to give the liquid the color of dark beer." This solution may be approximated by dissolving I/3 gram of iodin in Ioo grams of 30 per cent alcohol. The stained leaves are put in a white porcelain dish filled with distilled water, and the dish placed in the strong diffuse light of a window. The colored leaf stands out sharply against the porcelain, and the degree of coloration, and hence of starch content, is determined by the following table:

${ }^{1}$ SACHs, J. Ein Beitrag zur Kenntniss der Ernährungsthätigkeit der Blätter. Gesammelte Abhandlungen über Pflanzenphysiologie. 1:355. 1892. 
I. bright yellow or leather yellow (no starch in the chlorenchym)

2. blackish (very little starch in the chlorenchym)

3. dull black (starch abundant)

4. coal black (starch very abundant)

5. black, with metallic Juster (maximum starch-content)

ADAPTATION

177. Influence of chloroplasts upon form and structure. The begin ning of all modifications produced by light stimuli must be sought in the chloroplast as the sensitized unit of the protoplasm. Hence, it seems a truism to say that the number and arrangement of the chloroplasts determine the form of the cell, the tissue, and the leaf, although it has not yet been possible to demonstrate this connection conclusively by means of experiment. In spite of the lack of experimental proof, this principle is by far the best guide through the subject of adaptations to light, and in the discussion that follows, it is the fundamental hypothesis upon which all others rest. The three propositions upon which this main hypothesis is grounded are: (I) that the number of chloroplasts increases with the intensity of the light; (2) that in shaded habitats chloroplasts arrange themselves so as to increase the surface for receiving light; (3) that chloroplasts in sunny habitats place themselves in such fashion as to decrease the surface, and consequently the transpiration due to light. In these, there can be little doubt concerning the facts of number and arrangement, since they have been repeatedly verified. The purpose of epistrophe and apostrophe, however, can not yet be stated with complete certainty.

The stimulus of sunlight and of diffuse light is the same in one respect, namely, the chloroplasts respond by arranging themselves in rows or lines on the cell wall. The direct consequence of this is to polarize the cell, and its form changes from globoid to oblong. This effect is felt more or less equally by both palisade and sponge cells, but the disturbing influence of acration has caused the polarity of the cells to be much less conspicuous in the sponge than in the palisade tissue. While the cells of both are typically polarized, however, they assume very different positions with reference to incident light. This position is directly dependent upon the arrangement of the plastids as determined by the light intensity. In consequence, palisade cells stand at right angles to the surface and parallel with the impinging rays; the sponge cells, conversely, are parallel with the epidermis and at right angles to the light ray. Some plants, especially monocotyledons, exhibit little or no polarity in the chlorenchym. As a result the leaf does not show a differentiation into spcnge and palisade, and the leaves of sun and shade ecads are essentially alike in form and structure. The form of 
the leaf is largely determined by the chloroplasts acting through the cells that contain them. A preponderance of sponge tissue produces an extension of leaf in the direction determined by the arrangement of the plastids and the shape of the sponge cells, viz., at right angles to the light. Shade leaves are in consequence broader and thinner, and sometimes larger, than sun leaves of the same species. A preponderance of palisade likewise results in the extension of the leaf in the line of the plastids and the palisade cells, i. e., in a direction parallel with the incident ray. In accordance, sun leaves are thicker, narrower, and often smaller than shade leaves.

178. Form of leaves and stems. In outline, shade leaves are more nearly entire than sun leaves. This statement is readily verified by the comparison of sun and shade ecads, though the rule is by no means without exceptions. In the leaf prints shown in figures $I_{4}$ and 15 , the modification of form is well shown in Bursa and Thalictrum; in Capnoides the change is less evident, while in Achilleia and Machaeranthera lobing is more pronounced in the shade form, a fact which is, however, readily explained when other factors are taken into account. The leaf prints cited serve as more satisfactory examples of the increase of size in consequence of an increase in the surface of the shade leaf, although the leaves printed were selected solely with reference to thickness and size or outline. In all comparisons of this kind, however, the relative size and vigor of the two plants must be taken into account. This precaution is likewise necessary in the case of thickness, which should always be considered in connection with amount of surface. The relation between surface and thickness is shown by the following species, in all of which the size of the leaf is greater in the shade than in the sun. In Capnoides aureum, the thickness of the shade leaf is $1 / 2(6: 12)$ that of the sun leaf; in Galium boreale the ratio is $5: 12$, and in Allionia linearis it is $3: 12$. The ratio in Thalictrum sparsifiorum is $9: 12$, and in Machaeranthera aspera II:I2. The thickness of sun and shade leaves of Bursa bursa-pastoris is as $14: 12$, but this anomaly is readily explained by the size of the plants; the shade form is ten times larger than the sun form. Certain species, e. g., Erigeron speciosus, Potentilla bipinnatifida, etc., show no change in thickness and but little modification in size or outline. They furnish additional evidence of a fundamental principle in adaptation, namely that the amount of structural response is profoundly affected by the stability of the ancestral type.

The effect of diffuse light in causing stems to elongate, though known for a long time, is still unexplained. The old explanation that the plant stretches up to obtain more light seems to be based upon nothing more than the coincidence that the light comes from the direction toward which the stem grows. Later researches have shown that the stretching of the stem is due 
to the excessive elongation of the parenchyma cells, but the cause of the latter is far from apparent. It is generally assumed to be due to a lack of the tonic action of sunlight, which brings about a retardation of growth in
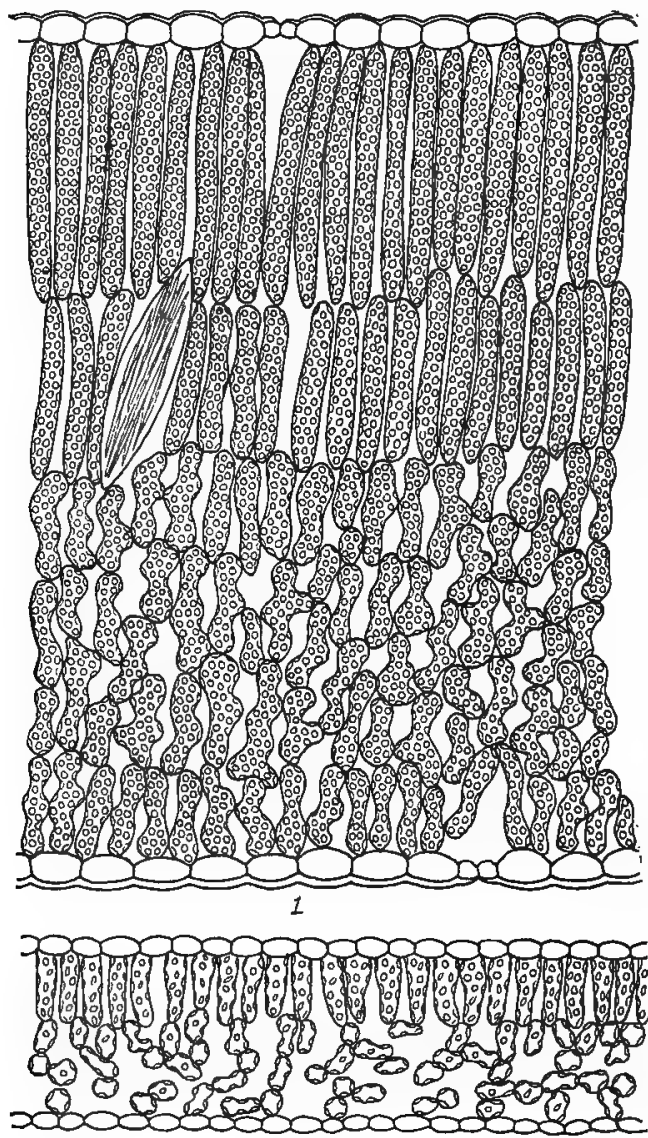

2.

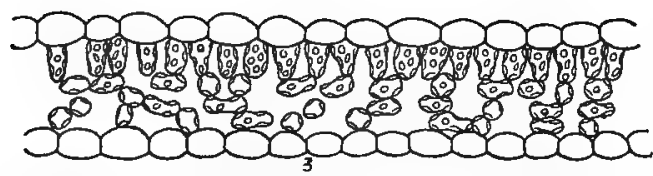

Fig. 40. Isophotophyll of Allionia linearis, showing diphotic ecads: 1 , light $1 ; 2$, light .012; 3, light.003. $\times 130$.

slight development of hairs in sciophilous plants is an advantage, but it must be referred to the factors that determine water loss. The significance favor of this view is not conclusive, and it seems probable at least that the elongation of the parenchyma cells takes place under conditions which favor the mechanical stretching of the cell wall, but inhibit the proper growth of the wall by intussusception. It is hardly necessary to state that the reduced photosynthetic activity of shade plants favors such an explanation. Whatever the cause, the advantage that results from the elongation of the internodes is apparent. Leaves interfere less with the illumination of those below them, and the leaves of the branches are carried away from the stem in such a way as to give the plant the best possible exposure for its aggregate leaf surface.

179. Modification of the epidermis. The development of epidermal chloroplasts in diffuse light is the only change which is due to the direct effect of light. This does not often occur in the shade ecads of sun species, but chloroplasts are regularly present in the epidermis of woodland ferns and of submerged plants. The sun plants. The evidence in 
of epidermal papillae in increasing the absorption of light by shade plants has already been discussed. The questions as to what factor has called forth these papillae and what purpose they serve must still be regarded as unsettled. The increased size of the epidernal cells, which is a fairly constant feature of shade ecads, seems to be for the purpose of increasing translocation and transpiration, and to bear no relation to light. The extreme development of the cells of the epidermis in 'Streptopus and Limnorchis, which grow at the edge of mountain brooks, has been plausibly explained by E. S. Clements as a contrivance to increase water loss. The presence of a waxy coating, such as that found upon the leaves of Impatiens aurea and $I$. pallida, is clearly to prevent the wetting of the leaf and the consequent stoppage of the stomata. In regard to the latter, different observers have noted that the number of the stomata is greater in sun than in shade leaves. This holds generally for sun and shade species, but it is most clearly indicated by different ecads of the same species. In Scutellaria brittonii, the sun form possesses IOO stomata per square millimeter, but in the shade these are reduced to 40 per square millimeter; the sun leaf of $\mathrm{Al}$ lionia linearis has $\mathrm{I} 80$ stomata to the square millimeter, the shade leaf 9o: In the stable leaf of Erigeron speciosus,

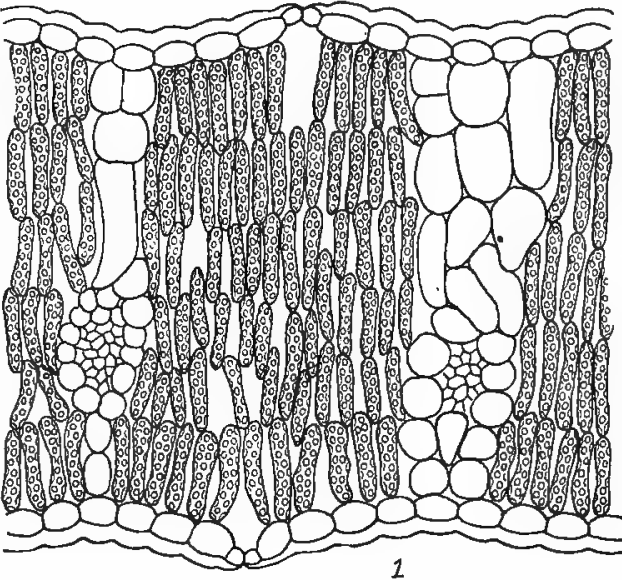

1

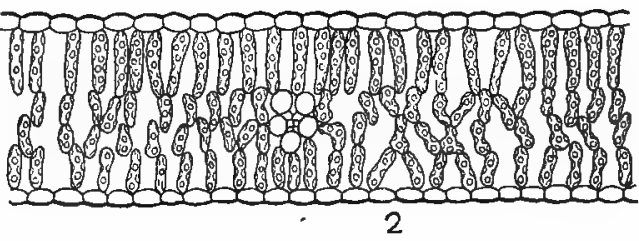

Fig. 41. Isophotophyll of Helianthus pumilus, showing isophotic ecad: 1 , sun leaf; 2 , shade leaf (light .012). $\times 130$.

however, the number of stomata is the same, 180 per square millimeter, for sunlight and for diffuse light. The presence of the larger number of stomata in the plant exposed to greater loss, which at first thought seems startling, is readily explained by the more intense photosynthetic activity in the sun. Since the absorption of gases is the primary function of the stomata, and transpiration merely secondary, it is evident that sun plants must have more stomata than shade plants. This is further explained by the fact that the small air passages of sun leaves necessitate frequent inlets, which are less necessary in shade leaves with their larger air spaces. In 
shade plants, moreover, the decrease in the number is compensated in some measure by the ability of the epidermal cells to absorb gases directly from the air.

180. The differentiation of the chlorenchym. The division of the chlorenchym into two tissues, sponge and palisade, is the normal consequence of the tunequal illumination of the leaf surfaces. Exceptions to this rule occur only in certain monocotyledons, in which the leaf tissue consists of sponge-like cells throughout, and in those stable species that retain more or less palisade in spite of their change to diffuse light. The difference in the illumination of the two surfaces is determined by the position of the leaf. Leaves that are erect or nearly so usually have both sides about equally illuminated, and they may be termed isophotic. Leaves that stand more

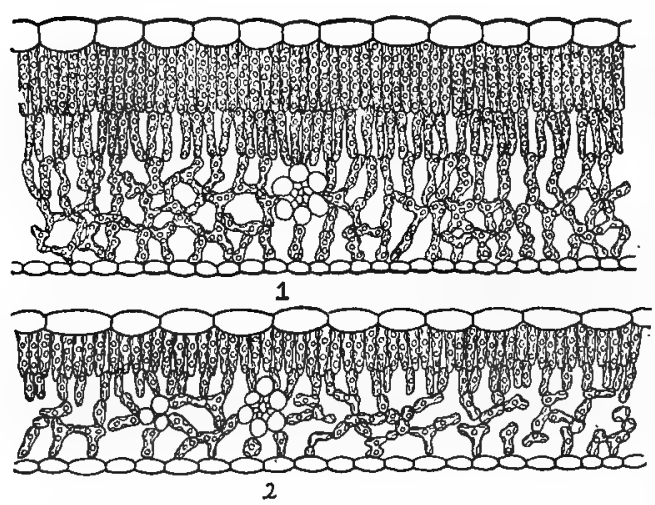

Fig. 42. Diphotophylls of Quercus novimexicana: 1 , sun leaf; 2 , shade leaf of the same tree (light .06). $\times 130$. or less at right angles to the stem receive much more light upon the upper surface than upon the lower, and may accordingly be termed diphotic. Certain dorsiventral leaves, however, absorb practically as much light on the lower side as upon the upper. This is true of sun leaves with a dense hairy covering, which screens out the greater part of the light incident upon the upper surface. It occurs also in xerophytes which grow in light-colored sands and gravels that serve to reflect the sun's rays upon the lower surface. In deep shade, moreover, there is no essential difference in the intensity of the light received by the two surfaces, and shade leaves are often isophotic in consequence. From these examples it is evident that isophotic and diphotic leaves occur in both sun and shade, and that the intensity of the light is secondary to direction, in so far as the modification of the leaf is concerned.

The essential connection of sponge tissue with diffuse light is conclusively shown by the behavior of shade ecads, but further evidence of great value is furnished by diphotic leaves, and those with hairy coverings. The sponge tissue, which in the shade leaf is due to the diffuse light of the habitat, is prodiced in the hairy leaf as a consequence of the absorption and diffraction of the light by the covering. In ordinary diphotic leaves, the 
absorption of light in the palisade reduces the intensity to such a degree that the cells of the lower half of the leaf are in diffuse light, and are in consequence modified to form sponge tissue. The sponge tissue of the diphotic leaf is just as clearly an adaptation to diffuse light as it is in those plants where the whole chlorenchym is in the shade of other plants or of a covering of hairs. As is indicated later, all these relations permit of ready confirmation by experiment, either by changing the position of the leaf or by modifying the intensity or direction of the light.

The preceding discussion makes it fairly clear that sponge tissue is developed primarily to increase the lightabsorbing surface. Because of its direct connection with photosynthesis, the sponge tisstue is the especial organ of aeration, also, and since it shows a high development of air spaces for this purpose, it is inevitably concerned in transpiration. It seems to be partly a coincidence, however, that the sponge is found next to the lower surface upon which the stomata are most numerous. This is indicated by artificial ecads of Ranunculus sceleratus, in which sponge tissue is unusually developed, although the stomata are much more numerous upon the upper surface. Palisade tissue is apparently developed
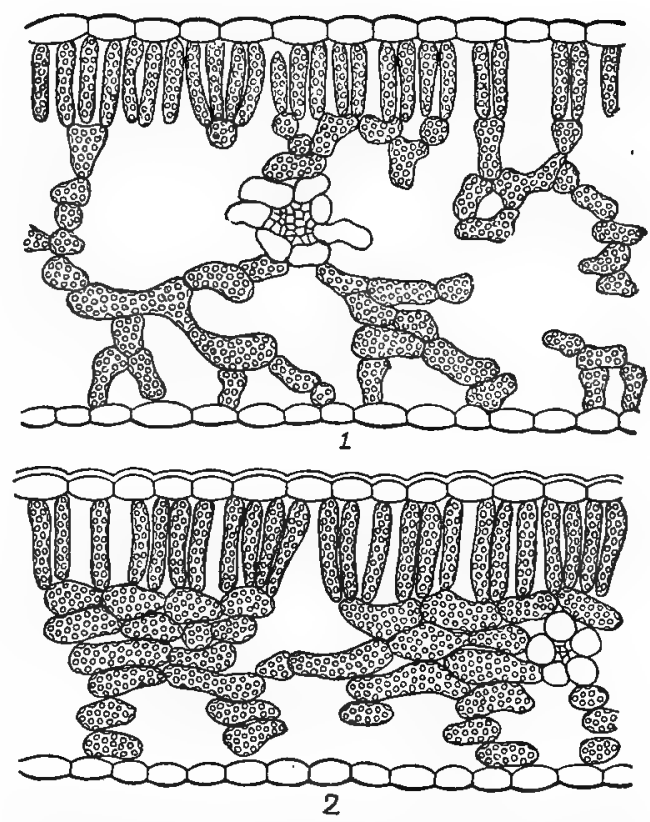

Fig. 43. A plastic species, Mertensia polyphylla, showing the effect of water upon the sponge: 1, chresard 25\%; 2, chresard 12\%. $\times 130$.

primarily as a protection against water loss, particularly that due to the absorption of light by the chloroplast. The small size of the intercellular passages between palisade cells likewise aids in decreasing transpiration. The fact that leaves with much palisade tissue transpire twice a much as shade leaves is hardly an objection to this view, as Hesselmann (l. c., 442) would think. It is readily explained by the intense photosynthesis of sun plants, which makes necessary an increase, usually a doubling, in the number of stomata, in consequence of which the transpiration is increased. 
181. Types of leaves. Isophotic leaves are equally illuminated and possess more or less uniform chlorenchym. Diphotic leaves are unequally illuminated, and exhibit a differentiation into palisade and sponge tissues. They may be distinguished as isophotophylls and diphotophylls respec-

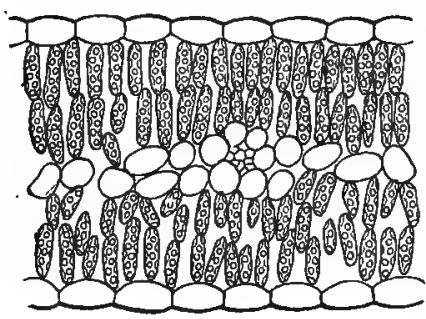

1

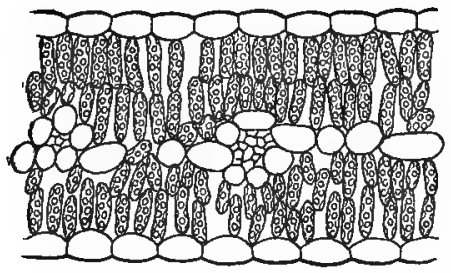

2

Fig. 44. A stable species, Erigeron speciosus: 1, sun leaf; 2 , shade leaf (light .03). $\times 130$. tively. ${ }^{1}$ Isophotic leaves fall into three types based upon the intensity of the light. The staurophyll, or palisade leaf, is a sun type in which the equal illumination is due to the upright position or to the reflection from a light soil, and in which the chlorenchym consists wholly of rows of palisade cells. The diplophyll is a special form of this type in which the intense light does not penetrate to the middle of the leaf, thus resulting in a central sponge tissue, or waterstorage tissue. The spongophyll, or sponge leaf, is regularly a shade type; the chlorenchym consists of sponge cells alone. For the present at least it is also necessary to refer to this group those monocotyledons which grow in the sun but contain no palisade tisstue. Diphotic leaves always contain both palisade and sponge, though the ratio between them varies considerably. Diphotophylls are characteristic of surny mesophytic habitats. They are frequent in xerophytic habitats as well as in woodlands where the light is not too diffuse. In the case of stable species, this type of structure sometimes persists in the diffuse light of coniferous forests. Floating leaves, in which the light is almost completely cut off from the lower surface, are also members of this group. Stubmerged leaves, on the other hand, are spongophylls.

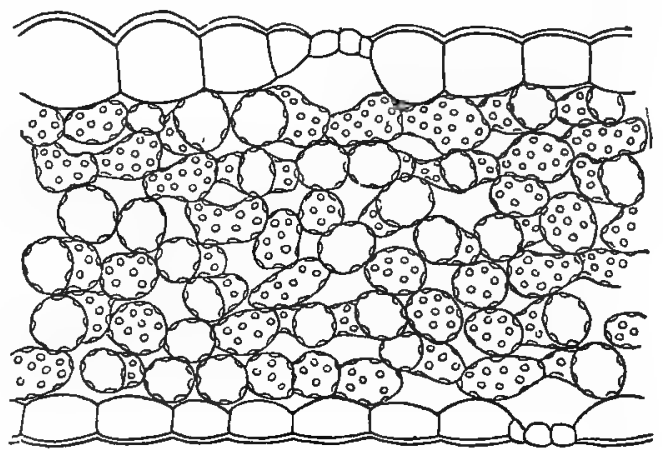

Fig. 45. Spongophyll of Gyrostachys stricta (light 1$). \times 130$.

182. Heliophytes and sciophytes. The great majority of sun plants possess diphotophylls. This type is represented by Pedicularis procera

${ }^{1}$ Clements, E. S. The Relation of Leaf Structure to Physical Factors. 1905. 
(fig. 32). Plants with isophotophylls are found chiefly in xerophytic places, though erect leaves of this type occur in most sunny habitats. The staurophyll, in which the protection is due to the extreme development of palisade tissue, is illustrated by Allionia linearis (fig. 40) and Bahia dissecta (fig. 33). The diplophyll, which is characterized by a central band of sponge tissue or storage cells, is found in Mertensia linearis (fig. 34). The form of the spongophyll that is found in certain monocotyledons is shown by Gyrostachy's stricta (fig. 45). The spongophyll (fig. 38:3, 39:2) is frequent among plants of deep shade, but as the leaf sections of Allionia (figs. 38, 40) and Quercus (fig. 42) show, the diphotophyll. is the rule in shade ecads.

\section{Experimental Evolution}

183. Scope. The primary task of experimental evolution is the detailed study, under measured conditions, of the origin of new forms in nature. As a department of botanical research that is as yet unformed, it has little concern with the host of hypotheses and theories which rest merely upon general observation and conjecture. A few of these constitute good working hypotheses or serve to indicate possible points of attack, but the vast majority are worthless impedimenta which should be thrown away at the start. It is the general practice to speak of evolution as founded upon a solid basis of incontestible facts, but a cursory examination of the evidence shows that it is drawn, almost without exception, from observaton alone, and has in consequence suffered severely from interpretation. With the exception of DeVries's work on mutation, sustained and accurate investigation of the evolution of plants has been lacking. As a result, botanical research has been built high upon an insecure foundation, nearly every stone of which must be carefully tested before it can be left permanently in place. In a field so vast and important as evolution, experiment should far outrun induction, and deduction should enter only when it can show the way to a working hypothesis of real merit. The great value of DeVries's study of mutation as an example of the proper experimental study of evolution has been seriously reduced by the fact that the "mutation theory" has carried induction far beyond the warrant afforded by experiment. The investigator who plans to make a seriotis study by experiment of the origin of new. plant forms should rest secure in the conviction that the most rapid and certain progress can be made only by the accumulation of a large number of unimpeachable facts, obtained by the most exact methods of experimental study.

The general application of field experiment to evolution will render the current methods of recognizing species quite useless. It will become imperative to establish an experimental test for forms and species, and to 
apply this test critically to every "new species." Descriptive botany, as practiced at present, will fall into disuse, as scientific standards come to prevail, and in its place will appear a real science of taxonomy. In the latter the criteria upon which species are based will be obtained solely by experiment.

184. Fundamental lines of inquiry. There are two primary and sharply defined fields of research in experimental evolution, namely, adaptation in consequence of variation (and mutation), and hybridization. The latter constitutes a particular field of inquiry, which is not intimately connected with the problems of evolution in nature. In the study of specific adaptation, two questions of profound importance appear. One deals with the effects of ancestral fixity or plasticity in determining the amount of modification produced by the habitat. These are fundamental problems, and a solution of them can not be hoped for until exact and trustworthy data have been provided by numerous experimental researches. It thus becomes clear that the principal, if not the sole task of experimental evolution for years to come is the diligent prosecution of accurate and prolonged experiment in the modification of plant forms. It seems inevitable that this will be carried on along the lines that have already been indicated. Plants will be grown in habitats of measured value, or in different intensities of the same factor. The relation between stimulus and adjustment will form the basis of careful quantitative study, and the final expression of this relation in structural modifications will find an exact record in drawings, photographs, exsiccati, and biometrical measures. The making of an accurate and complete record of the whole course of each experiment of this sort is an obligation that rests upon every investigator. Studies in experimental evolution will prove time-consuming beyond all other lines of botanical research, and the work of one generation should appear in a record so perfect that it can be used without doubt or hesitation as a basis for the studies of the succeeding generation.

185. Ancestral form and structure. The significance of the fact that some species have been found to remain unaltered structurally under changes of liabitats that produced striking modifications in others has already been commented upon. It is hardly necessary to indicate the important bearing which this has upon evolution. The very ability of a plant to undergo modification, and hence to give rise to new forms, depends upon the degree of fixity of the characters which it has inherited. Stable plants are less susceptible of evolution than plastic ones. The latter adapt themselves to new habitats with ease, and in each produce a new form, which may serve 
as the starting point of a phylum. There is at present no clue whatever as to what calls forth this essential difference in behavior. This is not surprising in view of the fact that there have been no comparative experimental studies oi stable and plastic species. Until these have been made, it is impossible to do more than to formulate a working hypothesis as to the effect of stability, and an explanation of the forces which cause or control it is altogether out of the question.

186. Variation and mutation. New forms of plants are known to arise by three methods, viz., variation, mutation, adaptation. The evidence in support of these is almost wholly observational, and consequently more or less inexact, but for each there exist a few accurate experiments which are conclusive. Origin by variation and subsequent selection is the essence of the Darwinian theory of the origin of species. According to this the appearance of a new form is due to the accumulation, and selection, through a long period, of minute differences which prove advantageous to the plant in its competition with others in nature, or are desirable under cultivation. Slight variations appear indiscriminately in every species. Their cause is not known, but since they are found even in the most uniform habitats, it is impossible to find any direct connection between them and the physical factors. In the case of origin by mutation, the new form appears suddenly, with definite characteristics fully developed. Selection, in the usual sense of the term, does not enter into mutation at all, though the persistence of the new form is still to be determined by competition. Mutations are known at present for only a few species, and their actual appearance has been studied in a very few cases. Like variations, they are indiscriminate in character. The chief difference between them is apparently one of degree. Indeed, mutation lends itself readily to the hypothesis that it is simply the sudden appearance of latent variations which have accumulated within the plant. DeVries regards constancy as an essential feature of mutation, but the evidence from the mutants of Onagra is not convincing. Indeed, while there can be no question of the occurrence of mutation in plants, a fact known for many years, the facts so far brought forward in support of the "mutation theory" fall far short of proving "the lack of significance of individual variability, and the high value of mutability for the origin of species." Mutations do not show any direct connection with the habitat, but their sudden appearance suggests that they may be latent or delayed responses to the ordinary stimuli. Origin by adaptation is the immediate consequence of the stimuli exerted by the physical factors of a

IDE VRIES, H. Die Mutationstheorie, 1:6. 1901. 
habitat. This fact distinguishes it from origin by variation, or by mutation. The new form may appear suddenly, often in a single generation, or gradually, but in either case it is the result of adaptation that is necessarily advantageous, because it is the result of adjustment to controlling physical factors. Origin by adaptation is perhaps only a special kind of origin by variation, but this might be said with equal truth of mutation. New forms resulting from adaptation are like those produced from mutation, in that they appear suddenly as a rule and without the agency of selection. They are essentially different, inasmuch as their cause may be found at once in the habitat, and since a reversal of stimuli produces, in many cases at least, a reversion in form and structure to the ancestral type.

A valid distinction between forms or species upon the basis of constancy is impracticable at the present time. It is doubtful that such a distinction can ever be made in anything like an absolute sense, since all degrees of fluctuation may be observed between constancy and inconstancy. In all events, it is gratuitous to make constancy the essential criterion in the present state of our knowledge. So little is certainly known of it that it is equally unscientific to affirm or to deny its value, and even a tentative statement can not be ventured until a vast amount of evidence has been obtained from experiment. Accordingly, there is absolutely no warrant, other than tradition, for limiting the term species to a constant group. In the evolutionary sense, a species is the aggregate ancestral group and the new forms which have sprung from it by variation, mutation, or adaptation. It should not be regarded as an isolated unit for purposes of descriptive botany; indeed, its use in this connection is purely secondary. It is properly the unit to be used in indicating the primary relationships which are the result of evolution.

On the basis of their actual behavior in the production of new forms, species may be distingurshed as variable, mutable, or adaptable. The new form which results from variation is a zariant; the product of mutation is a mutant, and that of adaptation, an ecad. The following examples serve to illustrate these distinctions. Machaeranthera canescens, judging from the numerous minute intergrades between its many forms, is a variable species, i. e., one in which forms are arising by the gradual selection of small variations. It apparently comprises a large number of variants, $M$. canescens aspera, superba, ramosa, viscosa, etc. Onagra lamarckiana is a mutable species : it comprises many mutants, e. g., Onagra lamarckiana gigas, $O$. l. nanella, $O$. . l. lata, etc. Galium boreale is an adaptable species: it possesses one distinct ecad, Galium boreale hylocolum, which is the shade form of the species. 
18\%. Methods. The best of all experiments in evolution are those that are constantly being made in nature. Such experinents are readily discovered and studied in the case of origin by adaptation; variants present much greater difficulties, while mutants are very rare under natural conditions. The method which makes use of these experiments may be termed the method of natural experiment. The number of ecads which appear naturally in vegetation is limited, however, and it is consequently very desirable to produce them artificially, by the method of habitat culture. This method, while involving more labor than the preceding, yields results that are equally conclusive, and permits the study of practically every species. The method of control culture, which is carried on in the planthouse, naturally does not possess the fundamental value of the field methods. It is an invaluable aid to the latter, however, since it permits the physical factors to be readily morlified and controlled. All these methods are based on the indispensable use of instruments for the measurement of physical factors.

\section{METHOD OF NATURAL F.XPERIMENT}

188. Selection of species. Species that are producing variants or ecads are found everywhere in nature; those which give rise to mutants seem, however, to be extremely rare. Consequently, mutants can not be counted upon for experimental work, and their study scarcely needs to be considered. When a mutant is discovered by some fortunate chance, the mutable species from which it has sprung, and related species as well, should be subjected to the most critical surveillance, in the hope that new mutants will occur or the original one reappear. On account of the suddenness with which they appear, mutants do not lend themselves readily to natural experiment, and after they have once been discovered, inquiry into the causes and course of mutation is practicable only by means of habitat and control cultures. Among variable species, those are most promising that show a wide range of variation and are found in abundance over extensive areas. A species which occurs in widely separated, or more or less isolated areas, furnishes especially favorable material for investigation, since distance or physical barriers partly eliminate the leveling due to constant cross-fertilization. The individuals or groups which show appreciable departure from the type are marked and observed critically from year to year. The direction of the variation and the rapidity with which small changes are accumulated can best be determined by biometrical methods. Representative individuals of the species and each of its variants should likewise be selected from year to year. After heing photograpled, these are preserved as exsiccati, and with the photographs constitute a complete 
graphic record of the course of variation. When the latter is made evident in structural feature also, histological slides are an invaluable part of the record.

Polydemic species are by far the best and most frequent of all natural experiments. In addition to plants that are strictly polydemic, i. e., grow in two or more distinct habitats, there are a large number which occur in physically different parts of the same habitat. The recognition of polydemics is the simplest of tasks. As a rule, it requires merely a careful examination of contiguous formations in order to ascertain the species common to two or more of them. The latter are naturally most abundant along the ecotones between the habitats, and, as a result, transition areas and mixed formations are almost inexhaustible sources of ecads. Many adaptable species are found throughout several formations, however, and such are experiments of the greatest possible value. Not infrequently species of the manuals are seen to be ecads, in spite of their systematic treatment, and to constitute natural experiments that can be readily followed. Finally, it must be kept in mind that some polydemics are stable, and.do not give rise to ecads by structural adaptation. They not only constitute extremely interesting experiments in themselves, but they should also be very carefully followed year by year, since it seems probable that the responses are merely latent, and that they will appear suddenly in the form of mutants. In natura! experiments it is sometimes difficult to distinguish which form is the ecad and which the original form of the species. As a rule, however, this point can be determined by the relative abundance and the distribution, but in cases of serious rloubt, it is necessary to appeal to experimental cultures.

Although habitats differ more or less with respect to all their factors, the study of polydemics needs to take into account only the direct factors, water-ccntent, humidity, and light. Humidity as a highly variable factor plays a secondary part, and in consequence the search for ecads may be entirely confined to those habitats that show efficient differences in the amount of water-content or of light. Temperature, wind, etc., do not produce ecads, and may be ignored, except in so far as they affect the direct factors. Complexes of factors, such as altitude, slope, and exposure, are likewise effective oniy through the action of the component simple factors upon water and light. The influence of biotic factors is so remote as to be negligible, especially in view of the fact that ecads are necessarily favorable adaptations, and are in consequence little subject to selective agencies. The essential lest of a habitat is the production of a distinguishable ecad, but a knowledge of the water-content and light values of the habitats under examination is a material aid, since a minute search of each formation is necessary to reveal all the ecads. It is evident that habitats or 
areas that do not show efficient differences of water or light will contain no ecads of their common species, and also that extreme differences in the amount of either of these two factors will preclude origin by adaptation to a large degree, on account of the need for profound readjustment. The general rule followed by most polydemics is that sun species will give rise to shade forms, and vice versa, and that xerophytes will produce forms of hydrophytic tendency, or the converse, when the areas concerned are not too remote, and the water or light differences are efficient, but not inhibitive. Some species are capable of developing naturally two series of ecads, one in response to light, the other to water-content, but they, unfortunately, have been found to be rare. Greatly diversified regions, such as the Rocky mountains, in which alternation is a peculiarly striking feature of the vegetation, are especially favorable to the production of ecads, and hence for the study of natural experiments in origin by adaptation.

189. Determination of factors. For the critical investigation of the origin of new forms, an exact knowledge of the factors of the habitat, both physical and biotic, is imperative. In the case of variable species, these factors determine what variations are of advantage, and thereby the direction in which the species can develop. They are the agents of selection. With mutants, the factors of the habitat are apparently neither causative nor selective, thongl it seems probable that further study of mutants will show an essential connection between mi1tant and factor. In any event, the persistence of a mutant in nature, and its corresponding ability to initiate new lines of development, is as much dependent upon the selection exerted by physical and biotic factors as is the origin of variants. Physical factors are causative agents in the production of ecads, as has been shown at length elsewhere. The form and structure of the ecad are the ultimate responses to the stimuli of light or water-content, and the quantitative determination of the latter is accordingly of the most fundamental importance. The measurement of factors has been treated so fully in the preceding chapters that it is only necessary to point out that the thorougl investigation of habitats by instruments is as indispensable for the study of experimental evolution as for that of the development and structure of the formation. Furthermore, it is evident that a knowledge of physical factors is as imperative for habitat and control cultures as for the method of natural experiment. In the latter, however, the biotic factors demand unusual attention, since pollination, isolation, etc., are often decisive factors in origin by variation and in the persistence of mutants.

Measurements of adjustment, i. e., functional response to the direct factor concerned, are extremely valuable, but not altogether indispensable 
to research in experimental evolution. This is due to the fact that a knowledge of adjustment is important in tracing the origin of new forms only when adjustment is followed by adaptation, and in all such cases the ratio between the two processes seems to be more or less constant. In the present rudimentary development of the subject, however, it is very desirable to make use of all methods of measuring functional responses to water and light that are practicable in the field. Certain methods that are difficult of application in nature may be used to advantage in control cultures, and the results thus secured can be used to interpret those obtained from natural experiments and field cultures.

190. Method of record. As suggested elsewhere, there are four inportant kinds of records, which should be made for natural experiments, and likewise for habitat and control cultures. These are exsiccati, photographs, biometrical formulae and curves, and histological sections. These serve not merely as records of what has taken place, but they also make it possible to trace the course of evolution through a long period with an accuracy otherwise impossible, and even to foreshadow the changes which will occur in the future. The possibility of doing this depends primarily upon the completeness of the record, and for this reason the four methods indicated should be used conjointly. In the case of ecads and mutants, exsiccati, photographs, and sections are the most valuable, and in the majority of cases are sufficient, since both ecads and mutants bear a more distinctive impress than variants do. On the other hand, since variatious are more minute, the deternination of the mean and extreme of variation by biometrical methods is almost a prerequisite to the use of the other three methods, which must necessarily be applied to representative individuals.

Exsiccati and photographs are made in the usual way for plants, but it is an advantage to photograph each ancestral form alongside of its proper ecads, mutants, or variants, in addition to making detail pictures of each form and of the organs which show modification. In the collection of material for histological sections, which deal primarily with the leaf or with stems in the case of plants with reduced leaves, a few simple precautions have been found necessary. Whenever possible, material should be killed where it is collected, since in this way the chloroplasts are fixed in their normal position. In case. leaves that can not be replaced easily have become wilted, an immersion of 5-6 hours in water will make it possible to kill them without shrinkage. In selecting leaves, great pains must be taken to collect only mature leaves. When the plants have a basal rosette, or distinct radical leaves, mature leaves are taken from both stem and base. In all cases where the two surfaces of the leaf can not be readily. distinguished, the upper one is clearly marked. 
191. Scope and advantages. By means of experiments actually made in the field, practically every species that is capable of modification can be made to produce new forms, the origin of which can be traced in the manner already indicated. Field experiments of this sort are especially favorable to the production of ecads from adaptable species. No attempt has yet been made to apply it to mutable or variable species, but its ultimate application to these does not seem at all impossible. The chief advantage of the method of habitat cultures is seen in the great range of choice in selecting the plant for experiment, and the habitat or area in which the experiment is carried out. A polydemic species which already has one or more ecads can be extended to a number of different habitats of known value, and a complete series of ecads obtained, based either upon water-content, or light, or upon both. On the other hand, an endemic species, or one brought from a remote flora, can be placed in as many habitats as desired, and the appearance of ecads followed in each. Frequently, results of much value are obtained in a diversified habitat by growing its most plastic species in those areas which show the greatest differences in water-content or light intensity. Habitat cultures give results which are practically as perfect as those obtained from nattural experiments, since the course of adaptation in no wise depends upon whether the agent by which the seed or propagule is carried into the new habitat is natural or artificial. Cultures of this kind further possess the distinct advantage of permitting more or less modification of the physical factors themselves. However, when it is desirable to have the factors under as complete control as possible, it is necessary to use the method of control caltures in the planthouse.

192. Methods. All field experiments in evolution are based upon a change of habitat. The latter is accomplished by the modification of the habitat itself, or by the transfer of the species to one or more different habitats, or to different areas of the same habitat. In both cases the choice of habitats is made upon the basis of efficient differences of water-content or light. Saline situations do not constitute an exception, since the chresard is really the effective stimulus. Cultures at different altitudes, which afford striking results, appear to concern several factors, but in the final analysis, water-content and humidity are alone found to be really formative. Cultures may furthermore be distinguished as simple or reciprocal. Simple cultures are those in which a species is transferred to one or more habitats, or in which a habitat is modified in one or more ways. Reciprocal cultures are possible only with polydemic species, or with endemics after ecads have 
been produced by experiment. Modification or transfer is made in the usual way, but reciprocally, i. e., the original form is transferred to the habitat of the ecad, and the latter to the habitat of the former; or the shade in which some individuals of the ecad are growing may be destroyed, and at the same time individuals of the type may be shaded. Both transfer and modification may be applied to the same species, but since the same measured change of factor can be obtained in either way, the use of both is undesirable, with the exception of the rare cases where they serve as checks upon each other. The transfer of a seed or plant is so much simpler and more convenient that this method is the one regularly used. It sometimes happens, however, that a change of water-content or light intensity is readily and conveniently made, and is desirable for other reasons.

It is evident that both transfer. and modification require that the factor records of the various habitats or areas be as full as possible, at least so far as water-content, humidity; and light are concerned. In the case of the areas that are to be modified, these factors are determined before the change is made. Afterward they are read from time to time during the growing season, and are also checked by readings made near at hand in the unmodified formation. The readings made in the beginning should correspond closely to the check readings, but in case of disagreement the latter are to be taken as conclusive.

193. Transfer. After the species to be used for experiment has been chosen, the various habitats or areas selected, and the direct factors measured by instruments, the actual transfer of the individuals is made by means of seeds, preferably in autumn, though the results are, practically the same if seeds are kept over the winter and planted at the opening of spring. The natural method is to scatter the seeds in the place selected, as though they had been carried by the usual agents of migration. The mortality is usually great in such case, however, and the chances of success are increased by actually planting the seeds. This is the method which has been used in making cultures of species of the European Alps on the summit of Mount Garfield in the Rocky mountains. The number of seeds used is recorded in order to obtain some estimate of germination and competition. While the use of the seed or disseminule possesses the great advantage of making the experiment essentially a natural one, the transfer of rosettes, seedlings, or young plants makes the results more certain, and consequently saves time, even though the actual transfer is somewhat more difficult. It is hardly uecessary to point out that the removal of the plant should be made with the greatest care. The best success is obtained by making the transfer on cloudy or rainy days, and when shade plants are to be placed in sunny situations, they should be transplanted late in the afternoon. When the task of carry- 
ing them is not too great, it is a distinct advantage to move a number of individuals in the same block of earth. The transfer of mature plants is inadvisable, except for those perennials which can not readily be secured in an early stage. This naturally does not apply to woody plants, evergreen herbs, mosses and lichens; the last two may be transferred at any time with satisfactory results. Each culture is carefully marked with stakes, and definitely located by means of landmarks.

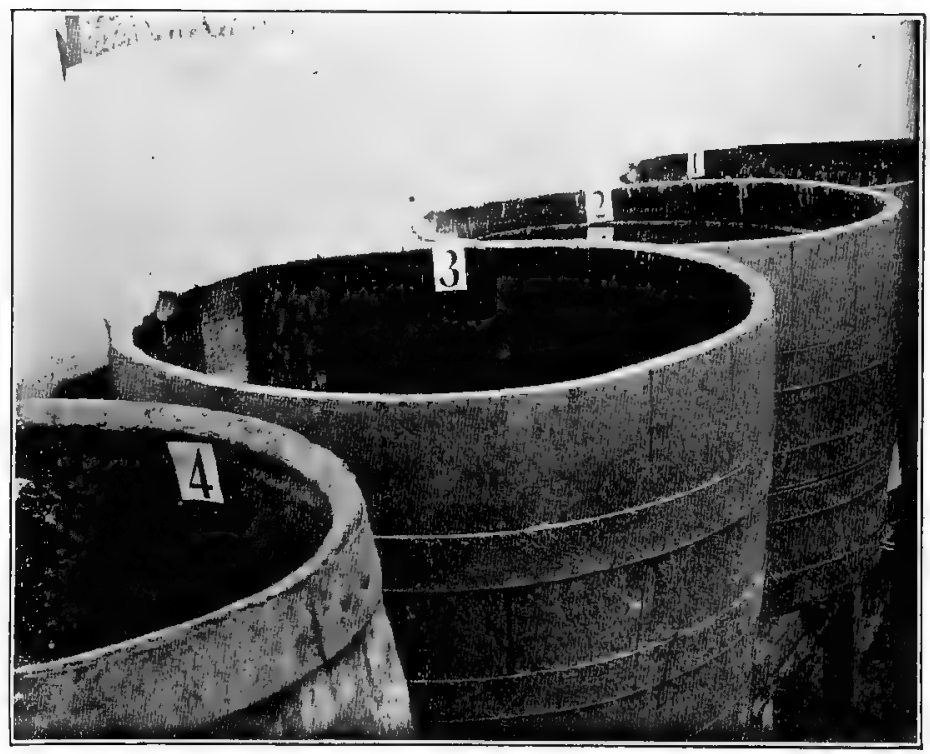

Fig. 46. Series for producing hydrophytic forms under control: 1, amphibious; 2 , floating; 3 , competition; 4, submerged.

Reciprocal transiers may be made by means of seed or plant. Since the experiment is a complex one, all the care possible should be taken to make sure that the plants become established in the reciprocal situations, and consequently, it is often advisable to transfer both seeds and plants. Reciprocal transfer is of paramount value in solving the problem which bog plants present. A slight modification of the method makes it possible to obtain experimental eviclence of the polyphyletic origin of species in consequence of adaptation. In an experiment mentioned elsewhere, the transfer of Kuhnistera purpurea to the area occupied by $K$. candida, and vice versa, is designed to show whether one has been derived from the other. If the two species are moved into an area which contains more water than that usualls occupied by $K$. purpurea, and less water than is found where $K$. candida habitually grows, the resulting modifications will throw much light upon the 
origin of polyphyletic species. In this connection, it hardly needs to be pointed out that this simple transfer of a species to several separated areas of a new habitat may often furnish complete proof that a new form may arise at different-times, and at different places.

194. Modification of the habitat. Efficient changes in the habitat are brought about by increasing or decreasing the water-content, or by varying the light intensity between sunshine and the diffuse light of deep forests. Humidity can not well be regulated except in so far as it is connected with water-content. Since its effects merge with those of the latter, its modification is unnecessary. An increase in water-content is readily brought about by irrigation. A stream may be dammed and its water allowed to spread over the area to he studied, or the water may be carried to the proper place

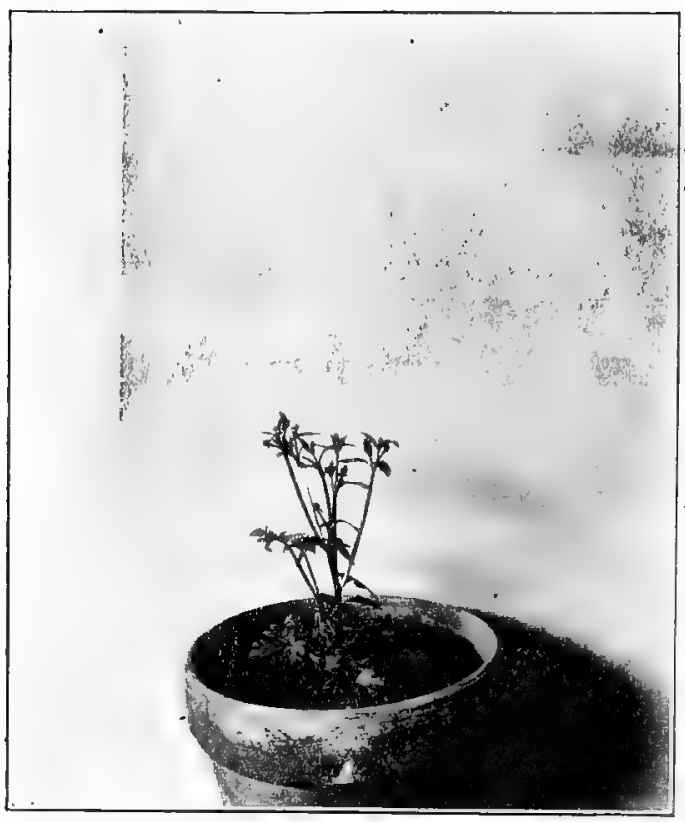

Fig. 47. Control ecad of Ranunculus sceleratus, holard $10 \%$ (50 cc.). by deflecting the stream or by digging a canal. The construction of earth reseryoustis. makes it possible to obtain almost any per cent of soil water by varying the size of the reservoir or the height of the wall or bank. Near a base station, such as Minneliaha, where there is a simple system of waterworks, the experimental area may be watered whenever desirable by means of a hose. Water-content may be readily decreased by drainage, or by the deflection of a stream. When such means are not available, as in the case of extensive marshes, hummocks may be used or constructed, and the soil blocks containing plants placed upon them. By the use of sand or gravel, the water-content of mesophytic areas can be reduced in a similar manner, or by surrounding the plant in situ with either of these soils which hold little water. In meadows, especially, the addition of a large quantity of alkaline salts decreases the amount of available water, while the holard may be reduced by denuding the soil about the plants concerned. 
In sunny habitats, the light intensity is most easily reduced by means of cloth awnings, which can be put in place conveniently. It is not a difficult matter to produce effective shade by using shrubs or small trees for this purpose. This plan is especially advantageous in habitats too remote to make frequent visits feasible. When a shrub or tree is used, the experiment necessarily requires a longer time, though this disadvantage is partly compensated by the fact that the shelter requires practically no attention after the shrub is once established. Forest plantations furnish excellent examples of this kind of experiment. On the other hand, clearings afford the only examples of habitats modified in such manner as to increase the light. In nature, the diffuse light in. which shade plants grow is due to the presence of tall plants, chiefly shrubs and trees, and an increase in the light intensity is possible only through the thinning-out or removal of the plant screen. This is a task of considerable magnitude in for-

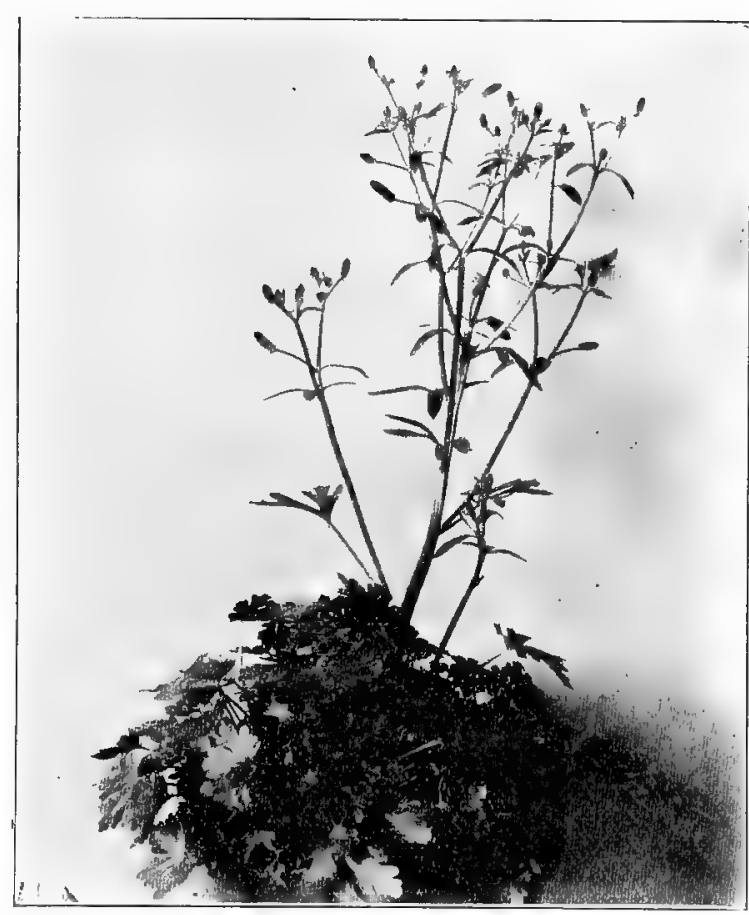

Fig. 48. Control ecad of Ranunculus sceleratus, holard $40 \%$ ( $200 \mathrm{cc}$.).

ests, but it can be readily accomplished in thickets and at the edges of woodlands. It is quite practicable to establish a series of awnings or clearings of various light values, but the labor required is hardly worth while when it is recalled that the method of transfer makes it possible to take advantage of the various intensities already found in nature.

METHOD OF CONTROL CULTURES

195. Scope and procedure. Control experiments are necessarily carried on in the planthouse, since factors can be controlled in the field only with great difficulty. Their greatest value is in connection with 
experiments that are being carried on in the habitat, but they also constitute an invaluable means of independent research, since it is not at all difficult to approximate the conditions of a habitat, especially with reference to water-content and light. The essential feature of the method is that the less important factors are equalized as far as possible, while the direct factors, water-content and light, are under the complete control of the investigator. By the equalization of humidity and temperature is meant experimentation in which all the plants of each experiment are subjected to the same amounts of these factors. It is a matter of no importance whatever whether the humidity and temperature are constant or variable. In the case of soil, which is not a variable, it naturally happens that the plants are placed once for all in the same soil mixture. Batteries consisting of thermograph and psychrograph have been kept in the different control houses, but although used at first to give some idea of the hourly and daily fluctuations of temperature and humidity, they have slight bearing upon the evolution of new forms under control. For use in connection with supplementary experiments in adjustment and adaptation, the batteries have proved to be indispensable. Control experiments are regularly made in series which are planned with reference to as many modifications as the efficient difference of the factor and the plasticity of the species concerned permit.

196. Water-content series. An account of the experiments which have been carried on for four generations with Ranunculus sceleratus will serve to show the application of culture methods to the origin of new forms in response to varying water-content. This species was chosen because it grows readily in the planthouse, is plastic, and, since it is naturally amphibious, permits of much modification in both directions. The smallest amount of water per day under which the seedlings would grow was found to be $25 \mathrm{cc}$. This was taken as one extreme for the series, and deep water in which the plant could be submerged as the other. An arbitrary series was tentatively made as follows: $25 \mathrm{cc}$., $50 \mathrm{cc}$., IOO cc., I $50 \mathrm{cc}$, $200 \mathrm{cc}$., mud, shallow water. and deep water. Further study justified these divisions, since the first six gave efficient differences in water-content, and the resulting forms all showed differences of structure as well as of growth and form. Seedlings of the same age, and as nearly alike as possible, were transplanted to large pots of which there were four for each of the first six; they were placed in half-barrels for mud and floating forms, and in a barrel for submerged forms. After a few days, when they had become well established, the plants in the pots were watered in the amounts indicated, as often as was necessary to keep the most xerophytic form alive; the soil for 
the mud form was kept covered with a thin film of water; the leaves of the form in shallow water were kept floating on the surface, and those of the last form submerged just below the surface. The water in which the submerged form grew was aerated by means of a spigot near the bottom of the barrel. From time to time water-content determinations were made of the soil in the pots until it was definitely ascertained that the holard was practically constant. The nine new forms obtained by adaptation showed striking differences in vigor and growth, as may be seen from the figures. In all cases, these were accompanied by distinct and often striking differences in the number and position of the stomata, the amount of sponge and palisade tissues, and the development of air passages. Photographs were made of a typical plant of each form, and the different leaf structures

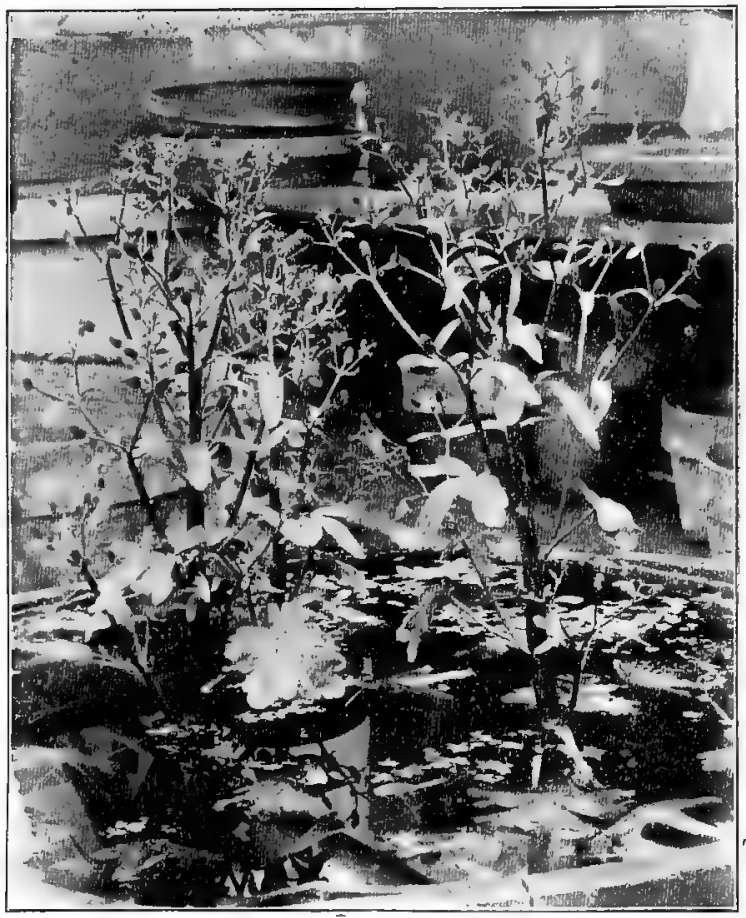

Fig. 49. Floating form of Ranunculus sceleratus grown under control.

were preserved in permanent mounts. The xerophytic and the submerged form were unable to produce flowers, and it was necessary to develop them anew in each generation. The other forms fruited abundantly, and the succeeding generations of each form were produced from plants which had grown the year before in the same conditions. In addition to the development of a series of new water-content forms, this experiment was begun in the hope of determining whether the modifications of a plastic species tend to become fixed if each new form is grown constantly under the same conditions. A period of four years is too short, however, to throw much light upon this problem. 
Helianthus anmuns has been used for other series of experiments, in which alkaline salts or different soils are employed to vary the water-content. These are more complex and hence are not as satisfactory as the series described above, but they are valuable for the light they throw upon the behavior of plants in similar conditions in nature. In the case of soil, however, the adaptation may be referred to water-content alone, if thoroughly leached sands and gravels are used, so that the difference is solely one of water-retaining power.

197. Light series. Cloth tents have been found the most satisfactory means of obtaining different light intensities in the planthouse. The cloth permits the air to circulate to a considerable degree, and in consequence the equalization of humidity and temperature is much more complete than in the glass houses first employed. The cloth tents, or shade tents as they are called, are cubical, each dimension being I meter. The series which has been most used consists of three tents: the first is made of cheesecloth and has a light value of .I ; the second is of thin muslin, and has a value of .04, while the third is made of dark cambric and the light is reduced to .or. A more desirable series is one with five tents, which have approximately the following light intensities: .I, .05, .01, .007, .003. Plants grown in shade tents should be repotted as often as they will permit in order to increase the aeration of the soil. The amount of water given them must also be decreased as the shade increases. "Mesophytic species give the best results in shade tents, xerophytes thrive less well, and amphibious plants do not grow at all except in the brightest light. Excellent results have been obtained with Helianthus, Taraxacum, Gaura, and Onagra, while Ranunculus sceleratus is unable to produce flowers and seeds in a light intensity of .OI.

A number of important supplementary experiments have been made in connection with light tents. These do not result in the production of new forms, but they throw much light upon it. Plants have been placed in the shade tents so that certain leaves would be in the sun and others in the shade. Young leaves have been fixed at various angles with the stem, and they have been revolved $90^{\circ}$ or $180^{\circ}$ in order to change the relation of their surfaces. Soils of different colors, e. g., loam and sand, have been used to determine the effect of light reflected from their surfaces. Shade tents make it possible to illuminate plants from the top, bottom, or side, and to carry on a large number of fundamental experiments in adjustment and adaptation. 


\section{CHAPTER IV. THE PLANT FORMATION}

\section{Methods of Investigation and Record}

198. The need of exact methods. The use of instruments in the study of the habitat has made it evident that the loose methods of descriptive ecology were altogether inadequate to the accurate investigation of the formation. This feeling has been heightened by the recognition of the fact that vegetation exhibits both development and structure, and is, in consequence, open to exact methods of inquiry. In the search for feasible methods, it was quickly seen that the quadrat, first ${ }^{ \pm}$used for determining the abundance of species, furnished the key to the problem. Accordingly, the principle underlying it, viz., that of intimate detailed study and record, was developed and extended in such a way as to give rise to a number of methods of precision. These have been applied in the field for several years with signal success, and they are here described in the conviction that they constitute a satisfactory svstem, if not, indeed, the only one for the exact study of formations.

There has been a growing appreciation of the fact that the superficial methods of descriptive ecology made it impossible to build upon such a foundation, and they, indeed, were making actual progress in the field of ecology more and more difficult. Ecologists have now begun to see clearly that precise methods are as indispensable in the habitat as they are to the study of the structure and modification of the plant. For some reason, however, they have been slow to perceive that accuracy in the investigation of the cause, the habitat, is a fruitless task unless it be followed by corresponding exactness in the study of the effect, the formation. After having utged the fundamental necessity of instrumental methods, for six or seven years, both in season and out of season, the writer does not feel called upon to further plead the cause of the quadrat. The final acceptance of the instrument was inevitable if progress were to be made in the habitat, and it is just as obvious that the quadrat must be accepted if the study of the habitat is to bear fruit in the interpretation of the formation. The use of the quadrat does not mean that the general methods of descriptive ecology are all to be discarded, whether they have value or not. The statement that quadrat methods are indispensable signifies merely that they must be used for research work in the development and structure of vegetation. They are

1 Pound And Clements. A Method of Determining the Abundance of Secondary Species. Minn. Bot. Studies, 2:19. 1898. 
not necessary in reconnaissance, nor do they displace general methods of real value. The use of the latter in even a supplementary way will gradually be discontinued, however, as fields become smaller by reason of increase in the number of workers, and as the need for precise methods becomes more universally felt.

The quadrat constitutes the initial concept from which all the methods have grown. In itself, it has given rise to a variety of quadrats applicable to the most fundamental problems of vegetation. From it have come, on the one hand, the migration circle, and on the other, the transect. The latter in turn has yielded the ecotone chart, and the layer chart. All of these are based upon direct and detailed contact with vegetation itself, and permit accurate recording of all the results obtained.

\section{QUADRATS}

199. Uses. In its simplest form, the quadrat, as the name implies, is merely a square area of varying size marked off in a formation for the purpose of obtaining accurate information as to the number and grouping of the plants present. As indicated above, it was first used for determining the abundance of the various species of a formation. This made it possible to ascertain the relative rank of the species of layers and formations, and enabled one for the first time to gain some idea of the minute structure of a bit of vegetation. The results were at once applied to the task of establishing a ntumerical basis for abundance, and of working out a new system of abundance to correspond. The quadrat method was also used to determine the character of seasonal aspects, and to yield a knowledge of the exact differences in diverse areas of the same formation. Incidentally, the determinations of abundance were made the basis of an actual census of certain alpine formations. This, while it was extremely interesting to find that a square mile of alpine meadow contained approximately I,500,000,000 plants, was confessedly destitute of ecological value. The most important applications of the quadrat idea were made by Clements ${ }^{1}$ in the chart, the permanent and the denuded quadrats. The development of these was due to the fact that zones or formations permit of comparison upon floristic as well as physical grounds, and that a detailed record of their structure is necessary for this purpose. Similar comparisons are necessary for the consocies, zones, and patches of the same formation, and the quadrat becomes an indispensable means for studying alternation and zonation. For the investi-

1 The Development and Structure of Vegetation, 84. 1904.

Thornber, J. J. The Prairiegrass Formation in Region I. Rep. Bot. Surv. Neb., 5:29. 1901. 
gation of invasion year by year, and especially for succession, the method of permanent quadrats is imperative, ard the denucled quadrat an invaluable aid. Changes, which would otherwise be incompletely observed and imperfectly recorded, are followed in the minutest detail and recorded with perfect accuracy.

200. Possible objections. The use of the quadrat has led to the criticism that it is needlessly detailed and thorough, and that, after all, the space covered is but a minute part of the entire formation. The first objection is one that has also been urged against the use of instruments of precision in the habitat. It is always brought forward by those who have not used instruments, and as witnesses they are of necessity incompetent. No one who is familiar with the instrument or the quadrat by actual practice has felt that the methods based upon them were too thorough. In no case has the writer ever listed or mapped a quadrat without discovering some new fact or relation, or clearing up an old question. It can not be denied that quadrat methods require both time and patience, but this is true of any kind of research work that is at all worth while. Every ecologist, moreover, that has the interests of his field at heart and deprecates the present slipshod work, will appreciate the necessity of methods which seem like drudgery to the mere dabbler.

The second objection, that the quadrat is at best but a small bit of the area under investigation, seems at first to be a valid one. It can not be gainsaid that the actual space studied is insignificant as compared with the whole formation; still, it must be obvious that even a single quadrat can add at least some facts of value, which can never be obtained by the best of general methods. Furthermore, if the formation be an actual and not an imaginary one, a single quadrat will be in some measure representative. In the more homogeneots ones, it will have much the same value that a type specimen bears to the species established upon it. In formations which are less uniform, its value is correspondingly reduced, so that in formations which show marked zones, consocies, or patches, it becomes necessary to locate a quadrat in each. In the matter of representation alone, the graphic method of the quadrat map with its close-focus detail photograph, is far superior to anything that can be obtained by the ordinary description and photograph. Finally, the scientific study and recording of succession, and particularly of competition, is an impossibility without the aid of the permanent and denuded quadrat. The stoutest champion of the practice of walking through a formation, and jotting down impressions, can not avoid their use if he would attack these problems, and, once familiar with the quadrat, his objections to the drudgery of thoroughness will soon vanish. 


\section{Kinds of Quadrats and Their Use}

201. Size and kinds. The unit size of quadrat is the meter, and when the term is used without qualification, it refers to the meter quadrat. To make them strictly comparable, and exactly divisible, unit quadrats are always grouped in squares; thus a major quadrat is a square of four units, and a perquadrat one of sixteen units, or four meters square. Quadrats of greater size are necessary in woodland and forest, where the rule, however, is that the woody plants alone are recorded for the whole quadrat, the herbaceous growth being listed or mapped for but one or two representative units. For special purposes, quadrats of 3, 5, 6, etc., meters may be used, but they are much less convenient. Quadrats are further distinguished with respect to their use. A list quadrat is one in which the plants are merely listed and the number of individuals of each species indicated. Chart quadrats are those in which the area concerned is accurately mapped on plotting paper. Both list and chart quadrats are rendered permanent by careful labeling, so that their changes can be followed from year to year. The greater value of the chart causes practically all permanent quadrats to be of this type, and for the same reason only permanent chart quadrats are converted into denuded ones.

202. Tapes and stakes. The lines for marking out quadrats are made of strong white tape, $5 / 8$ inches wide. This is doubled and sewed firmly at both edges. Under moderate stretching, the tape is carefully marked off into decimeters, and eyelets $5 \mathrm{~mm}$. in diameter are set in at each end and at the marks. This can readily be done by any shoemaker at slight expense. The usual lengths are one and two meters, as these are most frequently used, and they can also be easily combined to make larger quadrats. The tapes are slightly longer than one meter in order that the distance between the end eyelets may be exact. The tapes of the larger forest quadrats should be divided into lengths of one meter, as these permit ready plotting and also make it possible to interpolate a meter quadrat for the study of the undergrowth at any point. The intervals of the tape are ntumbered from left to right, as conspicuously and clearly as possible. For this a waterproof ink or paint is very desirable. For holding the tapes in position, hatpins, nails, and meat-skewers have been used with more or less satisfaction. The ideal stake, however, is one which holds the tape close to the ground, and can be readily moved. It is merely a stout wire, $3 \mathrm{~mm}$. in diameter and 8 inches long, looped at the top, sharpened at the tip, and with a small ring of solder 3 inches from the tip. 
203. Locating quadrats. In staking a quadrat, the end tapes are invariably placed so that the numbers read from left to right, and the side tapes so that they read down. In mapping, a fifth tape is stretched parallel to the top, and as each decimeter strip is marked, the outer tape is shifted to delimit the new strip. Indeed, the side tapes can be placed alone, and the plotting tapes moved down one at a time as the mapping proceeds, but it is usually more satisfactory to locate the quadrat exactly and to square it first, a task most easily done by enclosing the whole quadrat, and then using a fifth tape. In the case of list quadrats in open vegetation, the measuring strip is unnecessary, but as a rule it facilitates counting, as well as mapping.

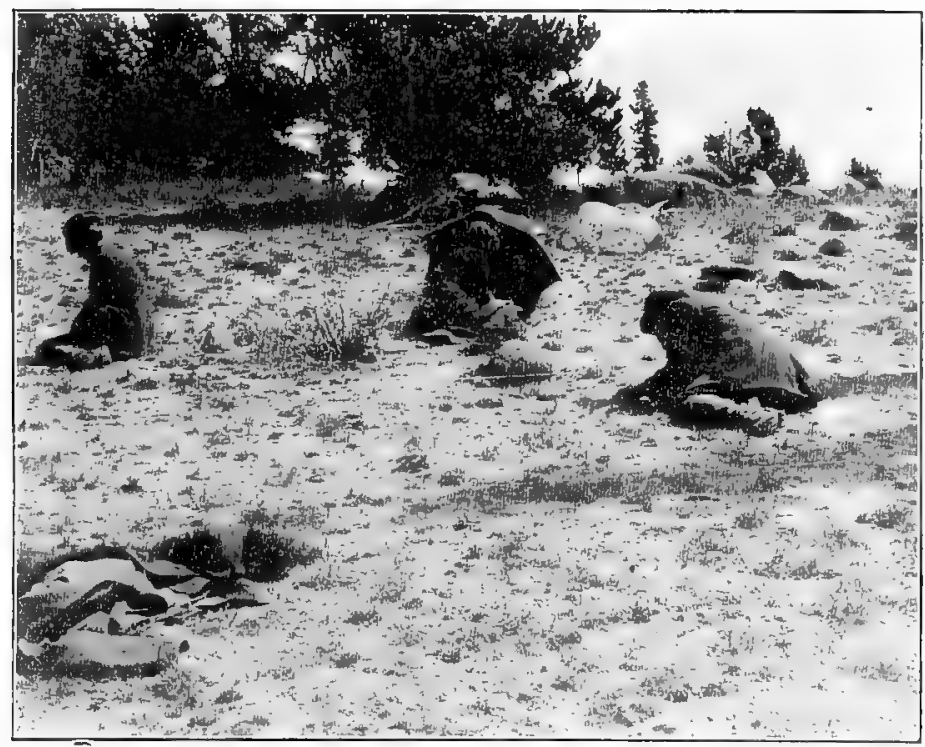

Fig. 50. Mapping a major quadrat on Mount Garfield at $3,600 \mathrm{~m}$.

\section{The List Quadrat}

204. Description. This, as the simplest form of quadrat, is employed primarily to ascertain the abundance of species in a formation or during a. particular aspect of it. Since this can be obtained readily from the chart, the list quadrat has fallen more and more into disuse, except where it is desired to determine abundance alone, or to aid in deciding whether a chart is really representative. The size depends almost wholly upon the nature of the vegetation. When the number of trees is to be determined, a quadrat of 10 or 50 meters is necessary. In ordinary herbaceous formations, the usual size is 2 meters, while the meter quadrat is used when the 
plants are especially small and crowded, as in alpine meadows. The location of the quadrat is based upon the general rule, but since its especial task is the determination of the greatest variable in vegetation, viz., number, it is necessary to use more quadrats, and to place them in areas which show the greatest differences in the mixture of species. For example, it was found that a half dozen list quadrats, when carefully located in the prairie formation, gave results almost identical with those obtainable from a larger number. With a little experience, the various degrees of mixture can be picked out superficially, and the corresponding number of quadrats established. If a single list quadrat is to be made for a formation or station, such a time should be selected as will make it possible to cover the greatest number of plants. Fortunately, this usually falls near the middle of the summer, when the remains of spring plants are still in evidence, and the autumn ones are sufficiently developed to be recognizable. In taking the census of different aspects, the quadrat should be made as near the middle of the period as is possible.

205. Manner of use. In listing a quadrat, i. e., counting the individuals of each species, the plan followed is to list the smaller, less conspicuous plants first, since they are apt to be tramped down. As a rule, the outside tapes and the taller species afford sufficient landmarks. When this is not the case, the measure tape is used, and the individuals of all species are checked as they are found, while in the first method one species, rarely two, is taken at a time. In cases of peculiar difficulty, it may be permissible to pull or break plants as they are counted, but ordinarily this can and should be avoided. Clusters, and bunches of stems from the same root are counted as single plants, and the number of stems indicated by an exponent. In the case of bunch grasses, each bunch counts as one plant.

206. Table of abundance. The species are arranged in the final list in the order of their numerical importance, and are divided into groups which correspond to the different degrees of abundance. The latter are arranged in two series, based upon the fact that association is by groups or by individuals. The table of abundance, based upon a 2-meter quadrat rather than upon the 5 -meter one, by means of which the earlier results were obtained, is as follows:

Social exclusive, no other species of vascular plants present social inclusive, above IOO

\begin{tabular}{|c|c|c|}
\hline gr $^{1}$ gregarious ${ }^{1}$ & $100-50$ & copious $^{1}$ \\
\hline $\mathrm{gr}^{2}$ gregarious $^{2}$ & $5^{\circ-25}$ & copious $^{2}$ \\
\hline $\mathrm{gr}^{8}$ gregarious $^{8}$ & $25-10$ & copious $^{3}$ \\
\hline subgregarious & $10-5$ & subcopious \\
\hline vixgregarious & $5-I$ & sparse \\
\hline
\end{tabular}


It is obvious that the above outline is faulty inasmuch as it takes no account of the height and width of the individuals. This is a serious defect, and it constitutes one of the many reasons why the list quadrat should be replaced by the chart quadrat. The prairie formation affords an unusually striking illustration of this. A single quadrat may be filled by ten plants of Psoralea floribunda, and at the same time contain 22,000 plants of Festuca octoflora. Yet the former is conspicuous and controlling; the latter plays an altogether insignificant role. This difference is readily shown by comparing a plant of each. The one is $3 \times 3$ feet, the other $3 \times 1 / 4$ inch. Such figures furnish a valuable check upon mere number, but make the brief, graphic designation of abundance difficult. An attempt has been made to solve this problem by roughly determining the space occupied by the plant, by means of the formula, height $\left(\pi R^{2}\right) \times$ abundance. This would give Psoralea a value of 2 Io, and Festuca one of 1.6, which much more nearly represents their real importance in the formation. Abundance or numerical value is a floristic concept entirely, and has . little place in ecology unless checked in the way indicated. The whole problem, ecologically, depends upon an intimate knowledge of competition, and its solution in consequence is at present impossible.

\section{The Chart Quadrat}

207. Description and use. The detailed labor required in mapping makes it advisable to use the meter quadrat. An additional reason of much importance is furnished by the desirability of securing a detail photograph of the quadrat. This is impossible with field cameras, which should not exceed $61 / 2 \times 8 \frac{1}{2}$ inches, and are incleed most serviceable in the $4 \times 5$ size, if the area be larger. In open formations, the major quadrat of 2 meters can be used if necessary, but this is very rarely the case. Forest quadrats of ten meters square are easily charted, but detail photographs can not be made of them. Larger quadrats are impracticable; they can be counted but not mapped to advantage. The location of the chart quadrat must be decided by the structure to be studied. Its greatest service is in connection with zcries and societies of the same formation, which can be easily compared in the chart form. In fact, the chart quadrat may well be regarded as the fundamental method for inquiry into zonation and alternation. It is an important aid in deliniting areas from the contiguous formations, and in determining the relationships of mixed formations. It is also used to record the character of the different aspects, but this is done more satisfactorily by the permanent quadrat. 
208. The chart used is a decimeter square, and the scale is consequently IO:I. It is outlined on centimeter plotting paper, and the centimeter squares are numbered at the edges to correspond to the intervals of the quadrat, i. e., the top and bottom lines are numbered from left to right, and the side lines from top to bottom. These outlines are ruled in quantity and used as needed, or the forms can be furnished by the printer. In practice, a special quadrat book the size of the chart has been used. The need of a second book may be avoided by outlining two charts on the plotting sheet, and filing the latter in the field record book. In the few cases where 2-meter quadrats are desirable, four charts are used, care being taken to label them so that they can be combined whenever necessary. Ten-meter quadrats are recorded on the decimeter chart also, each meter interval corresponding to a centimeter, i. e., the scale is IOO:I.

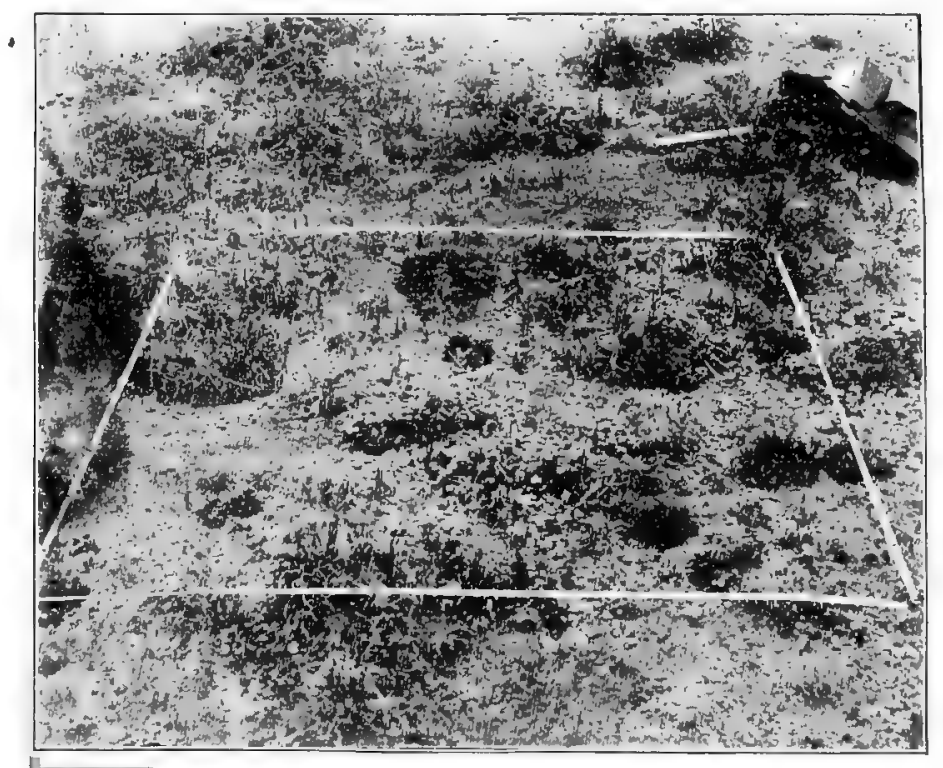

Fig. 51. Permanent chart quadrat, Andosacile, Carex-Campanulacoryphium.

209. Mapping is invariably begun at the upper left-hand corner of the chart, and is carried across the strip marked off by the plotting tape, decimeter by decimeter. As soon as this strip is completed, a second one is formed by moving the top tape to a position one decimeter below the plotting tape, which then becomes the upper one. This is repeated until the last strip is reached. Little difficulty is experienced in locating each plant exactly, as 
the decimeter interval is small, and the centimeter square which corresponds is divided into twenty-five tiny squares. Each plant is put in whenever possible, but mats, turfs, and mosses are merely outlined in mass if the individuals are not distinguishable. This holds true of all large rosettes and mats, even when they are single plants. Symbols were formerly used for indicat-

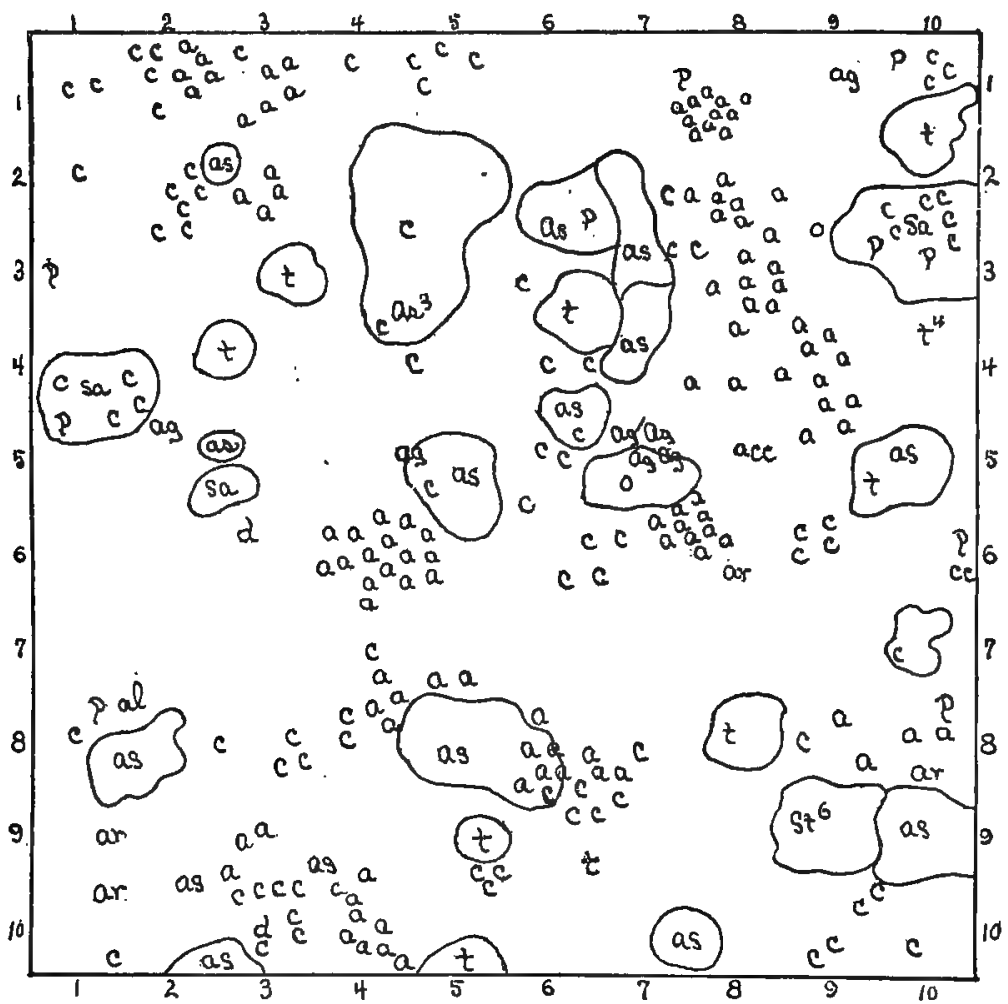

Fig. Chart of the quadrat shown in figure 51. Legend: a, Androsace chamaejasine; c, Carex rupestris; t, Tetraneuris lanata; $\mathrm{p}$, Potentilla rubricaulis; as, Arenaria sajonensis; ar, Artemisia scopulorum; ag, Agropyrum scribneri; sa, Silene acaulis; st, Sicversia turbinata; d, Dasyphora fruticosa; al, Allium reticulatum; o, Oreoxis alpina.

ing the various species. They have the advantage of requiring little space on the chart, and the disadvantage of necessitating constant reference to the legend. They are at present replaced by initials. By this plan, the decapitalized first letter of the generic name is tused if no other genus found in the quadrat begins with the same letter. If, however, two or more genera begin with a, for example Agropyrum, Anemone, and Allium, the most abundant । 
one is indicated by $a$, and the others by the first two letters, as an, al. In case two species of the same genus are present, the species initial is used in connection with that for the genus, as ac and ar for Agropyrum caninum and Agropyrum richardsonii respectively. It is rarely necessary to exceed two letters for any species. Plants which regularly have several stems from the same root are indicated by the initial and an exponent as $a^{3}$. Seedlings are represented by a line drawn through the letter. Usually the chart sheet affords sufficient space below the chart for the legend. When the list of species is long, the back of the sheet is used.

210. Factors and photographs. Each chart is numbered, and the formation, station, and date indicated. The constant factors, altitude, slope, and exposure are ascertained and recorded on the sheet. The variable factors are read in each quadrat whenever possible, and in addition to being preserved in the record book, are noted on the chart sheet along with the base reading in the formation for the same time. This facilitates the interpretation of the differences found when two or more charts are compared. Chart quadrats are regularly photographed. For this purpose a long-focus $4 \times 5$ camera with a telephoto lens is used. At the proper distance this will make a view of the same size as the chart, thus making possible an exact comparison of the two. The chart and photograph serve as mutual checks, as well as complements, since the former shows number, position, and arrangement, and the latter, height, form, position, and arrangement. The view is usually made by placing the camera directly in front of the middle of the lower tape, at such a distance that the side tapes fall just within the limits of the ground glass. The swing is always used in order that the focus may be uniformly sharp. Surface views of the quadrat can be taken by means of a device which permits the camera to hang downward from the tripod, or by means of a tripod with a swinging platform. Such views are especially valuable for the study of competition, since they give a clear idea of the spread and density of the various plants. They are difficult to make unless the vegetation is low and nearly uniform in height. The usual photograph is much more serviceable in regular quadrat work.

\section{The Permanent Quadrat}

211. Description and uses. As stated heretofore, either list or chart quadrats may be rendered permanent in order that they may be followed from season to season or from year to year. As a matter of fact, however, an area which is to be studied repeatedly really demands charting, and in practice chart quadrats alone are made permanent. This is done simply by driving 
a labeled stake at one corner of the quadrat, and locating the latter definitely in relation to a conspicuous landmark. When one is in residence for several years, practically all chart quadrats are converted into permanent ones, since the work already done in the chart quadrat is so much accomplished towards the permanent one. This is not necessary when one wishes merely to compare different areas of stable formations. As a rule, however, some change is constantly being wrought by invasion or competition, and the amount and direction of this can only be revealed by the permanent quadrat. The latterhas a fundamental value for all kinds of invasion, but it is absolutely indis-

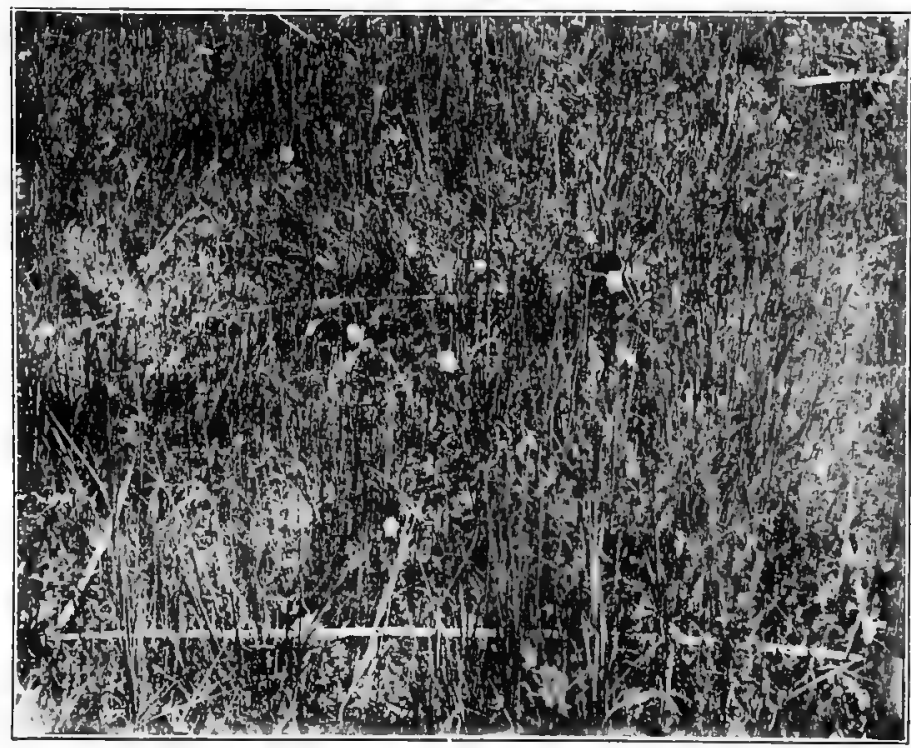

Fig. 53. Permanent quadrat, Polygonile (Polygonum bistortoides) Ruxton Park; mapped and photographed July 22, denuded September 8, 1903.

pensable in studying complete invasion or succession, and in discovering and recording the gradual effects of competition. It is in the detailed investigation of these dynamic phenomena that the paramount importance of the quadrat is most evident. If the experience of several years be taken as conclusive, no other method is capable of revealing the minute changes as they are occurring.

The permanent quadrat is regularly I meter square, a size determined both by the exigencies of charting and photographing. When ecograph batteries are used, the quadrat is located as close to the latter as is possible. Other- 
wise, the quadrat itself should constitute a station for making factor observations. This connection is absolutely essential, since the quadrat is used expressly to determine the structural changes, which are produced by physical factors, and the reaction of vegetation upon them. Permanent quadrats are established in different formations or stages of a succession to trace the invasion of new species and the dropping out of old ones in response to competition. They serve to distinguish the proper formation, which represents a particular stage of development, from the mixed formations which precede and follow, and also to determine the exact course as well as the rapidity of the change that follows each reaction. When applied to different examples of the same stage, and to all the different stages of a succession, the whole development of the latter may be minutely traced and definitely recorded. The importance of following the changes from aspect to aspect is much less, since these are periodical rather than dynamic. They are an essential feature of structure, however, and it has been the practice to make at least one series of aspect charts from each permanent quadrat.

For tracing the invasion and competition of lichens and mosses, which play a primary role in initial formations, a subquadrat is used. The size varies, but it is usually smaller than the quadrat, although the latter is entirely available in the case of the large foliose lichens. For the crustose and smaller foliose forms, a subquadrat 2 decimeters square is used, and for the larger forms and tufted mosses, one of 5 decimeters. In the case of ground forms. tapes are employed, and the quadrat is permanentiy staked. On rocks and cliffs, where moss and lichen stages are most common, tapes are impracticable, and the quadrat is permanently outlined with paint. Charts of lichen quadrats are made to the usua! scale of IO:I.

212. Manner of use. Permanent quadrats are mapped and photographed in exactly the same way as chart quadrats. As soon as this has been done, a labeied stake is driven at the upper left-hand corner, so that its edge indicates the exact position of the quadrat stake, and a smaller one is placed at the opposite corner to facilitate the task of setting the tapes accurately in later readings. The label stake bears merely the number of the quadrat and the date when it was first established. It is firmly fixed and allowed to project just enough to enable it to be located readily. Its position requires careful landmarking when the quadrat is to be visited year by year. In forest formations, this is readily done by blazing, but in grassland it is necessary to have recourse to compass and pacing, or to erect an artificial landmark. After several charts have been made, a permanent quadrat attains a high value, and every precaution must be taken to prevent losing its exact location. At the second reading of a quadrat, whether in the succeeding aspect 
or year, the tapes are placed with reference to the stakes, and a chart and photograph are made in the ustral manner. These are labeled and dated like the original ones, but they are numbered to indicate both the quadrat and the series, e. g., $\mathrm{I} 5^{2}$ indicates the second chart, and photograph made of quadrat I5. The date indicates whether the readings are by the aspect or the year, though this may be shown also in the name of the series itself. It is clearly an advantage to have the two successive charts of a quadrat upon the same sheet, and to file all the charts and photographs of the same permanent quadrat together, and in the proper order.

Since much of the value of a permanent quadrat depends upon its use as a station for observing physical factors, it is unprofitable to establish a large number. The results of invasion and competition can be ascertained by the quadrat alone, but these should be merely preliminary to seeking for their causes. Clearly, a quadrat should be established for each battery of instruments, while additional ones should be located only in so far as they can be visited often enough to give an insight into the factors that control them. In view of the fact that the most important factors, water-content and light, are less variable than humidity, temperature, and wind, it will suffice if visits are made once a week. This is especially true when it is possible to refer the more variabie factors to the continuous records of a base station. While all the results determined for permanent quadrats are preserved in the field record, a record of them is also kept on the reverse of the chart sheet for convenience in interpreting the different charts.

\section{The Denuded Quadrat}

213. Description. This is primarily a permanent quadrat from which the plant covering has been removed, after it has been charted and photographed. What is practically the same thing is obtained by establishing a permanent quadrat in a new soil, or in one recently laid bare and not yet reclothed with plants. These, however, are merely permanent quadrats, in which the first chart and photograph furnish a record of the habitat alone. They are of great importance in succession, and will be more fully discussed under experimental vegetation. The denuded quadrat is of the usual size, I meter, though the smaller lichen quadrats are also denuded. The location is subject to the conditions already indicated, especially with reference to physical factors. The denuded quadrat, however, is particularly adapted to the study of invasion and the resulting competition. Consequently, when migration is markedly from one direction, a series of denuded quadrats throws a flood of light upon the actual steps in invasion. Denuding is a valuable aid in succession, but it must be clearly recognized that, while permanent quadrats 
register the exact course of the succession, denuded ones can merely furnish facts as to the probable courses of stages not now in evidence.

214. Methods of denuding and recording. Permanent quadrats may be denuded at any time during the time they are under observation. The best results, however, are to be obtained by establishing the two side by side, or at least close together. In this way, they are mutually supplementary, and furnish the most evidence possible with regard to the procedure of invasion and competition. Another advantage is found in that the same observations of climatic factors will do for both, though water-content and soil temperatures

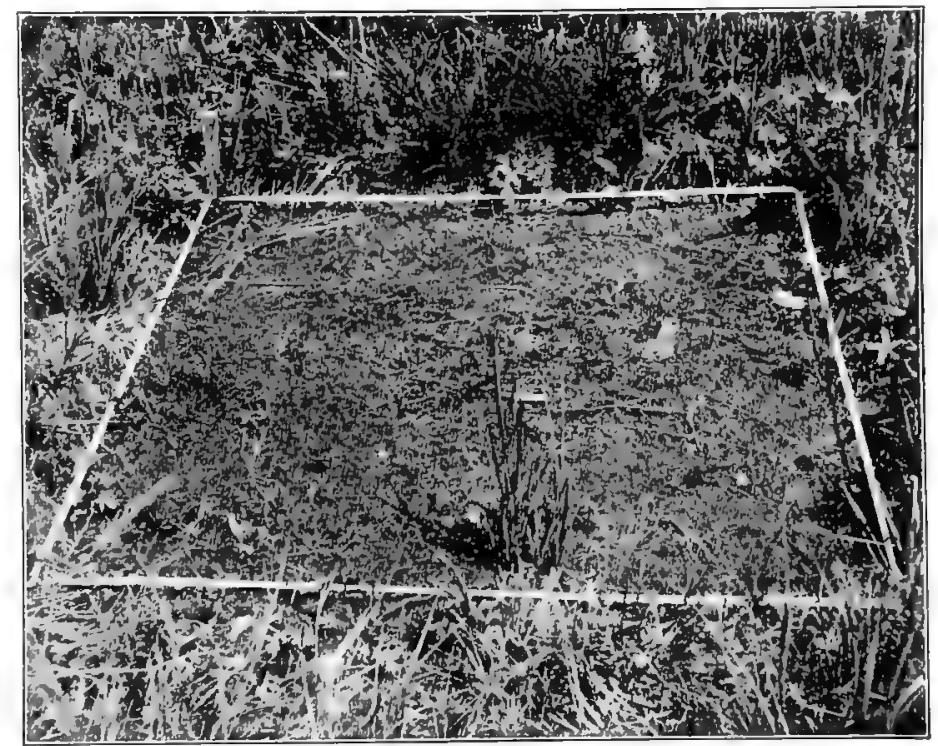

Fig. 54. Denuded quadrat; this is the quadrat shown in figure 53 ; photographed September 7, 1904.

are necessarily different. A quadrat which is to be denuded is first mapped, photographed, and labeled exactly like a permanent quadrat. The vegetation is then destroyed. This is usually done by removal, though it may also be burnt, destroyed by flooding, or in some other manner. The method will depend upon the use which the quadrat is to serve. If it is to throw light upon the vegetation of an area in which denudation has affected the surface alone, the aerial parts only are removed by paring the surface with a spade. When the disturbance is to be more profound, the upper seed-bearing layer is removed, and the underground parts dug up. 'In the interpretation of a 
secondary succession, the denuding cause is made use of in a fashion as nearly natural as possible. Ordinarily, the plants are removed just below the top of the ground by a spade, leaving the underground parts undisturbed. This method has yielded very interesting results.

Quadrats have been denuded in the fall after the majority of the plants have completed their growth. This is largely owing to the fact that other field work is less pressing at this time. Denudation can be done as well in the spring, though the invasion will be slower in this case, since the seeds which have accumulated will be partly or entirely removed. During the first season the denuded quadrat should be mapped every month, and, if the invasion be rapid, photographed also. In open formations, especially those of a xerophytic nature, a single chart and photograph made at the end of the season are sufficient. In a few cases of this sort, indeed, no invaders have appeared until the second year. Beginning with the second season, a single record taken near the close of the growing period will suffice. Denucled quadrats are labeled, dated, and filed exactly as other permanent quadrats, but it should be noted that the first member of the chart and photograph series is that which records the original vegetation of the area denuded.

215. Physical factors. When denuded quadrats are single, their physical factors must be observed in the usual way. If they are associated with permanent ones, the ordinary readings are made for the latter, and those factors which are affected by exposing the soil are alone taken for the denuded area. These are the water-content, soil and surface temperatures, and in some stations at least the humidity near the surface. As everywhere, water-content is the most important, but the temperature at or near the surface has a marked effect upon germination. Because of its bearing upon the latter, the surface water-content is usually determined also. This has been done by taking a surface sample 2 inches square and I inch deep. Denuded quadrats naturally show considerable differences from year to year as the action of the invaders becomes more pronounced. To this fact is due much of their value as aids in interpreting succession.

\section{Aquatic Quadrats}

216. Scope. The preceding discussion of quadrat methods is based wholly upon their use in terrestrial formations. Wet meadow and dry bog are the wettest places in which quadrats have been used. It is clear, however, that with certain necessary modifications, quadrats can be used as successfully, though not as conveniently, in many water formations as in land ones. The tapes need to be raised above the surface of the water by longer stakes, and 
photographs often taken from a boat, but otherwise the usual methods apply, at any rate for bogs and shallow bodies of water. In lakes or streams the tapes might be attached to buoys or floats. The determination of factors is made as usual. Permanent quadrats are feasible in many cases at least, and denuded quadrats are not altogether impossible.

\section{TRANSECTS}

217. The transect is essentially a cross section through the vegetation of a station, a formation, or a series of formations. It is designed primarily to show the order of arrangement of species in zones and societies, but it also serves as a record of the heterogeneity of any area. In the form of the layer transect, it furnishes a graphic method of representing the spatial relations of the species in layered formations, e. g., forests, ponds, and lakes. It is merely a logical extension of the idea underlying the quadrat, and the transect is, indeed, little more than an elongated quadrat. An important difference, however, lies in the fact that the former normally traverses areas more or less unlike, while the latter is always located in a homogeneous one. Furthermore, the transect is plotted with especial reference to the topography. With respect to dimension, transects are classified as line, layer, and belt transects, and the latter may also be permanent or denuded.

\section{The Line Transect}

218. Description and method. A simple transect is sometimes made by establishing the points between which it is to be run, and then recording the plants pace by pace along this line. This is satisfactory where the striking changes in structure are desired. A more accurate method is ordinarily used, since it gives detailed results, and at the same time brings out the more general features. For this, use is made of a tape of proper length which is divided into decimeters. Tapes of 10, 50, and Ioo meters are used, and if they are furnished with eyelets, transects of intermediate lengths may be run with them. When longer transects are desired, as in the case of forest formations, tapes of 500 or 1,000 meters should be used with eyelets a meter apart. The transect is located in the area to be studied by running the tape from one landmark to another, fastening it here and there by means of quadrat stakes. Previous to this, the shortest distance between landmarks is ascertained when the transect runs through a depression or upon a level surface. In the case of an elevation, the height is ascertained by a barometer, the length and angle of the two slopes obtained, and the length of the base line determined from these data. The field record of the arrangement of the 


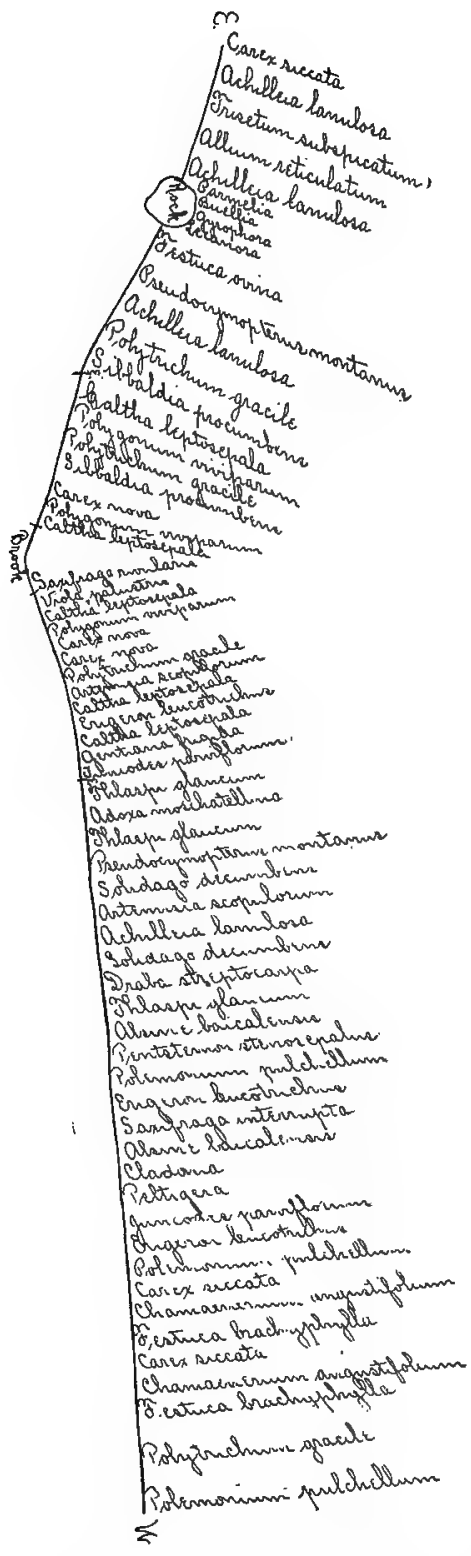

Fig. 55. Line transect running east and west in the Picea-Pinus-hylium, showing the relation of the herbaceous layer to the Carex-Catha-helium, invading along the brook; ecotones at $e$.

plants is made entirely without reference to the surface line. The vertical lines on the centimeter sheet are taken to correspond with the tape, and the individual which touches the latter on either side is recorded to the right or left respectively and within the proper square: The species are indicated as for quadrats. A single row on either side may be taken alone, but the double series serves as a desirable check. After the record is made, the topography of the transect is drawn carefully to scale. This drawing is made upon the scale of Ioo:I for transects of io meters or less, and of I000:I for those that are longer. The combination of this drawing with the line series of plants can not be made advantageously in the field. For the shorter transects, meter sizes of centimeter plotting paper can often be used to advantage. In this event, the topographic line is drawn to the scale of IO:I and the series of plants transferred directly to it. In the case of transects between Io and Ioo meters, the scale of the drawing is increased from IOOO:I to IOO:I, so that each decimeter of the original series is compressed into a centimeter. For the longest transects, corresponding reductions must be made, but in these it will be remembered that the series is plotted by meter instead of decimeter.

219. The location and size of line transects are determined by the purpose for which they are designed. Short transects are valuable for detail, but they can be used to advantage only where 
changes in arrangement are taking place rapidly. They are especially adapted to the study of minute alternations and to the zonation of small ponds, st:"eams, ditches, roads, blowouts, etc. Longer transects can not furnish the same detail, on account of the amount of time necessary, but they are invaluable for the zonation and alternation of larger areas, such as the consocies, formation, and formation series. They are of particular importance for the record of zonation, since they afford a clue to the topographic symmetry of the area. The location of a transect depends upon the area to be studied, though it should always run through a portion as typical as possible. The general direction is ascertained by means of the compass, and when there is a measurable clifference in elevation it is taken by the barometer or otherwise.

The points at which ecotones cross the transect are carefully indicated upon the chart. They serve as stations for simultaneous readings of physical factors, though in the majority of cases water-content readings alone will determine the reason for the ecotone. Photographs of line transects should be made while the tape is in position, in order that the superficies of the series may be as evident as possible.

\section{The Belt Transect}

220. Details. This differs from the line transect in that it is wider, and consequently affords a more accurate record of the arrangement of plants. While both give the actual facts of distribution, the line transect necessarily ignores the minor lateral deviations in position. These are brought out in a strip of some width, and the belt transect thus gives a more correct view of the variations which result from competition in an area physically homogeneous. The width of such transects depends upon the length, and the character of the vegetation. The standard width is one decimeter in herbaceous formations, and one meter in the long transects which are used in woodlands. In open vegetation, especially in the initial stages of successions, the width may often be increased to advantage, but ordinarily the amount of work necessary to run a belt transect of some length limits the width to one decimeter.

The location of a belt transect, the choice of landmarks, the determination of direction and elevation are made exactly as for the line transect. The topographic map is made in precisely the same way also, the scale used depending upon the length. Two tapes, however, are employed, and these are placed so that they mark off a strip just one decimeter wide. Every few meters, or oftener if need be, they are checked by a decimeter rule, and fixed firmly in place by quadrat stakes. The arrangement of the plants is recorcied 
as for the line transect, except that the record covers a decimeter strip just as in quadrat work. Accordingly, an interval of a centimeter is left on the sheet between the successive portions of the strip, in order that the latter may be put together without confusion when the topographic map and the plant series are conibined. The record should invariably start in the upper left-hand corner and read down. The map and the centimeter strip recording the plants of the transect are combined on a common scale as already indicated for the line transect.

The ecotones of zones are shown on belt transects by single cross lines, and those of consocies by paraliel cross lines. In taking photographs of the transect, it is desirable to use guidons to mark these points clearly. The same device may also be used to indicate the course of the transect, when the tapes are completely hidrlen by the plants. Physical factor readings should always be taken, and, as before, they are best made at the intersections of the ecotones.

\section{The Permanent Transect}

221. Advantages. Both line and belt transects, after they have been recorded, should be rendered permanent, in order that they may serve to indicate the changes of a heterogeneous area from year to year in the same detailed fashion that the permanent quadrat does for homogeneous ones. For historical as well as for physical reasons, the ecotones of zones and of consocies are subject to change from year to year, and the amount and direction of this change can cnly be ascertained from annual records made in exactly the same spot. By means of the permanent transect alone the very origin of such areas can be followed from one stage to another of the succession. Moreover, the transect is equally valuable with the quadrat in making it possible to follow every step of the minute changes wrought by competition.

222. Details. The transect is made permanent by blazing the landmarks at aither end, if these already exist, or by erecting them when it is necessary. A label stake is driven at each end, on which is painted the number and date of the transect and its length. Each stake should also indicate the exact direction in which the other lies. The position of the ecotone is indicated by smaller stakes bearing the number of the transect and the date when the ecotone was found at that point. These are left in place, and in a few years show very graphically the change in position of the zones. For the first season, permanent transects afiord results of great value when recorded for each aspect, but after this an annual visit will suffice. The details of mapping, plotting, etc., are identical with those indicated above, with the addition 
that all charts and photographs must bear the number of the reading as well as that of the transect. Physical factor observations are taken as often as the charts are made, and the results noted on the back of the chart sheet for purposes of ready comparison.

\section{The Denuded Transect}

223. The denuded transect bears exactly the same relation to a permanent one as that which exists between the denuded and the permanent quadrat. While the permanent transect records the actual mutations due to changing physical factors or to competition, the denuded transect throws needed light upon the mobility and ecesis of the various species, and upon the nature of the competition between them. Denuded transects may be established wherever it seems desirable, after the strip has been properly charted and photographed. The most valuable results, however, are secured by locating each one alongside of a permanent one. The best plan is to locate and chart two permanent transects a meter apart. A single view is then made of the two. One of them is denuded together with a strip 2 decimeters on either side, resulting in a denuded transect 5 . decimeters wide. In charting this during succeeding years, the entire width may well be plotted as long as the vegetation is open, but after it has again become well established, it is necessary to save time by confining one's attention to the central decimeter strip. Photographs can be made either of the permanent and denuded transects singly, or of the two together. The latter method has certain obvious advantages. Climatic factor readings can be made for both transects in common, but all those factors which are affected by the exposure of the soil surface must be observed in each.

\section{The Layer Transect}

224. This is a modification of the line transect, by means of which the vertical relations of plants are also shown, especially the tendency to form layers which is so regular a feature of forest formations. . Owing to the difficulty of charting in three planes, belt transects do not lend themselves' to this purpose. Because of the greater complexity, layer transects can rarely exceed ten meters in length except in those formations where layering is little or not at all developed. The simplest method is to establish a line transect in the ordinary way, and then to record the height of each plant as its position is noted. This is done by means of a measuring stick ruled in decimeters, which can be moved from interval to interval along the tape, or better, by two such sticks connected by tapes a meter long at every five deci- 
meters of the sticks. These should be two meters high for woodland, and one meter for grassland. Layer transects often run on even surfaces, but if this is not the case, the usual data for a topographic map should be taken. The final chart is constructed on the scale of Io:I, the height of each plant being indicated by a vertical line equal to.I of the observed height. A photograph of a representative meter of the transect is taken when the measuring sticks and rods indicated above are in position. Physical factor readings, principally of light, but often also of humidity, temperature, and wind are made at the height of the various layers when these are present.

\section{ECOTONE CHARTS}

225. The contour lines of zones and consocies are of the utmost importance in recording the structure of vegetation. They do not permit such accuracy as do quadrats and transects, but this is hardly to be considered a disadvantage in view of the fact that ecotones are rarely sharply defined. In establishing the ecotones of zonation, the width and the length of the base, i. e., the area of excess or deficiency, or as much of it as is to be considered, are determined. This base may be road, ditch, pool, lake, or stream, or the peak or crest of a hill, ridge, or mountain. When the zonation is bilateral, meter tapes are run at right angles to the base, at proper intervals, and the points and the distances where the ecotones cross are noted. In the case of radial zones, the tapes are run in the four cardinal directions, and if the base be large, in the four intermediate ones also, the intersections being likewise noted. From the data thus obtained, the zones may be outlined with a fair degree of accuracy. If the series be an extensive one, it is charted to the scale of I00:I ; in cases of small areas, however, the scale of IO:I will give better results. Whenever the zones show clearly enough to warrant, a photograph is also taken. Water-content readings are of paramotnt importance in the interpretation of zones. Samples should be taken at all intersections, and the resulting values indicated at the corresponding points upon the chart. When the zones are broken up into alternating patches in consequence of asymmetry in the topography, the ecotones of the latter are traced in a similar fashion from the center of each as a base, the absolute position of which is ultimately determined with reference to the ecotone lines already establisherl. 
226. Purpose. The migration circle is designed to record the invasion of species, since it operates outward from an individual or a group of plants as a center. As migration takes place to a certain degree in all directions, a circle is better adapted to the purpose than the quadrat. From the very nature of invasion, migration circles should always be permanent in order that the yearly advance may be accurately noted. Circles of this character are important aids in the study of any vegetation, except, perhaps, one that has practically become stabilized. Their great value, however, is found in succession, where it is necessary to trace the movement of new individuals away from the original invaders as centers of colonization.

227. Location and method. The size of the migration circle is largely controlled by the density of the vegetation, and in some degree by the height of the species also, since this determines the trajectory of the disseminule. In close formations, a circle of 1 -, rarely of 5 -meter radius can best be used, but in the more open initial stages of succession a radius of 5 , Io, or, in exceptional cases such as open woodland, even 25 meters, affords the best results. The location should always be made with a plant or group of plants of the species to be studied as a center. This migration circle differs from the quadrat in that it is used to show the movement of one, rarely two or three species, and not the position of all the plants within it. The center is permanently fixed by driving a labeled stake with the number of the circle and the data. Two tapes the length of the radius are used for recording. These are provided with the usual eyelets, 5 decimeters apart, and are fastened on a peg in the top of the central stake so that they move readily. At the outer ends they are staked 5 decimeters apart by a tape of this length when the radius is I meter, and I meter when the radius is 5 or to meters. The record forms must be especially prepared on blank sheets about 9 inches square. The scale is IO:I for circles of I meter, and IoO:I for those of $5^{-}$and Io-meter radius. In the former, concentric circles are drawn about the center at intervals of 5 decimeters, and radii are drawn to the circumference at the same interval. In the larger circles, the intervals are $\mathrm{I}$. meter. Each segment of the circle is read by means of the two tapes, and the position indicated with reference to the concentric lines and radii. When but one species is read, a tiny circle is used to denote the position of each plant. If more than one is used, the symbols are those already indicated for the quadrat. One tape is left in place and the other with the segment tape is shifted to a new position, and the resulting segment is read as before. The exact position of the base radius is fixed by a label stake; 
in order that the segments of successive years may exactly correspond. The record sheet is labeled, dated, and filed. By folding at one edge, it may be filed in the regular field book.

228. The denuded circle is established in the same way as a permanent one. The original position of the individuals of the species under consideration may be recorded or not, depending upon the use to be made of the results. The safest plan is first to read the circle in the usual way, and then to denude it. The latter should be done in such a way as to remove all the disseminules from the surface in so far as possible. It is essential also that this be done before the seeds are mature and begin to be scattered. The central plant or cluster is of course not removed. In special cases, all the plants of the species are allowed to remain to serve as centers of colonization. The successive yearly readings of the denuded area are made exactly as for a permanent circlc. Permanent and clenuded circles, like quadrats, should always be established near each other so that they permit of ready comparison under similar conditions.

229. Photographs of migration circles furnish the most detail when the camera is placed just behind the central group in such a way as to show its relation to the other individuals or clusters of the circle. In the denuded circle, or when the plants stand out conspicuously from the bulk of the vegetation, it is not necessary to use guidons, but in other cases the latter greatly increase the value of the picture. Factor readings are less important for migration circles than for quadrats and transects. The factors of principal importance are those that deal with migration and ecesis, $i$. e., wind, water-content, and soil temperatures. The former may be determined for both circles in common, but the conditions that affect ecesis must be observed separately for each.

\section{CARTOGRAPHY}

230. Value of cartographic methods. Chart, map, and photograph aré records indispensable to the systematic study of vegetation. They serve not merely to preserve the facts ascertained, and to permit their ready comparison, but they also put a premium upon accurate methods, and consequently bring to light many points otherwise overlooked. For ecology, they have the value which drawings possess in taxonomy, in that they make clear at a glance what pages of description fail to indicate. They are the fundamental material of comparative phytogeography, and in all careful vegetational study their use is no longer optional but obligatory. 
Hence it is obvious that cartographic methods should be clear and simpie, and that they shoulci be uniform, so that charts and maps of widely separated formations may be directly compared without difficulty. It is not to be expected that uniform methods will come into general use immediately, but a proper appreciation of the obligation that rests upon every ecologist to make his results both easily comprehensible and usable will serve to procluce this very necessary result. In the treatment that follows, as elsewhere, no attempt is made to describe the general cartographic methods used by other: ecologists, notably Flahault. The methods employed by the author form a complete system, which has proved valuable, and for various rcasons it alone is discussed here.

231. Standard scale. The question of the scale to which charts and maps are to be made is of primary importance. The general principle is that the ratio betwcen area and drawing should be as small as possible. Moreover, charts and maps of the same character should always be drawn to the same scale, unless a good reason to the contrary exists. The ideal scale is I:I, which is manifestly an impossibility. This is approached most nearly in the quadrat chart where the scale is IO:I. Charts of definite areas are made on a scale as large as possible, while maps of formations, regions, etc., are necessarily drawn upon a very small scale. General maps designed to show the distribution of species and formations, or the vegetation of continents, are usually not drawn with reference to a scale at all. While it is manifestly impossible to use the same scale for charts and maps, it is feasible and desirable that they be constructed upon scales readily convertible into each other. This is most satisfactorily accomplished by means of the decimal system, and the various type scales are Io:I, IOO:I, I000:I, etc. The first two or three scales are used for charts of quadrats, transects, and circles; the remaining ones are employed in making maps of large areas. No attempt has been made to draw an absolute line between charts and maps, but an endeavor is made to restrict the term chart to the record of the number and position of plants, while maps deal with the arrangement and location of formational areas. It is hardly necessary to point out the reasons why all charts and naps should be based upon the decimal system of scales. Experience will furnish the very best of arguments.

232. Color scheme. The first requisite for the graphic representation of formations, regions, etc., is that each class of formations be invariably indicated by the same color. It is also necessary that the colors and shades be easily distinguishable, and it is at least desirable that they be referred to the different classes in some consistent sequence. Uniformity in all these 
points is greatly to be desired at the hands of all ecologists. Here, as in the case of the standard scale, uniformity will be found the more desirable the more inpossible it is made by ignoring it. In the use of color to represent regions and provinces, on maps too small to indicate formations, the color of each division is represented by the color of its dominant formation; thus the prairie province is colored ochroleucus on account of the color used to represent prairie formations, the boreal-subalpine zone atrovirens on account of the typical coniferous forests, etc. No endeavor has been made to take account of the various types of formations, e. g., the different coniferous forests, as this is a problem to be worked out for more local maps in various sliades of dark green, etc. The following color scheme which has been based upon the points made above is proposed as a satisfactory solution of the problem. The color standard used is that of Saccardo's Chromotaxia.

I. Hydrophytic Formations: blue

I. Marine: cyaneus

2. Brackish: ardesiacus

3. Freshwater: caeruleus

4. Swamps and marshes: caesius

II. Mesophytic Formations

A. Forest formations: grecn

I. Coniferous forests: atrovirens

2. Broadleaved evergreen forests: viridis

3. Deciduous forests: fanlu-virens

B. Grassland formations: yellow

I. Meadows: melleus

2. Prairies: ochrolencus

C. Culture and waste formations: red

I. Fields : ruber

2. Groves and orchards: atropurpureus

3. Wastes: purpureus

III. Xerophytic Formations : brozen

r. Deserts : isabellinus

2. Plains and steppes: avellaneus

3. Saline formations: umbrinus

4. Arctic-alpine formations: testacens

233. Formation and vegetation maps are detailed maps of a single formation or a series of them, showing the formational limits, and when the scale is not too small, the ecotones of zones and consocies. In the cases 
where the topography is level, as sometimes happens in mapping single formations, the cluain and pedometer must be used to ascertain the size of the different areas. Indeed in all mapping of vegetation, the methods of surveying are directly applicable. Over large areas, however, it is not necessary that limits be drawn with mathematical accuracy, and for the purposes of the ecologist, the plane table and camera are satisfactory substitutes for the surveyor's transit, at least in the present aspect of the subject. When the formation or group of formations is commanded by an elevation of some height, the latter is used as a base. A plane table is established upon it and the topographical and vegetational features are recorded in the usual way. This map is usually supplemented by a series of views from the same base. Indeed it has come to be recognized that a complete series of photographs of this kind give a more valuable record than the plane table, and that the construction of an accurate map from them is an easy matter. Since the camera saves much time and energy also, it is used almost exclusively to furnish the data for map making. In hilly, and especially in mountainous regions, the photographic method is indispensable. Its application is extremely simple. A central hill or mountain is selected, and from it a series of views is taken so that the edge of one exactly meets the edge of the other. This is an extremely important matter, and cemands much nicety of judgment. The camera is kept in the same spot, and after each exposure it is turned as the operator ${ }^{\circ}$ looks through it until: a landmark at one edge just passes from view at the other. As soon as the new position is determined, the tripod screw is turned to hold the box firmly in position. In case of a slight jar, the exact position should again be obtained. If the series is accurately made, the resulting prints will give a complete panoramic view of the region, without overlap or omission. For this purpose, a $6 / 2 \times 8 \mathrm{~T} / 2$ camera is desirable, since the topographic and vegetational features are larger and stand out more distinctly. A large camera requires fewer changes of position, and hence saves time and reduces the chance of error. A 4. $\mathrm{x} 5$ camera serves the purpose sufficiently well, though it requires a little more care in operation on account of the greater number of exposures necessary. This may be avoided in some degree by the use of a wide-angle lens if the depth of the area is not too great. Whatever camera may be used, a telephoto lens is a very desirable adjunct, since it enables one to choose between three different sizes of the view without changing the position of the camera. To avoid possible confusion, the exposures are always made from right to left, and the plates are used in the numerical order of their holders. For the same reason the landmarks are described and numbered in their proper order. The prints obtained are mounted on a card in sequence. The view map may be preserved in this form, or it may be reduced or 
enlarged by making a copy to the size desired. Outline maps of topography may be traced from the resulting negative, and the formations filled in by means of the proper colors. The most satisfactory method, however, is to have the original views or the copy printed "light" and to color the formations just as they appear there, with all the wealth of topographic and vegetational cletail. If a detailed topographic map alone is desired, this is traced directly from the large copy.

234. Continental maps. A method of determining the general outlines of regions, provinces, and vegetational zones as a preliminary to their cletailed study has been used successfuily for several years. ${ }^{1}$ This is based upon provincial and continental maps on which are traced the geographical areas of the species of genera typical of the various formations. Detail topographic maps of the prairie province and the North American continent have been used for this purpose. A number of the facies of extensive and representative formations of the different portions of the continent are selected and grouped according to genera. One map is devoted to each genus, unless the number of species is large. In this case a number of maps are used, since the limits are apt.to become confused. The range of each species is determined from all the reliable sources, and a corresponding line is drawn upon the map to delimit its geographical area. The limits of the area of each species are drawn in a different color, and the name of the species printed in the same color in the legend. Although this work has as yet been done only for the trees of North America, and for the grasses and principal species of the prairie province, it promises to constitute a final method for the limitation of vegetational divisions. It is clear that if the original data concerning ranges are accurate, the increasing study of formations will do little more than rectify the detailed course of the liniting line, since in most cases facies and formations coincide in distribution. The limiting line or ecotone of a zone or province is a composite obtained from the limits of certain representative facies and principal species, and checked by the limits of species typical of the contiguous vegetations. Thus, the boreal-subalpine zone is clearly outlined by combining the limits of Populus tremuloides, Larix americana, Pinus bankiana, Abies balsamea, Picea mariana, Picea carladensis, and Betula papyracea, and checking the results by the areal limits of the hardwoods and grasses to the southward.

${ }^{2}$ Pound and Clements. The Vegetation Regions of the Prairie Province. Bot. Gaz., 25 :381. 1898. 


\section{PHOTOGRAPIY}

235. The camera is an indispensable instrument for the ecologist. Although it has too often been employed to give an air of thoroughness to work of no ecological value, it is as important for recording the structure of vegetation as the automatic instrument is for the study of the habitat. No ecologist is equipped for systematic field investigation until he is provided with a good camera and has become skilful in its use. For this reason, it is felt that a few hints concerning photographic methods and their application in ecology may not be out of place. No written advice can take the place of experience, but certain elementary suggestions and cautions will greatly shorten the apprenticeship of one who does not have the good fortune to be taught by a professional photographer. To the student of ecology, the camera is not a toy. It must be understood and operated with as much thoroughness as any other instrument, and when this is done, the results will be equaliy certain and desirable.

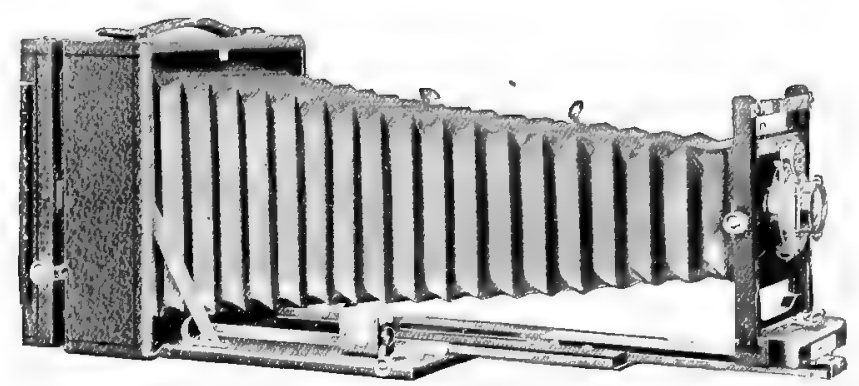

Fig. 56. $4 \times 5$ long focus "Korona" camera (series V).

236. The camera and its accessories. Although two cameras are desirable whenever it is possible to obtain them, a single one will meet all the requirements of field work. This should be $4 \times 5$ inches in size, since it is much more convenient and will do all the work that a larger camera can. In the comparatively few cases in which larger views are needed, the $4 \times 5$ negatives can be readily enlarged. The smaller instrument is less expensive in operation because of the cheapness of the plates, and it gives a negative of the proper size for lantern slides and for reproduction. A $6 \mathrm{I} / 2 \times 8 \mathrm{r} / 2$ camera is valuable in special cases, such as making a series of photographs for maps. In the writer's own experience, the $61 / 2 \times 81 / 2$ camera, although used exclusively at first, has been almost completely supplanted by the $4 \times 5$. The best field camera is of the folding type with a good stout box. It must be what is known technically as a long-focus instrument, which 
enables small objects to be taken natural size and permits the use of a telephoto lens. It should be provided with a swing and also a reversible back by which the position of the plates can be changed instantly. The lens must be of the telephoto pattern, which makes it possible to use the front or back lens either alone or in combination. The chief advantage of this is that the image, when distant, may be made of three different sizes without changing the position of the camcra. Generally speaking, the high-priced rapid lenses are the best, since it is exceptional to get the desired length of exposure in vegetation, on account of the ease with which the plants move in the wind. Before buying such a lens it is desirable to test its rapidity and depth of focus, since it is not necessarily better than some of the lenses furnished with good cameras. The lens should be provided with an iris diaphragm capable of being stopped down to 128 or 256 . The shutters furnished with the ordinary lenses are satisfactory, since "snap-shots,"

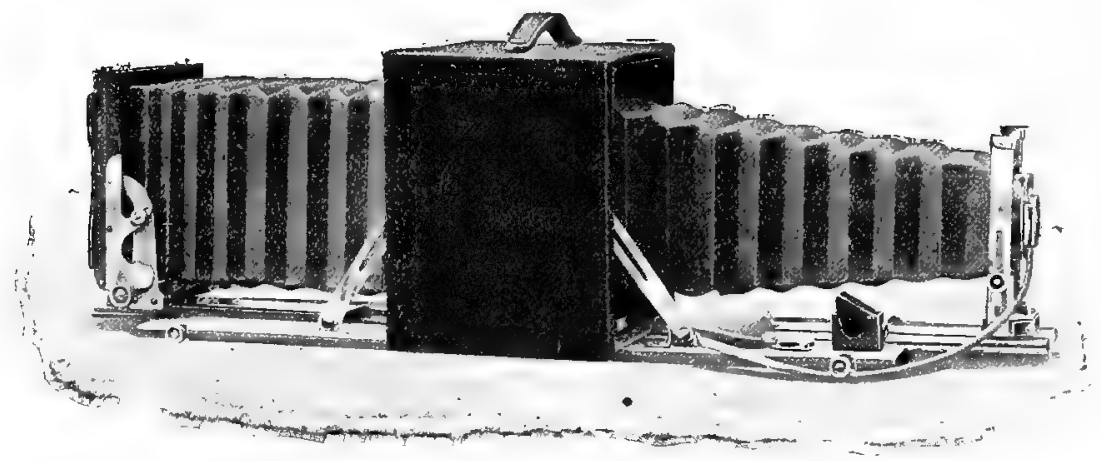

Fig. 57. $5 \times 7$ long focus "Premo" camera.

i. e., instantaneous exposures, are practically never possible for plants. The automatic shutter of the "Premo" camera is an especially convenient form. All shutters should be carefully tested before using to determine the exact time value of the exposures indicated. It is not uncommon for the exposure at I second, or at other points, to have a value quite different from the one indicated. When this is the case, it is evident that it can not be known too soon. The camera should have at least a half-dozen double plate-holders. These are numbered consecutively so that the figure uppermost when the holder is in the camera will indicate the number of the plate exposed. A carrying case is desiralle on a long trip when all the plate-holders must be taken, but ordinarily it is a disadvantage, since the camera box will carry two or three holders. The camera cloth should be as small and light as possible, and at the same time opaque. The most satisfactory one for the 
field is the rubber cloth. The tripod should be a happy combination of lightness and stability, a condition more nearly reached by the aluminum tripod than by any other. It should have not less than three joints in order to facilitate the use of the long focus upon objects near the ground.

237. Choice of a camera. There is not a great deal of choice between the moderate-priced cameras of the various makers. A field camera is restricted to certain special uses, and hence is more serviceable when attachments useful only in portraiture or instantaneous work are absent. Even the ray filter, which has some value in the indoor photography of flowers, is useless in the field on account of the long exposure required. From considerable experience, "Premo" and "Korona" cameras have been found to be very satisfactory instruments. Doubtless the same statement would be found trise of all the standard makes, but they have not been used by the

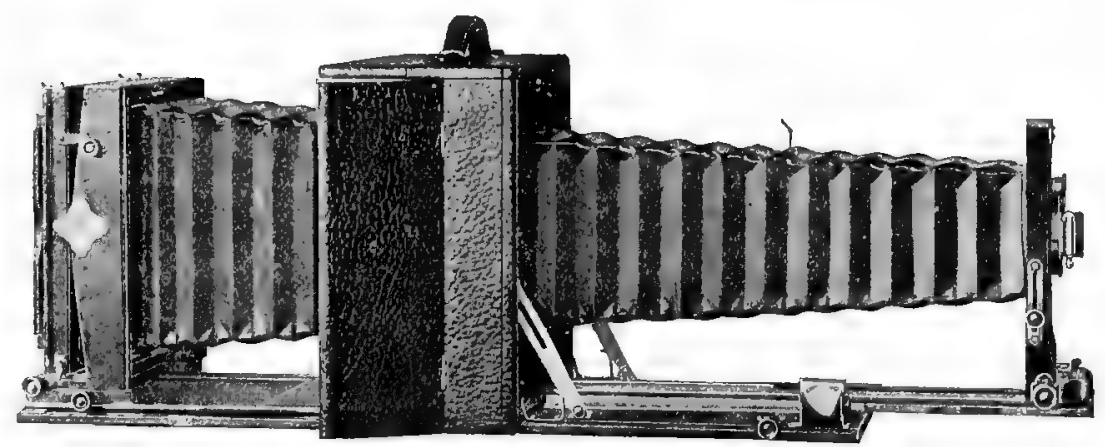

Fig. 58. $5 \times 7$ "Korona" Royal camera.

writer. "Premo" cameras are made by the Rochester Optical Co., Rochester, N. Y., and "Korona" cameras by the Gundlach-Manhattan Optical Co., Rochester, N. Y. When two or more cameras are used, the best results can be obtained if they are of the same make, since the details of operation are then the same. The reduced liability of making a blunder is often offset by the fact that a different pattern will permit of a wider range of use. Any standard brand of plates will produce good negatives when skilfully uscis; at least, this has been proved in the case of the Cramer, Hammer, Seed, and Stanley brands. Every professional photographer has his favorite brand of plate, but the ecologist will do well to give the various kinds a thorough trial, and then to invariably use the one which gives him the best results. Thus, while it seems to be less popular with the profession than the others mentioned, the writer has obtained at least as satisfactory results 
with the Stanley plate as with the others, and consequently now uses it exclusively, since it is cheaper. The one important point is to make a final choice only after personal experience, and then to always use plates of the same brand, and preferably of the same rate of speed.

238. The use of the camera. To the ecologist, objects to be photographed fall into two categories, viz., those that move, and those that do not move. For practical purposes, areas sufficiently distant to render the movement imperceptible belong to the latter, as well as those, such as rock lichens, many fungi, etc., which can not be stirred by ordinary winds. The treatment accorded the two is essentially different. A fundamental rule of ecological photography is that detail must receive the first emphasis. The ecological view should be a picture as well as a map, however, but when one must be sacrificed, artistic effect must yield to clearness, and accuracy, i. e., technically speaking, contrast must give way to detail. Leaving apart the necessity of securing a sharp focus, which holds for all work, detail or definition depends directly upon the aperture of the diaphragm. Detail is increased by decreasing the size of the aperture. This in turn increases the length of time necessary for a proper exposure, and consequently the danger that the plant will be moved in the midst of the exposure. When the movement is negligible, the invariable rule should be to reduce the aperture to its smallest size, and to expose for a corresponding time. In all cases where the plants are close enough to show even a slight blurring on account of the action of the wind, the time of exposure must be reduced, in the hope that a short period of quiet will suffice for it. This reduction in time must be compensated by increasing the aperture of the diaphragm, and hence the amount of light which strikes the plate. The proper balance between the two is a matter of considerable nicety. It depends much upon the vagaries of the wind, and can readily be determined only after considerable experience. Although regions naturally differ somewhat in the nature of their winds, much experience in prairie and mountain regions warrants the primary rule that views of vegetation and plants subject to movement are not to be attempted on windy or cloudy days when it can possibly be avoided. Even on reconnaissance, a poor picture is no better than none at all, while in resident work a time will come sooner or later which will permit the making of a view satisfactory in all respects. There may be occasional instances when one is rewarded for keeping the camera trained on a particular spot for hours, and for wasting several plates in the hope that still moments will prove to be of the requisite duration. As a regular procedure, however, this has nothing to commend it.

Various methods have been tried to reduce or eliminate the trouble caused by the wind. Canvas screens have been used for this purpose with some 
benefit. When the picture is worth the trouble, a tent may be erected to afford a very efficient protection. This is too prodigal of time and energy, however, to be practicable under the usual conditions. Flashlight exposures on still nights are sometimes feasible, but the disadvantages connected with them are too great to bring them into general use. The best procedure is to bide one's time, and to take quadrats, transects, and other detail areas, as well as many plant groups, at a time that promises to be most favorable. Single plants can often be moved in the field so that they are protected from the wind, or so that they are more strongly lighted. Slender, or feathery plants are usually very difficult to handle out of doors. The best plan is to photograph them in a room that is well and evenly lighted, or, best of all, in a stable, roomy tent.

239. The sequence of details. No photographer ever escapes blunders entirely. At the outset of his work, the ecologist must fully realize this, and accordingly plan a method of operating the camera which will reduce the chance of mistake to a minimum. The ustual blunders which every one makes sooner or later, such as making two exposures on one plate, drawing the slide before closing the shutter, allowing the light to strike the plate through the slit in the holder, etc., can be all but absolutely avoided by a fixed order of doing things. This order will naturally not be the same for different persons; it is necessary merely that each have his own invariable sequence. The following one will serve as an illustration. As a preliminary, the plate-holders are filled, after having been carefully dusted, and the slides are uniformly replaced with the black edge inward. It is a wise precaution to again see that all the slicles are in this position before leaving the dark room. This will cnsure that a black edge outward always means that the plate has been exposed. The tripod is first set up and placed in what seems about the proper position. The camera is next attached to it, and the front and back opened. The bellows is pulled out, a short distance for views, and a longer one for detail pictures, and fastened. It is necessary to move the diaphragm index to the largest aperture and to open the shutter at "time." The next steps are to orient the view or object, and to bring it into sharp focus upon the ground glass. The first is accomplished by moving the entire instrument, changing the position of the tripod legs, swinging the camera upon the tripod, or by raising or lowering the lens front. It is often desirable also to change the position of the object on the plate by use of the reversible back. In views with much distance, the foreground is brought into sharp focus. In close views, especially of quadrats, the swing is used to increase the distance for the foreground, and the focus is made upon the center. After focusing, the shutter is closed, the indicator set at the time 
desired, and the diaphragm "stopped down" as far as possible. Plate-holder I is slipped into place, care being taken not to move the camera by a sudden jar. The camera cloth is dropped above the holder and allowed to hang down over the slide end. The slide is drawn and put on top of the instrument, the black edge always up. The exposure is made and the slide replaced zeith the black edge outreard. This point shotild receive the most critical attention, as a blunder here will often cause the loss of two negatives. The plate-holcler is returned to the receptacle, or merely placed in the back of the camera, which is then closed. The number of the plate, the name of the view or object, the condition of the light, the length of exposure, and the aperture of the diaphragm, as well as the date, are recorded in a notebrook for this purpose. The shutter is then opened at "time," the diaphragm thrown wide open, and the front of the camera closed. When distances are short, the camera is often carried upon the tripod. As a rule, however, it is usually removed, and the tripod folded. In making subsequent pictures, the plates should always be used in their numerical order.

240. The time of exposure is obviously the most critical task in the manipulation of a camera. The time necessary for a proper exposure varies with the season, the hour, the condition of the sky, the light intensity of the formation, the color and size of the area to be photographed, and, finally, of course, with the aperture of the diaphragm. Fortunately for the ecologist, the variation in light intensity during the season, and even during the greater part of the day, is not great, and can ordinarily be ignored. The beginner will make the most progress by determining the exposure demanded by his instrument for taking a general view in full sunlight and with the smallest stop of the diaphragm. In standard cameras with lenses of ordinary rapidity, this is tusually about one second. This will serve as a basis from which all other exposures may be reckoned until one has worked through a wide range of conditions and can recall just what time each view requires. On completely cloudy days the time required is five to ten times that necessary on a clear day; filmy clouds and haze necessitate an exposture of two or three seconds. The more open forest formations demand an exposure of about five to ten seconds on a sunny day, while the deeper ones require two or three times as long. A close view requires more time than a distant one, since the light-reflecting surface is much smaller. Quadrats require two or three seconds, and individual groups frequently take a longer time. The color of the vegetation plays an important part also: a dark green spruce forest requires twice as long an exposure as the aspen forest, and a grassland quadrat taikes more time than one located in a gravel slide. In this connection, it is hardly necessary to point out that the lighted side of 
objects should always be taken, never the shaded one. The exposures indicated above are based upon the.smallest stop. The reasons for using this whenever possible have already been given. When a larger stop is necessary, the exposure is decreased to correspond; for example, a quadrat that takes three to four seconds at 256 can be taken at 64 in one second. As a rule, the sun should not be in front of the camera, but, when necessary, views can be made in this position if the sun is prevented from shining directly into the lens.

241. Developing is as important as exposing. Indeed, it may well be considered more important, since a properly exposed plate may be spoiled in developing, while an under-exposure or over-exposure may be saved. Owing to the ease with which plants move in the wind, the ecologist is obliged to reconcile himself to many under-exposures, which can be converted into good negatives only by skilful developing. Every base station should have a good dark room, equipped with running water when possible, a good ruby lantern, and the proper trays and chemicals. Prepared developing solutions are alluring because of their convenience, but after an extended trial of several kinds, the writer has reached the conviction that pyrogallic acid, or "pyro," is by far the most satisfactory in working with vegetation. Of almost innumerable formulae, the following gives excellent satisfaction and is convenient to use.

I.

II.

500 cc. water

3o grams sodium sulphite

30 grams sodium carbonate
500 cc. water

5 grams pyrogallic acid

For developing, equal parts of I and II are mixed, and a few drops of a Io per cent solution of potassium bromide added, unless there is reason to suspect that the plate has been seriously underexposed. The fixing bath is a concentrated solution of sodium hyposulphite, "hypo," to which a few drops of acetic acid are added. It should be replaced every week or two, depending upon how much it is usel. A tray of water is kept at hand for bringing out the detail in underexposed negatives, and a second tray is used for washing. The "pyro" and the bromide solution should always be within reach, the former for accelerating, and the latter for retarding the development of unsatisfactory plates.

The image will begin to show on a properly exposed plate within one to three minutes after it has been put in the developer. If the image appears almost instantly, and then recedes quickly, the plate is badly over- 
exposed, and should be thrown away. In case it "comes up" less quickly, indicating that it is not greatly overexposed, it can be saved by the addition of more bromide. When the image does not show till the end of five to ten minutes, the plate has been underexposed. It is then necessary to add more "pyro," taking care not to pour it on the plate, and, after the image appears with its striking contrast, to leave the plate in water until as much detail as possible is brought out in the shadows. In the case of a normal exposure, when greater detail is desired, the negative is left for some time in water, and when contrast is sought more "pyro" is used. Negatives with unusual detail lack "snap"; they are "flat," and fail to make artistic pictures. Contrast, on the other hand, often obscures detail, and the best results can only be obtained by a happy combination of the two. The most important maxim in developing is that the process shall be continted until the image has become indistinct. The universal tendency of the begiuner is to remove the riegative the moment the outlines grow dimmer, and the result is a thin, lifeless negative. It is almost impossible to cevelop too far, if the image is not allowed to disappear. Negatives of this sort are "thick," and though they print more slowly, produce brilliant pictures. A large quantity of the developing solution is used with single plates in small trays, and is allowed to act without rocking the tray. Much time is saved, however, by developing several plates together, and to avoid using a large quantity of the solution, the tray is gently rocked from time to time. This movement is particularly necessary at the beginning, in order that the plates may be covered evenly, and at once. Fifty cubic centimeters of the solution will develop three or four $6 \mathrm{r} / 2 \times 8 \mathrm{I} / 2$ plates, and twice as many $4 \times 5$ 's. After the developer has once been used, it is kept for several days to restrain overexposed plates. As soon as the plate is developed, it is rinsed in water, and placed in the fixing fluid, until the white opaqueness is entirely removed. The "hypo" is then washed out by immersing the negatives for one to two hours in running water. If the latter can not be secured, the water in which they are placed should be changed frequently. . The negatives are then air-dried within doors, in a place free from dust. Finally, they are filed away in negative envelopes, each bearing the name and number of the negative, and preferably also, the time and other exposure data.

242. Finishing. On account of the time demanded by other field tasks, it has not been found desirable to make and finish prints in the field. This, with the making of lantern slides, enlargements, etc., may well be turned over to a professional photographer. It is the custom to make a proof of each negative to meet the castlal needs that arise in the field. For this 
purpose, solio" "seconds" are used, since they are both cheap and satisfactory: When an urgent demand for a finished print does arise, it is met by using "velox" paper, which can be exposed in the dark room, and then developed and fixed exactly like a plate. Two standard papers for views are "solio" and "platina." The former gives brown tones, and is used for contrast and brilliancy, hence it is especially good for printing from negatives that have too much detail and too little contrast. "Platina," on the contrary, yields soft gray tones, and softens contrasts.

\section{FORMIATION AND SUCCESSION HERBARIA}

243. Concept and purpose. A formation herbarium is a collection of exsiccati, in which the species are arranged with respect to their position in the formation, instead of being grouped in genera and families. Its primary purpose is to furnish a record of the constitution and the structure of a formation or a series of formations. At the same time, it affords the basal material for developing the stibject of comparative phytogeography. It is impossible for one ecologist to visit many remote regions, to say nothing of spending a period sufficient for obtaining even a fair knowledge of the vegetation. He can at the best acquire an acquaintance with but few regions at first hand. In consequence, a method that brings a vegetation to him, with its structure carefully wrought out by years of study, is of the highest value. Time, as well as distance, sets a narrow limit to the number of formations which one man can investigate critically in a lifetime. It is no longer possible for a botanist to explore vast regions, and to bring back results which have anything more than a very general value. This fact, far from restricting the comparative study of vegetation, will serve to make it more accurate and systematic. The exact results of numerous resident investigators, expressed in formation herbaria, with the proper series of quadrat maps and photographs, will be worked over by men who are themselves specially acquainted with a particular vegetation. Comparisons will be founded upon a definite basis, and the relationship of various vegetations can then be expressed in precise rather than general terms. It is hardly too sweeping to assert that accurate work in the field of comparative phytogeography can be done only in this fashion. The value of formation herbaria_in class work is eviclent. On account of the limitations of time and distance, classes can touch but few formations, and these at every time except the growing period. For these reasons, an accurate and complete formational record that can be consulted or studied at any time is almost indispensable to class study in the development and structure of formations. 
244. Details of collecting. Formational collections, unlike the ordinary sets of exsiccati, can not be made upon the first visit to a region, or by a single journey through it. The determination of formation limits, and of developmental stages, of aspects, layers, abundance, etc., must necessarily precede, a work which alone takes several years. Moreover, collecting itself requires more than one year in a region containing numerous formations. This is exemplified by the Herbaria Formationum Coloradensium. ${ }^{1}$ The preliminary study for this was made from $1896-1899$, the collecting was done chiefly in IgO0 and IgOI, while additional numbers were added in I902-3. For the purposes of the formation herbarium, specimens should be collected and pressed in such fashion as to show all the ecological features possible. Plants must be collected both in flower and in fruit, with the underground parts as perfect as may be. Seedlings and rosettes should be included whenever present. In pressing, one or two leaves should be arranged with the lower side uppermost to admit of the ready comparison of both surfaces. Opened flowers are valuable for flower biology, while seeds and fruits are desirable for showing migration contrivances. The ferns, mosses, and lichens of the formation should be fully represented, together with the more important fungi and algae. The number of photographs taken for each herbarium should be limited only by considerations of time and expense. The ideal series consists of a general view of each formation, showing its physiographic setting, nearer views of each of its aspects, detail views of its consocies, societies, and layers, and flower portraits of all the constituent species. Such a series can only be obtained by resi- dence through a long term of years, and in most cases general and aspect views, with portraits of the facies and a few of the striking principal species, must suffice. Quadrat and transect charts, together with formational maps, are extremely desirable, and, indecd, all but indispensable.

245. Arrangement. The arrangement of species within each formation herbarium is based upon the structure of the vegetation. The primary groupings are made with reference to time of appearance and abundance; when definite zones, associations, or layers are present, they must likewise be taken into account. In the Colorado collection, the first division is into three aspects based upon the period of flowering (aspectus vernalis, aestivalis, autumnalis). Within each aspect, the species are arranged with respect to abundance in the groups, facies, principal species, and secondary species. Each group is placed in an ordinary manila cover, which bears a printed label indicating the aspect and the group. The species labels g-ve,

${ }^{1}$ Clements, F. E. and E. S. Herbaria Formationum Coloradensium. 1902. 
in addition to the name, date, and place of collection, the phyad or vegetation form, the geographical area, the rank of the species, the aspect, and the formation. To these may well he added data concerning migration contrivances, seed production, pollination, period of flowering, etc. The photographs are mounted on the usual herbarium sheets, and placed in the proper order in the various groups, and a similar disposition is made of quadrat and transect charts, and such physical factor summaries as seem desirable.

246. Succession herbaria. The arrangement of formation herbaria may follow the classification of formations with respect to character, region, or development. The first is the most convenient for purposes of instruction, and has distinct advantages in permitting a close comparison of the vegetation of different habitats. The second basis, which is the one used in the Herbaria Formationum Coloradensium, is peculiarly adapted to mountain vegetation in which the zones are usually very distinct. The arrangement of herbaria in a developmental series, however, is the most logical and the most illuminating, since the structure of the ultimate formations is not only made plain, but the stages in their development are also laid bare. Such succession herbaria are the natural outgrowth of formational ones. Indeed, the latter should be made merely the starting point for these in all regions where the causes which bring about successions are active. Where weathering is still an important factor, as in mountains, the initial and intermediate formations which lead to the final grassland or forest are often in evidence. After a formation herbarium of each stage has been made in the way indicated, a succession herbarium is obtained merely by arranging the various herbaria in the sequence of the developmental stages. Thus, in the Colorado collection, the subalpine formations are arranged according to altitude in the following series: (I) the pine formation, (2) the gravel slide formation, (3) the half gravel slide formation, (4) the aspen formation, (5) the balsam-spruce formation, (6) the spruce-pine formation, (7) the meadow thicket formation, (8) the brook bank formation. Of these, five belong to the same succession, and it is possible to indicate the development of the spruce-pine forest by arranging these five formations in their proper order in a succession herbarium, as follows: (I) the gravel slide formation, (2) the half gravel slide formation, (3) the pine formation, (4) the balsam-spruce formation, (5) the spruce-pine formation. 


\section{Development and Struciure}

247. Vegetation an organism. The plant formation is an organic unit. It exhibits activities or changes which result in development, structure, and reproduction. These changes are progressive, or periodic, and, in some degree, rhythmic, and there can be no objection to -regarding them as functions of vegetation. According to this point of view, the formation is a complex organism, which possesses functions and structure, and passes through a cycle of development similar to that of the plant. This concept may seem strange at first, owing to the fact that the common understanding of function and structure is based upon the individual plant alone. Since the formation, like the plant, is subject to changes caused by the habitat, and since these changes are recorded in its structure, it is evident that the terms, function and structure, are as applicable to the one as to the other. It is merely necessary to bear in mind that the functions of plants and of formations are absolutely different activities, which have no more in common than do the two structures, leaf and zone.

248. Vegetation essentially dynamic. As an organism, the formation is undergoing constant change. Constructive or destructive forces are necessarily at work; the former, as in the plant, predominate until maturity, when the latter prevail. Consequently, it no longer seems fruitful to classify the phenomena of vegetation as dynamic or static. The emphasis which has been placed upon dynamic aspects of vegetation has served a useful purpose by calling attention to the development of the latter. Although it is a quarter of a century since Hult, and more than a half century since Steenstrup, by far the greater number of ecological studies still ignore the problem of development. This condition, however, can be remedied more easily by insisting upon an exact understanding of the nature of the formation than in any other way. It is entirely superfluous to speak of dynamic and static effects in the plant, and the use of these terms with reference to the formation becomes equally unnecessary as soon as the latter is looked upon as an organism. The proper investigation of a formation can no more overlook development than structure, so closely are the two interwoven. Future research must rest squarely upon this fact.

249. Functions and structures. The functions of a formation are association, invasion, and succession: the second may be resolved into migration and ecesis, and the third, perhaps, into reaction and competition. Formational structures comprise zones, layers, consocies, societies, etc., all of which may be referred to zonation, or to alternation. The term associa- 
tion has been used in both an active and a passive sense. In the former, it applies to the inevitable grouping together of plants, by means of reproduction and immobility. Passively, it refers to the actual grouipings which result in this way, and in this sense it is practically synonymous with vegetation. Invasion is the function of movement, and of occupying or taking possession; with association, it constitutes the two fundamental activities of vegetation. It is the essential part of succession, but the latter is so distinctive, because of the intimate relation of competition and reaction, that clearness is gained by treating it as a separate function which is especially concerned with development. Association, zonation, and alternation are structural phenomena, which are in large part the immediate product of habitat and function, and in a considerable degree, also, the result of ancestral or historical facts. It is a difficult matter to determine in what measure the last factor enters, but it is one that must always be taken into account, particularly when the physical factors of the habitat are inadequate to explain the structures observed. Structurally, association regularly includes both zonation and alternation. As there are certain typical instances in which it exhibits neither, the treatment will be clearer"if each is considered separately.

\section{ASSOCIATION}

250. Concept. The principle of association is the fundamental law of vegetation. Indeed, association is vegetation, for the individual passes into vegetation, strictly speaking, at the moment when other individuals of the same kind or of different kinds become grouped with it. It is then (and the same statement necessarily holds for vegetation) the coming together and the staying together of individuals and, ultimately, of species. A concrete instance will illustrate this fact. In the development of the blowout formation of the Nebraska sand-hills (Redficldia-Muhlenbergia-anemin $m$ ), association begins only when the first plant of Redfieldia flexuosa is joined by other plants that have sprung from it, or have wandered in over the margin of the blowont. Henceforth, whatever changes the blowout formation may undergo, association is a settled characteristic of it until some new and overwhelming physical catastrophe shall destroy the associated individuals. It will readily be seen that association does not depend upon particular individuais, for these pass and others take their place, but that it does depend essentially upon number of individuals.

Association involves the idea of the relation of plants to the soil, as well as that of plants to each other. It is synonymous with vegetation only when the two relations are represented, since there may be association such 
as that of a parasite with its host, which does not constitute vegetation. But it will be seen that the relation of the parasite to the host is practically identical with the relation of the plant to the soil or stratum, and the two concepts mentioned above become merged in such a case. From this it follows that association results in vegetation only when the two ideas are distinct. The concept of association contains a fact that is everywhere significant of vegetation, namely, the likeness or unlikeness of the individuals which are associated. In the case of parasite and host, this unlikeness is marked; in vegetation, all degrees of similarity obtain. As will be evident when the causes which lead to association are considered, alternate similarity and dissimilarity of the constituent individuals or species is subordinate as a feature of vegetation only to the primary fact of association.

Since association contains two distinct, though related, ideas, it is of necessity ambiguous. It is very desirable that this be avoided, in order that each concept may be clearly delimited. For this reason, the act or process of grouping individuals is termed aggregation, while the word association is restricted to the condition or state of being grouped together. In a word, aggregation is functional, association is structural; the one is the result of the other. This distinction makes clear the difference between association in the active and passive sense, and falls in with the need of keeping function and structure in the foreground.

251. Causes. In considering the causes which produce association, it is necessary to call in evidence the primary facts of the process in concrete examples of this principle. These facts are so bound up in the nature of vegetal organisms that they are the veriest axioms. Reproduction gives rise immediately to potential, and ultimately, in the great majority of cases, to actual association. The degree and permanence of the association are then determined by the immobility of the individuals as expressed in terms of attachment to each other or to the stratum, such as sheath, thallus, haustoria, holdfasts, thizoids, roots, etc. The range of immobility is very great. In terrestrial plants, mobility is confined almost entirely to the period when the individual lies dormant in the seed, spore, or propagative part, which is alone nobile. In aquatic spermatophytes, the same is true of all attached forms, while free floating plants such as Lemna are mobile in a high degree, especially during the vegetative period. Among the algae and hydrophilous fungi, attached forms are mobile only in the spore or propagative condition, while the motile forms of the plancton typify the extreme development of mobility. The immediate result of reproduction in an immobile species is to produce association of like individuals, while in the case of a mobile species reproduction may or may not lead immediately 
to association. We may lay down the general principle that immobility tends to maintain the association of the individuals of the same generation, i. e., the association of like forms, while mobility tends to separate the similar individuals of one generation and to bring unlike forms together. With the mobile algae, separation of the members of each generation is the rule, unless the individuals come to be associated in a thallus, or are grouped in contact with the substratum. Flowering plants that are relatively immobile, especially in the seed state, drop their seeds beneath and about the parent plants, and in consequence dense association of the new plants is the rule. In.very many cases, however, this primitive tendency is largely or completely negatived by the presence of special dissemination contrivances, which are nearly, if not quite, as effective for many terrestrial plants as the free floating habit is for algae. From this point, the whole question of mobility belongs to migration, just as the adjustment between the parent plants and their offspring, or between plants established and the mobile plants to be established, belongs to competition.

If association were determined by reproduction and immobility alone, it would exhibit areas dissimilar in the mass of individuals, as well as areas dissimilar in the kinds of individuals. Some areas would be occtpied by plants of a single species, others by plants of several or many species. This tendency of association to show differences is, however, greatly emphasized by the fact that vegetation is fundamentally attached to and dependent upon a surface that exhibits the most extreme physical differences. For this reason, new differences in association appear, due not only to the morphological differentiation of vegetation forms, but also to the changes in the degree and manner of association produced directly by the different habitats. Association might then be defined as a grouping together of plant individuals, of parents and progeny, which is initiated by reproduction and immobility, and determined by environment. It is a resultant of differences and similarities. In consequence, association in its largest expression, vegetation, is essentially heterogeneous, while in those areas which possess physical or biological definiteness, habitats and vegetation centers, it is relatively homogeneous. This fundamental peculiarity has given us the concept of the formation, an area of vegetation, or a particular association, which is homogeneous within itself, and at the same time essentially different from contiguous areas, though falling into a phylogenetic series with some and a biological series with others. From its nature, the plant formation is to be considered the logical unit of vegetation, though it is not, of course, the simplest example of association. 
252. Aggregation. As indicated under the causes of association, the process by which groups of individuals are formed depends entirely upon reproduction and migration. In short, aggregation is merely a corollary of movement. The simplest example of this process occurs in forms like Gloeoccipsa, Tetraspora, and others, where the plants resulting from fission are held together by means of a sheath. Though called a colony, such a group of individuals is a family in the ordinary sense. Practically the same grouping resulis in the case of terrestrial plants, especially spermatophytes, when the seeds of a plant mature and fall to the ground about it. The relation in both instances is essentially that of parent and offspring, although the parent soon disappears in the case of annuals, while among the algae its existence is regularly terminated by fission. The size and the density of the family group are determined by the number of seeds produced, and by their mobility. These are further affected by the height and branching of the plant, and by the position of the seeds upon it. The disseminules of immobile species fall directly beneath the parent, and the resulting group is both uniform and definite. A similar arrangement is caused likewise by offshoots. An increase in mobility brings about a decrease of aggregation, since the disseminules are carried away from the parent plant. Perfectly mobile forms rarely produce family groups for this reason. It is evident, however, that mobile perennials sometimes arrange themselves in similar fashion in consequence of propagation by underground parts. Consequently, it is possible to state the law of single aggregation, viz., that immobility promotes the grouping of parent and offspring, and mobility hinders it.

If all species were immobile, the family group would be characteristic of vegetation. Since the great majority are more or less mobile, aggregates of this sort are the exception rather than the rule. Mobility not only decreases the number of offspring in the family group, but it also spreads disseminules broadcast to enter dissimilar groups. It leads directly to mixed aggregation, by which individuals of one or more species invade the family group. Once established, the newcomers tend also to produce simple groups, thus causing an arrangement corresponding essentially to a community. Such collections of family groups are extremely variable in size and definition. This arises in part from the nature of simple aggregation, and in part from the varying mobility of different species. Mobility alone often produces similar communities by bringing together the disseminules of different plants, each of which then becomes the center of a mixed group. In the case of permobile species, several disseminules of each may be brought together. The resulting area, though larger, is practically the same. At present, it is difficult to formulate the law for this method of grouping. It may be stated provisionally as follows: mixed aggregation is the direct result of mobility, and the greater the mobility the more heterogeneous the mixture. 
The constitution of all the major areas of a formation is to be explained upon the basis of aggregation by the two methods described. The relative importance of family groups and communities differs for every formation, and the exact procedure in each can be obtained only by the detailed study of quadrats. The problem is further complicated by competition and reaction, particularly in closed vegetation. For this reason, aggregation can be studied most satisfactorily in a new or denuded area, where these processes are not yet in evidence.

\section{Kinds of Association}

253. Categories. In the analysis of association, it must be kept clearly in mind that the concrete examples from which all generalizations must be drawn are often in very different stages of development, and are of correspondingly different ages. For this reason it has seemed best to consider the primary relations of association in general in this place, leaving the treatment of the effects of invasion, succession, alternation, and zonation to be taken up under these tcpics.

Various categories of association may be distinguished, according to the dominant physical factor concerned or the point of view taken. These will fall into two series, as we consider the relation of plant to plant with reference to some object or characteristic, or the grouping of plants together in response to some dominant factor. In the first series may be placed association with reference to substratum, to the ground (occupation), and to invasion; in the second belong light and water-content association. It should be noted that these are all actual associations in nature, and not concepts suchi as the vegetation form, within which plants from widely different associations may be classified. Naturally, it does not follow that it is not logical or valuable to group together those plants, such as hydrophytes, sciophytes, hysterophytes, etc., which have a common relation to some factor, but belong to different formations.

254. Stratum association. Plants manifest independent or dependent association with reference to the stratum to which they are attached and from which they derive food or support. Independent association is exhibited by those holophytic species of a formation which are entirely independent of each other with respect to mechanical support or nutrition. It is characteristic of the greater number of the constituent species of formations. Dependent association is manifested in the relation between host and parasite, stratum and epiphyte, support and liane. Warming ${ }^{-1}$ has distinguished six

'Lehrbuch der Ökologischen Pflanzengeographie, 97. 1896. 
kinds of associations: parasitism, helotism, mutualism, epiphytism, lianism, and commensalism. Commensalism corresponds to the primary principle of association which has given rise to vegetation. Homogeneous commensalism is the term applied to social exclusive plants, in which the patch is composed of a single species. Such association is extremely rare in nature, and if the most minute forms be considered, probably never occurs. On the other hand, heterogeneous commensalism, in which individuals of more than one species are present, is everywhere typical of vegetation. Warming regards saprophytism merely as a specialized kind of parasitism, an opinion that may well be defended. Helotism, however, is also a mere modification of parasitism, if it is not indeed parasitism pure and simple. Mutualism is an altogether vague concept, including parasites, epiphytes, and endophytes of doubtful physiological relation. Pound and Clements ${ }^{1}$ treated lianes, parasites, and saprophytes as vegetation forms, relating herbaceous creepers and twiners to the lianes, and dividing the fungi and lichens into nine groups. Whatever the value of these divisions may be from the standpoint of vegetation forms, they represent the same relation between plant and nutritive stratum, and with respect to association should be merged in one group. Schimper ${ }^{2}$ was the first to perceive the essential similarity of all such groups from the standpoint of association. He terms these plant societies (Genossenschaften), retaining the four groups already established, lianae, epiphyta, saprophyta, and parasiticae. It is evident that dependent association comprises extremely divergent forms, from the slightly clinging herb, such as Galitm, to the most intense parasite. The distinction, however, is a clear one, if restricted to that relation between plants in which one acts as a mechanical support or stratum or as a nutritive host for the other.

255. Ground association. The first division of formations into open and closed was made by Engler and Drude. ${ }^{3}$ Open formations were defined as those having incomplete stability and heterogeneous composition, while closed formations have a more definite uniform stamp. What is true of formations is equally true of vegetation, so that association may be regarded as open or closed with reference to the density and thoroughness with which the plants occupy the ground. In open association, the ground is slightly or partially occupied, readily permitting the entrance of new plants without the displacement of those already present. Such an arrangement is characteristic of the early stages of a formation, or of a succession of formations. It produces unstable open formations, which arise, usually

'Phytogeography of Nebraska, 1st ed, 101. 1898.

Pfanzengeographie auf physiologischer Grundlage, 208. 1898.

Die Vegetation der Erde. Engler Bot. Jahrb., 17 :b55. 1893. 
after denudation, in sand-hills, blowouts, gravel slides, dunes, flood plains, burned areas, etc. In closed association, occupation of the ground is complete, and the invasion of new species can occur only through displacement. Closed association results. in stable, closed formations, such as forest, thicket, meadow, and prairie. As open association characterizes the early stages of a succession of formations, so closed association is peculiar to the later or last stages of all such successions. In short, open formations represent certain phases of the development of vegetation, while closed formations correspond to the relatively final structural conditions. It is a fundamental principle of association that every succession from denudation, or from newly formed soils, begins with open formations and ends with a closed formation. The causes leading up to open and closed association are intimately connected with development, and hence are considered under invasion and succession.

256. Species guild association. Drude has distinguished a kind of association peculiar to invasion, in which there is a successive or concomitant movement of certain species of a formation into another formation or region, resulting in species guilds (Artengenossenschaften). The association in this case is largely one of community of origin or area, and of concomitant migration. It is especially characteristic of areas adjacent to formational and regional limits. Fundamentally, it is merely the grouping of plants which are invading at the same time, and consequently it differs only in degree from what occurs in every invasion where more than a single individual is concerned. Accordingly, this type of association has little more than historical interest. This must not be construed to mean that it does not occur, but that it differs in no essential from the ordinary grouping of invaders.

257. Light association. The constituent species of formations show two fundamentally different groupings with respect to light. In the one case, the individuals are on the same level, or nearly so, in such a way that each has direct access to sunlight. Such an arrangement is characteristic of most grassland and herbaceous formations. In the case of desert formations, there is often considerable difference in the height of the plants, but the distance between them is so great as to admit of direct illumination of all. This arrangement may be termed coordinate association. In forests, thickets, and many herbaceous wastes, the height and density of certain species enable them to dominate the formation. In a dense forest, the trees receive practically all the light incident upon the formation, and the shrubs, herbs, fungi, and algae of lower habit and inferior position must adapt themselves to the diffuse light which passes through or between the leaves. The same 
is equally true of dense thickets and wastes, except that the vertical distance is less, and the diffuseness of the light is correspondingly modified. In these formations, the dominant trees, shrubs, or herbs, the facies, constitute a primary or superior layer. The degree of subordinate association, as a result of which inferior layers will arise, is entirely determined by the density of the facies. In open woodlands, which are really mixed formations of woodland and grassland, the intervals, and ustually the spaces beneath the trees also, are covered with poophytes, showing an absence of subordination due to light. This is the prevailing condition in the pine formation (Pimus pondercsa-xerohylium) of the ridges and foot-hills of western Nebraska. When, however, the trees stand sufficiently close that their shadows meet or overlap throughout the day, the increasing 'diffuseness begins to cause modification and rearrangement of the individuals. By photometric methods, the light in a forest is found to be least diffuse just below the facies, while the diffuseness increases markedly in passing to the ground. The taller, stronger indivicluals are consequently in a position to assimilate more vigorously, and to become still taller and stronger as a result. Just as these have taken up a position inferior to that of the facies, so the shorter or weaker species must come to occupy a still more subordinate position. This results, not only because the light is primarily weaker nearer the ground, but also because the taller plants interpose as a second screen. The complete working out of this arrangement with reference to light produces typical strbordinate association, which finds its characteristic expression in the layering of forests and thickets. Layers tend to appear as soon as open woodland or thicket begins to pass into denser conditions, and up to a certain point, at which they disappear, they become the more numerous and the more marked, the denser the forest.

In the Otowanie woods near Lincoln (Quercus-Hicoria-hylium), layering usually begins at a light value of .I ( $I=$ normal sunshine in the open). Thornber ${ }^{1}$ has found the same value to obtain in the thickets of the Missouri bluffs. In these, again, layers disappear at a value of .005 , the extreme diffuseness making assimilation impossible except for occasional mosses and algae. A number of herbaceous plants are present in the spring, but these are all prevernal or vernal bloomers, which are safely past flowering before shade conditions become extreme. In the Fraxinus-Catalpa-alsium, all inferior holophytic vegetation disappears between the light value of .004 and that of .oo3. The spruce-pine formation (Picea-Pinus-hylium) of the Rocky mountains, with a light value of .or, usually contains but a few scattered herbs, mostly evergreen; in some cases there are no subordinate plants

${ }^{1}$ Thornber, J. J. The Prairiegrass Formation in Region I. Rep. Bot. Surv. Nebr., $5: 36,46.1901$. 
other than mosses and hysterophytes. The lodge-pole pine formation (Pinus murrayana-hylium), with light values often less than .005, is nearly or quite destitute of all but hysterophytic undergrowth. Such extremely dense formations are examples of coordinate association merely, since the formation is reduced to a single superior layer, in which the individuals of the facies bear the same spatial relation to incident light. In layered formations, in addition to the subordinate relation of other species to the facies, there is, of course, a kind of coordinate association manifested in each layer.

258. Water-content association. Schouw ${ }^{1}$ was the first to give definite expression to the value of the water-content of the soil for the grouping of plants. He established four groups: (1) water plants, (2) swamp plants, (3) plants of moist meadows, (4) plants of dry soils. The first he termed hydrophytes, introducing the term halophytes to include all saline plants. Thurmann ${ }^{2}$ recognized the fundamental influence of water-content upon association, and further perceived that the amount of water present was determined primarily by the physical nature of the soil. He distinguished plants which grow in soils that retain water as hygrophilous, and those found upon soils that lose water readily as xerophilous. Those which seemed to grow indifferently upon either were termed ubiquitous. The latter correspond in some measure to mesophytes, but they are really plants possessing a considerable range of adaptability, and do not properly constitute a natural group. Warming ${ }^{3}$ proposed the term mesophytes to include all the plants intermediate between hydrophytes and xerophytes. He recognized the paramount value of water-content association as the basis of ecology, and upon this made a logical and systematic treatise out of the scattered results of many workers. Schimper ${ }^{4}$ placed the study of vegetation upon a new basis by drawing a distinction between physical and physiological water-content, and $\mathrm{r} y$ pointing out that the last alone is to be taken into account in the study of plant life, and hence of plant geography. Accepting the easily demonstrable fact that an excess of salts in the soil water, as well as cold, tends greatly to diminish the available water of the soil, i. e., the chresard, it is at once seen why saline and arctic plants are as truly xerophytic as those that grow on rocks or in desert sands. An anomalous case which, however, physical factor records have explained fully, is presented by many plants growing in alpine gravel slides, strands, blowouts, sandbars, etc., in which the water-content is considerable, but the water loss excessive, on account

${ }^{1}$ Grundzüge einer allgemeinen Pflanzengeographie, 157. 1823.

${ }^{2}$ Essai de phytostatique, etc. 1849.

${ }^{3} l . c_{\text {. }}, 116.1896$.

4. c.. 3. 1898. 
of extreme heat or reduced air pressure. The effect of these conditions is to produce a plant xerophytic as to its aerial parts, and mesophytic or even hydrophytic as to subterranean parts. Such plants may, from their twofold nature, be termed dissophytes; they are especially characteristic of dysgeogenous soils in alpine regions where transpiration reaches a maximum, but are doubrless to be found in all gravel and sand habitats with high watercontent. With these corrections, the concept of water-content association, which owes much to both Warming and Schimper, but is largely to be credited to Thurnann, becomes completely and fundamentally applicable to all vegetation.

Up to the present time, the general character of the habitat, together with the gross appearance of the plant itself, has been thought sufficient to determine the proper position of a plant or a formation in the water-content classification. Such a method is adequate, however, only for plants and formations which bear a distinct impress. For an accurate classification into the three categories, hydrophytes, mesophytes, and xerophytes, it is necessary to make exact determinations of the normal holard and chresard of the habitat, and to supplement this, in some degree at least, by histological studies. Except in the case of saline, acid, and frozen soils, the holard alone will be a fairly accurate index, especially in habitats of similar soil composition. For an exact and comprehensive classification, however, and particularly in comparative work, the chresard must constitute the sole criterion. As the latter has been ascertained for very few formations, and in Nebraska and Colorado alone, the present characterization of many plants and formations as hydrophytic, mesophytic, or xerophytic must be regarded as largely tentative, and the final classification will be possible only after the thorough quantitative investigation of their habitats.

The water-content groups, hydrophytia, mesophytia, and xerophytia, include all formations found upon the globe. The exactness with which this classification applies to vegetation is made somewhat more evident by dividing mesophytia into forest and grassland. This is based primarily upon light association, but it also reflects water-content differences in a large degree. The groups thus constituted represent the fundamental zonation of the vegetative covering with respect to water-content. Ocean, forest, grassland, and desert correspond exactly to hydrophytia, hylophytia, poophytia, and xerophytia. The difference is merely one of terminology: the first series takes into account the physiognomy of the vegetation itself, while the other emphasizes the causative factors. 


\section{THE DEVELOPMENT OF THE FORMATION}

259. A strict account of development should trace the results of the various activities of vegetation in their proper sequence. This is aggregation, migration, ecesis, reaction, and competition. These functions are so intimately and often so inextricably associated that it is hardly feasible to discuss development by treating each one separately. In consequence, the two fundamental phenomena, invasion and succession, which they produce, are taken as the basis of the discussion. These, moreover, are different only in degree; succession is merely complete, periodic invasion. Nevertheless, the subject gains in clearness by a separate treatment of each.

\section{INVASION}

260. By invasion is understood the movement of plants from an area of a certain character into one of a different character, and their colonization in the latter. This movement may concern an individual, a species, or a group of species. From the nature of invasion, which contains the double idea of going into and taking possession of, it usually operates between contiguous formations, but it also takes place between formational zones and patches. More rarely and less noticeably, there may be invasion into a remote vegetation, as a result of long carriage by wind, water, birds, railroads, or vessels. Movement or migration, however, represents but one of the two ideas involved in invasion. Migration merely, carries the spore, seed, or propagt1le into the area to be invaded. In ecesis, the spores or seeds germinate and grow, after more or less adjustment, and in case the latter becomes sufficiently complete, the new plants reproduce and finally become established. With all terrestrial plants, invasion is possible only when migration is followed by ecesis, because of the inherent differences of formations or of areas of the same formation. In the case of surface floating forms, such as Lemnaceae, and of the plancton, ecesis is of much less importance, on account of the uniformity of the medium and the lack of attachment, and migration is often practically synonymous with invasion.

\section{MIGRATION}

261. Migration has been sometimes used loosely as a synonym for invasion, but it is here employed in its proper sense of removal or departure, i. e., movement, and is contrasted with ecesis, the making of a home, the two ideas being combined in invasion, which is a moving into and a taking possession of. An analysis of migration reveals the presence of four factors, mobility, agency, proximity, and topography. Not all of these are present 
in every instance of migration, as for example in the simple elongation of a rootstalk, but in the great majority of cases each plays its proper part. Mobility represents the inherent capacity of a plant for migration, and in its highest expression, motility, is in itself productive of movement. As a general rule, however, modifications for securing mobility are ineffective in the absence of proper agents, and the effective operation of the two will be profoundly influenced by distance and topography.

262. Mobility denotes potentiality of migration as represented by modifications for this purpose. It corresponds, in a sense, to dissemination, though seed production also enters into it. Its most perfect expression is found in those plants which are themselves motile, Bacteriaceae, Oscillatoria, Volvocaceae, and Bacillariaceae, or possess motile propagules, such as most Phycophyta. On the other hand, it is entirely undeveloped in many plants with heavy unspecialized seeds and fruits. Between these two extremes lie by far the greater number of plants, exhibiting the most various degrees. of mobility, from the motile though almost immobile offshoots of many Liliaceae to the immotile but very mobile spores of fungi. It is thus seen that motility plays a relatively small part in migration, being practically absent in terrestrial forms, and that it bears a very uncertain relation to mobility. In analyzing the latter, contrivances for dissemination are seen to determine primarily the degree of mobility, while the number of seeds produced will have an important effect in increasing or decreasing it. A third factor of considerable importance is also involved, namely, position with reference to the distributive agent, but any exact knowledge of its importance must await systematic experiment somewhat after the methods of Dingler, but with aircurrents, etc., of known velocity and direction. The time is not distant when by such methods it will be possible to establish a coefficient of mobility, derived from terms of position, weight, resistant surface, and trajectory for definite wind velocities or for particular propulsive mechanisms.

263. Organs for dissemination. Plants exhibit considerable diversity with reference to the part or organ modified, or at least utilized, for dissemination. This modification, though usually affecting the particular product of reproduction, may, in fact, operate on any part of the plant, and in certain cases upon the entire plant itself. In the majority of plants characterized by alternation of generations, the same individual may be disseminated in one generation by a reproductive body, and in the other by a propagative one, as is the case in the oogones and conidia of Peronospora, the spores and gemmae of Marchantia, the fruits and runners of Fragaria, etc. Special modifications have, as a rule, been developed in direct connection with spores 
and seeds, and mobility reaches its highest expression in these. It is, on the other hand, greatly restricted in offshoots and plant bodies, at least in terrestrial forms, though it will now and then attain a marked development in these, as shown by the rosettes of Sempervivum and the tumbling plants of Cycloloma. For the sake of convenience, in analyzing migration, all plants may be arranged in the following groups with reference to the organ or part distributed.

I. Spore-distributed, sporostrotes. This includes all plants possessing structures which go by the name of spore, such as the acinetes of Nostoc and Protococculs, the zoogonidia of Ulothrix, Ectocarpus, etc., the conidia, ascospores, and basidiospores of fungi, the tetraspores of red seaweeds, and the gemmae and spores proper of liverworts, mosses, and ferns. These are almost always without especial contrivances for dissemination, but their extreme minuteriess results in great mobility.

2. Seed-distributed, spermatostrotes. This group comprises all flowering plants in which the seed is the part modified or at least disseminated. The mobility of seeds is relatively snall, except in the case of minute, winged or comate seeds.

3. Fruit-distributed, carpostrotes. The modifications of the fruit for distribution exceed in number and variety all other modifications of this sort. All achenes, perigynia, utricles, etc., properly belong here.

4. Offshoot-distributed, thallostrotes. To this class are referred those plants, almost exclusively cormophytes, which produce lateral, branch-like propagules, such as root-sprouts, rhizomes, runners, stolons, rosettes, etc. Migration with such plants is extremely slow, but correspondingly effective, since it is almost invariably followed by ecesis.

5. Plant-distributed, phytostrotes. This group includes all plancton and surface forms, whether motile or non-motile, and those terrestrial plants in which the whole plant, or at least the aerial part, is distributed, as in tumbleweeds and in many grasses.

264. Contrivances for dissemination. Any investigation of migration to be exact must confine itself to fixed forms. For these the degree of perfection shown by dissemination contrivances corresponds almost exactly to the degree of mobility. Because of the difficulty of ascertaining the effect of ecesis, it is impossible to determine the actual effectiveness in nature of different modifications, and the best that can be clone at present is to regard mobility, together with the occurrence and forcefulness of distributive agents, as an approximate measure of migration. The general accuracy of such a measure will be more or less evident from the following. Of I 8 species common to the foot-lill and sand-hill regions of Nebraska, regions which are 
sufficiently diverse to indicate that these common species must have entered either one by migration from the other, 83 exhibit modifications for dissemination, while 8 others, though without special contrivances, are readily distributed by water, and 4 more are mobile because of minuteness of spore or seed. Some degree of mobility is present in 73 per cent of the species common to these regions, while of the total number of species in which the mode of migration is evident, viz., 95, 66 per cent are wind-distributed, 20 per cent animal-distributed, and $\mathbf{4} 4$ per cent are water-distributed. It need hardly be noted that this accords fully with the prevalence and forcefulness of winds in these regions. Of the species peculiar to the foot-hill region, many are doubtless indigenous, though a majority have come from the montane regions to the westward. The number of mobile species is $\mathrm{I} 2 \mathrm{I}$, or 60 per cent of the entire number, while the number of wind-distributed ones is 85 , or 70 per cent of those that are mobile. Among the 25 species found in the widely separated wooded bluff and foot-hill regions, 2 only, Amorpha nana and Roripa nasturtium, are relatively immobile, but the minute seeds of the latter, however, are readily distributed, and the former is altogether infrequent.

The following groups of plants may be distinguished according to the character of the contrivance by which dissemination is secured:

I. Saccate, saccospores. Here are to be placed a variety of fruits, all of which agree, however, in having a membranous envelope or an impervious, air-containing pericarp. In Ostrya, Phy'salis, Staphylea, the modification is for wind-distribution, while in Carex, Nymphaea; etc., it is for watertransport.

2. Winged, pterospores. This group includes all winged, margined, and flattened fruits and seeds, such as are found in Acer, Betula, Rumex, many Unbelliferae, Graminaceac, etc.

3. Comate, comospores. To this group belong those fruits and seeds with long silky hairs, Gossypium, Anemone, Asclepias, etc., and those with straight capillary hairs or bristles not confined to one end, Typha, Salix, etc.

4. Parachute, petasospores. The highly developed members of this group, Taraxacum, Lactuca, and other Liguliflorae are connected through Senecio and Eriophorum with the preceding. These represent the highest development of mobility attained by special modification.

5. Chaffy-pappose, carphospores. In this group are placed those achenes with a more or less scaly or chaffy pappus with slight mobility, as in $R u d$ beckia, Branneria, Helianthus, etc.

6. Plumed, lophospores. In the fruits of this class, the style is the part itsually modified into a long plumose organ, possessing a high degree of mobility, as in Pulsatilla, Sieversia, and Clematis. 
7. Awned, acospores. These are almost exclusively grasses, in which the awns serve for distribution by wind, water, or animals, and even, according to Kerner, by hygroscopic creeping movements. The mobility in many cases is great.

8. Spiny, centrospores. This group contains a few representatives which possess a moderate degree of mobility by attachment, as in Tribulus and Cenchrus.

9. Hocked, oncospores. The members of this group are extremely numerous, and the degree of mobility as a rule is very high. All exhibit in common the development of hooks or barbs, by which they are disseminated in consequence of attachment, though the number, size, and disposition of the hooks vary exceedingly.

IO. Viscid, gloeospores. In these, the inflorescence is more or less covered with a viscid substance, as in species of Silene, or the fruit is beset with glandular hairs, as in Cerastium, Salvia, etc.

II. Fleshy, sarcospores. These are intended for dissemination by deglutition, largely by birds; the effectiveness of the modification, depends in a large degree upon the resistance of the seed envelope to digestion. The mobility varies greatly, but the area over which migration may be effected is large.

12. Nut-fruited, creatospores. This group includes those plants with nut fruits which are carried away and secreted by animals for food.

I3. Flagellate, mastigospores. These are plants with ciliate or flagellate propagative cells, i. e., zoogoniclia, as in Protococcus, Ulothrix, Oedogonium, Ectocarpus, etc., or with plant bodies similarly motile, Bacteriaceae and Volvocaceae.

265. Position of disseminule. The position on the plant of the organ to be disseminated, i. e., its exposure to the distributing agent, plays a considerable part in determining the degree of mobility. In the majority of plants, the position of the inflorescence itself results in maximum exposure, but in a large number of forms special modifications have been developed for placing the spores or seeds in a more favorable position. In both cases, there are often present also devices for bringing about the abscission of the seed or fruit. It is, moreover, self-evident that the height of the inflorescence above ground or above the surrounding vegetation is likewise of considerable importance in increasing the trajectory. It is yet too early to make a complete classification of contrivances for placing disseminules in the most favorable exposure, but the following will serve as a basis for future arrangements.

I. In all operculate Discomycetes, and especially in the Ascobolaceae, where the asci project above the hymenium, the spores are raised above the 
surface by tensions within the apothecium. This might be regarded as dissemination by expulsion, if it were not for the fact that the spores fall back into the cup, unless carried away by the wind.

2. In Gasteromycetes and in certain Hepaticae, the spores are not only elevated slightly above the sporophore by the expanding capillitium or by the mass of elaters, but they are also held apart in such a way that the wind blows them out much more readily.

3. In Bryophyta, the sporophore regularly dehisces by a slit, or is provided with a peristome. Both structures are for the purpose of sifting the spores out into the wind; by reason of their hygroscopicity, they also insure that the spores will not be shaken out in wet weather.

4. In a few grasses, such as Stipa and Aristida, the twisting and intertwining of the awns lift the floret ont of the glumes, and at the same time constitute a contrivance readily blown away by the wind or carried by attachment.

5. In certain Compositae, the involucral scales are reflexed at maturity, and at the same time the dișk becomes more or less convex, serving to loosen the achenes. This result is also secured in certain species by the drying and spreading of the pappus hairs.

6. The scapose Liguliflorae, Tara.racum. Agoseris, etc., are characterized by the elongation of the scape after anthesis, with the result that the head is raised to a considerable height by the time the achenes are mature.

7. Carpotropic movements, though primarily for another purpose, often serve to bring seeds and fruits into a better position for dissemination.

266. Seed production. The relation of spore or seed-production to mobility is obvious in the case of mobile species; in the case of immobile ones, it is just as evident that it has no effect, though it may still have considerable influence in increasing migration. In the case of two species with equally effective dissemination contrivances, the one with the largest seed-production will be the more mobile. On the basis of the relation of seeds to flower, two groups of plants may be distinguished, one, Polyanthae, in which the flowers are many and the seeds few or single, as in Compositae, and the other, Polyspermatae, Portulaca, Yucca, etc., in which the number of seeds to each flower is large. So far as the actual number of seeds produced is concerned, polyanthous plants may not differ from polyspermatous ones, but, as a rule, they are much more highly specialized for dissemination and are more mobile. The number of fertile seeds is also much greater, a fact which is of great importance in ecesis, and which, taken in connection with mobility, partially explains the supremacy of the composites. Among the fungi and algae, the amount of spore-production in a large degree determines the mobility, since these forms are intrinsically permobile. 
267. Agents of migration. In the last analysis, however, the possibility ot migration clepends upon the action of distributive agents; in the absence of these, even the most perfect contrivance is valueless, while their presence brings about the distribution of the most immobile form. In short, migration depends much more upon such agents than upon mobility, however perfect the latter may be. It is, moreover, evident, that the amount and extent of migration will be determined primarily by the permanence and forcefulness of the agent, as indicated by its ability to bring about transportation. Finally, as will be shown later, the direction and rapidity of migration depend directly upon the direction and intensity of the agent.

Migration results when, spores, seeds, fruits, offshoots, or plants are moved out of their home by water, wind, animals, man, gravity, glaciers, growth, or mechanical propulsion. Correspending to these agents, there may be recognized the following groups:

I. Water, hydrochores. These comprise all plants distributed exclusively by water, whether the latter acts as ocean currents, tides, streams, or surface run-off. In the case of streams and run-off, especially, mobility plays little part, provided the disseminules are impervious or little subject to injury by water. Motile plants, or those with motile cells, which belong entirely to this group, may be distinguished as autochores, which correspond closely to mastigospores.

2. Wind, anemochores. This group includes the majority of all permobile terrestrial plants, i. e., those in which modifications for increasing surface have been carried to the extreme, or those which are already permobile by reason of the minuteness of the spore or seed. Saccate, winged, comate, parachute, pappose, plumed, and, to a certain extent, awned seeds and fruits represent the various types of modifications for wind-distribution.

3. Animals, zoochores. Among terrestrial plants, dissemination by attachment represents essentially the same degree of specialization as is found in wind-distributed plants. The three types of contrivances for this purpose are found in spinose, hooked, and glandular fruits. Dissemination by cleglutition and by carriage, either intentional or unintentional, though of less value, play a striking part on account of the great distance to which the seeds may be carried. Dissemination by deglutition is characteristic of sarcospores, and distribution by carriage of creatospores.

4. Man, brotochores. Dissemination by man has practically no connection with mobility. It operates through great distances and over immense areas as well as near at hand. It may be intentional, as in the case of cultivated species, or unintentional, as in thousands of native or exotic species. No other disseminating agent is comparable with man in respect to universal and obvious migration. 
5. Gravity, clitochores. The members of this group are exclusively colline, montane, and alpine plants, growing on rocks, cliffs, and gravel-slides (talus), etc., in which the seeds reach lower positions merely by falling, or more frequently by the breaking away and rolling. down of rock or soil masses and particles. Dissemination by this method is relatively insignificant, though it plays an important part in the rock fields and gravel slides of mountain regions, particularly in the case of immobile species.

6. Glaciers, crystallochores. At the present time, transport by glaciers is of slight importance, because of the restriction of the latter to alpine and polar regions, where the flora is poorly developed. In the consideration of migrations during the glacial epoch, however, it plays an important point.

7. Growth, blastochores. The mobility of species disseminated by offshoots is extremely slight, and the annual movement relatively insignificant. The certainty of migration and of ecesis, is, however, so great, and the presence of offshoots so generally the rule in terrestrial plants that growth plays an important fart in migration, especially within formations.

8. Propulsion, bolochores. Like growth, clissemination by mechanical propulsion, though operating through insignificant distances, exerts an important effect in consequence of its cumulative action. The number of plants, however, with contrivances for propulsion is very much smaller than the number of blastochores. A!l bolochorous species agree in having modifications by means of which a tension is established. At maturity, this tension suddenly overcomes the resistance of sporangium or fruit, and throws the enclosed spores or seeds to some distance from the parent plant. In accordance with the manner in which the tension is produced, sling-fruits may be classified as follows :

(a) Hygroscopicity, pladoboles. These include the ferns with annulate sporangia, in which the expansion of the annulus by the absorption of moisture bursts the sporangium more or less suddenly, though the actual propulsion of the spores seems to come later as a result of dessication.

(b) Turgescence, edoboles. Dissemination by turgescence is highly developed in Pilobolus and in Discomycetes, though in the latter turgescence results rather in placing the spores in a position to be readily carried by the wind. Impatiens and Oxalis furnish familiar examples of fruits which dehisce in consequence of increased turgidity.

(c) Dessication, xerioboles. The number of fruits which dehisce upon drying is very large, but only a small portion of these expel their seeds forcibly. Geranizu, Viola, Erysimum, and Lotus illustrate the different ways in which dessication effects the sudden splitting of fruits.

(d) Resilience, tonoboles. In some plants, especially composites, labiates, and borages, the achenes or nutlets are so placed in the persistent calyx or 
involucre that the latter serves as a sort of mortar for projection, when the stem of the plant is bent to one side by any force, such as the wind or an animal. It will be noticed that two separate agents are actually concerned in dissemination of this sort.

Frequently, two or more agents will act upon the same disseminule, usually in succession. The possibility of such combinations in nature is large, but actual cases seem to be infrequent, except where the activities of man enter into the question. Some parts, moreover, such as awned inflorescences, are carried almost equally well by wind or animals, and may often be disseminated by the cooperation of these two agents. The wind also often blows seeds and fruits into streams by which they are carried away, but here again, parts adapted to wind-dissemination are injured as a rule by immersion in water, and the number of plants capable of being scattered by the successive action of wind and water is small.

In the present state of our knowledge of migration, it is impossible to establish any definite correspondence between dissemination-contrivance, agent, and habitat. As a general rule, plants growing in or near the water, in so far as they are morlified for this purpose at all, are adapted to watercarriage. Species which grow in exposed grassy or barren habitats are for the most part anemochores, while those that are found in the shelter of forests and thickets are usually zoochorous, though the taller trees and shrtibs, being exposed to the upper air currents, are generally wind-distributed. There is then a fair degree of correspondence, inasmuch as most hydrophytes are hydrochorous, most hylophytes, zoochorous, and the majority of poophytes and xerophytes, anemochorous. Definite conclusions can be reached, however, only by the statistical study of representative formations.

With respect to their activity, agents may be distinguished as constant, as in the case of currents, streams, winds, slope, growth, and propulsion, or intermittent, animals and man. In the former, the direction is more or less determinate, and migration takes place year by year, i. e., it is continuous, while in the latter dissemination is largely an accidental affair, indeterminate in direction, and recurring only at indefinite intervals. The effective conversion of migration into invasion is greatest when the movement is continuous, and least when it is discontinuous, since, in the latter, species are usually carried not only out of their particular habitat but even far beyond their geographical area, and the migration, instead of being an annual one with the possibility of gradual adjustment, may not recur for several years, or may, indeed, never take place again. The rapidity of migration is greatest in the case of intermittent agents, while the distance of migration is variable, being great chiefly in the case of man, ocean-cur- 
rents, and wind, and slight when the movement is due to slope, growth, or propulsion. Disregarding the great distances over which artificial transport may operate, seeds may be carried half way across the continent in a week by strong-flying birds, while the possibilities of migration by growth or expulsion are limited to a few inches, or at most to a few feet per year. This slowness, however, is more than counterbalanced by the enormously greater number of disseminules, and their much greater chance of becoming established.

268. The direction of migration is determinate, except in the case of those distributive agents which act constantly in the same direction. The general tendency is, of course, forward, the lines of movement radiating in all directions from the parent area. This is well illustrated by the operation of winds which blow from any quarter. In the case of the constant winds, migration takes a more or less definite direction, the latter being determined to a large degree by the fruiting period of any particular species. In this connection, it must be kept clearly in mind that the position of new areas with reference to the original home of a species does not necessarily indicate the direction of migration, as the disseminules may have been carried to numerous other places in which ecesis was impossible. The local distribution of zoochorous species is of necessity indeterminate, though distant migration follows the pathways of migratory birds and animals. In so far as dissemination by man takes place along great commercial routes, or along highways, it is determinate. In ponds, lakes, and other bodies of standing water, migration may occur in all directions, but in ocean currents, streams, etc., the movement is determinate, except in the case of motile species. The dissemination of plants by slopes, glaciers, etc., is local and definite, while propulsion is in the highest degree indeterminate. Migration by growth is equally indefinite, with the exception that hydrotropism and chemotropism result in a radiate movement away from the mass, while propulsion throws seeds indifferently into or away from the species-mass. From the above it will be seen that distant migration may take place by means of water, wind, animals or man, and, since all these agents act in a more or less definite direction over great distances, that it will be in some degree determinate. On the other hand, local migration will as regularly be indeterminate, except in the case of streams and slopes. The direction of migration, then, is controlled by these distributive agents, and the limit of migration is determined by the intensity and duration of the agent, as well as by the character of the space through which the latter operates. 


\section{ECESIS}

269. Concept. By the term ecesis is designated the series of phenomena exhibited by an invarling disseminule from the time it enters a new formation until it becomes thoroughly established there. In a word, ecesis is the adjustment of a plant to a new habitat. It comprises the whole process covered more or less incompletely by acclimatization, naturalization, accommodation, etc. It is the decisive factor in invasion, inasmuch as migration is entirely ineffective without it, and is of great value in indicating the presence and direction of migration in a great number of species where the disseminule is too minute to be detected or too little specialized to be recognizable.

The relation of migration to ecesis is a most intimate one: the latter depends in a large measure upon the time, direction, rapidity, distance, and amount of migration. In addition, there is an essential alternation between the two, inasmuch as migration is followed by ecesis, and the latter then establishes a new center from which further migration is possible, and so on. The time of year in which fruits mature and distributive agents act has a marked influence upon the establishment of a species. Disseminules designed to pass through a resting period are often brought into conditions where they germinate at once, and in which they perish because of unfavorable physical factors, or because competing species are too far advanced. On the other hand, spores and propagules designed for immediate germination may be scattered abroad at a time when conditions make growth impossible. The direction of movement is decisive in that the seed or spore is carried into a habitat sufficiently like that of the parent to secure establishment, or into one so dissimilar that germination is impossible, or at least is not followed by growth and reproduction. The rapidity and distance of migration have little influence, except upon the less resistant disseminules, conidia, gemmae, etc. Finally, the amount of migration, i. e., the number of migrants, is of the very greatest importance, affecting directly the chances that vigorous disseminules will be carried into places where ecesis is possible.

Normally, ecesis consists of three essential processes, germination, growth, and reproduction. This is the rule among terrestrial plants, in which migration regularly takes place by means of a resting part. In free aquatic forms, however, the growing plant or part is usually disseminated, and ecesis consists merely in being able to continue growth and to insure reproduction. Here establishment is practically certain, on account of the slight differences in aquatic habitats, excepting of course the extremes, fresh water and salt water. 'The ease indeed with which migration and ecesis are effected in the water often makes it impossible to speak properly of invasion in this connection, since aquatics are to such a large extent cosmopolitan. 
In dissemination by offshoots, the conditions are somewhat similar. Here, also, ecesis comprises the sequence of growth and reproduction, and invasion, in the sense of passing from one habitat to another, is of rare occurrence, as the offshoot grows regularly under the same conditions as the parent plant. The adjustment of growing plants and parts is so slight, and their establishment so certain on account of their inability to migrate into very remote or different habitats, that they may be ignored in the following discussion.

In accordance with the above, it would be possible to distinguish three groups of terrestrial plants: (I) those migrants which germinate and disappear, (2) those which germinate and grow but never reproduce, (3) those which reproduce, either by propagation or generation, or both. Such a classification has little value, however, since the same species may behave in all three fashions, depending upon the habitat to which it has migrated, and since invasion does not occur unless the plant actually takes possession, i. e., reproduces. From the latter statement, it follows that invasion occurs only when a species migrates to a new place, in which it germinates, matures, and reproduces. Maintenance by annual invasion simply, in which the plants of each year disappear completely, can not then be regarded as invasion proper. On the other hand, though such instances are rare, it is not necessary that the invaders produce fruit, provided they are able to maintain themselves, or to increase by propagation. Furthermore, if a plant germinate, grow, and reproduce, it is relatively immaterial whether it persist for a few years or for many, since, as we shall see under Succession, the plants of one invasion are displaced by those of the next, the interval between invasions increasing with the stabilization.

270. Germination of the seed. The germination of seed or spore is determined by its viability and by the nature of the habitat. Viability depends upon the structural characters of fruit, seed-coat, and endosperm, and to a degree upon the nature of the protoplasm or embryo. The first three affect the last directly, by protecting the embryo against dryness, against injury due to carriage by water, or by deglutition, and probably in. some cases against excessive heat or cold. Marloth ${ }^{1}$ has investigated the structure of seed coats, establishing the following groups, which are summarized here somewhat fully because of their bearing upon ecesis: seed coats without protective elements, endosperm absent or rudimentary, Epilobium, Impatiens, Parnassia, Sasittaria, etc.; (2) protective elements lacking or few, endosperm highly developed with thick-walled cells,

${ }^{2}$ Uber mechanische Shutzmittel der Samen gegen schädliche Einfüsse von aussen. Engler Bot. Jahrb., $5: 56.1883$. 
Liliaceae, Primulaceae, Rubiaceae, etc.; (3) protective cells present in the seed coats, endosperm. little or none, Boraginaceae, Crassulaceae, Cruciferae, Labiatae, Papilionaceae, etc.; (4) protective elements present, Asclepias, Campanula, Gentiana, Silcne, Saxifraga, etc.; (5) protective cells present, endosperm thick-walled, Euonymus, Helianthemum, Ribes. The protective cells are of various kinds: (I) epidermal cells strongly cuticularized, Caryophyllaceae, Crassulaceae, Fumariaceae, Saxifragaceae; (2) parenchyma thick-walled, several-layered, Aesculus, Castanea, Fagus; parenchyma cells with the inner or radial walls thickened, Campanula, Erythraea, Gentiana; (4) epidermal cells cup-shaped, thick-walled, Cruciferae, Ribes, Vaccinium; (5) parenchyma with thickened, cellulose walls, Geranium, Viburnum; (6) a single row of stone-cells, Labiatae; (7) tissue of stone-cells, Hippuris, Naias, Potamogeton; (8) elongate stone-cells, Coniferae, Cupuliferae, Euphorbia, Limum, Malva, Viola; (9) short, columnar, thick-walled branched cells, Cucurbitaceae, Datura, Hypericum; (Io) frosenchyma with cellulose walls, Clematis; (II) prosenchyma with lignified walls, Fraxinus, Rhomnus, Ranunculus. The seed-coats have a certain influence in determining germination at the proper time, inasmuch as they make it difficult for the seed to germinate under the stimulus of a quantity of warmth and moisture insufficient to support the seedling. The effect of the endosperm, as well as that of other food-supply in the seed, upon germination and the establishment of the seedling is obvious.

The behavior of seed or spore with respect to germination depends in a large degree upon the character of the protoplasm or embryo, though in just what way is at present a matter of conjecture. It is evident that many seeds are not viable because fertilization has not been effected, and in consequence no embryo has developed. This is the usual explanation of the low germinating power of the seeds of some species, especially polyspermatous ones. But even in viable seeds the behavior is always more or less irregular. The seeds of some species will grow immediately after ripening, while others germinate only after a resting period of uncertain duration. The same is true of spores. Even in the case of seeds from the same parent, under apparently similar conditions, while the majority will germinate the first year, some will lie dormant for one or more years. The precise reason why many seeds and spores germinate more readily after being frozen is equally obscure. The period of time for which disseminules may remain viable is extremely diverse, though, as would be expected, it is much longer as a rule for seeds than for spores. The greater vitality of seeds in the case of ruderal plants suggests that this diversity may be due simply to variation in the vigor of the embryos. It would seem that under proper conditions seeds may retain their viability for an indefinite period. 
The influence of habitat upon germination is of primary importance, though the manner in which its influence is exerted is by no means as evident as might be supposed. In the case of seeds sown in the planthouse, it is almost universally the case that germination is less than in nature, notwithstanding the fact that temperature and moisture appear to be optinum. In nature, the seeds of the species nay be carried into a number of different formations, any one or all of which may present conditions unfavorable to germination. With respect to probability of germination, habitats are of two sorts: those which are denucled and those which bear vegetation. It is impossible to lay down general propositions with respect to either group, since germination will vary with the character of the invading species, the annual distribution of heat and moisture in the habitat, etc. In a general way, however, it may be stated that the chances for germination are greater in vegetation than in denuded areas, chiefly because the latter are usually xerophytic. On the other hand, the lack of competition in the clenuded area tends to make ultimate establishment much more certain. Here, as elsewhere when exact statistical results are desired, the use of the quadrat, and especially of the permanent quadrat, is necessary to determine the comparative germination of the invading species in relation to denudation and vegetation.

271. Adjustment to the habitat. The seedling once established by germination, the probability of its growing and maturing will depend upon its habitat form, plasticity, and vegetation form. Even though it may germinate under opposite conditions, a typical hylophyte, such as Impatiens for example, will not thrive in an open meadow, nor will characteristic poophytes, such as most grasses, srow in deep shade. In the same way, xerophytes do not adapt themselves to hydrophytic habitats, nor hydrophytes to xerophytic conditions. Many mesophytes, however, possess to a certain degree the ability to adjust themselves to somewhat xerophytic or hydrophytic situations, while woodland plants often invade either forest or meadow. This capability for adjustment, i. e., plasticity, is greatest in intermediate species, those that grow in habitats not characterized by great excess or deficiency of some factor, and it is least in forms highly specialized in respect to water-content, shade, etc. It may then be established as a fundamental rule that ecesis is cletermined very !argely by the essential physical similarity of the old and the new habitat, except in the case of plastic forms, which admit of a wider range of accomodation. The plasticity of a plant is not necessarily indicated by structural modification, though such ad.justment is usually typical of plastic species, but it may sometimes arise from a functional adaptation, which for some reason does not produce 
concomitant structural changes. The former explains such various habitat forms of the same species as are found in Galium boreale, Gentiana acuta, etc., and the latter the morphological constancy of plants like Chamaenerium, which grow in very diverse habitats.

The vegetation form of the invading species is often of the greatest importance in determining whether it will become established. The vegetation form represents those modifications which, produced in the original home by competition, i. e., the struggle for existence, are primarily of value in securing and maintaining a foothold. These comprise all structures by means of which the plant occupies a definite space in the air, through which the necessary light and heat reach it, and in the soil, from which it draws its food supply. These structures are all organs of duration or of perennation, such as root, rootstalk, bulb, tuber, woody stem, etc., which find their greatest development among trees and shrubs, and their least among annual herbs. But while the invaders are aided in securing possession by the proper vegetation form, the occupation of the plant already in possession is increased by the same means, and the outcome is then largely determined by other factors. To avoid repetition, the bearing of occupation upon invasion will be considered under succession.

\section{BARRIERS}

272. Concept. De Canclolle ${ }^{1}$ seems to have been the first to use the term barrier and to distinguish the various kinds, though Hedenberg ${ }^{2}$ clearly saw that stations of one kind were insurmountable obstacles to plants belonging ic a very different type. De Candolle pointed out that the natural barriers to continuous invasion ("transport de proche en proche") are: (I) seas, which decrease invasion almost in inverse proportion to their extent; (2) deserts; (3) mountain ranges, which are less absolute on account of passes, valleys, etc.; (4) vegetation, marshes being barriers to dry land plants, forests to those that fear the shade, etc. Grisebach ${ }^{3}$, in discussing the effect of barriers upon the constitution of vegetation, laid down the fundamental rule that: "The stpreme law which serves as the basis of the permanent establishment of natural floras is to be recognized in the barriers which have hindered or completely prevented invasion."

Any feature of the topography, whether physical or biological, that restricts or prevents invasion, is a barrier. Such features are usually permanent and produce permanent barriers, though the latter may often be temporary, existing for a few years only, or even for a single season. In

${ }^{1}$ Essai Elémentaire de Géographie Botanique, 45.

${ }^{2}$ Stationes Plantarum Amoen. Acad., $4: 64.1754$.

${ }^{3}$ Die Vegetation der Erde, 4. 1872.

1820. 
this last case, however, they are as a rule recurrent. Barriers may furthermore be distinguished as complete or incomplete with respect to the thoroughness with which they limit invasion. Finally, the consideration of this subject gains clearness if it be recognized that there are barriers to. migration as well as to ecesis, and if we distinguish barriers as physical or biological with reference to the character of the feature concerned.

273. Physical barriers are those in which limitation is produced by some marked physiographic feature, such as the ocean or some other large body of water, large rivers, mountain ranges and deserts (including ice and snow fields). All of these are effective by virtue of their dominant physical factors; hence they are barriers to the ecesis of species coming from very different habitats, but they act as conductors for species from similar vegetation, especially in the case of water currents. A body of water, representing maximum water-content, is a barrier to mesophytic and xerophytic species, lut a conductor for hydrophytic ones; deserts set a limit to the spread of mesophytic and hydrophytic plants, while they offer conditions favorable to the invasion of xerophytes; and a high mountain range, because of the reduction of temperature, restricts the extension of macrothermal and mesothermal plants. A mountain range, unlike other physical barriers, is also an obstacle to migration, inasmuch as natural distributive agents rarely act through it or over it.

274. Biological barriers include vegetation, man and animals, and plant parasites. The limiting effect of regetation is exhibited in two ways. In the first place, a formation acts as a barrier to the ecesis of species invading it from the formations of another type, on account of the physical differences of the habitats. Whether such a barrier be complete or partial will depend upon the degree of dissimilarity existing between the formations. Hylophytes are unable to invade a prairie, though open thicket plants may do so to a certain degree. In the same way, a forest formation on account of its diffuse light is a barrier to poophytes; and a swamp, because of the amount and character of the water-content, sets a limit to both hylophytes and poophytes. Formations, such as forests, thickets, etc., sometimes act also as direct obstacles to migration, as in the case of tumbleweeds and other anemochores, clitochores, etc. A marked effect of vegetation in decreasing invasion arises from the closed association typical of stable formations and of social exclusive species. In these, the occupation is so thorough and the struggle for existence so intense that the invaders, though fitted to grow under the physical factors present, are unable to compete with the species in possession for the requisite amount of some neces- 
sary factor. Closed associations usually act as complete barriers, while open ones restrict invasion in direct proportion to the degree of occupation. To this fact may be traced a fundamental law of succession, viz., the number of stages in a succession is determined largely by the increasing difficulty of invasion as the habitat becomes stabilized. Man and animals affect migration directly, though not obviously, by the destruction of disseminules. They operate as a pronounced barrier to ecesis wherever they alter conditions in such a way as to make them unfavorable to invading species, or when, by direct action upon the latter, such as grazing, tramping, parasitism, etc., they turn the scale in the struggle for existence. The absence of insects adapted to insure fertilization is sometimes a serious barrier to the establishment of adventitious or introduced plants. The presence of parasitic fungi, in so far as they destroy the seeds of plants, acts as an obstacle to migration, and restricts or prevents ecesis in so far as the fungi destroy the invaders, or place them at a disadvantage in the struggle for existence.

275. Influence of barriers. Physical barriers are typically permanent in character, while biological ones are either permanent or temporary, depending upon the permanence of the formation and the constancy of the physical factors which determine it. A stable formation, such as a forest or meadow, which acts as a decided barrier to invasion from adjacent vegetation, may disappear completely, as a result of a landslide, flood, or burn, or through the activity of man, and may leave an area into which invaders crowd from every point. Often, without undergoing marked change, a formation which has presented conditions unfavorable to the ecesis of species of mesophytic character may, by reason of a temporary change in climate, become suffciently modified to permit the invasion of mesophytes. On the other hand, a meadow ceases to be a barrier to prairie xerophytes during a period of unusually dry years. A peculiar example of the modification of a barrier is afforded by the defoliation of aspen forests in the mountains as a result of which poophytes have been enabled to invade them. Nearly all xerophytic stretches of sand and gravel, dunes, blowouts, gravel slides, etc., and even prairies to a certain degree, exhibit a recurrent seasonal change in spring, as a result of which the hot, dry surface becomes sufficiently moist to permit the germination and growth of invaders, which are entirely barred out during the remainder of the year. In an absolute sense, no barrier is complete, since the coldest as well as the dryest portions of the earth's surface are capable, at times at least, of supporting the lowest types of vegetation. Relatively, however, in connection with the natural spread of terrestrial plants, it is possible to distinguish partial barriers from complete ones. Such a distinction is of importance in the consideration of invasions from 
a definite region, as it is only in this restricted sense that complete barriers have produced endemism.

Distance, though hardly to be considered a barrier in the strict sense of the word, unquestionably plays an important part in determining the amount of invasion. The effect of distance is best seen in the case of migration, as it influences ecesis only in those rare cases where viability is affected. The importance of distance, or take the converse, of proximity, is readily ascertained by the study of any succession from denudation. It has been established that the contiguous vegetation furnishes $75-90$ per cent of the constituent species of the initial formation, and in mountainous regions, where ruderal plants are extremely rare, the percentage is even higher. The reason for this is to be found not only in the fact that the adjacent species have a much shorter distance to go, and hence will be carried in much greater quantity, but also in that the species of the formations beyond must pass through or over the adjacent ones. In the latter case, the number of dissemintules is relatively small on account of the distance, while invasion through the intermediate vegetation, if not entirely impossible, is extremely slow, so that plants coming in by this route reach the denuded area only to find it already occupied. It is as yet impossible to give a definite numerical value to proximity in the various invasions that mark any particular succession. This will not be feasible until a satisfactory method has been found for determining a coefficient of mobility, but, this once done, it will be a relatively simple matter, not merely to trace the exact evolution of any succession of formations, but actually to ascertain from the adjacent vegetation the probable constitution of a particular future stage.

From what has been said, it follows that the primary effect of barriers upon vegetation is obstruction. Where the barrier is in the pathway of migration, however, it causes deffection of the migrant as a rule, and sets up migration in a new direction. This is often the case when the strong winds of the plains carry disseminules towards the mountains and, being 11nable to cross the range, drop them at the base, or, being deflected, carry them away at right angles to the original direction. The same thing happens when resistant fruits and seeds borne by the wind fall into streams of water. or into ocean currents. The direction of migration is changed, and what is normally a barrier serves as an agent of dissemination.

\section{ENDEMISM}

276. Concept. Since its first use by DeCandolle, the term endemic has been employed quite consistently by phytogeographers with the meaning of "peculiar to a certain region." Some confusion, however, has arisen from the fact that a few authors have made it more or less synonymous 
with indigenous and autochthonous, while others have regarded it as an antonym of exotic. In its proper sense, endemic refers to distribution, and not to origin. Its exact opposite will be found then in Fenzl's term polyclemic, dwelling in severa! regions. Indigenous (autochthonous) and exotic, on the contrary, denote origin, and are antonyms, indigenous signifying native, and exotic foreign. As Drude has shown, endemic plants may be either indigenous, as in the case of those species that have never moved out of the original habitat, or exotic, as in the much rarer instances where a polydemic species has disappeared from its original home and from all regions into which it has migrated except one. It is understood that not all indigenous or exotic species are endemic. The proportion of endemic to polydemic species is a variable and somewhat artificial one, depending upon the size of the divisions employed.

277. Causes. The primary causes of endemism are two, lack of migration and presence of barriers. Since distributive agents are practically tiniversal, lack of migration corresponds essentially to immobility, a fact which decreases the difficulty of ascertaining the immediate causes of endemism in any particular species. Either immobility or a barrier may produce endemism: extremely immobile plants, for example, liliaceous species propagating almost wholly by underground parts, are as a rule endemic, while alpine plants and those of oceanic islands are endemic in the highest degree, regardless of their mobility. When the two conditions act concomitantly upon a species, endemism is almost inevitable. It can not be supposed, however, that immobility or natural barriers alone, or the concomitance of the two, must invariably give rise to endemic species; the most immobile plant may be carried into another region by unusual or accidental agencies, or the most formidable barrier to migration may be overcome by the intensity of an agent or through the action of man. Endemism is also brought about by the modification of species; new or nascent species are as a rule endemic. Whether they will remain endemic or not will depend upon the perfection of their contrivances for dissemination and upon the presence of harricrs to migration or ecesis. Finally, as D'rude was the first to point out, the disappearance of a polydemic species in all regions but one, owing to the struggle for existence or to changed physical conditions, will result in endemism.

278. Significance. Endemism is readily recognized by methods of distributional statistics, applied to areas limited by natural barriers to migration or ecesis. For political areas, it has no significance whatever, unless the boundaries of these coincide with barriers. It determines in the 
first degree the validity of regions, though the latter are often recognized also by the presence of barriers and by the character of the vegetation. Endemism may occur in areas of vegetation of any rank from a formation to a zone. When the term is not qualified, however, it should be used of species with reference to formations alone. Comparisons to be of value, however, can be instituted only between areas of the same order, i. e., between two or more formations, two or more regions, provinces, etc. In the same way, taxonomic groups of the same rank should be used in such comparisons, i. e., species should be contrasted with species, genera with genera, and families with families, except when it is desired to obtain some measure of the age of the vegetation by the differentiation of the endemic phyla within it. There will be seen to exist a funclamental correspondence between the rank of the floral division and the taxonomic group, though the apparent exceptions to this are still too numerous to warrant its expression in a general law. As a rule, however, formations most frequently show endemic habitat forms and species, more rarely endemic genera; regions and provinces commonly exhibit endemic species and genera, rarely endemic families; while zones and hemispheres contain endemic orders as well as families. This correspondence is readily seen to depend primarily upon the fact that increased differentiation in the taxonomic sense is a concomitant of the increased invasion of endemic species, measured in terms of distance and difference in habitat.

It is too early to decide satisfactorily whether it is proper to speak of formations as endemic. At first thought it would seem that all formations, with the exception of ruderal ones, were endemic, but a study of almost any transition area between regions would seem to point to the opposite conclusion, viz., that no formations are properly endemic. It is equally impossible at present to distinguish different types of endemics, such as relictae, etc., as any such classification must await the elaboration of a method for determining the phylogeny of a natural group of species by an investigation of their comparative differentiation in connection with their migration in all directions from the vegetation center into new habitats. In short, it will not be possible to make a thorough study of endemism and to postulate its laws until modern methods of research have been extended to a much larger portion of the vegetation of the globe. The final task of phytogeography is the division of the earth's vegetation into natural areas. It will be at once evident that most plants can not properly be called endemic until the natural regions in which they are found have been accurately defined, a work which has barely begun. In the much simpler matter of distribution, upon which the accuracy of statistical methods depends directly, there are few regions sufficiently well known at the present time to yield anything like permanent results. 


\section{POLYPHYLESIS AND POLYGENESIS}

279. Concept. The idea of polyphylesis, as advanced by Engler, contains two distinct concepts: (I) that a species may arise in two different places or at two different times from the same species, and (2) that a genus or higher group may arise at different places or times by the convergence of two or more lines of origin. It is here proposed to restrict polyphylesis, as its meaning would indicate, to the second concept, and to employ for the first the term polygenesis, ${ }^{1}$ first suggested by Huxley in the sense of polyphylesis. The term polyphylesis is extended, however, to cover the origin of those species which arise at different places or times from the convergence of two or more different species, a logical extension of the idea underlying polyphyletic genera, though it may seem at first thought to be absurd. Polygenesis may be formally defined as the origin of one species. from another species at two or more distinct places on the earth's surface, at the same time or at different times, or its origin in the same place at different times. Polyphylesis, on the contrary, is the origin of one species from two or more different species at different places, at the same time or at different times. It is evident that what is true of species in this connection will hold equally well of genera and higher groups. Opposed to polygenesis is monogenesis, in which a species arises but once from another species; with polyphylesis is to be contrasted monophylesis, in which the species arises from a single other species. It will be noticed at once that these two concepts are closely related. The following diagrams will serve to make the above distinctions more evident:

I. Polygenesis

II. Polyphylesis

III. Monogenesis
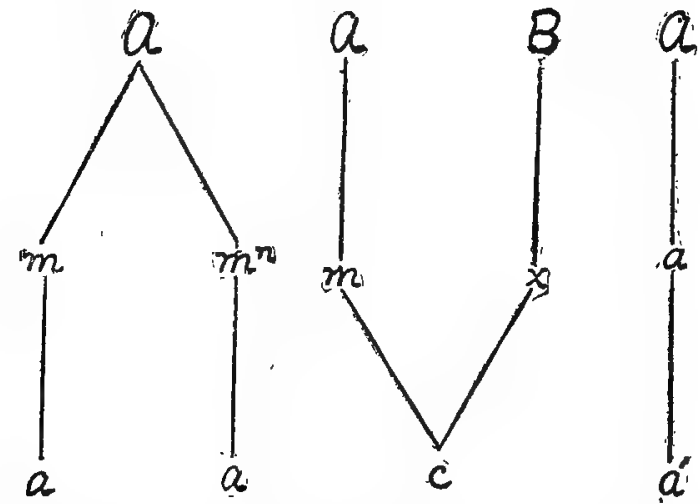

(Monophylesis)

${ }^{2}$ When this word was first proposed, the author did not know that Briquet had already applied the term polytopism to this concept (Ann. Conserv. Bot. Gen., $5: 73$. 
In I, a species $A$, becomes scattered over a large area in a series of places, $m$. . . $m^{n}$, with the same physical. factors, in any or all of which may arise the new species $a$. In II, a species with xerophytic tendency, $A$, and one with mesophytic tendency, $B$, in the course of migration find themselves respectively in a more mesophytic habitat, $m$, and a more xerophytic one, $x$, in which either may give rise to the new form, $c$, which is more or less intermediate between $A$ and $B$. In III, the method of origin is of the simplest type, in which a species is modified directly into another one, or is split up into several.

280. Proofs of polygenesis. In affirming the probability of a polygentic origin of species, there is no intention of asserting that all species origi, ate in this way. It seems evident that a very large number of species of restricted range are certainly monogenetic, at least as far as origin in space is concerned. It is possible that any species may arise at two or more distinct times. Polygenesis can occur readily only in species of more or less extensive area, in which recur instances of the same or similar habitat. The relative frequence and importance of the two methods can hardly be conjectured as yet, but origin by monogenesis would seem to be the rule.

The arguments adduced by Engler in support of polygenesis are in themselves conclusive, but the investigations of the past decade have brought to light additional proofs, especially from the experimental side. In determining the physical factors of prairie and mountain formations, and especially by methods of experimental ecology, the author has found that habitats are much less complex than they are ordinarily thought to be, since water-content and humidity, and to a less degree light, constitute the only factors which produce direct modification. In addition, it has been ascertained that the minimum difference of water-content, humidity, or light, necessary to produce a distinguishable morphological adjustment is much greater than the unit differences recorded by the instruments. In short, the differences of habitats, as ascertained by thermograph, psychrometer and photometer, are much greater than their efficient differences, and, with respect to their ability to produce modification, habitats fall into relatively few categories. A striking illustration of this is seen in the superficially very different habitats, desert. strand, alkali plain, alpine moor, and arctic tundra, all of which are capable of prodticing the same type of xerophyte. It follows from this that many more or less plastic species of extensive

1901). Since polygenesis expresses the idea of origin, and applies to multiple origin in time as well as in space, it is retained as the name of this concept. Polytopic and monotopic are adopted for multiple and single origin in space respectively, and polychronic and monochronic are proposed for similar origin in time. 
geographical area will find themselves in similar or identical situations, measured in terms of efficient differences, and will be modified in the same way in two or more of these. In mountain regions, where interruption of the surface and consequent alternation are great, the mutual invasion of contiguous formations is of frequent occurrence, often resulting in labitat forms. The spots in which these nascent species, such as Galium boreale hylocolum, Aster levis lochmocolus, etc., are found, are often so related to the area of the parent species as to demonstrate conclusively that these forms are the result of polygenesis and not of migration. $\mathrm{Na}$ turally, what is true of a small area will hold equally well of a large region, and the recurrence of the same habitat form may be accepted as conclusive proof of polygenesis. The most convincing evidences of multiple origin, however, are to be found in what DeVries has called "mutations." It makes little difference whether we accept mutations in the exact sense of this author, or regard them as forms characterized by latent variability. The evidence is conclusive that the same form may arise in nature or in cultivation, in Holland or in America, not merely once, but several or many times. In the presence of such confirmation, it is unnecessary to accumulate proofs. Polygenesis throws a new light upon many difficult problems of invasion and distribution, and, as a working principle, admits of repeated tests in the field. It obviates, moreover, the almost insuperable difficulties in the way of explaining the distribution of many polygenetic species on the basis of migration alone.

281. Origin by polyphylesis. In $I 898$, the author first advanced a tentative hypothesis to the effect that a species homogeneous morphologically may arise from two distinct though related species. During subsequent years of formational study, the convinction has grown in regard to the probability of such a method of origin. Since the appearance of Engler's work, a polyphyletic origin for certain genera has been very generally accepted by botanists, but all have ignored the fact that the polyphylesis of genera carries with it the admission of such origin for species, since the former are merely groups of the latter. I can not, however, agree with Engler, that polyphyletic genera, and hence species also, are necessarily unnatural. If the convergence of the lines of polyphylesis has been great, resulting in essential morphological harmony, the genus is a natural one, even though the ancestral phyla may be recognizable. If, on the other hand, the convergence is more or less imperfect, resulting in subgroups of species more nearly related within the groups than between them, the genus can hardly be termed natural. This condition may, however, prevail in a monophyletic genus with manifest divergence and still not be an indication that it is artificial. 
Darwin", in spcaking of convergence, has said: "If two species, belonging to two distinct though allicd genera, had both produced a large number of new and divergent forms, it is conceivable that these might approach each other so closely that they would have all to be classified under the same genus; and thus the descendants of two distinct genera would converge into one." The application of this statement to species would at once show the possibility of polyphylesis in the latter, and a further examination of the matter will demonstrate its probability. It is perfectly evident that a species may be split into two or more forms by varying the conditions, let us say of water-content, and that the descendants of these forms may again be changed into the parent type by reversing the process. This has, in fact, been done experimentally. Since it is admittedly impossible to draw any absolute line between forms, varieties, and species, it is at once clear that two distinct though related species, especially if they are plastic, may be caused to converge in such a way that the variants may constitute a new and homogeneous species. This may be illustrated by a concrete case at present under investigation. Kuhnistera purpurea differs from $K$. candida in being smaller, in having fewer, smaller, and more narrow leaflets, and a globoid spike of purple flowers in place of an elongated one of white flowers; in a word, it is more xerophytic. This conclusion is completely corroborated by its occurrence. On dozens of slopes examined, Kuhnistera purpurea has never been found mingling with $K$. candida on lower slopes, except where an accident of the surface has resulted in a local decrease of water-content. The experiment as conducted is a simple one, consisting merely in sowing seed of each in the zone of the other, and in growing $K$. purpurea under controlled mesophytic conditions, and $K$. candida under similarly measured xerophytic conditions in the planthouse.

While the polyphyletic origin of species is in a fair way to be decided by experiment, it receives support from several well-known phenomena. The striking similarity in the plant body of families taxonomically so distinct as the Cactaceae, Stapeliaceae, and Euphorbiaceae, or Cyperaceac and Inncaceae, indicates that a vegetation form may be polyphyletic. On the other hand, the local appearance of zygomorphy, of symphysis, and of aphanisis in the floral types of phylogenetically distinct families is a proof of the operation of convergence in reproductive characters. To be sure, the convergence is never so great as to produce more than superficial similarity, but this is because the groups are markedly. different in so many fundamental characters. The same tendency in closely related species would easily result

${ }^{1}$ The Origin of Species, 186. 1850. 
in indentity. As in the case of polygenesis, the relatively small number of typically distinct habitats makes it clear that two different species of wide distribution, bearing to each other the relations of xerophyte to mesophyte, of hydrophyte to mesophyte, or of poophyte to hylophyte, might often find themselves in reciprocal situations, with the result that they would give rise to the same new form. The final proof of the polyphylesis of species is afforded by the experiments of DeVries in mutation. DeVries found that Oenothera nanella arose from $O$. Lamarckiana, $O$. laevifolia, and $O$. scintillans; Oenothera scintillans arose from $O$. lata and $O$. Lamarckiana; Oenothera rubrinervis from $O$. Lamarckiana, $O$. laevifolia, $O$. lata, $O$. oblonga, $O$. nanella, and $O$. scintillans, etc. Whatever may be the rank assigned to these mutations, whether form, variety, or species, there can be no question of their polyphyletic origin, nor, in consequence of the connection of mutations with variations through such inconstant forms as $O$. scintillars, $O$. elliptica, and $O$. sublinearis, of the possibility of polyphylesis in any two distinct though related species or genera.

\section{KINDS OF INVASION}

282. Continuous and intermittent invasion. With respect to the frequency of migration, we may distinguish invasion as contimous, or intermittent. Continuous invasion, which is indeed usually mutual, occurs between contiguous formations of more or less similar character, in which there is an annual movement from one into the other, and at the same time a forward movement through each, resulting from the invaders established the preceding year. By far the greater amount of invasion is of this sort, as many readily be seen from the fact that migration varies inversely as the distance, and ecesis may decrease even more rapidly than the distance increases. The significant feature of continuous invasion is that an outpost may be reinforced every year, thus making probable the establishment of new outposts from this as a center, and the ultimate extension of the species over a wide area. The comparatively short distance and the regular alternation of migration and ecesis render invasion of this sort very effective. An excellent illustration of this is seen in transition areas and regions, which are due directly to continuous and usually to mutual invasion. Intermittent invasion results commonly from distant carriage, though it may occur very rarely between dissimilar adiacent formations, when a temporary swing in the physical factors makes ecesis possible for a time. It is characterized by the fact that the succession of factors which have brought about the invasion is more or less accidental and may never recur. Intermittent invasion is relatively rare, and from the small number of disseminules affected, it is of little importance in modifying vegetation quantitatively. On the other 
hand, since a species may often be carried far from its geographical area, it is frequently of great significance in distribution.

283. Complete and partial invasion. When the movement of invaders into a formation is so great that the original occupants arc finally driven out, the invasion may be termed complete. Such invasion is found regularly in the case of many ruderal formations, and is typical of the later stages of many successions. It is ordinarily the result of continuous invasion. If

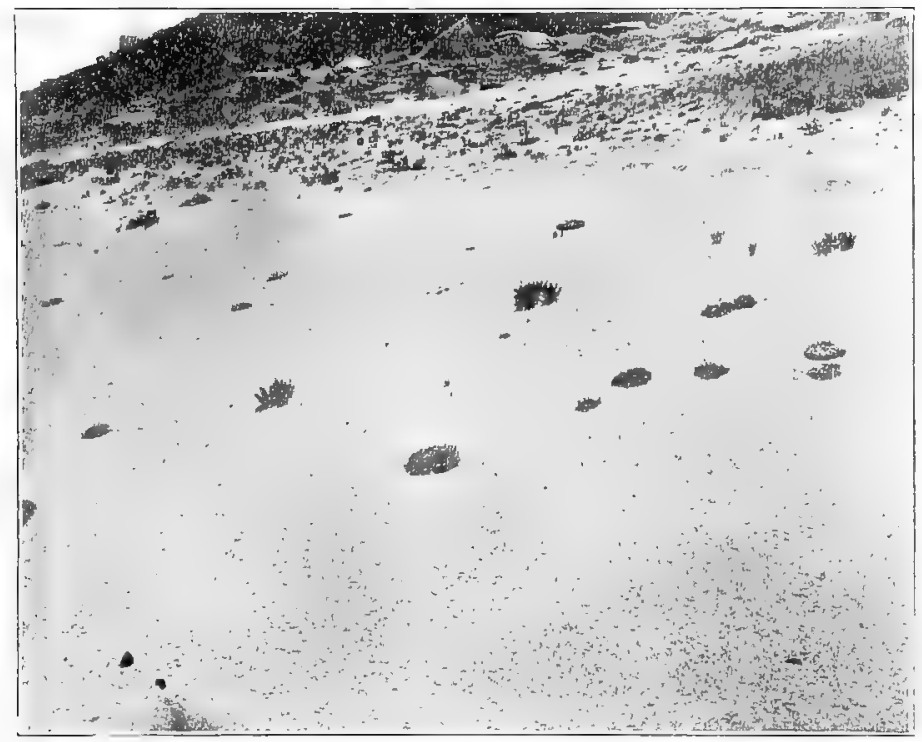

Fig. 59. Continuous invasion into a new area; mats of Arenaria sajunensis. Silcne acaulis and Sieversia turbinata invading an alpine gravel slide.

the number of invaders is sufficiently small that they may be adopted into the formation without radically changing the latter, the invasion is partial. This is doubtless true of the greater number of invasions, though these are regularly much less striking and important than instances of complete invasion.

284. Permanent and temporary invasion. The permanence of invasion depends upon the success attending ecesis, and upon the stability of the formation. It has already been noticed that under certain conditions plants may germinate and grow, and if they are perennials, even become established, 
and still ecesis be so imperfect that reproduction is impossible. Others may find the conditions sufficiently favorable for propagation, but unfavorable for the formation of flowers and fruits. Finally there are plants which seem to be perfectly established for a few years, only to disappear completely. The latter are examples of temporary invasion. It is necessary to draw clearly the line between complete and partial invasion in this connection. The former is temporary in the initial or intermediate stages of nearly all-successions, as compared with the ultimate stages, though it is in a large degree permanent in comparison with the partial invasion of species which are able to maintain themselves for a few years. In a sense, there is a real distinction between the two, inasmuch as a particular stage of succession is permanent as long as the habitat remains essentially the same. A critical study of the species of such stages shows, however, that they manifest very different degrees of permanence. Species which invade stable vegetation temporarily have been termed adventive by A. DeCandolle. Permanent inrasion occurs when a species becomes permanently established in a more or less stable formation. It is characteristic of the great majority of invaders found in the grassland and forest stages of successions.

Plants which have arisen within a formation or have been a constituent part of it since its origin are indigenous. Contrasted with these are the species which have invaded the formation since it received its distinctive impress: these are derived. The determination of the indigenous and derived species of a formation or larger division is of the utmost importance, as it enables us to retrace the steps by which the formation has reached its present structure, and to reconstruct formations long since disappeared. To render it less difficult, it is necessary to scrutinize the derived elements closely, first, because it is easiest to recognize the indigenous species by eliminating the derived, and second, because this analysis will show that not all derived species have entered the formation at the same time and from the same sources. Derived species may be termed vicine, when they are fully established invaders from adjacent formations or regions, and adventitions, when they have come from distant formations and have succeeded in establishing themselves. Finally, those derived species which are unable to establish themselves permanently are adventive.

\section{MANNER OF INVASION}

285. Entrance into the habitat. Since the ecesis of invaders depends in large measure upon the occupation of the plants in possession, the method and degree of invasion will be determined by the presence or absence of vegetation. Areas without vegetation are either originally naked or de- 
sunded, while vegetation with respect to the degree of occupation is open (sporadophytia), or closed (pycnophytia). Each type of area presents different conditions to invaders, largely with respect to the factors determining ecesis. Naked habitats, rocks, talus, gravel slides, and dunes, while they offer ample opportunity for invasion on account of the lack of occupation, are really invaded with the greatest difficulty, not only because they contain originally few or no disseminules, but also because of their xerophytic character and the diffictlty of obtaining a foothold, on account of the extreme density or instability of the soil. Denuded habitats, blowouts, sand draws, ponds, flood plains, wastes, fields, and burns, ustually afford maximum opportunity for invasion. They invariably contain a large number of disseminules ready to spring up as soon as the original vegetation is destroyed. The surface, moreover, is usually such as to catch disseminules and to offer them optimum conditions of moisture and nutrition. Open formations are readily invaded, though the increased occupation renders entrance more difficult than it is in denuded areas. Closed formations, on the other hand, are characterized by a minimum of invasion, partly because invaders from different formations find unfavorable conditions in them, but chiefly because the occupation of the inhabitants is so complete that invaders are unable to establish themselves.

Invasion takes place by the penetration of single individuals or groups of individuals. This will depend in the first place upon the character of the disseminule. It is evident that, no matter how numerous the achenes may be, the invasion of those anemochorous species with comate or winged seeds or one-seeded fruits will be of the first type, while all species in which the disseminule is a several or many-seeded fruit or plant, as in hooked fruits, tumble-weeds, etc., will tend to produce a group of invaders. Occasionally of course, the accidents of migration will bring together a few one-seeded disseminules into a group, or will scatter the seeds of a many-seeded fruit, but these constitute relatively rare exceptions. This distinction in the matter. of invasion is of value in studying the relative rapidity of the latter, and the establishment of new centers, but it is of greatest importance in explaining the historical arrangement of species in a formation, and hence has a direct bearing upon alternation. It is entirely independent of the number of invaders, which, as we have seen, depends upon seed-production, mobility, distance, occupation, etc., but is based solely upon mode of arrangement, and will be found to underlie the primary types of abundance, copious, and gregarious. In this connection, it should also be noted that the contingencies of migration, especially the concomitant action in the same direction of two or more distributive agencies, often results in the penetration of a group of individuals belonging to two or more species. This may well be 
termed mass invasion; it is characteristic of transition areas or regions, and along valleys or other natural routes for migration it gives rise to species guilds. The movement of species guilds constitutes one of the most complex and interesting problems in the whole field of invasion, the solution of which can be attempted only after the thorough analysis of the simpler invasions between formations. A better understanding of the meaning of invasion by species guilds is imperative for the natural limitation of regions, as at present such groups constitute alien associations in many regions otherwise homogeneous.

286. Influence of levels. The invasion of a formation may occur at three different levels: (I) at the level of the facies, (2) below the facies, (3) above the facies, depending directly upon the relative height of invaders and occupants. The invasion level is an extremely simple matter to determine, except in the case of woody plants, such as shrubs and trees, which attain their average height only after many years. Its importance is fundamental. The level at which invasion occurs not only determines the immediate constitution of the formation, whether its impress shall still be given by the occupants or by the invaders or by both together, but it also clecides the whole future of the formation, i. e., whether the invaders or occupants shall persist unmodified or modified. The problem is an extremely complex one, but the careful analysis of invasion at each level throws a flood of light upon it. The entrance of invaders of the same general height as the facies of a formation results regularly in mixed formations. This is well illustrated by the structure of the transition areas between two formations of the same categary, i. e., forests, meadows, etc. It is seldom, however, that the facies and invaders are so equally matched in height and other qualities that they remain in equilibrium for a long period. One or the other has a slight advantage in height, or the one suffers shading or .crowding better than the other, is longer-lived or faster-growing, with the result that invader yields to occupant, or occupant to invader. It is a wellknown fact that many mixed formations represent intermediate stages of development.

Invasion at a level different from that of the facies is inevitably followed by modification. If the invasion takes place below the facies, the invaders will be exterminated gradually, or slowly assimilated. In either case, there is little structural change in the formation, and its stability is affected slightly or not at all. If the invaclers overtop the facies in any considerable number, the entire formation undergoes partial or complete modification, or in extreme cases it disappears, as is typically the case in succession. A peculiar variation of invasion at a level above the facies is seen where woody 
plants invade grassland, when the trees or shrubs become more or less uniformly scattered in an open woodland or open thicket. Here the grassland takes on an altogether different appearance superficially, though it is usually unchanged, except beneath and about the invaders, where either adaptation or extermination results. Finally, it should be borne in mind that the invasion of a particular formation, especially in the case of layered thickets and forests, often takes place at two levels, at the height of the facies and below the facies.

\section{INVESTIGATION OF INVASION}

287. The methods to be used in the study of invasion are those already described elsewhere. The migration circle is of the first importance because it makes it possible to secure an accuratc record of actual movement. Quadrat and transect are valuable, but from their nature they are more serviceable for ecesis than for migration. All of these should be of the permanent type, in order that the fate of invaders may be followed for several years at least. Permanent areas furnish evidence of the changes, wrought in the actual vegetation, while denuded ones can serve only to show the potential migration and ecesis of the constituent species. Transition zones and areas are special seats of invasion; they are best studied by means of the belt transect and the ecotone chart. The movement of a line of invaders or of scattered outposts is traced by the use of labeled stakes at the points concerned. It is clear that this method will yield conclusive data in regard to the great invasions between regions, such as the movement of species guilds, the advance of the forest frontier, etc. When invasion is scattered, factor instruments can not be used to advantage, but where the invading line is well marked, or where extra-formational areas occur, a knowledge of the physical factors is a great aid.

An invasion that has been completed can not be studied in the manner indicated. A method of comparison must be used, in order to determine the original home of the invaders. For this an exact knowledge of the contigtous formations and of the abundance of the species common to all is a prerequisite. With this as a basis, it is usually a simple task to refer all the species of the formation concerned to their proper place in the groups, indigenous, derived, and adventitious.

\section{SUCCESSION}

288. Concept. Succession is the phenomenon in which a series of invasions occurs in the same spot. It is important, however, to distinguish clearly between succession and invasion, for, while the one is the direct result of the 
other, not all invasion produces succession. The number of invaders must be large enough, or their effect must be sufficiently modifying or controlling to loring about the gradual decrease or disappearance of the original occupants, or a succession will not be established. Partial or temporary invasion can never initiate a succession unless the reaction of the invaders upon the habitat is very great. Complete and permanent invasion, on the other hand, regularly produces successions, except in the rare cases where a stable formation entirely replaces a less stable one without the intervention of other stages. Succession depends in the first degree upon invasion in such quantity and of such character that the reaction of the invaders upon the habitat will prepare the way for further invasion. The characteristic presence of stages in a succession, which normally correspond to formations, is due to the peculiar operation of invasion with reaction. In the case of a denuded habitat, for example, migration from adjacent formations is constantly taking place, but only a small number of migrants, especially adapted to somewhat extreme conditions, are able to become established in it. These reach a maximum development in size or number, and in so doing react upon the habitat in sich a way that more and more of the dormant disseminules present, as well as those constantly coming into it, find the conditions favorable for germination and growth. The latter, as they in turn attain their maximum, cause the gradual disappearance of the species of the first stage, and at the same time prepare the way for the individuals of the stcceeding formation. It is at present impossible to determine to what degree this substitution is due to the struggle for existence between the individuals of each species and between the somewhat similar species of each stage, and to what degree it arises out of the physical reaction.

It is evicient that geological succession is but a larger expression of the same phenomenon, dealing with infinitely greater periods of time, and pro-duced by physical changes of such intensity as to give each geological stage its peculiar stamp. If, however, the geological record were sufficiently complete, we should find unquestionably that these great successions merely represent the stable termini of many series of smaller changes, such as are found cverywhere in recent or existing vegetation.

289. Kinds of succession. The fundamental causes of succession are invasion and reaction, but the initial causes of a particular succession are to be sought in the physical or biological disturbances of a habitat or formation. With reference to the initial cause, we may distinguish normal succession, which begins with nudation, and ends in stabilization, and anomalous succession, in which the facies of an ultimate stage of a normal succession are replaced by other species, or in which the direction of movement is radically 
changed. The former is of universal occurrence and recurrence; the latter operates upon relatively few ultimate formations. In the origin of normal successions, nudation may be brought about by the production of new soils or habitats, or by the destruction of the formation which already occupies a habitat. In a few cases, the way in which the habitat arises or becomes denuded is not decisive as to the vegetation that is developed upon it, but as a rule the cause of nudation plays as inportant a part in the development of a succession as does the reaction exerted by the invaders. The importance of this fact has been insisted upon under invasion. New soils present extreme conditions for ecesis, possess few or no dormant disseminules, and in consequence their successions take place slowly and exhibit many stages. Denuded soils as a rule offer optimum conditions for ecesis as a result of the action of the previous succession, dormant seeds and propagules are abundant, and the revegetation of such habitats takes place rapidly anci shows few stages. The former may be termed primary succession, the latter secondary succession.

\section{PRIMARY SUCCESSIONS}

290. These arise on newly formed soils, or upon surfaces exposed for the first time, which have in consequence never borne vegetation before. In general they are characteristic of mountain regions, where weathering is the rule, and of lowlands and shores, where sedimentation or elevation. constantly occur. The principal physical phenomena which bring about the formation of new soils are: (I) elevation, (2) volcanic action, (3) weathering, with or without transport.

291. Succession through elevation. Elevation was of very frequent occurrence during the earlier, more plastic conditions of the earth, and the successions arising as a result of it must have been important features of the vegetation of geological periods. To-day, elevation is of much less importance in changing physiography, and its operation is confined to volcanic islands, coral reefs, and islets, and to rare movements or displacements in seacoasts, lake beds, shore lines, etc. There has been no investigation of the development of vegetation on islands that are rising, or have recently been elevated, probably because of the slow growth of coral reefs and the rare appearance of volcanic islands. On coral reefs, the first vegetation is invariably marine. but as the reef rises higher above the surf line and the tide, the vegetation passes into a xerophytic terrestrial type adapted to an impervious rock soil, and ultimately becomes mesophytic. In volcanic islands, unless they are mere rocks over which the waves rush, the succession must always begin with a xerophytic rock formation. The best known example of a rising coast line is found in Norway and Sweden, where the southeast- 
ern coast is rising at the rate of five or six feet a century. There can be little question that such changes of level will produce marked changes in vegetation, but the modification will be so gradual as to be scarcely perceptible in a single generation. It is probable that the forests of the Atlantic coastal plains are the ultimate stages of successions initiated at the time of the final elevation of the sea bottom along the coast line.

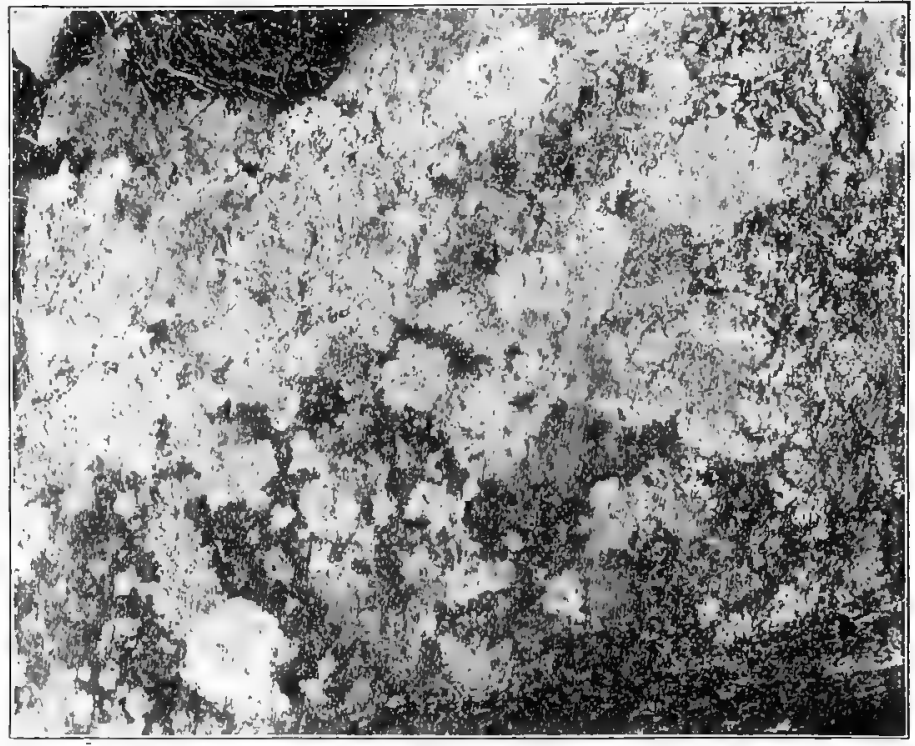

Fig. 60. A lichen formation (Lecanora-Physcia-petrium), the first stage of the typical primary succession (Lecanora-Picea-sphyrium) of the Colorado motuntains.

292. Succession through volcanic action. The deposition of volcanic ashes and flows of lava are relatively infrequent at present, occurring only in the immediate vicinity of active volcanoes, chiefly in or near the tropics. Successicns of this sort are in consequence not only rare, but they are also relatively inaccessible to investigators. They have been studied in a few cases, for example, those of Krakatoa by Treub, but this study has been confined to the general features of revegetation. Ash fields and lava beds are widely different in compactness, but they agree in having a low water- and nutrition-content. The pioneer plants in both will be intense xerophytes, but the soil differences will determine that these slall be sandbinders in the former, and rock-weathering plants in the latter. 
293. Weathering. Practically all primary successions start on soils produced by weathering. This is also true of coral or volcanic islets and of lava beds, for no terrestrial vegetation can secure a foothold upon them until the surface of the rock has been to some extent decomposed or disintegrated. Weathering, as is well known, consists of two processes, disintegration and decomposition, which usually operate successively, though they are sometimes concomitant. Disintegration usually precedes, especially in rock masses, and unless it is soon followed by decomposition, restults in dysgeogenous soils. Decomposition often goes hand in hand with disintegration, or it takes place so rapidly and perfectly that it alone seems to be present. In either case, the resulting soil is eugeogenous. The relation of decompositicn to disintegration determines the size and compactness of the soil particles, and upon the latter depend the porosity, capillarity, and hygroscopicity of the soil. These control in large degrce the character of the first vegetation to appear on the soil.

Another point of fundamental value in determining revegetation is the disposition of the weathered rock. If it remains in situ, it will evidently differ in respect to compactness, homogeneity, nutrition-content, water-conlent, disseminules, etc., from weathered material which has been transported. An essential difference also arises from the fact that a rock may be weathered a long distance from the place where the decomposed particles are finally deposited, and in the midst of a vegetation very different from that found in the region of deposit. The disposition of the weathered material affords in consequence a satisfactory basis for the arrangement of primary successions. The following classification is proposed, based upon the soil groups established by Merrill. ${ }^{1}$

294. Succession in residuary soils. Residuary soils are always sedentary, i. e., they are formed in situ. They show certain differences dependent upon the rock from which they originate, which may be mixed crystalline shale, sandstone, or limestone, but the thoroughness of decomposition causes these differences to be comparatively small. Residuary soils are typically eugeogenous; their successions in consequence ustrally begin with mesophytes, and consist of a few stages. The soluble salt-content is comparatively low, since all soluble matters are readily leached out. Successions in these soils are especially characteristic of shale, sandstone, and limestone ledges or banks. Cumulose deposits, like residuary ones, are sedentary in character, but as they are produced by the accumtilation of organic matter, they will be considered under reactions of vegetation upon habitat.

${ }^{2}$ Rocks, Rock-weathering, and Soils, 300. $189 \%$. 
295. Succession in colluvial soils. Colluvial deposits owe their aggregation solely or chiefly to the action of gravity. They are the immediate result of the disintegration of cliffs, ledges, and mountain sides, decomposition appearing later as a secondary factor. The masses and particles arising from disintegration are extremely variable in size, but they agree as a rule in their angular shape. The typical example of the colluvial deposit is the talus, which may originate from any kind of rock, and contains pieces of all sizes. Gravel slides differ from ordinary talus in being composed of more uniform particles, which are worn round by slipping down the slope in response to gravity and surface wash. Boulder fields are to be regarded as talus pro-

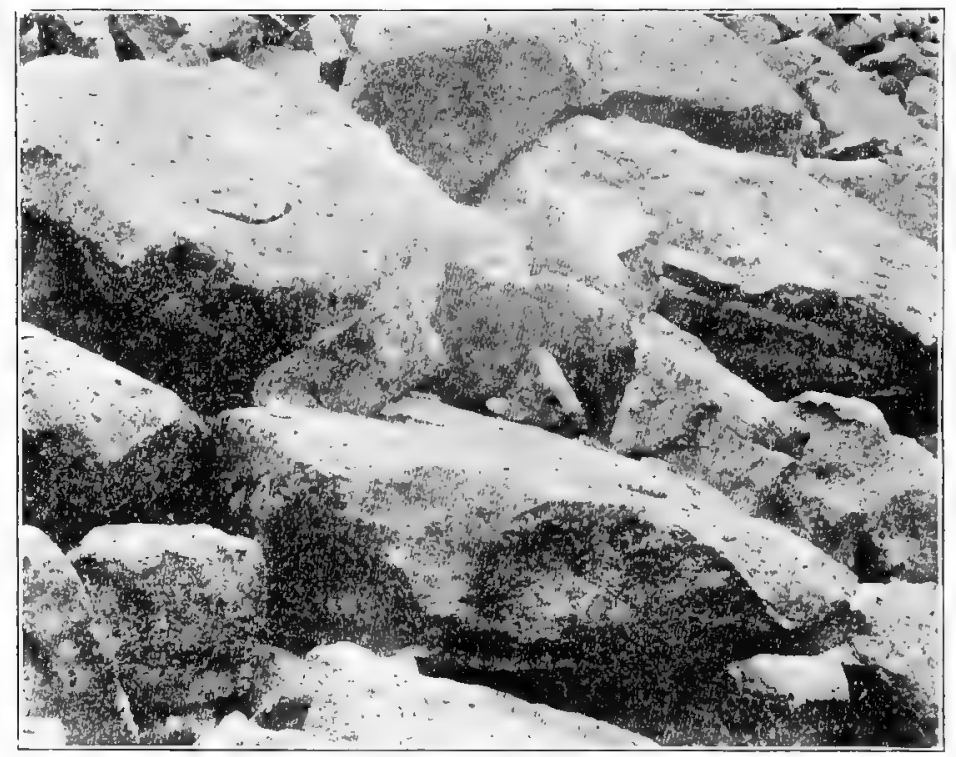

Fig. 61. Talus arising from the disintegration of a granitic cliff; the rocks are covered with crustose lichens.

duced by weathering under the influence of joints, resulting in huge boulders which become more and more rounded under the action of water and gravity. This statement applies to those fields which are in connection with some cliff that is weathering in this fashion; otherwise, boulder fields are of aqueous or glacial origin. The character of the successions in talus will depend upon the kind of rock in the latter. If the rock is igneous or metamorphic, decomposition will be slow, and the soil will be dysgeogenous. Successions on such talus consist of many stages, and the formations are for a long time open and xerophytic. In talus formed from sedimentary rocks, 
especially shales, linestones, and calcareous sandstones, decomposition is much more rapid, and the successions are simpler and more mesophytic.

296. Succession in alluvial soils. Alluvial soils are fluvial when laid down by streams and rivers, and litoral when washed up by the waves or tides. They are formed when any obstacle retards the movement of the water, decreasing its carrying power, and causing the deposit of part or all of its load. They consist of more or less rounded, finely comminuted particles, mingled with organic matter and detritus. Alluvial deposits are especially frequent at the mouth of streams and rivers, on their terraces and flood plains, and along silting banks as compared with the erosion banks of meanders. The filling of ponds by the erosion due to surface drainage, and of lakes by the deposition of the loads of streams that enter them, results in the formation of new alluvium. A similar phenomenon occurs along coasts, where bays and inlets are slowly converted into marshes in consequence of being shallowed by the material washed in by the waves and tides. Such paludal deposits are invariably salt water or brackish. Contrasted with these, which are uniformly black in consequence of the large amount of organic matter present, are the sandbars and beaches, which, though due to the same agents, are light grey or white in color, because of the constant leaching by the waves. Two kinds of alluvial deposits may accordingly be distinguished: (I) those black with organic matter, and little disturbed by water, and (2) those of a light color, which are constantly swept by the waves. The successions corresponding to these are radically different. In the first, the pioneer vegetation is hydrophytic, consisting largely of amphibious plants. The pioneer stages retard the movement of the water more and more, and correspondingly hasten the deposition of its load. The marsh bed slowly rises in consequence, and finally the marsh begins to dry out, passing first into a wet meadow, and then into a meadow of the normal type. A notable exception to this sequence occurs when the swamp contains organic matter or salts in excess, in which case the vegetation consists indefinitely of swamp xerophytes, or halophytes. The first vegetation on fresh water sandbars is xerophytic, or, properly, dissophytic, unless they remain water-swept, and the ultimate stages of their successions are mesophytic woodlands composed of water-loving genera, Populus, Salix, etc. It seems certain, however, that these will finally give way to longer-lived hardwoods. Maritime sandbars and beaches are always saline, and their successions run their short course of development entirely within the group of halophytes, inless the retrcat of the sea or fresh-water floods change the character of the soil. The chemical action of underground waters also produces new soils, which might be classed as alluvial. These soils are essentially rock deposits, travertine, sili- 
cious sinter, etc., made by iron and lime springs and by geysers, and they must be changed by decomposition into soils proper to be comparable with alluvial soils.

297. Succession in aeolian soils. The only wind-borne soils of geological importance at the present time are those which form dunes, both inland and coastal. Aeolian deposits consist largely of rounded sand particles, which are of almost uniform size in any particular dune, but vary. greatly in dunes nf different ages. The reaction of the pioneers on dunes plays an important

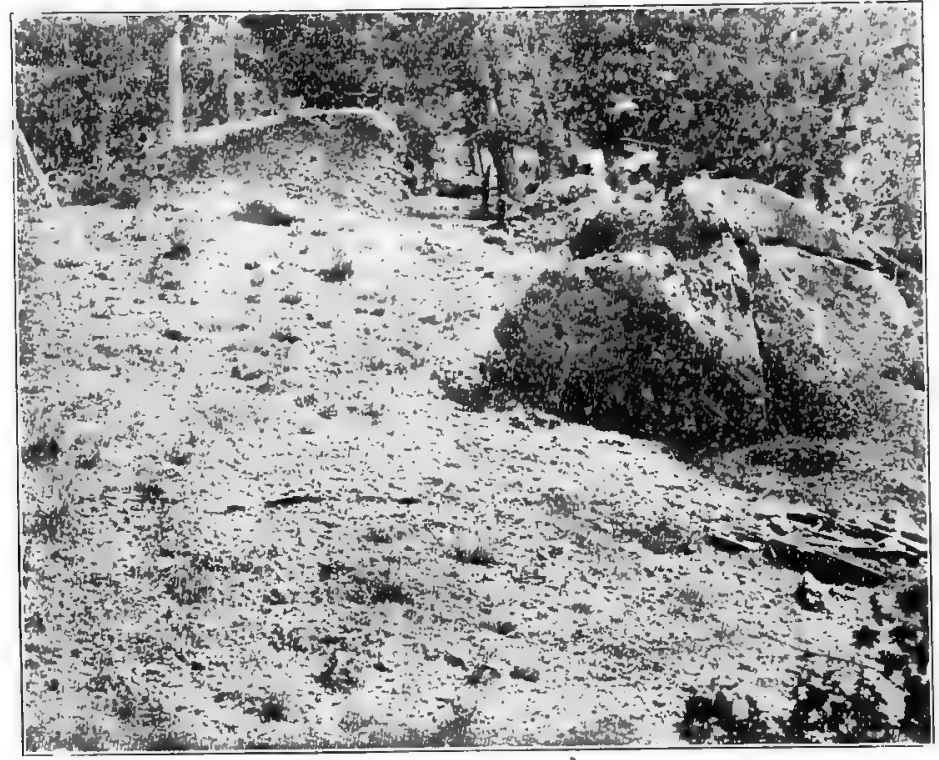

Fig. 62. Talus arising from the decomposition of granite; the gravel is covered with a formation of foliose lichens (Parmelia-chalicium), the second stage of the primary talus succession; the herbs are pioneers of the next stage.

part in building the latter, but the immense dunes of inland deserts, which are entirely destitute of vegetation, seem to indicate that its value has been overestimated. The first stages in dune successions are dissophytic, i. e., the plants grow in a soil of medium or high water-content, but in an atmosphere that is extremely xerophytic. The ultimate stages vary widely in accordance with the region in which they occur; they may be xerophytic heaths or mesophytic meadows and forests. Because of their striking character and economic significance, dunes have received much attention, with the result 
that their successions are the most thoroughly known of all. Prairie and steppe formations are probably to be regarded as the ultimate stages of successions established on wind-borne loess, and it is possible that the same is true of sand-hill vegetation in the prairie province.

298. Succession in glacial soils. The formation of glacial deposits is at present confined to alpine and arctic regions. Recent successions in such soils are localized in these regions, and are in consequence relatively unimportant. There can be little question, however, that the thorough investigation of succession in and near the moraines of existing glaciers will throw much light upon the successions of the glacial period. Moraines, drumlins, eskars, and alluvial cones represent the various kinds of glacial deposits. They agree in being heterogeneous in composition, and are covered to-day with ultimate stages of vegetation, except in the immediate vicinity of glaciers.

\section{SECONDARY SUCCESSIONS}

299. Generally speaking, all successions on denuded soils are secondary. When vegetation is completely removed by excessive erosion, it is an open question whether the resulting habitat is to be regarded as new or denuded. Erosion is rarely so extreme and so rapid, however, as to produce such a condition, even when it results from cultivation or deforestation. It is, moréover, especially characteristic of newly formed soils, and in studying succession in eroded habitats, it is fundamentally important to determine whether erosiun has produced denudation, or has operated upon a new soil. The great majority of secondary successions owe their origin to floods, animals, or the activities of man, and they agree in occurring upon decomposed soils of medium water-content, which contain considerable organic matter, and a large number of dormant migrants. These successions consist of relatively few stages, and are rarely of extreme character.

300. Succession in eroded soils. Eroded soils show considerable differences, as they arise in consequence of erosion by water or by wind, though the initial stages of revegetation derive their character more from the aggregation of the soil than from the nature of the erosive agent. Eroded soils are as a rule xerophytic. In the case of erosion by water, dysgeogenous soils are readily worn away in consequence of their lack of cohesion, as in sanddraws, etc., while eugeogenous soils are easily eroded only on slopes, as in the case of ravines, hillsides, etc. In the former, the extreme porosity and slight capillarity of the sand and gravel result in a low water-content. In the finer sails, the water-content is also low, on account of the excessive run- 
off, due to compactness of the particles and to the slope. The erosive action of winds upon soils bearing vegetation is not very general; it is found to some extent in more or less established dunes, and exists in a marked degree in buttes, mushroom rocks, and blowouts. The first two are regularly xerophytic, the last as a rule, dissophytic. The early stages of successions in eroded soils are composed of xerophytes. In loose soils, these are forms capable of binding the soil particles together, this preventing wash, and increasing the accumulation of fine particles, especially of organic matter. In compact soils, the effect is much the same; the pioneers not only decrease erosion, but at the same time also increase the water-content by retarding the movement of the run-off.

301. Succession in flooded soils. The universal response of vegetation to floods is found in the amphibious plant, which is a plastic form capable of adjustment to very different water-contents. Floods are confined largely to river basins and coasts. In hilly and mountainous regions, where the slope is great, any considerable accumulation of flood waters is now impossible, although of frequent occurrence when land forms were more plastic.

In all streams that have become graded, the fall is insufficient to carry off the surplus water in the spring when snows are melting rapidly, or at times of unusual precipitation. These waters accumulate, and, overflowing the banks, spread out over the lowlands, resulting in the formation of a welldefined food plain. This is a periodical occurrence with mature streams, and it occurs more or less regularly with all that are not torrent-like in character. The effect of the overflow is to destroy or to place at a disadvantage those plants of the flood plain that are not hydrophytes. At the same time, a thin layer of fresh silt is deposited upon the valley floor of sand or alluvium. Flooding is most frequent and of longest duration near the banks of the stream. It extends more or less uniformly over the flood plain, and disappears gradually or abruptly as the latter rises into the bench above. Floods destroy vegetation and make a place for secondary successions by drowning out mesophytic species, by washing away the aquatic forms of ponds and pools, and by the erosion of banks and sandbars. They affect the amphibious vegetation of swamp and shore to a certain extent, but, unless the period of flooding is long, they tend to emphasize such formations rather than to destroy them. The still-water formations of many cutoff and oxbow lakes owe their origin to a river which cuts across a meander in time of flood. This result is more often attained by the alternate silting and erosion of a meandering river by which it cuts across a bend in its channel. The usual successions in floorled lands are short as a rule; amphibious algae, liverworts; and mosses soon give way to ruderal plants, and these in turn to the original 
mesophytes of meadows, or dissophytes of sandbars. In the case of ponds and pools, the process of washing-out or silting-up merely removes or destroys the vegetation, without effectively modifying the habitat, and the secondary successions that follow are extremely short.

302. Succession by subsidence. Subsidence is a factor of the most profound importance in changing vegetation. It operates over vast areas through immense periods of time. For these reasons, the changes are so slow as to be almost imperceptible, and the resulting successions can be studied only in the geological record. Extensive subsidence is confined today to coastal plains, as in Greenland, the south Atlantic coast, and the region of the Mississippi delta, where its effects are merged with the paludation of tidal rivers, and the wave and tide erosion of the sea shore. Such successions are unique, inasmuch as the denuding force operates very slowly instead of quickly, and the first pioneers of the new vegetation appear before. the original formation has been destroyed. In all cases, the succession is from mesophytic or halophytic formations to paludose, and, finally, marine vegetation. In small areas of subsidence, such as shore slips along lakes and streams, sink holes, and sunken bogs, the succession is usually both short and simple, mesophytes giving place to amphibious and ultimately to aquatic forms.

303. Successions in landslips. Landslips occur only in montane and hilly regions, and here they are merely of local importance. In many respects, they are not unlike talus; they show essential differences, however, in that they are not sorted by gravity, and in that they destroy vegetation almost instantly. The succession arises as a rule, not upon the original soil, but upon that of the landslip, and, as pointed out elsewhere, might well be regarded as primary.

304. Succession in drained, or dried soils. In geological times, the subsidence of barriers must often have produced drainage and drying-out, just as elevation frequently resulted in flooding and lake formation. At the present time, the drying-out of lakes and ponds is the result of artificial drainage, or of climatic changes. The former will be considered under successions brought about by the agency of man. Climatic changes when general operate so slowly that the stages of such successions are perceptible only when recorded in strata. More locally, climate swings back and forth through a period of years, with the result that in dry years the swamps and ponds of wetter seasons are dried out, and the vegetation destroyed or changed. If the process be gradual, the succession passes from hydrophytic through am- 
phibious to mesophytic, and, in dry regions, xerophytic conditions. When the process of drying out occurs rapidly, as in a single summer, the original formation is destroyed, and the new vegetation consists largely of rudera! plants. A peculiar effect of climate occurs in regions with poor drainage, where the result of intense evaporation is to produce alkaline basins and salt lakes, in which the succession becomes more and more open, and is finall! represented by a few stabilized halophytes, or disappears completely.

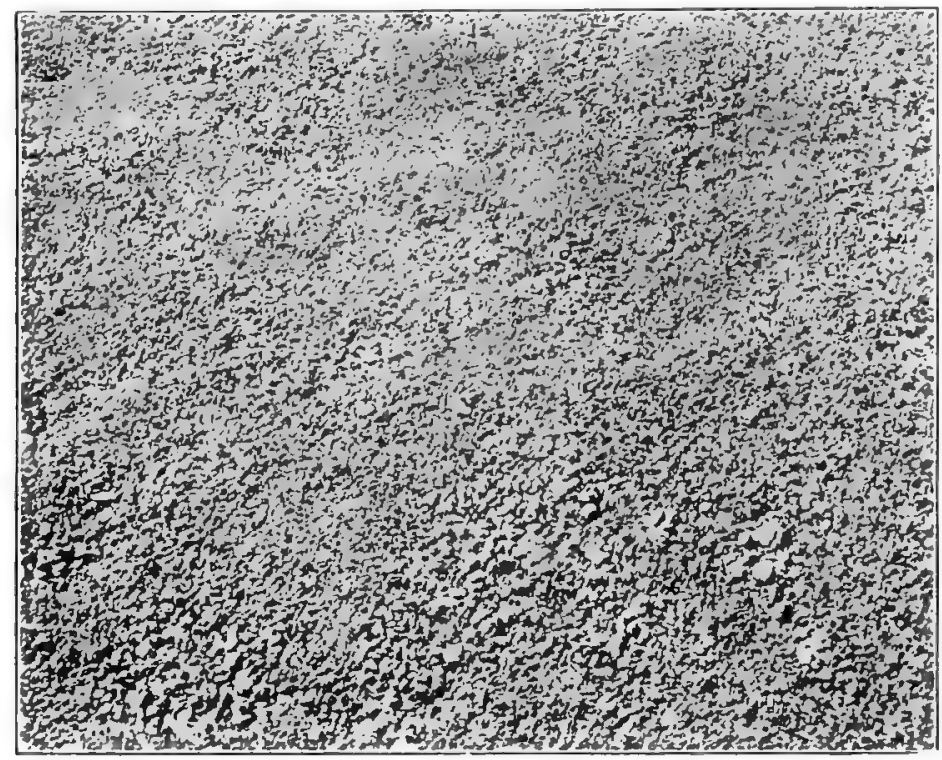

Fig. 63. A typical gravel slide (talus) of the Rocky mountains, before invasion.

305. Succession by animal agency. Successions of this class are altogether of secondary importance, the instances in which animals produce denudation being relatively few. Such are the heaps of dirt thrown up by prairie dogs and other burrowing animals, upon which ruderal plants are first established, to be finally crowded out by the species of the original formation. Buffalo wallows furnish examples of similar successions in which the initial stages are subruderal, while overstocking and overgrazing frequently produce the same result with ruderal plants.

306. Succession by human agency. The activities of man in changing the surface of the earth arc so diverse that it is impossible to fit the resulting successions in a natural system. While man does not exactly make new soils, he exposes soils in various operations: mining, irrigation, railroad 
building, etc. He destroys vegetation by fires, lumbering, cultivation, and drainage, and if he can not control climate, he at least modifies its natural effects by irrigation and the conservation of moisture. The operations of man extend from seacoasts and swampy lowlands through mesophytic forests and prairies to the driest uplands and inlands. Since the adjacent formations determine in large degree the course and constitution of a succession, it will be seen that the effects of any particular activity upon vegetation will differ greatly in different regions. For convenience, all classes of successions arising from the presence and activity of man will be considered in this place, though, as indicated above, some might well be regarded as producing primary successions, while others produce anomalous ones.

307. Succession in burned areas. It will suffice merely to point out that "burns" may arise naturally through lightning, volcanic cinders, lava flows, etc., but the chances are so slight that these causes may be ignored. The causes of fires are legion, and as they have little or no effect upon results, they need not be considerecl. From their nature, fires are of little significance in open vegetation, deserts, polar barrens, alpine fields, etc., since the area of the burn can never be large. In closed formations, the extent of fires is limited only by the area of the vegetation, and the effect of wind, rain, and other forces. Forest fires usually occur during the resting period, except in the case of coniferous forests. In grassland, the living parts are underground during autumn and winter, when prairie fires commonly occur. As a consequence, the repeated annual burning of meadow or prairie does not result in denudation and subsequent succession. On the contrary, it acts in part as a stabilizing agent, inasmuch as it injures the typical vegetation forms of grassland much less than it does the woody invaders. All formations with perennial parts above ground, viz., thicket, open woodland, and forest, are seriously injured by fire. A severe general fire destroys the vegetation completely; a local fire destroys the formation in restricted areas; while a slight or superficial burn removes the undergrowth and hastens the disappearance of the weaker trees. In the latter case, while the primary layer of the forest remains the same, succession takes place in the herbaceous and shrubby layers. These successions are peculiar in that they are composed almost wholly of the proper species of the forest, and that they are very short, showing only a few poorly defined stages. A local fire initiates a succession in which the pioneers are derived largely from the original formation, particularly when the latter encloses the burned area more or less completely. The constitution of the intermediate and ultimate stages will depend in a larger degree still upon the size and position of the burn. When a particular formation is destroyed wholly or in large part, the first stages of the new vegetation are made up by invaders from the adjacent formations. 
In the most perfect types of succession, this dissimilarity between the new and the old vegetation continues to the last stage, in which the reappearance of the facies precedes that of the subordinate layers. In many forest successions, however, the general physical similarity of the ultimate stages permits the early reappearance of the herbaceous and shrubby species, and the final stages affect the facies alone. Successions in burned areas operate usually within the water-content groups. The reconstruction of a mesophytic forest takes place by means of mesophytes : of the rarer xerophytic and hydrophytic forests, through xerophytes and hydrophytes respectively. This is due to the fact that the alteration of the soil is slight, except where the burning of the vegetation permits the entrance of erosion, as on mountain slopes.

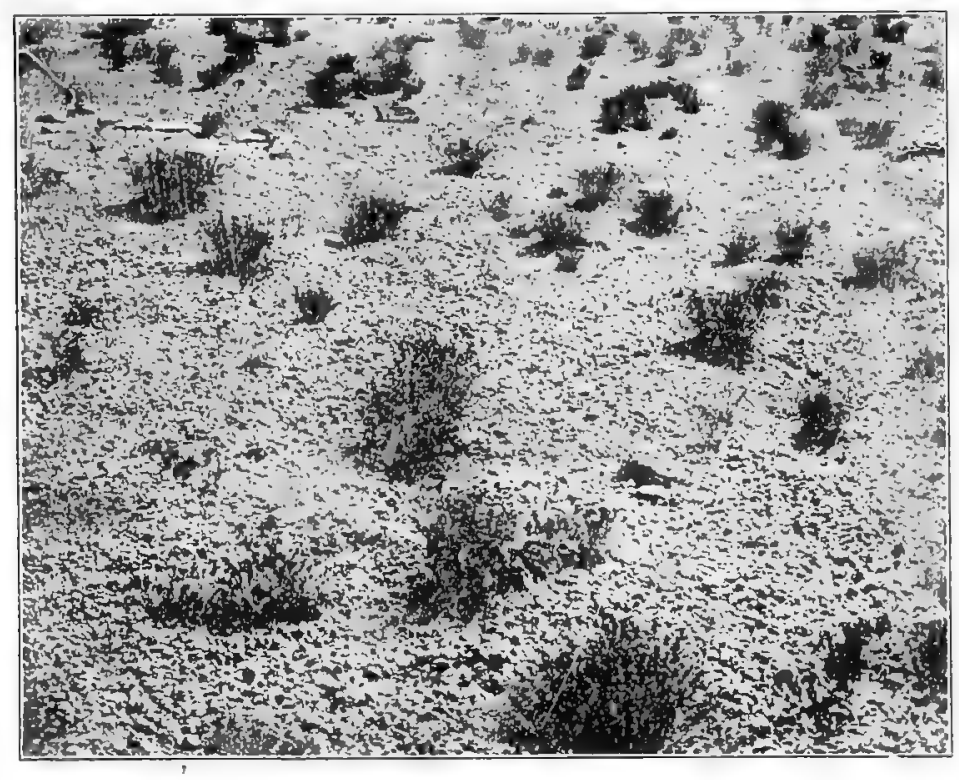

Fig. 64. Gravel slide formation (Pseudocymopterus-Mentzelia-chal icium), stage III of the talus succession.

308. Succession in lumbered areas. Commercial lumbering, especially where practiced for wood-pulp as well as for timber, results in complete or nearly complete destruction of the vegetation by removal and the change from diffuse light to sunlight, or by the action of erosion upon the exposed surface. In the first place, short mesophytic successions will result; in the second, the successions will be long and complex, passing through decreasingly xerophytic conditions to a stable mesophytic forest. Where a forest 
is cut over for certain species alone, the undisturbed trees soon take full possession, though the causes effective in the beginning will ultimately restore the original facies in many instances. Such successions are anomalous, and will be treated under that head.

309. Succession by cultivation. The clearing of forests and the "breaking" of grassland for cultivation destroy the original vegetation; the temporary or permanent abandonment of cultivated fields then permits the entrance of ruderal species, which are the pioneers of new successions. This phenomenon takes place annually in fields after harvest, resulting in the secondary formations of Warming, in which practically the same species reappear year after year. In fields that lie fallow for several years, or are permanently abandoned, the first ruderal plants are displaced by newcomers, or certain of them become dominant at the expense of others. In a few years, these are crowded out by invaders from the adjacent formations, and the field is tultimately reclaimed by the original vegetation, unless this has entirely disappeared from the region. The number of stages depends chiefly upon whether the final formation, is to be grassland or woodland. Other activities of man, such as the construction of buildings, roads, railways, canals, etc., remove the native vegetation, and make room for the rapid development of ruderal formations. In and about cities, where the original formations have entirely disappeared, the chance for succession is remote, and the initial rtteral stages become more or less stabilized. Elsewhere the usual successions are established, and the ruderal formation finally gives way to the dominant type. In mountain and desert regions, where ruderal plants are rare or lacking, their place is taken by subruderal forms, species of the native vegetation capable of rapid movement in them. These, like ruderal plants, are gradually replaced by other native species of less mobility, but of greater persistence, resulting in a short succession operating often within a single formation. From the nature of cultivated plants, succession after cultivation generally operates within the mesophytic series.

310. Succession by drainage. Successions of this kind show. much the same stages as are found in those due to flooding. They proceed from aquatic or swamp formations to mesophytic termini, either grassland or woodland. When drainage takes place rapidly and completely, the pioneer stages are usually xerophytic; cases of this sort, however, are infrequent.

311. Succession by irrigation. Irrigation produces short successions of peculiar stamp along the courses of irrigating canals and ditches, and in the vicinity of reservoirs. These are recent, as a rule, and are usually found in 
the midst of cultivated lands, so that their complete history is still a matter of conjecture. The original xerophytes are forced out not only by the disturbance of the soil, but also by its increased water-content. A few of them often thrive under the new conditions, and, together with the usual ruderal plants and a large number of lowland mesophytes and amphibious forms derived from the banks of the parent stream, constitute a heterogeneous association. This is doubtless to be regarded as an initial stage of a succession, but it is an open question whether the succession will early be stabilized as a new formation, or whether the original vegetation will sooner or later le reestahlished under somewhat mesophytic conditions. From the number of mesophytes and from the behavior of valleys, it seems certain that the banks of such canals will ultimately be occupied by a formation more mesophytic than hydrophytic, into which some of the surrounding xerophytes of plastic nature have been aclopted.

312. Anomalous successions are those in which the physical change in the habitat is relatively slight, resulting in a displacement of the ultimate stage, or the disturbance of the usual sequence, merely, instead of the destruction and reconstruction of a formation, or the gradual development of a new series of stages on new soil. In nature, the ultimate grass or forest stage of a normal succession is often replaced by a similar formation, especially if the facies be few or single. It is evident that certain trees naturally replace others in the last stages of a forest succession, without making the latter anomalous. The last occurs only when a normal stage is replaced by one belonging properly to an entirely different succession, as when a coniferous forest replaces a deciduous one in a hardwood region. The presence and development of such successions can be determined only after the nornal types are known. The interpolntion of a foreign stage in a natural succession, or a change of direction, by which a succession that is mesotropic again becomes hydrophytic, is easily explained when it is the result of artificial agents, as is often the case. In nature, anomalous successions are conmonly the result of a slow backward and forward swing of climatic conditions.

313. Perfect and imperfect successions. A normal succession will regularly be perfect; it passes in the usual sequence from initial to ultimate conditions without interruption or omission. Imperfect succession results when one or more of the ordinary stages is omitted anywhere in the course, and a later stage appears before its turn. It will occur at any time when a new or denuded habitat becomes so surrounded by other vegetation that the formations which usually furnish the next invaders are unable to do so, or when 
the abundance and mobility of certain species enable them to take possession before their proper turn, and to the exclusion of the regular stage. Incomplete successions are of great significance, inasmuch as they indicate that the stages of a succession are often due more to biological than to physical causes, the proximity and mobility of the adjacent species being more determinative than the physical factors. Subalpine gravel slides regularly pass through the rosette, mat, turf, thicket, woodland, and forest stages; occasionally, however, they pass immediately from the rosette, or mat condition, to an aspen thicket which represents the next to the last stage. Sucl successions are by no means infrequent in hilly and montane regions; in regions physiographically more mature or stable, perfect successions are almost invariably the rule.

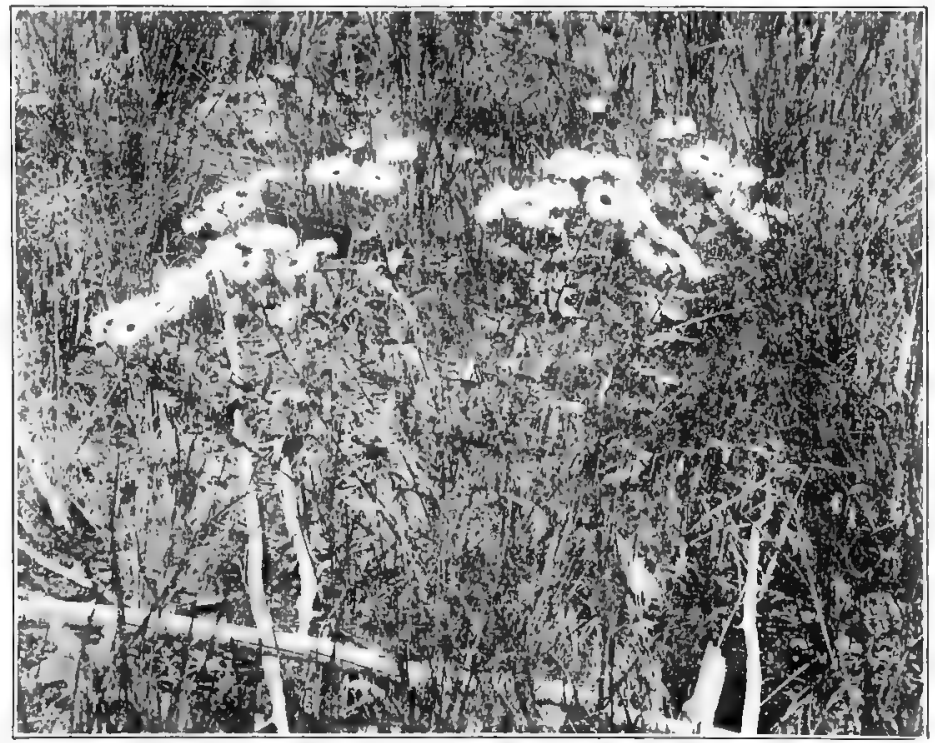

Fig. 55. Half gravel slide formation (Elymus-Muhlenbergia-chalicium), stage IV of the talus succession.

314. Stabilization. It may be stated as a general principle that vegetation moves constantly and gradually toward stabilization. Each successive stage modifies the physical factors, and dominates the habitat more and more, in such a way that the latter seems to respond to the formation rather than this to the habitat. The more advanced the succession, i. e., the degree of stabilization, the greater the climatic or physiographic change necessary to disturb it, with the result that such disturbances are much more frequent in 
the earlier stages than in the later development. Constant, gradual movement towarc a stable formation is characteristic of continuous succession. Contrasted with this is intermittent succession, in which the succession swings for a time in one direction, from xerophytic to mesophytic for example, and then moves in the opposite direction, often passing through the same stages. 'This phenomenon usually is characteristic only of the less stable stages, and is generally produced by a climatic swing, in which a series of hot or dry years is followed by one of cold or wet years, or the reverse. The same effect upon a vast scale is produced by alternate elevation and subsidence, but these operate through such great periods of time that one can not trace, but can only conjecture their effects. A normal continuous succession frequently changes its direction of movement, or its type, in transition regions or in areas where the outposts of a new flora are rapidly advancing, as in wide mesophytic valleys that run down into or traverse plains. Here the change is often sudden, and grass and desert formations are replaced by thickets and forests, resulting in abrupt succession. Species guilds are typical examples of this. More rarely, a stage foreign to the succession will be interpolated, replacing a normal stage, or slipping in between two such, though finally disappearing before the next regular formation. This may be distingtuished as interpolated succession.

The apparent terminus of all stabilization is the forest, on account of the thoroughness with which it controls the habitat. A close examination of vegetation, however, will show that its stable terms are dependent in the first degree upon the character of the region in which the formation is indigenous. It is obviously impossible that successions in desert lands, in polar barrens, or upon alpine stretches should terminate in forest stages. In these, grassland $n$ ust be the ultimate condition, except in those extreme habitats, alpine and polar, where mosses and lichens represent the highest type of existing vegetation. Forests are ultimate for all successions in habitats belonging to a region generally wooded, while grassland represents the terminus of prairie and plains successions as well as of many arctic-alpine ones.

\section{CAUSES AND REACTIONS}

315. The initial cause of a succession must be sought in a physical change in the habitat; its continuance depends upon the reaction which each stage of vegetation exerts upon the physical factors which constitute the habitat. A single exception to this is found in anomalous successions, where the change of formation often hinges upon the appearance of remote or foreign disseminules. The causes which initiate successions have already been considered; they may be summarized as follows: (I) weathering, (2) erosion, (3) elevation, (4) subsidence, (5) climatic changes, (6) artificial changes. 
-The effect of succeeding stages of vegetation upon a new or denuded habitat ustally finds expression in a change of the habitat with respect to a particular factor, and in a definite direction. Often, there is a primary reaction, and one or more secondary ones, which are corollaries of it. Rarely, there are two or more coordinate reactions. The general ways in which vegetation reacts upon the habitat are the following: (I) by preventing weathering, (2) by binding aeolian soils, (3) by reducing run-off and preventing erosion, (4) by filling with silt and plant remains, (5) by enriching the soil, (6) by exhausting the soil, ( 7 ) by accumulating humus, (8) by modifying atmospheric factors. The direction of the movement of a succession is the immediate result of its reaction. From the fundamental nature of vegetation, it must be expressed in terms of water-content. The reaction is often so great that the habitat undergoes a profound change in the course of the succession, changing from hydrophytic to mesophytic or xerophytic, or the reverse. This is characteristic of newly formed or exposed soils. Such successions are xerotropic, mesotropic, or hydrotropic, according to the ultimate condition of the habitat. When the reaction is less marked, the type of habitat does not change materially, and the successions are xerostatic, mesostatic, or hydrostatic, depending upon the water-content. Such conditions obtain for the most part only in denuded habitats.

316. Succession by preventing weathering. Reactions of this nature occur especially in alpine and boreal regions, in the earlier stages of lichenmoss successions. They are typical of igneous and metamorphic rocks in which disintegration regularly precedes decomposition. The influence of the vegetation is best seen in the lichen stages, where the crustose forms make a compact layer, which diminishes the effect of the atmospheric factors producing disintegration. In alpine regions especially, this protection is so perfect that the crustose lichens may almost be regarded as the last stage of a succession. There are no recorded observations which bear upon this point, but it seems certain that the pioneer rock lichens, Lecanora, Lecidea, Biatora, $\dot{B} u e l l i a$, and Acarospora, cover alpine rocks for decades, if not for centuries. Ultimately, however, the slow decomposition of the rock surface beneath the thallus has its effect. Tiny furrows and pockets are formed, in which water accumulates to carry on its ceaseless work, and the compact crustose covering is finally ruptured, permitting the entrance of foliose forms. The latter, like the mosses, doubtless protect rock surfaces, especially those of the softer rocks, in a slight degree against the influence of weathering, but this is more than offset by their activity in hastening decomposition, and thus preparing a field for invasion. Rocks and boulders (petria, petrodia, phellia) furnish the best examples of this reaction; cliffs (cremnia) usually have a lichen 
covering on their faces, while the forces which produce disintegration operate from above or below.

317. Succession by binding aeolian soils. Dunes (thinia) are classic examples of the reaction of pioneer vegetation upon habitats of wind-borne sand. The initial formations in such places consist exclusively of sandbinders, plants with masses of fibrous roots, and usually also with strong rootstalks, long, erect leaves, and a vigorous apical growth. They are almost exclusively perennial grasses and sedges, possessing the unique prop-

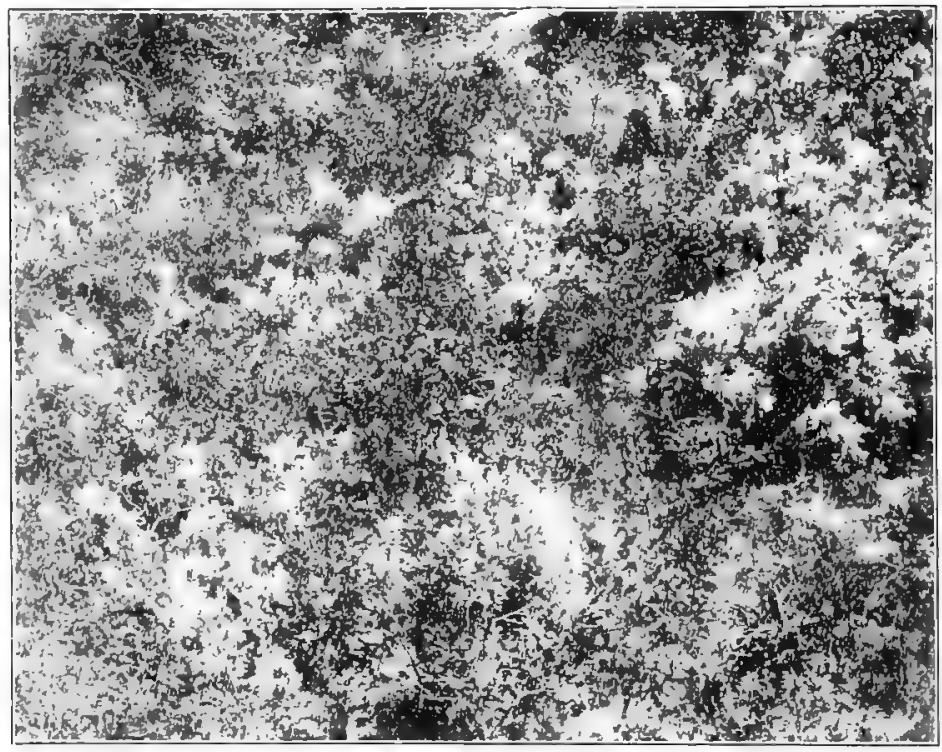

Fig. 06. Thicket formation (Qucrcus-Holodiscus-driodium), stage V of the talus sticcession.

erty of pushing up rapidly through a covering of sand. They react by fixing the sand with their roots, thus preventing its blowing about, and also by catching the shifting particles among their culms and leaves, forming a tiny area of stabilization, in which the next generation can establish a foothold. The gradual accumulation of vegetable detritus serves also to enrich the soil, and makes possible the advent of species requiring better nourishment. Blowouts (anemia) are almost exact duplicates of dunes in so far as the steps of revegetation are concerned; while one is a hollow, and the other a hill, in both the reaction operates upon a wind-swept slope. Sand-hills (amathia) and deserts (eremia) show similar though less marked 
reactions, except where they exhibit typical inland dunes. Sand-binders, while usually classed as xerophytic or halophytic, are in reality dissophytes. Their roots grow more or less superficially in moist sand, and are morphologically mesophytic while their leaves bear the stamp of xerophytes. The direction of movement in successions of this kind is normally from xerophytes to mesophytes, i. e., it is mesotropic. In sand-hills and deserts, the succession operates wholly within the xerophytic (dissophytic) series. Along seacoasts, the mesophytic terminus is regularly forest, except where forests are remote, when it is grassland.

318. Succession by reducing run-off and erosion. All bare or denuded habitats that have an appreciable slope are subject to erosion by surface water. The rapidity and degree of erosion depend upon the amount of rainfall, the inclination of the slope, and the structure of the surface soil. Regions of excessive rainfall, even where the slope is slight, show great, though somewhat uniform erosion; hill and mountain are deeply eroded even when the rainfall is small. Slopes consisting of compact eugeogenous soils, notwithstanding the marked adhesion of the particles, are much eroded where the rainfall is great, on account of the excessive run-off. Porous dysgeogenous soils, on the contrary, absorb most of the rainfall; the run-off is small and erosion slight, except where the slope is great, a rare condition on account of the imperfect cohesion of the particles. In compact soils, the plants of the initial formations not merely break the impact of the raindrops, but, what is much more important, they delay the downward movement of the water, and produce numberless tiny streams. The delayed water is largely absorbed by the soil, and the reduction of the run-off prevents the formation of rills of sufficient size to cause erosion. As in dunes, such plants are ustrally perennial grasses, though composites are frequent; the, root system is, however, more deeply seated, and a main or tap root is often present. On sand and gravel slopes, the loose texture of the soil results generally in the production of sand-binders with fibrous roots. Unlike dunes, such slopes exhibit a large number of mats and rosettes with tap-roots, which are effective in preventing the slipping or washing of the sand, and run little danger of being covered, as is the case with duneformers. In both instances, each pioneer plant serves as a center, of comparative stabilization for the establishment of its own offspring, and of such unvaders as find their way in. From the nature of these, slopes almost invariably pass througl grassland stages before finding their termini in thickets or forests. Bad lands (tiria) furnish the most striking examples of eroded habitats. The rainfall in the bad lands of Nebraska and South Dakota is small (300 mm.) ; yet the steepness of the slope and the compact- 
ness of the soil render erosion so extreme that it is all but impossible for plants to obtain a foothold. Their reaction is practically negligible, and the vegetation passes the pioneer stages only in the relatively stable valleys. Mountain slopes (ancia), and ridges and hills (lophia) are readily eroded in new or denuded areas. This is especially true of hill and mountain regions which have been stripped of their forest or thicket cover by fires, lumbering, cultivation, or grazing. Where the erosion is slight, the resulting succession may show initial xerophytic stages, or it may be completely mesostatic. Excessively eroded habitats are xerostatic, as in the case of bad lands, or, more frequently, they are mesotropic, passing first through a long series of xerophytic formations. Sandbars (cheradia, syrtidia) should be considered here, though they are eroded by currents and waves, and not by run-off. They are fixed and built up by sand-binding grasses and sedges, usually of a hydrophytic nature, and pass ultimately into mesophytic forest.

319. Succession by filling with silt and plant remains. All aquatic habitats into which silt, wash, or other detritus is borne by streams, currents, floods, waves, or tides are slowly shallowed by the action of the water plants present. These not only check the movement of the water, thus greatly decreasing its carrying power, and causing the deposition of a part or all of its load, but they also retain and fix the particles deposited. In accordance with the rule, each plant becomes the center of a stabilizing area, which rises faster than the rest of the floor, producing the well-known hummocks of lagoons and swamps. All aquatics produce this reaction. It is more pronounced in submerged and amphibious forms than in floating ones, and it takes place more rapidly with greatly branched or dissected plants than with others. In pools (tiphia) and lakes (7imnia), debouching streams and surface waters deposit their loads in consequence of the check exerted by the still water and the marginal vegetation, and delta-like marshes are quickly built up by filling. Springs (crenia) likewise form marshes where they gush forth in sands, the removal of which is impeded by vegetation. The flood plains and deltas of rivers show a similar reaction. The heavily laden flood waters are checked by the vegetation of meadows and marshes, and deposit most of their load. The banks of streams (ochthia) and of ditches (taphria) are often built $u p$ in the same fashion by the action of the marginal vegetation upon the current. The presence of marginal vegetation often determines the checking or deflecting of the current in such a way as to initiate meanders, while natural levees owe their origin to it, in part at least. Along low seacoasts, waves and tides hasten the deposit of river-borne detritus, causing the water to spread over the lowlands and form swamps. They 
often throw back also the sediment that has been deposited in the sea, the marsh vegetation acting as a filter in both cases. Successions of the kind indicated above are regularly mesotropic. Where the soil is sandy, and the filling-up process sufficiently great, or where salts or humus occur in excess, xerophytic formations result. In certain cases, these successions appear to be permanently hydrostatic, changing merely from floating or submerged to amphibious conditions, but this is probably due to the slowness of the reaction. As a rule, the accumulation of plant remains is relatively slight, and

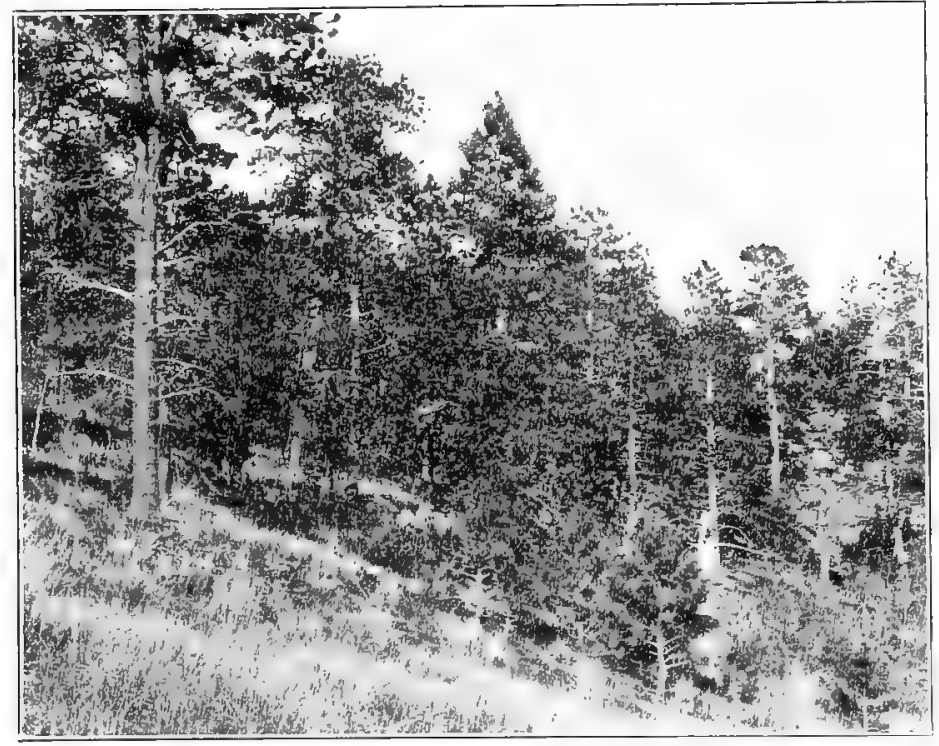

Fig. 67. Pine forest formation (Pinus-serohylium), stage VI of the talus succession.

plays an unimportant part in the reaction. In peat bogs and other extensive swamps, the amount of organic matter is excessive, and plays an important role in the building up of the swamp bed.

320. Succession by enriching the soil. This reaction occurs to some degree in the great majority of all successions. . The relatively insignificant lichens and mosses produce this result upon the most barren rocks, while the higher forms of later stages, grasses, herbs, shrubs, and trees, exhibit it in marked progression. The reaction consists chiefly in the incorporation of the decomposed remains of each generation and each stage in the soil. A very important part is played by the mechanical and chemical action of the 
roots in breaking up the soil particles, and in changing them into soluble substances. Mycorrhizae, bacterial nodules; and especially soil bacteria play a large part in increasing the nutrition-content of the soil, but the extent to which they are effective in succession is completely unknown. The changes in the color, texture, and food value of the soil in passing from the initial to ultimate stages of a normal succession are well known, and have led many to ,think them the efficient reactions of such successions. It seems almost certain, however, that this is merely a concomitant, and that, even in anomalous successions where facies replace each other without obvious reasons, the reactions are concerned more with water-content, light, and humidity than with the food-content of the soil.

321. Succession by exhausting the soil. This is a reaction not at all understood as yet in nature. A number of phenomena, such as the "fairy rings" of mushrooms and other fungi, the peripheral growth and central decay of lichens, Lecanora, Placodium, Parmelia, and of matforming grasses, such as Muhlenbergia, and the circular advance of the rootstalk plants, indicate that certain plants at least withdraw much of the available supply of some essential soil element, and are forced to move away from the exhausted area. It is probable that the constant shifting of the individuals of a formation year after year, a phenomenon to be discussed under alternation, has some connection with this. It will be impossible to establish such a relation, however, until the facts are exactly determined by the inethod of quadrat statistics. So far as native formations are concerned, there can not be the slightest question that prairies and forests have existed over the same area for centuries without impoverishing the soil in the least degree, a conclusion which is even more certain for the open vegetation of deserts and plains. With cuiture formations, the case is quite different. The exhaustion of the soil by continuous or intensive cultivation is a matter of common experience in all lands settled for a long period. Calcium, phosphorus, and nitrogen compounds especially are used up by crops, and must be supplied artificially. The reason for this difference in reaction between native and culture formations seems evident. In harvesting, not merely the grain, but the stems and leaves, and in gardening often the root also, are removed, so that the plant makes little or no return to the soil. In nature, annual plants return to the ground every year all the solid matter of roots, stems, leaves, and fruits, with the exception of the relatively small number of seeds that germinate. Perennial herbs return everything but the persistent underground parts. Shrubs and trees replace annually an immense amount of material used in leaves and fruits, and sooner or later, by the gradual decay of the individuals or by the destruction of the whole 
formation, they restore all that they have taken from the soil. This balance is further maintained to an important degree by the activity of the roots, which take from the deep-seated layers of the soil the crude materials necessary for the formation of leaves and fruits. Upon the fall and decay of these, their materials are incorporated with the upper layers of the formation floor, from which they may be absorbed by the undergrowth, or find their way again into the layers permeated by the tree roots. From the universal occurrence of weeds in cultivated regions, the pioneers in impoverished or exhausted fields are uniformly ruderal plants. As is well known, the seed

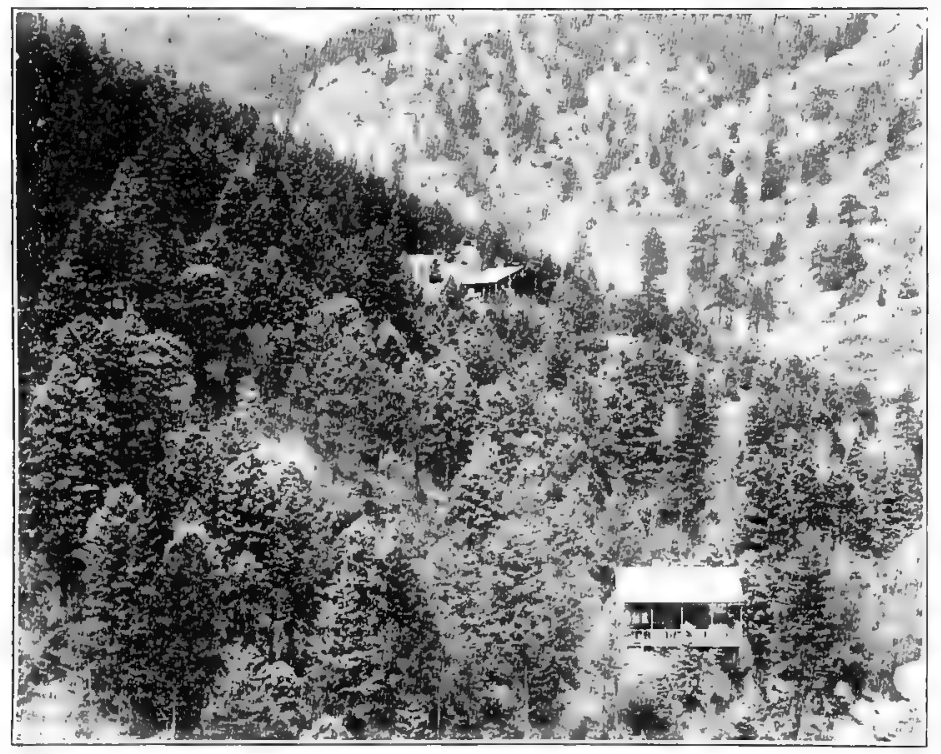

Fig. 68. Spruce forest formation (Picea-Pseudotsuga-hylium), stage VII, the ultimate stage of the talus succession.

production and ecesis of these forms are such that they take possession quickly and completeiy, while their demands upon the soil are of such a nature that the most sterile field can rapidly be covered by a vigorous growth of weeds. As indicated elsewhere, ruderal formations ultimately yield to the native vegetation, though in regions so completely given over to culture that native formations are lacking or remote, it is probable that successions reach their final stage within the group of ruderal plants.

322. Succession by the accumulation of humus. This is the characteristic reaction of peat bogs and cypress swamps (oxodia), in which the 
accumulation of vegetable matter is enormous. The plant remains decompose slowly and incompletely under the water, giving rise to the various liumic acids. These possess remarkable antiseptic qualities, and have an injurious effect upon protoplasm. They affect the absorption of water by the root hairs, though this is also influenced by poor aeration. The same acids are found in practically all inland marshes and swamps, but the quantity of decomposing vegetation in many is not great enough to produce an efficient reaction. Formations of this type usually start as freshwater swamps. The succession is apparently hydrostatic, but no thorough study of its stages has as yet been made.

323. Succession by modifying atmospheric factors. All layered formations, forests, thickets, many meadows and wastes, etc., show reactions of this nature, and are in fact largely or exclusively determined by them. The reaction is a complex one, though it is clear that light is the most efficient of the modified factors, and that humidity, temperature, and wind, while strongly affected, play subordinate parts. In normal successions, the effect of shade, i. e., diffuse light, enters with the appearance of bushes or shrubs, and becomes more and more pronounced in the ultimate forest stages. The reaction is exerted chiefly by the facies, but the effect of this is to cause increasing diffuseness in each successively lower layer, in direct ratio with the increased branching and leaf expansion of the plants in the layer just above. In the ultimate stage of many forests, especially where the facies are reduced to one, the reaction of the primary layer is so intense as to preclude all undergrowth. Anomalous successions often owe their origin to the fact that certain trees react in such a way as to cause conditions in which they produce seedlings with increasing difficulty, and thus offer a field favorable to the ecesis of those species capable of enduring the dense shade. Successions of this kind are almost invariably mesostatic, as it is altogether exceptional that layered formations are either xerophytic or hydrophytic.

\section{LAWS OF SUCCESSION}

324. The investigation of succession has so far been neither sufficiently thorough nor systematic to permit the postulation of definite laws. Enough has been done, however, to warrant the formulation of a number of rules, which apply to the successions studied, and afford a convenient method for the critical investigation of all successions upon the basis of initial causes, and reactions. Warming has already brought together a few such rules, and an attempt is here made to reduce the phenomena of succession, including its catises and effects, to a tentative system. At present it is difficult to 
make a thoroughly satisfactory classification of such rules, and they are here arranged? in general conformity with the procedure in succession.

I. Causation. The initial cause of a succession is the formation or appearance of a new habitat, or the efficient change of an existing one.

II. Reaction. Each stage reacts upon the habitat in such a way as to produce physical conditions more or less unfavorable to its permanence, but advantageous to the invaders of the next stage.

III. Proximity and mobility.

(I) The pioneers of a succession are those species nearest at hand that are the most mobile.

(2) The number of migrants from any formation into a habitat varies inversely as the square of the distance.

(3) The pioneer species are regularly derived from different formations, as the latter nearly always contain permobile species capable of effective ecesis.

(4) The plants of the initial stages are normally algae and fungi, with minute spores, composites, and grasses, which possess permobile fruits, or ruderal plants, on account of their great seed production.

IV. Ecesis.

(I) All the migrants into a new, denuded, or greatly modified habitat are sorted by ecesis into three groups: (I) those that are unable to germinate or grow, and soon die; (2) those that grow normally under the conditions present; (3) those that pass through one or more of the earlier stages in a dormant state to appear at a later stage of the succession.

(2) Wherever ruderal vegetation is present, it contributes a large number of the pioneer species of each succession, on account of the thorough ecesis. In other regions this part is played by subruderal native species.

(3) Annuals and biennials are characteristic of tile early stages of secondary successions, on account of their great seed production and ready ecesis.

(4) In layered formations, heliophytes appear before sciophytes; they ultimately yield to the latter, except where they are able to maintain a position in the primary layer.

(5) Excessive seed production and slight mobility lead to the imperfect ecesis of individuals in dense stands, and in consequence ustally produce great instability. 
(6) Each pioneer produces about itself a tiny area of ecesis and stabilization for its own offspring, for the disseminules of its fellows, or of invaders.

(7) Species propagating by offshoots, or producing relatively immobile disseminules in small number, usually show effective ecesis, as the offspring appear within the area of the reaction of the parent forms.

V. Stabilization.

(I) Stabilization is the universal tendency of vegetation.

(2) The ultimate stage of a succession is determined by the dominant vegetation of the region. Lichen formations are often ultimate in polar and niveal zones; grassland is the final vegetation for plains and alpine stretches, and for much prairie, while forest is the last stage for mesophytic midlands and lowlands, as well as for subalpine regions.

(3) Grassland or forest is the usual terminus of a succession; they predominate in lands physiographically mature.

(4) The limit of a succession is determined in large part by the progressive increase in occupation, which makes the entrance of invaders more and more difficult.

(5) Stabilization proceeds radiately from the pioneer plants or masses. The movement of offshoots is away from the parent mass, and the chances of ecesis are greatest near its edges, in a narrow area in which the reaction is still felt, and the occupation is not exclusive.

VI. General laws.

(I) The stages, or formations, of a succession are distinguished as initial (prodophytia), intermediate (ptenophytia), and ultimate (aiphytia).

(2) Initial formations are open, ultimate formations are closed.

(3) The number of species is small in the initial stages; it attains a maximum in intermediate stages; and again decreases in the ultimate formation, on account of the dominance of a few species.

(4) The normal sequence of vegetation forms in succession is: ( $I$ ) algae, fungi, mosses; (2) annuals and biennials; (3) perennial herbs; (4) bushes and shrubs; (5) trees.

(5) The number of species and of individuals in each stage increases constantly up to a maximum, after which it gradually decreases before the forms of the next stage. The interval between two maxima is occupied by a mixed formation. 
(6) A secondary succession does not begin with the initial stage of the primary one which it replaces, but usually at a much later stage.

(7) At present, successions are generally mesotropic, grassland and fcrest being the ultimate stages, though many are xerostatic or hydrostatic. If erosion continue until the sea level is reached, the ultimate vegetation of the globe will be hydrophytic. Should the heat of the sun decrease greatly before this time, the last vegetation will be xerophytic, i. e., crymophytic.

(8) The operation of succession was essentially the same during the geological past as it is to-day. From the nature of their vegetation forms, the record deals largely with the ultimate stages of such successions.

\section{CLASSIFICATION AND NOMENCLATURE}

325. Basis, New or clenuded habitats arise the world over by the operation of the same or similar causes, and they are revegetated in consequence of the same reactions. Similar habitats produce similar successions. The vegetation forms and their sequence are usually identical, and the genera are frequently the same, or corresponding in regions not entirely unrelated. The species are derived from the adjacent vegetation, and, except in alpine and coast regions, are normally different. The primary groups of successions are determined by éssential identity of habitat or cause, e. g., aeolian successions, erosion successions, burn successions, etc. When they have been more generally investigated, it will be possible to distinguish subordinate groups of successions, in which the degree of relationship is indicated by the similarity of vegetation forms, the number of common genera, etc. For example, burn successions in the Ural and in the Rocky mountains show almost complete similarity in the matter of vegetation forms and their sequence, and have the majority of their genera in common. A natural classification of successions will divide them first of all into normal and anomalous. The former fall into two classes, primary and secondary, and these are subdivided into a number of groups, based upon the cause which initiates the succession.

326. Nomenclature. The need of short distinctive names of international value for plant formations is obvious; it has become imperative that successions also should be distinguished critically and designated clearly. From the very nature of the case, it is impossible to designate each formation or succession by a single Greek or Latin term, as habitats of the same 
character will show in different parts of the world a vegetation taxonomically very different. It may some day be possible to use a binomial or trinomial for this purpose, somewhat after the fashion of taxonomy, in which the habitat name will represent the generic idea as applied to formations, and a term drawn from the floristic impress the specific idea. Such an attempt would be futile or valueless at the present time; it could not possibly meet with success until there is more uniformity in the concept of the formation, and until there has been much accurate and thorough investigation of actual formations, a task as yet barely begun. At present, it seems most feasible as well as scientific to designate all formations occupying similar habitats

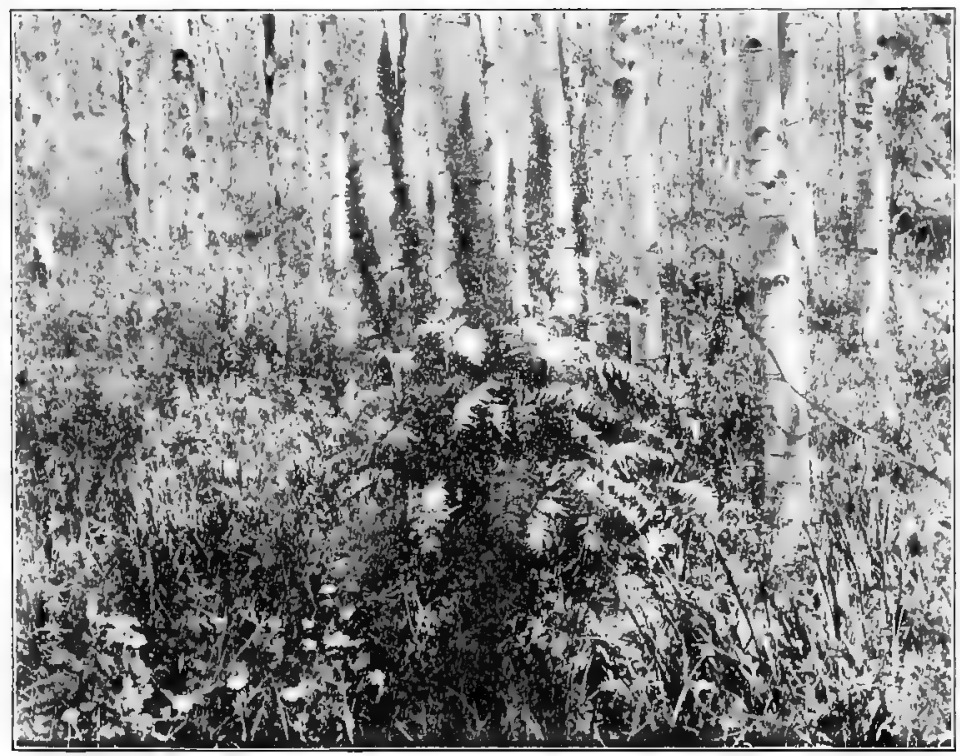

Fig. 69. Aspen forest formation (Populus-hylium), the typical stage of burn successions in the Rocky mountains; it is sometimes an anomalous stige in primary successions, interpolated in place of the thicket formation.

by a name drawn from the character of the latter, such as a meadow formation, poium, a forest formation, hylium, a desert formation, eremium, etc. A particular formation is best designated by using the generic name of one or two of its most inportant species in conjunction with its habitat term, as Spartina-Elymus-poium, Picea-Pinus-hylium, Cereus-Yucca-eremium, etc. Apparently a somewhat similar nomenclature is adapted to successions. The cause which produces a new habitat may well furnish the basis for the name of the general groups of successions, as pyrium (literally, a place 
or a habitat burned over), a burn succession, tribium, an erosion succession, etc. A burn succession consists of a sequence of certain formations in one part of the world, and of a series of quite different ones, floristically, in another. A particular burn succession should be designated by using the names of a characteristic facies of the initial and ultimate stages in connection with the general term, e. g., Bryum-Picca-pyrium, etc. A trinomial constructed in this way represents the desirable mean between definition and brevity. Greater definiteness is possible only at the expense of brevity, while to shorten the name would entirely destroy its precision. The following classification of successions is proposed, based upon the plan outlined above. The termination -ium ( $\epsilon$ iov) has been used throughout in the construction of names for successions, largely for reasons of euphony. If it should become desirable to distinguish the names of formations and successions by the termination, the locative suffix -on ( $-\omega v$ ) should be used for the latter. The terms given below would then be hypson, rhyson, hedon, sphyron, prochoson, pnonn, pagon, tribon, cly'son, repon, olisthon, xerasion, theron, broton, pyron, ecballon, camnon, ocheton, ardon.

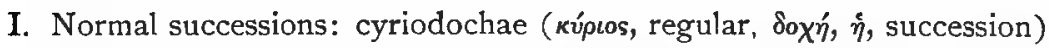

a Primary successions: protodochae ( $\pi \rho \hat{\omega} \tau o s$, first, primary)

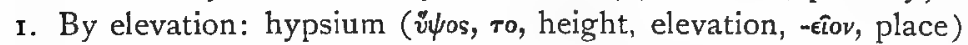

2. By volcanic action: rhysium ( $\dot{p} v \sigma i s \quad \dot{\eta}$, flowing, especially of fire)

3. In residuary soils: hedium ( $\tilde{\delta} \delta$ os, $\tau o ́$, a sitting base)

4. In colluvial soils: sphyrium ( $\sigma \phi \dot{v} \rho o v$, tó, ankle, talus)

5. In alluvial soils: prochosium ( $\pi \rho o ́ x \omega \sigma \iota s, \dot{\eta}$, a deposition of mud)

6. In aeolian soils: pnoium ( $\pi \nu \eta_{\eta}^{\prime} \dot{\eta}$, blowing, blast)

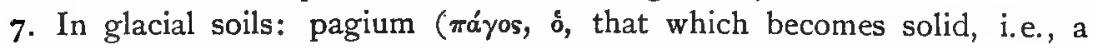
glacier)

b. Secondary successions: hepodochae ( $\pi \omega \omega$, to follow)

8. In eroded soils: tribium ( $\tau \rho i \beta \omega$, wear or rub away)

9. In flooded soils: clysium ( $\kappa \lambda v ́ \sigma \iota s, \delta$, a drenching, flooding)

ı. By subsidence: repium ( $\rho \dot{\varepsilon} \pi \omega$, incline downwards, sink)

I I. In landslips: olisthium (ö $\lambda \iota \sigma \theta o s, \dot{\delta}$, slip)

I2. In drained and dried out soils: xerasium ( $\xi_{\eta p \alpha \sigma i ́} \alpha, \dot{\eta}$, drought)

I3. By animal agencies: therium ( $\theta \dot{\eta} \rho, \delta$, wild animal)

14. By liuman agency: brotium ( $\beta$ porós, $\delta$, a mortal)

a. Burns: pyrium ( $\pi \hat{\imath} \rho, \tau \dot{o}$, fire)

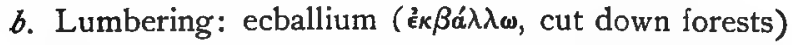

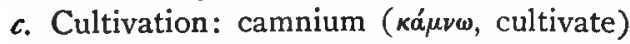

$d$. Drainage: ochetium (ỏxєтós, $\delta$, drain)

e. Irrigation: ardium (ă $\rho \delta \omega$, irrigate) 
II. Anomalous successions: xenodochae ( $\xi^{\prime} \varepsilon^{\prime}{ }^{\prime}$, st strange, unusual)

327. Illustrations. The following series will illustrate the application of this systen of nomenclature to particular successions, and their stages, or formations.

Thlaspi-Picea-sphyritum: pennycress-spruce talus succession

Thlaspi-Eriogonum-chaliciım: pennycress-eriogonum gravel slide formation.

Elymus-Gilia-chalicium: wildrye-gilia half gravel slide formation

Quercus-Holodiscus-driodium: oak-fringewood dry thicket formation

Pinus-xerohylium: pine dry forest formation

Picea-Pseudotsuga-hylium: spruce-balsam forest formation

Bryum-Picea-pyrium : moss-spruce burn succession

Bryum-telmatium: moss meadow formation

Aster-Chamaenerium-poium: aster-fireweed meadow formation

Deschampsia-Carex-poium: hairgrass-sedge meadow formation

Salix-Betula-helodritm: willow-birch meadow thicket formation

Populus-hylium: aspen forest formation

Picea-hylium: spruce forest formation

Lecanora-Carex-hedium : lichen-carex resicluary succession

Lecanora-Gyrophora-petrium: crustose lichen rock formation

Parmelia-Cetraria-chalicium: foliose lichen gravel slide formation

Paronychia-Silene-chalicium: nailwort-campion gravel slide formation

Carex-Campantula-coryphium: sedge-bluebell alpine meadow formation

Eragrostis-Helianthus-xerasium: eragrostis-sunflower drainage succession

Eragrostis-Polygonum-telmatium: eragrostis-heartsease wet meadow formation

Helianthus-Ambrosia-chledium: sunfiower-ragweed waste formation

\section{INVESTIGATION OF SUCCESSION}

328. General rules. The study of succession must proceed along two fundamental lines of inquiry: it is necessary to investigate quantitatively the physical factors of the initial stages and the reactions produced by the subsequent stages. This should be done by automatic instruments for humidity, light, temperature, and wind, in order that a continuous record may be obtained. Water-content is taken daily or even less frequently, while soil properties, and physiographic factors, altitude, slope, surface, and exposure are determined once for all. It is equally needful to determine the development and structure of each stage with particular reference to the adjacent formations, to the stage that has just preceded, and the one that is 
to follow. For this, the use of the permanent quadrat is imperative, as the sequence and structure of the stages can be understood only by a minute study of the shifting and rearrangement of the individuals. Permanent migration circles are indispensable for tracing movement away from the pioneer areas by which each stage reaches its maximum. Denuded quadrats are a material aid in tinat they furnish important evidence with respect to migration and ecesis, By means of them, it is possibie to determine the probable devel-

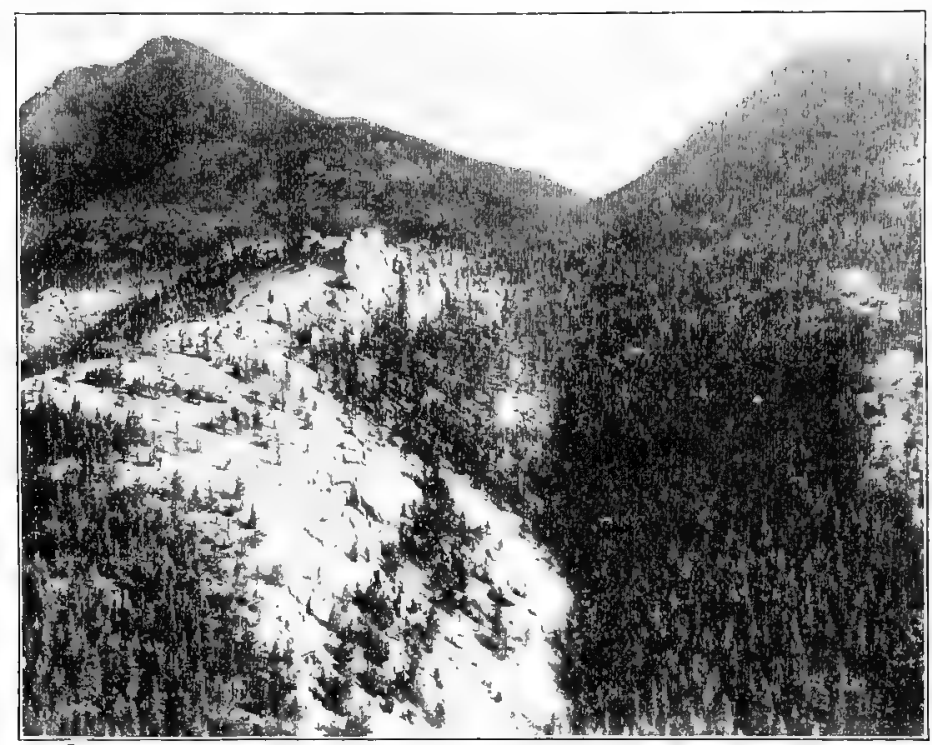

Fig. 70. Alternating gravel slides on Mounts Cameron and Palsgrove, from the comparison of which the initial development of the talus succession has been reconstructed.

opment of stages which reach back a decade or more into the past. In the examination of successions, since cause and effect are so intimately connected in each reaction, it is especially important that general and superficial observations upon structure and sequence be replaced by precise records, and that vague conjectures as to causes and reactions be supplanted by the accurate determination of the physical factors which underlie them.

329. Method of alternating stages. The period of time through which a primary succession operates is usually too great to make a complete study possible within a single lifetime. Secondary successions run their course much more quickly, and a decade will sometimes suffice for stabiliza- 
tion, though even here the period is normally longer. The longest and most complex succession, however, may be accurately studied in a region, where several examples of the same succession occur in different stages of development. In the same region, the physical factors of one example of a particular succession are essentially identical with those of another example. in the same stage. If one is in an initial stage, and the other in an intermediate conclition, the development of the former makes it possible to reestablish more or less completely the life history of the latter. The same connection may be made between intermediate and ultimate stages, and it is thus possible to determine with considerable accuracy and within a few years the sequence of stages in a succession that requires a century or more for its complete development. In the Rocky mountains, gravel slides (talus slopes) are remarkably frequent. They occur in all stages of development, and the alternating slides of different ages furnish an almost perfect record of this succession. This method lacks the absolute finality which can be obtained by following a succession in one spot from its inception to final stabilization, but it is alone feasible for long successions, i. e., those extending over a score or more of years. When it comes to be universally recognized as a plain duty for each investigator to leave an exact and complete record in quadrat maps and quadrat photographs of the stages studied by him, it will be a simple task for the botanists of one generation to finish the investigations of succession begun by their predecessors.

330. The relict method of studying succession is next in importance to the method of aiternating areas. The two in fact are supplementary, and should be used together whenever relicts are present. This method is based upon the law of successive maxima, viz., the number of species and of individuals in each stage constantly increases up to a certain maximum, after which it gradtully decreases before the forms of the next stage. In accordance with this, secondary species usually disappear first, principal species next, and facies last of all. There are notable exceptions to this, however, and the safest plan is to use the relict method only when principal species or facies are left as evidence. An additional reason for this is that secondary species are more likely to be common to two or more formations. In the majority of cases, the relict is not modified, and is readily recognized as belonging properly to a previous stage. This is true of herbs in all the stages of grassland, and in the initial ones of forest succession. The herbs and shrubs of earlier stages, which persist in the final forest stages, are necessarily morlified, often in such a degree as to become distinct ecads, or species. The facies of the stages which precede the ultimate forest are rarely modified. The application of the relict method, together with the modifica- 
tion just described, is nicely illustrated by the balsam-spruce formation at Minnehaha. Of the initial gravel slide stage, the relicts are Vagnera stellata and Galium boreale, the one modified into Vagnera leptopetala, and the other into G. boreale hylocolum. The thicket stage is represented by Holodiscus dumosa, greatly changed in form and branching, and in the shape and structure of the leaf. The most striking relict of the aspen formation is the facies itself, Populus tremuloides. The tall slender trunks of dead aspens are found in practically every balsam-spruce forest. In many places, living trees are still found, with small, straggling crowns, which are vainly trying to outgrow the surrounding conifers. Of the aspen undergrowth,

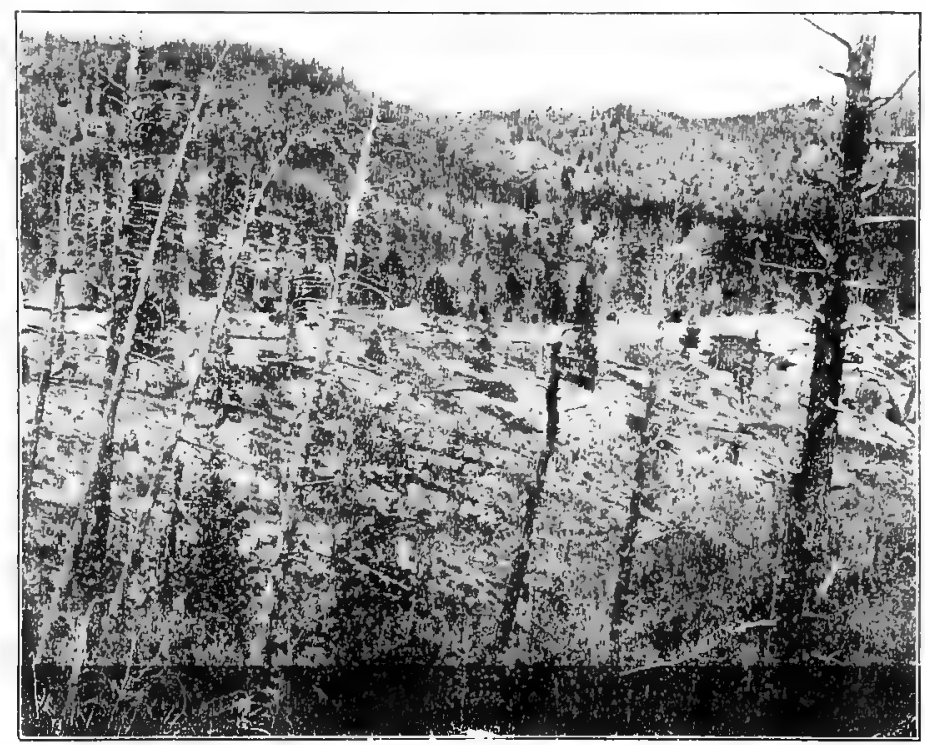

Fig. 71. Relict spruces and aspens, showing the character of the succession immediately preceding the burn succession now developing.

Rosa sayii, Helianthella parryi, Frasera speciosa, Zygadenus elegans, Castilleia confusa, Gentiana acuta, and Solidago orophila remain more or less modified by the diffuse light. It is still a question whether the aspen stage passes directly into the balsam-spruce forest, or whether a pine.forest intervenes. The presence of both Pinus ponderosa and $P$. Alexilis, which are scattered more or less uniformly through the formation, furnishes strong evidence for the latter view.

The lifetime of forest and thicket stages of successions is ascertained by counting the annual rings of the stumps of facies. This is a perfectly feasible 
method for many woodland formations where stumps already abound or where a fire has occurred, and it is but rarely necessary to cut down trees for this purpose. When trees or shrubs are present as relicts, the same method is used to determine the length of time taken by the development of the corresponding stages.

\section{THE STRUCTURE OF THE FORMATION}

331. Since all the structures exhibited by formations, such as zones, layers, consocies, etc., are to be referred to zonation or alternation, these principles are first considered in detail. This, then, constitutes the basis for a consideration of the structure of a normal formation, with special reference to the different parts that compose it. The investigation of formational structure, since the latter is the result of aggregation, invasion, and succession, is accomplished by instruments, quadrats, etc., in the manner already indicated under development, and no further discussion of it is necessary here.

\section{ZONATION}

332. Concept. The recognition of vegetation zones dates from Tournefort $^{1}$, who found that, while the plants of Armenia occupied the foot of Mount Ararat, the vegetation of the slopes above contained many species of southern Europe. Still higher appeared a flora similar to that of Sweden, and on the sumnit grew arctic plants, such as those of Lapland.

As the historical summary shows, the concept of zonation is the oldest in phytogeography. Notwithstanding this, it has never been clearly defined, nor has there been any detailed investigation of the phenomenon itself, or of the causes which produce it. Zones are so common, and often so clearly marked, that they invite study, but no serious attempt has heretofore been made to analyze zonation, or to formulate a definite method of investigating it. Zonation is the practically universal response of plants to the quantitative distribution of physical factors in nature. In almost all habitats, one or more of the physical factors present decreases gradually in passing away from the point of greatest intensity. The result is that the plants of the labitat arrange themselves in belts about this point, their position being determined by their relation to the factor concerned. Close investigation will show that there is hardly a formation that is entirely without zonation, though in many cases the zones are incomplete or obscure for various reasons. Zonation is as characteristic of vegetation as a whole as it is of its unit, the formation, a fact long ago recognized in temperature zones. A conti-

'Relation d'un Voyage dı Levant. 1717. 
nental climate, however, often results in the interruption of these, with the consequence that these belts of vegetation are not always continuous.

\section{CAUSES OF ZONATION}

333. Growth. The causes that produce zones are either biological or physical: the first have to do with some characteristic of the plant, the second with the physical features of the habitat. Biological causes arise from the method of growth, from the manner of dissemination, or from the reaction of the species upon the habitat. The formation of circles as a result of radial growth is a well-known occurrence with certain plants, but it is much more common than is supposed. In the case of agarics, this phenomenon has long been known under the name of "fairy-rings." It is found in a large number of moulds, and is characteristic of early stages of the mycelium of the powdery mildews. It occurs in nearly all maculicole fungi, and is exhibited by certain xylogenous fungi, such as Hysterographium. Among the foliose lichens, it is a common occurrence with the rock forms of Parmelia, Placodium, Physcia, and Lecanora, and with the earth forms of Parmelia and Peltigera. The thalloid liverworts show a similar radial growth. The flowering plants, and many mosses also, furnish good examples of this sort of growth in those species which simulate the form of the mycelium or thallus. These are the species that form mats, turfs, or carpets. Alpine mat formers, such as Silene acaulis, Paronychia pulvinata, Arenaria sajanesis, etc., are typical examples. Xerophytic, turf-forming species of Muhlenbergia, Sporobolus, Boutelona, Festuca, Poa, and other grasses form striking ring-like mats, while creeping species of Euphorbia, Portulaca, Amarantus, etc., produce circular areas. Rosettes, bunch-grasses, and many ordinary rootstalk plants spread rapidly by runners and rhizomes. The direction of growth is often indeterminate in these also, and is in consequence more or less bilateral or unilateral. Growth results in zonation only when the older central portions of the individual or mass die away, leaving an ever-widening belt of younger plants or parts. This phenomenon is doubtless due in part to the greater age of the central portion, but seems to arise chiefly from the demands made by the young and actively growing parts upon the water of the soil. There may possibly be an exhaustion of nutritive cortent, as in the case of the fungi, but this seems improbable for the reason that young plants of the same and other species thrive in these areas. It must not be inferred that these miniature growth zones increase in size until they pass into zones of formations. Growth contributes its share to the production of these, but there is no genetic connection between a tiny plant zone and a zone of vegetation. 
Radial and bilateral growth play an important part in formational zones in so far as they are related to migration. The growth of the runner or rhizome itself is a very effective means of dissemination, while the seeding cf the plants thus carried away from the central mass is most effective at the edge of the newly occupied area. This holds with equal force for plants with a mycelium or a thallus. The circular area becomes larger year by year. Sooner or later, the younger, more vigorous, and more completely occupied circumference passes into a more or less complete zone. This will result from the reaction of the central individuals upon the habitat, so that they are readily displaced by invaders, or from their increasing senility and dying out, or from the invasion of forms which seed more abundantly and successfully. This result will only be the more marked if the radiating migrants reach a belt of ground especially favorable to their ecesis. In this connection it must be carefully noted that vegetation pressure, before which weaker plants are generally supposed to flee, or by which they are thought to be forced out into less desirable situations, is little more than a fanciful term for radial growth and migration. It has been shown under invasion that disseminules move into vegetation masses, as well as away from them, the outward movement alone being conspicuous, because it is only at the margin and beyond that they find the necessary water and light for growth.

334. Reactions. Certain reactions of plants upon habitats produce zonation. The zones of fungi are doubtless caused by the exhaustion of the organic matter present, while in lichens and mosses the decrease in nutritive content has something to do with the disappearance of the central mass. In the mats of flowering plants, the connection is much less certain. The reaction of a forest or thicket, or even of a tall herbaceous layer, is an extremely important factor in the production of zonation. The factor chiefly concerned here is light. Its intensity is greatest at the edge of the formation and just below the primary layer; the light becomes increasingly diffuse toward the center of the forest, and toward the ground. In response to this, both lateral and vertical zones appear. The former are more or less incomplete, and are only in part due to differences in illumination. The vertical zones or layers are characteristic of forest and thickets, and are caused directly by differences in light intensity.

335. Physical factors. The physical causes of zonation are by far the most important. They arise from differences in temperature, water, and light. In the large, temperature differences are the most important, producing the great zones of vegetation. In a particular region or habitat, variations of water-content and humidity are controlling, while light, as 
shown above, is important in the reactions of forest and thicket. Physical factors produce zonation in a habitat or a series of habitats, when there is either a gradual and cumulative, or an abrupt change in their intensity. Gradual, slight changes are typical of single habitats; abrupt, marked changes of a series of habitats. This modification of a decisive factor tends to operate in all directions from the place of greatest intensity, producing a characteristic symmetry of the habitat with reference to the factor concerned. If the area of greatest amount is linear, the shading-out will take

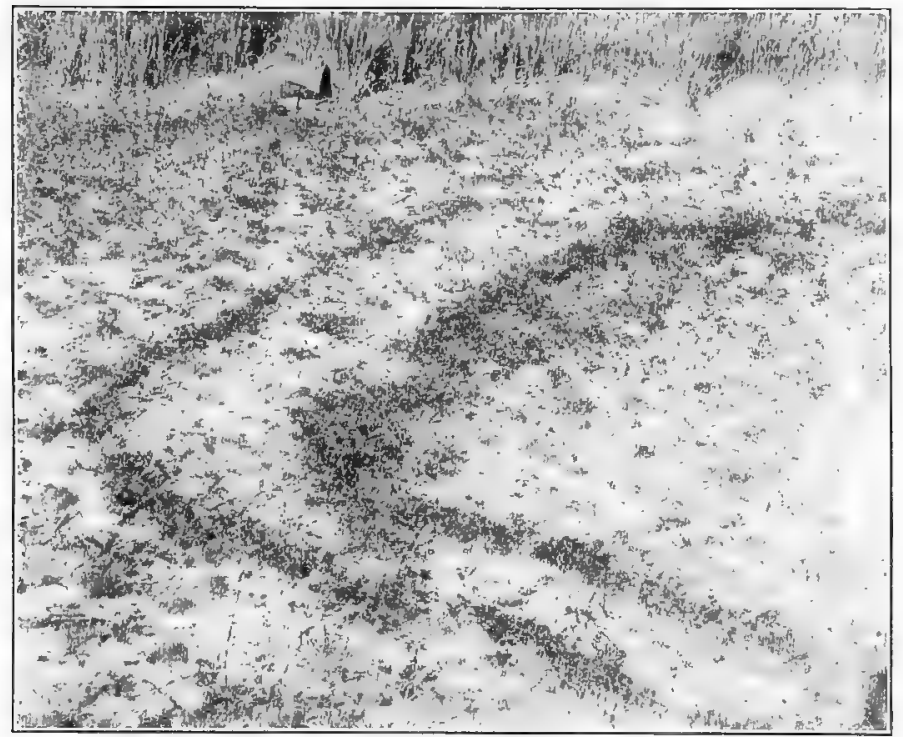

Fig. 72. Zones of Cyperus erythrorrhizus produced by the recession of the shore-line.

place in two directions, and the symmetry will be bilateral, a condition well illustrated by rivers. On the other hand, a central intense area will shade out in all directions, giving rise to radial symmetry, as in ponds, lakes, etc. The essential connection between these is evident where a stream broadens into a lake, or the latter is the source of a stream, where a mountain ridge breaks up into isolated peaks, or where a peninsula or landspit is cut into islands. The line that connects the points of accumulated or abrupt change in the symmetry is a stress line or ccotone. Ecotones are well-marked between formations, particularly where the medium changes; they are less distinct within formations. It is obvious that an ecotone separates two 
different series of zones in the one case, and merely two distinct zones in the other.

336. Physiographic symmetry. The physical symmetry of a habitat depends upon the distribution of water in it, and this is profoundly affected by the soil and the physiography. The influence of precipitation is slight or lacking, as it is nearly uniform throughout the habitat; the effects of wind and humidity are more localized. Differences of soil rarely obtain within a single habitat, though often occurring in a zoned series. The strikingly

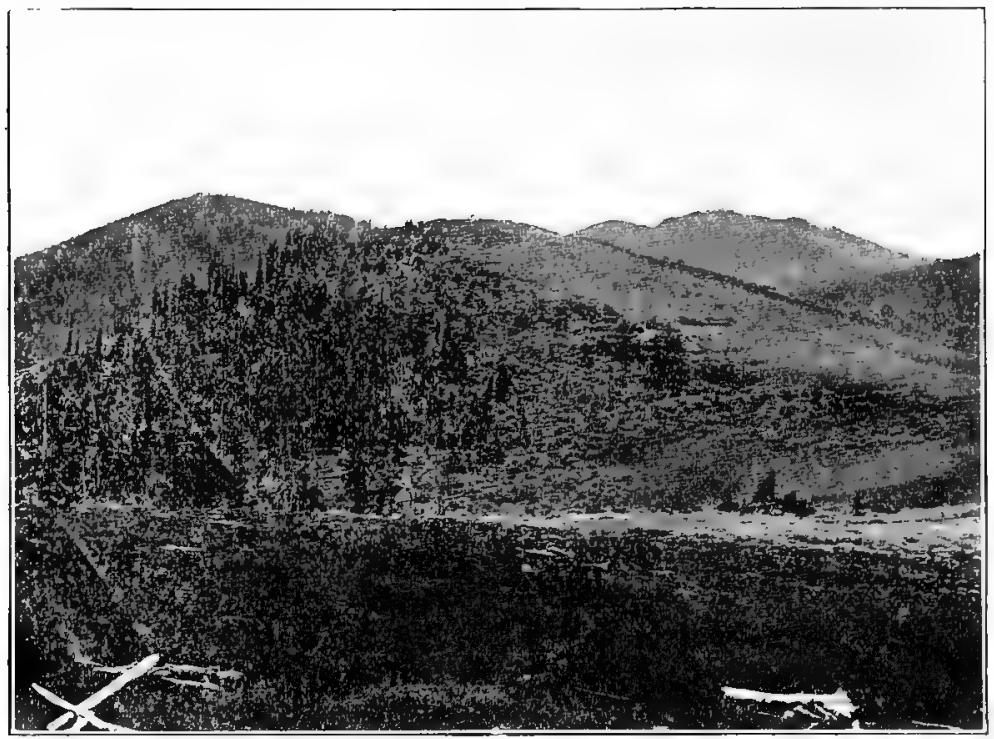

Fig. 73. Regional zones on a spur of Pike's Peak $(3,800 \mathrm{~m}$.); the forest consists of Picea engelmannii and Pinus aristata, the forewold is Salix pseudolapponum, and the grassland, alpine meadow (Carex-Campanula-coryphium).

zonal structure or arrangement of habitats is nearly always due to differences in water-content produced by physiographic factors, slope, exposure, surface, and altitude. The effect of these upon water-content and humidity is obvious. Wherever appreciable physiographic differences occur, there will be central areas of excess and deficiency in water-content, between which there is a symmetrical modification of this factor. Peaks are typical examples of areas of deficiency, lakes and oceans of areas of excess. When these areas are extreme and close to each other, the resulting zonation will be marked; when they are moderate, particularly if they are widely separated, 
the zones produced are obscure. Asymmetry of a habitat or a region practically does not exist. Central areas of excess and deficiency may be very large and in consequence fail to seem symmetrical, or the space between them so great that the symmetry is not conspicuous, but they are everywhere present, acting as foci for the intervening areas.

The response of vegetation to habitat is so intimate that physiographic symmetry everywhere produces vegetational symmetry, which finds its ready expression in plant zones. The reaction of vegetation upon habitat causes biolugical symmetry, typical of growth zones and light zones. From these facts it is clear that zonation will be regularly characteristic of the vegetative covering. The zonal arrangement of formations is usually very evident; the zones of a formation are often obscured, or, where the latter occupies a uniform central area of excess or deficiency, they are rudimentary or lacking, as in shallow ponds. Tones are frequently imperfect, though rarely entirely absent in new soils, such as talus. They are rendered obscure in several ways. In the initial stages of a succession, as well as in the transitions between the various stages, the plant population is so scattered, so transient, or so dense as to respond not at all to a degree of symmetry which produces marked zonation in later formations. The alternation of conspicuous species not only causes great interruption of zones, but often also completely conceals the zonation of other species, such as the grasses, which, though of more importance in the formation, have a lower habit of growth. Furthermore, the ecotones of one factor may run at right angles to those of another, and the resulting series of zones mutually obscure each other. Finally, such a physiographic feature as a hill may have its symmetry interrupted by ridges or ravines, which deflect the zones downward or upward, or cause them to disappear altogether, while the shallows or depths of a pond or lake may have the same effect. An entire absence of zones, i. e., azonation, is exceptional in vegetation. Almost all cases that seem to exhibit it may be shown by careful examination to arise in one of the several ways indicated above.

\section{KINDS OF ZONATION}

337. Two kinds of zonation are distinguished with reference to the direction in which the controlling factor changes. When this is horizontal, as with water-content and temperature, zonation will be lateral; when it is vertical, as in the case of light, the zonation is vertical. There exists an intimate connection between the two in forests, where the secondary layer of small trees and shrubs is continuous with a belt of trees and shrubs around the central nucleus, and the lower layers of bushes and herbaceous plants with similar zones still further out. This connection doubtless arises from 
the fact that conditions are unfavorable to the facies, outside of the nucleus as well as beneath it. Floristically, each layer and its corresponding zone are distinct, as the one consists of shade, the other of sun species. Lateral zonation is radial when the habitat or physiographic feature is more or less circular in form, and it is bilateral, when the latter is elongated or linear. Vertical zonation is unilateral.

338. Radial zonation is regularly characteristic of elevations and depressions. From the form of the earth, it reaches its larger expression in the girdles of vegetation corresponding to the zones of temperature. The zones of mountain peaks are likewise due largely to temperature, though humidity is a very important factor also. Mountain zones are normally quite perfect. The zonation of islands, hills, etc., is due to water-content. In the former, the zones are usually quite regular and complete; in the latter, they are often incomplete or obscured. Prairies and steppes are not zoned as units, but are complexes of more or less zonal hills and ridges. Ponds, lakes, and seas regularly exhibit complete zones, except in those shallow ponds where the depth is so slight that what is ordinarily a marginal zone is able to extend over the entire bottom. The line between an elevation and a depression, i. e., the edge of the water level, is the most sharply defined of all ecotones. It separates two series of zones, each of which constitutes a formation. One of these is regularly hydrophytic, the other is usually mesophytic. The line between the two can rarely be drawn at the water's edge, as this is not a constant, owing to waves, tides, or periodical rise and fall. There is in consequence a more or less variable transition zone of amphibious plants, which are, however, to be referred to the hydrophytic formation. Nearly all forest formations serve as a center about which are arranged several somewhat complete zones. As a rule, these merge into a single heterogeneous zone of thickets.

339. Bilateral zonation differs from radial only in as much as it deals with linear elevations and depressions instead of circular ones. With this difference, the zones of ranges and ridges correspond exactly to those of peaks and hills, while the same relation is evident between the zones of streams, and of lakes and ponds. The ecotones are identical except as to form; they are linear in the one and circular in the other. Incompleteness is more frequently found in bilateral zonation, though this is a question of distance or extent, rather than one of symmetry.

340. Vertical zonation is peculiar in that there is no primary ecotone present, on either side of which zones arrange themselves with reference to 
the factor concerned. This arises from the fact that the controlling factor is light, which impinges upon the habitat in such manner as to shade out in but one direction, i. e., downward. Vertical zones appear in bodies of water, on account of the absorption of light by the water. In a general way, it is possible to distinguish bottom, plancton, and surface zones, consisting almost wholly of algae. There is little question that minor zones exist, especially in lakes and seas, but these await further investigation. The most characteristic vertical zones occur in forests, where the primary layer of trees acts as a screen. The density of this screen determines the number of zones found beneath it. In extreme cases the foliage is so dense that the light beneath is insufficient even for mosses and lichens. As a rule, however, there will be one or more zones present. In an ordinary deciduous forest, the layers below the facies are five or six in number: (I) a secondary layer of small trees and shrubs, (2) a tertiary layer of bushes, (3) an upper herbaceous layer of tall herbs, (4) a middle herbaceous layer, (5) a lower herbaceous layer, (6) a ground layer of mosses, lichens, other fungi, and algae. The upper layers are often discontintous, the lower ones are more and more continuous. As a forest becomes denser, its layers disappear from the upper downward, the ground layer always being the last to disappear because of its ability to grow in very diffuse light. A vertically zoned formation shows a complex series of reactions. The primary layer determines the amount of heat, light, water, wind, etc., for the subordinate layers in general. Each of these layers then further determines the amount for those below it, the ground layer being subject in some degree to the control of every layer above it. This accounts probably for the definiteness and permanence of this layer. The degree to which the lower layers influence the upper by reacting upon the habitat is not known. It is evident that this influence must be considerable by virtue of their control of the water supply in the upper soil strata, by virtue of their transpiration, their decomposition, etc.

The ecotone between two formations is never a sharp line, but it is an area of varying width. The edge of this area which is contiguous to one formation marks the limit for species of the other. Both formations disappear in this transition zone, but in opposite directions. The overlapping which produces such zones arises from the fact that the physical factors tend to approach each other at the line of contact between formations, and that many species are more or less adjustable to conditions not too dissimilar.

341. Vegetation zones. As a fundamental expression of progressive change in the amount of heat and water, zonation is the most important feature of vegetation. It constitutes the sole basis for the division of continental as well as insular vegetation. The continent of North America 
furnishes striking proof of the truth of this. Conforming to the gradual cecrease of temperature and water-content northward, three primary belts of vegetation stretch across the continent from east to west. These are forest, grassland, and polar desert. The first is further divided into the secondary zones of broad-leaved evergreen, deciduous, and needle-leaved forests. At right angles to this temperature-water symmetry lies a symmetry due to water alone, in accordance with which forest belts touch the oceans, but give way in the interior to grasslands, and these to deserts. It is at once evident that the mutual interruption of these two series of zones has produced the primary features of North America vegetation, i. e., tropical forests where heat and water are excessive, deserts where either is unusually deficient, grassland when one is low, the other moderate, and deciduous and coniferous forests, where the water-content is as least moderate and the temperature not too low. Sich a simple yet fundamental division has been modified, however, by the disturbing effect which three continental mountain systems have had upon humidity and upon temperature symmetry. The two are intimately interwoven. The lowering of temperature due to altitude produces the precipitation of the wind-borne moisture upon those slopes which look toward the quarter from which the prevailing winds blow. A mountain range thus makes an abrupt change in the symmetry, and renders impossible the gradual change from forest to grassland and desert. The Appalachian system is not sufficiently high to produce a pronounced effect, and forests extend far beyond it into the interior before passing into prairies and plains. On the other hand, the influence of the Rocky mountains and the Sierra Nevada is very marked. The latter rise to a great height relatively near the coast, and condense upon their western slopes nearly all of the moisture brought from the Pacific. The Rocky mountains have the same effect upon the much drier winds that blow from the east, and the two systems in consequence enclose a parched desert. This series of major zones thus becomes, starting at the east, forest, grassland, desert, and forest, instead of the more symmetrical series, forest, grassland, desert, grassland, forest, which would prevail were it not for these barriers. This actual series of major zones undergoes further interruption by the action of these mountain systems in deflecting northern isotherms far to the south. This action is greatest in the high ranges, the Rocky mountains and the Sierras, and least in the lower Appalachians. Its result is to carry the polar deserts of the north far southward along the crests of the mountains, and to extend the borea! coniferous forests much further south along their slopes. In the Appalachians, this means no more than the extension of a long tongue of conifers into the mass of deciduous forests, and the occasional appearance of an isolated peak. In the western ranges, it produces two symmetrical 
series of minor mountain zones, forest, alpine grassland or desert, and forest, to say nothing of the foot-hill and timber-line zones of thicket.

There seems to be no good reason for distinguishing the zones of mountains as regions. The term itself is inapplicable, as it has no reference to zonation, and is used much more frequently as a term of general application. Its use tends to obscure also the essential identity of the so-called vertical zones of mountains with the major continental zones, an identity which can not be insisted. upon too strongly. For the sake of clearness, it is important to distinguish all belts of vegetation as zones, though it is evident that these are not all of the same rank. The following division of the vegetation of North America is based upon the fundamental principles of continental symmetry and the community of continental and mountain zones.

I. Polar-niveal zone-zona polari-nivalis

II. Arctic-alpine zone-zona arctici-alpina

Arctic province-provincia arctica

Alpine province-provincia alpina

III. Boreal-subalpine zone-zona boreali-subalpina

Alaska province-provincia alaskana

Cordilleran province-provincia cordillerana

Ontario province-provincia ontariensis

IV. Temperate zone-zona temperata

Atlantic province-provincia atlantica

Appalachian province-provincia appalachiana

Nebraska province-provincia nebraskensis

Utah province-provincia tutahensis

Coast province-provincia litoralis

Pacific province-provincia pacifica

V. Subtropical zone-zona subtropicalis

Florida province--provincia floridana

Mexican province-provincia mexicana

VI. Tropical zone-zona tropicalis

Antilles province-provincia antilleana

Andean province-provincia andeana

ALTERNATION

342. Concept. The term alternation is used to designate that phenomenon of vegetation, in which a formation recurs at different places in a 
region, or a species at separate points in a formation. Although it is a fundamental feature of vegetation, it has been recognized but recently. ${ }^{1}$

Alternation is the response of vegetation to the heterogeneity of the surface of the earth. It is in sharp contrast to zonation, inasmuch as it is directly caused by asymmetry in the topography. In consequence, it deals with the subdivisions of zones, arising from physical differences within the symmetrical area. It deals with vegetation areas of every rank below that of major zone, with the habitat and geographical areas of species, and, in a certain way, with the correspondence of vicarious genera. The breaking up of vegetation into formations is a striking example of alternation. The same phenomenon occurs in every formation, producing consocies and minor plant groups, and everywhere giving variation to its surface and structure. The essential idea involved in this principle is the recurrence of like formations, consocies, or groups, which are more or less separated by formations, consocies, or groups differing from them. It is an exact expression of the primary law of association that heterogeneity of structure varies directly as the extent and complexity of the habitat, or the series of habitats. Vegetation is made up of what are superficially homogeneous formations, but upon analysis these are seen to contain consocies. The latter, though more uniform than formations, break up into groups, each of which still shows a characteristic heterogeneity arising from the varying number and arrangement of its constituent species.

343. Causes. The primary cause of alternation is physical asymmetry, which is everywhere present within the symmetrical areas which produce zones. This is influenced so strongly, however, by migration and plant competition (phyteris) that the consideration of this subject will gain in clearness if these are treated as separate causes. The essential relation between them must not be lost sight of, however. Migration carries disseminules into all, or only some of the different areas of a formation, or into different formations, with little respect to the physical nature of these. The physical claracter of these asymmetrical areas determines that some of these plants shall be established in one series of places, and some in another, while the competition between the individuals in the various areas determines the numerical value of each species as well as its persistence. These three causes are invariably present in the production of alternating areas, and originally, i. e., in new or denuded soils, the sequence is constant, viz., migration, ecesis in asymmetrical areas, and competition.

${ }^{1}$ Clements, F. E. The Development and Structure of Vegetation. Rep. Bot. Surv. Nebr., $7: 16: 3.1904$. 
With respect to the different portions of an asymmetrical area, migration will have one of three effects: (I) it will carry disseminules into both favorable and unfavorable areas, (2) into favorable ones only, or (3) into unfavorable ones alone. From the radial nature of migration, the first case is far the most frequent; it is typical of sporostrotes, and the highly specialized spermatostrotes and carpostrotes. The effect of migration is uniform here, and alternation arises in consequence of the selective power of ecesis. It is evident that migration does not have an even indirect effect, when the disseminules are carried into none but unfavorable situations. Where the movement is into favorable places alone, alternation is the immediate result. The intermittent operation of migration and the presence of barriers are responsible for the absence of plants in situations favorable to them, and in consequence bring about a certain alternation between corresponding species.

The selective operation of phyșical factors upon the disseminules carried into the different parts of an asymmetrical area is the usual cause of alternation. Asymmetry alone is universal within the more conspicuous structures termed zones, down to the smallest areas which a group of plants can occupy. The difference between contiguous areas, particularly within the same habitat, is often small. It sometimes seems inefficient in the initial stages of a succession when a single species is present, but even in extreme cases its effect will be recognizable in the size and density of the individuals. Asymmetry is clearly evident in vegetation where two symmetrical series cross each other, or when a symmetry is interrupted by barrier-like elevations or depressions. Within formations, it arises from differences, often very slight, in slope, exposture, elevation, from irregularities of surface, differences in soil structure, or composition, in the amount of cover, and in the reactions of the living plants. At the last point, it is in direct connection with plant competition.

344. Competition. Much uncertainty, as well as diversity of opinion, seems still to exist in regard to the precise nature of the competition between plants that occupy the same area. It has long been admitted that the phrase, "struggle for existence," is true of this relation only in the most figurative sense, but the feeling still prevails that, since plants live in associations, there must be something mysterious and vitalistic in their relation. No one has been able to discover anything of this nature, but nevertheless the impression remains. Such a direct relation exists only between parasites, epiphytes, and lianes, and the plants which serve to nourish or support them. In the case of plants growing on the same stratum, actual competition between plant and plant does not occur. One individual can affect another only in as much as if changes the physical factors that influence the latter. Competition is a question of the reaction of a plant upon the physical factors which encompass it, and of the effect of these modified 
factors upon the adjacent plants. In the exact sense, two plants do not compete with each other as long as the water-content and nutrition, the heat and light are in excess of the needs of both. The moment, however, that the roots of one enter the area from which the other draws its water supply, or the foliage of one begins to overshade the leaves of the other, the reaction of the former modifies unfavorably the factors controlling the latter, and competition is at once initiated. The same relation exists throughout the process; the stronger, taller, the more branched, or the better rooted plant reacts upon the habitat, and the latter immediately exerts an unfavorable effect upon the weaker, shorter, less branched, or more poorly rooted plant. This action of plant upon habitat and of habitat upon plant is cumulative, however. An increase in the leaf surface of a plant not merely reduces the amount of light and heat available for the plant near it or beneath it, but it also renders necessary the absorption of more water and other nutritive material, and correspondingly decreases the amount available. The inevitable result is that the successful individual prospers more and more, while the less successful one loses ground in the same degree. As a consequence, the latter disappears entirely, or it is handicapped to such an extent that it fails to produce seeds, or these are reduced in number or vitality.

Competition in vegetation furnishes few instances as simple as the above, but this will serve to make clear the simplest case of ordinary competition, i. e., that in which the individuals belong to a single species. The various individuals of one species which grow together in a patch show relatively slight differences, in height, width, leaf expanse, or root surface. Still, some will have the largest surfaces for the impact of water, heat, and light, while others will have the smallest; the majority, perhaps, will occupy different places between the extremes. The former will receive more than their share of one or more factors. The reaction thus produced will operate upon the plants subject to it inversely as the amount of surface impinged upon. The usual expression of such competition is seen in the great variation in height, branching, etc., of the different individuals, and in the inability of many to produce flowers. This is particularly true of annuals, and of perennials of the same generation. In the competition between parents and offspring of the same perennial species, the former. usually have so much the advantage that the younger plants are often unable to thrive or even germinate; and disappear, leaving a free space beneath and about the stronger parents. This illustrates the primary law of competition; viz., that this is closest when the individuals are most similar. Similar individuals make nearly the same demands upon the habitat, and adjust themselves least readily to their mutual reactions. The more unlike plants are, the greater the difference in their needs, and some are able to adjust themselves to the reactions of others with little or no disadvantage. 
In accordance with the above principles, the competition is closer between species of like form than between those that are dissimilar. This similarity must be one of vegetation or habitat form, not one of systematic position. The latter is in fact of no significance, except where there is a certain correspondence between the two. Leaf, stem, and root characters determine the outcome, and those species most alike in these features will be in close competition, regardless of their taxonomic similarity or dissimilarity. This is as conclusive of the competition between the species of the same genus as it is between those belonging to genera of widely separated families. From this may be deduced a second principle of competition, viz., the closeness of the competition between the individuals of different species varies directly with their similarity in vegetation or habitat form. This principle is of primary importance in the competition which arises between occupants and invaders in the different stages of succession. Those invading species that show the greatest resemblance to occupants in leaf, stem, and root form experience the greatest difficulty in establishing themselves. The species, on the contrary, which are so unlike the occupants that they come in at a clear advantage or uisadvantage, estahlish themselves readily, in the one case as a result of the reaction, in the other by taking a subordinate position. This principle lies at the base of the changes in succession which give a peculiar stamp to each stage. A reaction sufficient to bring about the disappearance of one stage can be produced only by the entrance of invaders so different in form as to materially or entirely change the impress of the formation. Stabilization results when the entrance of invaders of such form as to exert an efficient reaction is no longer possible. In forests, while many vegetation forms can still enter, none of these produce a reaction sufficient to place the trees at a disadvantage, and the ultimate forest stage, though it may change in composition, can not be displaced by another.

It is obvious that the vegetation forms and habitat forms of associated species are of fundamental importance in determining the course and result of competition. Identity of vegetation form regularly produces close competition, and the consequent numerical reduction or disappearance of one or more species. Dissimilarity, on the other hand, tends to eliminate competition, and to preserve the advantage of the superior form. Species of trees compete sharply with each other when found together; the same is true of shrubs, or rosettes, etc. The relation of the shrubs to the trees, or of the rosettes to the shrubs of a formation is one of subordination rather than of competition. The matter of height and width often enters here also to such a degree that the tallest herbs compete with the bushes and shrubs, and rosettes with mats or grasses. The amount and disposition of the leaf surface are decisive factors in the competition between species of the sanne 
vegetation form, in so far as this is governed by light. In those plants in which the leaves are usually erect, notably the grasses and sedges, the competition between the aerial parts is relatively slight, and the result is determined by the reactions of the underground stens and roots.

The position of the competing individuals is of the greatest importance. The distance between the plants affects directly the degree of competition, while their arrangement, whether in groups according to species or singly, exerts a marked influence by determining that the contest shall be between like forms, or unlike forms. Position is controlled primarily by the relation existing between seed-production and dissemination. It is of course influenced in large measure by the initial position taken by the invaders into a nudate area, but this is itself a result of the same phenomena. The individuals of species with great seed-production and little or no mobility u1sually occur in dense stands. In these, the competition is fierce, for the two reasons of similarity and density, and the result is that the plants fall far below the normal in height and width. This is an extreme example of the group arrangement. When the seed-production is small, the mobility may be great or little without seriously affecting the result. The individuals of a species of this kind will be scattered among those of other species, and the closeness of competition will depend largely upon the similarity existing between the two. The arrangement in such cases is sparse. A species with great seed-production and great mobility usually shows both kinds of arrangement, the position of the individuals and the competition between them varying accordingly. This is due to the intermittent action of distributing agents, making it possible for the seeds to fall directly to the ground during the times that winds, etc., are absent. The three types of arrangement indicated above are termed gregarious, copious, and gregario-copious. They furnish the basis for the investigation of abundance which deals essentially with the number and arrangement of the individuals of competing species. The effect of distance, i. e., the interval between individuals, upon competition is fundamental. The competition increases as the interval diminishes, and the reverse.

The view here advanced, i. e., that competition is purely physical in nature, renders untenable the current conceptions of vegetation pressure, occupation, etc. Masses of vegetation are thought to force the weaker species toward the edge, thus initiating an outward or forward pressure. As has been shown above, no such phenomenon occurs in vegetation. This movement is nothing but simple migration, followed by ecesis, and has no connection with "weaker" species, or the development of a vital pressure. The direction taken by the migrating disseminules is essentially indeterminate. Migration seems to be outward, or away from the mass, merely because the 
ecesis is greater at the edge, where the increased dissimilarity between plant forms diminishes the competition. The actual movement is outward, but it takes place through the normal operation of competition. In this connection, it should be pointed out that the common view that plants require room is inexact, if not erroneous. This is difficult of proof, as it is impossible to distinguish room as such from the factors normally present, light, heat, water, and nutrient salts, but it seems obvious that the available amounts of these will determine the space cccupied by a plant, irrespective of the room adjacent plants may allow it. The explanation of competition upon physical grounds likewise invalidates the view that plants possess spheres of influence other than the areas within which they exert a demonstrable reaction upon the physical factors present.

Competition plays a very important role in alternation. It produces minor examples of alternation in the physical units of an asymmetrical series. Its greatest influence, however, is exerted in modifying the effects of asymmetry. The reaction of occupants "emphasizes or reduces the effect of asymmetry, and has a corresponding action upon alternation. This result of competition is typical of succession, in which the sequence of stages arises from the interaction of occupant and invader.

345. Kinds of alternation. Alternation involves two ideas, viz., the al- ternation of different species or formations with each other, and the alternation of the same species or formation in similar but separate situations. This is the evident result of asymmetry, in response to which contiguous areas are dissimilar and remote ones often similar. Individuals of the same species or examples of the same formation may be said to alternate between two or more similar sittlations, while different species or formations are said to alternate reith each other, occurring tsully in situations different in character. From the nature of altemation, the two phenomena are invariably found together.

It is possible to distinguish three kinds of alternation: (I) of a formation, consocies, layer, facies, or species in similar situations; (2) of similar or corresponding formations, species, etc., in similar situations; (3) of facies and other species with respect to number. The last two are merely variations of the first, arising out of slight differences in the physical factors of the alternating areas, the adjacent flora, or the course of competition. The alternation of different examples of the same formation is a significant feature of greatly diversified areas, such as mountains. It is naturally much less characteristic of lands physiographically more uniform. A xerophytic formation will alternate from ridge to ridge, a mesophytic formation between the intermediate valleys; aquatic vegetation will alternate from pond to pond, 
or stream to stream. The appearance of new or denuded soils upon which successions establish themselves is the most important cause of the alternation of formations. The weathering of rocks in different areas of the same region produces in each a sequence of similar or identical formations. The same statement is true in general of other causes of succession, such as erosion, flooding, burning, cultivation, etc., wherever they operate upon areas physically similar and surrounded by the same type of vegetation. The areas of more or less heterogeneous formations characterized by major physical differences are occupied by consocies. In an extensive formation, the same consocies alternates from one to another of these areas that are similar. When the formation is interrupted and occurs here and there in separate examples, a consocies often alternates from one to another of these. A consocies regularly derives its character from the fact that one or more of the facies of the formation is more intimately connected with certain areas of the latter than with others. This explains why the alternations of consocies and facies are usually identical. Layers sometimes alternate between different examples of the same forest or thicket formation, when they are suppressed in sonie by the diffuseness of the light.

The alternation of species is a typical feature of formations; it is absent only in those rare cases where the latter consist of a single species. The areas of a habitat which show ninor physical or historical (i. e., competitive) differences are occupied by groups of individuals belonging to one or more species responsive to these differences. Each of these groups will recur in all areas essentially similar, the intervals being occupied of course by slightly different groups. Such groups are constituted by gregarious or copious species of restricted adjustability. Sparse plants likewise alternate, but they necessarily play a much less conspicuous part. In habitats not too heterogeneous, a large number of species are sufficiently adjustable to the slight differences so that they occur throughout the formation. Often, to be sure, they show a characteristic response, expressed in the size or number. This is illustrated by the facies and many of the principal species of the prairie formation. Festuca, Koelera, Panicum, and Andropogon occur throughout, except in the moist ravines which are practically meadows. Astragalus, Psoralea, Erigeron, and Aster grow everywhere on slopes and crests, but they are much more abundant in certain situations. Other plants, Lomatium, Meriolix, Anemone, Pentsiemon, etc., recur in similar or identical situations upon different hills. Lomatium alternates between sandy or sandstone crests, Meriolix and Pentstemon occur together upon dry upper slopes, while Anemone alternates between dry slopes and crests.

Owing to the accidents of migration and competition, similar areas within a habitat are not occupied by the same species, or group of species. A spe- 
cies found in one area will be replaced in another by a different one of the same or a different genus. The controlling factors of the area render imperative an essential identity of vegetation and habitat form, though in systematic position the plants may be very diverse. Such genera and species may be termed corresponding. The relation between such plants is essentially alternation; it should, perhaps, be distinguished from alternation proper as corresponsive. The prairie formation furnishes a good example of this on exposed sandy crests, upon which Lomatium, Comandra, and Pentstemon alternate. Formations exhibit a similar correspondence.

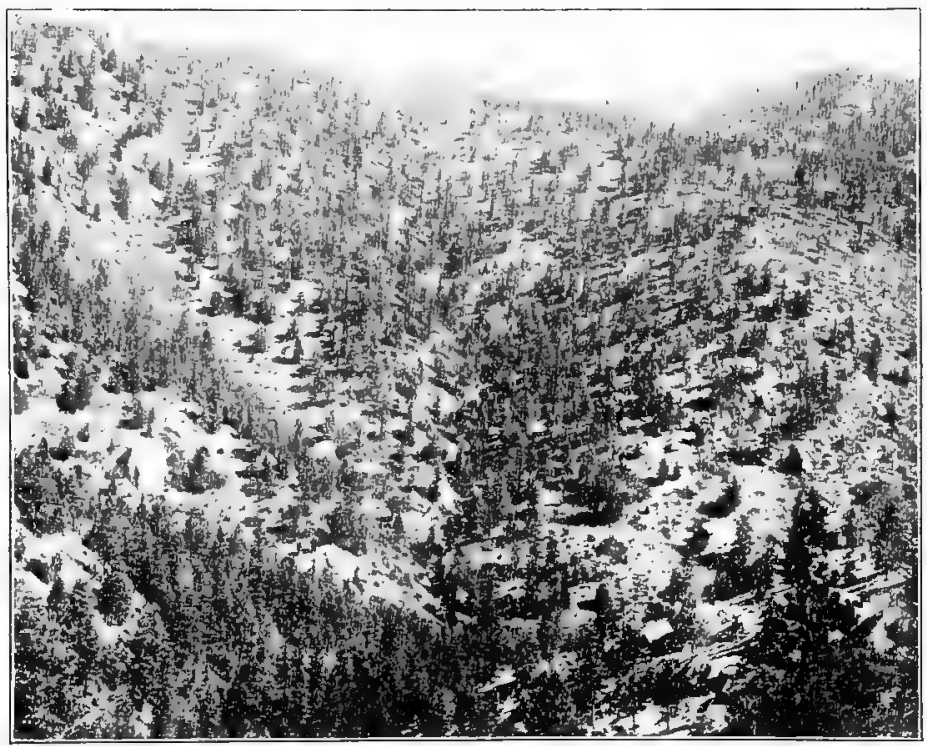

Fig. 74. Numerical alternation of Pinus and Pseudotsuga upon east and west slopes.

All species that alternate show a variation in abundance from one area to another. Frequently, the difference is slight, and may be ignored, except in determining abundance. Very often, however, the variation is so great that a facies may be reduced, numerically, to the rank of a principal species, or one of the latter to a secondary species. This phenomenon is distinguished as numerical alternation. It arises from the fact that the similar areas are sufficiently different to affect the abundance, without producing complete suppression. It is probable that this result is due almost entirely to competition. Astragalus crassicarpus grows on all the slopes of the prairie formation, but on some it has the abundance of a facies, while on others it is repre- 
sented by a few scattered individuals: This difference is much more striking in separate examples of the same formation, particularly when a normal facies is reduced to the numerical value of a secondary species. This is a matter of great importance in the study of formations, for it has doubtless often resulted in mistaking a consocies for a formation.

Alternation furnishes the logical basis for what may be called comparative phytogeography. The latter is of much broader scope than the old subject of geographical distribution, for it treats not only of the distribution of formations and associations as well as of species, but it also seeks to explain this by means of principles drawn from the relation between irabitat and vegetation. When the latter come to be fully based upon physical factor investigations, and upon the effects of migration and competition as shown in alternation, the comparative study of formations will represent the highest type of phytogeographical activity.

THE FORMATION IN DETAIL

346. The rank of the formation. There have been as many different opinions in regard to the application of the term formation as there are concerning the group which is to be called a specics. In taxonomy, however, the concept of the species is purely arbitrary, and agreement can not be hoped for. In vegetation, on the contrary, the connection between formation and habitat is so close that any application of the term to a division greater or smaller than the habitat is both illogical and unfortunate. As effect and cause, it is inevitable that the unit of the vegetative covering, the formation, should correspond to the unit of the earth's surface, the habitat. This places the formation upon a basis which can be accurately determined. It is imperative, however, to have a clear understanding of what constitutes the difference between habitats. A society is in entire correspondence with the plysical factors of its area, and the same is true of the vegetation of a province. Nevertheless, many societies usually occur in a single habitat, and a province contains many habitats. The final test of a habitat is an efficient difference in one or more of the direct factors, water-content, humidity, and light, by virtue of which the plant covering differs in structure and in species from the areas contiguous to it. A balsam-spruce forest shows within itself certain differences of physical factors and of structure. The watercontent will range from 20-25 per cent, and the light from .02-.003. One portion may consist chiefly of Pscudotsuga mucronata, another of Picea engelmannii, and a third of Picea parryana, or these species may be intermingled. If, however, this forest is compared with the gravel slide, which touches it on one side, and the meadow thicket, which meets it on another, 
the physical factors and the species both demonstrate that it is the forest, and not its parts, which corresponds to a distinct physical entity, the habitat. This test of a formation is superfluous in a great many cases, where the physiognomy of the contiguous areas is conclusive evidence of their difference. It is evident also that remote regions which are floristically distinct, such as the prairies and the steppes, may possess areas physically almost identical and yet be covered by different formations. This point is further discussed under classification.

The existing confusion in the matter of formations is due to two causes. The first arises from the fact that much ecological work has been hasty. Little or no attention has been given to development, and in consequence rudimentary and transitory stages of succession have often been described as formations. Mixed areas in particular have caused trouble. In the second place, there has been a marked tendency to minimize the need of thoroughness and training by calling every slightly different area a formation. A failure to recognize the primary value of alternation has also contributed materially to this. Alternating facies, and principal species, when separated from each other, have often been mistaken for formations. This is a danger that must be fully appreciated and guarded against. In practically all regions, the same formation is represented by numerous scattered areas, all showing greater or less differences arising from alternation. This is especially true of thickly populated regions where virgin areas are rare. The fact that twenty-five miles intervene to-day between two small stretches of primitive prairie is permitted to unduly emphasize their differences. It requires the study of a number of such examples to counteract this tendency, and to cause one to see clearly that they must have been at one time merely so many bits of the prairie formation.

In this connection, the lichen and moss groups which are found on rocks constitute an interesting problem. It is clear that Peltigera and Cladonia, which grow on the forest floor, and Evernia, Ramalina, and Physcia, which are found on the trees, are merely constituent species of the forest formation. The same is true of Cladonia, Urceolaria, and Parmelia, which are found among the sedges and grasses of alpine meadows. The physical conditions are essentially those of the formation, and the lichens themselves are more or less peculiar to it. This is particularly true of the forest, in which the two strata, bark and moist shaded soil, are present because of the trees. In the case of granitic rocks, the circumstances are very different. The species of lichens found on the rocks are not peculiar to the formation, but they also occur elsewhere. In the forest, Parmelia, Placodium, Physcia, Rinodina, Urceolaria, Lecanora, Lecidea, etc., occur on the rocks. In the alpine meadows, the rock groups are composed of Parmelia, Gyrophora, 
Cetraria, Acarospora, Lecanora, Lecidea, Buellia, etc. The stratum itself is physically very different and constitutes a distinct habitat. These groups are really small formations, which are quite distinct from the surrounding forest or meadow. This is proven conclusively in many places in the mountains where areas of the characteristic lichen formations of cliffs are carried by the fall of rock fragments into forest and meadow, where they persist without modification. This also shows clearly that the groups on scattered rocks in the same area are to be regarded as examples of the same cliff formation, except where the differences are evidently to be ascribed to develop-

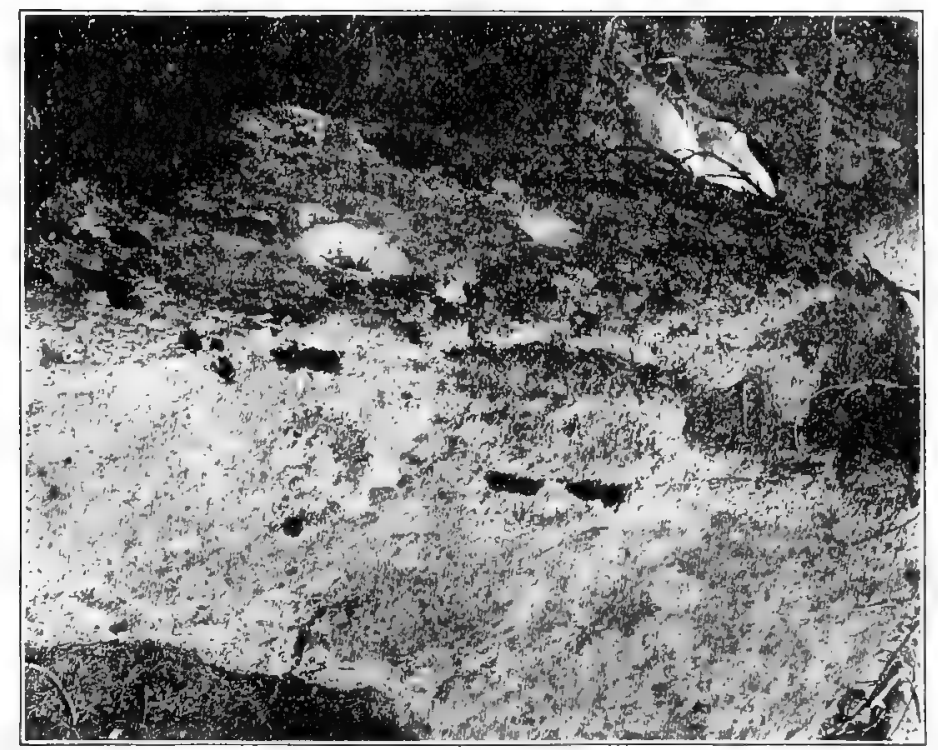

Fig. 75. Relict lichen formation in a spruce forest, invaded by rock mosses.

ment and not to alternation. Where these rock formations can not be traced to cliffs or magmata with certainty, they must be considered as antedating the vegetation in which they occur. Often, indeed, especially in igneous areas, they are relicts of the initial stage of a primary succession. Finally, they prove their independence of the forest or meadow formation by initiating a distinct succession within these. Crustaceous groups or formations yield to foliose ones, and these in turn give way to formations of mosses, particularly in the forest where the effect of the diffuse light is felt. From the above, the following rule of formational limitation is obtained: any area, which shows an essential difference in physical character, composition, or development from the surrounding formation is a distinct formation. 
347. The parts of a formation. All the parts which make up the structure of a formation are directly referable to zonation and alternation, alone or together, or to the interaction of the two. The principles which underlie this have already been discussed under the phenomena concerned. It is necessary to point out further that the structure may be produced in several ways: (I) by zonation alone, (2) by alternation alone, (3) by zonation as primary and alternation as secondary, (4) by primary alternation and secondary zonation, (5) by the interaction of the two, as in layered formations. Though all these methods occur, the first two are relatively rare, and the

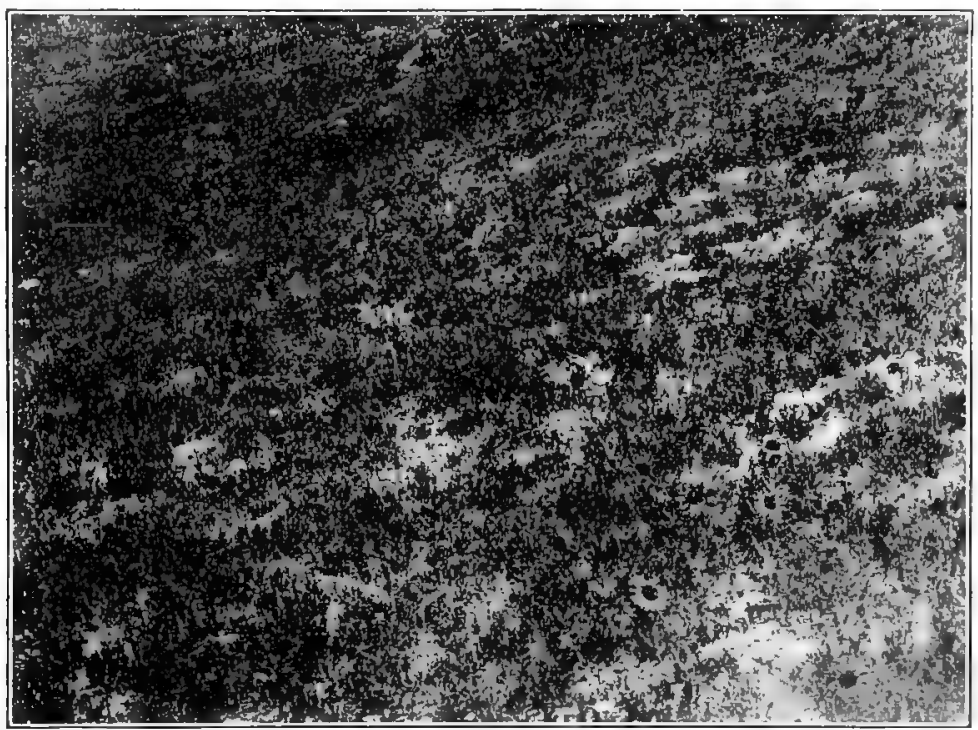

Fig. 76. Early (prior) aspect of the alpine meadow formation ( $\mathrm{Ca}$ rex-Campanula-coryphium), characterized by Rydbergia grandiflora.

resulting structure comparatively imperfect. The typical structure of formations can best be made clear by the consideration of a prairie which belongs to the fourth group, and a forest which represents the last.

The major divisions of prairie and forest formations are regularly due to alternation. There is an inherent tendency to the segregation of facies, arising out of physical or historical reasons, or from a combination of both. Not all formations show this, but it is characteristic of the great majority of them. The primary areas which thus arise have been called associations: they are naturally subordinate to the formation. To avoid the confusion which inevitably results from using the word association in two different 
senses, it is proposed to term this primary division of the formation, a consociation, or better, a consocies. This term is applied only to an area characterized by a facies, or less frequently, by two or more facies uniformly commingled. The consocies of grassland are determined by grasses, those of forests by trees, etc. From the different position of the facies in these two types of vegetation such areas are readily seen at all times in the forest, but they are often concealed in grassland by the tall-growing principal species of the various aspects. When definite consocies are present, they are often found to mingle where they touch, producing miniature transition areas, and, very rarely, they sometimes leave gaps in which no facies appears.

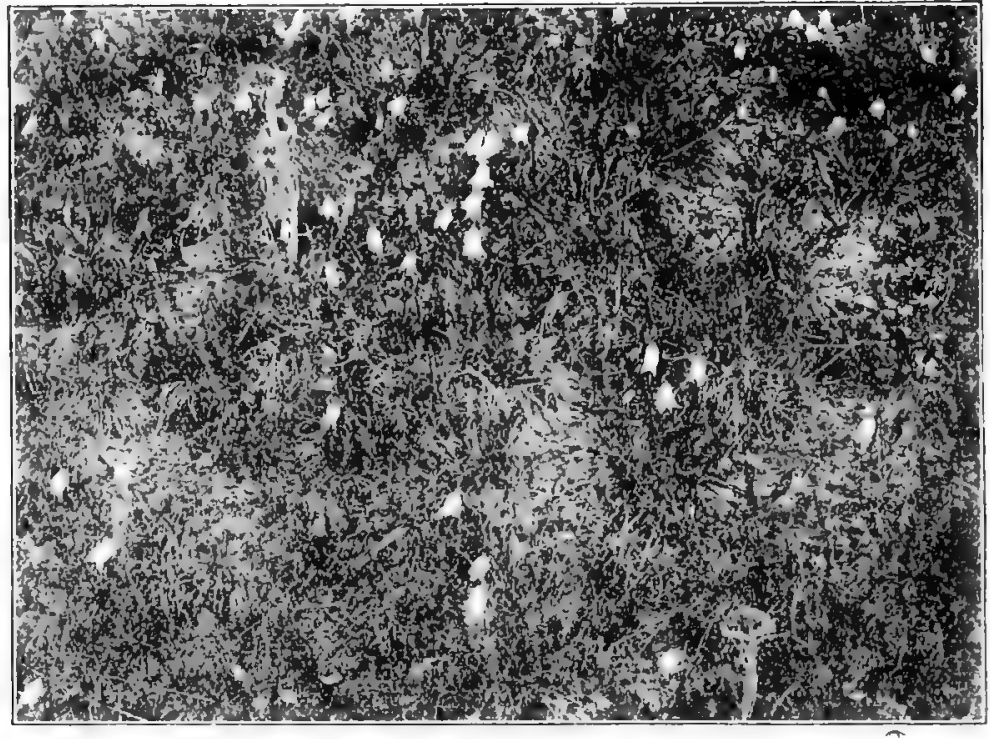

Fig. 77. Late (serotinal) aspect of the alpine meadow, characterized by Campanula petiolata, Rydbergia in fruit.

The seasonal changes of a formation, which are called aspects, are indicated by changes in composition or structure, which ordinarily correspond to the three seasons, spring, summer, and autumn. The latter affect the facies relatively !ittle, especially those of woody vegetation, but they influence the principal species profoundly, causing a grouping typical of each aspect. For these areas controlled by principal species, but changing from aspect to aspect, the term socicty is proposed. They are prominent features of the majority of herbaceous formations, where they are often more striking than the facies. In forests, they occur in the shrubby and herbaceous layers, and 
are consequently much less conspictous than the facies. A close inspection of the societies formed by principal species shows that they are far from uniform. Since they usually fail to exhibit distinct parts, it becomes necessary to approach the question of their structure from a new standpoint. Such is afforded by aggregation, which yields the simplest group in vegetation, i. e., that of parent and offspring. This is so exactly a family in the ordinary sense that there seems to be ample warrant for violating a canon of terminology by using the word for this group, in spite of its very different application in taxonomy. It has already been shown that aggregation further produces a grótiping of families, which may properly be called a com-

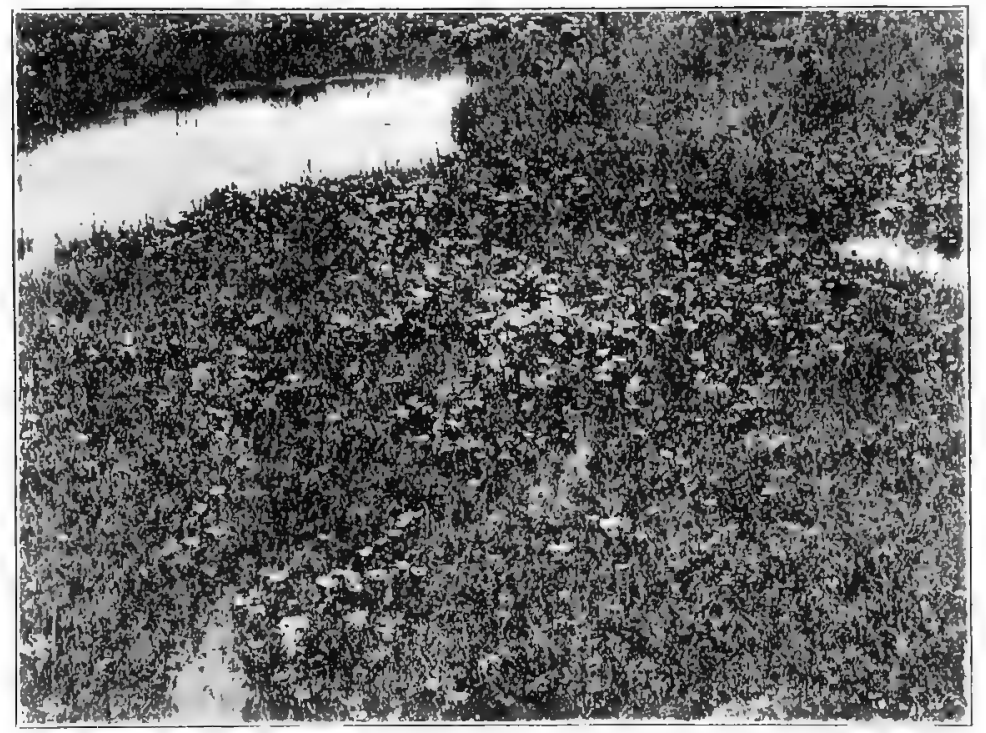

Fig. 78. Calthetum (Caltha leptosepala), a consocies of the alpine bog formation.

munity. As they are used here, family and community become equally applicable to the association of plants, animals, or man. Both families and communities occur regularly in each society of the formation, and they represent its two structures. In some cases, all the families are grouped in communities, two or more of which then form the society. Very frequently, however, families occur singly, without reference to a community, and the two then constitute independent parts of the same area. This is typically the case wherever gregarious species are present, since these are merely family groups produced by aggregation. 
Objection may be made that this analysis of formational structure has been carried too far, and that some of the structures recognized are mere interpretations, and not actual facts. Such a criticism will not come from one who has got beyond the superficial study of formations, for he will at once recognize that certain probable features of structure have not been considered. On the other hand, the ecologist or the botanist who has not made a careful investigation from the standpoints of development and structure will naturally refrain from expressing an opinion, until he has obtained an acquaintance at first hand with the facts. Over-refinement is the usual pen-

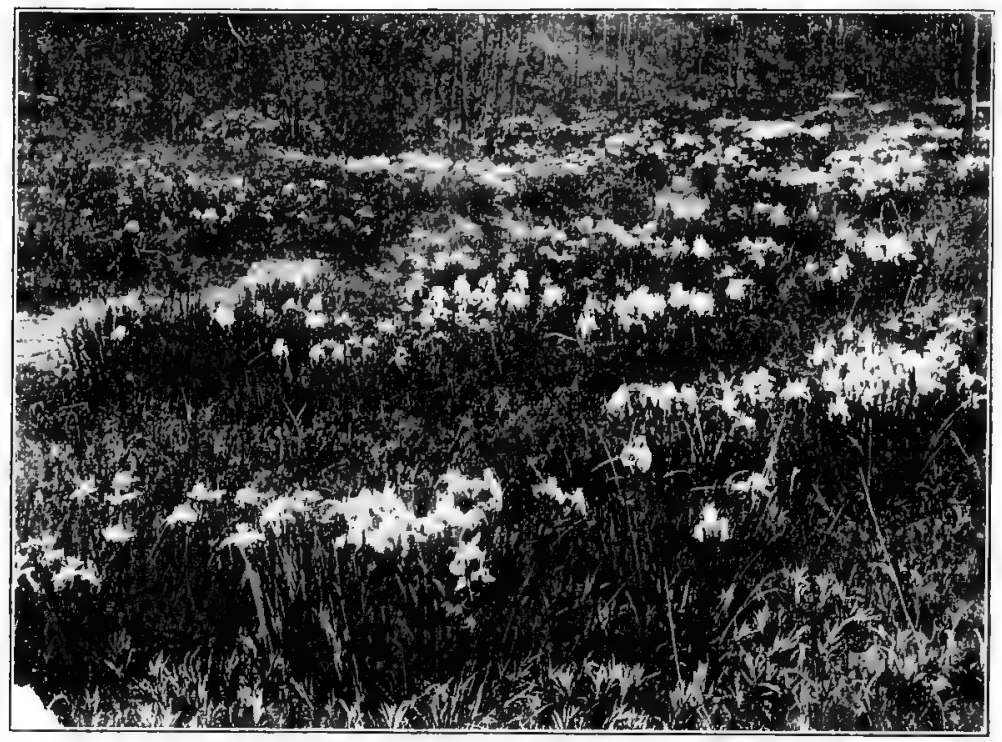

Fig. 79. Iridile (Iris missonriensis), a society of the aspen formation.

alty of intensive work. The unbiased investigator, however, will not be misled by the suddenness with which new concepts appear. It seems plausible that the structure of a formation, if not as definite, is at least nearly as complex as that of an individual plant. Few botanists will insist that the refinement of tissues and tissue systems has been carried further than the differentiation of the plant warrants. Yet, if these had been defined within a period of a few years rather than slowly recognized during more than a century, they would have been called serioutsly in question. As a matter of fact, the consocies, under the term association, and the society, under various names, have been recognized by ecologists for several years. They are defi- 
nite phenomena of alternation which can be found anywhere. The family and the community, though the latter is less distinct in outline, are equally valid structures, the proof of which anyone can obtain by thorough methods of study.

348. Nomenclature of the divisions. The suffix -etum is used to designate a consocies of a formation, e. g., Picetum, Caricetum, etc. When two or more species characterize the area, the most important, or more rarely, the two are used. The termination used to designate a society is -ile, as Asterile, Sedile, Rosile. The suffix which denotes the community is -are, and for the family, it is -on, viz., Giliare, Bromare, Bidenton, Helianthon, etc. Layers are indicated by the affix -anum, as Opulasteranum, Verbesina-Rudbeckianum, etc. It is evident that these suffixes, like the terms to which they refer, must be used always for the proper divisions if they are to have any value at all. There has been a marked tendency, for example, to use -etum in connection with the names of groups of very different rank. It is hardly necessary to point out that such a practice does not promote clearness. The following tabular statement will illustrate the application of both terms and suffixes:

Picea-Psendotsuga-hylinm formation(-ium) Paronychia-Silene-chalicium

Picetum consocies (-etum)

Opulaster-Ribesanum

Opulasterile

Thalictrare

Pirolon

layer(-anum)
society (-ile)
community (-are)
family(-on)

Paronychietum .

Androsacile

Festucare

Arenarion

349. The investigation of a particular formation. A comprehensive and thorough study of a formation should be based upon as many examples of it as are accessible. The example which is at once the most typical and the most accessible is made the base area. This plan saves time and energy, reduces the number of instruments that are absolutely necessary, and establishes a common basis for comparison. The inquiry should be made along four lines, all fundamental to a proper knowledge of the formation. These lines are: (I) the determination of the factors of the habitat, (2) a quadrat and a transect study of the structure of the formation, (3) a similar investigation of development, (4) a floristic study of the contiguous formation, with special reference to migration. The sequence indicated has proven to be the most satisfactory, and is to be regarded as all but absolutely essential. Naturally, this applies only to the order in which the various lines are to be taken up, as they are carried on together when the work is fully under way. Since instrument and quadrat methods have already been given in detail, it 
is unnecessary that they be repeated. Similarly, the questions which pertain to structure and development and to the surrounding vegetation are considered in detail in the pages which precede.

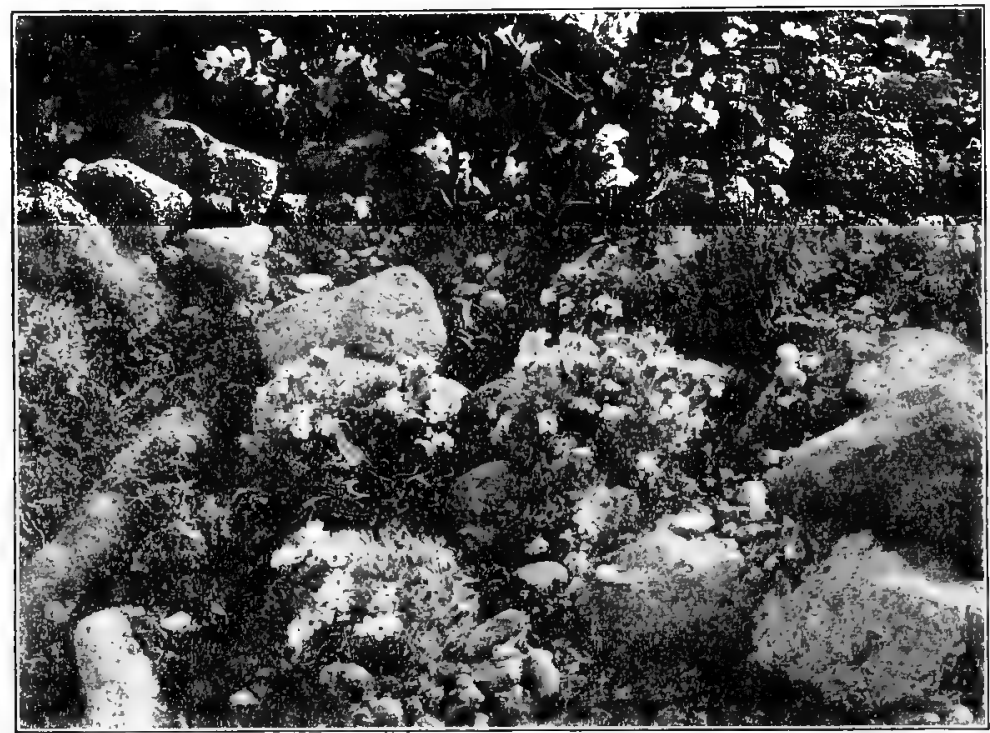

Fig. 80. Eritrichiare (Eritrichinm aretioides), a community of the alpine meadow formation.

350. Bases. Formations may be grouped with reference to habitat or kind, development or position. Classification upon the basis of habitat places together formations which are sinilar in physiognomy and structure. Developmental classification is based upon the fact that the stages of a particular succession are organically connected or related, though they are normally different in both physiognomy and structure. Grouping with respect to position is made solely upon occurrence in the same division of vegetation. The formations thus brought together usually possess neither similarity of kind or structure, nor do they have any necessary developmental connection. Habitat and developmental classification are of fundamental value; regional arrangement is more superficial in character. All serve, however, to emphasize different relations, and, while the developmental system expresses the most, they should all be used to exhibit the vegetation of a region, province, or zone. 
351. Habitat classification. In arranging formations with reference to habitats, the direct factors, water and light, can alone be used to advantage. Such a system is fundamental, because it is founded upon similarity of habitat and of structure. Proposed groupings based upon nutrition-content, or upon the division of factors into climatic and edaphic, have elsewhere ${ }^{1}$ been shown to be altogether of secondary importance, if not actually erroneous. The basis of the habitat grouping is water-content. which is supplemented by light whenever the factor is decisive. The primary divisions thus obtained are water, forest, grassland, and desert, which are characterized re-

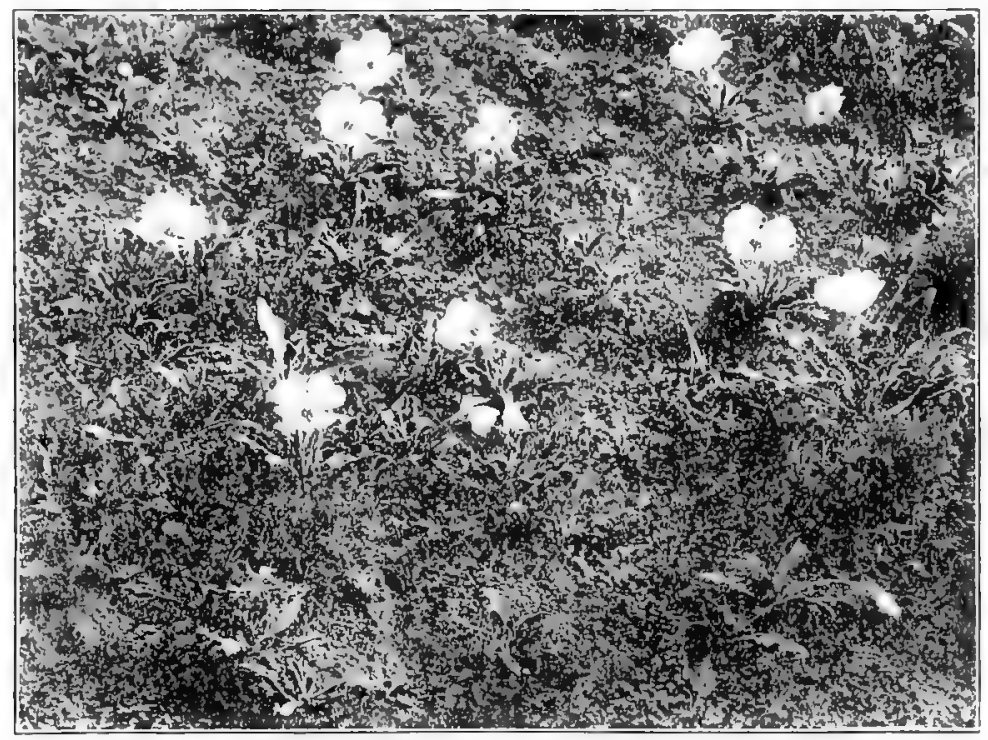

Fig. 81. Pachylophon (Pachylophus caespitosus), a family of the gravel slide formation.

spectively by associations of hydrophytes, mesophytes, hylophytes, poophytes, and xerophytes respectively. Within these, formations are arranged according to the type of habitat, i. e., pond, meadow, forest, dune, etc. These divisions comprise all fornations which belong to the type by virtue of their physiognomy and structure. Such formations differ from each other very considerably or completely in the matter of floristic, i. e., component species, but they still belong to the same type. A dune formation in the interior and one on the coast may not have a single species in common, and yet they are essentially alike in habitat, development, and structure.

${ }^{2}$ Clements, F. E. The Development and Structure of Vegetation, 24, 27. 1904. 
352. Nomenclature. The names of formations are taken from the habitats which they occupy. Each formation should have a vernacular and a scientific name. The latter is especially important since it ensures brevity and uniformity, and obviates the obscurity and confusion that arise from vernacular terms in many tongues. Scientific names have been made uniformly from Greek words of proper meaning by the addition of the suffix -ium ( $\varepsilon \hat{\varepsilon} o v$ ), which denotes place. ${ }^{1}$ The following list gives the English and the scientific name of the various habitats, and their corresponding formations, and indicates the primary divisions into which these fall.

I. Hydrophytia : water plant formations

I. ocean : oceanium: oceanad, $^{2}$ oceanophilous, etc.

2. sea: thalassium surface of the sea: pelagium deep sea: pontium

3. lake: limnium, limnad

4. pond, pool, tiphium, tiphad

5. stagnant water: stasium: stasad

6. salt marsh: limnodium, limnodad

7. fresh marsh: helium

8. wet meadow : telmatium

9. river: potamium

Io. creek, rhoium

II. brook: namatium

I2. torrent: rhyacium

I3. spring: crenium

I4. warm spring: thermium

I5. ditch: taphrium

I6. sewer: laurium
I7. swamp forest: helohylium

I8. swamp open woodland: helodium

I9. meadow thicket: helodrium

20. bank: ochthium rock bank: petrochthium

sand bank: ammochthium

mud bank: pelochthium

2I. rocky seashore: actium

22. sandy seashore: agium

23. sandbar: cheradium

24. tank: phretium

II. Mesophytia: middle plant formations

a. Sciophytia: shade plant formations

26. forest: hylium

27. grove: alsium

28. orchard: dendrium

29. canyon: ancium

3o. open woodland: orgadium

${ }^{1}$ Clements, F. E. A System of Nomenclature for Phytogeography. Engler Jahrb., 31 :b70:1. 1902.

"The terms, oceanad, hylad, poad, eremad, etc., are proposed in place of oceanophyte, hylophyte, etc. They are much shorter and make consistent groups under the general term, ecad., i. e., habitat form. 
3I. thicket: lochmium

b. Heliophytia: sun plant formations

32. meadow: poium

33. pasture: nomium

34. culture land: agrium

35. waste place: chledium

III. Xerophytia: dry plant formations

36. desert: eremium

37. sand-hills, sandy plain: amathium

38. prairie, plains : psilium

39. dry, open woodland: hylodium

40. dry thicket: driodium

4I. dry forest: xerohylium

42. gravel slide: chalicium

43. sandbar: syrtidium

44. sand draw: enaulium
45. blowout: anemium

46. strand: psamathium

47. dune: thinium

48. badlands: tirium

49. hill, ridge: lophium

50. cliff : cremnium

5I. rock field: phellium

52. boulder field: petrodium

53. rock, stone: petrium

54. humus marsh: oxodium

55. alkali area: drimium

56. heath, dry meadow: xeropoium

57. moor: sterrhium

58. alpine meadow: coryphium

59. polar barrens : crymium

6o. snow : chionium

6I. wastes: chersium

Particular formations are indicated by means of floristic distinctions. Thus, Populus-hylium is the aspen forest as distinguished from the PiceaPsendotsuga-hylium, or the balsam-spruce forest; and the Bulbilis-psilium, or buffalo-grass prairie, from the Bontelona-Andropogon-psilium, or gramabluestem prairie. Similarly, the aspen formation of the Old World and of the New may be distinguished as Populus-tremula-hylium and Populustrenuloides-hylinut, respectively. In all formational names, the facies alone should be used. Frequently, a single facies will suffice for clearness. As a rule, however, the two most important facies should be employed; in rare cases only is it necessary to use the names of three. When it is desirable to refer to two or more examples of the same formation, a geographical term is added, e, g., (I) Populus-hylium (Crystal Pàrk), (2) Populus-hylium (Cabin Canyou).

353. Developmental classification. This is based upon succession as the record of development. Upon the basis of development, all the formations which belong to the same succession are classed together. They are arranged within each group in the sequence found in the particular succession. From its nature, developmental classification is of primary importance in exhibiting the history of vegetational changes. It has less value than the 
habitat system for stmmarizing the essential structure of a vegetation, inasmuch as it places the emphasis upon historical rather than structural features. It is evident that both deal with the same formations, and that the difference is merely one of viewpoint. The habitat classification is simpler in that it considers only those formations actually on the ground, while development has regularly to take into account stages which have clisappeared. The groups of the developmental system, and the arrangement of formations within them have already been indicated under the nomenclature of succession (sections 326 ani 327 ).

354. Regional classification. The grouping of formations with respect to the divisions of vegetations is chiefly of geographical value. It indicates a certain general relationship, but its principal use is to summarize the structure of the vegetative covering of a region. The arrangement of formations in the various divisions is made with reference to the outline of North American vegetation (section 34I). This is naturally based upon the identity of altitude and latitude zones. In the study of mountain countries, it is often desirable to group formations with reference to altitude alone. In this case, the grouping is based upon the following divisions: (I) bathyphytia, lowland plant formations: (2) mesiophytia, midland formations; (3) pediophytia, upland formations; (4) pagophytia, foothill formations; (5) orophytic, subalpine formations; (6) acrophytia, alpine formations; (7) chionophytia, niveal formations.

355. Mixed formations. These are mixtures of two, rarely more, adjacent formations, or of two consecutive stages of the same sttccession. Mixed formations are really transitions in space or in time between two distinct formations. Theoretically, they are to be referred to one or the other, according to the preponderance of species. Actually, however, they often persist in an intermediate condition for many years, and it becomes necessary to devote considerable attention to them: In some cases, there is good reason to think that the species of two contiguous formations have become per-. manently associated, and thus constitute a new formation. This is often apparently true in succession, when the change from one stage to the next requires a long term of years, but it is really true only of the very rare cases in which a succession becomes stabilized in a transition stage. When the mixture is due to development, the formations concerned are often quite dissimilar, e. g., grassland and thicket, thicket and forest. If it is the result of position, the formations are usually similar, i. e., both are grassland, thicket, or forest, since the plants of the lower level are regularly assimilated or destroyed, when invasion occurs at two levels. The term mictium ( $\mu$ iкrív, 
mixture) is here proposed for the designation of all mixed formations, whether they arise from succession or from juxtaposition. Thus, the Mentzeliu-Elymus-mictium is the transition between the Mcntzclia-Psendocymopterus-chalicium and the Elymus-Muhlenbersia-chalicium. Similarly, the Populus-Picea-mictium and the Pinus-Psendotsuga-mictium are transition stages in the development of the Picea-hylinm. On the other hand, the Andropogon-Bulbilis-mictinm is a mixture produced by the mingling of two contiguous prairie formations. In the future development of this subject, it will probably become desirable to name mixed formations on the basis of

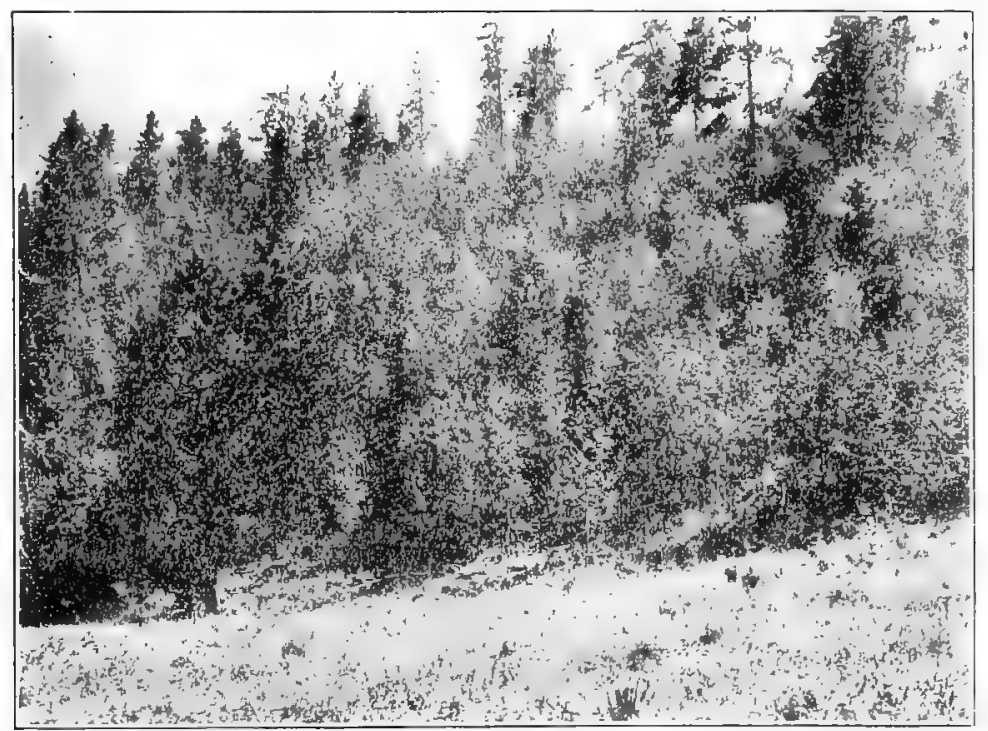

Fig. 82. A mixed formation of aspens and spruces (Populus-Picedmictium), preceding the final spruce forest of a burn succession.

origin, but at present this is unnecessary. Both in classification and in description they should be considered between the formations which give rise to them, and this will at once indicate their origin.

Puzzling cases of mixture resulting from position occur toward the limits of facies which occupy extensive areas. Boutelona oligostachy'a, and Andropogon scoparins extend from the prairies through the sand-hills and plains, and into the foot-hills of the Rocky mountains. Their abundance at once raises a question as to the validity of the prairie, sand-hill, plain, and foothill formations. If these two grasses were controlling, and equally characteristic throughout, then the entire stretch would have to be regarded as a 
single formation. Since they are often absent, or mixed with other facies of greater importance, they can not be considered the sole tests of the formation. This view is reinforced by the fact that prairie, sand-hill, plains, and foot-hill all have their characteristic principal and secondary species, in addition to facies that are more or less typical. In certain formations, doubtless, Bouteloua and Andropogon are relicts, in others invaders, while in the formations actually constituted by them they are dominant. The final solution of such problens is quite impossible, however, until the comparative study of large areas can be based upon the accurate detailed investigation of the component formations.

\section{EXPERIMENTAL VEGETATION}

356. Scope and methods. The experimental study of the formation as a complex organism rests upon methods essentially similar to those discussed under experimental evolution. The scope of the two fields is practically the same, moreover, in that both deal with the experimental development of an organism and the structures that result. The actual problems are naturally very different, since the formation is a complex of individual plants, but the fupdamental bis of habitat, function, and structure is common to both. However, the functions now to be considered are aggregation, invasion, competition, etc., and the structures, zones, consocies, societies, communities, and families. The latter may properly be regarded as adaptations called forth by the adjustment, i. e., aggregation, migration, ecesis, etc., of the formation to the physical factors of the habitat. As consequences of measured factors, formational adjustment and adaptation must themselves be carefully measured and recorded. For these purposes, the methods of quadrat and transect, of chart, photograph, and formation herbarium are used. Invaluable as they are for any scientific inquiry into vegetation, such methods form the very foundation of experimental study in which accuracy is the first desideratum.

It has already been shown that nature's own experiments in the production of new forms furnish the best material for experimental evolution. This statement is equally true of experimental vegetation. The formation of new habitats by weathering and transport, and the denuding of old ones, yield experimental plats of the greatest value. This is likewise the case in the great majority of formations, where invasion or competition is active. These are the phenomena that must be considered in any careful study of vegetation, but in taking them up from the experimental standpoint, greater attention must be paid to detail, and the changes must be followed closely for a longer time. The method that makes use of existing changes in vegetation 
is designated the method of nalural habitats. In contrast with this is the method of artificial habitats, in which the habitat itself is definitely modified, or a group of species actually transferred to a different habitat. Many problems of vegetation can be attacked with greater success under control than in the field. "This is particularly true of competition, in which results can be obtained most readily by means of the method of control habitats, as carried on in the plant house.

\section{METHOD OF NATURAL HABITATS}

357. Natural experiments. Every family as well as every community constitutes an experiment in competition; the same statement necessarily holds for the larger groups, society, consocies, and formation, which are composed of families and communities. The last also make it possible to study competition in two typical instances, viz., in the family, where the individuals are of one kind, and in the community, where they belong to two or more different species. The community, moreover, is a product of invasion, and it furnishes material for the study of this function, as well as for that of aggregation and competition. Practically every formation shows some invasion, but as a rule stable formations contain so few invaders that they are relatively unimportant in this connection. Invasion is most active in transition areas and in mixed formations, whether produced by juxtaposition or by succession, and its study in these places yields by far the largest number of valuable results.

As typical complete invasion, a succession is the best of all natural experiments in aggregation, migration, ecesis, and competition. This is especially true of the initial stages in which changes in the number and position are most readily followed. The methods used in studying successions have been given elsewhere. In addition, it should be pointed out that one of the first tasks in taking up the ecological investigation of a region is to make a careful search for all new and denuded areas, as well as for those in which succession is taking place. The phenomena in these areas can not be explained until the habitats and formations have been worked over critically, hut the facts must be collected at the earliest possible moment, since the stages of the succession are constantly changing, while the stable formations are not.

\section{METHOD OF ARTIFICIAL HABITATS}

358. Modification of habitat. As the final factors in ecesis and competition, water, light, and temperature control the grouping of plants into vegetation. An efficient change in one of these, or in all of them, brings about a visible adjustment in the structure of the plant group concerned. Modifica- 
tions of water-content and light are readily produced in the field by drainage, irrigation, shading, clearing, etc. In fact, all the changes of habitat indicated under experimental evolution serve equally well to initiate experiments in experimental vegetation; indeed, the same experiment covers both fields. It is impracticable, however, to modify the temperature of a habitat without changing its water-content or light, and consequently the influence of temperature can not be determined through experiment by modification. The extent of the area modified should be as large as convenience will permit, in order that the number of individuals may be large enough to indicate clearly the resulting adjustment in position and arrangement. The best results can be obtained where a small separate area of a formation can be modified, e. g., where a small swamp can be drained, or a depression flooded. In the case of light, however, it is ustually impossible to clear or to shade a large area, and the study must be restricted to a relatively small group of plants. In regions where lumbering is actively carried on, the consequent clearing initiates invaluable experiments over large areas, and this is likewise true of forest plantations. M.odification of a large area has decided advantages in bringing out the changes in the more prominent structural features, - but the causes and the details of the adjustment can be worked out much more satisfactorily in a small area.

359. Denuding. The modification of the habitat by denuding is the sole method of initiating succession by experiment. It is consequently of the most fundamental importance in investigating aggregation, ecesis, and competition, as well as the reactions exerted by the invaders of the different stages. The possibilities of denuding an entire habitat or an extensive area are not great, and the investigator must content himself with denuded quadrats, transects, and migration circles, which are small enough to permit a critical study of all the factors in succession. It is of course unnecessary that the denuding be done by the ecologist himself, provided he is able to follow the succession from the very beginning. Accordingly, it becomes possible for him to make the very best use of all those changes wrought by man in which the vegetation is destroyed over considerable areas. These are essentially natural experiments, and at this point the methods of natural. and artificial habitats merge.

The manner of denuding depends in a degree upon the nature of vegetation, but, when time, convenience, and safety are all taken into account, the actual removal of the vegetation as indicated under the denuded quadrat is by far the most satisfactory. Under certain conditions, flooding or burning can be used to advantage, but cases of this kind are infrequent. The purpose of the experiment determines the kind of area to be denuded. 
Quadrat, transact, and migration circle are equally valuable for ecesis and competition. The quadrat is best adapted to work in a homogeneous area, while the transect is suited to a heterogeneous one characterized by zones, societies, or communities. It is an advantage to replace the denuded transect by a series of denuded quadrats, one for each zone or society, when the transect would be too long for convenience. The denuded migration circle is invaluable for aggregation and ecesis, since it makes possible the study of migration as a distinct function. A series of denuded quadrats, consisting of one or more in the different stages of a succession, furnishes important evidence concerning the development of each stage. By far the best method, however, for making a comparative study of the stages of a succession is the quadrat sequence. A quadrat is denuded each year, thus yielding a complete sequence of miniature stages through the whole course of succession. This method is especially valuable when a succession is represented by a single example, and there is no opportunity of reconstructing it by the comparison of various stages. 'A quadrat sequence is naturally of the greatest value if begun at the time when the first invaders appear.

360. Modification of the formation by transfer. The study of partial and intermittent invasion into an established vegetation is made through the transfer of a species or group of species by means of seeding or planting. The process differs in no way from that described for experimental evolution, except in so far that an endeavor is made to establish a family or a community, and not merely a few individuals. Transfer makes possiple the critical investigation of ecesis under conditions of intense competition, as well as the study of aggregation and the origin of plant groups under these conditions. Perhaps its greatest value is in the experimental study of alternation and zonation, especially the former. It is practically impossible to determine whether alternation, especially when corresponsive, is due to plysical or historical causes, i. e., migration and competition, except by means of the reciprocal transfer of the species concerned.

Field cultures for the careful study of ecesis and competition are made by transferring seeds or plants to new or denuded soils. This is practically a combination of the methods of modification and transfer. It has a unique value in making it possible to initiate artificial successions of almost any character that is desired, and to carry them out with the reactions more or less under control. This opens up an extremely important field of experimental inquiry, which promises to put the study of succession upon a much more exact basis. Competition cultures in the field are not essentially different from those under control, and they will be considered under the next method. 
361. Competition cultures. Although it is quite possible to carry on experiments in invasion and succession in the planthouse, the limited space usually available makes this undesirable, except in a few problems where control is necessary. Competition cultures, on the other hand, yield better results in the planthouse than in the field, since the physical factors and the appearance of unwelcome migrants are much more easily controlled. The possibilities of the culture method in the study of competition seem inexhaustible, and the author has found it necessary to confine his own investi-

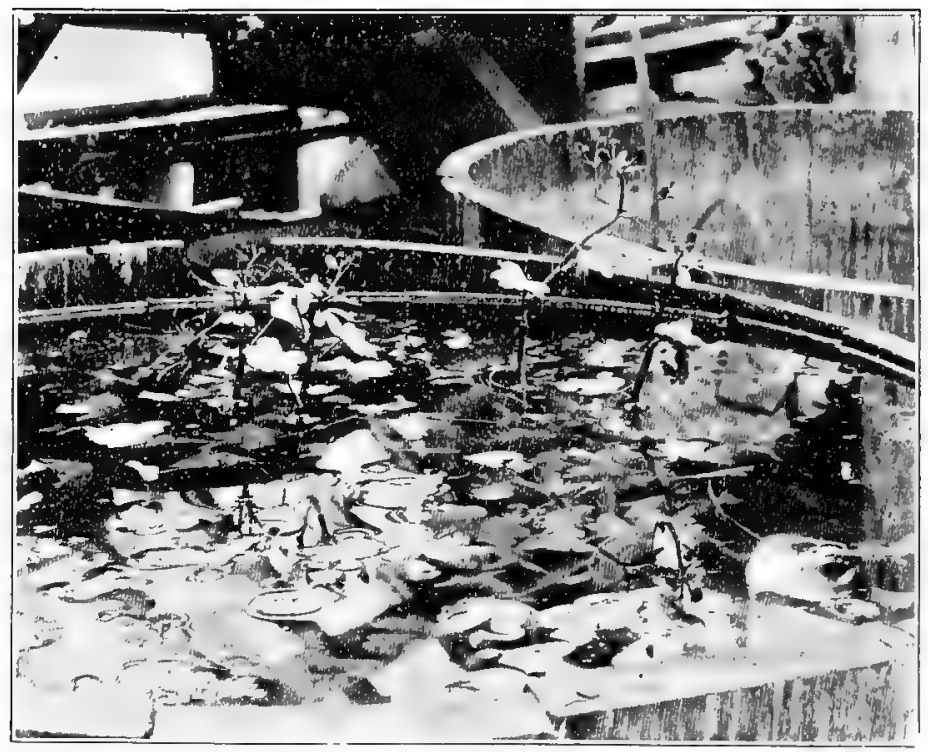

Fig. 83. Simple culture of floating ecads of Ranunculus sceleratus.

gations to a few of the fundamental problems. In this work, he has distinguished several kinds of cultures, based chiefly upon the species concerned and the arrangement of the individuals. Simple cultures are those in which a single species is used. The restlting group is a family, and the competition is between like individuals. In such cultures, the problem of the factors in competition is reduced to its simplest terms. Mixed cultures are based upon two or more species, and the problem is correspondingly complicated. As a rule, all the seeds have been sown at the same time in both simple and mixed cultures, but it has been found desirable to make some heterochronous cultures, in which seeds are also sown after the plants have appeared. Mixed cultures are distinguished as layered cultures, when 
the species are of very different height. Thus, rosettes have been grown with stemmed plants, tall slender forms with low branching ones, erect plants with twining and climbing plants, etc. Further evidence as to the nature of competition has been sought by means of ecad cultures, and factor cultures. In the former, plants of different response to water and light are grown together under the same conditions, in order to evaluate the part played by the nature of the piant. In a factor culture, the area is divided into two or more parts which are given different amounts of water or of light, in order to determine the influence of slight variations upon the same competitors. In somewhat similar fashion, an attempt has been made to

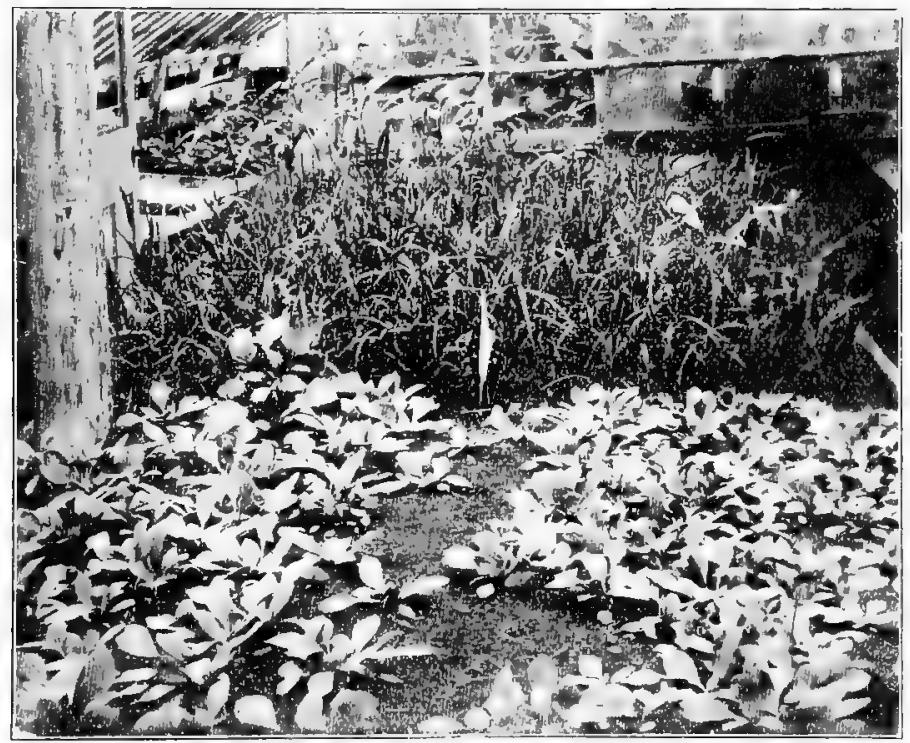

Fig. 84. Mixed culture of Solidago rigida and Onagra biennis.

ascertain the bearing of biotic factors upon competition. Cultures are easily made in which Cuscuta or parasitic fungi are used to place certain species at a disadvantage. Permenent cultures are obtained by allowing the plants to ripen and drop their seeds for several generations, just as in nature. 'They are indispensable for determining the final outcome of the competition between different species.

362. Details of culture methods. All competition cultures have been made I meter square. In other words, they are quadrats, and they are treated exactly as denuded quadrats in the field with respect to factor readings, charts, and photographs. In the writer's studies, germination 
lests were made of a large number of species, and those selected which showed a high per cent of germinability. Since this was the first experimental study of competition, this test was deemed necessary, but it is quite evident that no such selection is made in nature. Consequently, when the seeds used are known to be fresh, a germination test is usually superfluous. Considerable care was taken also to select species known to be vigorous growers, with the result that practically all the species used for experiment

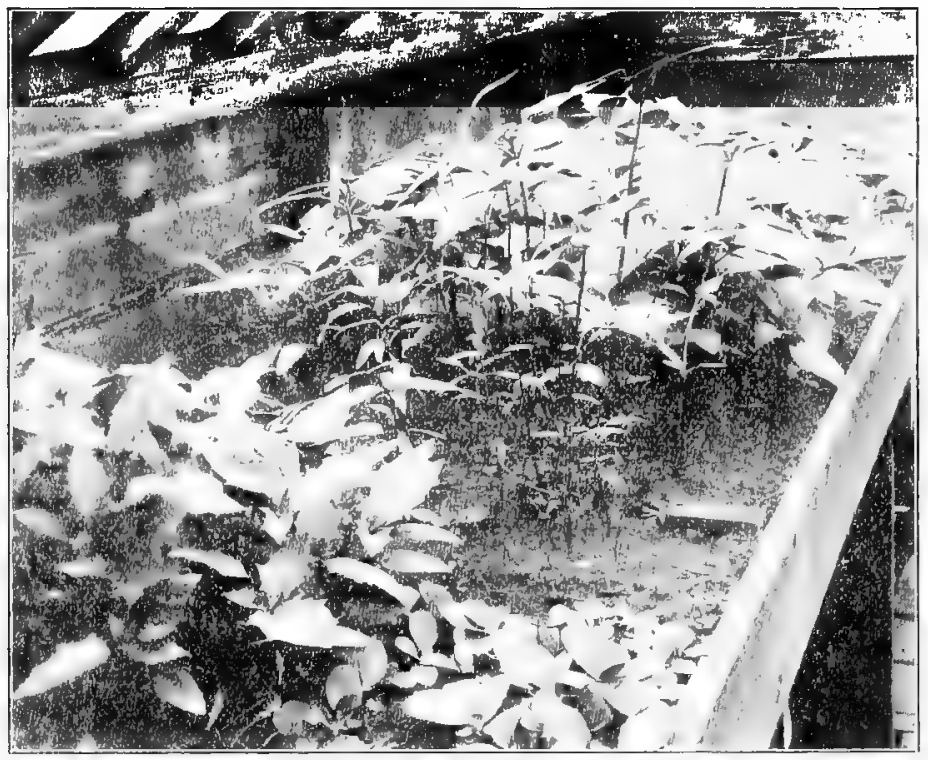

Fig. 85. Heterochronous culture of Helianthus annums and Datura stramonium. Family culture of Datura, Verbascum, etc., in the foreground.

were ruderal or subruderal. The species employed, and the kinds of cultures in which they were grouped were as follows:

I. Simple culture of Hclianthus annuus. The culture plot was divided into four equal parts; I2 seeds were planted in one, 25 in another, 50 in the third, and 100 in the fourth.

2. Mixed culture of Helianthus annuus, Panicum virgatum, and Elymus canadensis. Twenty-five seeds each of Helianthus and Panicum were planted alternately at equal distances in one-half of the plot, while the other half was planted similarly with Helianthus and Elymus.

3. Mixed culture of Solidago rigida and Onagra biennis. Over one-half of the plot were scattered 50 seeds of Solidago and I00 of Onagra; over the other, IOO and 200 seeds respectively. 
4. Layered culture of Laciniaria punctata, Bidens frondosa, Salvia pitcheri, Cassia chamaecrista and Kuhnia glutinosa. Fifty seeds of each species were scattered more or less uniformly over the entire plot.

5. Layered culture of Silphium laciniatum, Datura stramonium and Lactuca ludoriciana. Fifty seeds of Datura and Lactuca, and 25 of Silphium were sown uniformly in one-half of the plot. In the other half, 25 holes were made at equal intervals, and one seed of each of the three planted in each hole.

6. Ecad culture of Oenothera rhombipetala (xerophytic), Verbascum thapsus (mesophytic), and Penthorum sedoides (hydrophytic). One hundred seeds of Oenothera and 200 each of $l^{\prime}$ erbascum and Penthorum were scattered over the plot.

7. Heterochronous culture of Helianthus annuns and Datura stramonium. One hundred seeds of Helianthus were scattered over one half, and the same number of Datura seeds over the other half of the plot. In both, also, 50 seeds were sown in one 4 -inch circle, and 25 seeds in a second circle at some distance. A month later, Ioo seeds of Helianthus were sown in the Datura plot, and vice versa.

8. Family' culture of Helianthus, Kuhnia, Panicum, Bidens, Onagra, Datura, Penthorum, Solidago and Verbascum. The plot was divided into 9 squares and in each were sown 50 seeds of one of these plants.

9. Community culture. The sowing was made exactly as for the family culture, except that 20 seeds of each plant were used. In the middle of each square, 5 seeds of a different species were planted. For the Helianthus, Kuhnia, and Panicum groups, Onagra was used; for Bidens, Onagra, and Datura, Helianthus was used, and for Penthorum, Solidago, and Verbascum, Panicum.

At the time the cultures were started, check plants were sown in pots. The most vigorous seedlings were transplanted singly to large pots, and grown under conditions of water, light, and soil as similar as possible to those of the competition plots. Photographs of check plants and plots were made at the proper intervals, and the plots were charted in quadrats to show the course of competition. The factors which control competition were sought in a critical study of water-content and light values, which is still in process. This work has gone far enough to indicate the correctness of the view $^{1}$ that competition is purely physical in character. It has, moreover, been demonstrated that "room" in competition is merely a loose expression for the relation between the number of individuals in a given space, and the amount of water, light, and temperature available in the same space.

${ }^{1}$ Clements, F. E. The Development and Structure of Vegetation, 166. 1904, 


\section{GLOSSARY}

Note: Last terms frequent in compounds are found in their proper place alphabetically. The accent is indicated only in those words accented on the penult; all others are accented on the antepenult, or recessively.

abundance, the total number of individuals in an area.

acospore ( $\alpha \kappa \eta^{\prime}$, point), a plant with awned disseminules.

acrophyti'um (ắkpov, peak), an alpine plant formation.

acti'um (ג $\alpha \tau \dot{\eta}$, rocky coast), a rocky seashore formation; actad, plant of a rocky seashore.

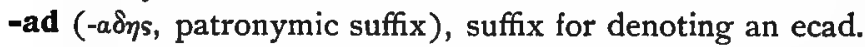

adaptable, able to originate ecads; adaptation, the structural response to stimuli.

adjustment, the functional response to stimuli.

adventicious (adventicius, foreign), invading from distant formations.

adventive (adventivus, accidental), established temporarily.

aggregation, the coming together of plants into groups.

agi'um ( $\boldsymbol{\alpha} \gamma \dot{\eta}$, beach), a beach formation; agad, a beach plant.

agri'um (å $\gamma$ oós, field), a culture formation; agrad, a cultivated plant.

aiphyti'um (åki, permanent), an ultimate formation.

alsi'um (å $\lambda \sigma o s$, grove), a grove formation; alsad, a grove plant.

alternation, the heterogeneous arrangement of plant groups and formations universally present in vegetation.

amathi'um (ä $\mu a \theta 0 s$, sand of the plain), a sandhill or sandplain formation; amathad, a sandhill plant.

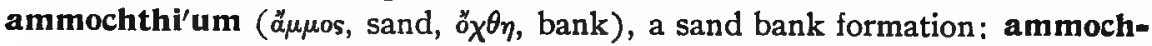
thad, a sand bank plant.

anci'um ( $\ddot{\alpha} \gamma \kappa o s$, mountain glen), a canyon formation; ancad, a canyon plant.

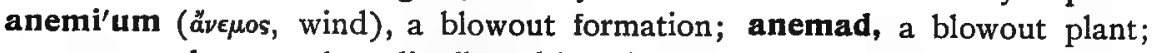
anemochore, a plant distributed by wind.

-anum (locative suffix), a suffix denoting a layer.

apostrophe ( $\dot{a} \pi \delta^{\prime}$, away from, $\sigma \tau \rho \circ \phi \eta^{\prime}$, a turning), the arrangement of the row of chloroplasts parallel to the rays of light.

apparent noon, the time when the sun crosses the meridian, i.e., sun noon as distinguished from noon, standard time.

-ard (äpdov, water of the land), combining term for water-content; ardium, a succession due to irrigation.

ardesiacus, slate colored. 
-are (locative suffix), suffix denoting a community.

aspect (aspectus, appearance), the seasonal impress of a formation, e.g., the spring aspect.

association, the arrangement of individuals in vegetation.

atmometer (ả $\tau \mu o ́ s$, vapor), an instrument for measuring evaporation.

atropurpureus, dark purple.

atrovirens, dark green.

autochore (aviós, self), motile plants, or those with motile spores; autochthonous ( $\chi \theta \dot{\theta} v$, ground), native.

avellaneus, drab.

barrier, a physical or biological obstacle to migration or ecesis.

bathyphyti'um ( $\beta a \theta$ ís, low), a lowland plant formation.

blastochore ( $\beta \lambda \alpha \dot{\sigma} \sigma \eta$, growth), a plant distributed by offshoots.

-bole ( $\beta$ o $\eta^{\prime}$, a throw), combining term for propulsion; bolochore, a plant distributed by propulsion.

broti'um (ßporós, mortal), a succession caused by man; brotochore, a plant distributed by man.

caeruleus, pale blue.

caesius, eye-blue.

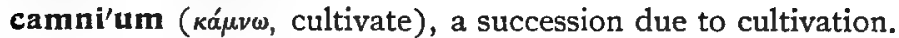

carphospore ( $\alpha \dot{\rho} \rho \phi o s$, scale), a plant with disseminules possessing a scaly or chaffy pappus.

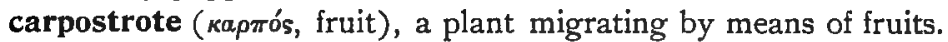

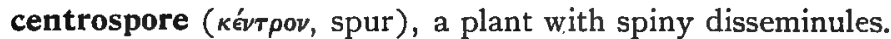

chalici'um ( $\chi^{\prime} \lambda \iota \xi$, gravel), a gravel slide formation; chalicad, a gravel slide plant.

cheradi'um ( $\chi$ ćpa $\delta$ os, a sandbar), a wet sandbar formation cheradad, a wet sandbar plant.

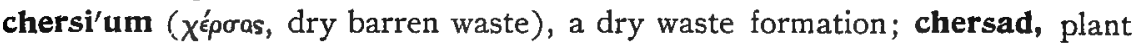
of a dry waste.

chioni'um ( $\chi \chi^{\iota} v^{\prime}$, óvos, snow), a snow formation; chionad, a snow plant; chionophyti'um, a niveal plant formation.

chledi'um ( $\chi \lambda \hat{\eta} \delta$ os, rubbish), a ruderal formation; chledad, a ruderal plant.

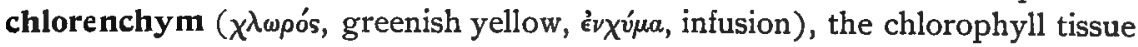
of the leaf.

-chore ( $\chi \omega \rho \epsilon^{\omega} \omega$, to spread abroad), combining term to denote agent of migration. chresard ( $\chi p \hat{\eta} \sigma \iota s$, use), the available water of the soil, the physiological watercontent.

clitochore ( $\kappa$ íitos, slope), a plant distributed by gravity. 
clysi'um ( $\kappa \lambda \dot{v} \sigma \iota s$, a flooding), a succession in a flooded soil.

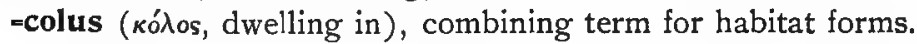
community, a mixture of the individuals of two or more species, a group of families.

comospore ( $\kappa^{\prime} \mu \eta$, hair) a plant with hairy or silky disseminules.

competition, the relation between plants occupying the same area, and depend. ent upon the same supply of physical factors.

consocies, that subdivision of a formation controlled by a facies.

copious, used of species in which the individuals are arranged closely but uniformly.

coryphi'um (кopvф $\eta$, peak), an alpine meadow formation; coryphad, an alpine meadow plant.

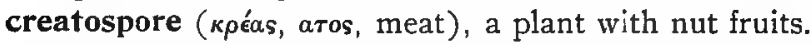

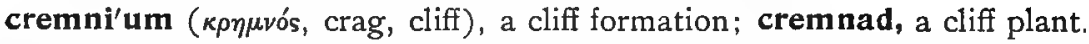

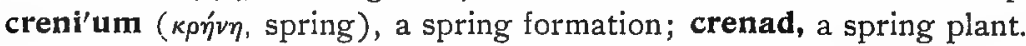

crymi'um (крvнós, frost), a polar barren formation; crymad, a polar plant; crymophytic, pertaining to polar plants.

crystallochore ( $\kappa \rho v_{\sigma \tau} \tau_{\lambda \lambda \lambda o_{5}}$, ice), a plant distributed by glaciers.

cyaneus, azure.

cyriodoche ( $\kappa$ v́pos, regular), a normal succession.

dendri'um ( $\delta$ évopa, fruit trees), an orchard formation; dendrad, an orchard plant.

derived, coming from other formations or regions, not native.

diphotic ( $\delta t-$ two), the two surfaces unequally lighted; diphotophyll, a leaf dif-

ferentiated into palisade and sponge tissues owing to unequal illumination.

diplophyll ( $\delta$ เл $\lambda$ ós, two-fold), an isophotic leaf with water-storage cells in the middle.

disseminule (semen, seed), a seed fruit modified for migration.

dissophyte ( $\delta \sigma^{\prime} \sigma o$ s, double), a plant with xerophytic leaves and stems, and mesophytic roots.

=doche ( $\delta o \chi \eta^{\prime}$, succession), succession.

drimi'um ( $\delta \rho \mu v^{\prime}$, biting, pungent), an alkaline habitat, and the corresponding formation; drimad, a plant of such a formation.

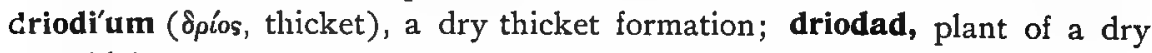
thicket.

dysgeogenous ( $\delta v s^{-}$, bad, $\gamma \hat{\eta}$, soil), weathering with difficulty to form soil.

ecad (oíkos, home), a habitat form due to origin by adaptation; ece'sis (oîñ act of coming to be at home), the germination and establishment of invaders; ecograph, an instrument for measuring a physical factor of a habitat; ecotone (róvos, tension), the tension line between two zones, formations, consocies, etc. 
ecballi'um, ('̌́ $\kappa \beta \alpha \lambda \lambda \omega$, cut down forests), a succession due to lumbering.

echard ( ${ }^{\prime} \chi \omega$, to withhold), the non-available water of the soil.

edobole (oìos, swelling), a plant whose seeds are scattered by propulsion through turgescence.

efficient difference, the amount of a physical factor necessary to produce a change in the response.

enauli'um ('̌vavios, hollow channel), a sanddıaw formation; enaulad, a sanddraw plant.

ende'mic ( $\boldsymbol{\epsilon} \nu$, within $\delta \hat{\eta} \mu o s$, district), occurring in a single formation, or natural region; ende'mism, the condition of growing in but one natural area.

epistrophe ('ं $\pi \dot{i}$, towards, $\sigma \tau \rho \circ \phi \eta \dot{\eta}$, a turning), the arrangement of the row of chloroplasts at right angles to the incident light.

eremi'um ( ${ }^{\prime} p \eta \mu o s$, desert), a desert formation; eremad, a desert plant.

estival, pertaining to summer.

-etum (locative suffix), suffix used to denote a consocies.

eugeogenous ( $\epsilon \hat{v}-$, well, $\gamma \hat{\eta}$, soil), weathering readily to form soil.

facies, a dominant species of a formation: a distinct area controlled by it is a consocies.

family, a group of individuals belonging to one species.

fixity, the condition characterized by little or no response to stimuli.

flavovirens, yellow green.

forewold, equivalent to the German "vorwald," the thicket zone bordering a forest.

-genous ( $\gamma^{\prime} v \omega$, to produce), producing.

geotome ( $\gamma \hat{\eta}$, earth, $\tau o \mu \dot{\eta}$, edge), an instrument for obtaining soil samples.

gloeospore ( $\gamma$ גoós, sticky stuff), a plant with viscid disseminules.

-graph ( $\gamma \rho \alpha \phi \eta^{\prime}$ a writing), combining term for a recording instrument.

gregarious (gregarius, grouped in herds), used of species in which the individuals occur in groups.

habitat, a definite physical area characterized by a formation; habitat form, the impress given the plant by the habitat.

harmosis (a $\tilde{a} \rho \mu \sigma \tau \iota s$, an adapting), response to stimuli, comprising both adjustment and adaptation.

hedi'um (๕̌̀os, a sitting, base), a succession in a residuary soil.

heliad ( $\ddot{\lambda} \iota$ os, sun), a heliophyte; heliophyll, the leaf of a sun plant; heliophyte, a sun plant; heliophytíum, a sun plant formation; heliophilous, sun-loving. 
heli'um (๕̈ $\lambda$ os, marsh), a marsh formation; helad, a marsh plant; helodi'um

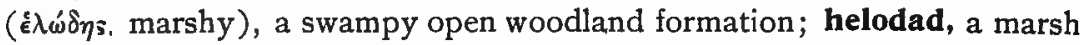
plant; helodrium ( $\delta$ pios, thicket), a thicket formation; helodrad, a plant of a marshy thicket; helohyli'um ( $\nu^{\prime} \lambda \eta$, forest) a marsh forest formation; helohylad, a marsh forest plant.

hepodoche ( $\boldsymbol{\epsilon} \pi \omega$, follow), a secondary succession. hizometer ( $I \zeta \omega$, to sink), an instrument for measuring gravitation water. holard (ö $\lambda$ os, whole), the total water-content of the soil.

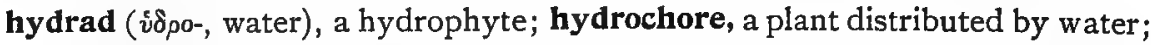
hydroharmose, response to water stimuli; hydrophyll, the leaf of a hydrophyte; hydrophyte, a water plant; hydrophyti'um, a water plant

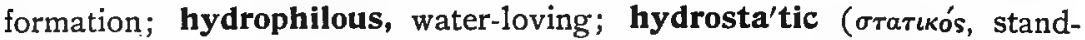
ing), completing the succession under hydrophytic conditions; hydrotropic ( $\tau \rho \circ \pi \iota \kappa o ́ s$, turning), applied to successions which become mesophytic. hygrome'tric (íypis, wet), measuring or absorbing water; hygroscopic ( $\sigma \kappa о \pi \epsilon \epsilon$, look), measurable only by a hygroscope; able to absorb moisture. hyli'um ( $\tilde{\nu} \lambda \eta$, forest), a forest formation; hylad, a forest plant; hylocolum,

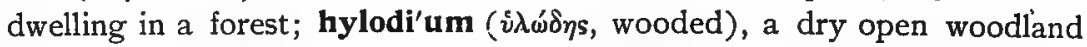
formation; hylodad, a plant of this formation; hylophyte, a forest plant. hypsi'um (vं\%os, elevation), a succession caused by elevation.

-ile (locative affix), suffix denoting a society.

immobile, without effective devices for migration.

indigenous (indigena, sprung from the land), native.

insolation, exposure to intense heat and light.

isabellinus, leather-colored.

isolation, separation by barriers.

isopho'tic ("loos, equal), equally illuminated; isophotophyll, a leaf in which both halves of the chlorenchym are alike, due to equal illumination.

-ium (-€̂́ov, locative affix), suffix denoting a formation.

labile, plastic, easily modified.

lauri'um ( $\lambda a v ́ p a$, drain), a drain formation; laurad, a drain plant. limni'um ( $\lambda^{\prime}(\mu \nu \eta$, lake), a lake formation; limnad, a lake plant; limnodium

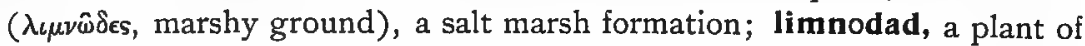
a salt marsh.

lochmi'um ( $\lambda o_{\chi} \chi \eta$, thicket), a thicket formation; lochmad, a thicket plant. lophi'um ( $\lambda$ ó spore, a plant with plumose disseminules.

mastigospore ( $\mu \alpha \dot{\sigma} \sigma \tau \iota \xi, \iota \gamma \circ s$, lash), a plant with ciliate or flagellate disseminules. melleus, honey-colored. 
meridian, used chiefly as a synonym for apparent noon; also an imaginary line of longitude.

mesad ( $\mu$ ́́бos, middle), a mesophyte; mesophilous, growing in moist soils; mesophyll, the leaf of a mesophyte; mesophyte, a plant of moist soils; mesophyti'um, a mesophytic formation; mesosta'tic (orarıkís, standing), completing the succession under mesophytic conditions; mesotro'pic ( $\tau \rho \circ \pi$ เós, turning), applied to successions which become mesophytic. -meter ( $\mu \dot{\epsilon} \tau \rho o \nu$, measure), combining term for instrument. micti'um ( $\mu \kappa \tau o ́ v$, mixture), a mixed formation. migrant, a plant that is migrating or invading.

migration (migratio, removal), the movement of plants into new areas; migration circle, a circle employed to measure migration.

mobile, able to be moved, i. e., modified for migration.

monochronic ( $\mu$ '́vos, single, xpóvos, time), arising but once; monogenesis ( $\gamma^{\prime}$ verıs, origin), the origin of a new form at a single place or time; monophyle'sis ( $\phi \hat{v} \lambda \_v$, race), origin from a single ancestral type; monoto'pic (то́тоs, place), arising at one place only.

motile, able to move by growth, by means of cilia, etc.

mutable, able to produce mutants; mutant, a form arising by mutation; mutation, the sudden appearance of new forms.

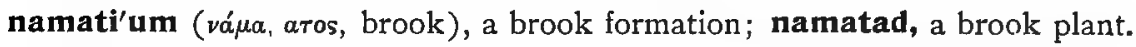
nomi'um (vopós, pasture), a pasture formation; nomad, a pasture plant.

occupation, possession of the ground by plants.

oceani'um ('ُאєavós, ocean), an ocean formation; oceanad, an ocean plant; oceanophyte, an ocean plant; oceanophilous, ocean-dwelling. ocheti'um (óxєrós, drain), a succession due to artificial drainage. ochroleucus, yellowish white. ochthi'um (ö $\chi \not \eta$, bank), a bank formation; ochthad, a bank plant.

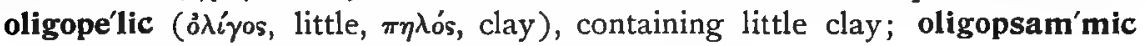
( $\psi a ́ \mu \mu o s$, sand), containing little sand.

olisthi'um (öㅅ $\sigma \theta 0$, slip), a succession in a landslip. ombrometer (o $\mu \beta$ pos, a rainstorm), a rain gauge. -on (-ẃv, locative suffix), suffix used to denote a family. oncospore (ŏ $\gamma \kappa o s$, hook), a plant with hooked disseminules. orgadi'um (ỏpyás, áóos, meadowland partially wooded), an open woodland - formation; orgadad, an open woodland plant. orophyti'um (öpos, mounta'n), a subalpine plant formation. oxodi'um ( $o \xi \omega ́ \delta \eta \xi$, sour), a humus marsh formation; oxodad, a plant of a humus marsh. 
pagi'um ( $\pi a ́ \gamma o s$, rocky hill, glacier), a succession in a glacial soil; pagophyti'um, a foothill plant formation.

pediophyti'um ( $\pi \epsilon \delta i o v$, plain), an upland plant formation.

pelagi'um ( $\pi$ élayos, surlace of the sea), a surface sea formation; pelagad, a plant of the sea surface.

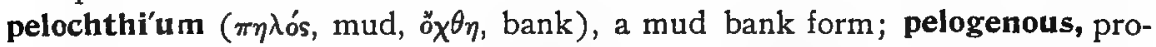
ducing clay; pelopsammic ( $\psi a ́ \mu \mu o s$, sand), composed of mixed clay and sand; pelopsammogenous, producing clay and sand.

permobile, extremely mobile.

perquadrat, a quadrat of $\mathrm{i} 6$ square meters or more.

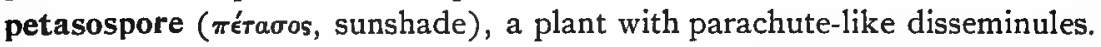

petrium ( $\pi$ ḱтрa, rock, stone), a rock formation; petrad, a rock plant; petroch thi'um (ö $\chi \theta \eta$, bank), a rock bank formation.

petrodi'um ( $\pi \epsilon \tau \rho \omega ́ \delta \eta \xi$, abounding in boulders), a boulder field formation; petrodad, a plant of a boulder field.

phelli'um ( $\phi \epsilon \lambda \lambda \epsilon v^{\prime}$, stony ground), a rock field formation; phellad, a rock field plant.

-philous ( $\phi i ́ \lambda o s)$, loving, dwelling in.

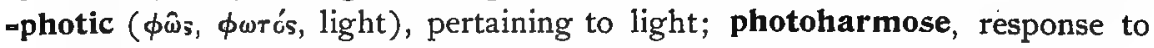

light stimuli; photometer, an instrument for measuring light.

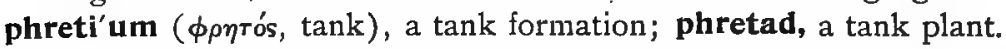

phyad ( $\phi v \eta^{\prime}$, form of growth), a vegetation form, e. g., tree, shrub, etc.

-phyll ( $\phi u ́ \lambda \lambda o v$, leaf), combining term for leaf.

-phyte ( $\phi v \tau_{o} v$, plant), combining term denoting plant; phyteris (

plant competition; =phyti'um ( $\phi u \tau e \hat{o} o v$, place covered with plants), com-

bining term for formation; phytostrote, a species migrating by means of the plant body.

pladobole ( $\pi \lambda a ́$ óos, moisture), a plant whose seeds are scattered by propulsion due to moisture.

plasticity. the condition characterized by ready response to stimuli. pnoi'um ( $\pi \nu v^{\prime}$, blast), a succession in an aeolian soil.

poi'um (róa, meadow), meadow formation; poad, a meadow plant; poophyte, a meadow plant.

polyan'thous ( $\pi 0 \lambda$ ús, many, aै $v \theta 0 s$, flower), producing many flowers; polychrónic ( $\chi$ p'vos, time), arising at two or more times; polyde'mic ( $\delta \hat{\eta} \mu \circ$, district), occurring in two or more formations or natural regions; polygenesis ( $\gamma^{\prime} v \epsilon \iota \iota s$, origin), the origin of a new form at two or more places or times; polyphyle'sis ( $\phi \hat{v} \lambda o v$, race), the origin of a form, species, or genus from two or more ancestral types; polyspermatous ( $\sigma \pi \dot{\epsilon}^{\prime} \rho \mu a$, seed),

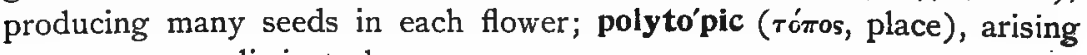
at two or more distinct places. 
ponti'um ( $\pi$ ćvтos, deep sea), a deep sea formation.

potami'um (потано́, river), a river formation; potamad, a river plant. potometer ( $\pi$ oróv, drink), an instrument for measuring absorption.

prevernal, pertaining to early spring.

prior, earlier, used of alpine aspects.

prochosi'um ( $\pi \rho{ }^{\prime} \chi \omega \sigma \iota 5$, a deposition of mud), a succession in an alluvial soil. prodophyti'um ( $\pi \rho o_{o \delta} o s$, pioneer), an initial formation.

protodoche ( $\pi \rho \hat{\omega} \tau o s$, first), a primary succession.

proximity (proximitas, nearness), nearness to the area invaded.

psamathi'um ( $\psi \alpha$ á $\theta_{0}$ os, sand of the seashore), a strand formation; psamathad,

a strand plant; psammogenous ( $\psi$ áumos, sand), producing a sandy soil.

psili'um ( $\psi \lambda \lambda a ́$, land without trees), a prairie formation; psilad, a prairie plant.

psychrometer ( $\psi v x$ pós, chill), an instrument that measures humidity by means of a fall in temperature; psychrograph, a psychrometer that records automatically.

ptenophyti'um ( $\pi \tau \eta v o ́ s$, passing), an intermediate formation.

pterospore ( $\pi \tau \epsilon \rho o ́ v$, wing), a plant with winged disseminules.

purpureus, purple.

pycnophyti'um ( $\pi v \kappa \nu o ́ s$, thick), a closed formation.

pyríum ( $\pi \hat{v} \rho$, fire), a burn succession.

quadrat (quadratum, a square), a square meter of vegetation marked off for counting, mapping, etc.; major, a quadrat of 2-I4 square meters.

reaction, the effect of the formation upon the habitat.

relict (relictus, left), a species belonging properly to an earlier type of succession than the one in which it is found.

repi um ( $\rho ́ \epsilon \omega, \operatorname{sink}$ ), a succession due to subsidence.

rhoi'um ( óos, stream), a creek formation; rhoad, a creek plant.

rhoptometer ( $\rho \circ \pi \tau o ́ v$, something absorbed), an instrument to measure absorption of water by the soil.

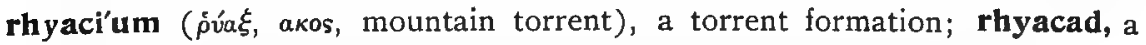
torrent plant.

rhysi'um ( $\dot{\rho} v \sigma i s$, a flowing of fire), a succession due to volcanic action.

ruber, red.

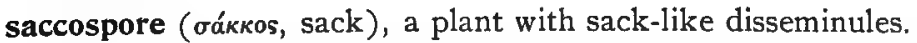

sarcospore ( $\sigma a ́ \rho \xi \xi$ баркós, flesh), a plant with fleshy disseminules.

sciad ( $\sigma \kappa \iota \dot{\alpha}$, shade), a sciophyte; sciophyll, the leaf of a shade plant; sciophyte, a shade plant; sciophyti'um, a shade plant formation; sciophilous, shade-loving. 


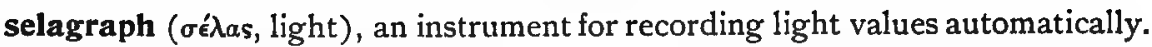
serotinal, late, pertaining to autumn.

social, used of plants in which the individuals are compactly grouped; exclusive, excluding individuals of ot her species; inclusive, permitting the entrance of individuals of other species.

society, a subdivision of the formation, characterized by a principal species. sparse, scattered singly.

spermatostrote ( $\sigma \pi \epsilon^{\prime} \rho \mu \alpha, a \tau o s$, seed), a plant migrating by means of seeds.

sphyri'um ( $\sigma \phi v i p o v$, ankle, talus), a succession in a talus soil.

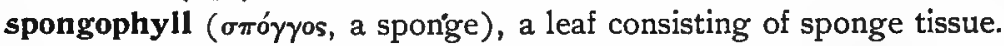

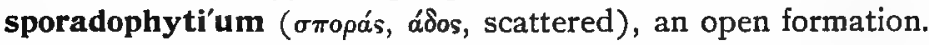

-spore ( $\sigma \pi 0 p a ́$, seed, fruit), combining term for migration contrivance; sporostrote, a plant migrating by means of spores.

stability, the condition in which the plant makes little or no response.

stabilization, the tendency typical of succession, in which the successive stages become more stable.

stasi'um ( $\sigma \tau a ́ \sigma \iota s$, a standing), a stagnant pool formation; stasad, a plant of stagnant water.

staurophyll (бravpós, a pale), a leaf consisting of palisade tissue.

sterrhi'um ( $\sigma \tau \epsilon \rho p o ́ s$, barren), a moor formation; sterrhad, a moor plant.

-strote ( $\sigma \tau$ púros, strewn), combining term for means of migration.

subcopious, scattered somewhat loosely.

subgregarious, arranged in loose groups.

subquadrat, a quadrat of $I-8$ decimeters.

succession, complete and continuous or repeated invasion, in consequence of which formations succeed each other.

symmetry, used of topography when it shows uniform changes; radial, a condition in which the different areas are concentric; bilateral, where the areas occur in two similar rows.

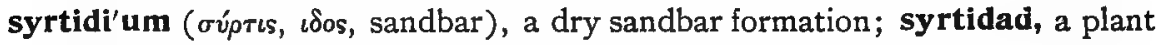
of a dry sandbar.

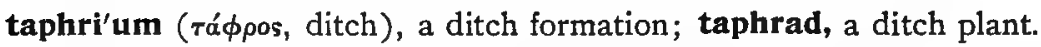

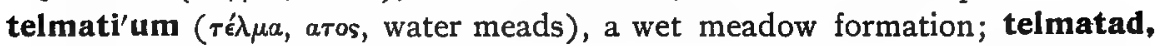
a wet meadow plant.

testaceus, pale brick colored.

thalassi'um ( $\theta \dot{\alpha} \lambda \alpha \sigma \sigma a$, sea), a sea formation; thalassad, a sea plant. thallostrote ( $\theta a \lambda \lambda o ́ s$, shoot), a species migrating by means of offshoots. theri'um ( $\theta \dot{\eta} \rho$, wild animal), a succession due to animals.

thermi'um ( $\theta^{\prime} \rho \mu \eta$, hot spring), a hot spring formation; thermad, a hot spring plant. 
thini'um ( $\theta i_{s}^{\prime}, \theta t v o$ s, a dune), a dune formation; thinad, a dune plant.

tiphi'um ( $\tau \hat{\imath} \phi o s$, pool), a pool formation; tiphad, a pond plant.

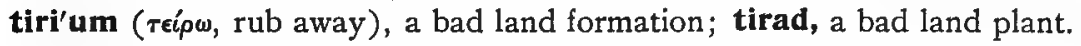

tonobole ( (óvos, tension), a plant whose seeds are scattered by projection from calyx or involucre.

transect (transeclus, cut through), a cross-section of vegetation.

trechometer ( $\tau \rho \rho^{\prime} \chi \omega$, to run off), an instrument for measuring run-off.

tribi'um ( $\tau \beta^{\prime} \beta \omega$, wear or rub away), a succession in an eroded soil.

umbrinus, umber.

variable, able to produce variants; variant, a form arising from origin by variation; variation, the origin of new forms by the action of selection upon minute differences.

vegetation form, a characteristic plant form, e..g., tree, rosette, etc.

vernal, pertaining to spring.

vicine (vicinus, neighboring), invading from adjacent formations.

viridis, green.

vixgregarious, arranged in small or indistinct groups.

water-content, the water of the soil or habitat; physiological, the available soil water; physical, the total amount of soil water.

xenodoche ( $\xi$ évos, strange), an anomalous succession.

xerad ( $\xi \eta p o ́ s, d r y)$, a xerophyte; xerasi'um ( $\xi \eta p a \sigma i a$, drought), a succession due to drainage or drought; xeriobole ( $\xi \eta p i a$, dryness), a plant whose seeds are scattered by dehiscence due to dryness; xerohyli'um ( $\tilde{\nu} \lambda \eta$, forest), a dry forest formation; xerohylad, a dry forest plant; xerophyll, the leaf of a xerophyte; xerophyte, a dry soil plant; xerophyti'um, a xerophytic formation; xerophilous, dwelling in a dry habitat; xero-

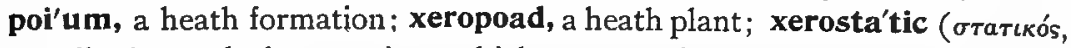
standing), used of successions which are completed under xerophytic conditions; xerotro'pic ( тротскós, turning), applied to successions which become xerophytic.

zonation, that condition in which plant groups or formations appear in belts or zones.

zone, a belt of more or less uniform vegetation.

zoochore ( $\zeta \omega$ ov, animal), a plant distributed by animals. 


\section{BIBLIOGRAPHY}

Ascherson, $P$.

Vón cler Verbrcitung der Pflanzen. Leunis Synopsis, I :724. 1883.

BACHMANN, E. T.

Darstellung der Entwicklungsgeschichte und des Baues der Samenschale der Scrofularineen. Just '8I :494. I88I.

BERG, E. v.

Das Verdrängen der Laubwälder durch die Fichte und Kiefer. Grise- bach Berichte, I844: I5. I844.

BIEIRG, I. J.

Oeconomia Naturae. Amoen. Acad., 4:I. I749.

Blytt, A.

Ueber zwei Kalktuffbildungen in Gudbrandsclalen. Engler Jahrb., I6:bi. I 892.

Essay on the Immigration of the Norwegian Flora. Just '76:693. 1876.

Brisseau-Mirbel, C. F.

Elémens de Physiologie Végétale et de Botanique. I8I5.

BuCH, C. L. v.

Allgemeine Uebersicht der Flora auf den Canarischen Inseln. I8I9.

Buchenau, F.

Monographie der Juncaceen. Engler Jahrb., I :I24. I88r.

BUCHWALD, J.

Die Verbreitungsmittel der Leguminosen der tropischen Afrika. Engler Jahrb., I9:494. I 893 .

Burgerstein, A.

Die Transpiration der Pflanzen. I9O4.

CALLmé, A.

Om de nybildade Hjelmaröarnes vegetation. Bihang Sv. Vet. Akad. Handl., XII. I887.

Clements, E. S.

The Relation of Leaf Structure to Physical Factors. Trans. Am. Micro. Soc., 25. 1905 . 
Clements, F. E.

Peculiar Zonal Formations of the Great Plains. Am. Nat., 3I :968. I897.

A System of Nomenclature for Phytogeography. Engler Jahrb., 3I : bi. 1902.

Greek and Latin in Biological Nomenclature. Uni. Nebr. Studies, 3 :I. r.902.

The Development and Structure of Vegetation. Rep. Bot. Surv. Nebr., 7. 1904.

Formation and Succession Herbaria. Uni. Nebr. Studies, 4:329. I904. Ciements, F. E. and E. S.

Herbaria Formationum Coloradensium. Igo2.

Contejean, C.

De l'influence du terrain sur la végétation. Ann. Nat. Sci., 5 :20:266. I874.

Costantin, J.

Accommodation des plantes aux climats froids et chauds. Bull. Soc. Bot. Fr., 3 I :490. 1897.

Coville, F. V.

Botany of the Death Valley Expedition. I6. I893.

Cowles, H. C.

The Ecological Relations of the Sanddunes of Lake Michigan. Bot. Gaz., 27 :95. I899.

The Physiographic Ecology of Chicago and Vicinity. Bot. Gaz., 3 I :73. I901.

DAMMER, U.

Die Verbreitungsausrüstungen der Polygonaceen. Engler Jahrb., I5: 260. 1893 .

Darwin, C.

Origin of Species. I859.

De Candollee, A.

Géographie Botanique Raisonnée. I855.

Constitution dans le regne végétal de groupes physiologiques applicables à la géographie ancienne et moderne. Arch. Sci. Phys. Nat., I874:5. I874.

Sur les causes de l'inegale distríbution des plantes rares dans la chaîne des Alpes. Just 3:663. 1875 . 
De Candolle, A. P.

Essai Elémentaire de Géographie Botanique. 1820.

Physiologie. 1832.

Delpino, F.

Studi di geografia botanica secondo un nuovo indirizzo. Mem. R. Acc. Sci., $5: 7: 329$. 1898.

DEVRIES, $\mathrm{H}$.

Die Mutationstheorie. IgOI.

Dingler, $\mathrm{H}$.

Die Bewegung der pflanzlichen Flugorgane. I889.

Douglas, $R$.

Succession of Forest Growth. Gard. Chron. '89:Io. Just '89:I72. I889.

DRUde, O.

Anwendung physiologischer Gesetze zur Erklärung Vegetationslinien. Just $4: 674 . \quad \mathbf{1} 876$.

Die Vertheilung und Zusammensetzung östlicher Pflanzengenossenschaften in der Uimgebung von Dresden. Isis. I885.

Die'systematische und geographische Anordnung der Phanerogamen. Schenck Handbuch, 3:487. 1886.

Atlas der Pflanzenverbreitung. I887.

Ueber die Principien in der Unterscheidung von Vegetationsformationen. Engler Jahrb., II :2I. I889.

Handbuch der Ptlanzengeographie. I890.

Deutschlands Pflanzengeographie. I896.

Der Hercynische Florenbezirk. I902.

Elmore, C. J.

Flora of a Dried-up Millpond. Proc. Nebr. Acad. Sci., $7: 29,69 . \quad$ rgor.

ENGLER, A.

Verstıch einer Entwicklungsgeschichte der Pflanzenwelt. 1879.

ENGLeR \& DRUDE

Die Vegetation der Erde. Engler Jahrb., I7 :b55. 1893.

EwaikT, A. J.

On the Physics and Physiology of Protoplasmic Streaming in Plants. 1903 . 
Fenzl, E.

Versuch einer Darstellung der geographischen Verbreitungs- und Vertheilungsverhältnisse der natürlichen Familien der Alsineen. I833.

Fratault, C. '

Les zones botaniques dans le Bas-Languedoc et les pays voisins. Bull. Bot. Soc. Fr., 40:36. 1893 .

Flahault, C., \& Combres, P.

Sur la flore de la Camargue. Bull. Soc. Bot. Fr., 4I :37. I894.

Fliche, M. P.

Un reboisement, étude botanique et forestière. Engler Jahrb., I I :I04. I 888 .

GarN, E.

Recherches sur le role physiologique de l'eau dans la végétation. Ann. Nat. Sci., $7: 20: 63$. 1895 .

GerhardT, J.

Handbutch des deutschen Dünenbaues. Igoo.

Goebel, K.

Pflanzenbiologische Schilderungen II. I892.

Organography of Plants. English edition. Igoo.

Goeze, E.

Pflanzengeographie. I882.

Graebner, P.

Studien, über die norddeutschen Heide. Engler Jahrb., 30:500. I895.

Ueber die Bildung natürlichen Vegetationsformationen in norddeutschen

Flachlande. Bot. Cent., $77: 212 . \quad$ I 898.

Gliederung der Westpreussischen Vegetationsformationen. Schrift. Nat. Ges. Danzig, 9:43. I898.

Die Heide Norddeutschlands. I902.

Grevillius, A. Y.

Om vegetationens utveckling pa de nybildade Hjelmaröarne. Bot. Cent. Beih., 5:36. I89?.

Studier öfver vegetationens sammansättning. Sver. Geol. Unders., I44. 1895.

Grisebach, A

Bericht über die Leistungen in der Pflanzengeographie. I843.

Die Vegetation der Erde. I872. 
Groenlund, C.

Planteväxten paa Island. Nat. For. Festschr. I884-90.

Haeerlandt, G.

Physiologische Pflanzenanatomie. I904.

Hansgirg, A.

Phyllobiologie. I903.

Henenberg, A.

Stationes Plantarum. Amoen. Acad., $4: 64$. I754.

Hegel meyer, F.

Ueber Bau und Entwickelung einiger Cuticulargebilde. Pringsheim Jahrb., $9: 286 . \quad$ I 873.

Henfrey, A.

The Vegetation of Europe. 1852.

Hensed, E. P.

An Investigation of the Movements of Petals. Uni. Nebr. Studies, 5 . I905.

Hesselmann, $\mathrm{H}$.

Zur Kenntnis des Pflanzenlebens schwedischer Laubwiesen. Beih. Bot. Cent., I8:3II. I904.

Hildebrand, F.

Die Schleuderfrüchte und ihr in anatomischen Bau begründeter Mechanismus. Pringsheim Jahrb., 9:235. 1873.

Der Verbreitungsmittel der Pflanzen. Just '73:224. I873.

Die Lebensdauer und Vegetationsweise. Engler Jahrb., 2:5I. Just '8I :302.' I88I.

Hildebrandt, $\mathrm{H}$.

Einige Beobachtungen über den Witterungs-Einfluss auf die Lebensdauer und Vegetationsweise der Pflanzen. Engler Jahrb., 4:I. 1883 .

HILL

Reise in Sibirien. 1858.

HoffmanN, $\mathrm{H}$.

Ueber Accommodation. Just '76:952. 1876.

Der nördliche Ural und das Küstengebirge Pae-Choi. $\quad$ I856. 
Hult, R.

Försok till analytisk behandlung af formationerna. Medd. Soc. Fenn., 8. $188 \mathrm{I}$.

Blekinges vegetation. Just '85:312. I885.

Die alpinen Pflanzenformationen des nördlichstens Finnlands. Engler Jahrb., II :b6I. I887.

Moosfloran i traktan mellan Aavasaksa och Pallastunturi. Engler Jahrb., 8 :bi. I887.

Humboldt, A. v.

Essai sur la Géographie des Plantes. I805.

Views of Nature. I848.

Humboldt, A. v., \& Bonpland, A.

Tableau Physique des Régions Equatoriales. I805.

Huтh, E.

Die Klettpflanzen mit besonderer Berüchsichtigung ihrer Verbreitung durch Tiere. Just '87:432. 1887.

JACCARD, $P$.

Étude Comparative de la Distribution Florale dans une portion des Alpes et du Jura. Bull Soc. Vaud., 37:547. Igor.

Gesetze der Pflanzenvertheilung in der alpinen Region. Flora, 90:349. I902.

KABSCH, W.

Das Pflanzenleben der Erde. I865.

KeRNER, A.

Das Pflanzenleben der Donauländer. I863.

Pflanzenleben. I888, I89I.

KimLMANN, A. O.

Pflanzenbiologische Studien aus Russisch-Lappland. I890.

KLINGE, J.

Ueber den Einfluss der mittleren Windrichtung auf das Verwachsen der Gewässer. Engler Jahrb., II :264. 1890.

KOEPPEN, W.

Die Wärmezonen der Erde. I :2I 5. I884.

Korschinsky, S.

Ueber die Entstehung und das Schicksal der Eichenwälder in mittleren

Russland. Engler Jahrb., I3:47r. I89r. 
Krasan, F.

Ueber die Bedeutung der gegenwärtigen Verticalzonen der Pflanzen, etc. Engler Jahrb., $4: 266$. I883.

Krassnotr, A.

Bemerkungen über die Vegetation des Altai. Engler Jahrb., 9:53. 1888.

Krause, E. H. L.

Beitrag zur Geschichte der Wiesenflora in Norddeutschland. Engler Jahrb., I5:387. I892.

Die Heide. Engler Jahrb., I4:5I5. I892.

Kurtz, F.

Die Flora des Chilcatgebietes im südostlichen Alaska. Engler Jahrb., I9:327. I895.

LEGrand, A.

Statistique Botanique de Ferez. Ann. Soc. Agr. Loire, 17:290. 1873.

Link, H. F.

Die Urwelt und das Alterthum. I820.

LINNÉ, C. v.

Flora Suecica. I745.

Philosophia Botanica. I75I.

LOHDE, G.

Ueber die Entwicklungsgeschichte und den Bau einiger Samenschalen. Just $2: 44$ I. I 874 .

Mac Millan, C.

Metaspermae of the Minnesota Valley. I892.

On the Occurrence of Sphagnum Atolls in Central Minnesota. Minn. Bot. Studies, I :I. I894.

On the Formation of Circular Muskeag in Tamarack Swamps. Torr. Bull., $23: 500 . \quad$ I896.

Observations on the Distribution of Plants along Shore at Lake of the

Woods. Minn. Bot. Studies, I :968. 1897.

Maginin, A.

Recherches sur lia végétation des lacs du Jura. Rev. Gen. Bot., 5 :24I, 303, 515. 1893 .

Martoth, R.

Ueber mechanische Schutzmittel der Samen gegen schädliche Einflüsse von aussen. Engler Jahrb., 4:247. I883. 
Meigen, F.

Beobachtungen ïber Formationsfolge im Kaiserstuhl. Deut. Bot. Monat., I8:I45. I900.

Beobachtungen über Formationsfolge bei Freyburg a. d. Unstrut. Deut. Bot. Monat., I3. I89'5.

Die erste Pflanzenansiedelung auf den Reblausherde, ib.

Die Besiedlung der Reblausherde in der Provinz Sachsen. Engler Jahrb., 21 :21.2. I8g6.

MERRIL.L, G. P.

Rocks, Rock-weathering and Soils. I897.

Meyen, F. J. F.

Grundriss der Pflanzengeographie. 1836.

MiddendorfF, A.

Die Gewächse Sibiriens. I864.

Mueller, P. E.

Bjärgfyrren (Pinus montana) Tidskr. pop. Fremst. I87r.

NaEgeli, C.

Das gesellschaftliches Entstehen neuer Species. Bot Mitth., 3:365. I873.

Verdrängung der Pflanzenformen durch ihrer Mitbewerber. Bot. Mitth., 3:205. I874.

NiLsson, A.

Om örtrika barrskogar. Tids. Skog., 93. 1896.

Nagra drag ur de svenska växtamhällenas utvecklingshistoria. I899. Bot. Cent. Beih., 9:370. I900.

Nilsson, N. H.

Einiges über die Biologie der schwedischen Sumpfpfianzen. Bot. Cent., 76 :9. $\quad$ I898.

Palacky, J.

Pflanzengeographische Studien. Just '89:160. I864.

Pfeffer, W.

Pflanzenphysiologie. I, I897; II, I904.

Pieters, A. J.

The Plants of Lake St. Clair. Bull. Mich. Fish Com. I894. 
Pound, R., \& Clements, F. E.

The Phytogeography of Nebraska. 1898. 2d ed. 1900.

A Method of Determining the Abundance of Secondary Species. Minn. Jot. Studies, $2: 19 . \quad 1898$.

The Vegetation Regions of the Prairie Province. Bot. Gaz., 25:381. I898.

Ramond, L. F. E.

Voyages au Mont-Perdu et dans la partie adjacente des Haut-Pyrénées. I 8 OI.

Rauniatar, C.

Vesterhavets Ost- og Sydkysts Vegetation. Bot. Cent., 4I :36I. I889. Saccardo, P. A.

Chromotaxia seu Nomenclator Colorum. r891:

SACHS, J.

Abhandlungen ïber Pflanzenphysiologie. 1892.

SCIJMPER, A. F. W.

Ueber Schutzmittel des Laubes gegen Transpiration. Monatsb. Akad. Berl., 7. I890.

Die indo-malayische Strandflora. I891.

Pflanzengeographie auf physiologischer Grundlage. $\quad$ I898.

Schouw, J. P.

Grundzüge einer Allgemeinen Pflanzengeographie. $\quad$ I823

Sendtner, O.

Die Vegetationsverhältnisse des Bayerischen Waldes. I860.

Senft, F.

Der Erdboden. Just '88:45. I888.

SERnANDER, R.

Die Einwanderung der Fichte in Skandinavien. Engler Jahrb., I5:I. I893:

SERNANDER, R., \& KI ELLMARK, K.

Eine Torfmoor Untersuchung aus dem nördlichen Nerike. Bull. Geol. Inst. Ups., 2 :4. 1895.

SOLEREDER, $\mathrm{H}$.

Systematische Anatomie der Dicotyledonen. I899.

Stebjer, F. G., \& Schroeter, C.

Beiträge zur Kenntniss der Matten und Weiden der Schweiz. Engler Jahrb., I7:bi7. 1893 . 
Steenstrup, J.

Geognostisk-geologisk -Undersögelse i det nordlige Själland. I842.

Stossner, A.

Vegetationsvcrhältnisse von Annaberg und Umgebung. I859.

Swartz, O.

Observationes Botanicæ. I79I.

TANFILjEw, G.

Ueber die Sümpfe des Gouv. Petersburgs. Engler Jahrb., 24:68. I 889 .

THORNBER, J. J.

The Prairiegrass Formation in Region l. Rep. Bot. Surv. Nebr., $5: 29$. I90I.

Thurmann, $T$.

Essai de phytostatique appliquée à la chaine du Jura et aux contrées voisines. $\mathrm{I} 849$.

TOURnEForT, J. P.

Relation d'un Voyage du Levant. I7x 7.

Treub, M.

Notice sur la nouvelle flore de Krakatau. Ann. Jard. Buit., 7. I888.

Treviranus, G. R.

Biologie. I802.

Vaupell, C.

De Nordsjaellandske Skovmoser. I851.

VINES, S. H.

Lectures on the Physiology of Plants. I886.

Wahlenßerg, G.

De Vegetatione et Climate Helvetia Septentrionalis. I8rз.

WARMING, E.

Ueber Sprossbau, Ueberwinterung und Verjüngung. Engler Jahrb., $5: 56$. I 884 .

Fra Vesterhavskystens Marskegne. Vid. Medd. Foren. I89o.

De psammofile Vegetationer in Danmark. Vid. Medd. Foren. I89I.

Plantesamfund, Grundtrok af den oekologiske Plantegeografi. I 895 .

WATSON, H. L.

Cybele Britannica: or, British plants and their geographical relations. 1847. 
WEBER, C.

Ueber die Zusammensetzung des natürlichen Graslandes. Engler Jahrb., $16: b 56 . \quad 1892$.

Ueber Veränderungen in der Vegetation der Hochmoore unter dem Einflusse der Cultur. Eng:er Jahrb., 23:bi5. I894.

Ueber die Erhaltung von Mooren und Heiden Norddeutschlands in Naturzustande. Abh. Nat. Ver. Bremen, I5:263. Igor.

Ueber die Vegetation und Entstehung des Hochmoores von Augstumal. I902.

WhitFoRd, H. N.

The Genetic Development of the Forests of Northern Michigan. Bot. Gaz., 31 :289. Igor.

WhITNEY, M. D.

Instructions to Field Parties and Descriptions of Soil Types. I903.

WIESNER, J.

Biologie der Pflanzen. I889.

WILLDENOW, K. L.

Allgemeine Bemerkungen über den Unterschied der Vegetation auf der nördlichen und südlichen Halblkugel der Erde. I8II.

WILLKOMLM, M.

Grundzüge der Pflanzenverbreitung anf der Iberischen Halbinsel. I896. WоJеIKоW, A.

Die Einwirkung des Menschen auf die Natur. Engler Jahrb., 24:69. I 894 .

ZIMMER,

Ueber Pflanzenwanderungen. $187 \mathrm{x}$. 





